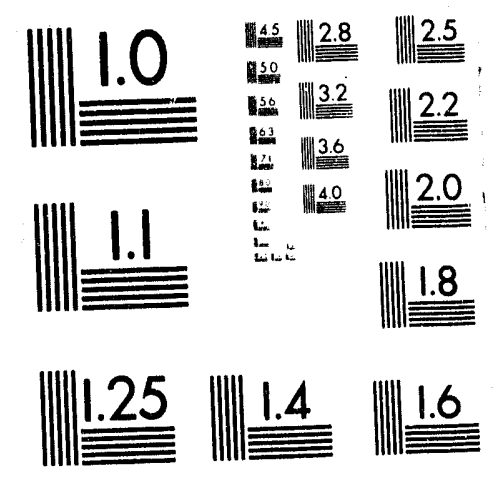



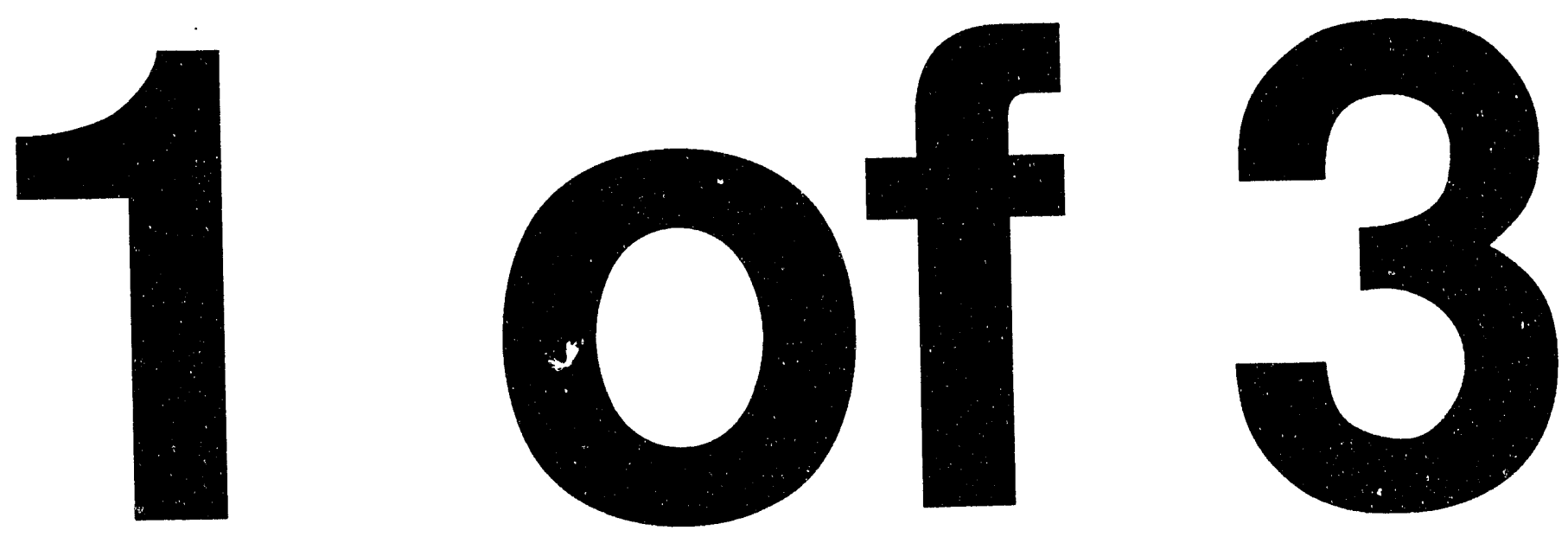
WHC-EP-0727

UC-516

\title{
Processing of FENDL-PA/1.1
}

\author{
F. M. Mann \\ D. E. Lessor \\ L. L. Carter
}

Date Published

February 1994

Prepared for the U.S. Department of Energy Assistant Secretary for Energy Research

\footnotetext{
(2) Westinghouse Hanford Company Richland, Washington 99352

Hanford Operations and Engineering Contractor for the

U.S. Department of Energy under Contract DE-AC06-87RL10930
}

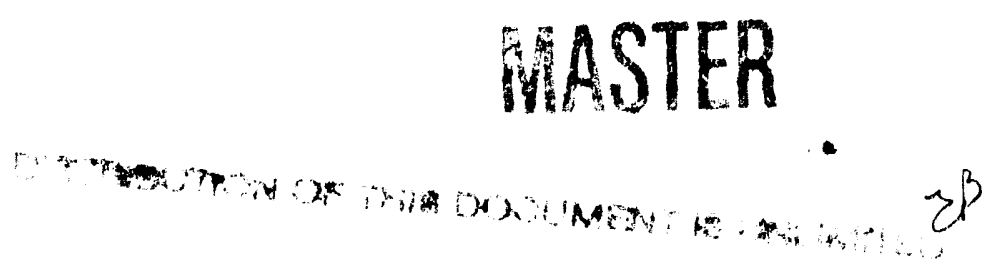

Approved for Public Release 


\section{RELEASE AUTHORIZATION}

Document Number: WHC-EP-0727

Document Title: Processing of FENDL-PA/1.1

Release Date: $\quad$ February 10, 1994

$* * * * * * * * * * * * *$

This document was reviewed following the procedures described in WHC-CM-3-4 and is:

APPROVED FOR PUBLIC RELEASE

$* * * * * * * * * * * * *$

WHC Information Release Administration Specialist: MN Boston

M. Bostan

February 10, 1994 
Document Title: Processing of FENDL-PA/1.1

Prepared by :

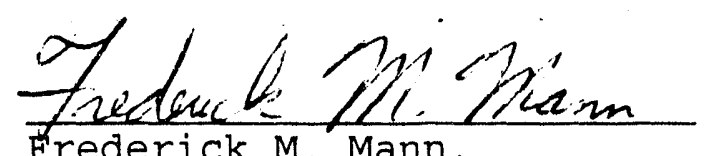

Frederick M. Mann,

Fellow Scientist

Environmental Risk \& Performance

Assessment

Prepared by :

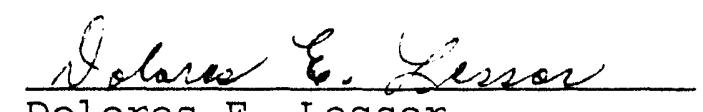

Dolores E. Lessor,

Engineering Technician

Radiation Physics and Shielding

$\frac{12 / 27 / 93}{\text { Date }}$

Prepared by:

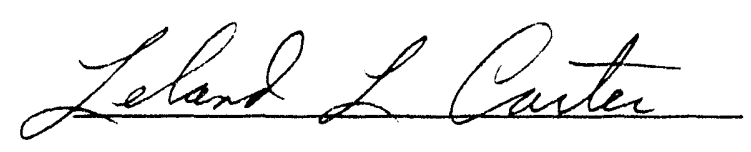

Leland I. Carter,

Fellow Engineer

Radiation Physics and Shielding

$\frac{12 / 27193}{\text { Date }}$

$12 / 28 / 93$

Date

Approved by :

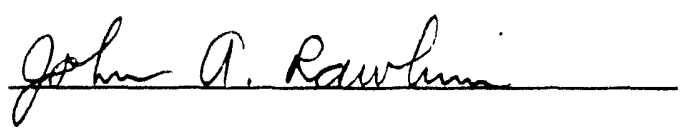

John W. Rawlins, Manager

Environmental Risk \& Performance Assessment 
WHC-EP-0727

This page intentionally left blank. 


\section{CONTENTS}

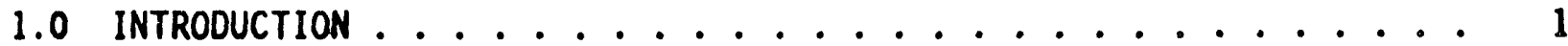

2.0 PROCESSING ....................... 1 2.1 FILE RETRIEVAL ..................

2.2 CONTINUOUS ENERGY MCNP FORMAT $\ldots \ldots \ldots \ldots \ldots$

2.3 MULTIGROUP REAC FORMAT $\ldots \ldots \ldots \ldots \ldots$

3.0 VERIFICATION

3.1 CONTINUOUS ENERGY MCNP FORMAT $\ldots \ldots \ldots \ldots \ldots \ldots \ldots \ldots$

4.0 AvaIlable filles $\ldots \ldots \ldots \ldots$

5.0 REFERENCES ..................... 5

\section{LIST OF TABLES}

1175 Group Energy Structure . . . . . . . . . . . . . 3

2 Location of filles .................. 4 
This page intentionally left blank. 


\section{PROCESSING OF FENDL-PA/1.1}

\subsection{INTRODUCTION}

- The Fusion Evaluated Nuclear Data Library (FENDL) is an evaluated nuclear data library based upon the best evaluations from the world community. The library is maintained by the Nuclear Data Section of the International Atomic Energy Agency (IAEA). However, before the data can be used in computer codes, it must be processed into the appropriate format.

The library containing the transmutation (or activation) cross sections that are part of FENDL is designated as FENDL-PA ( $P$ designating pointwise). The evaluations in Version 1.1 of this activation library were selected in the following two steps.

1. For the most important reactions ( 250$)$, consultants to the IAEA compared evaluations submitted for consideration with experimental data. These evaluations included those from ENDF/B, JEF, BROND, JENDL, as well as "complete" activation libraries, such as EAF and REAC. The consultants then chose the "best" evaluation for each of the reactions.

2. The evaluations for the remaining $(\sim 12,000)$ reactions were taken from EAF-3 (Reference 1).

The pointwise FENDL-PA/1.1 activation library was processed into two formats.

1. Continuous energy format as used by the Monte Carlo neutron/photon transport code MCNP (Reference 2).

2. ASCII 175 group multigroup format as used by the transmutation code REAC $* 2 / 3$ (Reference 3 ).

\subsection{PROCESSING}

\subsection{FILE RETRIEVAL}

FENDL-PA/1.1 was transferred over INTERNET from the Nuclear Data Section using FTP.

The list of reactions processed is displayed in Appendix A.

\subsection{CONTINUOUS ENERGY MCNP FORMAT}

A FORTRAN code was written to process the evaluated data into the ASCII format needed by MCNP (i.e., a directory file and the actual cross section file with pointers). The code is based upon previous work. 
Because the base libraries have already converted resonance parameters and other data into a linear-linear representation, there are no choices to be made except for thinning. No thinning of the cross sections was performed in this processing.

Because of the large size of the resulting cross section files, the processed library is divided into 8 sections:

1. Isotopes of elements $H \quad(Z=1)$ to Sc $(Z=21)$

2. Isotopes of elements $\mathrm{Ti} \quad(Z=22)$ to $Z n \quad(Z=30)$

3. Isotopes of elements $\mathrm{Ga}(Z=31)$ to $Y \quad(Z=39)$

4. Isotopes of elements $\mathrm{Zr} \quad(Z=40)$ to $\mathrm{Pd} \quad(Z=46)$

5. Isotopes of elements $\mathrm{Ag} \quad(Z=47)$ to $\mathrm{Te} \quad(Z=52)$

6. Isotopes of elements I $(Z=53)$ to $\mathrm{Sm} \quad(Z=62)$

7. Isotopes of elements $E u(Z=63)$ to $L u(Z=71)$

8. Isotopes of elements $\mathrm{Hf}(Z=72)$ to Po $(Z=84)$.

The suffix used is $.66 y$ for those targets in the ground state and $.67 y$ for those targets in an isomeric state. The use of different suffixes follows the convention established by Los Alamos National Laboratory (LANL).

\subsection{MULTIGROUP REAC FORMAT}

The multigroup cross sections were calculated by the MULTAV code from point-wise?? or pointwise as above?? cross sections described above. MULTAV is a FORTRAN code that reads ENDF/B file 3-formatted cross section files and a user-supplied weighting function. MULTAV then calculates the group-averaged value either analytically or using adaptive Romberg integration. Calculations were made for the 175-group?? Vitamin-J structure, which is displayed in Table 1. The flux used for group weighting is that used in the Vitamin-E group generation (Reference 4) as implemented in the NJOY processing code (Reference 5).

The 175-group Vitamin-J structure has been informally adopted as a standard for fusion transport studies. Thus, it is a natural choice. Similarly, the choice of the Vitamin-E weighting flux, which contains thermal, fast, and fusion components, is a natural one given the fusion experience.

All of the multigroup data are on one file. 
Table 1. 175 Group Energy Structure.

\begin{tabular}{|c|c|c|c|c|c|}
\hline $1.000 \mathrm{E}-5$ & $1.000 \mathrm{E}-1$ & $4.140 \mathrm{E}-1$ & $5.316 \mathrm{E}-1$ & $6.826 \mathrm{E}-1$ & $8.764 \mathrm{E}-1$ \\
\hline $1.1254 \mathrm{EO}$ & $1.4450 \mathrm{EO}$ & $1.8554 E 0$ & $2.3824 \mathrm{EO}$ & $3.0590 \mathrm{EO}$ & $3.9279 \mathrm{EO}$ \\
\hline $5.0435 \mathrm{E} 0$ & $6.4760 \mathrm{EO}$ & $8.3153 \mathrm{E} 0$ & $1.0677 \mathrm{E} 1$ & $1.3710 \mathrm{E} 1$ & $1.7603 \mathrm{E} 1$ \\
\hline $2.2603 \mathrm{El}$ & $2.9023 \mathrm{E} 1$ & $3.7267 E 1$ & $4.7851 \mathrm{E} 1$ & $6.1442 E 1$ & $7.8893 E 1$ \\
\hline $1.0130 \mathrm{E} 2$ & $1.3007 \mathrm{E} 2$ & $1.6702 \mathrm{E} 2$ & $2.1445 E 2$ & $2.7536 \mathrm{E} 2$ & $3.5358 \mathrm{E} 2$ \\
\hline $4.5400 \mathrm{E} 2$ & $5.8295 \mathrm{E} 2$ & $7.4852 \mathrm{E} 2$ & $9.6112 \mathrm{E} 2$ & $1.2341 \mathrm{E} 3$ & $1.5846 \mathrm{E} 3$ \\
\hline $2.0347 \mathrm{E} 3$ & $2.2487 \mathrm{E} 3$ & $2.4852 E 3$ & $2.6126 \mathrm{E} 3$ & $2.7465 \mathrm{E} 3$ & $3.0354 \mathrm{E} 3$ \\
\hline $3.3546 E 3$ & $3.7074 \mathrm{E} 3$ & $4.3074 E 3$ & $5.5308 \mathrm{E} 3$ & $7.1017 \mathrm{E} 3$ & $9.1188 \mathrm{E} 3$ \\
\hline $1.0595 \mathrm{E} 4$ & $1.1709 \mathrm{E} 4$ & $1.5034 E 4$ & $1.9305 \mathrm{E} 4$ & $2.1875 E 4$ & $2.3579 E 4$ \\
\hline $2.4176 \mathrm{E} 4$ & $2.4788 E 4$ & $2.6058 E 4$ & $2.7000 E 4$ & $2.8500 E 4$ & $3.1828 E 4$ \\
\hline $3.4307 E 4$ & $4.0868 E 4$ & $4.6309 E 4$ & $5.2475 E 4$ & $5.6562 \mathrm{E} 4$ & $6.7379 E 4$ \\
\hline $7.2000 E 4$ & $7.9500 \mathrm{E} 4$ & $8.2500 E 4$ & $8.6517 E 4$ & $9.8037 \mathrm{E} 4$ & $1.1109 E 5$ \\
\hline $1.1679 E 5$ & $1.2277 E 5$ & $1.2907 E 5$ & $1.3569 E 5$ & $1.4264 E 5$ & $1.4996 E 5$ \\
\hline $1.5764 \mathrm{E} 5$ & $1.6573 E 5$ & $1.7422 \mathrm{E} 5$ & $1.8316 \mathrm{E} 5$ & $1.9255 E 5$ & $2.0242 E 5$ \\
\hline $2.1280 E 5$ & $2.2371 E 5$ & $2.3518 E 5$ & $2.4724 E 5$ & $2.7324 \mathrm{E5}$ & $2.8725 E 5$ \\
\hline $2.9452 E 5$ & $2.9720 E 5$ & $2.9850 \mathrm{E} 5$ & $3.0197 E 5$ & $3.3373 E 5$ & $3.6883 E 5$ \\
\hline $3.8774 E 5$ & $4.0762 E 5$ & $4.5049 E 5$ & $4.9787 E 5$ & $5.2340 E 5$ & $5.5023 E 5$ \\
\hline $5.7844 \mathrm{E} 5$ & $6.0810 \mathrm{E} 5$ & $6.3928 \mathrm{E} 5$ & $6.7206 E 5$ & $7.0651 \mathrm{E} 5$ & $7.4274 E 5$ \\
\hline $7.8082 E 5$ & $8.2085 E 5$ & $8.6294 E 5$ & $9.0718 E 5$ & $9.6164 E 5$ & $1.0026 E 6$ \\
\hline $1.1080 \mathrm{E} 6$ & $1.1648 \mathrm{E} 6$ & $1.2246 \mathrm{E} 6$ & $1.2873 \mathrm{E} 6$ & $1.3534 \mathrm{E} 6$ & $1.4227 \mathrm{E} 6$ \\
\hline $1.4957 E 6$ & $1.5724 \mathrm{E} 6$ & $1.6530 \mathrm{E} 6$ & $1.7377 \mathrm{E} 6$ & $1.8268 \mathrm{E} 6$ & $1.9205 \mathrm{E} 6$ \\
\hline $2.0190 \mathrm{E} 6$ & $2.1225 \mathrm{E} 6$ & $2.2313 \mathrm{E} 6$ & $2.3069 \mathrm{E} 6$ & $2.3457 \mathrm{E} 6$ & $2.3653 \mathrm{E} 6$ \\
\hline $2.3852 E 6$ & $2.4660 \mathrm{E} 6$ & $2.5924 E 6$ & $2.7253 \mathrm{E} 6$ & $2.8650 \mathrm{E} 6$ & $3.0119 E 6$ \\
\hline $3.1664 \mathrm{E} 6$ & $3.3287 \varepsilon 6$ & $3.6788 \mathrm{E} 6$ & $4.0657 \mathrm{E} 6$ & $4.4933 E 6$ & $4.7237 \mathrm{E} 6$ \\
\hline 4.9659E6 & $5.2205 E 6$ & $5.4881 \mathrm{E} 6$ & $5.7695 \mathrm{E} 6$ & $6.0653 \mathrm{E} 6$ & $6.3763 \mathrm{E} 6$ \\
\hline $6.5924 \mathrm{E} 6$ & $6.7032 \mathrm{E} 6$ & $7.0469 \mathrm{E} 6$ & $7.4082 \mathrm{E} 6$ & $7.7880 \mathrm{E} 6$ & $8.1873 E 6$ \\
\hline $8.6071 \mathrm{E} 6$ & $9.0484 \mathrm{E} 6$ & $9.5123 \mathrm{E} 6$ & $1.0000 \mathrm{E} 7$ & $1.0513 \mathrm{E} 7$ & $1.1052 E 7$ \\
\hline $1.1618 \mathrm{E} 7$ & $1.2214 \mathrm{E} 7$ & $1.2480 \mathrm{E} 7$ & $1.2523 \mathrm{E} 7$ & $1.3499 \mathrm{E} 7$ & $1.3840 E 7$ \\
\hline $1.4191 \mathrm{E} 7$ & $1.4550 \mathrm{E} 7$ & $1.4918 \mathrm{E} 7$ & $1.5683 \mathrm{E} 7$ & $1.6487 \mathrm{E} 7$ & $1.6905 \mathrm{E} 7$ \\
\hline $1.7333 \mathrm{E} 7$ & $1.9640 \mathrm{E} 7$ & & & & \\
\hline
\end{tabular}


WHC-EP-0727

\subsection{VERIFICATION}

\subsection{CONTINUOUS ENERGY MCNP FORMAT}

Simple MCNP test runs were made using ${ }^{55} \mathrm{Mn}(n, y)$ and ${ }^{56} \mathrm{Fe}(n, p)$ activation reactions. Fine-group values of the cross sections from the MCNP output agreed with the values from the unprocessed files. format.

In addition, the ASCII files were successfuily processed into binary

\subsection{MULTIGROUP REAC FORMAT}

The ASCII file was processed into binary format using the FORTRAN code BINLIB, a module of REAC $\star 3$. The transmutation of ${ }^{56} \mathrm{Fe}$ using the spectrum from the first wall of the STARFIRE reactor was compared successfully using the FENDL and REAC cross sections.

\subsection{AVAILABLE FILES}

The files that are available are shown in Table 2. At the Hanford Site the files are kept on the Centralized File Storage (CFS) system directory /v92515/fendl/monp.

Table 2. Location of Files.

\begin{tabular}{|l|l|}
\hline \multicolumn{1}{|c|}{ File name } & \multicolumn{1}{|c|}{ Description } \\
\hline xsdir.zz & ASCII files containing directory information for MCNP \\
\hline actxsl.zz & $\begin{array}{l}\text { ASCII files containing pointer and continuous energy cross } \\
\text { section values for MCNP }\end{array}$ \\
\hline cross & ASCII files containing multigroup data in REAC format \\
\hline out.zz & $\begin{array}{l}\text { ASCII files listing the reactions which are included and } \\
\text { the number of energy/cross section points }\end{array}$ \\
\hline
\end{tabular}

Note: $z z$ is a suffix indicating the range of isotopes in the cross section set: h.sc, ti.zn, ga.y, zr.pd, ag.te, i.sm, eu.lu, and hf.po. 


\subsection{REFERENCES}

1. Kopecky, J., H. A. J. van der Kamp, H. Gruppelaar, and Nierop, The European Activation File EAF-3 with Neutron Activation and Transmutation Cross Sections, ECN-C-92-058, Netherlands Energy Research Foundation ECN, Petten, the Netherlands, September 1992.

2. Briesmeister, J. F., Editor, MCNP - A General Monte Carlo N-Particle Transport Code, Version 4A, LA-12625 (Manual), Los Alamos National Laboratory, Los Alamos, New Mexico, 1993.

3. Mann, F. M., REAC*2: Users Manual and Code Description, WHC-EP-0282, Westinghouse Hanford Company, Richland, Washington, Decembar 1989.

4. Weisbin, C. R., R. W. Roussin, J. J. Wagschal, J. E. White, and R. Q. Wright, Vitamin-E: An ENDF/B-V Multigroup Cross Section Library for LMFBR Core and Shield, Dosimetry, and Fusion Blanket Technology, ORNL-5505, Oak Ridge National Laboratory, Oak Ridge, Tennessee, February 1979.

5. MacFarlane, R. E., D. W. Muir, and R. M. Boicourt, The NJOY Nuclear Data Processing System, Volume I: User's Manual, LA-9303-M, Los Alamos National Laboratory, Los Alamos, New Mexico, May 1982. 
WHC-EP-0727

This page intentionally left blank. 
WHC-EP-0727

Appendix $\mathrm{A}-1$

List of Reactions on Files *.h.sc

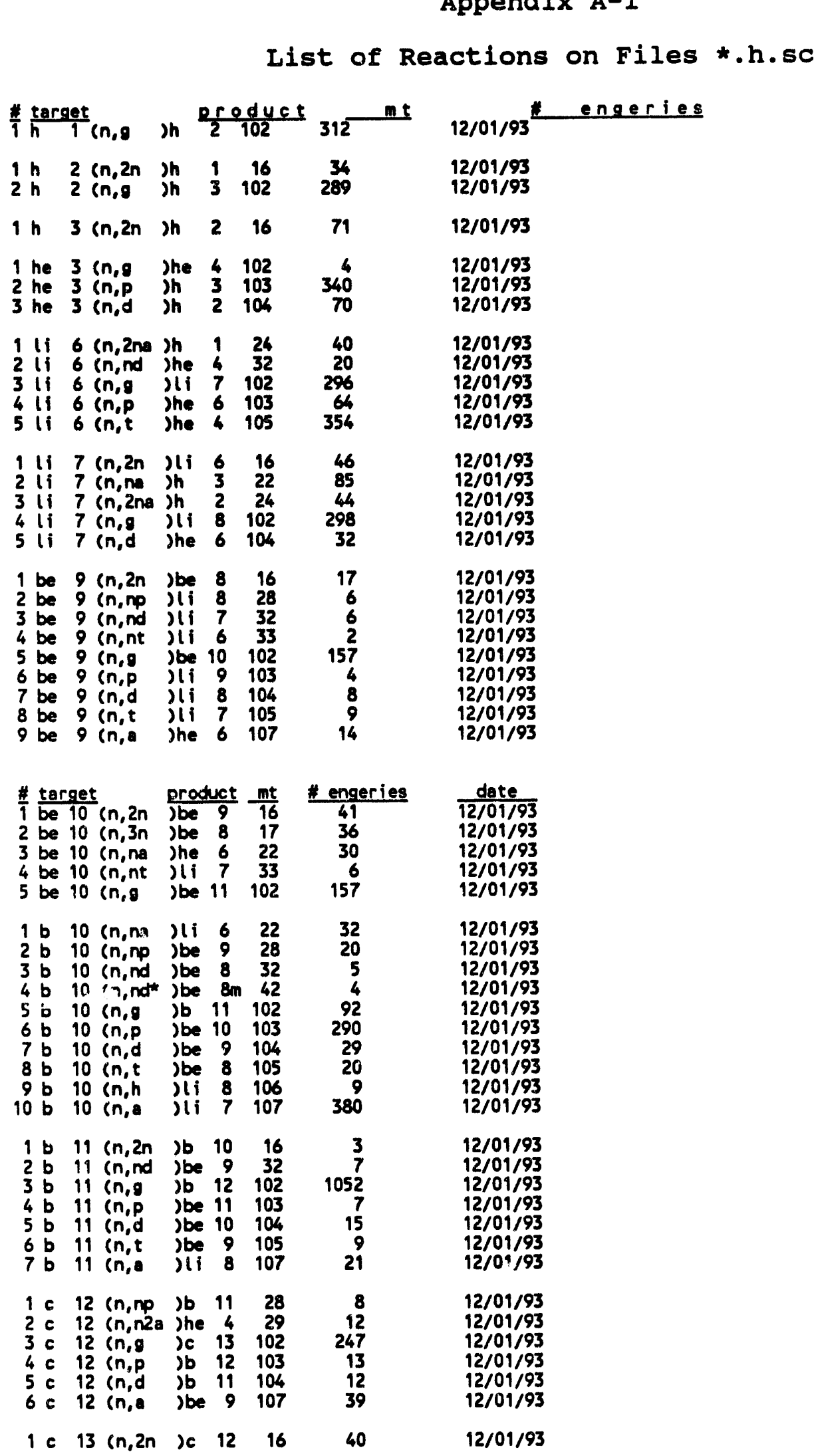

$A-1.1$ 


\begin{tabular}{|c|c|c|c|c|c|}
\hline \multicolumn{2}{|c|}{ E torget } & \multicolumn{2}{|c|}{ product mt } & * engeries & \multirow[b]{2}{*}{$\begin{array}{c}\text { date } \\
12 / 01 / 93 \\
12 / 01 / 93 \\
12 / 01 / 93 \\
12 / 01 / 93 \\
12 / 01 / 93 \\
12 / 01 / 93 \\
12 / 01 / 93\end{array}$} \\
\hline 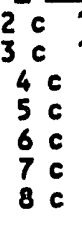 & $\begin{array}{c}13(n, n) \\
13(n, n p) b \\
13(n, g) \\
13(n, p) \\
13(n, d) \\
13(n, t) \\
13(n, d)\end{array}$ & $\begin{array}{ll}\text { be } & 9 \\
\text { b } & 12 \\
\text { jc } & 14 \\
\text { jb } & 13 \\
\text { jb } & 12 \\
\text { jb } & 11 \\
\text { jbe } & 10\end{array}$ & $\begin{array}{l}22 \\
28 \\
102 \\
103 \\
104 \\
105 \\
107\end{array}$ & $\begin{array}{c}17 \\
6 \\
176 \\
15 \\
11 \\
16 \\
39\end{array}$ & \\
\hline $\begin{array}{ll}1 & c \\
2 & c \\
3 & c \\
4 & c \\
5 & c \\
6 & c \\
7 & c\end{array}$ & 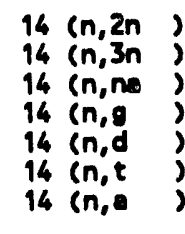 & $\begin{array}{ll}\text { jc } & 13 \\
\text { jc } & 12 \\
\text { be } & 10 \\
\text { jc } & 15 \\
\text { jb } & 13 \\
\text { jb } & 12 \\
\text { jbe } & 11\end{array}$ & $\begin{array}{r}16 \\
17 \\
22 \\
102 \\
104 \\
105 \\
107\end{array}$ & $\begin{array}{r}33 \\
25 \\
16 \\
165 \\
2 \\
7 \\
21\end{array}$ & $\begin{array}{l}12 / 01 / 93 \\
12 / 01 / 93 \\
12 / 01 / 93 \\
12 / 01 / 93 \\
12 / 01 / 93 \\
12 / 01 / 93 \\
12 / 01 / 93\end{array}$ \\
\hline $\begin{array}{ll}\frac{\#}{1} & \text { ta } \\
2 & n \\
3 & n \\
4 & n \\
5 & n \\
6 & n \\
7 & n \\
8 & n \\
9 & n \\
10 & n \\
11 & n\end{array}$ & $\begin{array}{l}\frac{\text { rget }}{14} \text { (n,2n } \\
14 \text { (n,ne } \\
14 \text { (n,np } \\
14 \text { (n,nd } \\
14 \text { (n,g } \\
14 \text { (n,p) } \\
14 \text { (n,d } \\
14 \text { (n,t } \\
14 \text { (n,h } \\
14 \text { (n,a } \\
14 \text { (n,2a }\end{array}$ & 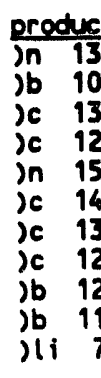 & $\begin{array}{c}\frac{m t}{16} \\
22 \\
28 \\
28 \\
32 \\
102 \\
103 \\
104 \\
105 \\
106 \\
107 \\
7 \\
108\end{array}$ & $\begin{array}{c}* \text { enperies } \\
14 \\
19 \\
23 \\
2 \\
310 \\
615 \\
39 \\
48 \\
8 \\
255 \\
14\end{array}$ & $\begin{array}{l}\text { date } \\
12 / 01 / 93 \\
12 / 01 / 93 \\
12 / 01 / 93 \\
12 / 01 / 93 \\
12 / 01 / 93 \\
12 / 01 / 93 \\
12 / 01 / 93 \\
12 / 01 / 93 \\
12 / 01 / 93 \\
12 / 01 / 93 \\
12 / 01 / 93\end{array}$ \\
\hline $\begin{array}{rl}1 & n \\
2 & n \\
3 & n \\
4 & n \\
5 & n \\
6 & n \\
7 & n \\
8 & n \\
9 & n \\
10 & n\end{array}$ & $\begin{array}{l}15 \text { (n, 2n } \\
15 \text { (n,na } \\
15 \text { (n,np } \\
15 \text { (n, nd } \\
15 \text { (n,nt } \\
15 \text { (n, g } \\
15 \text { (n,p } \\
15 \text { (n,d } \\
15 \text { (n,t } \\
15 \text { (n, a }\end{array}$ & 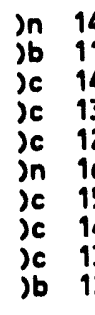 & $\begin{array}{r}16 \\
22 \\
28 \\
32 \\
33 \\
102 \\
103 \\
104 \\
105 \\
107\end{array}$ & $\begin{array}{r}15 \\
18 \\
20 \\
9 \\
9 \\
283 \\
22 \\
24 \\
18 \\
26\end{array}$ & $\begin{array}{l}12 / 01 / 93 \\
12 / 01 / 93 \\
12 / 01 / 93 \\
12 / 01 / 93 \\
12 / 01 / 93 \\
12 / 01 / 93 \\
12 / 01 / 93 \\
12 / 01 / 93 \\
12 / 01 / 93 \\
12 / 01 / 93\end{array}$ \\
\hline $\begin{array}{ll}1 & 0 \\
2 & 0 \\
3 & 0 \\
4 & 0 \\
5 & 0 \\
6 & 0 \\
7 & 0 \\
8 & 0 \\
9 & 0\end{array}$ & 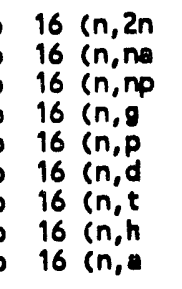 & $\begin{array}{ll}\text { jo } & 1 \\
\text { jc } & 1 \\
\text { in } & 1 \\
\text { jo } & 1 \\
\text { on } & 1 \\
\text { on } & 1 \\
\text { in } & 1 \\
\text { jc } & 1 \\
\text { jc } & 1\end{array}$ & $\begin{array}{rr}5 & 16 \\
2 & 22 \\
5 & 28 \\
7 & 102 \\
16 & 103 \\
15 & 104 \\
14 & 105 \\
14 & 106 \\
13 & 107\end{array}$ & $\begin{array}{r}3 \\
11 \\
4 \\
164 \\
45 \\
21 \\
13 \\
15 \\
173\end{array}$ & $\begin{array}{l}12 / 01 / 93 \\
12 / 01 / 93 \\
12 / 01 / 93 \\
12 / 01 / 93 \\
12 / 01 / 93 \\
12 / 01 / 93 \\
12 / 01 / 93 \\
12 / 01 / 93 \\
12 / 01 / 93\end{array}$ \\
\hline $\begin{array}{ll}1 & 0 \\
2 & 0 \\
3 & 0 \\
4 & 0 \\
5 & 0 \\
6 & 0 \\
7 & 0 \\
8 & 0 \\
9 & 0 \\
10 & 0 \\
11 & 0 \\
12 & 0\end{array}$ & 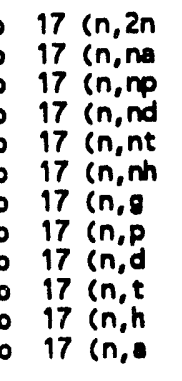 & 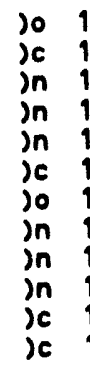 & $\begin{array}{rr}16 & 16 \\
13 & 22 \\
16 & 28 \\
15 & 32 \\
14 & 33 \\
14 & 34 \\
18 & 102 \\
17 & 103 \\
16 & 104 \\
15 & 105 \\
15 & 106 \\
14 & 107\end{array}$ & $\begin{array}{r}13 \\
15 \\
8 \\
10 \\
4 \\
3 \\
358 \\
14 \\
10 \\
18 \\
6 \\
270\end{array}$ & $\begin{array}{l}12 / 01 / 93 \\
12 / 01 / 93 \\
12 / 01 / 93 \\
12 / 01 / 93 \\
12 / 01 / 93 \\
12 / 01 / 93 \\
12 / 01 / 93 \\
12 / 01 / 93 \\
12 / 01 / 93 \\
12 / 01 / 93 \\
12 / 01 / 93 \\
12 / 01 / 93\end{array}$ \\
\hline $\begin{array}{l}0 \\
0 \\
0 \\
0 \\
0\end{array}$ & 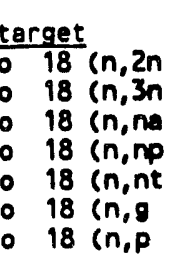 & $\begin{array}{l}\text { prod } \\
\text { jo } \\
\text { jo } \\
\text { on } \\
\text { on } \\
\text { jo } \\
\text { in }\end{array}$ & \begin{tabular}{lr} 
uct & mt \\
\cline { 2 - 2 } 17 & 16 \\
16 & 17 \\
14 & 22 \\
17 & 28 \\
15 & 33 \\
19 & 102 \\
18 & 103
\end{tabular} & $\begin{array}{c}\text { * engeries } \\
35 \\
30 \\
33 \\
8 \\
10 \\
166 \\
17\end{array}$ & $\begin{array}{l}\frac{\text { date }}{12 / 01 / 93} \\
12 / 01 / 93 \\
12 / 01 / 93 \\
12 / 01 / 93 \\
12 / 01 / 93 \\
12 / 01 / 93 \\
12 / 01 / 93\end{array}$ \\
\hline
\end{tabular}

A-1. 2 


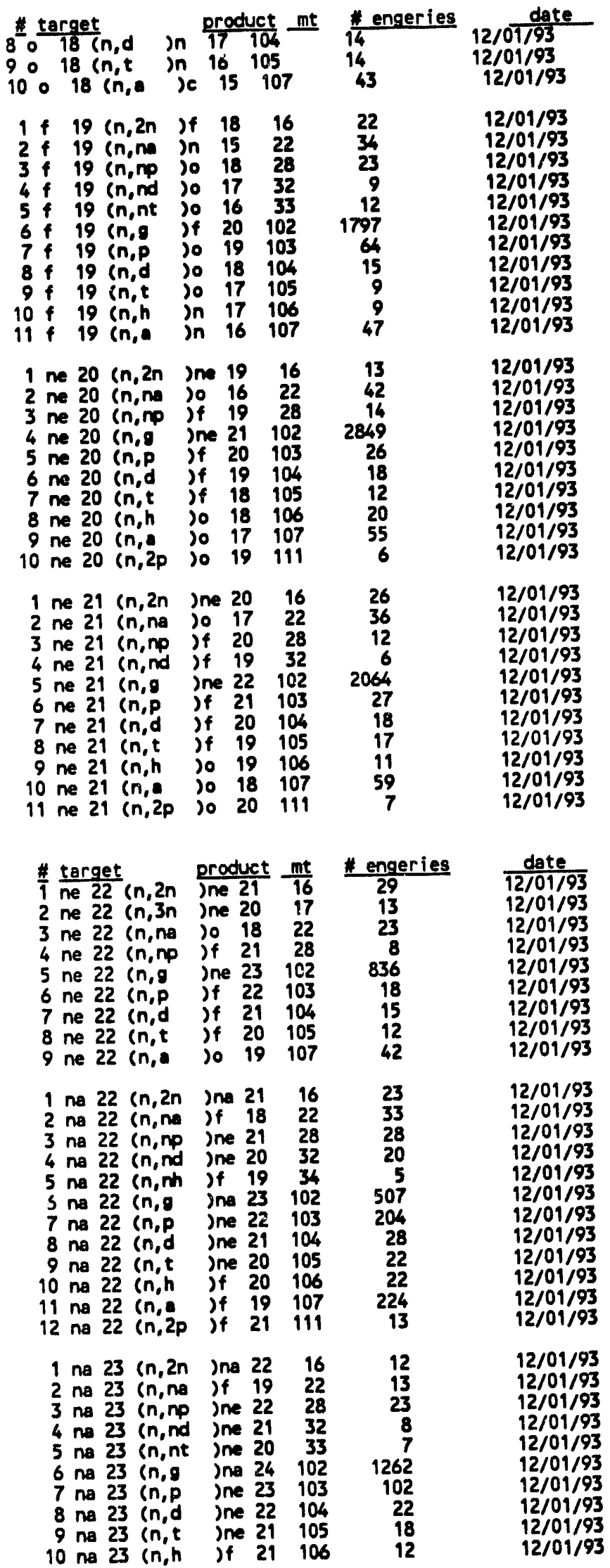

$A-1.3$ 


\begin{tabular}{|c|c|c|c|c|}
\hline $\begin{array}{l}\frac{n}{11} \frac{\text { target }}{\text { na } 23}(n, \mathrm{e} \\
12 \text { na } 23(n, 2 p \\
13 \text { na } 23\left(n, 9^{*}\right.\end{array}$ & 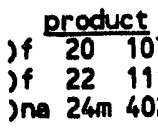 & & $\begin{array}{c}\frac{* \text { engerie }}{45} \\
2 \\
1262\end{array}$ & $\begin{array}{l}\frac{\text { date }}{12 / 01 / 93} \\
12 / 01 / 93 \\
12 / 01 / 93\end{array}$ \\
\hline 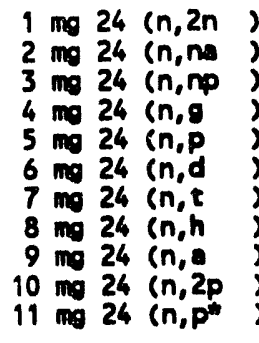 & $\begin{array}{ll}\text { ing } 23 & 1 \\
\text { ine } 20 & 2 \\
\text { ind } 23 & 2 \\
\text { ind } 25 & 10 \\
\text { ind } 24 & 10 \\
\text { ind } 23 & 10 \\
\text { ind } 22 & 10 \\
\text { ine } 22 & 10 \\
\text { ine } 21 & 10 \\
\text { ine } 23 & 11 \\
\text { ine } 24 m & 40\end{array}$ & $\begin{array}{l}16 \\
22 \\
28 \\
02 \\
03 \\
04 \\
05 \\
06 \\
07 \\
11\end{array}$ & $\begin{array}{r}4 \\
35 \\
5 \\
547 \\
45 \\
22 \\
11 \\
24 \\
9 \\
10 \\
44\end{array}$ & $\begin{array}{l}12 / 01 / 93 \\
12 / 01 / 93 \\
12 / 01 / 93 \\
12 / 01 / 93 \\
12 / 01 / 93 \\
12 / 01 / 93 \\
12 / 01 / 93 \\
12 / 01 / 93 \\
12 / 01 / 93 \\
12 / 01 / 93 \\
12 / 01 / 93\end{array}$ \\
\hline 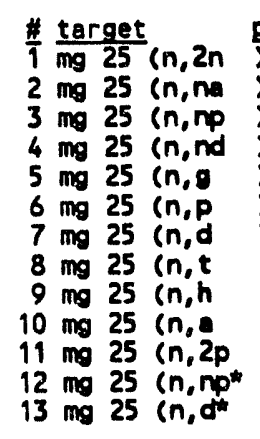 & $\begin{array}{l}\text { product } \\
\text { ing } 24 \\
\text { ine } 21 \\
\text { ind } 24 \\
\text { ind } 23 \\
\text { img } 26 \\
\text { ind } 25 \\
\text { ind } 24 \\
\text { ind } 23 \\
\text { ine } 23 \\
\text { ine } 22 \\
\text { ine } 24 \\
\text { Ind } 24 \mathrm{~m} \\
\text { Ind } 24 \mathrm{~m} \\
4\end{array}$ & $\begin{array}{l}\frac{m t}{16} \\
22 \\
28 \\
32 \\
102 \\
103 \\
104 \\
105 \\
106 \\
107 \\
111 \\
328 \\
404\end{array}$ & $\begin{array}{c}\text { \# engeries } \\
15 \\
30 \\
20 \\
8 \\
620 \\
12 \\
21 \\
18 \\
16 \\
16 \\
12 \\
18 \\
20\end{array}$ & $\begin{array}{l}\frac{\text { date }}{12 / 01 / 93} \\
12 / 01 / 93 \\
12 / 01 / 93 \\
12 / 01 / 93 \\
12 / 01 / 93 \\
12 / 01 / 93 \\
12 / 01 / 93 \\
12 / 01 / 93 \\
12 / 01 / 93 \\
12 / 01 / 93 \\
12 / 01 / 93 \\
12 / 01 / 93 \\
12 / 01 / 93\end{array}$ \\
\hline 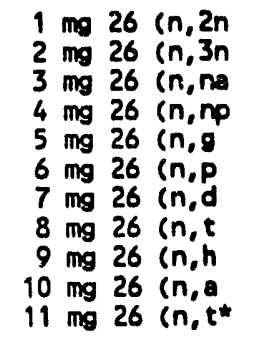 & 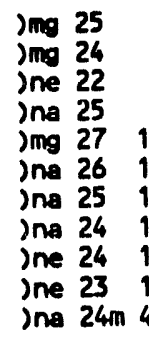 & $\begin{array}{l}16 \\
17 \\
22 \\
28 \\
102 \\
103 \\
104 \\
105 \\
106 \\
107 \\
405\end{array}$ & $\begin{array}{r}19 \\
5 \\
29 \\
14 \\
418 \\
16 \\
18 \\
13 \\
9 \\
8 \\
11\end{array}$ & $\begin{array}{l}12 / 01 / 93 \\
12 / 01 / 93 \\
12 / 01 / 93 \\
12 / 01 / 93 \\
12 / 01 / 93 \\
12 / 01 / 93 \\
12 / 01 / 93 \\
12 / 01 / 93 \\
12 / 01 / 93 \\
12 / 01 / 93 \\
12 / 01 / 93\end{array}$ \\
\hline 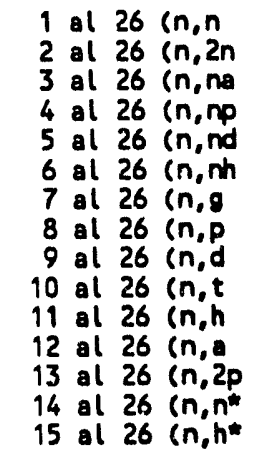 & 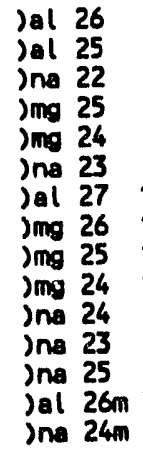 & $\begin{array}{r}4 \\
16 \\
22 \\
28 \\
32 \\
34 \\
102 \\
103 \\
104 \\
105 \\
106 \\
107 \\
111 \\
304 \\
406\end{array}$ & $\begin{array}{r}1 \\
25 \\
32 \\
29 \\
20 \\
7 \\
305 \\
37 \\
31 \\
24 \\
28 \\
51 \\
18 \\
53 \\
26\end{array}$ & $\begin{array}{l}12 / 01 / 93 \\
12 / 01 / 93 \\
12 / 01 / 93 \\
12 / 01 / 93 \\
12 / 01 / 93 \\
12 / 01 / 93 \\
12 / 01 / 93 \\
12 / 01 / 93 \\
12 / 01 / 93 \\
12 / 01 / 93 \\
12 / 01 / 93 \\
12 / 01 / 93 \\
12 / 01 / 93 \\
12 / 01 / 93 \\
12 / 01 / 93\end{array}$ \\
\hline 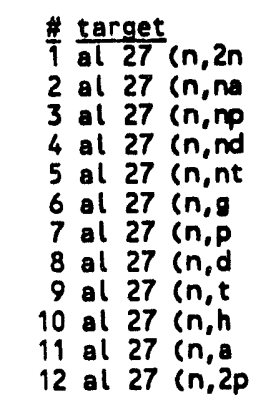 & 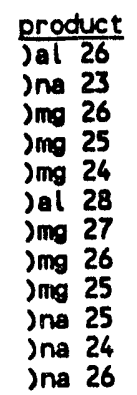 & $\begin{array}{l}\frac{m t}{16} \\
22 \\
28 \\
32 \\
33 \\
102 \\
103 \\
104 \\
105 \\
106 \\
107 \\
111\end{array}$ & $\begin{array}{c}\text { *engeries } \\
16 \\
29 \\
29 \\
9 \\
6 \\
292 \\
101 \\
28 \\
16 \\
20 \\
48 \\
9\end{array}$ & $\begin{array}{l}\text { date } \\
12 / 01 / 93 \\
12 / 01 / 93 \\
12 / 01 / 93 \\
12 / 01 / 93 \\
12 / 01 / 93 \\
12 / 01 / 93 \\
12 / 01 / 93 \\
12 / 01 / 93 \\
12 / 01 / 93 \\
12 / 01 / 93 \\
12 / 01 / 93 \\
12 / 01 / 93\end{array}$ \\
\hline
\end{tabular}

A-1. 4 


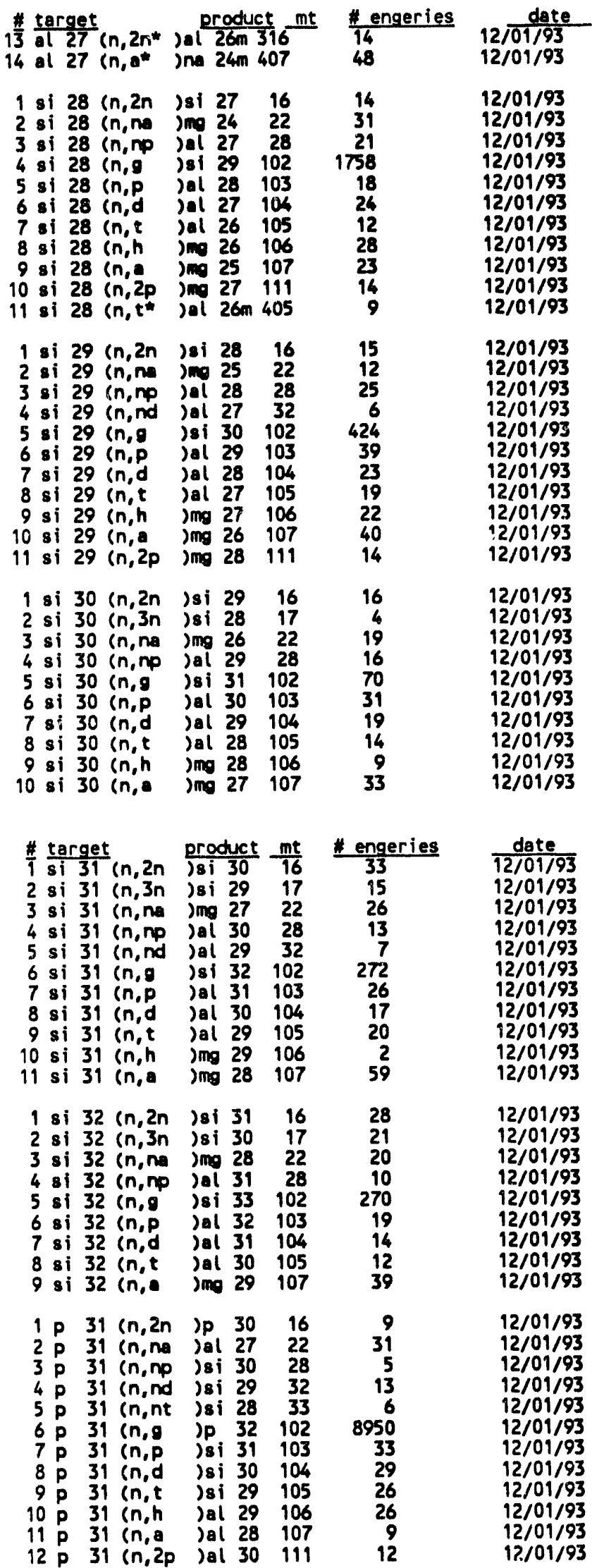

$\mathrm{A}-1.5$ 
WHC-EP-0727

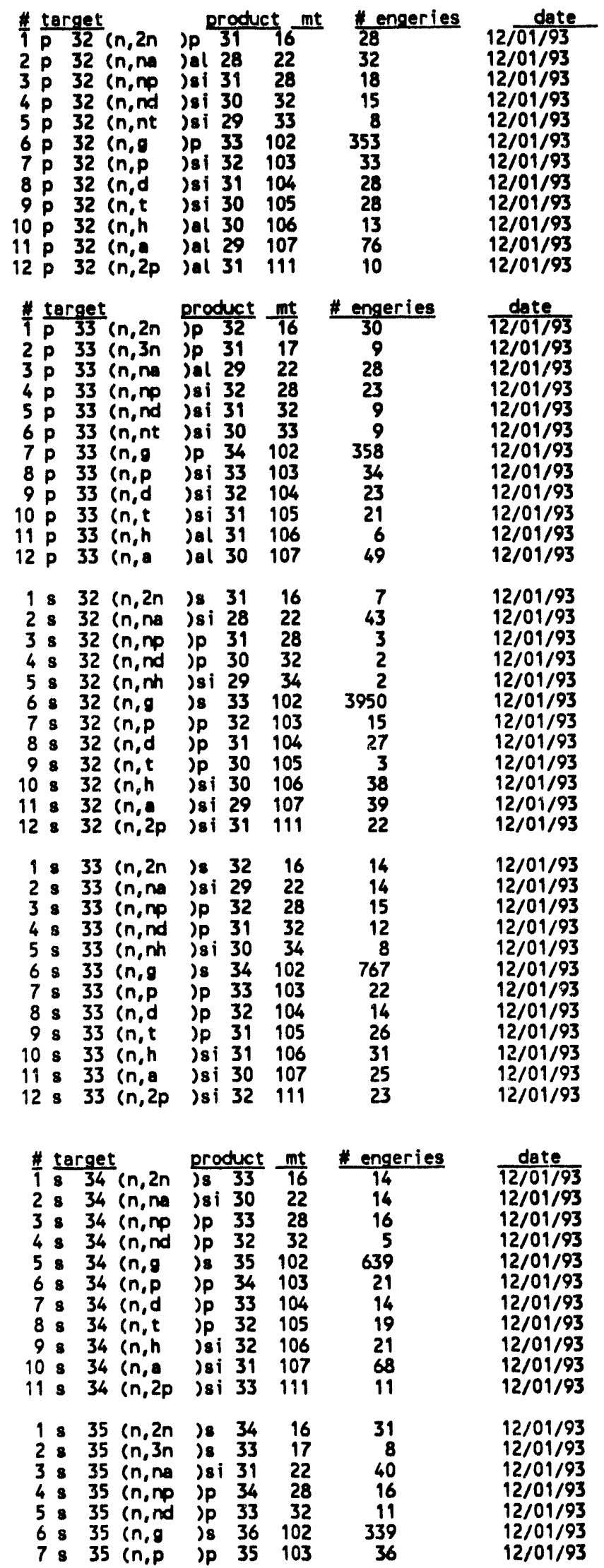

$A-1.6$ 


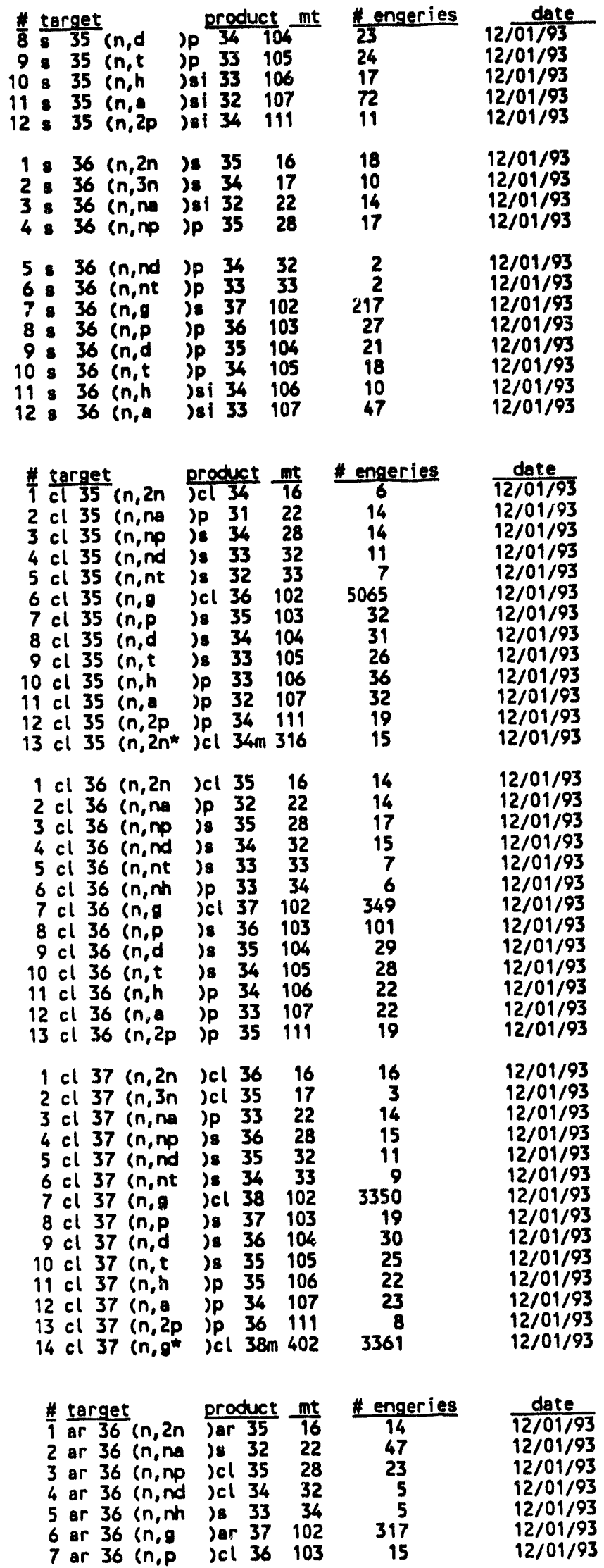

A-1.7 


\begin{tabular}{|c|c|c|c|}
\hline 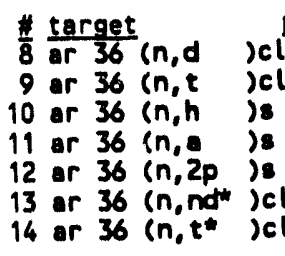 & 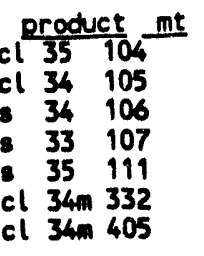 & $\begin{array}{l}\text { yngeries } \\
31 \\
18 \\
43 \\
16 \\
25 \\
8 \\
18\end{array}$ & $\begin{array}{l}\frac{\text { date }}{12 / 01 / 93} \\
12 / 01 / 93 \\
12 / 01 / 93 \\
12 / 01 / 93 \\
12 / 01 / 93 \\
12 / 01 / 93 \\
12 / 01 / 93\end{array}$ \\
\hline $\begin{array}{r}1 \text { or } 37(n, 2 n) \\
2 \text { ar } 37(n, n) \\
3 \text { ar } 37(n, n p) \\
4 \text { or } 37(n, n d) \\
5 \text { ar } 37(n, n h) \\
6 \text { ar } 37(n, 8) \\
7 \text { ar } 37(n, p) \\
8 \text { ar } 37(n, d) \\
9 \text { ar } 37(n, t) \\
10 \text { ar } 37(n, n) \\
11 \text { ar } 37(n, a) \\
12 \text { or } 37(n, 2 p)\end{array}$ & $\begin{array}{lrr}\text { ar } & 36 & 16 \\
\text { s } & 33 & 22 \\
c l & 36 & 28 \\
c l & 35 & 32 \\
\text { s } & 36 & 34 \\
\text { ar } & 38 & 102 \\
c l & 37 & 103 \\
c l & 36 & 104 \\
\text { cl } & 35 & 105 \\
8 & 35 & 106 \\
8 & 34 & 107 \\
8 & 36 & 111\end{array}$ & $\begin{array}{r}25 \\
46 \\
26 \\
13 \\
11 \\
335 \\
216 \\
29 \\
28 \\
38 \\
218 \\
27\end{array}$ & $\begin{array}{l}12 / 01 / 93 \\
12 / 01 / 93 \\
12 / 01 / 93 \\
12 / 01 / 93 \\
12 / 01 / 93 \\
12 / 01 / 93 \\
12 / 01 / 93 \\
12 / 01 / 93 \\
12 / 01 / 93 \\
12 / 01 / 93 \\
12 / 01 / 93 \\
12 / 01 / 93\end{array}$ \\
\hline $\begin{array}{l}1 \text { ar } 38(n, 2 n) \\
2 \text { ar } 38(n, n a) \\
3 \text { ar } 38(n, n p) \\
4 \text { ar } 38(n, n d) \\
5 \text { ar } 38(n, g) \\
6 \text { ar } 38(n, p) \\
7 \text { ar } 38(n, d) \\
8 \text { ar } 38(n, t) \\
9 \text { ar } 38(n, n) \\
10 \text { ar } 38(n, 8) \\
11 \text { ar } 38(n, 2 p) \\
12 \text { ar } 38\left(n, p^{4}\right)\end{array}$ & 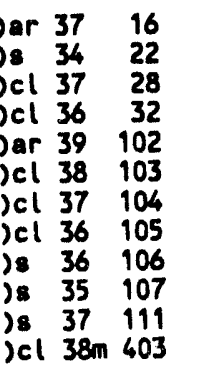 & $\begin{array}{r}14 \\
45 \\
20 \\
6 \\
404 \\
15 \\
28 \\
22 \\
32 \\
15 \\
13 \\
15\end{array}$ & $\begin{array}{l}12 / 01 / 93 \\
12 / 01 / 93 \\
12 / 01 / 93 \\
12 / 01 / 93 \\
12 / 01 / 93 \\
12 / 01 / 93 \\
12 / 01 / 93 \\
12 / 01 / 93 \\
12 / 01 / 93 \\
12 / 01 / 93 \\
12 / 01 / 93 \\
12 / 01 / 93\end{array}$ \\
\hline $\begin{array}{l}\frac{\#}{\#} \frac{\text { target }}{1} \text { ar } 39(n, 2 n) \\
2 \text { ar } 39(n, 3 n) \\
3 \text { ar } 39(n, n a) \\
4 \text { ar } 39(n, n p) \\
5 \text { ar } 39(n, n d) \\
6 \text { ar } 39(n, n t) \\
7 \text { ar } 39(n, n n) \\
8 \text { ar } 39(n, d) \\
9 \text { ar } 39(n, p) \\
10 \text { ar } 39(n, d) \\
11 \text { ar } 39(n, t) \\
12 \text { ar } 39(n, n) \\
13 \text { ar } 39(n, 1) \\
14 \text { ar } 39(n, 2 p) \\
15 \text { ar } 39\left(n, n p^{\star}\right) \\
16 \text { ar } 39\left(n, d^{\star}\right)\end{array}$ & 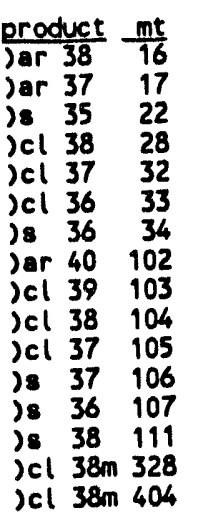 & $\begin{array}{c}\text { \#engeries } \\
19 \\
6 \\
46 \\
16 \\
14 \\
5 \\
8 \\
334 \\
15 \\
26 \\
28 \\
24 \\
15 \\
16 \\
14 \\
25\end{array}$ & $\begin{array}{l}\text { date } \\
\frac{1201 / 93}{12 / 01 / 93} \\
12 / 01 / 93 \\
12 / 01 / 93 \\
12 / 01 / 93 \\
12 / 01 / 93 \\
12 / 01 / 93 \\
12 / 01 / 93 \\
12 / 01 / 93 \\
12 / 01 / 93 \\
12 / 01 / 93 \\
12 / 01 / 93 \\
12 / 01 / 93 \\
12 / 01 / 93 \\
12 / 01 / 93 \\
12 / 01 / 93\end{array}$ \\
\hline 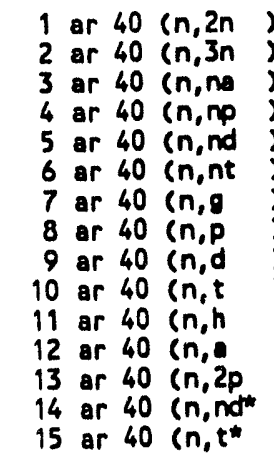 & 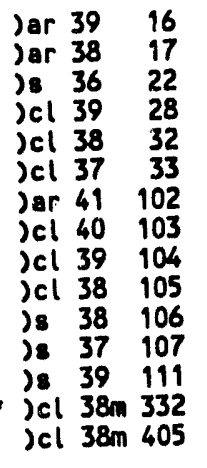 & $\begin{array}{r}21 \\
10 \\
47 \\
17 \\
7 \\
6 \\
3209 \\
14 \\
24 \\
19 \\
20 \\
14 \\
5 \\
8 \\
16\end{array}$ & $\begin{array}{l}12 / 01 / 93 \\
12 / 01 / 93 \\
12 / 01 / 93 \\
12 / 01 / 93 \\
12 / 01 / 93 \\
12 / 01 / 93 \\
12 / 01 / 93 \\
12 / 01 / 93 \\
12 / 01 / 93 \\
12 / 01 / 93 \\
12 / 01 / 93 \\
12 / 01 / 93 \\
12 / 01 / 93 \\
12 / 01 / 93 \\
12 / 01 / 93\end{array}$ \\
\hline $\begin{array}{l}1 \text { ar } 41 \text { (n, 2n } \\
2 \text { ar } 41 \text { (n,3n } \\
3 \text { ar } 41 \text { (n,no } \\
4 \text { ar } 41 \text { (n,np } \\
5 \text { ar } 49 \text { (n,nd }\end{array}$ & $\begin{array}{lll}\text { Jar } & 40 & 16 \\
\text { Jar } & 39 & 17 \\
\text { Jo } & 37 & 22 \\
\text { jcl } & 40 & 28 \\
\text { Jcl } & 39 & 32\end{array}$ & $\begin{array}{l}33 \\
20 \\
40 \\
17 \\
10\end{array}$ & $\begin{array}{l}12 / 01 / 93 \\
12 / 01 / 93 \\
12 / 01 / 93 \\
12 / 01 / 93 \\
12 / 01 / 93\end{array}$ \\
\hline
\end{tabular}

A-1. 8 


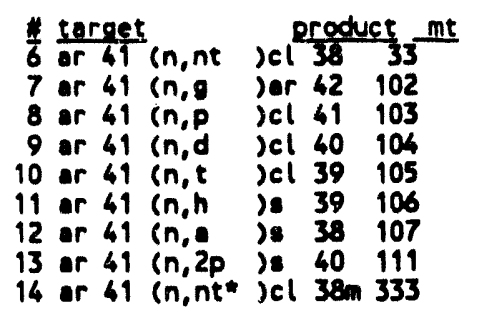

\begin{tabular}{cc} 
Hengeries & date \\
\hline 5 & $12 / 01 / 93$ \\
343 & $12 / 01 / 93$ \\
34 & $12 / 01 / 93$ \\
25 & $12 / 01 / 93$ \\
26 & $12 / 01 / 93$ \\
13 & $12 / 01 / 93$ \\
69 & $12 / 01 / 93$ \\
8 & $12 / 01 / 93$ \\
4 & $12 / 01 / 93$
\end{tabular}

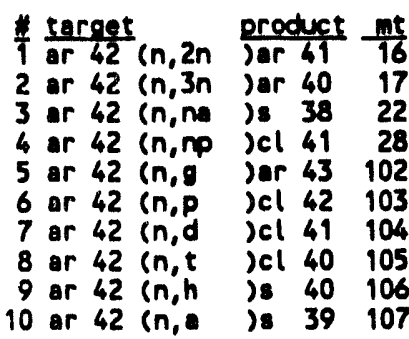

\begin{tabular}{cc} 
Eenperies & $\frac{1}{12 / 01 / 93}$ \\
\cline { 2 - 2 } 24 & $12 / 01 / 93$ \\
13 & $12 / 01 / 93$ \\
35 & $12 / 01 / 93$ \\
14 & $12 / 01 / 93$ \\
332 & $12 / 01 / 93$ \\
30 & $12 / 01 / 93$ \\
21 & $12 / 01 / 93$ \\
15 & $12 / 01 / 93$ \\
5 & $12 / 01 / 93$ \\
47 & $12 / 01 / 93$
\end{tabular}

$1 k 39(n, 2 n) k 38 \quad 16$

$2 k 39$ ( $n, n a)$ cl 3522

$3 k 39$ (n,np )ar 3828

$4 k 39$ ( $n$, nd ) ar 3732

$5 k 39$ (n, nt ) ar 3633

$6 k 39(n, n)$ ) cl $36 \quad 34$

$7 k 39(n, g) k 40 \quad 102$

$8 k 39$ (n,p ) or 39103

$9 k 39$ (n,d )ar 38104

$10 k 39(n, t)$ )ar $37 \quad 105$

$11 k 39$ (n,h ) cl $37 \quad 106$

$12 k 39(n, a)$ cl $36 \quad 107$

$13 k 39(n, 2 p)$ cl $38 \quad 111$

$14 k 39\left(n, 2 n^{*}\right) k 38 m 316$

$15 k 39$ (n,2p*) cl $38 m 411$

$\begin{array}{rr}16 & 12 / 01 / 93 \\ 45 & 12 / 01 / 93 \\ 14 & 12 / 01 / 93 \\ 11 & 12 / 01 / 93 \\ 6 & 12 / 01 / 93 \\ 2 & 12 / 01 / 93 \\ 2676 & 12 / 01 / 93 \\ 66 & 12 / 01 / 93 \\ 33 & 12 / 01 / 93 \\ 30 & 12 / 01 / 93 \\ 39 & 12 / 01 / 93 \\ 31 & 12 / 01 / 93 \\ 20 & 12 / 01 / 93 \\ 15 & 12 / 01 / 93 \\ 18 & 12 / 01 / 93\end{array}$

$1 k 40(n, 2 n) k 3916$

$2 k \quad 40$ (n,na )cl $36 \quad 22$

$3 k 40$ (n,np )ar 3928

$4 k 40$ ( $n$,nd ) ar 3832

$5 k 40$ (n,nt jar 3733

$6 k 40$ (n,nh $\mathrm{cl} 37 \quad 34$

7 k 40 (n,g) $k 41102$

$8 k 40 \mathrm{kn}, \mathrm{p}$ )ar 40103

$9 k 40 \mathrm{ln}, \mathrm{d}$ )ar 39104

$10 k 40(\mathrm{n}, \mathrm{t} \quad) \quad$ ar $38 \quad 105$

$11 k 40(n, h)$ cl 38106

$12 \mathrm{k} 40(\mathrm{n}, \mathrm{a}) \mathrm{cl} 37 \quad 107$

$13 k 40(n, 2 p) c(39111$

$14 k 40\left(n, h^{\star}\right)$ el $38 m 406$

$\begin{array}{rr}16 & 12 / 01 / 93 \\ 47 & 12 / 01 / 93 \\ 25 & 12 / 01 / 93 \\ 17 & 12 / 01 / 93 \\ 8 & 12 / 01 / 93 \\ 9 & 12 / 01 / 93 \\ 353 & 12 / 01 / 93 \\ 94 & 12 / 01 / 93 \\ 37 & 12 / 01 / 93 \\ 31 & 12 / 01 / 93 \\ 34 & 12 / 01 / 93 \\ 97 & 12 / 01 / 93 \\ 23 & 12 / 01 / 93 \\ 30 & 12 / 01 / 93\end{array}$

\begin{tabular}{|c|c|c|c|c|c|}
\hline $\begin{array}{ll}\frac{\#}{1} & \frac{t g}{k} \\
2 & k \\
2 & k \\
3 & k \\
4 & k \\
5 & k \\
6 & k \\
7 & k \\
8 & k \\
9 & k \\
10 & k \\
11 & k \\
12 & k \\
13 & k \\
14 & k\end{array}$ & 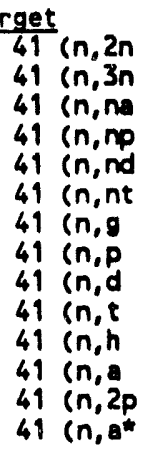 & $\begin{array}{ll}\text { proctuct } \\
\text { Jk } & 40 \\
\text { jk } & 39 \\
\text { jol } & 37 \\
\text { jar } & 40 \\
\text { jor } & 39 \\
\text { jar } & 38 \\
\text { jk } & 42 \\
\text { jar } & 41 \\
\text { jar } & 40 \\
\text { jar } & 39 \\
\text { jel } & 39 \\
\text { jcl } & 38 \\
\text { jcl } & 40 \\
\text { jcl } & 38 \mathrm{~m}\end{array}$ & $\begin{array}{l}\frac{m t}{16} \\
17 \\
22 \\
28 \\
32 \\
33 \\
102 \\
103 \\
104 \\
105 \\
106 \\
107 \\
111\end{array}$ & $\begin{array}{c}\text { \# engeries } \\
20 \\
8 \\
47 \\
36 \\
12 \\
11 \\
2308 \\
48 \\
35 \\
29 \\
28 \\
12 \\
14 \\
65\end{array}$ & $\begin{array}{l}\text { date } \\
12 / 01 / 93 \\
12 / 01 / 93 \\
12 / 01 / 93 \\
12 / 01 / 93 \\
12 / 01 / 93 \\
12 / 01 / 93 \\
12 / 01 / 93 \\
12 / 01 / 93 \\
12 / 01 / 93 \\
12 / 01 / 93 \\
12 / 01 / 93 \\
12 / 01 / 93 \\
12 / 01 / 93 \\
12 / 01 / 93\end{array}$ \\
\hline $\begin{array}{ll}1 & k \\
2 & k \\
3 & k\end{array}$ & $\begin{array}{l}42 \text { (n, 2n } \\
42 \text { (n, 3n } \\
42 \text { (n, na }\end{array}$ & $\begin{array}{ll}3 k & 41 \\
j k & 40 \\
j \mathrm{cl} & 38\end{array}$ & & $\begin{array}{l}28 \\
11 \\
47\end{array}$ & $\begin{array}{l}12 / 01 / 93 \\
12 / 01 / 93 \\
12 / 01 / 93\end{array}$ \\
\hline
\end{tabular}

A-1.9 


\begin{tabular}{|c|c|c|c|c|c|c|c|}
\hline $\begin{array}{rl} & 18 \\
\frac{1}{4} & \frac{1}{k} \\
5 & k \\
6 & k \\
7 & k \\
8 & k \\
9 & k \\
10 & k \\
11 & k \\
12 & k \\
13 & k \\
14 & k\end{array}$ & 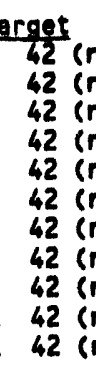 & 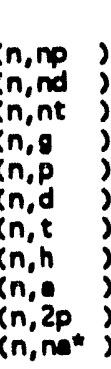 & $\begin{array}{l}\text { Pro } \\
\text { jer } 41 \\
\text { jor } 40 \\
\text { jar } 39 \\
\text { jk } 43 \\
\text { jor } 42 \\
\text { jor } 49 \\
\text { jar } 40 \\
\text { jol } 40 \\
\text { jel } 39 \\
\text { jel } 49 \\
\text { jel } 38\end{array}$ & $\begin{array}{ll}\text { oduct } \\
1 & 2 \\
0 & 3 \\
9 & 3 \\
3 & 10 \\
2 & 10 \\
1 & 10 \\
0 & 10 \\
0 & 10 \\
9 & 10 \\
1 & 11 \\
8 & 32\end{array}$ & $\begin{array}{l}28 \mathrm{mt} \\
32 \\
33 \\
02 \\
103 \\
104 \\
105 \\
106 \\
107 \\
111 \\
322\end{array}$ & $\begin{array}{l}\text { Henoeries } \\
\frac{15}{25} \\
17 \\
9 \\
357 \\
61 \\
35 \\
29 \\
21 \\
77 \\
16 \\
41\end{array}$ & $\begin{array}{l}\frac{\text { date }}{12 / 01 / 93} \\
12 / 01 / 93 \\
12 / 01 / 93 \\
12 / 01 / 93 \\
12 / 01 / 93 \\
12 / 01 / 93 \\
12 / 01 / 93 \\
12 / 01 / 93 \\
12 / 01 / 93 \\
12 / 01 / 93 \\
12 / 01 / 93\end{array}$ \\
\hline $\begin{array}{ll}1 & k \\
2 & k \\
3 & k \\
4 & k \\
5 & k \\
6 & k \\
7 & k \\
8 & k \\
9 & k \\
10 & k \\
11 & k \\
12 & k \\
13 & k\end{array}$ & 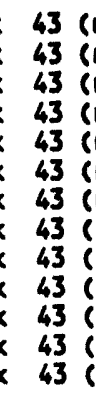 & 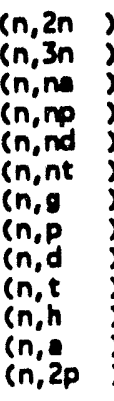 & 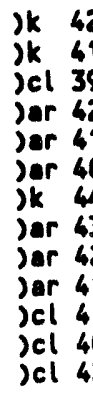 & $\begin{array}{l}2 \\
1 \\
2 \\
1 \\
0 \\
4 \\
3 \\
2 \\
1 \\
1 \\
0 \\
62\end{array}$ & $\begin{array}{l}16 \\
17 \\
22 \\
28 \\
32 \\
33 \\
102 \\
103 \\
104 \\
105 \\
106 \\
107 \\
111\end{array}$ & $\begin{array}{r}30 \\
13 \\
35 \\
24 \\
9 \\
10 \\
358 \\
42 \\
32 \\
24 \\
16 \\
58 \\
5\end{array}$ & $\begin{array}{l}12 / 01 / 93 \\
12 / 01 / 93 \\
12 / 01 / 93 \\
12 / 01 / 93 \\
12 / 01 / 93 \\
12 / 01 / 93 \\
12 / 01 / 93 \\
12 / 01 / 93 \\
12 / 01 / 93 \\
12 / 01 / 93 \\
12 / 01 / 93 \\
12 / 01 / 93 \\
12 / 01 / 93\end{array}$ \\
\hline $\begin{array}{rl}2 & c \\
3 & c \\
4 & c \\
5 & c \\
6 & c \\
7 & c \\
8 & c \\
9 & 0 \\
10 & c \\
11 & 0 \\
12 & 0 \\
13 & c \\
14 & \end{array}$ & $\begin{array}{ll}\text { te } & -\frac{g e t}{40} \text {, } \\
\text { ca } & 40 \\
\text { ca } & 40 \\
\text { ca } & 40 \\
\text { ca } & 40 \\
\text { ca } & 40 \\
\text { ca } & 40 \\
\text { ca } & 40 \\
\text { ca } & 40 \\
\text { ca } & 40 \\
\text { ca } & 40 \\
\text { ca } & 40 \\
\text { ca } & 40 \\
\text { ca } & 40\end{array}$ & 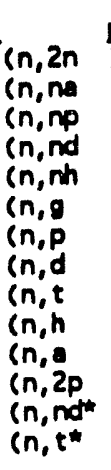 & $\begin{array}{l}\text { produ } \\
\text { Jca } 3 \\
\text { Jar } 3 \\
\text { Jk } 3 \\
\text { Jk } 3 \\
\text { Jar } 3 \\
\text { Jca } 4 \\
\text { Jk } 4 \\
\text { Jk } 3 \\
\text { Jk } 3 \\
\text { Jar } 3 \\
\text { Jar } 3 \\
\text { Jar } 3 \\
\text { Jk } 3 \\
\text { Jk } 3\end{array}$ & $\begin{array}{l}\frac{u c t}{39} \\
36 \\
39 \\
38 \\
37 \\
41 \\
40 \\
39 \\
38 \\
38 \\
37 \\
39 \\
38 m \\
38 m\end{array}$ & $\begin{array}{l}\frac{m t}{16} \\
22 \\
28 \\
32 \\
34 \\
102 \\
103 \\
104 \\
105 \\
106 \\
107 \\
111 \\
332 \\
405\end{array}$ & $\begin{array}{c}\text { \# engeries } \\
6 \\
4 \\
60 \\
2 \\
5 \\
2937 \\
10 \\
33 \\
3 \\
45 \\
33 \\
26 \\
2 \\
3\end{array}$ & $\begin{array}{c}\text { date } \\
12 / 01 / 93 \\
12 / 01 / 93 \\
12 / 01 / 93 \\
12 / 01 / 93 \\
12 / 01 / 93 \\
12 / 01 / 93 \\
12 / 01 / 93 \\
12 / 01 / 93 \\
12 / 01 / 93 \\
12 / 01 / 93 \\
12 / 01 / 93 \\
12 / 01 / 93 \\
12 / 01 / 93 \\
12 / 01 / 93\end{array}$ \\
\hline $\begin{array}{l}1 \\
2 \\
3 \\
4 \\
5 \\
6 \\
7 \\
8 \\
9 \\
10 \\
11 \\
12\end{array}$ & 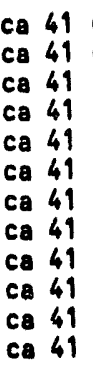 & $\begin{array}{l}\text { ln, 2n } \\
\text { in,na } \\
\text { (n,np } \\
(n, n d \\
\text { (n,nh } \\
\text { (n,g } \\
\text { in,p } \\
\text { in,d } \\
\text { (n,t } \\
(n, n \\
\text { in, } \\
\text { in, 2p }\end{array}$ & $\begin{array}{l}\text { Jca } \\
\text { Jar } \\
\text { jk } \\
\text { jk } \\
\text { Jar } \\
\text { jca } \\
\text { Jk } \\
\text { Jk } \\
\text { jk } \\
\text { Jar } \\
\text { Jar } \\
\text { Jar }\end{array}$ & $\begin{array}{l}40 \\
37 \\
40 \\
39 \\
38 \\
42 \\
41 \\
40 \\
39 \\
39 \\
38 \\
40\end{array}$ & $\begin{array}{c}16 \\
22 \\
28 \\
32 \\
34 \\
102 \\
103 \\
104 \\
105 \\
106 \\
107 \\
111\end{array}$ & $\begin{array}{r}25 \\
47 \\
25 \\
14 \\
12 \\
343 \\
49 \\
33 \\
31 \\
41 \\
55 \\
35\end{array}$ & $\begin{array}{l}12 / 01 / 93 \\
12 / 01 / 93 \\
12 / 01 / 93 \\
12 / 01 / 93 \\
12 / 01 / 93 \\
12 / 01 / 93 \\
12 / 01 / 93 \\
12 / 01 / 93 \\
12 / 01 / 93 \\
12 / 01 / 93 \\
12 / 01 / 93 \\
12 / 01 / 93\end{array}$ \\
\hline 11 & $\begin{array}{ll}\text { ca } & 42 \\
\text { ca } & 42 \\
\text { ca } & 42 \\
\text { ca } & 42 \\
\text { ca } & 42 \\
\text { ca } & 42 \\
\text { ca } & 42 \\
\text { ca } & 42 \\
\text { ca } & 42 \\
\text { ca } & 42 \\
\text { ca } & 42\end{array}$ & $\begin{array}{l}(n, 2 n \\
(n, n) \\
(n, n p \\
(n, n d \\
(n, g \\
(n, p) \\
l n, d \\
(n, t) \\
(n, h \\
i n, d \\
(n, 2 p\end{array}$ & 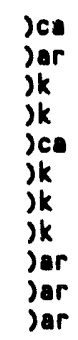 & $\begin{array}{l}41 \\
38 \\
41 \\
40 \\
43 \\
42 \\
41 \\
40 \\
40 \\
39 \\
41\end{array}$ & $\begin{array}{l}16 \\
22 \\
28 \\
32 \\
102 \\
103 \\
104 \\
105 \\
106 \\
107 \\
111\end{array}$ & $\begin{array}{r}24 \\
47 \\
23 \\
6 \\
2668 \\
49 \\
30 \\
22 \\
34 \\
34 \\
18\end{array}$ & $\begin{array}{l}12 / 01 / 93 \\
12 / 01 / 93 \\
12 / 01 / 93 \\
12 / 01 / 93 \\
12 / 01 / 93 \\
12 / 01 / 93 \\
12 / 01 / 93 \\
12 / 01 / 93 \\
12 / 01 / 93 \\
12 / 01 / 93 \\
12 / 01 / 93\end{array}$ \\
\hline
\end{tabular}




\begin{tabular}{|c|c|c|c|c|c|}
\hline $\begin{array}{ll}\frac{1}{1} & \mathrm{t} \\
2 & \mathrm{c} \\
2 & \mathrm{c} \\
3 & \mathrm{c} \\
4 & \mathrm{c} \\
5 & \mathrm{c} \\
6 & \mathrm{c} \\
7 & \mathrm{c} \\
8 & \mathrm{c} \\
9 & \mathrm{c} \\
10 & \mathrm{c} \\
11 & \mathrm{c} \\
12 & \mathrm{c} \\
13 & \mathrm{c}\end{array}$ & 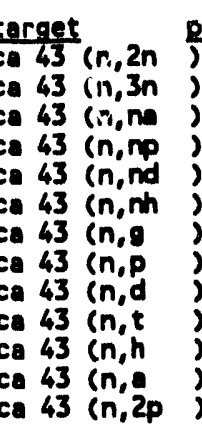 & 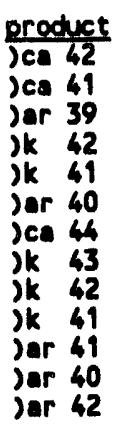 & $\begin{array}{l}\frac{m t}{16} \\
17 \\
22 \\
28 \\
32 \\
34 \\
102 \\
103 \\
104 \\
105 \\
106 \\
107 \\
111\end{array}$ & $\begin{array}{c}\text { Eengerieg } \\
23 \\
2 \\
45 \\
21 \\
11 \\
6 \\
2032 \\
54 \\
30 \\
30 \\
30 \\
70 \\
20\end{array}$ & $\begin{array}{l}\text { date } \\
\frac{12 / 01 / 93}{12 / 01 / 93} \\
12 / 01 / 93 \\
12 / 01 / 93 \\
12 / 01 / 93 \\
12 / 01 / 93 \\
12 / 01 / 93 \\
12 / 01 / 93 \\
12 / 01 / 93 \\
12 / 01 / 93 \\
12 / 01 / 93 \\
12 / 01 / 93 \\
12 / 01 / 93\end{array}$ \\
\hline $\begin{array}{rl}1 & c \\
2 & c \\
3 & c \\
4 & c \\
5 & c \\
6 & c \\
7 & c \\
8 & c \\
9 & c \\
10 & c \\
11 & c\end{array}$ & 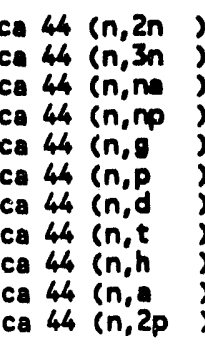 & $\begin{array}{ll}\text { Jca } & 43 \\
\text { Jca } & 42 \\
\text { Jar } & 40 \\
\text { Jk } & 43 \\
\text { Jca } & 45 \\
\text { Jk } & 44 \\
\text { jk } & 43 \\
\text { Jk } & 42 \\
\text { Jar } & 42 \\
\text { Jor } & 41 \\
\text { Jar } & 43\end{array}$ & $\begin{array}{r}16 \\
17 \\
22 \\
28 \\
102 \\
103 \\
104 \\
105 \\
106 \\
107 \\
111\end{array}$ & $\begin{array}{r}27 \\
6 \\
32 \\
18 \\
1702 \\
42 \\
28 \\
17 \\
23 \\
27 \\
11\end{array}$ & $\begin{array}{l}12 / 01 / 93 \\
12 / 01 / 93 \\
12 / 01 / 93 \\
12 / 01 / 93 \\
12 / 01 / 93 \\
12 / 01 / 93 \\
12 / 01 / 93 \\
12 / 01 / 93 \\
12 / 01 / 93 \\
12 / 01 / 93 \\
12 / 01 / 93\end{array}$ \\
\hline $\begin{array}{rl}1 & 0 \\
2 & 0 \\
3 & 0 \\
4 & 5 \\
5 & 5 \\
6 & \\
7 & \\
8 & \\
9 & \\
10 & \\
11 & \\
12 & \end{array}$ & 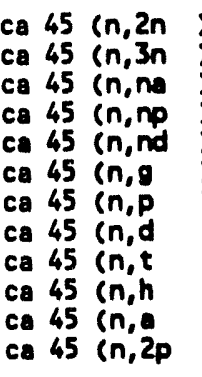 & $\begin{array}{ll}\text { Jca } & 44 \\
\text { jca } & 43 \\
\text { Jar } & 41 \\
\text { jk } & 44 \\
\text { jk } & 43 \\
\text { jca } & 46 \\
\text { jk } & 45 \\
\text { jk } & 44 \\
\text { jk } & 43 \\
\text { Jar } & 43 \\
\text { Jar } & 42 \\
\text { Jar } & 44\end{array}$ & $\begin{array}{l}16 \\
17 \\
22 \\
28 \\
32 \\
102 \\
103 \\
104 \\
105 \\
106 \\
107 \\
111\end{array}$ & $\begin{array}{r}25 \\
8 \\
33 \\
17 \\
8 \\
348 \\
43 \\
26 \\
25 \\
17 \\
35 \\
13\end{array}$ & $\begin{array}{l}12 / 01 / 93 \\
12 / 01 / 93 \\
12 / 01 / 93 \\
12 / 01 / 93 \\
12 / 01 / 93 \\
12 / 01 / 93 \\
12 / 01 / 93 \\
12 / 01 / 93 \\
12 / 01 / 93 \\
12 / 01 / 93 \\
12 / 01 / 93 \\
12 / 01 / 93\end{array}$ \\
\hline $\begin{array}{r}1 \\
2 \\
3 \\
4 \\
5 \\
6 \\
7 \\
8 \\
9 \\
10\end{array}$ & 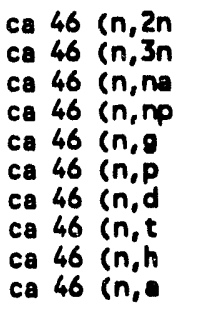 & $\begin{array}{ll}\text { Jca } & 45 \\
\text { Jca } & 44 \\
\text { Jar } & 42 \\
\text { Jk } & 45 \\
\text { Jca } & 47 \\
\text { Jk } & 46 \\
\text { Jk } & 45 \\
\text { Jk } & 44 \\
\text { Jar } & 44 \\
\text { Jar } & 43\end{array}$ & $\begin{array}{l}16 \\
17 \\
22 \\
28 \\
102 \\
103 \\
104 \\
105 \\
106 \\
107\end{array}$ & $\begin{array}{r}24 \\
11 \\
30 \\
15 \\
346 \\
34 \\
22 \\
15 \\
9 \\
47\end{array}$ & $\begin{array}{l}12 / 01 / 93 \\
12 / 01 / 93 \\
12 / 01 / 93 \\
12 / 01 / 93 \\
12 / 01 / 93 \\
12 / 01 / 93 \\
12 / 01 / 93 \\
12 / 01 / 93 \\
12 / 01 / 93 \\
12 / 01 / 93\end{array}$ \\
\hline $\begin{array}{r}\frac{\#}{1} \\
2 \\
3 \\
4 \\
5 \\
6 \\
7 \\
8 \\
9 \\
10 \\
11 \\
12\end{array}$ & 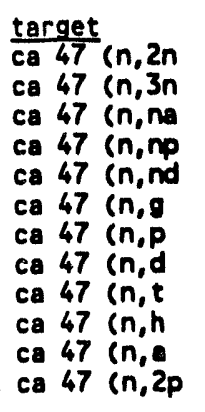 & $\begin{array}{ll}\text { product } \\
\text { Jcs } & 46 \\
\text { Jca } & 45 \\
\text { Jar } & 43 \\
\text { Jk } & 46 \\
\text { Jk } & 45 \\
\text { Jca } & 48 \\
\text { Jk } & 47 \\
\text { Jk } & 46 \\
\text { Jk } & 45 \\
\text { Jar } & 45 \\
\text { Jar } & 44 \\
\text { Jar } & 46\end{array}$ & $\begin{array}{l}\frac{m t}{16} \\
17 \\
22 \\
28 \\
32 \\
102 \\
103 \\
104 \\
105 \\
106 \\
107 \\
111\end{array}$ & $\begin{array}{c}\text { engeries } \\
32 \\
11 \\
26 \\
16 \\
5 \\
357 \\
37 \\
21 \\
20 \\
2 \\
51 \\
2\end{array}$ & $\begin{array}{l}\text { date } \\
12 / 01 / 93 \\
12 / 01 / 93 \\
12 / 01 / 93 \\
12 / 01 / 93 \\
12 / 01 / 93 \\
12 / 01 / 93 \\
12 / 01 / 93 \\
12 / 01 / 93 \\
12 / 01 / 93 \\
12 / 01 / 93 \\
12 / 01 / 93 \\
12 / 01 / 93\end{array}$ \\
\hline 9 & 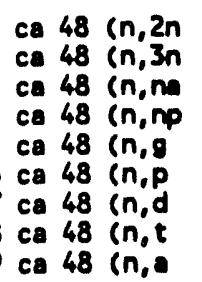 & 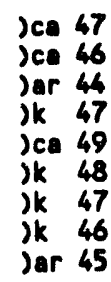 & $\begin{array}{r}16 \\
17 \\
22 \\
28 \\
102 \\
103 \\
104 \\
105 \\
107\end{array}$ & $\begin{array}{r}29 \\
13 \\
20 \\
11 \\
543 \\
24 \\
18 \\
12 \\
38\end{array}$ & $\begin{array}{l}12 / 01 / 93 \\
12 / 01 / 93 \\
12 / 01 / 93 \\
12 / 01 / 93 \\
12 / 01 / 93 \\
12 / 01 / 93 \\
12 / 01 / 93 \\
12 / 01 / 93 \\
12 / 01 / 93\end{array}$ \\
\hline
\end{tabular}

A-1. 11 


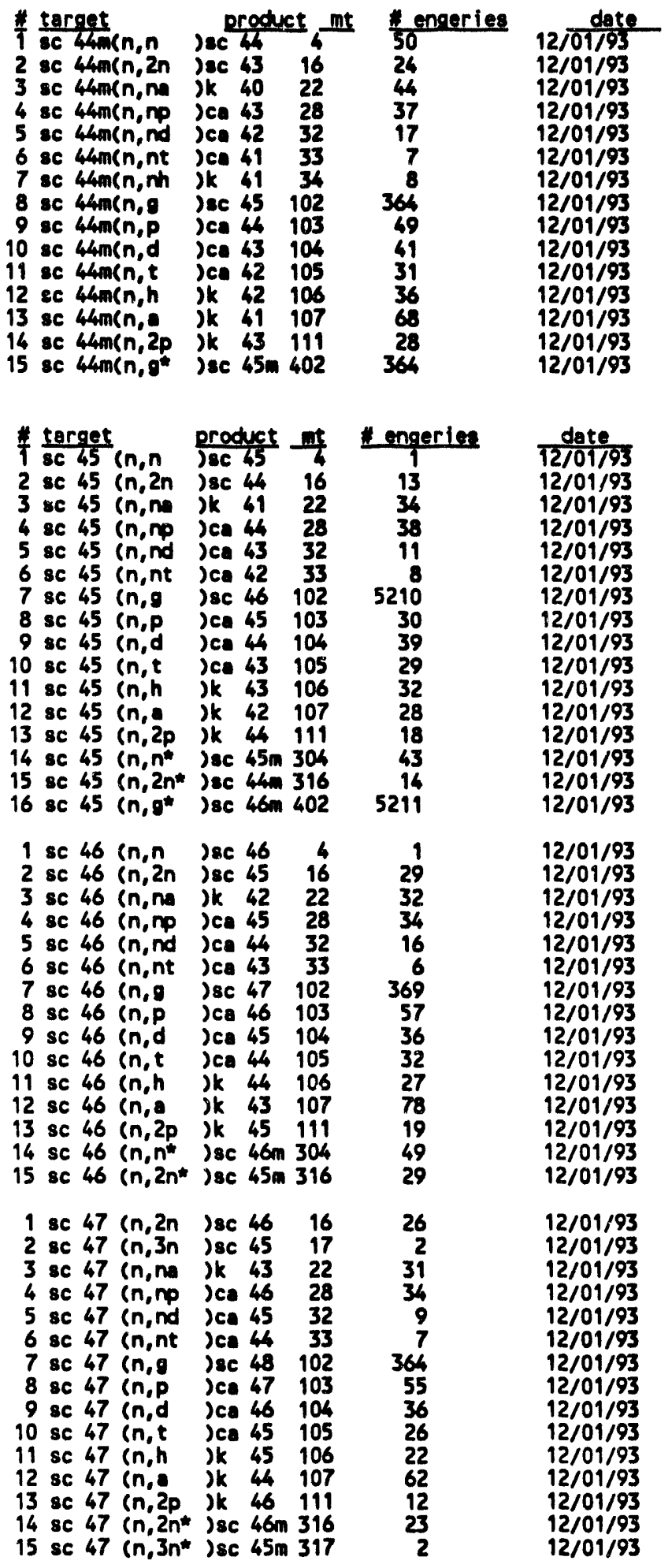


WHC-EP-0727

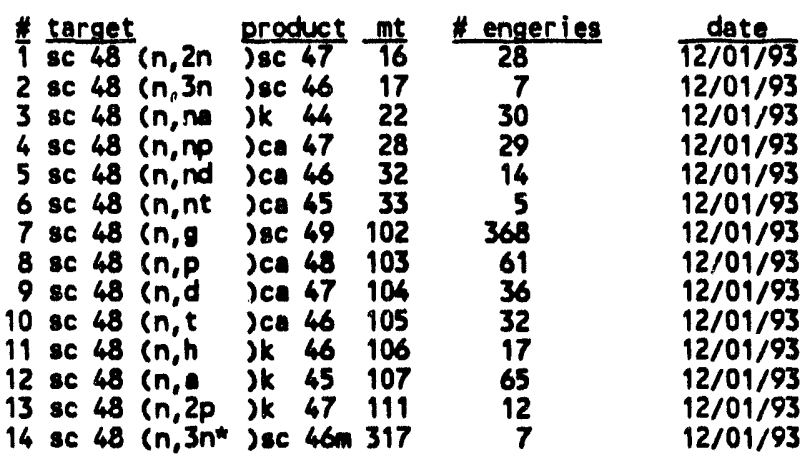

$A-1.13$ 
WHC-EP-0727

This page intentionally left blank.

$A-1.14$ 
WHC-EP-0727

Appendix $A-2$

List of Reactions on files *.ti.zn

岪 ti ti 44

2 ti 44 (n,ns) ca 4022

3 ti 44 (n,np) se 4328

4 ti 44 ( $n$,nd ) se 4232

5 ti 44 (n,mh )ea 41 34

6 ti $44\left(n_{0} g\right)$ ti 45102

7 ti 44 (n,p )ec 46103

$8 \mathrm{ti} 44 \mathrm{ln}, \mathrm{d}$ )se 43104

9 ti $44(n, t$ )ec 42105

10 ti $44(n, h$ )ea 42106

11 ti 44 (n,e jea 41 107

12 ti $44(n, 2 p)$ ca 43111

13 ti 44 (n,nd )ec 42m 332

14 ti $44\left(n, p^{*}\right)$ se 44403

15 ti 44 (n, t* ) se $42 m 405$

1 ti $45(n, 2 n) t i 4416$

2 ti 45 (n,in )ea 4122

3 ti 45 (n,no )ec 4428

4 ti 45 (n,nd) sc 4332

5 ti 45 (n,nh) ce 4234

$6 \mathrm{ti} 45 \mathrm{(n,g}) \mathrm{ti} 46 \quad 102$

7 ti $45 \mathrm{ln}, \mathrm{p}$ )sc 45103

8 ti 45 (n,d sc 44104

9 ti $45 \mathrm{kn}, \mathrm{t}$ ) $8 \mathrm{CC} 43105$

10 ti $45(n, h$ )ca 43106

11 ti 45 (n,a jea 42107

12 ti $45(n, 2 p) c e 44111$

13 ti 45 (n,np*) sc $44 m 328$

14 ti 45 (n,p) )sc $45 m 403$

15 ti 45 (n, d) sc 44404

$\frac{\#}{1} \frac{\text { target }}{\mathrm{ti} 46}\left(\mathrm{n}, 2 \mathrm{n} \frac{\text { product }}{\mathrm{jti} 45} \frac{\mathrm{mt}}{16}\right.$

$\begin{array}{lllll}1 & t i & 46 & \text { (n, 2n } & \text { (n, ns }) \text { ) } \\ 2 & \text { ca } 42 & 22\end{array}$

3 ti 46 (n,np jse 4528

4 ti 46 (n,nd) sc 4432

5 ti 46 (n,g) ti $47 \quad 102$

$6 \mathrm{ti} 46(\mathrm{n}, \mathrm{p}) \mathrm{sc} 46103$

7 ti 46 (n,d )sc 45104

8 ti $46(n, t)$ sc 44105

9 ti $46 \mathrm{cn,h}$ jes 44106

10 ti $46(n, a)$ ca 43107

11 ti 46 (n,2p )ca 45111

12 ti 46 ( $\left.n, n p^{*}\right) \mathrm{sc} 45 \mathrm{~m} 328$

13 ti $46\left(n, p^{*}\right)$ sc $46 m 403$

14 ti 46 ( $\left.n, d^{*}\right)$ sc 45m 404

15 ti 46 (n,t*) sc $44 \mathrm{~mm} 405$

1 ti $47(n, 2 n)$ ti $46 \quad 16$

2 ti 47 ( $n, n$ ) ce 4322

3 ti 47 (n,np) se 4628

4 ti 47 (n,nd) $8 c 4532$

5 ti $47(n, n h)$ ce 4434

6 ti $47 \mathrm{(n,g})$ ti 48102

7 ti 47 (n,p )sc $47 \quad 103$

$8 \mathrm{ti} 47 \mathrm{cn}, \mathrm{d}) \mathrm{isc} 46 \quad 104$

9 ti $47 \mathrm{kn,t}$ )sc 45105

10 ti $47 \mathrm{(n,h}) \mathrm{CB} 45106$

11 ti 47 (n,a ) )a 44107

12 ti 47 (n,2p )ca 46111

13 ti 47 ( $n, n p^{\star}$ ) sc $46 \mathrm{~m} 328$

14 ti 47 ( $n$, ndt )sc 45m 332

15 ti 47 ( n, it ) sc 465404

16 ti 47 (n,t*) sec 45m 405

\begin{tabular}{c} 
Engeries \\
\hline 11 \\
49 \\
36 \\
7 \\
8 \\
332 \\
56 \\
34 \\
21 \\
50 \\
67 \\
39 \\
8 \\
56 \\
19
\end{tabular}

$\frac{\text { date }}{12101 / 93}$

$12 / 01 / 93$

$12 / 01 / 93$

$12 / 01 / 93$

$12 / 01 / 93$

$12 / 01 / 93$

$12 / 01 / 93$

$12 / 01 / 93$

$12 / 01 / 93$

$12 / 01 / 93$

$12 / 01 / 93$

$12 / 01 / 93$

$12 / 01 / 93$

$12 / 01 / 93$

$12 / 01 / 93$

$\begin{array}{ll}24 & 12 / 01 / 93 \\ 49 & 12 / 01 / 93 \\ 35 & 12 / 01 / 93 \\ 11 & 12 / 01 / 93 \\ 12 & 12 / 01 / 93 \\ 360 & 12 / 01 / 93 \\ 51 & 12 / 01 / 93 \\ 35 & 12 / 01 / 93 \\ 31 & 12 / 01 / 93 \\ 44 & 12 / 01 / 93 \\ 56 & 12 / 01 / 93 \\ 43 & 12 / 01 / 93 \\ 35 & 12 / 01 / 93 \\ 51 & 12 / 01 / 93 \\ 33 & 12 / 01 / 93\end{array}$

\begin{tabular}{cc} 
\# engeries & date \\
\hline 27 & $\frac{12 / 01 / 93}{12}$ \\
12 & $12 / 01 / 93$ \\
15 & $12 / 01 / 93$ \\
2 & $12 / 01 / 93$ \\
2670 & $12 / 01 / 93$ \\
46 & $12 / 01 / 93$ \\
36 & $12 / 01 / 93$ \\
12 & $12 / 01 / 93$ \\
41 & $12 / 01 / 93$ \\
33 & $12 / 01 / 93$ \\
40 & $12 / 01 / 93$ \\
15 & $12 / 01 / 93$ \\
46 & $12 / 01 / 93$ \\
36 & $12 / 01 / 93$ \\
12 & $12 / 01 / 93$
\end{tabular}

$12 / 01 / 93$

$12 / 01 / 93$

$12 / 01 / 93$

$12 / 01 / 93$

$12 / 01 / 93$

$12 / 01 / 93$

$12 / 01 / 93$

$12 / 01 / 93$

$12 / 01 / 93$

$12 / 01 / 93$

$12 / 01 / 93$

$12 / 01 / 93$

$12 / 01 / 93$

$12 / 01 / 93$

$12 / 01 / 93$

$12 / 01 / 93$ 


\begin{tabular}{|c|c|c|c|}
\hline 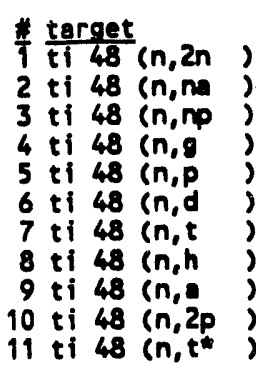 & 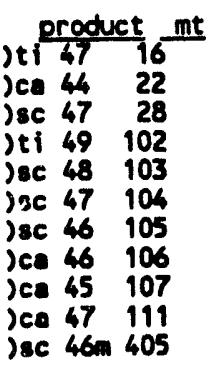 & $\begin{array}{l}\frac{4 \text { engeries }}{15} \\
9 \\
42 \\
872 \\
26 \\
44 \\
206 \\
30 \\
32 \\
30 \\
206\end{array}$ & $\begin{array}{l}\frac{\text { date }}{12 / 01 / 93} \\
12 / 01 / 93 \\
12 / 01 / 93 \\
12 / 01 / 93 \\
12 / 01 / 93 \\
12 / 01 / 93 \\
12 / 01 / 93 \\
12 / 01 / 93 \\
12 / 01 / 93 \\
12 / 01 / 93 \\
12 / 01 / 93\end{array}$ \\
\hline 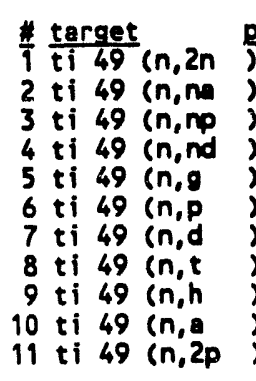 & 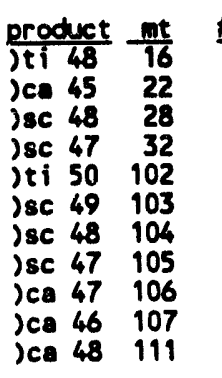 & $\begin{array}{c}\text { \# engeries } \\
16 \\
9 \\
16 \\
10 \\
1719 \\
21 \\
44 \\
36 \\
28 \\
31 \\
23\end{array}$ & $\begin{array}{l}\text { date } \\
12 / 01 / 93 \\
12 / 01 / 93 \\
12 / 01 / 93 \\
12 / 01 / 93 \\
12 / 01 / 93 \\
12 / 01 / 93 \\
12 / 01 / 93 \\
12 / 01 / 93 \\
12 / 01 / 93 \\
12 / 01 / 93 \\
12 / 01 / 93\end{array}$ \\
\hline 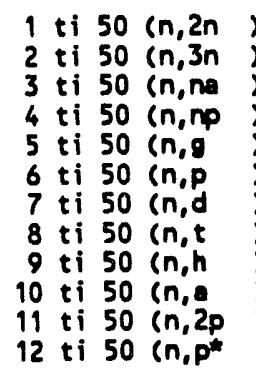 & 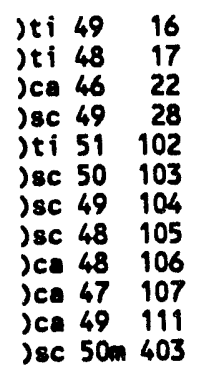 & $\begin{array}{r}17 \\
4 \\
9 \\
13 \\
79 \\
17 \\
40 \\
24 \\
25 \\
25 \\
14 \\
17\end{array}$ & $\begin{array}{l}12 / 01 / 93 \\
12 / 01 / 93 \\
12 / 01 / 93 \\
12 / 01 / 93 \\
12 / 01 / 93 \\
12 / 01 / 93 \\
12 / 01 / 93 \\
12 / 01 / 93 \\
12 / 01 / 93 \\
12 / 01 / 93 \\
12 / 01 / 93 \\
12 / 01 / 93\end{array}$ \\
\hline 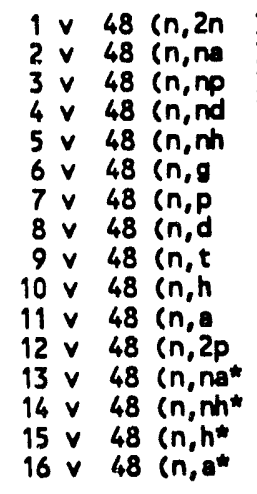 & 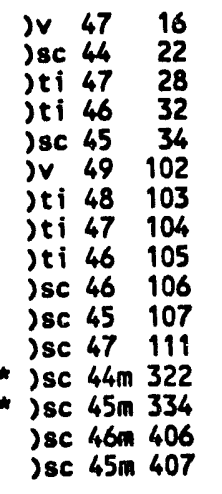 & $\begin{array}{r}24 \\
32 \\
38 \\
19 \\
6 \\
379 \\
52 \\
42 \\
36 \\
40 \\
76 \\
39 \\
31 \\
6 \\
40 \\
76\end{array}$ & $\begin{array}{l}12 / 01 / 93 \\
12 / 01 / 93 \\
12 / 01 / 93 \\
12 / 01 / 93 \\
12 / 01 / 93 \\
12 / 01 / 93 \\
12 / 01 / 93 \\
12 / 01 / 93 \\
12 / 01 / 93 \\
12 / 01 / 93 \\
12 / 01 / 93 \\
12 / 01 / 93 \\
12 / 01 / 93 \\
12 / 01 / 93 \\
12 / 01 / 93 \\
12 / 01 / 93\end{array}$ \\
\hline 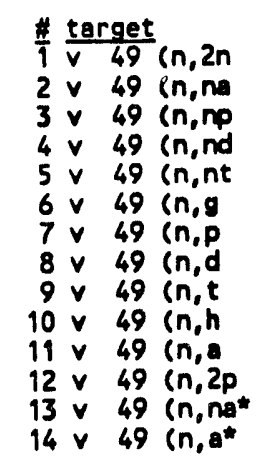 & 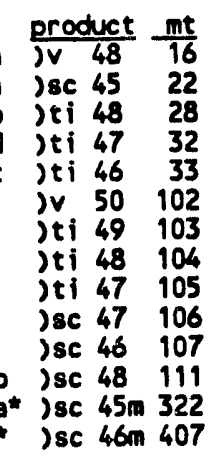 & $\begin{array}{c}\text { * engeries } \\
14 \\
9 \\
15 \\
11 \\
5 \\
372 \\
21 \\
43 \\
30 \\
35 \\
32 \\
23 \\
9 \\
32\end{array}$ & $\begin{array}{l}\frac{\text { date }}{12 / 01 / 93} \\
12 / 01 / 93 \\
12 / 01 / 93 \\
12 / 01 / 93 \\
12 / 01 / 93 \\
12 / 01 / 93 \\
12 / 01 / 93 \\
12 / 01 / 93 \\
12 / 01 / 93 \\
12 / 01 / 93 \\
12 / 01 / 93 \\
12 / 01 / 93 \\
12 / 01 / 93 \\
12 / 01 / 93\end{array}$ \\
\hline
\end{tabular}




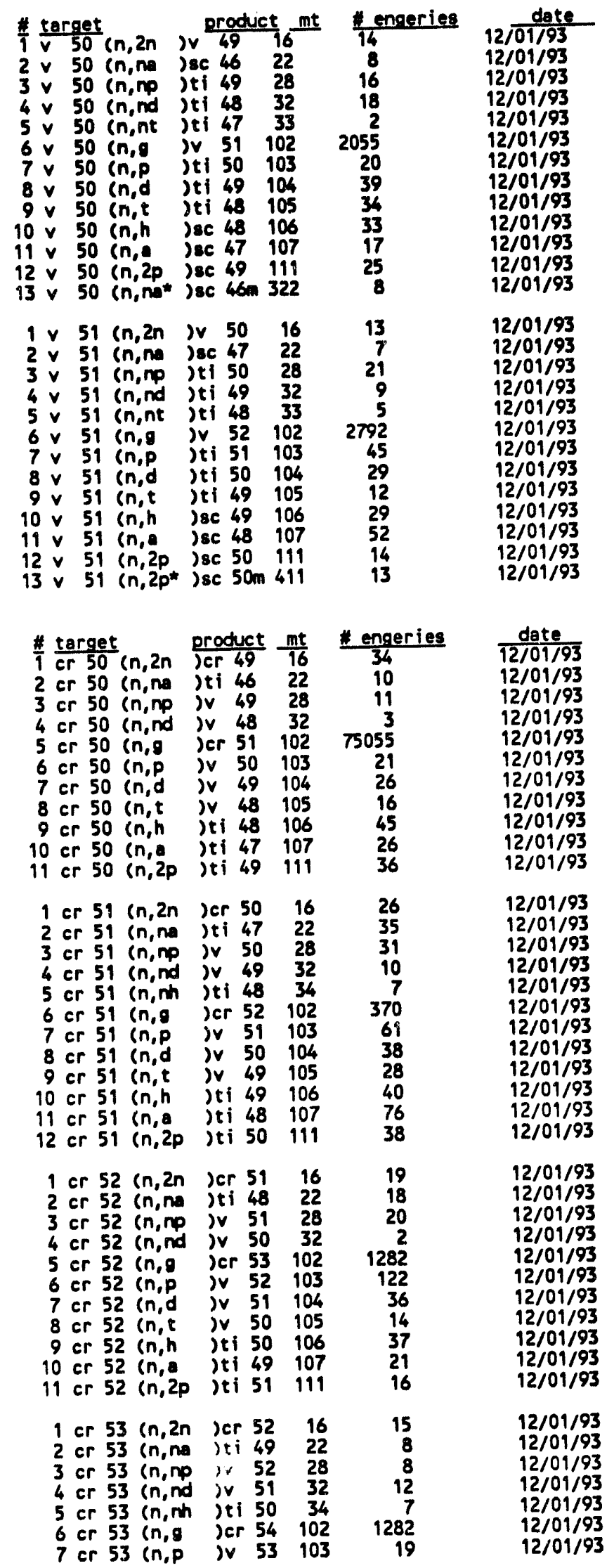

A-2.3 


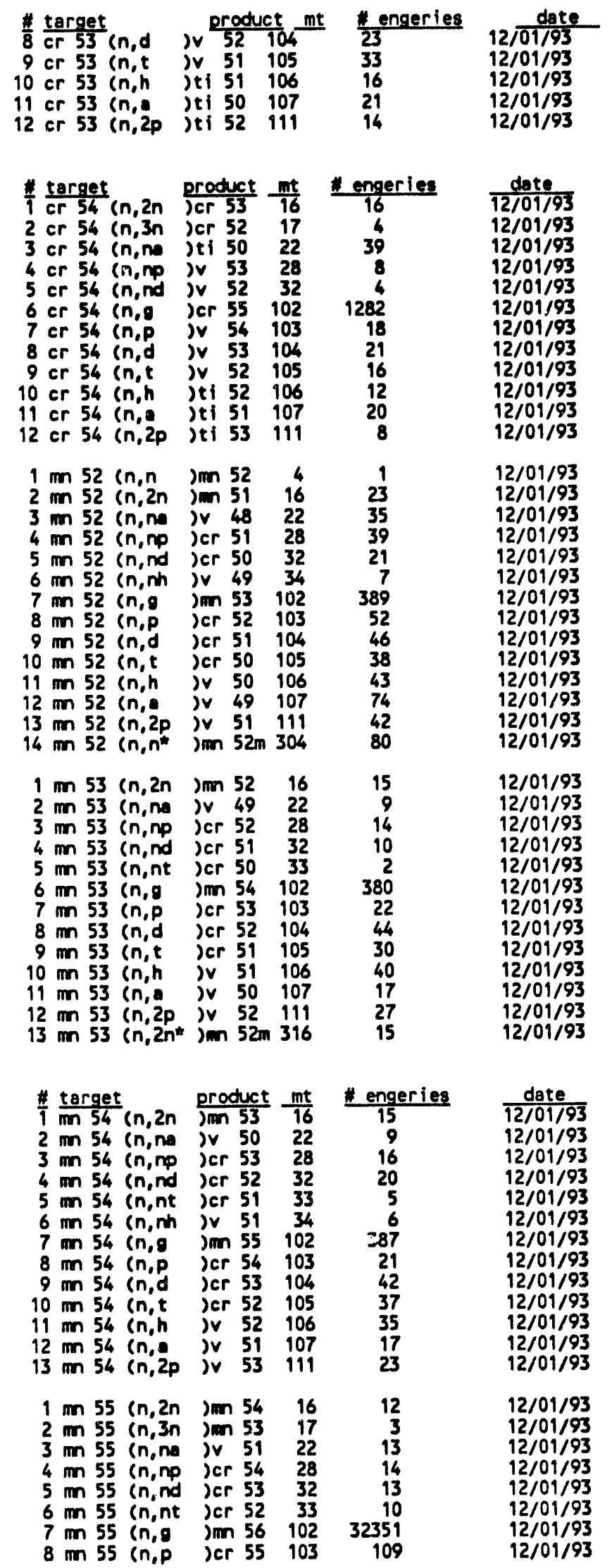

$$
\text { A-2. } 4
$$




\begin{tabular}{|c|c|c|c|c|}
\hline $\begin{array}{l}\frac{1}{9} \frac{\text { target }}{\operatorname{mn} 55} \text { (n,d } \\
10 \operatorname{mn} 55 \text { (n,t } \\
11 \text { m } 55 \text { (n, } \\
12 \text { mn } 55 \text { (n, } \\
13 \min 55 \text { (n, 2p }\end{array}$ & $\begin{array}{l}\text { produc } \\
\text { or } 54 \\
\text { or } 53 \\
\text { iv } 53 \\
\text { iv } 52 \\
\text { iv } 54\end{array}$ & $\begin{array}{l}\frac{\mathrm{ct}}{104} \mathrm{mt} \\
105 \\
106 \\
107 \\
111\end{array}$ & $\begin{array}{l}\text { engeries } \\
14 \\
33 \\
4 \\
161 \\
12\end{array}$ & $\begin{array}{l}\frac{\text { date }}{12 / 01 / 93} \\
12 / 01 / 93 \\
12 / 01 / 93 \\
12 / 01 / 93 \\
12 / 01 / 93\end{array}$ \\
\hline 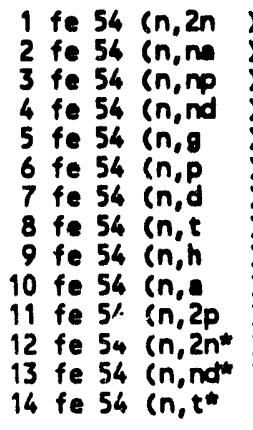 & 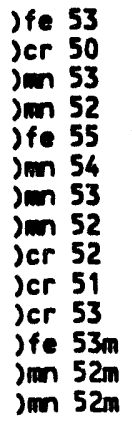 & $\begin{array}{r}16 \\
22 \\
28 \\
32 \\
102 \\
103 \\
104 \\
105 \\
106 \\
107 \\
111 \\
316 \\
332 \\
405\end{array}$ & $\begin{array}{r}81 \\
114 \\
63 \\
11 \\
778 \\
152 \\
26 \\
199 \\
49 \\
115 \\
40 \\
9 \\
11 \\
199\end{array}$ & $\begin{array}{l}12 / 01 / 93 \\
12 / 01 / 93 \\
12 / 01 / 93 \\
12 / 01 / 93 \\
12 / 01 / 93 \\
12 / 01 / 93 \\
12 / 01 / 93 \\
12 / 01 / 93 \\
12 / 01 / 93 \\
12 / 01 / 93 \\
12 / 01 / 93 \\
12 / 01 / 93 \\
12 / 01 / 93 \\
12 / 01 / 93\end{array}$ \\
\hline 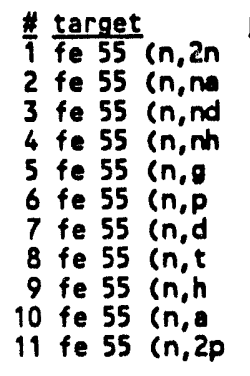 & 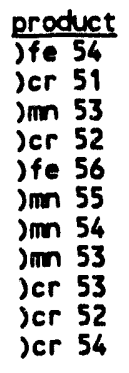 & $\begin{array}{l}\frac{m t}{16} \\
22 \\
32 \\
34 \\
102 \\
103 \\
104 \\
105 \\
106 \\
107 \\
111\end{array}$ & $\begin{array}{c}\text { engeries } \\
14 \\
9 \\
11 \\
9 \\
368 \\
19 \\
37 \\
33 \\
42 \\
18 \\
38\end{array}$ & $\begin{array}{l}\frac{\text { date }}{12 / 01 / 93} \\
12 / 01 / 93 \\
12 / 01 / 93 \\
12 / 01 / 93 \\
12 / 01 / 93 \\
12 / 01 / 93 \\
12 / 01 / 93 \\
12 / 01 / 93 \\
12 / 01 / 93 \\
12 / 01 / 93 \\
12 / 01 / 93\end{array}$ \\
\hline $\begin{array}{r}1 \text { fe } 56 \text { (n, 2n } \\
2 \text { fe } 56 \text { (n,na } \\
3 \text { fe } 56 \text { (n,np } \\
4 \text { fe } 56 \text { (n,nd } \\
5 \text { fe } 56 \text { (n, g } \\
6 \text { fe } 56 \text { (n,p } \\
7 \text { fe } 56 \text { (n,d } \\
8 \text { fe } 56 \text { (n, t } \\
9 \text { fe } 56 \text { (n, h } \\
10 \text { fe } 56 \text { (n,e } \\
11 \text { fe } 56 \text { (n,2p }\end{array}$ & 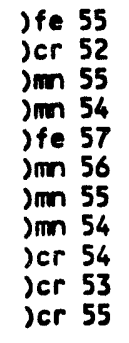 & $\begin{array}{r}16 \\
22 \\
28 \\
32 \\
102 \\
103 \\
104 \\
105 \\
106 \\
107 \\
111\end{array}$ & $\begin{array}{r}25 \\
21 \\
21 \\
13 \\
62638 \\
119 \\
39 \\
254 \\
39 \\
130 \\
9\end{array}$ & $\begin{array}{l}12 / 01 / 93 \\
12 / 01 / 93 \\
12 / 01 / 93 \\
12 / 01 / 93 \\
12 / 01 / 93 \\
12 / 01 / 93 \\
12 / 01 / 93 \\
12 / 01 / 93 \\
12 / 01 / 93 \\
12 / 01 / 93 \\
12 / 01 / 93\end{array}$ \\
\hline $\begin{array}{r}1 \text { fe } 57 \text { (n, 2n } \\
2 \text { fe } 57 \text { (n,3n } \\
3 \text { fe } 57 \text { (n,ne } \\
4 \text { fe } 57 \text { (n,np } \\
5 \text { fe } 57 \text { (n,nd } \\
6 \text { fe } 57 \text { (n,nt } \\
7 \text { fe } 57 \text { (n,mh } \\
8 \text { fe } 57 \text { (n,g } \\
9 \text { fe } 57 \text { (n,p } \\
10 \text { fe } 57 \text { (n,d } \\
11 \text { fe } 57 \text { (n,t } \\
12 \text { fe } 57 \text { (n,h } \\
13 \text { fe } 57 \text { (n, } \\
14 \text { fe } 57 \text { (n,2p }\end{array}$ & $\begin{array}{l}\text { Jfe } 56 \\
\text { Jfe } 55 \\
\text { Jcr } 53 \\
\text { Jm } 56 \\
\text { Jm } 55 \\
\text { Jm } 54 \\
\text { Jcr } 54 \\
\text { Jfe } 58 \\
\text { Jm } 57 \\
\text { Jm } 56 \\
\text { Jm } 55 \\
\text { Jcr } 55 \\
\text { Jer } 54 \\
\text { Jcr } 56\end{array}$ & $\begin{array}{l}16 \\
17 \\
22 \\
28 \\
32 \\
33 \\
34 \\
102 \\
103 \\
104 \\
105 \\
106 \\
107 \\
111\end{array}$ & $\begin{array}{r}28 \\
4 \\
41 \\
10 \\
15 \\
2 \\
2 \\
6 \\
18450 \\
20 \\
25 \\
35 \\
17 \\
205 \\
17\end{array}$ & $\begin{array}{l}12 / 01 / 93 \\
12 / 01 / 93 \\
12 / 01 / 93 \\
12 / 01 / 93 \\
12 / 01 / 93 \\
12 / 01 / 93 \\
12 / 01 / 93 \\
12 / 01 / 93 \\
12 / 01 / 93 \\
12 / 01 / 93 \\
12 / 01 / 93 \\
12 / 01 / 93 \\
12 / 01 / 93 \\
12 / 01 / 93\end{array}$ \\
\hline $\begin{array}{l}\# \frac{\text { target }}{1} \text { fe } 58 \text { (n, 2n } \\
2 \text { fe } 58 \text { (n,3n } \\
3 \text { fe } 58 \text { (n,na } \\
4 \text { fe } 58 \text { (n,np } \\
5 \text { fe } 58 \text { (n,nd } \\
6 \text { fe } 58 \text { (n,nt } \\
7 \text { fe } 58 \text { (n, } \\
8 \text { fe } 58 \text { (n,p } \\
9 \text { fe } 58 \text { (n,d } \\
10 \text { fe } 58 \text { (n,t }\end{array}$ & 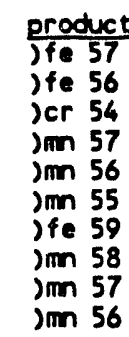 & $\begin{array}{r}\frac{\mathrm{mt}}{16} \\
17 \\
22 \\
28 \\
32 \\
33 \\
102 \\
103 \\
104 \\
105\end{array}$ & $\begin{array}{c}\text { - engeries } \\
28 \\
13 \\
43 \\
7 \\
4 \\
4 \\
3487 \\
20 \\
21 \\
16\end{array}$ & $\begin{array}{l}\text { date } \\
12 / 01 / 93 \\
12 / 01 / 93 \\
12 / 01 / 93 \\
12 / 01 / 93 \\
12 / 01 / 93 \\
12 / 01 / 93 \\
12 / 01 / 93 \\
12 / 01 / 93 \\
12 / 01 / 93 \\
12 / 01 / 93\end{array}$ \\
\hline
\end{tabular}

$A-2.5$ 


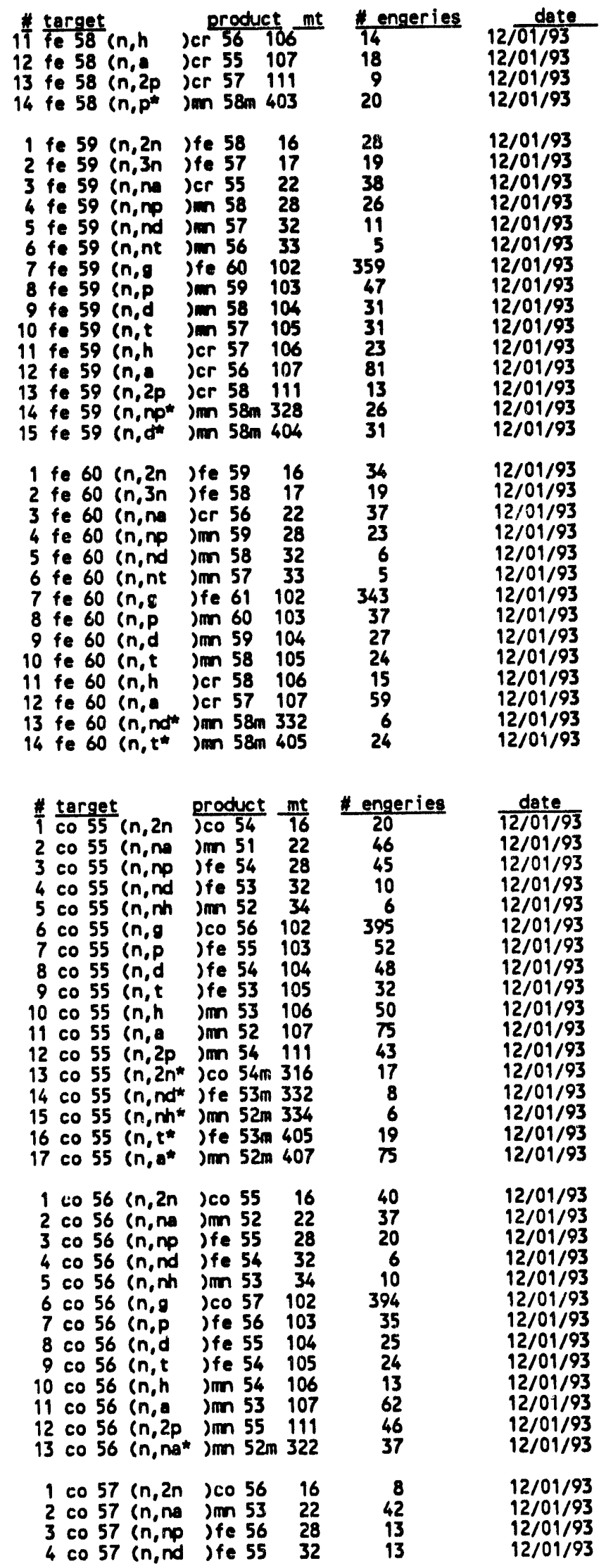

A-2. 6 


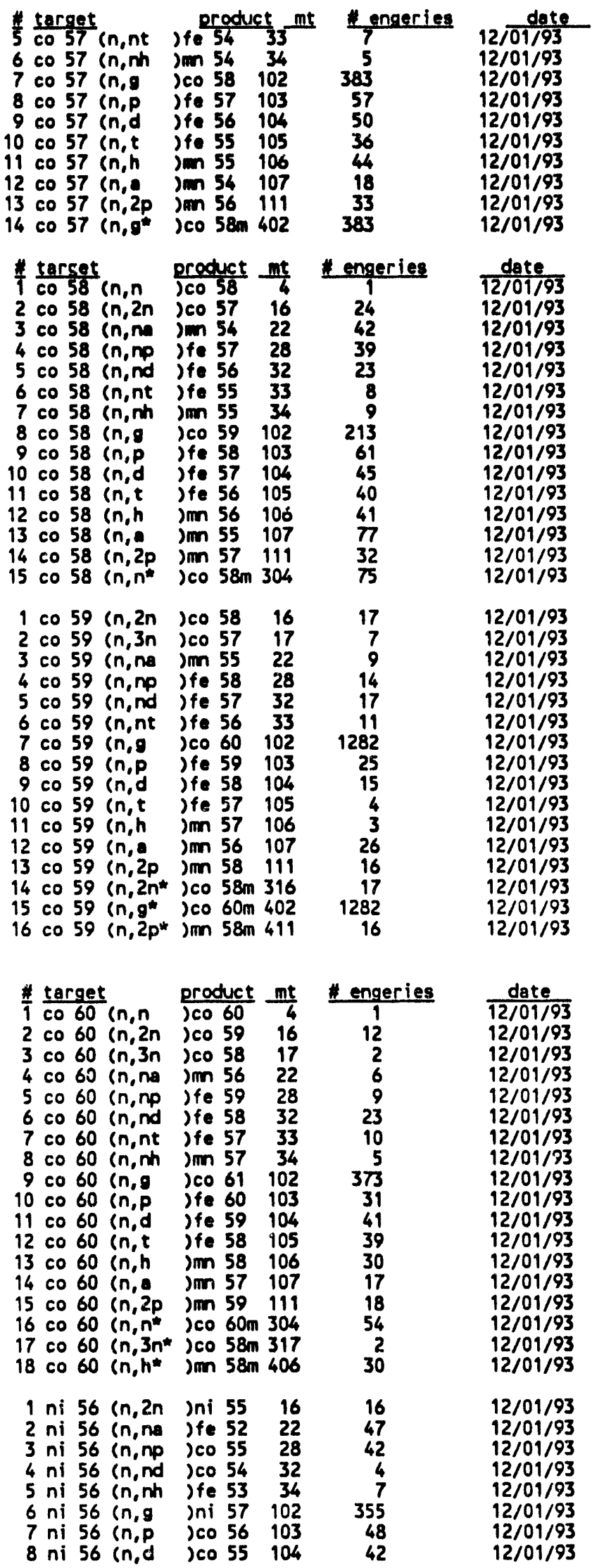




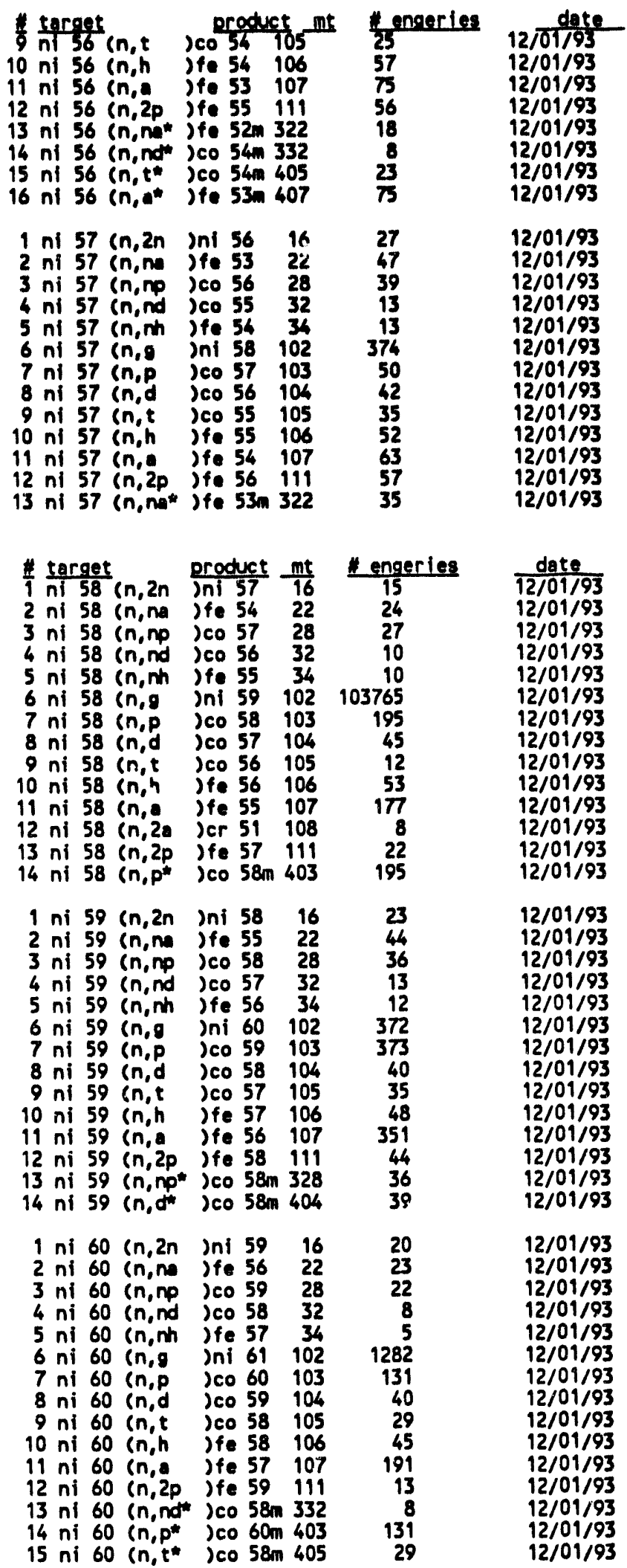




\begin{tabular}{|c|c|c|c|c|c|}
\hline 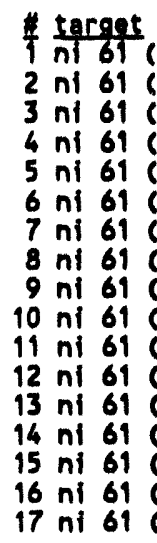 & $\begin{array}{l}(n, 2 n) \\
(n, 3 n) \\
(n, n \in) \\
(n, n p) \\
(n, n d) \\
(n, n d) \\
(n, n t) \\
(n, n) \\
(n, g) \\
(n, p) \\
(n, d) \\
(n, t) \\
(n, n) \\
(n, n) \\
(n, 2 p) \\
\left(n, n p^{*}\right) \\
(n, n t) \\
\left(n, d^{*}\right)\end{array}$ & $\begin{array}{l}\text { product } \\
\text { Pni } 60 \\
\text { ini } 59 \\
\text { jfe } 57 \\
\text { jco } 60 \\
\text { jco } 59 \\
\text { jco } 58 \\
\text { jfe } 58 \\
\text { jni } 62 \\
\text { jco } 61 \\
\text { jco } 60 \\
\text { jco } 59 \\
\text { jfe } 59 \\
\text { jfe } 58 \\
\text { jfe } 60 \\
\text { jco } 60 \mathrm{~m} \\
\text { ico } 58 \mathrm{~m} \\
\text { jco } 60 \mathrm{~m}\end{array}$ & $\begin{array}{l}\frac{m t}{16} \\
17 \\
22 \\
28 \\
32 \\
33 \\
34 \\
102 \\
103 \\
104 \\
105 \\
106 \\
107 \\
111 \\
328 \\
333 \\
404\end{array}$ & $\begin{array}{c}\text { engerles } \\
29 \\
2 \\
46 \\
12 \\
15 \\
5 \\
10 \\
6127 \\
34 \\
50 \\
36 \\
38 \\
205 \\
44 \\
7 \\
5 \\
50\end{array}$ & $\begin{array}{l}\text { date } \\
\text { 12/01/93 } \\
12 / 01 / 93 \\
12 / 01 / 93 \\
12 / 01 / 93 \\
12 / 01 / 93 \\
12 / 01 / 93 \\
12 / 01 / 93 \\
12 / 01 / 93 \\
12 / 01 / 93 \\
12 / 01 / 93 \\
12 / 01 / 93 \\
12 / 01 / 93 \\
12 / 01 / 93 \\
12 / 01 / 93 \\
12 / 01 / 93 \\
12 / 01 / 93 \\
12 / 01 / 93\end{array}$ \\
\hline $\begin{array}{ccc}1 & n i & 62 \\
2 & n i & 62 \\
3 & n i & 62 \\
4 & n i & 62 \\
5 & n i & 62 \\
6 & n i & 62 \\
7 & n i & 62 \\
8 & n i & 62 \\
9 & n i & 62 \\
10 & n i & 62 \\
11 & n i & 62 \\
12 & n i & 62 \\
13 & n i & 62 \\
14 & n i & 62 \\
15 & n i & 62 \\
16 & n i & 62\end{array}$ & 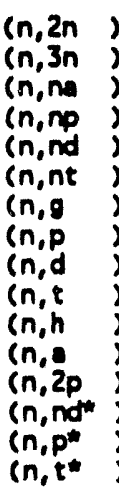 & 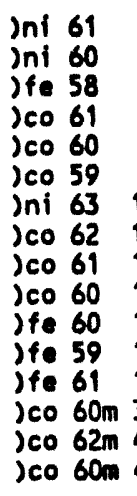 & $\begin{array}{l}16 \\
17 \\
22 \\
28 \\
32 \\
33 \\
102 \\
103 \\
104 \\
105 \\
106 \\
107 \\
111 \\
332 \\
403 \\
405\end{array}$ & $\begin{array}{r}28 \\
10 \\
47 \\
10 \\
6 \\
4 \\
3274 \\
24 \\
36 \\
32 \\
32 \\
29 \\
24 \\
6 \\
8 \\
32\end{array}$ & $\begin{array}{l}12 / 01 / 93 \\
12 / 01 / 93 \\
12 / 01 / 93 \\
12 / 01 / 93 \\
12 / 01 / 93 \\
12 / 01 / 93 \\
12 / 01 / 93 \\
12 / 01 / 93 \\
12 / 01 / 93 \\
12 / 01 / 93 \\
12 / 01 / 93 \\
12 / 01 / 93 \\
12 / 01 / 93 \\
12 / 01 / 93 \\
12 / 01 / 93 \\
12 / 01 / 93\end{array}$ \\
\hline 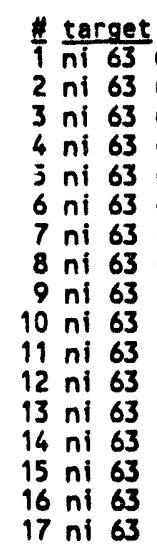 & 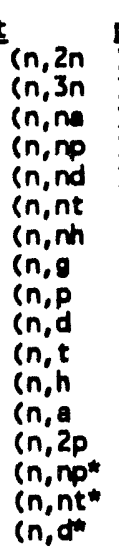 & $\begin{array}{l}\text { product } \\
\text { ini } 62 \\
\text { ini } 61 \\
\text { jfe } 59 \\
\text { jco } 62 \\
\text { jco } 61 \\
\text { jco } 60 \\
\text { jfe } 60 \\
\text { ini } 64 \\
\text { jco } 63 \\
\text { jco } 62 \\
\text { jco } 61 \\
\text { jfe } 61 \\
\text { jfe } 60 \\
\text { jfe } 62 \\
\text { jco } 62 \mathrm{~m} \\
\text { jco } 60 \mathrm{~m} \\
\text { jco } 62 \mathrm{~m}\end{array}$ & $\begin{array}{l}\frac{\mathrm{mt}}{16} \\
17 \\
22 \\
28 \\
32 \\
33 \\
34 \\
102 \\
103 \\
104 \\
105 \\
106 \\
107 \\
111 \\
328 \\
333 \\
404\end{array}$ & $\begin{array}{c}\text { engeries } \\
12 \\
3 \\
7 \\
5 \\
13 \\
5 \\
5 \\
359 \\
11 \\
32 \\
32 \\
27 \\
27 \\
17 \\
5 \\
5 \\
32\end{array}$ & $\begin{array}{l}\text { date } \\
\frac{12 / 01 / 93}{12 / 01 / 93} \\
12 / 01 / 93 \\
12 / 01 / 93 \\
12 / 01 / 93 \\
12 / 01 / 93 \\
12 / 01 / 93 \\
12 / 01 / 93 \\
12 / 01 / 93 \\
12 / 01 / 93 \\
12 / 01 / 93 \\
12 / 01 / 93 \\
12 / 01 / 93 \\
12 / 01 / 93 \\
12 / 01 / 93 \\
12 / 01 / 93 \\
12 / 01 / 93\end{array}$ \\
\hline $\begin{array}{ccc}1 & n i & 64 \\
2 & n i & 64 \\
3 & n i & 64 \\
4 & n i & 64 \\
5 & n i & 64 \\
6 & n i & 64 \\
7 & n i & 64 \\
8 & n i & 64 \\
9 & n i & 64 \\
10 & n i & 64 \\
11 & n i & 64 \\
12 & n i & 64 \\
13 & n i & 64 \\
14 & n i & 64 \\
15 & n i & 64\end{array}$ & 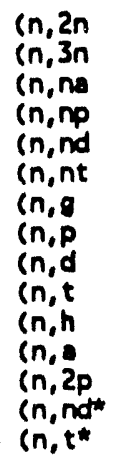 & $\begin{array}{l}\text { Jni } 63 \\
\text { jni } 62 \\
\text { jfe } 60 \\
\text { jco } 63 \\
\text { jco } 62 \\
\text { jco } 61 \\
\text { ini } 65 \\
\text { jco } 64 \\
\text { jco } 63 \\
\text { jco } 62 \\
\text { jfe } 62 \\
\text { jfe } 61 \\
\text { jfe } 63 \\
\text { jco } 62 \mathrm{~m} \\
\text { jco } 62 \mathrm{~m}\end{array}$ & $\begin{array}{c}16 \\
17 \\
22 \\
28 \\
32 \\
33 \\
102 \\
103 \\
104 \\
105 \\
106 \\
107 \\
111\end{array}$ & $\begin{array}{r}9 \\
19 \\
6 \\
4 \\
4 \\
4 \\
4338 \\
11 \\
40 \\
30 \\
20 \\
11 \\
13 \\
4 \\
30\end{array}$ & $\begin{array}{l}12 / 01 / 93 \\
12 / 01 / 93 \\
12 / 01 / 93 \\
12 / 01 / 93 \\
12 / 01 / 93 \\
12 / 01 / 93 \\
12 / 01 / 93 \\
12 / 01 / 93 \\
12 / 01 / 93 \\
12 / 01 / 93 \\
12 / 01 / 93 \\
12 / 01 / 93 \\
12 / 01 / 93 \\
12 / 01 / 93 \\
12 / 01 / 93\end{array}$ \\
\hline
\end{tabular}




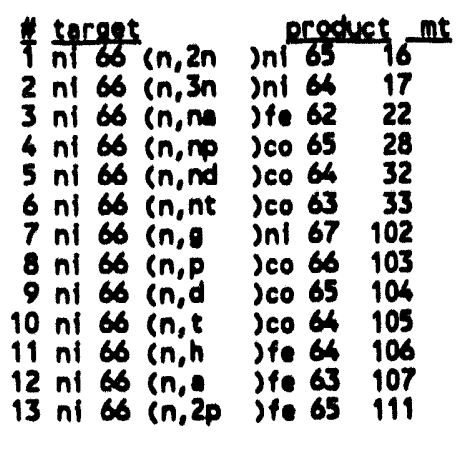

\begin{tabular}{rr}
\multicolumn{1}{c}{ enperies } & date \\
\hline 29 & $12 / 01 / 93$ \\
22 & $12 / 01 / 93$ \\
35 & $12 / 01 / 93$ \\
23 & $12 / 01 / 93$ \\
2 & $12 / 01 / 93$ \\
5 & $12 / 01 / 93$ \\
350 & $12 / 01 / 93$ \\
41 & $12 / 01 / 93$ \\
32 & $12 / 01 / 93$ \\
22 & $12 / 01 / 93$ \\
23 & $12 / 01 / 93$ \\
69 & $12 / 01 / 93$ \\
6 & $12 / 01 / 93$
\end{tabular}

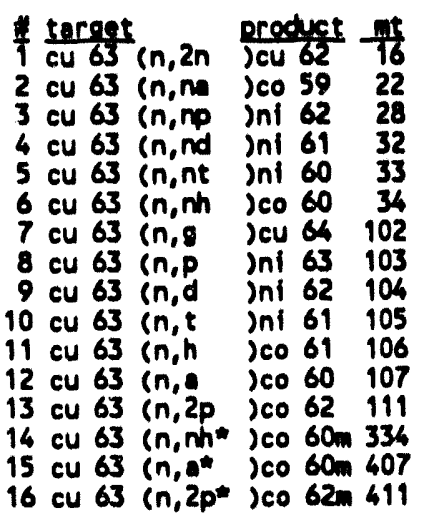

\begin{tabular}{rr} 
Engeries & date \\
\cline { 2 - 2 } 19 & $12 / 01 / 93$ \\
16 & $12 / 01 / 93$ \\
24 & $12 / 01 / 93$ \\
19 & $12 / 01 / 93$ \\
11 & $12 / 01 / 93$ \\
5 & $12 / 01 / 93$ \\
1892 & $12 / 01 / 93$ \\
24 & $12 / 01 / 93$ \\
52 & $12 / 01 / 93$ \\
38 & $12 / 01 / 93$ \\
42 & $12 / 01 / 93$ \\
216 & $12 / 01 / 93$ \\
26 & $12 / 01 / 93$ \\
5 & $12 / 01 / 93$ \\
216 & $12 / 01 / 93$ \\
26 & $12 / 01 / 93$
\end{tabular}

1 cu $64(n, 2 n)$ cu 6316

2 cu 64 ( $n, 3 n$ ) cu 6217

3 cu 64 (n,m) co 6022

4 cu 64 in,np ini 6328

$5 \mathrm{cu} 64$ ( $n$,nd )ni 6232

$6 \mathrm{cu} 64$ (n,nt ini 6133

7 cu 64 (n,nh )co $61 \quad 34$

$8 \mathrm{cu} 64$ (n,g) )eu 65102

9 cu 64 (n,p ini 64103

10 cu 64 (n,d ini 63104

$11 \mathrm{cu} 64$ (n,t )ni 62105

12 cu $64(n, h)$ co 62106

13 cu 64 (n,e )co 61107

$14 \mathrm{cu} 64(\mathrm{n}, 2 \mathrm{p}) \mathrm{co} 63111$

15 cu 64 ( $n, n a *) c 060 m 322$

$16 \mathrm{cu} 64$ (n,h*) joo $62 \mathrm{~m} 406$

$\begin{array}{rr}24 & 12 / 01 / 93 \\ 6 & 12 / 01 / 93 \\ 46 & 12 / 01 / 93 \\ 39 & 12 / 01 / 93 \\ 25 & 12 / 01 / 93 \\ 11 & 12 / 01 / 93 \\ 8 & 12 / 01 / 93 \\ 393 & 12 / 01 / 93 \\ 64 & 12 / 01 / 93 \\ 44 & 12 / 01 / 93 \\ 42 & 12 / 01 / 93 \\ 38 & 12 / 01 / 93 \\ 78 & 12 / 01 / 93 \\ 29 & 12 / 01193 \\ 46 & 12 / 01 / 93 \\ 38 & 12 / 01 / 93\end{array}$

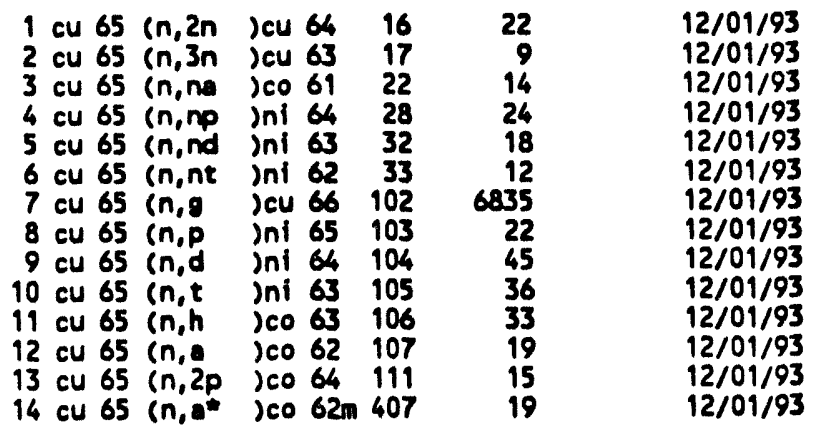

\begin{tabular}{|c|c|c|c|}
\hline $\begin{array}{l}\frac{7}{1} \text { target } \\
\frac{1}{c u} 67 \text { (n, 2n } \\
2 \text { cu } 67 \text { (n, } 3 n \\
3 \text { cu } 67 \text { (n, na } \\
4 \text { cu } 67 \text { (n,np } \\
5 \text { cu } 67 \text { (n, nd } \\
6 \text { cu } 67 \text { in,nt } \\
7 \text { cu } 67 \text { in, }\end{array}$ & $\begin{array}{l}\text { product } \\
\text { rcu } 66 \\
\text { jcu } 65 \\
\text { jeo } 63 \\
\text { oni } 66 \\
\text { oni } 65 \\
\text { oni } 64 \\
\text { jeu } 68\end{array}$ & $\begin{array}{r}\frac{m t}{16} \\
17 \\
22 \\
28 \\
32 \\
33 \\
102\end{array}$ & $\begin{array}{c}\text { * engeries } \\
30 \\
20 \\
42 \\
37 \\
15 \\
13 \\
396\end{array}$ \\
\hline
\end{tabular}

$A-2.10$ 


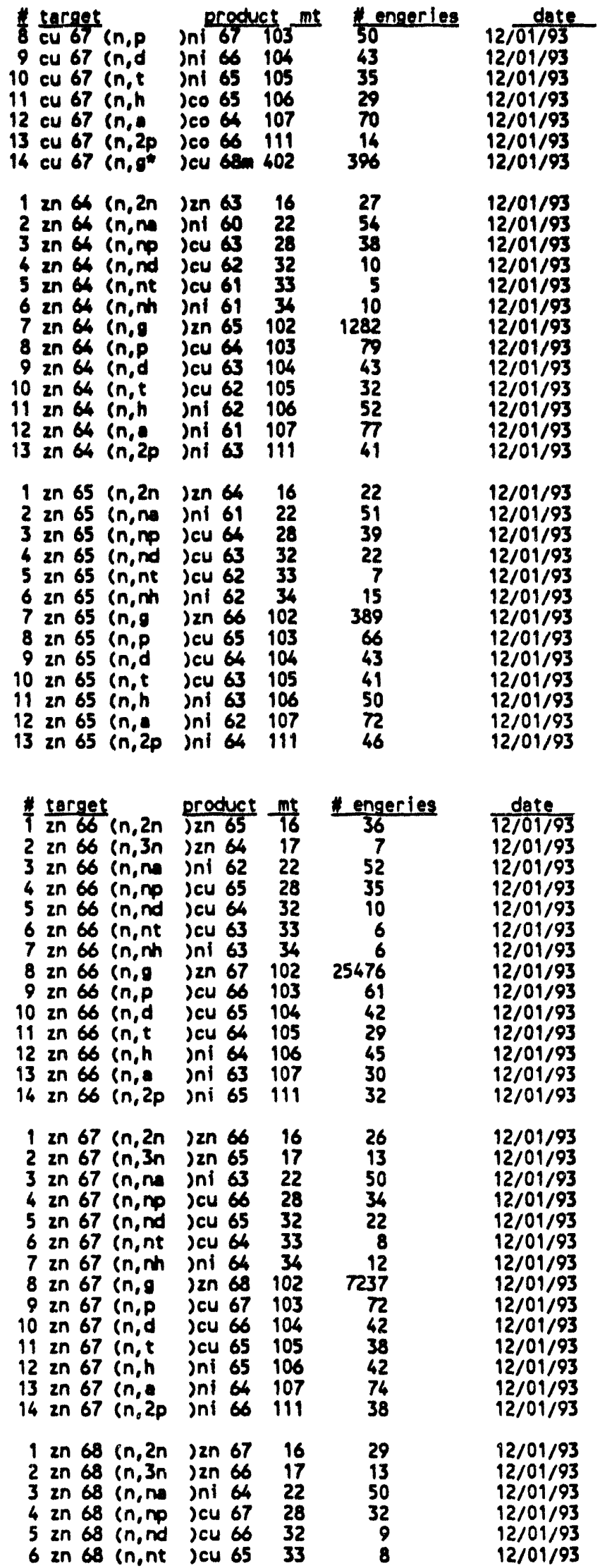

A-2. 11 


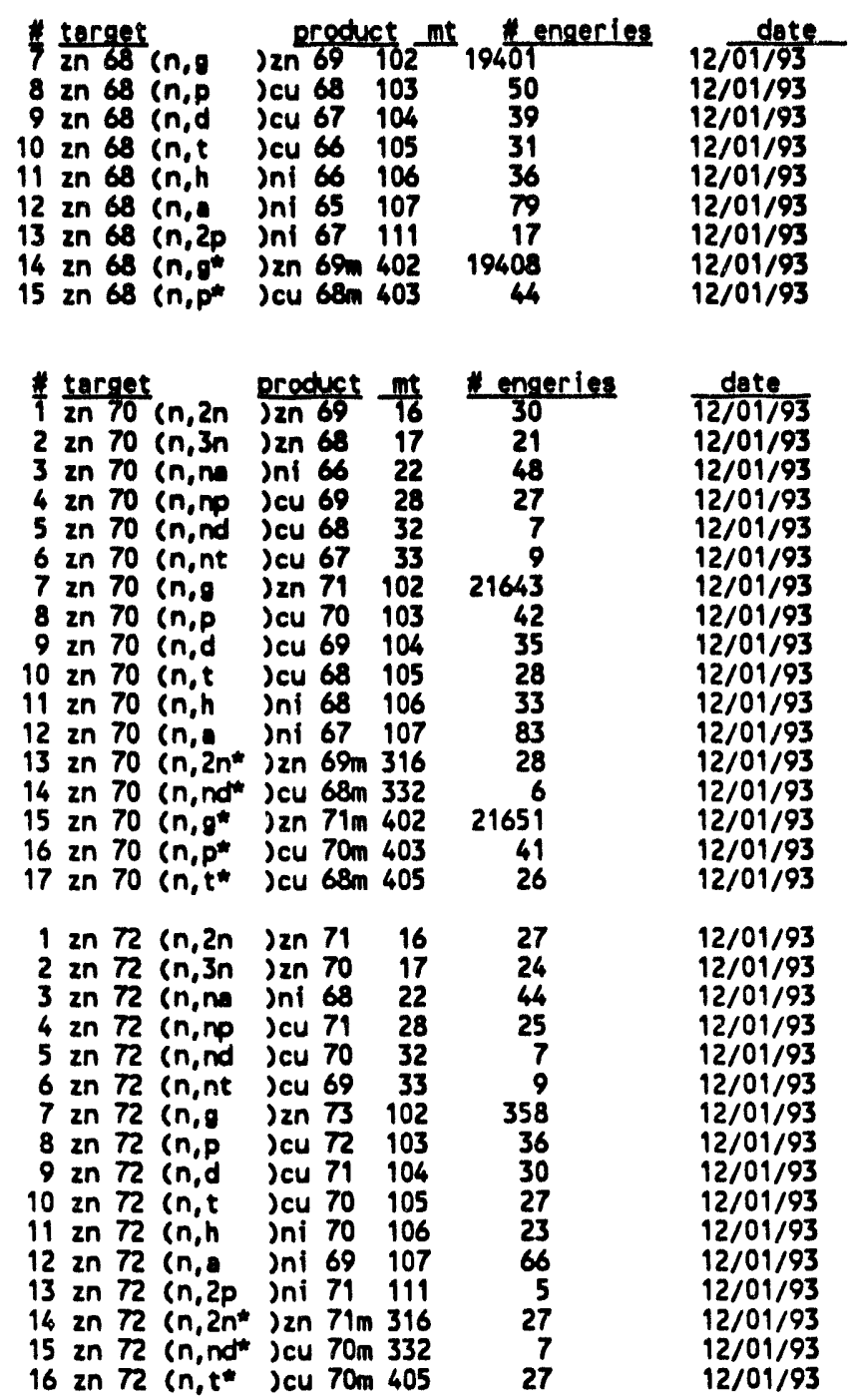


Appendix A-3

List of Reactions on files *.ga.y

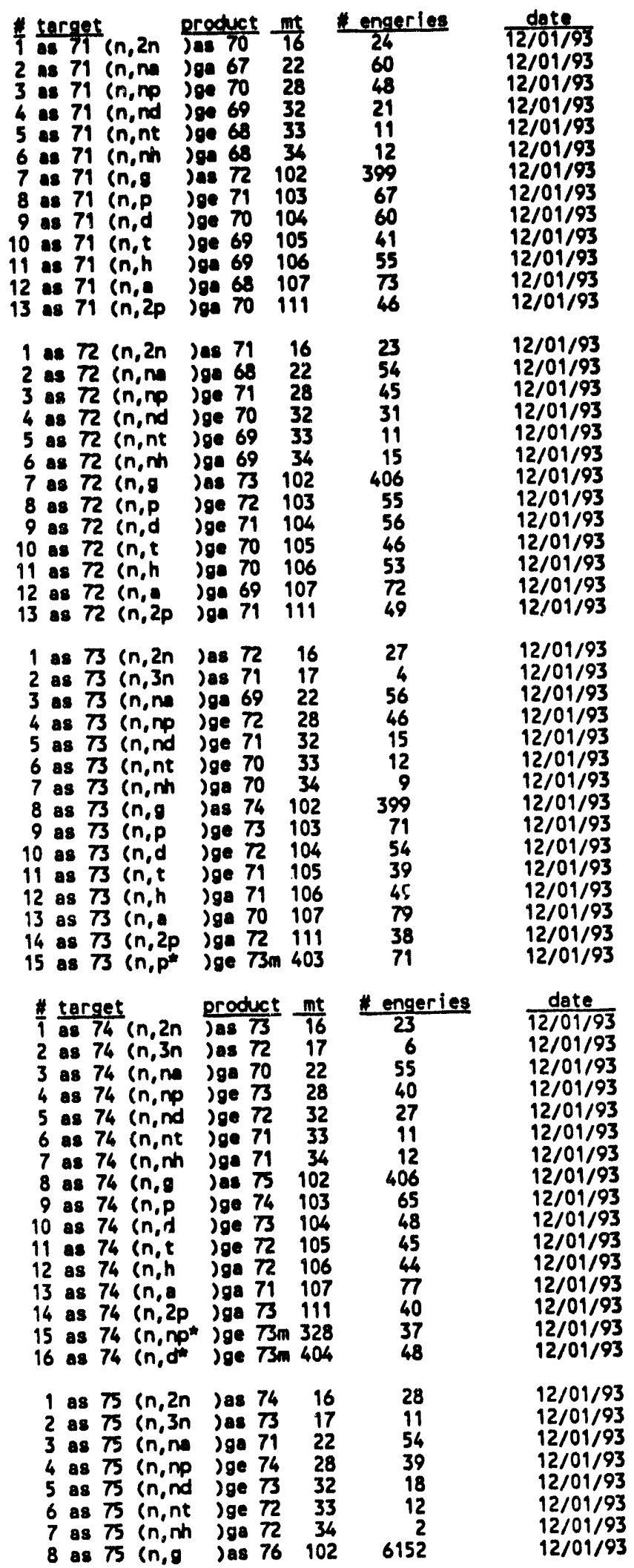

$A-3.1$ 


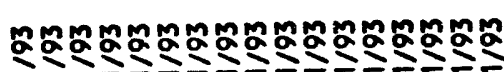

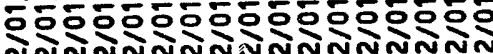

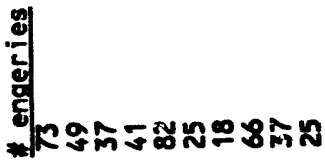

旬

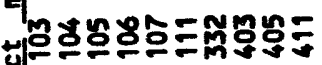

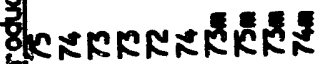

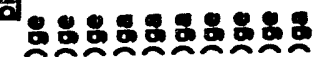

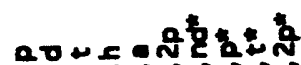

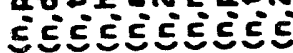
UKKKKKKKKKKR

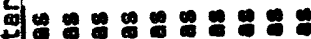
*10ำMำำะ
ㅎํำ

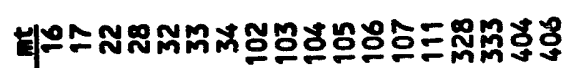
TRm

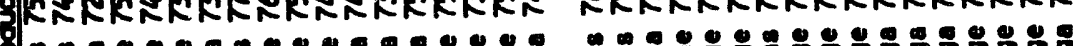

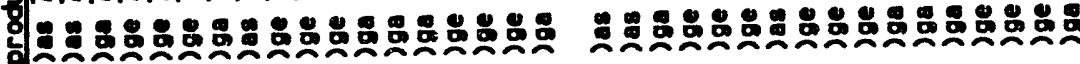

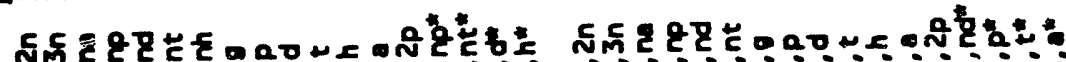

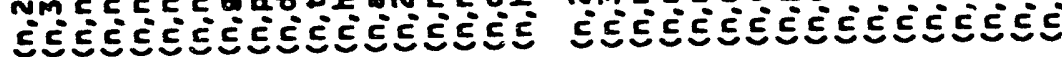

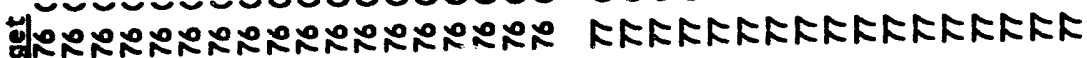
矛月:

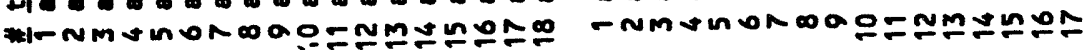
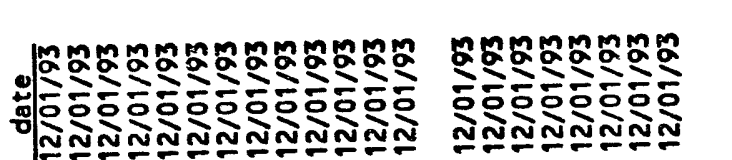

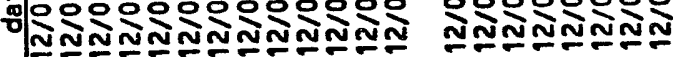

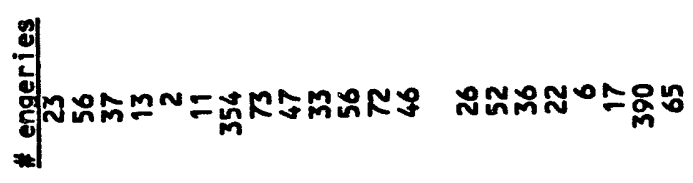

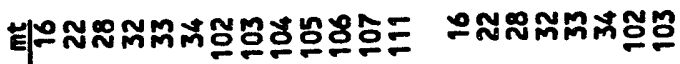
気รง

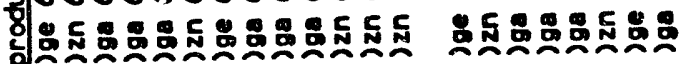
ㄷำใदृ

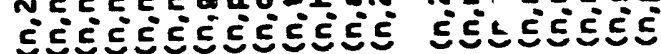

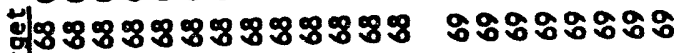

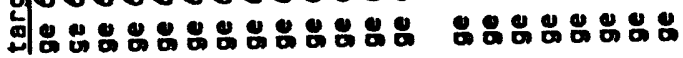

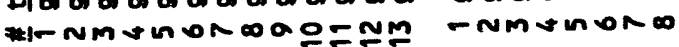




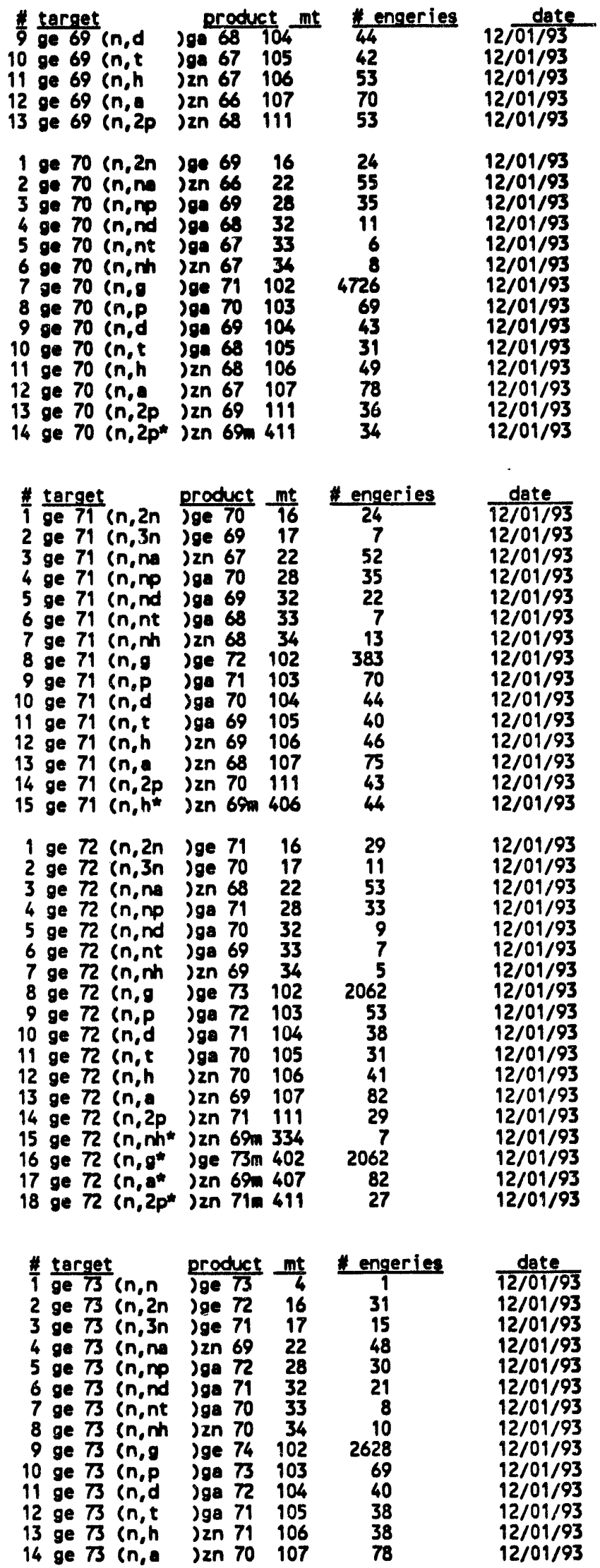

$A-3.3$ 


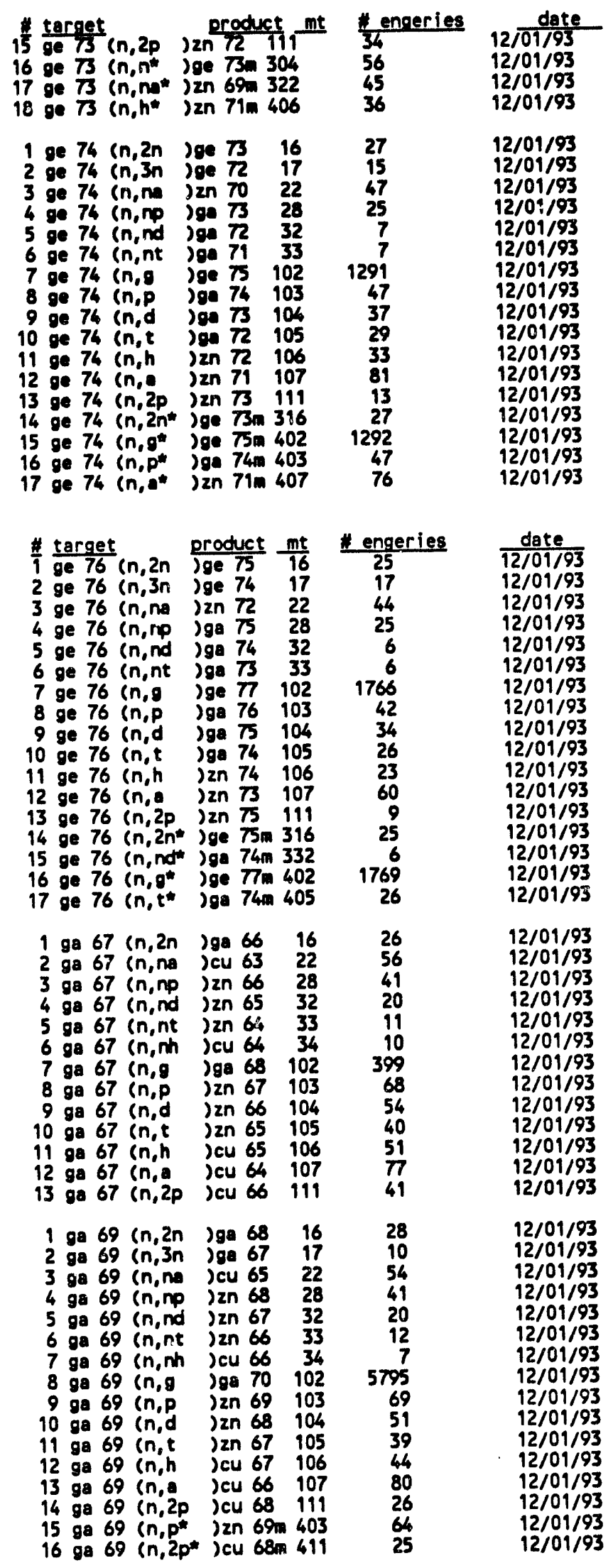




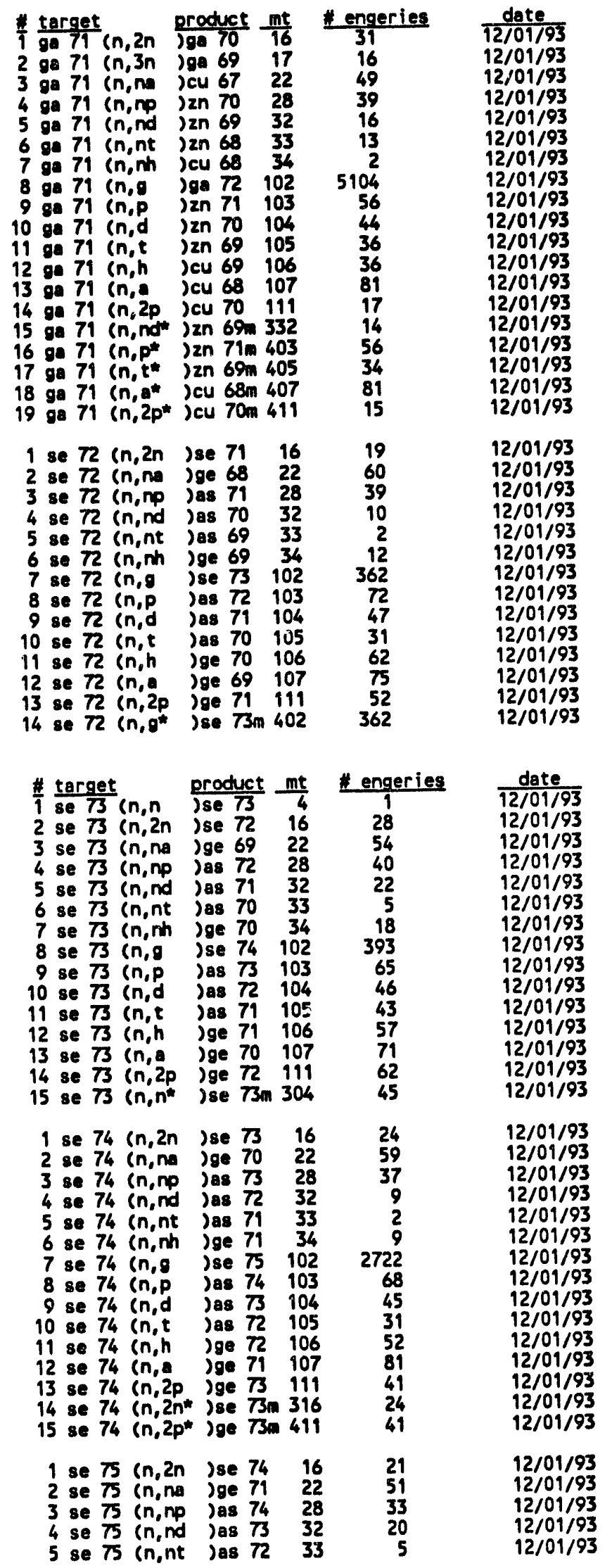

$A-3.5$ 


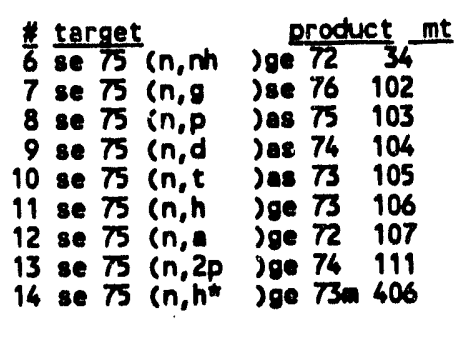

\begin{tabular}{cc} 
*engeries & date \\
\hline 14 & $12 / 01 / 93$ \\
388 & $12 / 01 / 93$ \\
71 & $12 / 01 / 93$ \\
45 & $12 / 01 / 93$ \\
40 & $12 / 01 / 93$ \\
50 & $12 / 01 / 93$ \\
73 & $12 / 01 / 93$ \\
47 & $12 / 01 / 93$ \\
50 & $12 / 01 / 93$
\end{tabular}

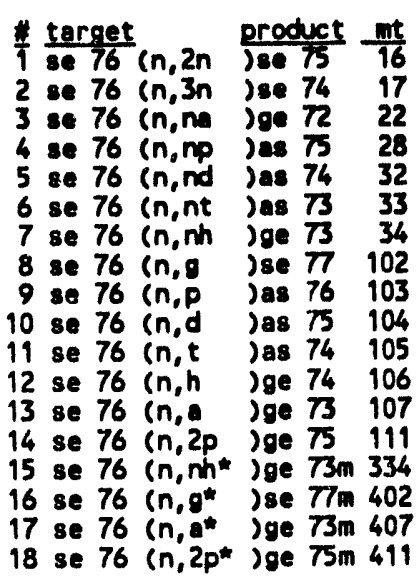

\begin{tabular}{cc} 
Eengeries & date \\
\cline { 2 - 2 } 24 & $12 / 01 / 93$ \\
4 & $12 / 01 / 93$ \\
51 & $12 / 01 / 93$ \\
32 & $12 / 01 / 93$ \\
8 & $12 / 01 / 93$ \\
2 & $12 / 01 / 93$ \\
5 & $12 / 01 / 93$ \\
2966 & $12 / 01 / 93$ \\
60 & $12 / 01 / 93$ \\
42 & $12 / 01 / 93$ \\
30 & $12 / 01 / 93$ \\
46 & $12 / 01 / 93$ \\
83 & $12 / 01 / 93$ \\
34 & $12 / 01 / 93$ \\
5 & $12 / 01 / 93$ \\
2965 & $12 / 01 / 93$ \\
83 & $12 / 01 / 93$ \\
34 & $12 / 01 / 93$
\end{tabular}

1 se $\pi(n, n)$ se $\pi \quad 4$

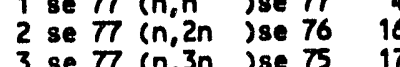

$\begin{array}{llll}3 & \text { se } 77(n, 3 n) \text { se } 75 & 17 \\ 4 & \text { se } 77 \text { (n,ne )ge } 73 \quad 22\end{array}$

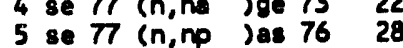

$\begin{array}{llll}5 & \text { se } 77 \text { (n,np )as } 76 & 28 \\ 6 & \text { se } & 77 & \text { (n,nd )as } \\ 75 & 32\end{array}$

7 se 7 (n,nt) as $74 \quad 33$

8 se 77 (n,nh) ge 74 34

9 se $77(n, s)$ se $78 \quad 102$

10 se 77 (n,p )as $77 \quad 103$

11 se 77 (n.d) as 76104

12 se 77 (n,t) as 75105

13 se $77(n, h)$ ge 75106

14 se 77 (n, a ) $74 \quad 107$

15 se $77(n, 2 p)$ ge $76 \quad 111$

16 se 77 (n, nt) se 77 m 304

17 se $\pi$ ( $n$, na*) ge $73 m 322$

18 se 77 (n,h ) ge $75 m 406$

$\begin{array}{rr}1 & 12 / 01 / 93 \\ 22 & 12 / 01 / 93 \\ 10 & 12 / 01 / 93 \\ 46 & 12 / 01 / 93 \\ 31 & 12 / 01 / 93 \\ 21 & 12 / 01 / 93 \\ 6 & 12 / 01 / 93 \\ 11 & 12 / 01 / 93 \\ 5719 & 12 / 01 / 93 \\ 74 & 12 / 01 / 93 \\ 42 & 12 / 01 / 93 \\ 38 & 12 / 01 / 93 \\ 43 & 12 / 01 / 93 \\ 79 & 12 / 01 / 93 \\ 41 & 12 / 01 / 93 \\ 51 & 12 / 01 / 93 \\ 46 & 12 / 01 / 93 \\ 43 & 12 / 01 / 93\end{array}$

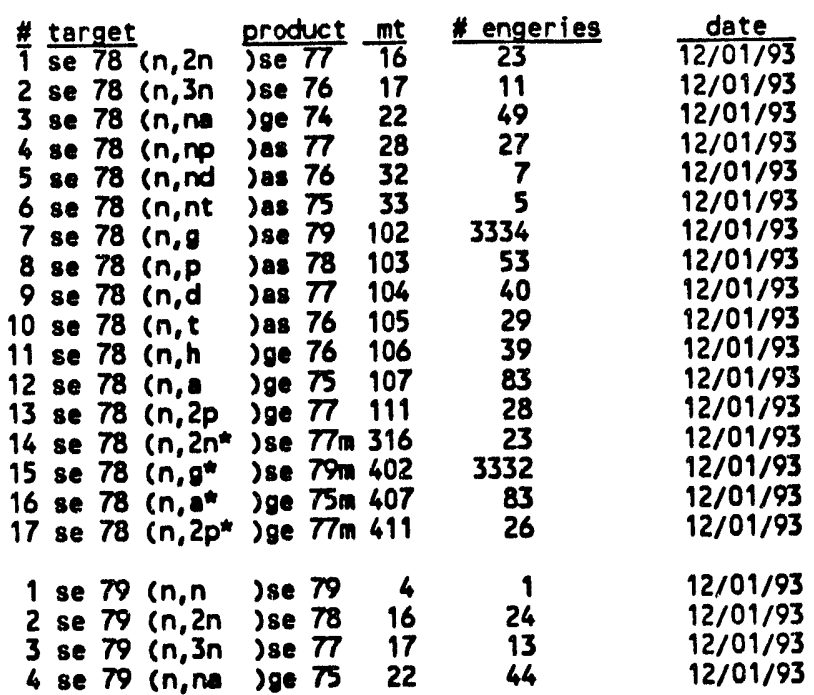

A-3. 6 


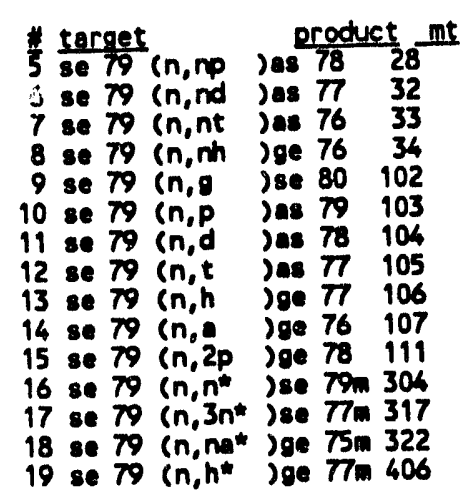

\begin{tabular}{cc} 
*enqeries & date \\
\hline 26 & $12 / 01 / 93$ \\
19 & $12 / 01 / 93$ \\
6 & $12 / 01 / 93$ \\
8 & $12 / 01 / 93$ \\
387 & $12 / 01 / 93$ \\
65 & $12 / 01 / 93$ \\
40 & $12 / 01 / 93$ \\
39 & $12 / 01 / 93$ \\
35 & $12 / 01 / 93$ \\
82 & $12 / 01 / 93$ \\
32 & $12 / 01 / 93$ \\
46 & $12 / 01 / 93$ \\
13 & $12 / 01 / 93$ \\
44 & $12 / 01 / 93$ \\
35 & $12 / 01 / 93$
\end{tabular}

\begin{tabular}{|c|c|c|c|c|}
\hline 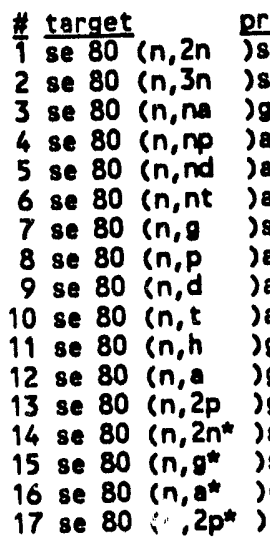 & $\begin{array}{l}\text { product } \\
\text { jse } 79 \\
\text { jse } 78 \\
\text { jge } 76 \\
\text { jas } 79 \\
\text { jas } 78 \\
\text { jas } 77 \\
\text { jse } 81 \\
\text { jas } 80 \\
\text { jas } 79 \\
\text { jas } 78 \\
\text { jge } 78 \\
\text { jge } 77 \\
\text { jge } 79 \\
\text { jee } 79 \mathrm{~m} \\
\text { jse } 81 \mathrm{~m} \\
\text { jge } 77 \mathrm{~m} \\
\text { jge } 79 \mathrm{~m}\end{array}$ & $\begin{array}{l}\frac{m t}{16} \\
17 \\
22 \\
28 \\
32 \\
33 \\
102 \\
103 \\
104 \\
105 \\
106 \\
107 \\
111 \\
316 \\
402 \\
407 \\
411\end{array}$ & $\begin{array}{c}\text { engeries } \\
25 \\
15 \\
47 \\
26 \\
7 \\
5 \\
1799 \\
48 \\
36 \\
28 \\
29 \\
77 \\
14 \\
25 \\
1796 \\
71 \\
12\end{array}$ & $\begin{array}{l}\text { date } \\
12 / 01 / 93 \\
12 / 01 / 93 \\
12 / 01 / 93 \\
12 / 01 / 93 \\
12 / 01 / 93 \\
12 / 01 / 93 \\
12 / 01 / 93 \\
12 / 01 / 93 \\
12 / 01 / 93 \\
12 / 01 / 93 \\
12 / 01 / 93 \\
12 / 01 / 93 \\
12 / 01 / 93 \\
12 / 01 / 93 \\
12 / 01 / 93 \\
12 / 01 / 93 \\
12 / 01 / 93\end{array}$ \\
\hline $\begin{array}{r}1 \text { se } 82(n, 2 n) \\
2 \text { se } 82(n, 3 n) \\
3 \text { se } 82(n, n n) \\
4 \text { se } 82(n, n p) \\
5 \text { se } 82(n, n d) \\
6 \text { se } 82(n, n t) \\
7 \text { se } 82(n, g) \\
8 \text { se } 82(n, p) \\
9 \text { se } 82(n, d) \\
10 \text { se } 82(n, t) \\
11 \text { se } 82(n, n) \\
12 \text { se } 82(n, a) \\
13 \text { se } 82(n, 2 p) \\
\left.14 \text { se } 82(n, 2)^{\star}\right) \\
15 \text { se } 82\left(n, g^{*}\right) \\
16 \text { se } 82\left(n, p^{\star}\right) \\
17 \text { se } 82\left(n, a^{*}\right)\end{array}$ & $\begin{array}{l}\text { jse } 81 \\
\text { jse } 80 \\
\text { jge } 78 \\
\text { jas } 81 \\
\text { jas } 80 \\
\text { jas } 79 \\
\text { jse } 83 \\
\text { jas } 82 \\
\text { jas } 81 \\
\text { jas } 80 \\
\text { jge } 80 \\
\text { jge } 79 \\
\text { jge } 81 \\
\text { joe } 81 \mathrm{~m} \\
\text { jse } 83 \mathrm{~m} \\
\text { jas } 82 \mathrm{~m} \\
\text { jge } 79 \mathrm{~m}\end{array}$ & $\begin{array}{l}16 \\
17 \\
22 \\
28 \\
32 \\
33 \\
102 \\
103 \\
104 \\
105 \\
106 \\
107 \\
111 \\
316 \\
402 \\
403 \\
407\end{array}$ & $\begin{array}{r}31 \\
17 \\
42 \\
25 \\
6 \\
5 \\
1662 \\
42 \\
34 \\
26 \\
21 \\
64 \\
8 \\
31 \\
1662 \\
42 \\
60\end{array}$ & $\begin{array}{l}12 / 01 / 93 \\
12 / 01 / 93 \\
12 / 01 / 93 \\
12 / 01 / 93 \\
12 / 01 / 93 \\
12 / 01 / 93 \\
12 / 01 / 93 \\
12 / 01 / 93 \\
12 / 01 / 93 \\
12 / 01 / 93 \\
12 / 01 / 93 \\
12 / 01 / 93 \\
12 / 01 / 93 \\
12 / 01 / 93 \\
12 / 01 / 93 \\
12 / 01 / 93 \\
12 / 01 / 93\end{array}$ \\
\hline 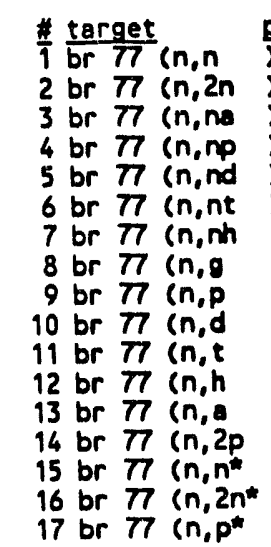 & $\begin{array}{l}\text { product } \\
\text { jbr } 77 \\
\text { jbr } 76 \\
\text { jas } 73 \\
\text { jse } 76 \\
\text { jse } 75 \\
\text { jse } 74 \\
\text { jas } 74 \\
\text { jor } 78 \\
\text { jee } 77 \\
\text { jse } 76 \\
\text { joe } 75 \\
\text { jas } 75 \\
\text { jas } 74 \\
\text { jas } 76 \\
\text { jbr } 77 m \\
\text { Jbr } 76 m \\
\text { jse } 77 m\end{array}$ & $\begin{array}{l}\frac{m t}{4} \\
16 \\
22 \\
28 \\
32 \\
33 \\
34 \\
102 \\
103 \\
104 \\
105 \\
106 \\
107 \\
111 \\
304 \\
316 \\
403\end{array}$ & $\begin{array}{c}\text { \# engeries } \\
1 \\
20 \\
54 \\
41 \\
21 \\
11 \\
8 \\
397 \\
70 \\
55 \\
39 \\
51 \\
83 \\
41 \\
55 \\
20 \\
69\end{array}$ & $\begin{array}{c}\text { date } \\
12 / 01 / 93 \\
12 / 01 / 93 \\
12 / 01 / 93 \\
12 / 01 / 93 \\
12 / 01 / 93 \\
12 / 01 / 93 \\
12 / 01 / 93 \\
12 / 01 / 93 \\
12 / 01 / 93 \\
12 / 01 / 93 \\
12 / 01 / 93 \\
12 / 01 / 93 \\
12 / 01 / 93 \\
12 / 01 / 93 \\
12 / 011 / 93 \\
12 / 01 / 93 \\
12 / 01 / 93\end{array}$ \\
\hline
\end{tabular}

$A-3.7$ 

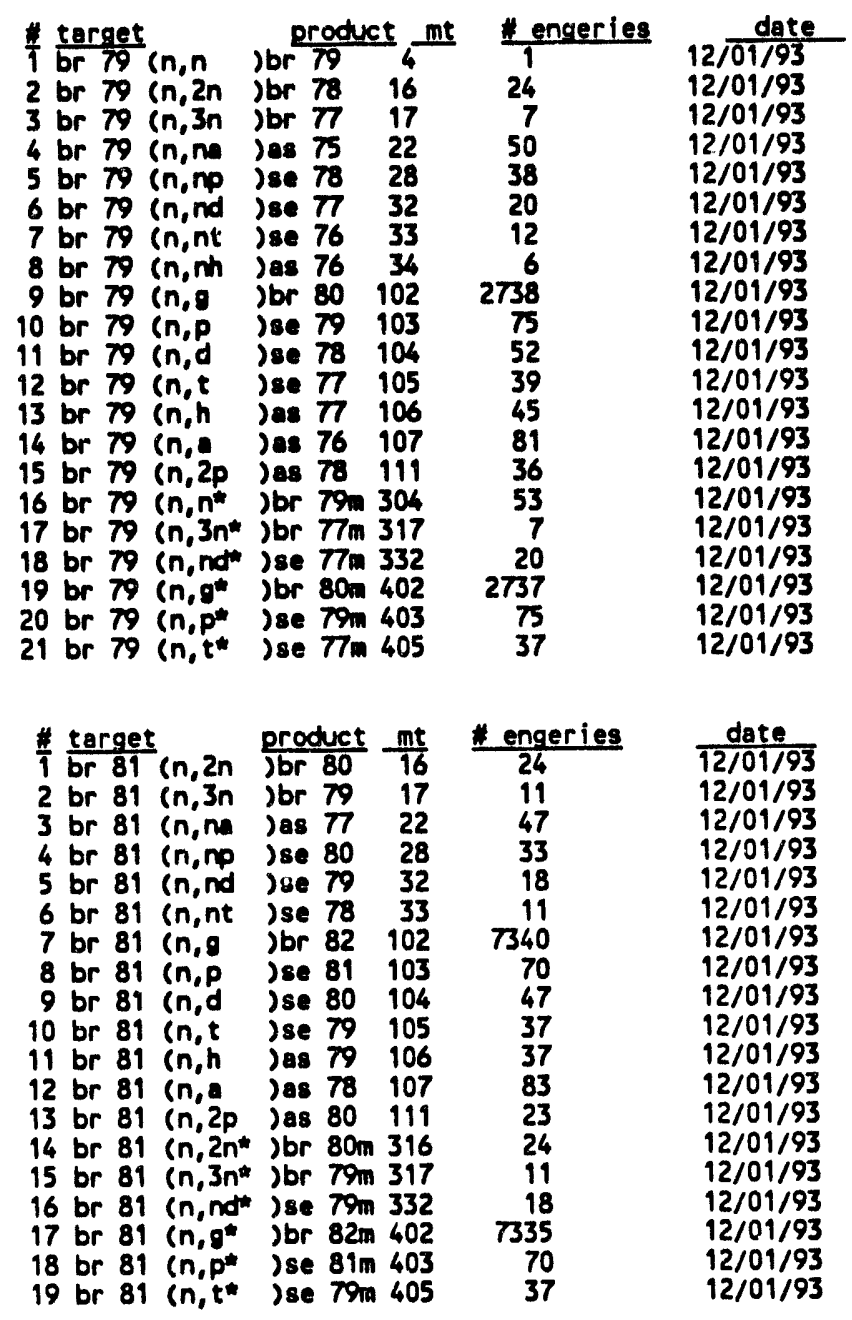

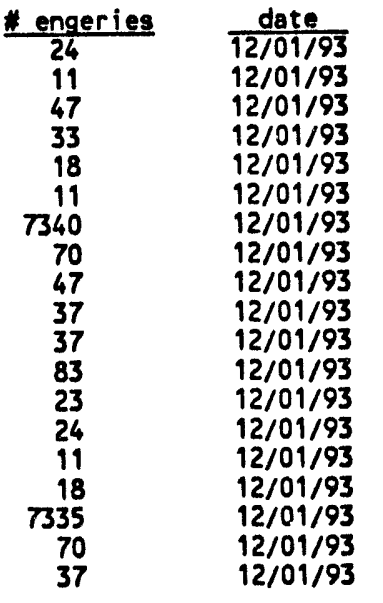

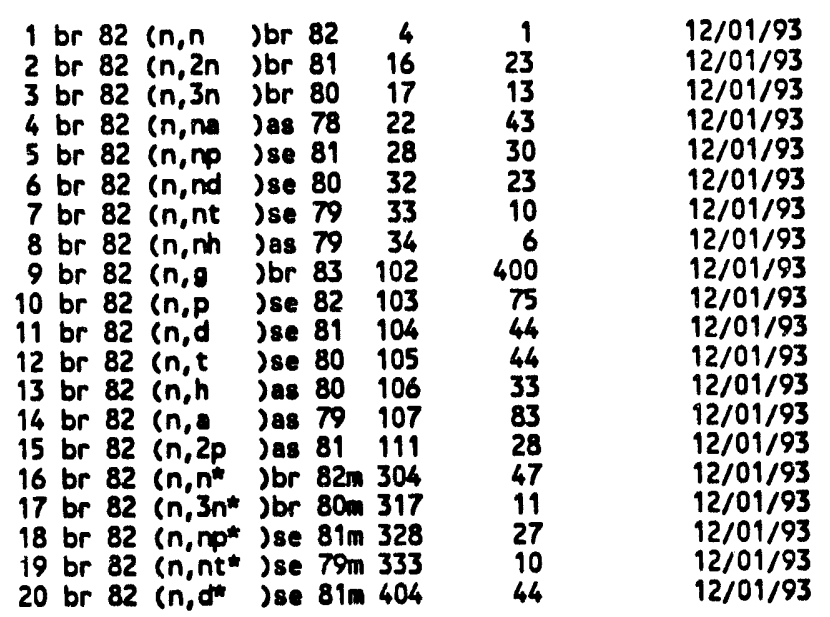

\begin{tabular}{|c|c|c|c|c|}
\hline 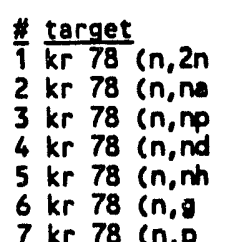 & $\begin{array}{l}\text { product } \\
\text { jkr } 77 \\
\text { 3se } 74 \\
\text { jbr } 77 \\
\text { Jbr } 76 \\
\text { jse } 75 \\
\text { Jkr } 79\end{array}$ & $\begin{array}{r}\frac{m t}{16} \\
22 \\
28 \\
32 \\
34 \\
102\end{array}$ & $\begin{array}{c}\text { * engeries } \\
20 \\
57 \\
32 \\
10 \\
9 \\
1368 \\
77\end{array}$ & $\begin{array}{l}\text { date } \\
12 / 01 / 93 \\
12 / 01 / 93 \\
12 / 01 / 93 \\
12 / 01 / 93 \\
12 / 01 / 93 \\
12 / 01 / 93 \\
12 / 01 / 93\end{array}$ \\
\hline
\end{tabular}

$A-3.8$ 


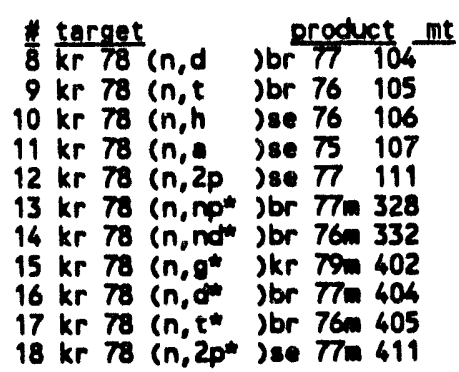

\begin{tabular}{cc} 
Hengeries & $\frac{\text { date }}{45}$ \\
\hline 33 & $12 / 1 / 93$ \\
33 & $12 / 1193$ \\
56 & $12 / 11 / 93$ \\
82 & $12 / 01 / 93$ \\
47 & $12 / 01 / 93$ \\
32 & $12 / 01193$ \\
10 & $12 / 01 / 93$ \\
1367 & $12 / 01 / 93$ \\
45 & $12 / 0193$ \\
33 & $12 / 01 / 93$ \\
47 & $12101 / 93$
\end{tabular}

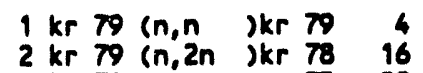

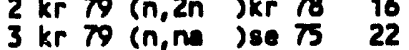
4 kr 79 (n,np )br 7828

$5 \mathrm{kr} 79$ (n, nd )br 732

$6 \mathrm{kr} 79$ (n,nt )br 7633

$7 \mathrm{kr} 79(\mathrm{n}, \mathrm{nh})$ se $76 \quad 34$

$8 \mathrm{kr} 79(n, 9) \mathrm{kr} 80 \quad 102$

$9 \mathrm{kr} 79(\mathrm{n}, \mathrm{p}) \mathrm{jr} 79103$

$10 \mathrm{kr} 79$ (n,d )br 78104

$11 \mathrm{kr} 79$ (n,t jbr 77105

$12 \mathrm{kr} 79(\mathrm{n}, \mathrm{h}$ ) se 77106

$13 \mathrm{kr} 79$ (n,e) se 76107

$14 \mathrm{kr} 79(n, 2 \mathrm{p})$ se 78111

$15 \mathrm{kr} 79$ (n, n* )kr 79m 304

$16 \mathrm{kr} 79$ (n, nd ) br $77 \mathrm{~m} 332$

$17 \mathrm{kr} 79$ (n,nt*) br $76 m 333$

$18 \mathrm{kr} 79$ (n, p* )br $79 m 403$

$19 \mathrm{kr} 79$ (n,t*) )br $77 \mathrm{~m} 405$

$20 \mathrm{kr} 79$ (n,h*) se $77 \mathrm{~m} 406$

$\begin{array}{rr}1 & 12 / 01 / 93 \\ 28 & 12 / 01 / 93 \\ 51 & 12 / 01 / 93 \\ 30 & 12 / 01 / 93 \\ 20 & 12 / 01 / 93 \\ 5 & 12 / 01 / 93 \\ 15 & 12 / 01 / 93 \\ 399 & 12 / 01 / 93 \\ 71 & 12 / 01 / 93 \\ 45 & 12 / 01 / 93 \\ 40 & 12 / 01 / 93 \\ 53 & 12 / 01 / 93 \\ 74 & 12 / 01 / 93 \\ 57 & 12 / 01 / 93 \\ 53 & 12 / 01 / 93 \\ 20 & 12 / 01 / 93 \\ 5 & 12 / 01 / 93 \\ 71 & 12 / 01 / 93 \\ 40 & 12 / 01 / 93 \\ 53 & 12 / 01 / 93\end{array}$

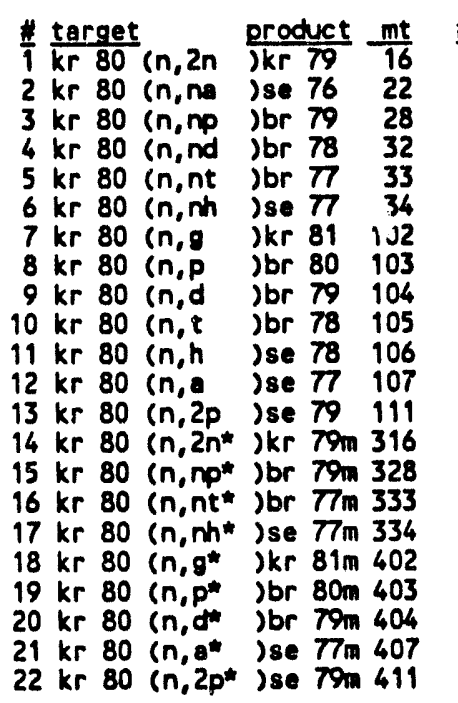

\begin{tabular}{cc} 
\# engeries & date \\
\cline { 2 - 2 } 20 & $12 / 01 / 93$ \\
53 & $12 / 01 / 93$ \\
31 & $12 / 01 / 93$ \\
8 & $12 / 01 / 93$ \\
2 & $12 / 01 / 93$ \\
7 & $12 / 01 / 93$ \\
1534 & $12 / 01 / 93$ \\
67 & $12 / 01 / 93$ \\
44 & $12 / 01 / 93$ \\
32 & $12 / 01 / 93$ \\
50 & $12 / 01 / 93$ \\
79 & $12 / 01 / 93$ \\
39 & $12 / 01 / 93$ \\
20 & $12 / 01 / 93$ \\
31 & $12 / 01 / 93$ \\
2 & $12 / 01 / 93$ \\
5 & $12 / 01 / 93$ \\
1531 & $12 / 01 / 93$ \\
67 & $12 / 01 / 93$ \\
42 & $12 / 01 / 93$ \\
79 & $12 / 01 / 93$ \\
39 & $12 / 01 / 93$
\end{tabular}

\begin{tabular}{|c|c|c|c|c|}
\hline 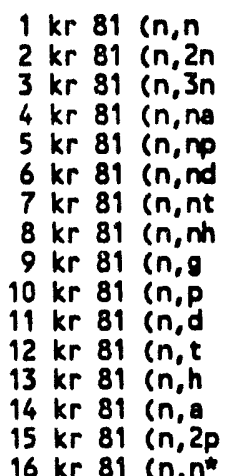 & $\begin{array}{l}\text { Jkr } 81 \\
\text { jkr } 80 \\
\text { jkr } 79 \\
\text { jse } 77 \\
\text { Jbr } 80 \\
\text { Jbr } 79 \\
\text { Jbr } 78 \\
\text { jse } 78 \\
\text { jkr } 82 \\
\text { jbr } 81 \\
\text { jbr } 80 \\
\text { jbr } 79 \\
\text { jse } 79 \\
\text { jse } 78 \\
\text { jse } 80 \\
\text { jkr } 81 \mathrm{~m}\end{array}$ & $\begin{array}{l}4 \\
16 \\
17 \\
22 \\
28 \\
32 \\
33 \\
34 \\
102 \\
103 \\
104 \\
105 \\
106 \\
107 \\
111 \\
304\end{array}$ & $\begin{array}{r}1 \\
29 \\
2 \\
47 \\
28 \\
19 \\
5 \\
12 \\
393 \\
73 \\
43 \\
39 \\
47\end{array}$ & $\begin{array}{l}12 / 01 / 93 \\
12 / 01 / 93 \\
12 / 01 / 93 \\
12 / 01 / 93 \\
12 / 01 / 93 \\
12 / 01 / 93 \\
12 / 01 / 93 \\
12 / 01 / 93 \\
12 / 01 / 93 \\
12 / 01 / 93 \\
12 / 01 / 93 \\
12 / 01 / 93 \\
12 / 01 / 93 \\
12 / 01 / 93 \\
12 / 01 / 93 \\
12 / 01 / 93\end{array}$ \\
\hline
\end{tabular}

$A-3.9$ 


\begin{tabular}{|c|c|c|c|c|c|}
\hline $\begin{array}{l}\text { target } \\
17 \mathrm{kr} 81 \\
18 \mathrm{kr} 81 \\
19 \mathrm{kr} 81 \\
20 \mathrm{kr} 81 \\
21 \mathrm{kr} 81 \\
22 \mathrm{kr} 81 \\
23 \mathrm{kr} 81\end{array}$ & $\begin{array}{l}\left(n, 3 n^{*}\right) \\
\left(n, n^{*}\right) \\
\left(n, n^{*}\right) \\
\left(n, n^{*}\right) \\
\left(n, d^{+}\right) \\
\left(n, t^{\star}\right) \\
\left(n, n^{*}\right)\end{array}$ & 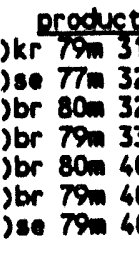 & $\begin{array}{l}51 \\
322 \\
328 \\
332 \\
604 \\
605 \\
606\end{array}$ & $\begin{array}{l}32 \\
47 \\
28 \\
17 \\
42 \\
39 \\
47\end{array}$ & $\begin{array}{l}\text { date } \\
12 / 01 / 93 \\
12 / 01 / 93 \\
12 / 01 / 93 \\
12 / 01 / 93 \\
12 / 01 / 93 \\
12 / 01 / 93 \\
12 / 01 / 93\end{array}$ \\
\hline 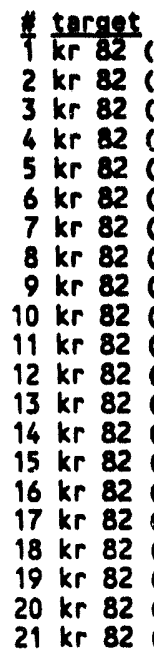 & 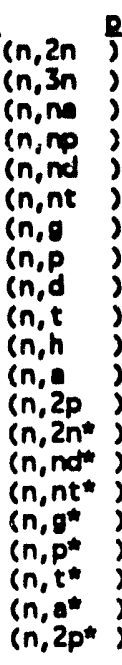 & 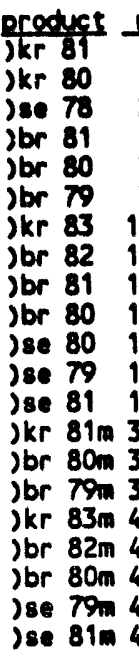 & $\begin{array}{l}\frac{\mathrm{m}}{16} \\
17 \\
22 \\
28 \\
32 \\
33 \\
102 \\
103 \\
104 \\
105 \\
106 \\
107 \\
111 \\
316 \\
332 \\
333 \\
402 \\
403 \\
405 \\
407\end{array}$ & $\begin{array}{c}\text { Aengeries } \\
21 \\
6 \\
50 \\
28 \\
7 \\
2 \\
621 \\
62 \\
42 \\
30 \\
42 \\
81 \\
33 \\
21 \\
7 \\
2 \\
621 \\
62 \\
30 \\
81 \\
33\end{array}$ & $\begin{array}{l}\text { date } \\
12 / 01 / 93 \\
12 / 01 / 93 \\
12 / 01 / 93 \\
12 / 01 / 93 \\
12 / 01 / 93 \\
12 / 01 / 93 \\
12 / 01 / 93 \\
12 / 01 / 93 \\
12 / 01 / 93 \\
12 / 01 / 93 \\
12 / 01 / 93 \\
12 / 01 / 93 \\
12 / 01 / 93 \\
12 / 01 / 93 \\
12 / 01 / 93 \\
12 / 01 / 93 \\
12 / 01 / 93 \\
12 / 01 / 93 \\
12 / 01 / 93 \\
12 / 01 / 93 \\
12 / 01 / 93\end{array}$ \\
\hline $\begin{array}{rll}1 & \mathrm{kr} & 83 \\
2 & \mathrm{kr} & 83 \\
3 & \mathrm{kr} & 83 \\
4 & \mathrm{kr} & 83 \\
5 & \mathrm{kr} & 83 \\
6 & \mathrm{kr} & 83 \\
7 & \mathrm{kr} & 83 \\
8 & \mathrm{kr} & 83 \\
9 & \mathrm{kr} & 83 \\
10 & \mathrm{kr} & 83 \\
11 & \mathrm{kr} & 83 \\
12 & \mathrm{kr} & 83 \\
13 & \mathrm{kr} & 83 \\
14 & \mathrm{kr} & 83 \\
15 & \mathrm{kr} & 83 \\
16 & \mathrm{kr} & 83 \\
17 & \mathrm{kr} & 83 \\
18 & \mathrm{kr} & 83 \\
19 & \mathrm{kr} & 83 \\
20 & \mathrm{kr} & 83 \\
21 & \mathrm{kr} & 83 \\
22 & \mathrm{kr} & 83\end{array}$ & 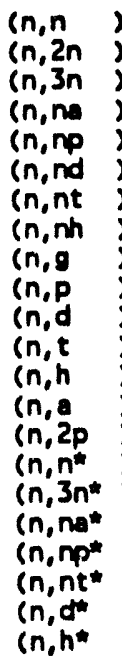 & 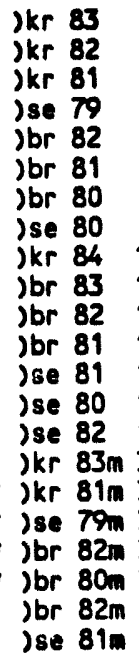 & $\begin{array}{l}4 \\
16 \\
17 \\
22 \\
28 \\
32 \\
33 \\
34 \\
102 \\
103 \\
104 \\
105 \\
106 \\
107 \\
111 \\
304 \\
317 \\
322 \\
328 \\
333 \\
404 \\
406\end{array}$ & $\begin{array}{r}1 \\
22 \\
9 \\
44 \\
27 \\
19 \\
5 \\
9 \\
1094 \\
77 \\
41 \\
38 \\
39 \\
81 \\
37 \\
50 \\
9 \\
44 \\
27 \\
5 \\
41 \\
39\end{array}$ & $\begin{array}{l}12 / 01 / 93 \\
12 / 01 / 93 \\
12 / 01 / 93 \\
12 / 01 / 93 \\
12 / 01 / 93 \\
12 / 01 / 93 \\
12 / 01 / 93 \\
12 / 01 / 93 \\
12 / 01 / 93 \\
12 / 01 / 93 \\
12 / 01193 \\
12 / 01 / 93 \\
12 / 01 / 93 \\
12 / 01 / 93 \\
12 / 01 / 93 \\
12 / 01 / 93 \\
12 / 01 / 93 \\
12 / 01 / 93 \\
12 / 01 / 93 \\
12 / 01 / 93 \\
12 / 01 / 93 \\
12 / 01 / 93\end{array}$ \\
\hline 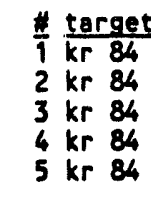 & $\begin{array}{l}\text { (n, 2n } \\
\text { (n, 3n } \\
\text { (n, nn } \\
\text { (n, np } \\
\text { in, nd }\end{array}$ & $\begin{array}{l}\text { product } \\
\text { jkr } 83 \\
\text { jkr } 82 \\
\text { jse } 80 \\
\text { jbr } 83 \\
\text { Jbr } 82\end{array}$ & $\begin{array}{l}\frac{m t}{16} \\
17 \\
22 \\
28 \\
32\end{array}$ & $\begin{array}{c}\text { Hengeries } \\
22 \\
11 \\
47 \\
25 \\
7\end{array}$ & $\begin{array}{l}\frac{\text { date }}{12 / 01 / 73} \\
12 / 01 / 43 \\
12 / 01 / 93 \\
12 / 01 / 93 \\
12 / 01 / 93\end{array}$ \\
\hline $\begin{array}{rrrr}6 & \mathrm{kr} & 84 \\
7 & \mathrm{kr} & 84 \\
8 & \mathrm{kr} & 84 \\
9 & \mathrm{kr} & 84 \\
10 & \mathrm{kr} & 84 \\
11 & \mathrm{kr} & 84 \\
12 & \mathrm{kr} & 84 \\
13 & \mathrm{kr} & 84 \\
14 & \mathrm{kr} & 84 \\
15 & \mathrm{kr} & 84 \\
16 & \mathrm{kr} & 84\end{array}$ & $\begin{array}{l}\text { ln,nt } \\
\text { (n,g } \\
\text { in,p } \\
\text { in,d } \\
\text { in,t } \\
\text { (n, } \\
(n, n \\
(n, 8 \\
(n, 2 p \\
(n, 2 n \star \\
\left(n, n d^{\star}\right. \\
\left(n, g^{\star}\right.\end{array}$ & $\begin{array}{l}\text { Jbr } 81 \\
\text { Jkr } 85 \\
\text { Jbr } 84 \\
\text { Jbr } 83 \\
\text { Jbr } 82 \\
\text { Jse } 82 \\
\text { Jse } 81 \\
\text { Jse } 83 \\
\text { Jkr } 83 m \\
\text { Jbr } 82 m \\
\text { Jkr } 85 m\end{array}$ & $\begin{array}{r}33 \\
102 \\
103 \\
104 \\
105 \\
106 \\
107 \\
111 \\
316 \\
332 \\
402\end{array}$ & $\begin{array}{r}1413 \\
50 \\
38 \\
30 \\
35 \\
81 \\
16 \\
22 \\
7 \\
1413\end{array}$ & $\begin{array}{l}12 / 01 / 93 \\
12 / 01 / 93 \\
12 / 01 / 93 \\
12 / 01 / 93 \\
12 / 01 / 93 \\
12 / 01 / 93 \\
12 / 01 / 93 \\
12 / 01 / 93 \\
12 / 01 / 93 \\
12 / 01 / 93 \\
12 / 01 / 93\end{array}$ \\
\hline
\end{tabular}

$A-3.10$ 


\begin{tabular}{|c|c|c|c|c|c|}
\hline 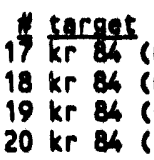 & $\begin{array}{l}\left(n, p^{\infty}\right. \\
\left(n, t^{\infty}\right. \\
\left(n, 0^{\infty}\right. \\
n n, 2 p^{*}\end{array}$ & 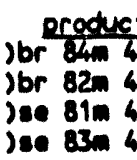 & $\begin{array}{l}\frac{6}{403} \\
405 \\
407 \\
411\end{array}$ & $\begin{array}{l}\frac{1}{49} \text { engeries } \\
30 \\
74 \\
74 \\
16\end{array}$ & $\begin{array}{l}\text { date } \\
12 / 01 / 93 \\
12 / 01 / 93 \\
12 / 01 / 93 \\
12 / 01 / 93\end{array}$ \\
\hline 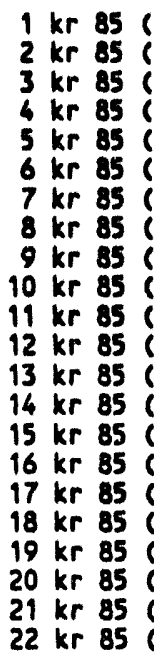 & 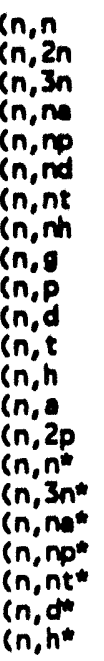 & 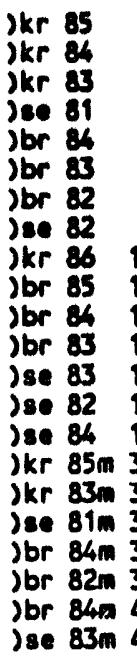 & $\begin{array}{l}6 \\
16 \\
17 \\
22 \\
28 \\
32 \\
33 \\
34 \\
102 \\
103 \\
104 \\
105 \\
106 \\
107 \\
111 \\
304 \\
317 \\
322 \\
328 \\
333 \\
404 \\
406\end{array}$ & $\begin{array}{r}1 \\
27 \\
15 \\
39 \\
28 \\
15 \\
5 \\
4 \\
417 \\
60 \\
37 \\
38 \\
29 \\
81 \\
27 \\
65 \\
15 \\
39 \\
28 \\
5 \\
36 \\
29\end{array}$ & $\begin{array}{l}12 / 01 / 93 \\
12 / 01 / 93 \\
12 / 01 / 93 \\
12 / 01 / 93 \\
12 / 01 / 93 \\
12 / 01 / 93 \\
12 / 01 / 93 \\
12 / 01 / 93 \\
12 / 01 / 93 \\
12 / 01 / 93 \\
12 / 01 / 93 \\
12 / 01 / 93 \\
12 / 01 / 93 \\
12 / 01 / 93 \\
12 / 01 / 93 \\
12 / 01 / 93 \\
12 / 01 / 93 \\
12 / 01 / 93 \\
12 / 01 / 93 \\
12 / 01 / 93 \\
12 / 01 / 93 \\
12 / 01 / 93\end{array}$ \\
\hline 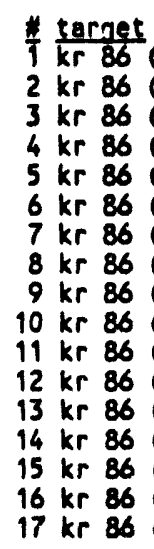 & 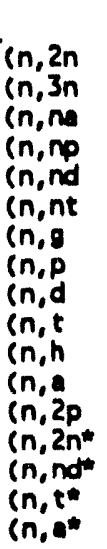 & 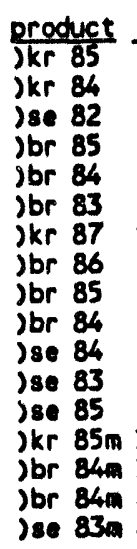 & $\begin{array}{l}\frac{m t}{16} \\
17 \\
22 \\
28 \\
32 \\
33 \\
102 \\
103 \\
104 \\
105 \\
106 \\
107 \\
111 \\
316 \\
332 \\
405 \\
407\end{array}$ & $\begin{array}{c}\text { Eengeries } \\
32 \\
15 \\
42 \\
28 \\
5 \\
5 \\
710 \\
42 \\
35 \\
27 \\
25 \\
65 \\
9 \\
27 \\
4 \\
27 \\
65\end{array}$ & $\begin{array}{c}\text { date } \\
12 / 01 / 93 \\
12 / 01 / 93 \\
12 / 01 / 93 \\
12 / 01 / 93 \\
12 / 01 / 93 \\
12 / 01 / 93 \\
12 / 01 / 93 \\
12 / 01 / 93 \\
12 / 01 / 93 \\
12 / 01 / 93 \\
12 / 01 / 93 \\
12 / 01 / 93 \\
12 / 01 / 93 \\
12 / 01 / 93 \\
12 / 01 / 93 \\
12 / 01 / 93 \\
12 / 01 / 93\end{array}$ \\
\hline $\begin{array}{ccc}1 & r b & 83 \\
2 & r b & 83 \\
3 & r b & 83 \\
4 & r b & 83 \\
5 & r b & 83 \\
6 & r b & 83 \\
7 & r b & 83 \\
8 & r b & 83 \\
9 & r b & 83 \\
10 & r b & 83 \\
11 & r b & 83 \\
12 & r b & 83 \\
13 & r b & 83 \\
14 & r b & 83 \\
15 & r b & 83 \\
16 & r b & 83 \\
17 & r b & 83 \\
18 & r b & 83 \\
19 & r b & 83 \\
20 & r b & 83 \\
21 & r b & 83 \\
22 & r b & 83 \\
23 & r b & 83\end{array}$ & 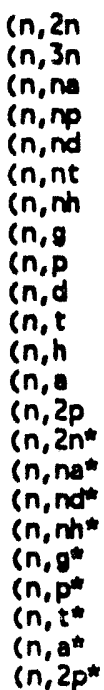 & 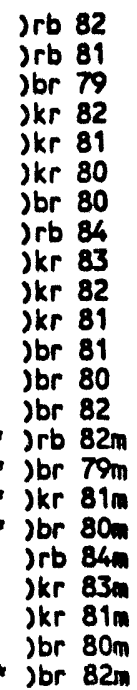 & $\begin{array}{r}16 \\
17 \\
22 \\
28 \\
32 \\
33 \\
34 \\
102 \\
103 \\
104 \\
105 \\
106 \\
107 \\
111 \\
316 \\
322 \\
332 \\
334 \\
402 \\
403 \\
405 \\
407 \\
411\end{array}$ & $\begin{array}{r}25 \\
2 \\
52 \\
38 \\
19 \\
11 \\
7 \\
404 \\
73 \\
55 \\
41 \\
49 \\
81 \\
40 \\
21 \\
49 \\
19 \\
7 \\
404 \\
73 \\
41 \\
81 \\
40\end{array}$ & $\begin{array}{l}12 / 01 / 93 \\
12 / 01 / 93 \\
12 / 01 / 93 \\
12 / 01 / 93 \\
12 / 01 / 93 \\
12 / 01 / 93 \\
12 / 01 / 93 \\
12 / 01 / 93 \\
12 / 01 / 93 \\
12 / 01 / 93 \\
12 / 01 / 93 \\
12 / 01 / 93 \\
12 / 01 / 93 \\
12 / 01 / 93 \\
12 / 01 / 93 \\
12 / 01 / 93 \\
12 / 01 / 93 \\
12 / 01 / 93 \\
12 / 01 / 93 \\
12 / 01 / 93 \\
12 / 01 / 93 \\
12 / 01 / 93 \\
12 / 01 / 93\end{array}$ \\
\hline
\end{tabular}




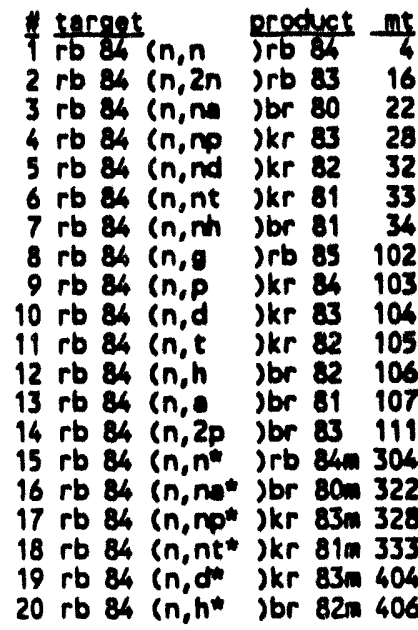

\begin{tabular}{cc} 
ongeries & date \\
\hline 1 & $12 / 01 / 93$ \\
25 & $12 / 01 / 93$ \\
46 & $12 / 01 / 93$ \\
33 & $12 / 01 / 93$ \\
26 & $12 / 01 / 93$ \\
9 & $12 / 01 / 93$ \\
10 & $12 / 01 / 93$ \\
406 & $12 / 01 / 93$ \\
232 & $12 / 01 / 93$ \\
49 & $12 / 01 / 93$ \\
45 & $12 / 01 / 93$ \\
44 & $12 / 01 / 93$ \\
82 & $12 / 01 / 93$ \\
43 & $12 / 01 / 93$ \\
49 & $12 / 01 / 93$ \\
46 & $12 / 01 / 93$ \\
33 & $12 / 01 / 93$ \\
9 & $12 / 01 / 93$ \\
49 & $12 / 01 / 93$ \\
44 & $12 / 01 / 93$ \\
19 & $12 / 01 / 93$ \\
2 & $12 / 01 / 93$ \\
47 & $12 / 01 / 93$ \\
33 & $12 / 01 / 93$ \\
17 & $12 / 01 / 93$ \\
10 & $12 / 01 / 93$ \\
1282 & $12 / 01 / 93$ \\
76 & $12 / 01 / 93$ \\
51 & $12 / 01 / 93$ \\
37 & $12 / 01 / 93$ \\
41 & $12 / 01 / 93$ \\
81 & $12 / 01 / 93$ \\
30 & $12 / 01 / 93$ \\
18 & $12 / 01 / 93$ \\
15 & $12 / 01 / 93$ \\
1282 & $12 / 01 / 93$ \\
76 & $12 / 011 / 93$ \\
37 & $12 / 01 / 93$ \\
81 & $12 / 01 / 93$ \\
30 &
\end{tabular}

1 rb 85 ( $n, 2 n)$ )rb 8416

2 rb 85 ( $n, 3 n)$ rb 8317

3 rb $85(n, n$ ) br 8122

4 rb 85 (n, np )kr 8428

5 rb 85 ( $n$, nd ) kr 8332

6 rb 85 (n, nt ) kr 8233

7 rb 85 (n,g) jrb $86 \quad 102$

8 rb 85 (n,p jkr 85103

9 rb 85 (n,d )kr $84 \quad 104$

10 rb 85 (n,t jkr 83 105

11 rb 85 (n,h jbr 83106

12 rb 85 (n, jbr 82107

13 rb 85 (n, 2p ) br 84111

$14 \mathrm{rb} 85\left(\mathrm{n}, 2 \mathrm{n}^{*}\right) \mathrm{rb} 84 \mathrm{~m} 316$

15 rb 85 ( $n$, nd ) kr 83m 332

$16 \mathrm{rb} 85\left(\mathrm{n}, \mathrm{g}^{\mathrm{t}}\right) \mathrm{rb} 86 \mathrm{~m} 402$

17 rb 85 (n, p )kr 85m 403

18 rb 85 (n,t ) kr 83m 405

19 rb 85 ( $\left.n . a^{*}\right)$ br $82 m 407$

20 rb 85 (n,2p*) br $84 m 411$

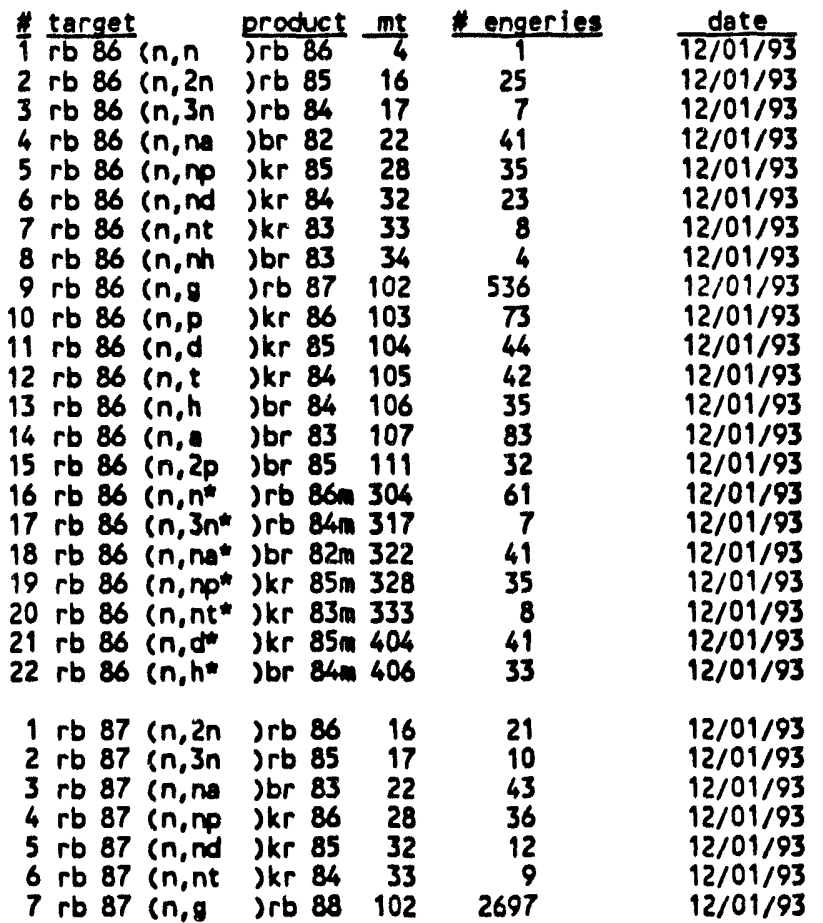

$A=3.12$ 


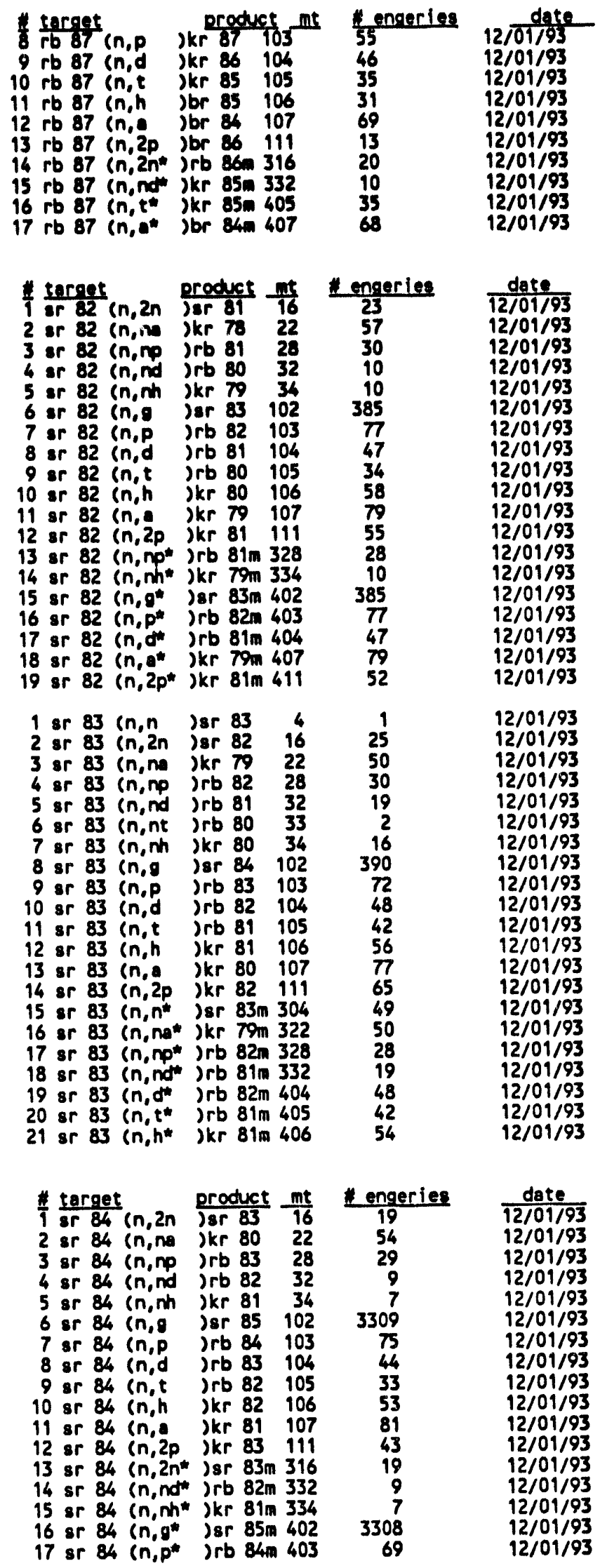




\begin{tabular}{|c|c|c|c|c|c|}
\hline $\begin{array}{l}18 \\
19 \\
20\end{array}$ & $\begin{array}{l}\operatorname{target} \\
\operatorname{sr} 84(n, t * \\
\operatorname{sr} 84(n,)^{*} \\
\operatorname{sr} 84\left(n, 2 p^{\star}\right.\end{array}$ & $\begin{array}{l}\text { Produc } \\
\text { Jrb } 82 \mathrm{~m} \\
\text { Jkr } 81 \mathrm{~m} \\
\text { Jkr } 83 \mathrm{~m}\end{array}$ & $\begin{array}{l}\frac{c t}{405} \\
405 \\
407 \\
411\end{array}$ & $\begin{array}{l}\text { engeries } \\
\frac{11}{31} \\
81 \\
44\end{array}$ & $\begin{array}{l}\frac{\text { date }}{12 / 01 / 93} \\
12 / 01 / 93 \\
12 / 01 / 93\end{array}$ \\
\hline $\begin{array}{l}1 \\
2 \\
3 \\
4 \\
5 \\
6 \\
7 \\
8 \\
9 \\
10 \\
11 \\
12 \\
13 \\
14 \\
15 \\
16 \\
17 \\
18\end{array}$ & 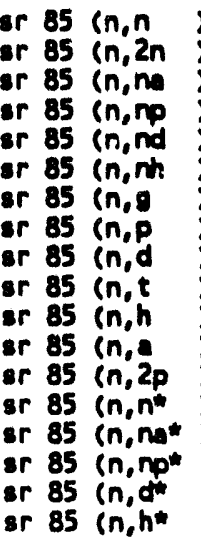 & 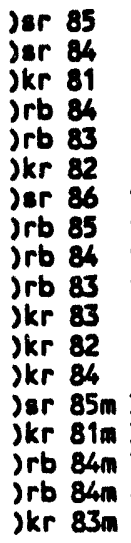 & $\begin{array}{r}4 \\
16 \\
22 \\
28 \\
32 \\
34 \\
102 \\
103 \\
104 \\
105 \\
106 \\
107 \\
111 \\
304 \\
322 \\
328 \\
404 \\
406\end{array}$ & $\begin{array}{l}1 \\
20 \\
46 \\
27 \\
18 \\
11 \\
390 \\
74 \\
46 \\
39 \\
48 \\
79 \\
51 \\
49 \\
43 \\
26 \\
44 \\
48\end{array}$ & $\begin{array}{l}12 / 01 / 93 \\
12 / 01 / 93 \\
12 / 01 / 93 \\
12 / 01 / 93 \\
12 / 01 / 93 \\
12 / 01 / 93 \\
12 / 01 / 93 \\
12 / 01 / 93 \\
12 / 01 / 93 \\
12 / 01 / 93 \\
12 / 01 / 93 \\
12 / 01 / 93 \\
12 / 01 / 93 \\
12 / 01 / 93 \\
12 / 01 / 93 \\
12 / 01 / 93 \\
12 / 01 / 93 \\
12 / 01 / 93\end{array}$ \\
\hline $\begin{array}{r}\frac{1}{1} \\
2 \\
3 \\
4 \\
5 \\
6 \\
7 \\
8 \\
9 \\
10 \\
11 \\
12 \\
13 \\
14 \\
15 \\
16 \\
17 \\
18\end{array}$ & 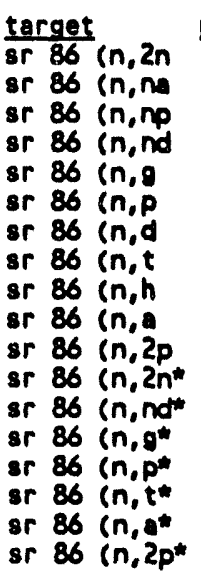 & 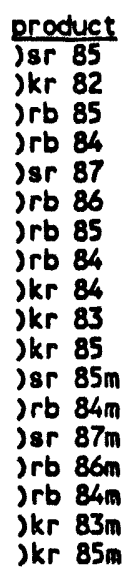 & $\begin{array}{l}\frac{m t}{16} \\
22 \\
28 \\
32 \\
102 \\
103 \\
104 \\
105 \\
106 \\
107 \\
111 \\
316 \\
332 \\
402 \\
403 \\
405 \\
407 \\
411\end{array}$ & $\begin{array}{c}\text { engeries } \\
19 \\
51 \\
27 \\
6 \\
4089 \\
67 \\
42 \\
31 \\
44 \\
81 \\
38 \\
19 \\
5 \\
4094 \\
63 \\
29 \\
81 \\
38\end{array}$ & $\begin{array}{l}\text { date } \\
12 / 01 / 93 \\
12 / 01 / 93 \\
12 / 01 / 93 \\
12 / 01 / 93 \\
12 / 01 / 93 \\
12 / 01 / 93 \\
12 / 01 / 93 \\
12 / 01 / 93 \\
12 / 01 / 93 \\
12 / 01 / 93 \\
12 / 01 / 93 \\
12 / 01 / 93 \\
12 / 01 / 93 \\
12 / 01 / 93 \\
12 / 01 / 93 \\
12 / 01 / 93 \\
12 / 01 / 93 \\
12 / 01 / 93\end{array}$ \\
\hline $\begin{array}{r}1 \\
2 \\
3 \\
4 \\
5 \\
6 \\
7 \\
8 \\
9 \\
10 \\
11 \\
12 \\
13 \\
14 \\
15 \\
16 \\
17 \\
18\end{array}$ & 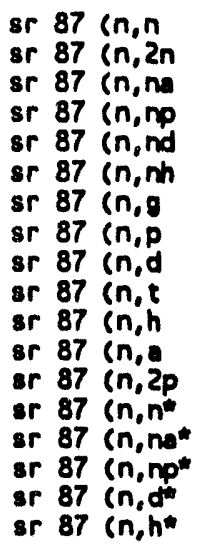 & 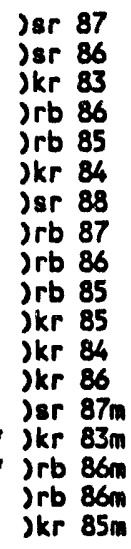 & $\begin{array}{r}4 \\
16 \\
22 \\
28 \\
32 \\
34 \\
102 \\
103 \\
104 \\
105 \\
106 \\
107 \\
111 \\
304 \\
322 \\
328 \\
404 \\
406\end{array}$ & $\begin{array}{r}1 \\
23 \\
43 \\
30 \\
14 \\
7 \\
3702 \\
74 \\
43 \\
37 \\
41 \\
82 \\
41 \\
49 \\
43 \\
29 \\
41 \\
39\end{array}$ & $\begin{array}{l}12 / 01 / 93 \\
12 / 01 / 93 \\
12 / 01 / 93 \\
12 / 01 / 93 \\
12 / 01 / 93 \\
12 / 01 / 93 \\
12 / 01 / 93 \\
12 / 01 / 93 \\
12 / 01 / 93 \\
12 / 01 / 93 \\
12 / 01 / 93 \\
12 / 01 / 93 \\
12 / 01 / 93 \\
12 / 01 / 93 \\
12 / 01 / 93 \\
12 / 01 / 93 \\
12 / 01 / 93 \\
12 / 01 / 93\end{array}$ \\
\hline & 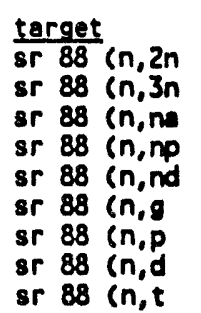 & $\begin{array}{l}\text { product } \\
\text { jor } 87 \\
\text { jsr } 86 \\
\text { jkr } 84 \\
\text { jrb } 87 \\
\text { jrb } 86 \\
\text { jsr } 89 \\
\text { jrb } 88 \\
\text { jrb } 87 \\
\text { jrb } 86\end{array}$ & $\begin{array}{l}\frac{m t}{16} \\
17 \\
22 \\
28 \\
32 \\
102 \\
103 \\
104 \\
105\end{array}$ & $\begin{array}{c}\text { engeries } \\
24 \\
2 \\
44 \\
29 \\
5 \\
1722 \\
50 \\
40 \\
29\end{array}$ & $\begin{array}{l}\frac{\text { data }}{12 / 01 / 93} \\
12 / 01 / 93 \\
12 / 01 / 93 \\
12 / 01 / 93 \\
12 / 01 / 93 \\
12 / 01 / 93 \\
12 / 01 / 93 \\
12 / 01 / 93 \\
12 / 01 / 93\end{array}$ \\
\hline
\end{tabular}




\begin{tabular}{|c|c|c|c|c|c|}
\hline $\begin{array}{l}\frac{1}{10} \\
11: \\
11: \\
13: \\
14: \\
15: \\
16:\end{array}$ & 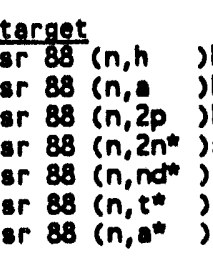 & 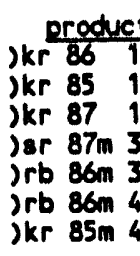 & $\begin{array}{l}\frac{5}{106} \\
107 \\
111 \\
316 \\
332 \\
405 \\
407\end{array}$ & $\begin{array}{l}\frac{4}{3} \text { engeries } \\
35 \\
75 \\
24 \\
21 \\
4 \\
27 \\
73\end{array}$ & $\begin{array}{l}\frac{\text { date }}{12 / 01 / 93} \\
12 / 01 / 93 \\
12 / 01 / 93 \\
12 / 01 / 93 \\
12 / 01 / 93 \\
12 / 01 / 93 \\
12 / 01 / 93\end{array}$ \\
\hline $\begin{array}{l}1 \\
2 \\
3 \\
4 \\
5 \\
6 \\
7 \\
8 \\
9 \\
10 \\
11 \\
12 \\
13 \\
14 \\
15 \\
16 \\
17\end{array}$ & 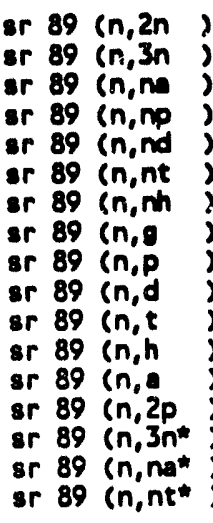 & $\begin{array}{l}\text { Jor } 88 \\
\text { jor } 87 \\
\text { jkr } 85 \\
\text { jrb } 88 \\
\text { jrb } 87 \\
\text { jrb } 86 \\
\text { jkr } 86 \\
\text { jor } 90 \\
\text { jrb } 89 \\
\text { jrb } 88 \\
\text { jrb } 87 \\
\text { jkr } 87 \\
\text { jkr } 86 \\
\text { jkr } 88 \\
\text { jor } 87 \mathrm{~m} \\
\text { jkr } 85 \mathrm{~m} \\
\text { Jrb } 86 \mathrm{~m}\end{array}$ & $\begin{array}{l}16 \\
17 \\
22 \\
28 \\
32 \\
33 \\
34 \\
102 \\
103 \\
104 \\
105 \\
106 \\
107 \\
111 \\
317 \\
322 \\
333\end{array}$ & $\begin{array}{r}32 \\
13 \\
41 \\
21 \\
14 \\
5 \\
4 \\
394 \\
54 \\
39 \\
38 \\
31 \\
85 \\
25 \\
9 \\
39 \\
4\end{array}$ & $\begin{array}{l}12 / 01 / 93 \\
12 / 01 / 93 \\
12 / 01 / 93 \\
12 / 01 / 93 \\
12 / 01 / 93 \\
12 / 01 / 93 \\
12 / 01 / 93 \\
12 / 01 / 93 \\
12 / 01 / 93 \\
12 / 01 / 93 \\
12 / 01 / 93 \\
12 / 01 / 93 \\
12 / 01 / 93 \\
12 / 01 / 93 \\
12 / 01 / 93 \\
12 / 01 / 93 \\
12 / 01 / 93\end{array}$ \\
\hline $\begin{array}{r}1 \\
2 \\
3 \\
4 \\
5 \\
6 \\
7 \\
8 \\
9 \\
10 \\
11 \\
12 \\
13 \\
14\end{array}$ & 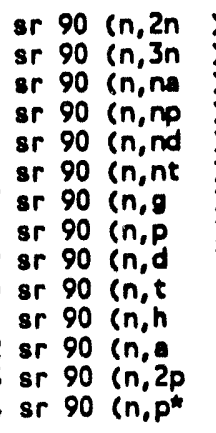 & $\begin{array}{l}\text { Jsr } 89 \\
\text { jsr } 88 \\
\text { jkr } 86 \\
\text { jrb } 89 \\
\text { jrb } 88 \\
\text { jrb } 87 \\
\text { jor } 91 \\
\text { jrb } 90 \\
\text { jrb } 89 \\
\text { jrb } 88 \\
\text { jkr } 88 \\
\text { jkr } 87 \\
\text { jkr } 89 \\
\text { jrb } 90 \mathrm{~m}\end{array}$ & $\begin{array}{l}16 \\
17 \\
22 \\
28 \\
32 \\
33 \\
102 \\
103 \\
104 \\
105 \\
106 \\
107 \\
111 \\
403\end{array}$ & $\begin{array}{r}35 \\
24 \\
53 \\
20 \\
10 \\
11 \\
408 \\
46 \\
38 \\
36 \\
27 \\
88 \\
12 \\
46\end{array}$ & $\begin{array}{l}12 / 01 / 93 \\
12 / 01 / 93 \\
12 / 01 / 93 \\
12 / 01 / 93 \\
12 / 01 / 93 \\
12 / 01 / 93 \\
12 / 01 / 93 \\
12 / 01 / 93 \\
12 / 01 / 93 \\
12 / 01 / 93 \\
12 / 01 / 93 \\
12 / 01 / 93 \\
12 / 01 / 93 \\
12 / 01 / 93\end{array}$ \\
\hline $\begin{array}{l}9 \\
10 \\
11 \\
12 \\
13 \\
14 \\
15 \\
16 \\
17 \\
18 \\
19 \\
20 \\
21 \\
22\end{array}$ & 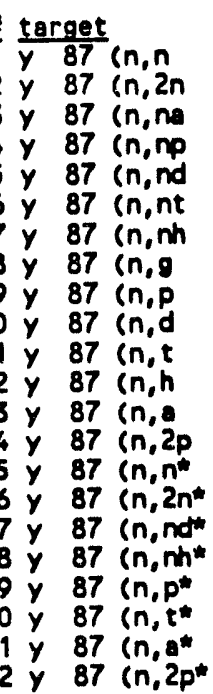 & $\begin{array}{l}\text { product } \\
\text { jy } 87 \\
\text { jy } 86 \\
\text { jrb } 83 \\
\text { j8r } 86 \\
\text { jsr } 85 \\
\text { jsr } 84 \\
\text { jrb } 84 \\
\text { jy } 88 \\
\text { jsr } 87 \\
\text { jsr } 86 \\
\text { jsr } 85 \\
\text { jrb } 85 \\
\text { jrb } 84 \\
\text { jrb } 86 \\
\text { jy } 87 n \\
\text { jy } 86 \pi \\
\text { jor } 85 \pi \\
\text { jrb } 84 \pi \\
\text { jor } 87 \pi \\
\text { jsr } 85 \pi \\
\text { jrb } 84 \pi \\
\text { jrb } 86 n\end{array}$ & $\begin{array}{c}\mathrm{mt} \\
4 \\
16 \\
22 \\
28 \\
32 \\
33 \\
34 \\
102 \\
103 \\
104 \\
105 \\
106 \\
107 \\
111 \\
304 \\
316 \\
332 \\
334 \\
03\end{array}$ & $\begin{array}{c}\text { \# engeries } \\
1 \\
25 \\
47 \\
36 \\
18 \\
8 \\
4 \\
399 \\
71 \\
55 \\
41 \\
50 \\
80 \\
44 \\
50 \\
21 \\
18 \\
4 \\
71 \\
38 \\
80 \\
43\end{array}$ & $\begin{array}{l}\text { date } \\
12 / 01 / 93 \\
12 / 01 / 93 \\
12 / 01 / 93 \\
12 / 01 / 93 \\
12 / 01 / 93 \\
12 / 01 / 93 \\
12 / 01 / 93 \\
12 / 01 / 93 \\
12 / 01 / 93 \\
12 / 01 / 93 \\
12 / 01 / 93 \\
12 / 01 / 93 \\
12 / 01 / 93 \\
12 / 01 / 93 \\
12 / 01 / 93 \\
12 / 01 / 93 \\
12 / 01 / 93 \\
12 / 01 / 93 \\
12 / 01 / 93 \\
12 / 01 / 93 \\
12 / 01 / 93 \\
12 / 01 / 93\end{array}$ \\
\hline
\end{tabular}




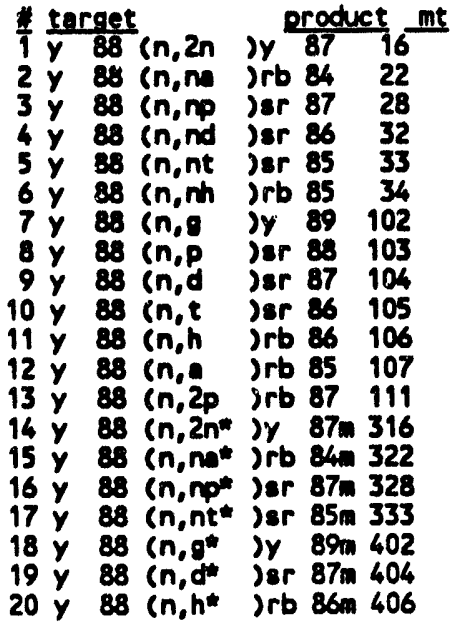

\begin{tabular}{cc} 
* engeries & date \\
\hline 13 & $12 / 01 / 93$ \\
39 & $12 / 01 / 93$ \\
16 & $12 / 01 / 93$ \\
23 & $12 / 01 / 93$ \\
7 & $12 / 01 / 93$ \\
7 & $12 / 01 / 93$ \\
395 & $12 / 01 / 93$ \\
20 & $12 / 01 / 93$ \\
50 & $12 / 01 / 93$ \\
46 & $12 / 01 / 93$ \\
46 & $12 / 01 / 93$ \\
83 & $12 / 01 / 93$ \\
47 & $12 / 01 / 93$ \\
24 & $12 / 01 / 93$ \\
38 & $12 / 01 / 93$ \\
39 & $12 / 01 / 93$ \\
5 & $12 / 01 / 93$ \\
395 & $12 / 01 / 93$ \\
50 & $12 / 01 / 93$ \\
44 & $12 / 01 / 93$
\end{tabular}

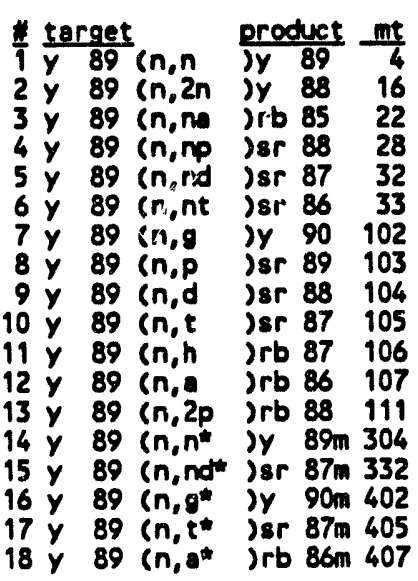

\begin{tabular}{cc} 
engeries & dste \\
\cline { 1 - 2 } 1 & $12 / 01 / 93$ \\
13 & $12 / 01 / 93$ \\
42 & $12 / 01 / 93$ \\
16 & $12 / 01 / 93$ \\
12 & $12 / 01 / 93$ \\
7 & $12 / 01 / 93$ \\
1706 & $12 / 01 / 93$ \\
20 & $12 / 01 / 93$ \\
52 & $12 / 01 / 93$ \\
37 & $12 / 01 / 93$ \\
41 & $12 / 01 / 93$ \\
85 & $12 / 01 / 93$ \\
31 & $12 / 01 / 93$ \\
59 & $12 / 01 / 93$ \\
10 & $12 / 01 / 93$ \\
1706 & $12 / 01 / 93$ \\
35 & $12 / 01 / 93$ \\
85 & $12 / 01 / 93$ \\
& \\
1 & $12 / 01 / 93$ \\
29 & $12 / 01 / 93$ \\
9 & $12 / 01 / 93$ \\
43 & $12 / 01 / 93$ \\
29 & $12 / 01 / 93$ \\
20 & $12 / 01 / 93$ \\
10 & $12 / 01 / 93$ \\
6 & $12 / 01 / 93$ \\
407 & $12 / 01 / 93$ \\
73 & $12 / 01 / 93$ \\
47 & $12 / 01 / 93$ \\
48 & $12 / 01 / 93$ \\
37 & $12 / 01 / 93$ \\
81 & $12 / 01 / 93$ \\
30 & $12 / 01 / 93$ \\
61 & $12 / 01 / 93$ \\
20 & $12 / 01193$ \\
42 & $12 / 01193$ \\
8 & $12 / 01193$ \\
405 & $12 / 01 / 93$ \\
& \\
&
\end{tabular}


WHC-EP-0727

\begin{tabular}{|c|c|c|c|c|}
\hline 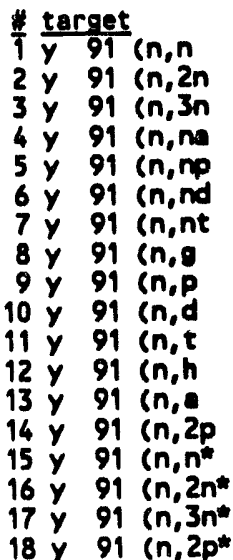 & $\begin{array}{l}\text { product } \\
\text { jy } 91 \\
\text { jy } 90 \\
\text { jy } 89 \\
\text { jrb } 87 \\
\text { jor } 90 \\
\text { jor } 89 \\
\text { jor } 88 \\
\text { jy } 92 \\
\text { jor } 91 \\
\text { jor } 90 \\
\text { jor } 89 \\
\text { jrb } 89 \\
\text { jrb } 88 \\
\text { jrb } 90 \\
\text { jy } 91 \mathrm{~m} \\
\text { jy } 90 \mathrm{~m}\end{array}$ & $\begin{array}{c}\text { mt } \\
16 \\
16 \\
17 \\
22 \\
28 \\
32 \\
33 \\
102 \\
103 \\
104 \\
105 \\
106 \\
107 \\
111\end{array}$ & $\begin{array}{c}\text { \# engeries } \\
1 \\
27 \\
19 \\
52 \\
35 \\
17 \\
16 \\
416 \\
65 \\
46 \\
44 \\
33 \\
84 \\
17 \\
63 \\
25 \\
14\end{array}$ & $\begin{array}{c}\text { date } \\
12 / 01 / 93 \\
12 / 01 / 93 \\
12 / 01 / 93 \\
12 / 01 / 93 \\
12 / 01 / 93 \\
12 / 01 / 93 \\
12 / 01 / 93 \\
12 / 01 / 93 \\
12 / 01 / 93 \\
12 / 01 / 93 \\
12 / 01 / 93 \\
12 / 01 / 93 \\
12 / 01 / 93 \\
12 / 01 / 93 \\
12 / 01 / 93 \\
12 / 01 / 93 \\
12 / 01 / 93 \\
12 / 01 / 93\end{array}$ \\
\hline
\end{tabular}

$A-3.17$ 
WHC-EP-0727

This pege intentionally left blank.

$A-3.18$ 
Appendix $\mathbf{A}-4$

List of Reactions on files *.2r.pd

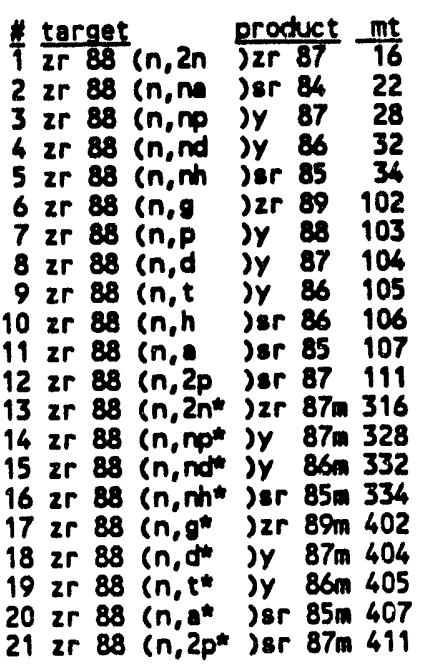

\begin{tabular}{c} 
\# engeries \\
\hline 24 \\
52 \\
29 \\
8 \\
6 \\
368 \\
76 \\
47 \\
33 \\
55 \\
80 \\
52 \\
21 \\
27 \\
8 \\
6 \\
368 \\
45 \\
31 \\
80 \\
49
\end{tabular}

$\frac{\text { date }}{12101 / 93}$

$12 / 01 / 93$

$12 / 01 / 93$

$12 / 01 / 93$

$12 / 01 / 93$

$12 / 01 / 93$

$12 / 01 / 93$

$12 / 01 / 93$

$12 / 01 / 93$

$12 / 01 / 93$

$12 / 01 / 93$

$12 / 01 / 93$

$12 / 01 / 93$

12/01/93

$12 / 01 / 93$

$12 / 01 / 93$

$12 / 01 / 93$

$12 / 01 / 93$

$12 / 01 / 93$

$12 / 01 / 93$

$12 / 01 / 93$

1 2r 89 (n.n )2r $89 \quad 4$

2 2r $89(n, 2 n) 2 r 88 \quad 16$

3 2r 89 (n, na )sr 8522

4 2r 89 ( $n, n$ ) iy 8828

5 zr 89 ( $n$,nd )y 8732

6 zr 89 (n,m ) $8 r 86 \quad 34$

7 zr 89 (n,g) zr 90102

8 zr 89 (n,p iy 89103

9 zr 89 (n,d iy 88104

10 2r $89(n, t)$ )y 87105

$11 \mathrm{zr} 89(\mathrm{n}, \mathrm{h} \quad) \mathrm{sr} 87106$

12 2r $89(n, a) 8 r \quad 86 \quad 107$

13 zr 89 (n,2p )sr 88111

14 zr $89\left(n, n^{*}\right) 2 r 89 m 304$

15 zr 89 ( $n, n n^{*}$ )sr $85 m 322$

16 2r 89 ( $n, n^{*}$ )y $87 m 332$

17 zr $89\left(n, g^{*}\right)$ zr $00 m 402$

18 zr 89 ( $n, p^{*}$ )y $89 m 403$

19 2r 89 (n,t* iy $87 \mathrm{~m} 405$

20 2r 89 (n, h* )sr $87 m 406$

$12 / 01 / 93$

$12 / 01 / 93$

$12 / 01 / 93$

$12 / 01 / 93$

$12 / 01 / 93$

$12 / 01 / 93$

$12 / 01 / 93$

$12 / 01 / 93$

$12 / 01 / 93$

$12 / 01 / 93$

$12 / 01 / 93$

$12 / 01 / 93$

$12 / 01 / 93$

$12 / 01 / 93$

$12 / 01 / 93$

$12 / 01 / 93$

$12 / 01 / 93$

$12 / 01 / 93$

$12 / 01 / 93$

$12 / 01 / 93$

\# target $\frac{\text { product }}{2 r 90} \frac{\mathrm{mt}}{4}$

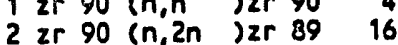

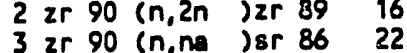

4 2r 90 (n,np) y 8928

5 zr 90 (n,nd oy 8832

6 zr 90 (n,g) izr 91102

7 2r 90 (n,p )y 90103

8 zr 90 (n,d )y 89104

9 $\operatorname{zr} 90(n, t$ )y 88105

$10 \mathrm{zr} 90(\mathrm{n}, \mathrm{h}$ ) $8 \mathrm{r} 88106$

11 zr $90(n, 8)$ sr $87 \quad 107$

12 zr $90(n, 2 p$ )sr 89111

13 zr 90 (n, n* )zr 90m 304

14 2r $90(n, 2 n *) 2 r 89 m 316$

15 zr 90 ( $n, n n^{*}$ )y $89 m 328$

16 zr 90 (n, p* )y $90 \mathrm{~m} 403$

17 zr 90 ( $n$, d) )y $89 \mathrm{~m} 404$

18 zr 90 ( $\left.n, a^{*}\right)$ sr $87 m 407$

\begin{tabular}{|c|c|}
\hline $\begin{array}{c}\text { \# engeries } \\
1 \\
21 \\
49 \\
16 \\
8 \\
2157 \\
28 \\
13 \\
33 \\
46 \\
21 \\
40 \\
59 \\
18 \\
16 \\
28 \\
13 \\
21\end{array}$ & $\begin{array}{c}\text { date } \\
12 / 01 / 93 \\
12 / 01 / 93 \\
1 / 01 / 93 \\
12 / 01 / 93 \\
12 / 01 / 93 \\
12 / 01 / 93 \\
12 / 01 / 93 \\
12 / 01 / 93 \\
12 / 01 / 93 \\
12 / 01 / 93 \\
12 / 01 / 93 \\
12 / 01 / 93 \\
12 / 01 / 93 \\
12 / 01 / 93 \\
12 / 01 / 93 \\
12 / 01 / 93 \\
12 / 01 / 93 \\
12 / 01 / 93\end{array}$ \\
\hline
\end{tabular}

1 zr $91(n, 2 n) 2 r 90 \quad 16$ 2 2r $91(n, 3 n$ ) $2 r 89 \quad 17$ $\begin{array}{llll}3 & 2 r & 91 & \text { (n, na } \\ \text { isr } 87 & 22\end{array}$

4 zr 91 en,np )y 9028

5 zr 91 ( $n$, nd )y 8932

6 2r $91(n, n t) y$

$12 / 01 / 93$

$12 / 01 / 93$

$12 / 01 / 93$

$12 / 01 / 93$

$12 / 01 / 93$

$12 / 01 / 93$

$A-4.1$ 


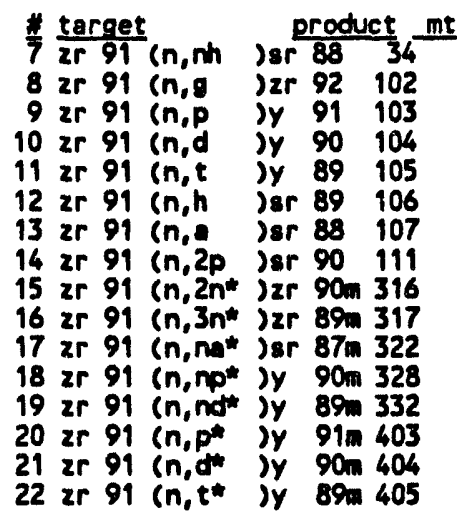

\begin{tabular}{cc} 
Hengeries & $\frac{\text { date }}{12 / 01 / 93}$ \\
\hline 8 & $12 / 01 / 93$ \\
2678 & $12 / 01 / 93$ \\
28 & $12 / 01 / 93$ \\
13 & $12 / 01 / 93$ \\
43 & $12 / 01 / 93$ \\
43 & $12 / 01 / 93$ \\
31 & $12 / 01 / 93$ \\
41 & $12 / 01 / 93$ \\
10 & $12 / 01 / 93$ \\
2 & $12 / 01 / 93$ \\
46 & $12 / 01 / 93$ \\
16 & $12 / 01 / 93$ \\
16 & $12 / 01 / 93$ \\
28 & $12 / 01 / 93$ \\
13 & $12 / 01 / 93$ \\
39 &
\end{tabular}

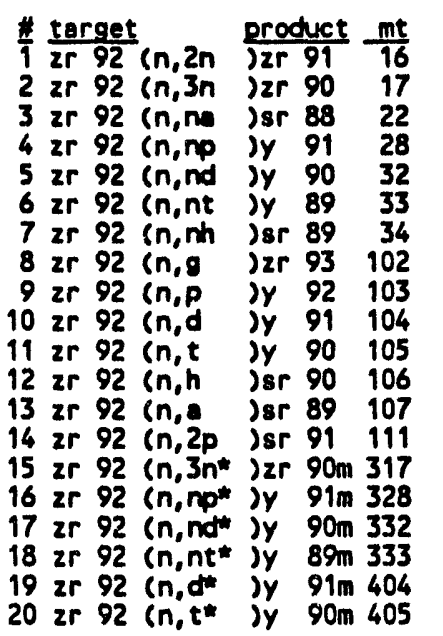

\begin{tabular}{|c|c|}
\hline $\begin{array}{c}\text { A engeries } \\
22 \\
13 \\
59 \\
13 \\
12 \\
14 \\
2 \\
22 \\
7292 \\
24 \\
13 \\
40 \\
39 \\
25 \\
33 \\
6 \\
13 \\
11 \\
9 \\
13 \\
35\end{array}$ & $\begin{array}{l}\text { date } \\
12 / 01 / 93 \\
12 / 01 / 93 \\
12 / 01 / 93 \\
12 / 01 / 93 \\
12 / 01 / 93 \\
12 / 01 / 93 \\
12 / 01 / 93 \\
12 / 01 / 93 \\
12 / 01 / 93 \\
12 / 01 / 93 \\
12 / 01 / 93 \\
12 / 01 / 93 \\
12 / 01 / 93 \\
12 / 01 / 93 \\
12 / 01 / 93 \\
12 / 01 / 93 \\
12 / 01 / 93 \\
12 / 01 / 93 \\
12 / 01 / 93 \\
12 / 01 / 93\end{array}$ \\
\hline
\end{tabular}

1 2r $93(n, 2 n) 2 r 92 \quad 16$ 2 zr $93(n, 3 n$ ) $2 r 91 \quad 17$ 3 zr 93 (n, ns ) $8 r 8922$ 4 2r 93 (n,np )y 9228

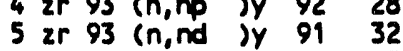
$6 \mathrm{zr} 93$ (n, nt )y 9033

7 zr $93 \mathrm{cn}, \mathrm{nh}) \mathrm{sr} 9034$

8 2r 93 (n,g) $2 r 94 \quad 102$

9 zr 93 (n,p )y 93103 10 zr 93 (n,d) )y 92104 11 zr $93(n, t)$ iy 91105 12 zr 93 (n,h )sr 91106 13 zr 93 (n,a )sr 90107 14 zr 93 (n,2p )sr 92111 15 er 93 (n, ndt )y $91 m 332$ 16 zr 93 (n, nt*) )y $90 m 333$ 17 zr 93 (n, p*) y $93 \mathrm{~m} 403$ $18 \mathrm{zr} 93\left(n, t^{*}\right.$ )y $91 \mathrm{~m} 405$

$\begin{array}{rr}24 & 12 / 01 / 93 \\ 14 & 12 / 01 / 93 \\ 53 & 12 / 01 / 93 \\ 25 & 12 / 01 / 93 \\ 15 & 12 / 01 / 93 \\ 11 & 12 / 01 / 93 \\ 7 & 12 / 01 / 93 \\ 710 & 12 / 01 / 93 \\ 17 & 12 / 01 / 93 \\ 44 & 12 / 01 / 93 \\ 42 & 12 / 01 / 93 \\ 37 & 12 / 01 / 93 \\ 24 & 12 / 01 / 93 \\ 34 & 12 / 01 / 93 \\ 13 & 12 / 01193 \\ 10 & 12 / 01 / 93 \\ 57 & 12 / 01 / 93 \\ 40 & 12 / 01 / 93\end{array}$

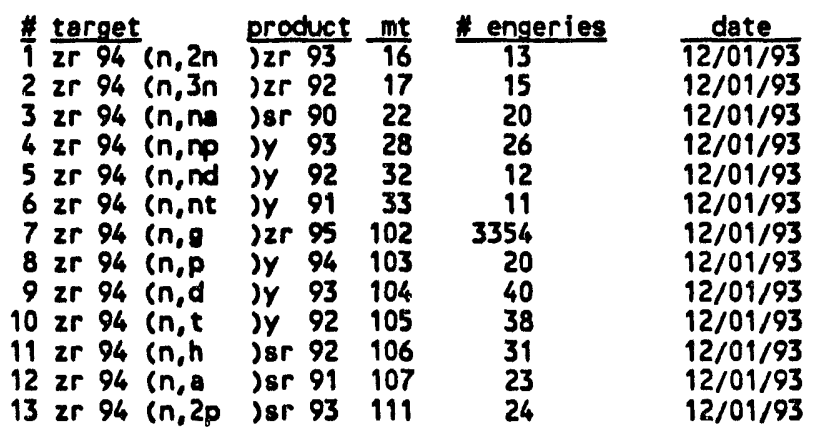




\begin{tabular}{|c|c|c|c|c|c|}
\hline $\begin{array}{l}1 \frac{1}{14} \frac{\text { target }}{14} 94 \\
15 \text { 2r } 94 \\
16 \text { 2r } 94\end{array}$ & $\begin{array}{l}\left(n, n p^{*}\right) y \\
(n, n t *) y \\
\left(n, d^{*}\right) y\end{array}$ & 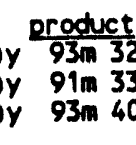 & & $\begin{array}{l}\text { enger i } \\
20 \\
9 \\
38\end{array}$ & $\begin{array}{l}\frac{\text { date }}{12 / 01 / 93} \\
12 / 01 / 93 \\
12 / 01 / 93\end{array}$ \\
\hline $\begin{array}{rlr}1 & 2 r & 95 \\
2 & 2 r & 95 \\
3 & 2 r & 95 \\
4 & 2 r & 95 \\
5 & 2 r & 95 \\
6 & 2 r & 95 \\
7 & 2 r & 95 \\
8 & 2 r & 95 \\
9 & 2 r & 95 \\
10 & 2 r & 95 \\
11 & 2 r & 95 \\
12 & 2 r & 95 \\
13 & 2 r & 95 \\
14 & 2 r & 95 \\
15 & 2 r & 95\end{array}$ & 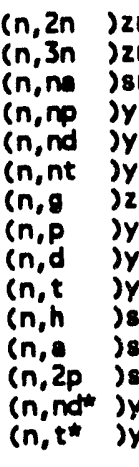 & 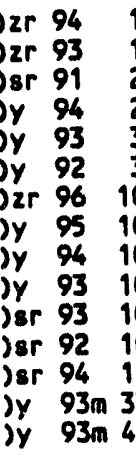 & $\begin{array}{l}16 \\
17 \\
22 \\
28 \\
32 \\
33 \\
102 \\
103 \\
104 \\
105 \\
106 \\
107 \\
111 \\
332 \\
405\end{array}$ & $\begin{array}{r}29 \\
23 \\
55 \\
22 \\
14 \\
12 \\
4016 \\
55 \\
41 \\
41 \\
29 \\
84 \\
26 \\
12 \\
37\end{array}$ & $\begin{array}{l}12 / 01 / 93 \\
12 / 01 / 93 \\
12 / 01 / 93 \\
12 / 01 / 93 \\
12 / 01 / 93 \\
12 / 01 / 93 \\
12 / 01 / 93 \\
12 / 01 / 93 \\
12 / 01 / 93 \\
12 / 01 / 93 \\
12 / 01 / 93 \\
12 / 01 / 93 \\
12 / 01 / 93 \\
12 / 01 / 93 \\
12 / 01 / 93\end{array}$ \\
\hline $\begin{array}{ccc}1 & \mathrm{zr} & 96 \\
2 & \mathrm{zr} & 96 \\
3 & \mathrm{zr} & 96 \\
4 & \mathrm{zr} & 96 \\
5 & \mathrm{zr} & 96 \\
6 & \mathrm{zr} & 96 \\
7 & \mathrm{zr} 96 \\
8 & \mathrm{zr} 96 \\
9 & \mathrm{zr} 96 \\
10 & \mathrm{zr} 96 \\
11 & \mathrm{zr} 96 \\
12 & \mathrm{zr} 96 \\
13 & \mathrm{zr} 96 \\
14 & \mathrm{zr} 96 \\
15 & \mathrm{zr} 96 \\
16 & \mathrm{zr} 96\end{array}$ & $\begin{array}{l}(n, 2 n) \\
(n, 3 n) \\
(n, n) \\
(n, n p) \\
(n, n d) \\
(n, n t) \\
(n, g) \\
(n, p) \\
(n, d) \\
(n, t) \\
(n, n) \\
(n, n) \\
(n, 2 p) \\
\left(n, n t^{*}\right) \\
\left(n, p^{*}\right) \\
\left(n, p^{* *)}\right)\end{array}$ & 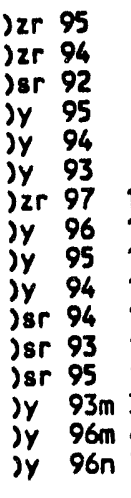 & $\begin{array}{l}16 \\
17 \\
22 \\
28 \\
32 \\
33 \\
102 \\
103 \\
104 \\
105 \\
106 \\
107 \\
111 \\
333 \\
403 \\
703\end{array}$ & $\begin{array}{r}12 \\
20 \\
54 \\
20 \\
11 \\
12 \\
5319 \\
46 \\
39 \\
35 \\
23 \\
23 \\
10 \\
8 \\
46 \\
46\end{array}$ & $\begin{array}{l}12 / 01 / 93 \\
12 / 01 / 93 \\
12 / 01 / / 93 \\
12 / 01 / 93 \\
12 / 01 / 93 \\
12 / 01 / 93 \\
12 / 01 / 93 \\
12 / 01 / 93 \\
12 / 01 / 93 \\
12 / 01 / 93 \\
12 / 01 / 93 \\
12 / 01 / 93 \\
12 / 01 / 93 \\
12 / 01 / 93 \\
12 / 01 / 93 \\
12 / 01 / 93\end{array}$ \\
\hline 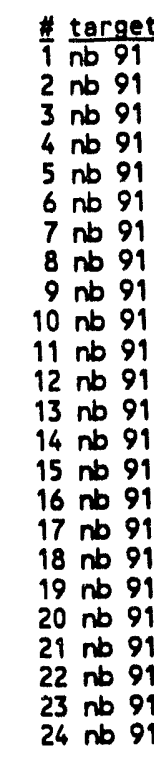 & 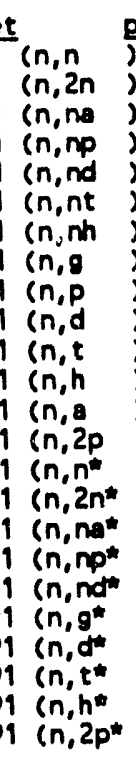 & $\begin{array}{l}\text { product } \\
\text { jnb } 91 \\
\text { jnb } 90 \\
\text { jy } 87 \\
\text { jr } 90 \\
\text { jzr } 89 \\
\text { jzr } 88 \\
\text { jy } 88 \\
\text { jnb } 92 \\
\text { jzr } 91 \\
\text { jzr } 90 \\
\text { jzr } 89 \\
\text { jy } 89 \\
\text { jy } 88 \\
\text { jy } 90 \\
\text { inb } 91 \mathrm{~m} \\
\text { jnb } 90 \mathrm{~m} \\
\text { jy } 87 \mathrm{~m} \\
\text { jzr } 90 \mathrm{~m} \\
\text { jzr } 89 \mathrm{~m} \\
\text { jnb } 92 \mathrm{~m} \\
\text { jzr } 90 \mathrm{~m} \\
\text { jzr } 89 \mathrm{~m} \\
\text { jy } 89 \mathrm{~m} \\
\text { jy } 90 \mathrm{~m}\end{array}$ & $\begin{array}{c}\frac{m t}{4} \\
16 \\
22 \\
28 \\
32 \\
33 \\
34 \\
102 \\
103 \\
104 \\
105 \\
106 \\
107 \\
111 \\
304 \\
316 \\
322 \\
\text { n } 328 \\
\text { n } 332 \\
\text { n } 402 \\
\end{array}$ & $\begin{array}{c}\text { \# engeries } \\
1 \\
14 \\
49 \\
43 \\
17 \\
7 \\
4 \\
380 \\
17 \\
62 \\
41 \\
55 \\
83 \\
45 \\
50 \\
19 \\
46 \\
32 \\
15 \\
380 \\
45 \\
38 \\
53 \\
43\end{array}$ & $\begin{array}{c}\text { date } \\
12 / 01 / 93 \\
12 / 01 / 93 \\
12 / 01 / 93 \\
12 / 01 / 93 \\
12 / 01 / 93 \\
12 / 01 / 93 \\
12 / 01 / 93 \\
12 / 01 / 93 \\
12 / 01 / 93 \\
12 / 01 / 93 \\
12 / 01 / 93 \\
12 / 01 / 93 \\
12 / 01 / 93 \\
12 / 01 / 93 \\
12 / 01 / 93 \\
12 / 01 / 93 \\
12 / 01 / 93 \\
12 / 01 / 93 \\
12 / 01 / 93 \\
12 / 01 / 93 \\
12 / 01 / 93 \\
12 / 01 / 93 \\
12 / 01 / 93 \\
12 / 01 / 93\end{array}$ \\
\hline $\begin{array}{cl}1 & n b \\
2 & n b \\
3 & n b \\
4 & n b \\
5 & n b \\
6 & n b \\
7 & n b \\
8 & n b \\
9 & n b \\
10 & n b\end{array}$ & $\begin{array}{l}n, n \\
n, 2 n \\
n, n= \\
n, n p \\
n, n d \\
n, n t \\
n, n n \\
n, g \\
n, p \\
n, d\end{array}$ & $\begin{array}{l}\text { Inb } 91 \\
\text { inb } 90 \\
\text { iy } 87 \\
\text { izr } 90 \\
\text { izr } 89 \\
\text { izr } 88 \\
\text { iy } 88 \\
\text { inb } 92 \\
\text { jzr } 91 \\
\text { jzr } 90\end{array}$ & $\begin{array}{r}4 \\
16 \\
22 \\
28 \\
32 \\
33 \\
34 \\
102 \\
103 \\
104\end{array}$ & $\begin{array}{r}50 \\
14 \\
49 \\
43 \\
17 \\
7 \\
4 \\
380 \\
17 \\
57\end{array}$ & $\begin{array}{l}12 / 01 / 93 \\
12 / 01 / 93 \\
12 / 01 / 93 \\
12 / 01 / 93 \\
12 / 01 / 93 \\
12 / 01 / 93 \\
12 / 01 / 93 \\
12 / 01 / 93 \\
12 / 01 / 93 \\
12 / 01 / 93\end{array}$ \\
\hline
\end{tabular}

$A-4.3$ 


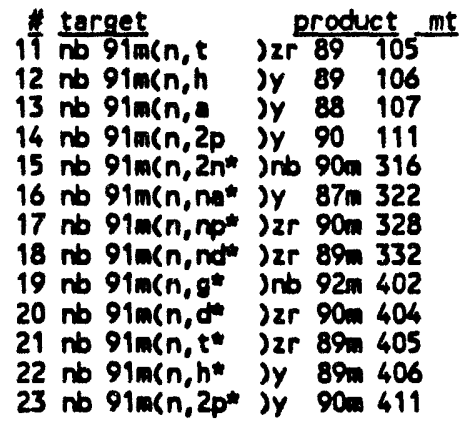

\begin{tabular}{cc} 
\# engeries & $\frac{\text { date }}{12 / 01 / 93}$ \\
\hline 41 & $12 / 01 / 93$ \\
55 & $12 / 01 / 93$ \\
83 & $12 / 01 / 93$ \\
46 & $12 / 01 / 93$ \\
19 & $12 / 01 / 93$ \\
46 & $12 / 01 / 93$ \\
32 & $12 / 01 / 93$ \\
15 & $12 / 01 / 93$ \\
380 & $12 / 01 / 93$ \\
45 & $12 / 01 / 93$ \\
38 & $12 / 01 / 93$ \\
53 & $12 / 01 / 93$ \\
41 &
\end{tabular}

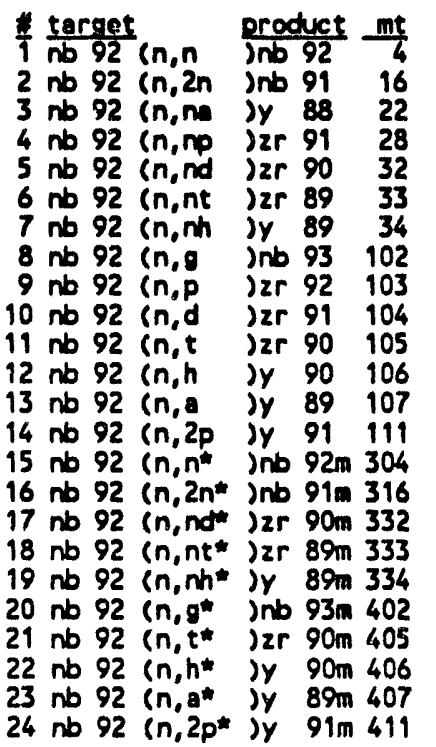

\begin{tabular}{|c|c|}
\hline $\begin{array}{c}\text { engeries } \\
1 \\
26 \\
50 \\
37 \\
24 \\
10 \\
12 \\
394 \\
17 \\
56 \\
51 \\
52 \\
76 \\
45 \\
48 \\
15 \\
16 \\
8 \\
13 \\
394 \\
42 \\
50 \\
76 \\
43\end{array}$ & $\begin{array}{l}\text { date } \\
12 / 01 / 93 \\
12 / 01 / 93 \\
12 / 01 / 93 \\
12 / 01 / 93 \\
12 / 01 / 93 \\
12 / 01 / 93 \\
12 / 01 / 93 \\
12 / 01 / 93 \\
12 / 01 / 93 \\
12 / 01 / 93 \\
12 / 01 / 93 \\
12 / 01 / 93 \\
12 / 01 / 93 \\
12 / 01 / 93 \\
12 / 01 / 93 \\
12 / 01 / 93 \\
12 / 01 / 93 \\
12 / 01 / 93 \\
12 / 01 / 93 \\
12 / 01 / 93 \\
12 / 01 / 93 \\
12 / 01 / 93 \\
12 / 01 / 93 \\
12 / 01 / 93\end{array}$ \\
\hline $\begin{array}{r}48 \\
21 \\
50 \\
34 \\
22 \\
10 \\
12 \\
394 \\
17 \\
56 \\
51 \\
52 \\
76 \\
45 \\
15 \\
16 \\
8 \\
13 \\
394 \\
40 \\
50 \\
76 \\
43\end{array}$ & $\begin{array}{l}12 / 01 / 93 \\
12 / 01 / 93 \\
12 / 01 / 93 \\
12 / 01 / 93 \\
12 / 01 / 93 \\
12 / 01 / 93 \\
12 / 01 / 93 \\
12 / 01 / 93 \\
12 / 01 / 93 \\
12 / 01 / 93 \\
12 / 01 / 93 \\
12 / 01 / 93 \\
12 / 01 / 93 \\
12 / 01 / 93 \\
12 / 01 / 93 \\
12 / 01 / 93 \\
12 / 01 / 93 \\
12 / 01 / 93 \\
12 / 01 / 93 \\
12 / 01 / 93 \\
12 / 01 / 93 \\
12 / 01 / 93 \\
12 / 01 / 93\end{array}$ \\
\hline
\end{tabular}

1 nb $92 m(n, n$ )nb 924 2 nb $92 m(n, 2 n$ )nb 9116 3 nb $92 m(n, n a$ )y 8822 4 nb $92 m(n, n p$ ) $2 r 91 \quad 28$ $5 \mathrm{nb} 92 \mathrm{~m}(\mathrm{n}$, nd ) $2 \mathrm{r} 90 \quad 32$ $6 \mathrm{nb} 92 m(n, n t) 2 r 89 \quad 33$ 7 no $92 \mathrm{~m}(\mathrm{n}, \mathrm{nh}$ )y 8934

8 nb $92 m(n, g)$ ) 93102

$9 \mathrm{nb} 92 \mathrm{~m}(\mathrm{n}, \mathrm{p}$ ) $2 \mathrm{r} 92103$

$10 \mathrm{nb} 92 \mathrm{~m}(\mathrm{n}, \mathrm{d}$ ) $2 \mathrm{r} 91104$

$11 \mathrm{nb} 92 \mathrm{~m}(\mathrm{n}, \mathrm{t}) 2 \mathrm{r} 90105$

$12 \mathrm{nb} 92 \mathrm{~m}(\mathrm{n}, \mathrm{h}$ )y 90106 $13 \mathrm{nb} 92 \mathrm{~m}(\mathrm{n}, \mathrm{a}$ iy 89107 $14 \mathrm{nb} 92 \mathrm{~m}(\mathrm{n}, 2 \mathrm{p}$ )y 91111

$15 \mathrm{nb} 92 \mathrm{~m}(\mathrm{n}, 2 \mathrm{n}$ ) $) \mathrm{nb} 91 \mathrm{~m} 316$

$16 \mathrm{nb} 92 \mathrm{~m}\left(\mathrm{n}, \mathrm{nd}^{\mathrm{t}}\right) \mathrm{zr} 90 \mathrm{~m} 332$

$17 \mathrm{nb} 92 \mathrm{~m}(\mathrm{n}, \mathrm{nt}$ *) $2 \mathrm{r} 89 \mathrm{~m} 333$

$18 \mathrm{nb} 92 \mathrm{~m}(\mathrm{n}, \mathrm{nm} *$ )y $89 \mathrm{~m} 334$

$19 \mathrm{nb} 92 \mathrm{~m}\left(\mathrm{n}, \mathrm{g}^{*}\right.$ ) $\mathrm{nb} 93 \mathrm{~m} 402$

20 nb $92 m(n, t *) 2 r 90 m 405$

$21 \mathrm{nb} 92 \mathrm{~m}(\mathrm{n}, \mathrm{h} *$ )y 90m 406

22 nb $92 \mathrm{~m}\left(n, a^{*}\right.$ )y $89 \mathrm{~m} 407$

$23 \mathrm{nb} 92 \mathrm{~m}\left(n, 2 p^{*}\right.$ )y $91 \mathrm{~m} 411$

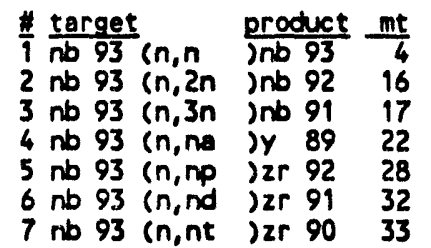

\begin{tabular}{c} 
Engeries \\
\hline 1 \\
18 \\
10 \\
8 \\
40 \\
18 \\
17
\end{tabular}

$\frac{\text { date }}{12 / 01 / 93}$
$12 / 01 / 93$
$12 / 01 / 93$
$12 / 01 / 93$
$12 / 01 / 93$
$12 / 01 / 93$
$12 / 01 / 93$

$A-4.4$ 


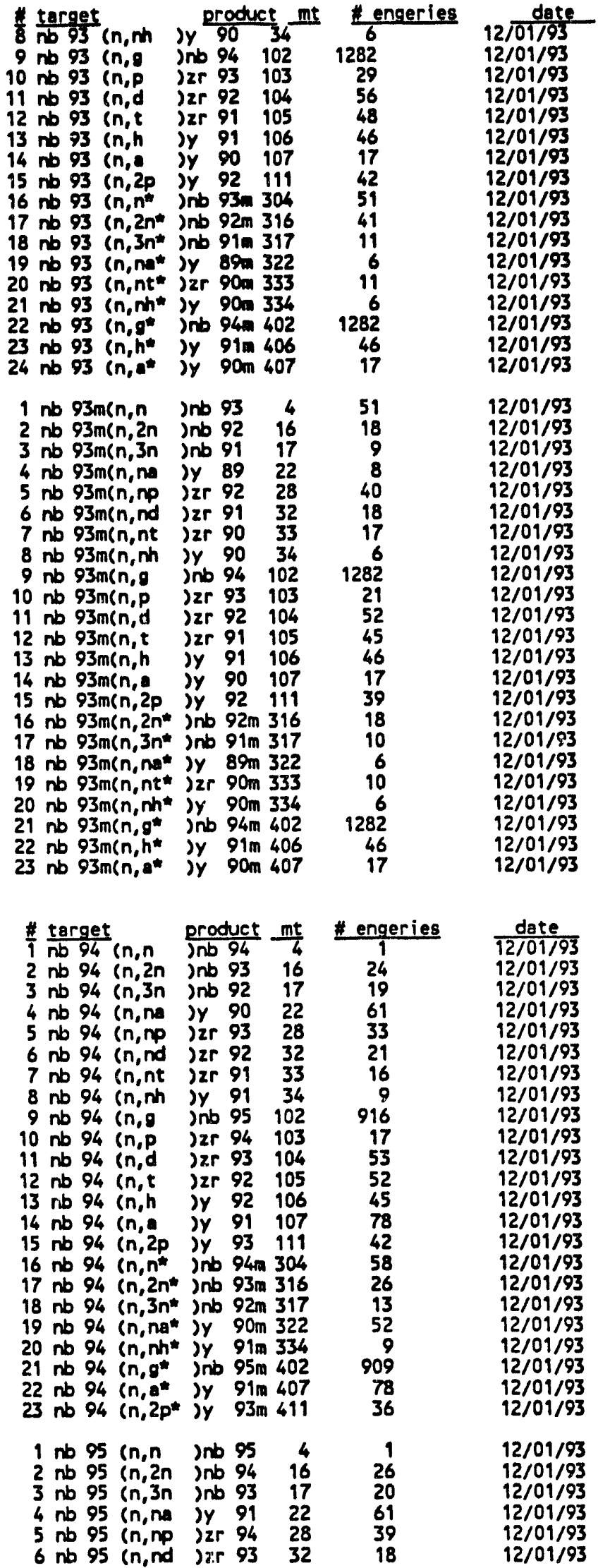




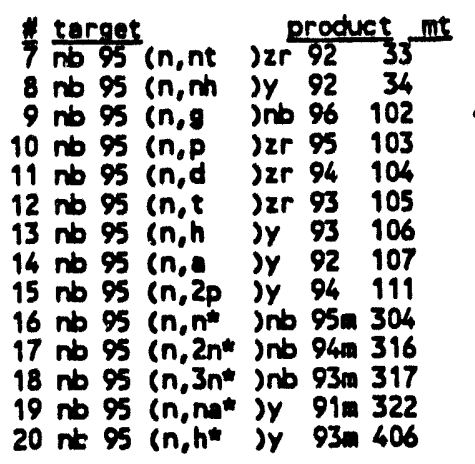

4 engeries
19
4
4094
76
50
45
41
83
33
49
26
16
57
41

$\frac{\text { date }}{12 / 01 / 93}$
$12 / 01 / 93$
$12 / 01 / 93$
$12 / 01 / 93$
$12 / 01 / 93$
$12 / 01 / 93$
$12 / 01 / 93$
$12 / 01 / 93$
$12 / 01 / 93$
$12 / 01 / 93$
$12 / 01 / 93$
$12 / 01 / 93$
$12 / 01 / 93$
$12 / 01 / 93$

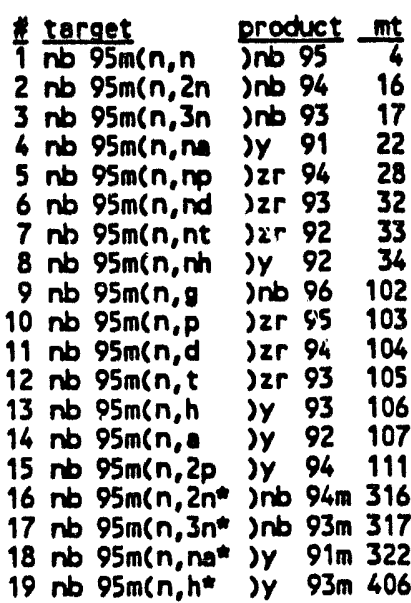

\begin{tabular}{c}
\hline engeries \\
\hline 49 \\
26 \\
16 \\
60 \\
36 \\
16 \\
17 \\
4 \\
4094 \\
67 \\
50 \\
45 \\
41 \\
83 \\
33 \\
26 \\
16 \\
55 \\
39
\end{tabular}

date
$\frac{12 / 01 / 93}{12 / 01 / 93}$
$12 / 01 / 93$
$12 / 01 / 93$
$12 / 01 / 93$
$12 / 01 / 93$
$12 / 01 / 93$
$12 / 01 / 93$
$12 / 01 / 93$
$12 / 01 / 93$
$12 / 01 / 93$
$12 / 01 / 93$
$12 / 01 / 93$
$12 / 01 / 93$
$12 / 01 / 93$
$12 / 01 / 93$
$12 / 01 / 93$
$12 / 01 / 93$
$12 / 01 / 93$

1 mo $92(n, 2 n)$ mo 9916

2 mo 92 ( $n, n$ ) $2 r$ s8 22

3 mo 92 ( $n, n p$ ) nb 9128

$4 \mathrm{mo} 92$ (n,nd) $n \mathrm{~b} 9032$

5 mo 92 ( $n, n h$ ) $2 r 89 \quad 34$

6 mo $92(n, g)$ mo 93102

7 mo 92 (n,p )nb 92103

8 mo 92 (n,d inb 91904

9 mo 92 (n,t )nb 90105

$10 \mathrm{mo} 92(\mathrm{n}, \mathrm{h}$ ) $2 \mathrm{r} 90106$

11 mo 92 (n,a) zer 89107

12 mo 92 ( $n, 2 p)$ zr 91111

$13 \mathrm{mo} 92\left(\mathrm{n}, 2 \mathrm{n}^{*}\right) \mathrm{mo} 91 \mathrm{~m} 316$

14 mo 92 ( $n, n n^{*}$ ) nb 91 m 328

15 mo 92 ( $n, n c^{m}$ )nb 90m 332

16 mo 92 ( $\left.n, n n^{*}\right) 2 r 89 m 334$

17 mo $92\left(n, g^{*}\right)$ mo $93 m 402$

18 mo 92 (n, p* inb $92 m 403$

19 mo 92 (n, d* ) nb $91 m 404$

20 mo 92 ( $n, t^{*}$ job $90 \mathrm{~m} 405$

21 mo 92 (n,h*) 2r 90m 406

22 mo $92(n, 4 *)$ ) 2 r 8907

$\begin{array}{rr}9 & 12 / 01 / 93 \\ 58 & 12 / 01 / 93 \\ 50 & 12 / 01 / 93 \\ 12 & 12 / 01 / 93 \\ 2 & 12 / 01 / 93 \\ 4379 & 12 / 01 / 93 \\ 23 & 12 / 01 / 93 \\ 15 & 12 / 01 / 93 \\ 36 & 12 / 01 / 93 \\ 66 & 12 / 01 / 93 \\ 15 & 12 / 01 / 93 \\ 59 & 12 / 01 / 93 \\ 8 & 12 / 01 / 93 \\ 15 & 12 / 01 / 93 \\ 10 & 12 / 01 / 93 \\ 2 & 12 / 01 / 93 \\ 4379 & 12 / 01 / 93 \\ 23 & 12 / 01 / 93 \\ 14 & 12 / 01 / 93 \\ 36 & 12 / 01 / 93 \\ 52 & 12 / 01 / 93 \\ 15 & 12 / 01 / 93\end{array}$

\begin{tabular}{|c|c|c|c|}
\hline $\begin{array}{l}\frac{\#}{1} \frac{\text { target }}{\text { mo } 93} \text { (n,n } \\
2 \text { mo } 93 \text { (n, 2n } \\
33 \text { mo } 93 \text { (n,na } \\
4 \text { mo } 93 \text { (n,np } \\
5 \text { mo } 93 \text { (n,nd } \\
6 \text { mo } 93 \text { in,nt } \\
7 \text { mo } 93 \text { in,nh } \\
8 \text { mo } 93 \text { in, } \\
9 \text { mo } 93 \text { (n,p } \\
10 \text { mo } 93 \text { (n,d } \\
11 \text { mo } 93 \text { (n,t } \\
12 \text { mo } 93 \text { (n,h }\end{array}$ & $\begin{array}{l}\text { product } \\
\text { imo } 93 \\
\text { jmo } 92 \\
\text { jzr } 89 \\
\text { inb } 92 \\
\text { inb } 91 \\
\text { inb } 90 \\
\text { jer } 90 \\
\text { jmo } 94 \\
\text { jnb } 93 \\
\text { inb } 92 \\
\text { inb } 99 \\
\text { izr } 91\end{array}$ & $\begin{array}{r}\frac{m t}{4} \\
16 \\
22 \\
28 \\
32 \\
33 \\
34 \\
102 \\
103 \\
104 \\
105 \\
106\end{array}$ & $\begin{array}{c}\text { \# engeries } \\
1 \\
19 \\
52 \\
31 \\
17 \\
5 \\
20 \\
379 \\
23 \\
47 \\
44 \\
53\end{array}$ \\
\hline
\end{tabular}

$A-4.6$ 


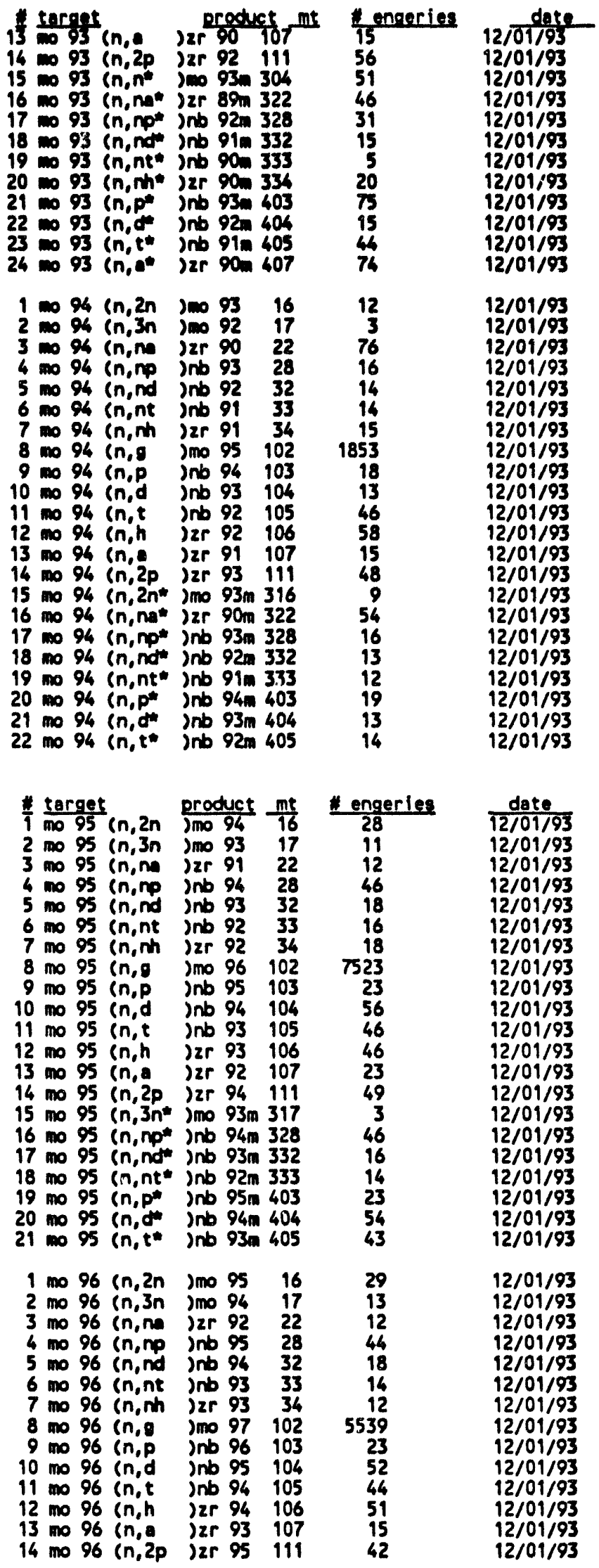

$$
A-4.7
$$


it thert

15 mo os (n, np* onb $95 \mathrm{sm} 328$

16 mo \% (n, ndw inb $94 m 332$

17 mo $\%$ en, nt* inb 93 m 333

18 mo 96 (n,d inb 95 m 404

19 no 96 in,t inb 94 m 405

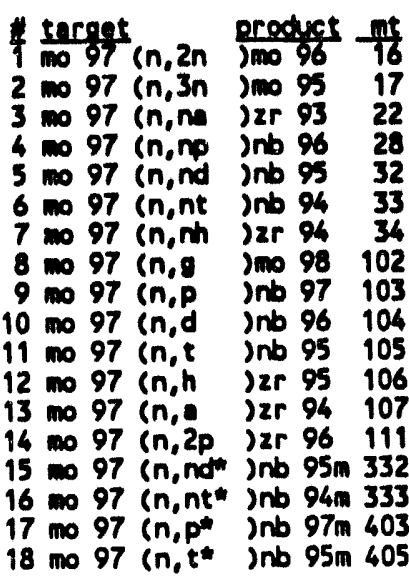

1 mo $98(n, 2 n)$ mo $97 \quad 16$

2 mo 98 (n, 3n )mo $96 \quad 17$

3 mo 98 (n,m )2r $94 \quad 22$

4 mo 98 ( $n, n$ inb 9728

5 mo 98 (n,nd inb 9632

6 mo 98 (n,nt inb 9533

7 mo 98 (n.) ino 99102

8 mo 98 (n,p inb 98103

9 mo 98 (n,d )nb 97104

10 mo 98 (n.t )nb 96105

11 mo $98 \mathrm{kn,h}$ )2r 96106

12 mo 98 (n,a jer 95107

13 mo 98 (n,2p )zr 97111

14 mo 98 (n,np*) nb $97 \mathrm{~m} 328$

15 mo 98 (n, nt*) )nb $95 \mathrm{~m} 333$

$16 \mathrm{mo} 98 \mathrm{cn} \mathrm{p*}^{*}$ ) nb $98 \mathrm{~m} 403$

17 mo 98 (n, d ) inb $97 \mathrm{~m} 404$

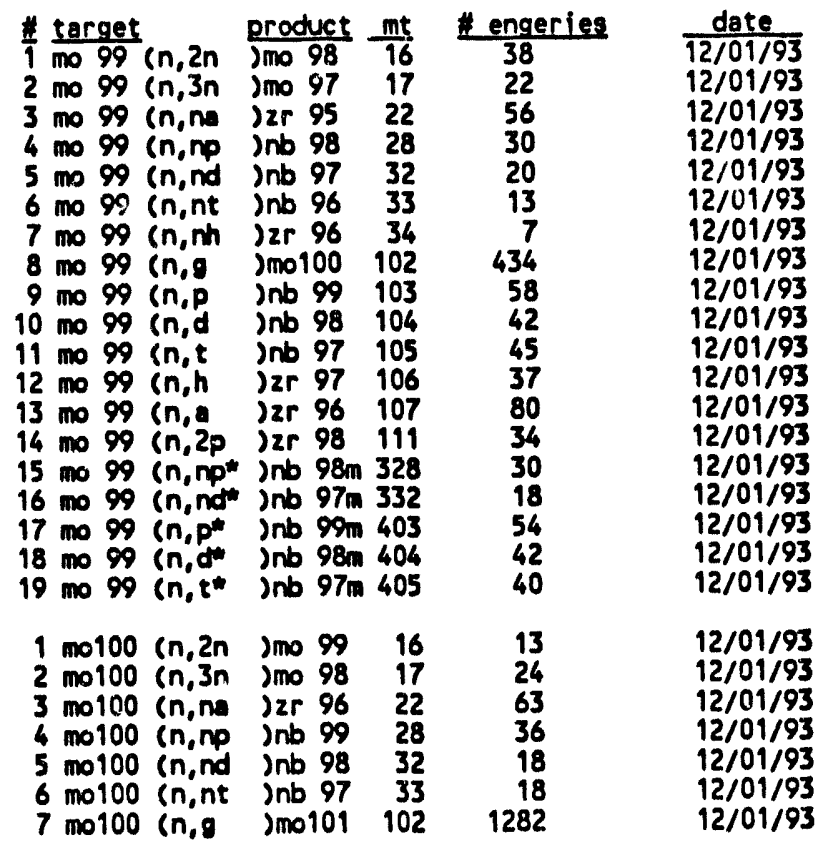

$A-4.8$

$12 /$ dits

$12 / 01 / 93$

$12 / 01 / 93$

$12 / 01 / 93$

$12 / 01 / 93$

$\begin{array}{cc}\text { engeries } & \text { dote } \\ 31 & 12 / 01 / 93 \\ 15 & 12 / 01 / 93 \\ 70 & 12 / 01 / 93 \\ 44 & 12 / 01 / 93 \\ 24 & 12 / 01 / 93 \\ 16 & 12 / 01 / 93 \\ 15 & 12 / 01 / 93 \\ 9086 & 12 / 01 / 93 \\ 22 & 12 / 01 / 93 \\ 52 & 12 / 01 / 93 \\ 50 & 12 / 01 / 93 \\ 40 & 12 / 01 / 93 \\ 15 & 12 / 01 / 93 \\ 45 & 12 / 01 / 93 \\ 24 & 12 / 01 / 93 \\ 16 & 12 / 01 / 93 \\ 23 & 12 / 01 / 93 \\ 50 & 12 / 01 / 93\end{array}$

$12 / 01 / 93$

$12 / 01 / 93$

$12 / 01 / 93$

$12 / 01 / 93$

$12 / 01 / 93$

$12 / 01 / 93$

$12 / 01 / 93$

$12 / 01 / 93$

$12 / 01 / 93$

$12 / 01 / 93$

$12 / 01 / 93$

$12 / 01 / 93$

$12 / 01 / 93$

$12 / 01 / 93$

$12 / 01 / 93$

$12 / 01 / 93$

date

2/01/93

2/01/93

$2 / 01 / 93$

$12 / 01 / 93$

$12 / 01 / 93$

12/01/93

2/01/93

(19193

$12 / 01 / 93$

101/93

$12 / 01 / 93$

12/01/93

12/01/93

$12 / 01 / 93$

$12 / 01 / 93$

$12 / 01 / 93$
$12 / 01 / 93$

2/01/93 


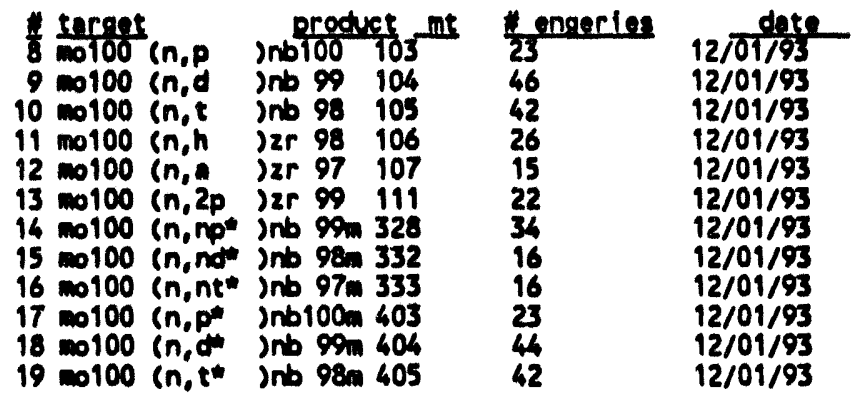

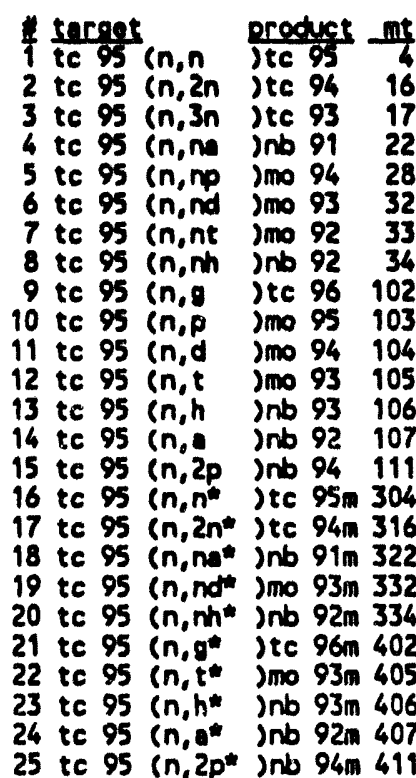

\begin{tabular}{cc} 
nengeries & date \\
\cline { 2 - 2 } $12 / 01 / 93$ \\
22 & $12 / 01 / 93$ \\
9 & $12 / 01 / 93$ \\
71 & $12 / 01 / 93$ \\
46 & $12 / 01 / 93$ \\
19 & $12 / 01 / 93$ \\
15 & $12 / 01 / 93$ \\
9 & $12 / 01 / 93$ \\
401 & $12 / 01 / 93$ \\
73 & $12 / 01 / 93$ \\
63 & $12 / 01 / 93$ \\
48 & $12 / 01 / 93$ \\
53 & $12 / 01 / 93$ \\
79 & $12 / 01 / 93$ \\
48 & $12 / 01 / 93$ \\
41 & $12 / 01 / 93$ \\
22 & $12 / 01 / 93$ \\
70 & $12 / 01 / 93$ \\
13 & $12 / 01 / 93$ \\
9 & $12 / 01 / 93$ \\
400 & $12 / 01 / 93$ \\
37 & $12 / 01 / 93$ \\
53 & $12 / 01 / 93$ \\
79 & $12 / 01 / 93$ \\
48 & $12 / 01 / 93$
\end{tabular}

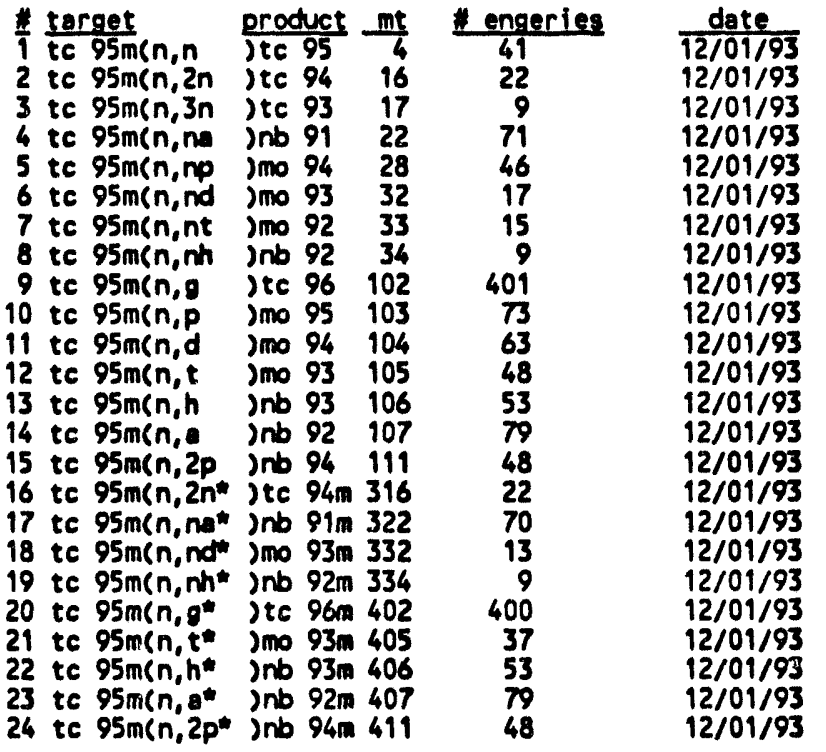




\begin{tabular}{|c|c|}
\hline $\begin{array}{l}4 \text { terget } \\
4 \text { tc } 96 \text { (n, 2n } \\
2 \text { tc } 96 \text { (n, 3n } \\
3 \text { tc } 96 \text { (n,n } \\
4 \text { tc } \% \text { (n, no } \\
5 \text { tc } 96 \text { in,nd } \\
6 \text { tc } 96 \text { in,nt } \\
7 \text { tc } 96 \text { in,nh }\end{array}$ & $\begin{array}{l}\text { prod } \\
\text { jte } 95 \\
\text { jte } 94 \\
\text { inb } 92 \\
\text { jmo } 95 \\
\text { ino } 94 \\
\text { jom } 93 \\
\text { jo } 93\end{array}$ \\
\hline
\end{tabular}

\begin{tabular}{ll} 
Hengeries & date \\
\hline 29 & $12 / 01 / 93$ \\
13 & $12 / 01 / 93$ \\
68 & $12 / 01 / 93$ \\
39 & $12 / 01 / 93$ \\
27 & $12 / 01 / 93$ \\
15 & $12 / 01 / 93$ \\
15 & $12 / 01 / 93$
\end{tabular}

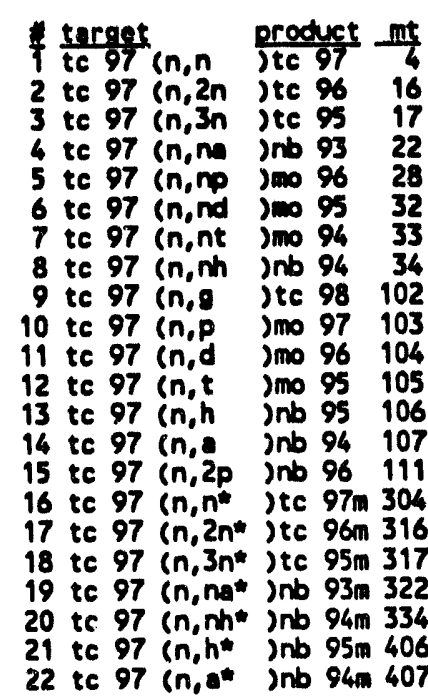

\begin{tabular}{cc} 
cengerles & date \\
\cline { 2 - 2 } $12 / 01 / 93$ \\
26 & $12 / 01 / 93$ \\
13 & $12 / 01 / 93$ \\
64 & $12 / 01 / 93$ \\
41 & $12 / 01 / 93$ \\
18 & $12 / 01 / 93$ \\
16 & $12 / 01 / 93$ \\
6 & $12 / 01 / 93$ \\
402 & $12 / 01 / 93$ \\
75 & $12 / 01 / 93$ \\
56 & $12 / 01 / 93$ \\
48 & $12 / 01 / 93$ \\
46 & $12 / 01 / 93$ \\
81 & $12 / 01 / 93$ \\
44 & $12 / 01 / 93$ \\
42 & $12 / 01 / 93$ \\
24 & $12 / 01 / 93$ \\
13 & $12 / 01 / 93$ \\
60 & $12 / 01 / 93$ \\
6 & $12 / 01 / 93$ \\
46 & $12 / 01 / 93$ \\
81 & $12 / 01 / 93$
\end{tabular}

1 tc $97 m(n, n)$ tc 97

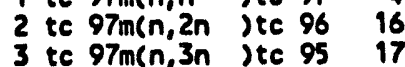

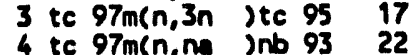
5 tc $97 m(n, n p$ ino 9628 6 tc $97 \mathrm{~m}(\mathrm{n}, \mathrm{nd}) \mathrm{mo} 9532$ 7 tc $97 m(n, n t)$ mo $94 \quad 33$ 8 tc $97 m(n, n h) n b 94 \quad 34$ 9 tc $97 \mathrm{~m}(\mathrm{n}, \mathrm{g}$ )tc 98102 10 tc $97 \mathrm{~m}(\mathrm{n}, \mathrm{p})$ mo $97 \quad 103$ 11 te $97 \mathrm{~m}(\mathrm{n}, \mathrm{d})$ mo 96104 12 tc $97 \mathrm{~m}(\mathrm{n}, \mathrm{t}) \mathrm{mo} 95105$ 13 tc $97 \mathrm{~m}(\mathrm{n}, \mathrm{h}$ ) $\mathrm{nb} 95106$ 14 tc $97 \mathrm{~m}(\mathrm{n}, \mathrm{s}$ inb 94107 15 tc $97 \mathrm{~m}(\mathrm{n}, 2 \mathrm{p}$ ) $n \mathrm{~b} 96111$ 16 tc $97 \mathrm{~m}(\mathrm{n}, 2 \mathrm{n}$ ) )tc $96 \mathrm{~m} 316$ 17 tc $97 \mathrm{~m}\left(\mathrm{n}, 3 \mathrm{n}^{\star}\right)$ tc $95 \mathrm{~m} 317$ 18 te $97 \mathrm{~m}(\mathrm{n}, \mathrm{ng}$ ) $\mathrm{nb} 93 \mathrm{~m} 322$ 19 tc $97 \mathrm{~m}(n, n)$ ) $n b 94 \mathrm{~m} 334$ 20 te $97 \mathrm{~m}(\mathrm{n}, \mathrm{h}$ ) $\mathrm{nb} 95 \mathrm{~m} 406$ 21 tc $97 \mathrm{~m}(n, a *)$ ) $b 94 \mathrm{~m} 407$

$\begin{array}{rr}42 & 12 / 01 / 93 \\ 24 & 12 / 01 / 93 \\ 13 & 12 / 01 / 93 \\ 60 & 12 / 01 / 93 \\ 41 & 12 / 01 / 93 \\ 18 & 12 / 01 / 93 \\ 16 & 12 / 01 / 93 \\ 6 & 12 / 01 / 93 \\ 402 & 12 / 01 / 93 \\ 75 & 12 / 01 / 93 \\ 56 & 12 / 01193 \\ 45 & 12 / 01 / 93 \\ 46 & 12 / 01 / 93 \\ 81 & 12 / 01 / 93 \\ 44 & 12 / 01 / 93 \\ 24 & 12 / 01193 \\ 13 & 12 / 01 / 93 \\ 60 & 12 / 01 / 93 \\ 6 & 12 / 01 / 93 \\ 46 & 12 / 01 / 93 \\ 81 & 12 / 01 / 93\end{array}$

\begin{tabular}{|c|c|c|c|}
\hline 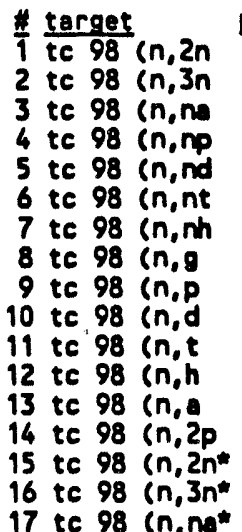 & 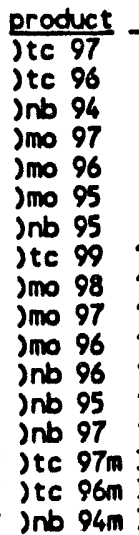 & $\begin{array}{c}\frac{m t}{16} \\
17 \\
22 \\
28 \\
32 \\
33 \\
34 \\
102 \\
103 \\
104 \\
105 \\
106 \\
107 \\
111\end{array}$ & $\begin{array}{c}\text { engeries } \\
28 \\
18 \\
61 \\
36 \\
28 \\
19 \\
8 \\
600 \\
72 \\
57 \\
52 \\
45 \\
79 \\
45 \\
28 \\
18 \\
61\end{array}$ \\
\hline
\end{tabular}

$$
A-4.10
$$




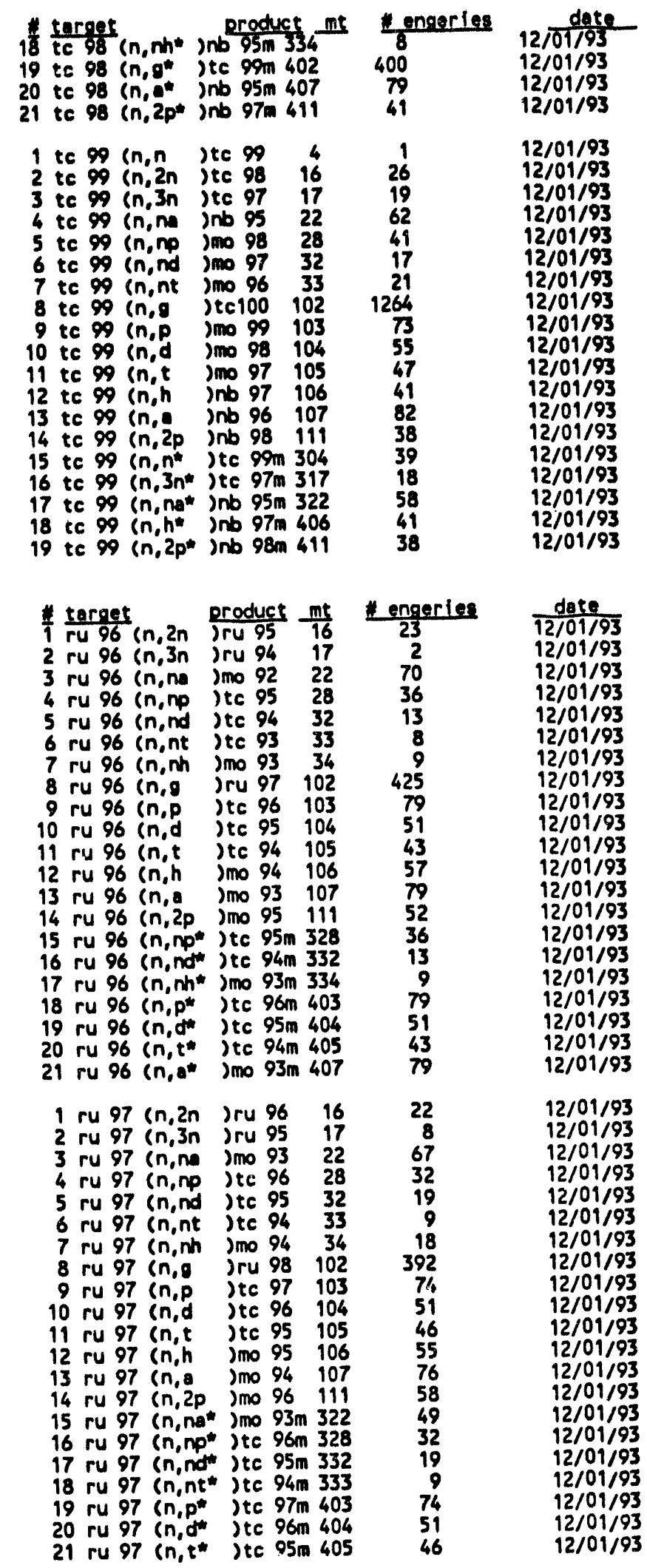

$A-4.11$ 


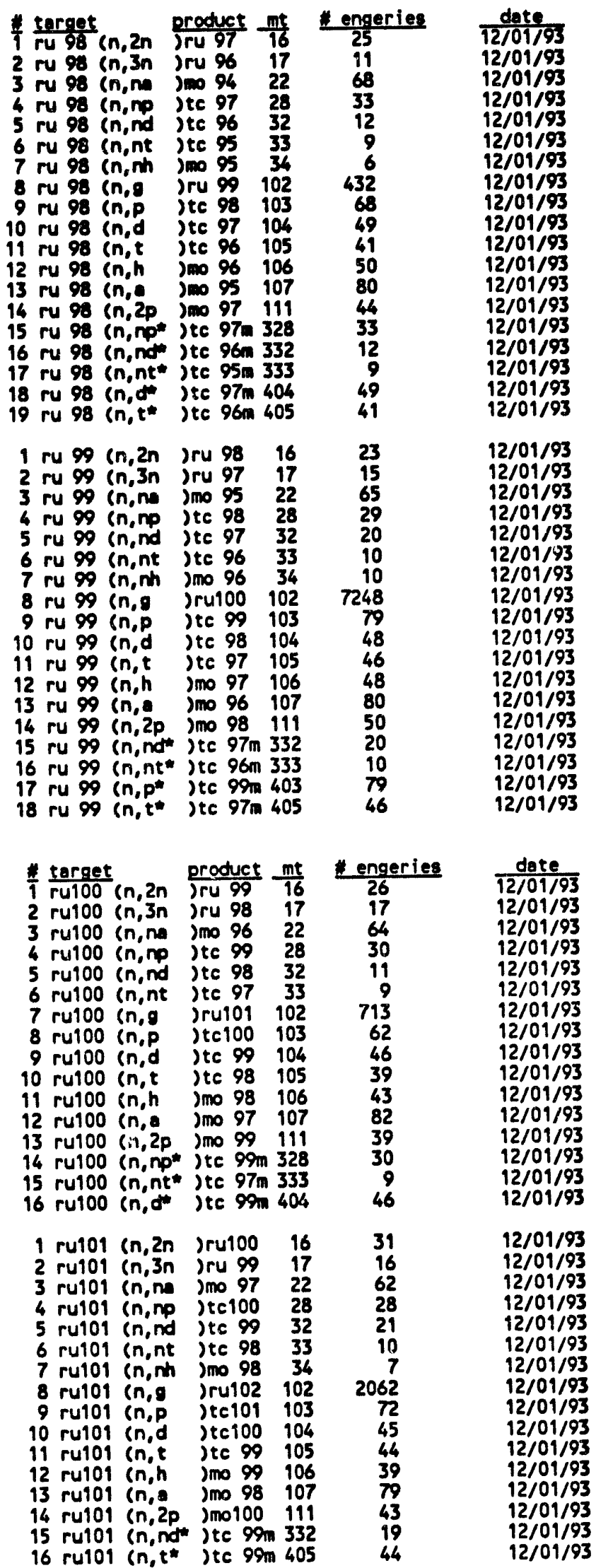

$A-4.12$ 


\begin{tabular}{|c|c|c|c|c|c|c|}
\hline & $\begin{array}{l}\text { target } \\
\text { ru102 } \\
\text { ru102 s } \\
\text { ru102 } \\
\text { ru102 } \\
\text { ru102 } \\
\text { ru102 } \\
\text { ru102 } \\
\text { ru102 } \\
\text { ru102 } \\
\text { ru102 } \\
\text { ru102 } \\
\text { ru102 } \\
\text { ru102 } \\
\text { ru102 } \\
\text { ru102 }\end{array}$ & 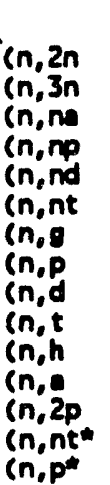 & $\begin{array}{l}\text { produc } \\
\text { jrui101 } \\
\text { jru100 } \\
\text { jmo } 98 \\
\text { jtc101 } \\
\text { jtc100 } \\
\text { jtc } 99 \\
\text { jru103 } \\
\text { jtc102 } \\
\text { jtc101 } \\
\text { jtc100 } \\
\text { jmo100 } \\
\text { jmo } 99 \\
\text { jmo101 } \\
\text { jtc } 99 \mathrm{~m} \\
\text { jtc102m }\end{array}$ & $\begin{array}{l}c t \\
16 \\
17 \\
22 \\
28 \\
32 \\
33 \\
102 \\
103 \\
104 \\
105 \\
106 \\
107 \\
111 \\
333 \\
403\end{array}$ & $\begin{array}{l}\text { \# engeries } \\
29 \\
17 \\
61 \\
28 \\
11 \\
10 \\
1317 \\
53 \\
44 \\
38 \\
35 \\
84 \\
31 \\
8 \\
50\end{array}$ & $\begin{array}{l}\frac{\text { date }}{12 / 01 / 93} \\
12 / 01 / 93 \\
12 / 01 / 93 \\
12 / 01 / 93 \\
12 / 01 / 93 \\
12 / 01 / 93 \\
12 / 01 / 93 \\
12 / 01 / 93 \\
12 / 01 / 93 \\
12 / 01 / 93 \\
12 / 01 / 93 \\
12 / 01 / 93 \\
12 / 01 / 93 \\
12 / 01 / 93 \\
12 / 01 / 93\end{array}$ \\
\hline 10 & $\begin{array}{l}\text { target } \\
\text { ru103 } \\
\text { ru103 } \\
\text { ru103 } \\
\text { ru103 } \\
\text { ru103 } \\
\text { ru103 } \\
\text { ru103 } \\
\text { ru103 } \\
\text { ru103 } \\
\text { ru103 } \\
\text { ru103 } \\
\text { ru103 } \\
\text { ru103 } \\
\text { ru103 } \\
\text { ru103 }\end{array}$ & 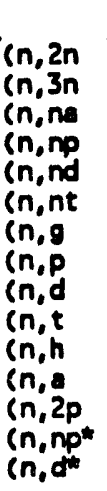 & $\begin{array}{l}\text { product } \\
\text { Jrul02 } \\
\text { jru101 } \\
\text { jmo } 99 \\
\text { jtc102 } \\
\text { jtc101 } \\
\text { jtc100 } \\
\text { jru104 } \\
\text { jtc103 } \\
\text { jtc102 } \\
\text { jtc101 } \\
\text { jmo101 } \\
\text { jmo100 } \\
\text { jmo102 } \\
\text { jtc102m } \\
\text { jtc102m }\end{array}$ & $\begin{array}{l}\frac{m t}{16} \\
17 \\
22 \\
28 \\
32 \\
33 \\
102 \\
103 \\
104 \\
105 \\
106 \\
107 \\
111 \\
328 \\
404\end{array}$ & $\begin{array}{c}\text { Eengeries } \\
31 \\
18 \\
53 \\
23 \\
20 \\
11 \\
1950 \\
65 \\
42 \\
44 \\
31 \\
79 \\
37 \\
23 \\
40\end{array}$ & $\begin{array}{c}\text { date } \\
\frac{12 / 01 / 93}{12 / 01 / 93} \\
12 / 01 / 93 \\
12 / 01 / 93 \\
12 / 01 / 93 \\
12 / 01 / 93 \\
12 / 01 / 93 \\
12 / 01 / 93 \\
12 / 01 / 93 \\
12 / 01 / 93 \\
12 / 01 / 93 \\
12 / 01 / 93 \\
12 / 01 / 93 \\
12 / 01 / 93 \\
12 / 01 / 93\end{array}$ \\
\hline & $\begin{array}{l}\text { ru104 } \\
\text { ru104 } \\
\text { ru104 } \\
\text { ru104 } \\
\text { ru104 } \\
\text { ru104 } \\
\text { ru104 } \\
\text { ru104 } \\
\text { ru104 } \\
\text { ru104 } \\
\text { ru104 } \\
\text { ru104 } \\
\text { ru104 } \\
\text { ru104 } \\
\text { ru104 }\end{array}$ & 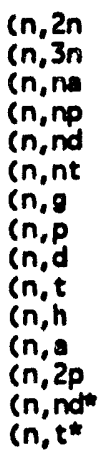 & $\begin{array}{l}\text { Jru103 } \\
\text { jru102 } \\
\text { jmo100 } \\
\text { jtc103 } \\
\text { jtc102 } \\
\text { jtc101 } \\
\text { jru105 } \\
\text { jtc104 } \\
\text { jtc103 } \\
\text { jtc102 } \\
\text { jmo102 } \\
\text { jmo101 } \\
\text { jmo103 } \\
\text { jtc102m } \\
\text { jtc102m }\end{array}$ & $\begin{array}{l}16 \\
17 \\
22 \\
28 \\
32 \\
33 \\
102 \\
103 \\
104 \\
105 \\
106 \\
107 \\
111 \\
332 \\
405\end{array}$ & $\begin{array}{r}34 \\
21 \\
58 \\
27 \\
10 \\
10 \\
2948 \\
49 \\
44 \\
36 \\
29 \\
79 \\
25 \\
8 \\
35\end{array}$ & $\begin{array}{l}12 / 01 / 93 \\
12 / 01 / 93 \\
12 / 01 / 93 \\
12 / 01 / 93 \\
12 / 01 / 93 \\
12 / 01 / 93 \\
12 / 01 / 93 \\
12 / 01 / 93 \\
12 / 01 / 93 \\
12 / 01 / 93 \\
12 / 01 / 93 \\
12 / 01 / 93 \\
12 / 01 / 93 \\
12 / 01 / 93 \\
12 / 01 / 93\end{array}$ \\
\hline $\begin{array}{l}12 \\
13 \\
14\end{array}$ & $\begin{array}{l}\text { ru105 } \\
\text { ru105 } \\
\text { ru105 } \\
\text { ru105 } \\
\text { ru105 } \\
\text { ru105 } \\
\text { ru105 } \\
\text { ru105 } \\
\text { ru105 } \\
\text { ru105 } \\
\text { ru105 } \\
\text { ru105 } \\
\text { ru105 } \\
\text { ru105 }\end{array}$ & 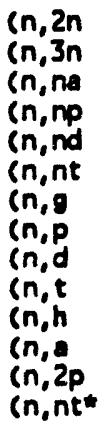 & $\begin{array}{l}\text { Jru104 } \\
\text { Jru103 } \\
\text { Jmo101 } \\
\text { jtc104 } \\
\text { jtc103 } \\
\text { jtc102 } \\
\text { jru106 } \\
\text { jtc105 } \\
\text { jtc104 } \\
\text { jtc103 } \\
\text { Jmo103 } \\
\text { Jmo102 } \\
\text { Jmo104 } \\
\text { Jtc102m }\end{array}$ & $\begin{array}{l}16 \\
17 \\
22 \\
28 \\
32 \\
33 \\
102 \\
103 \\
104 \\
105 \\
106 \\
107 \\
111 \\
333\end{array}$ & $\begin{array}{r}36 \\
19 \\
51 \\
21 \\
20 \\
11 \\
335 \\
61 \\
42 \\
44 \\
25 \\
83 \\
31 \\
9\end{array}$ & $\begin{array}{l}12 / 01 / 93 \\
12 / 01 / 93 \\
12 / 01 / 93 \\
12 / 01 / 93 \\
12 / 01 / 93 \\
12 / 01 / 93 \\
12 / 01 / 93 \\
12 / 01 / 93 \\
12 / 01 / 93 \\
12 / 01 / 93 \\
12 / 01 / 93 \\
12 / 01 / 93 \\
12 / 01 / 93 \\
12 / 01 / 93\end{array}$ \\
\hline
\end{tabular}


WHC-EP-0727

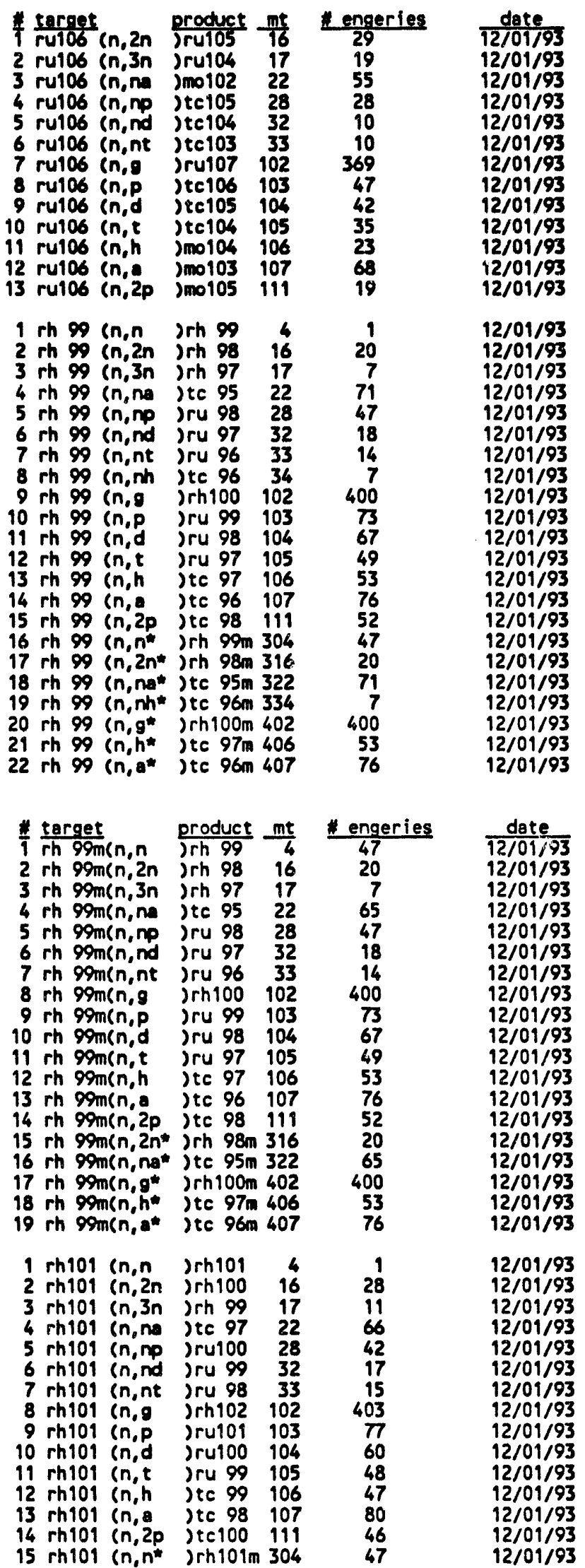

$A-4.14$ 


\begin{tabular}{|c|c|c|c|c|c|}
\hline & $\begin{array}{l}\frac{\text { target }}{\text { rh101 }}\left(n, 2 n^{*}\right. \\
\text { rh101 }\left(n, 3 n^{*}\right. \\
\text { rh101 (n, na* } \\
\text { rh101 (n, } g^{*} \\
\text { rh101 }\left(n, h^{*}\right.\end{array}$ & $\begin{array}{l}\text { produc } \\
\text { Orh } 100 \mathrm{~m} \\
\text { Jrh } 99 \mathrm{~m} \\
\text { jtc } 97 \mathrm{~m} \\
\text { jrh } 102 \mathrm{~m} \\
\text { Jtc } 99 \mathrm{~m}\end{array}$ & $\begin{array}{l}\text { ct } \\
316 \\
317 \\
322 \\
402\end{array}$ & $\begin{array}{l}\text { \# enqeri } \\
24 \\
11 \\
65 \\
403 \\
47\end{array}$ & $\begin{array}{l}\text { date } \\
12 / 01 / 93 \\
12 / 01 / 93 \\
12 / 01 / 93 \\
12 / 01 / 93 \\
12 / 01 / 93\end{array}$ \\
\hline $\begin{array}{l}\frac{\#}{1} \\
2 \\
3 \\
4 \\
5 \\
6 \\
7 \\
8 \\
9\end{array}$ & 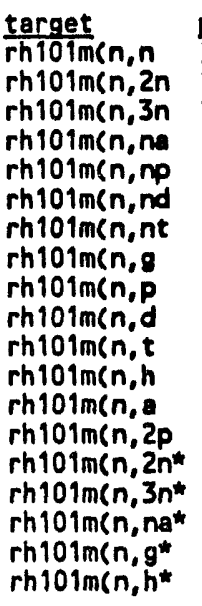 & $\begin{array}{l}\text { product } \\
\text { Orh101 } \\
\text { jrh100 } \\
\text { jrh } 99 \\
\text { jtc } 97 \\
\text { jru100 } \\
\text { jru } 99 \\
\text { jru } 98 \\
\text { jrh102 } \\
\text { jru101 } \\
\text { jru100 } \\
\text { jru } 99 \\
\text { jtc } 99 \\
\text { jtc } 98 \\
\text { jtc100 } \\
\text { jrh100m } \\
\text { jrh } 99 \mathrm{~m} \\
\text { jtc } 97 \mathrm{~m} \\
\text { jrh102m } \\
\text { jtc } 99 \mathrm{~m}\end{array}$ & $\begin{array}{c}m t \\
4 \\
16 \\
17 \\
22 \\
28 \\
32 \\
33 \\
102 \\
103 \\
104 \\
105 \\
106 \\
107 \\
111 \\
316 \\
317 \\
322 \\
402 \\
406\end{array}$ & $\begin{array}{c}\text { \# engeries } \\
50 \\
24 \\
11 \\
66 \\
42 \\
17 \\
15 \\
403 \\
77 \\
60 \\
48 \\
47 \\
80 \\
43 \\
24 \\
11 \\
65 \\
403 \\
47\end{array}$ & $\begin{array}{l}\text { date } \\
12 / 01 / 93 \\
12 / 01 / 93 \\
12 / 01 / 93 \\
12 / 01 / 93 \\
12 / 01 / 93 \\
12 / 01 / 93 \\
12 / 01 / 93 \\
12 / 01 / 93 \\
12 / 01 / 93 \\
12 / 01 / 93 \\
12 / 01 / 93 \\
12 / 01 / 93 \\
12 / 01 / 93 \\
12 / 01 / 93 \\
12 / 01 / 93 \\
12 / 01 / 93 \\
12 / 01 / 93 \\
12 / 01 / 93 \\
12 / 01 / 93\end{array}$ \\
\hline $\begin{array}{r}1 \\
2 \\
3 \\
4 \\
5 \\
6 \\
7 \\
8 \\
9 \\
10 \\
11 \\
12 \\
13 \\
14 \\
15 \\
16 \\
17 \\
18 \\
19\end{array}$ & 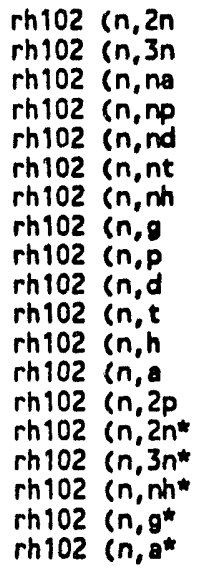 & $\begin{array}{l}\text { Jrh101 } \\
\text { Jrh100 } \\
\text { Jtc } 98 \\
\text { Jru101 } \\
\text { Jru100 } \\
\text { Jru } 99 \\
\text { jtc } 99 \\
\text { Jrh103 } \\
\text { Jru102 } \\
\text { Jru101 } \\
\text { Jru100 } \\
\text { Jtc100 } \\
\text { Jtc } 99 \\
\text { Jtc101 } \\
\text { Jrh101m } \\
\text { Jrh100m } \\
\text { Jtc } 99 \mathrm{~m} \\
\text { Jrh103m } \\
\text { Jtc } 99 \mathrm{~m}\end{array}$ & $\begin{array}{r}16 \\
17 \\
22 \\
28 \\
32 \\
33 \\
34 \\
102 \\
103 \\
104 \\
105 \\
106 \\
107 \\
111 \\
316 \\
317 \\
334 \\
402 \\
407\end{array}$ & $\begin{array}{r}22 \\
15 \\
63 \\
35 \\
29 \\
15 \\
7 \\
399 \\
73 \\
57 \\
55 \\
43 \\
79 \\
46 \\
22 \\
13 \\
7 \\
399 \\
79\end{array}$ & $\begin{array}{l}12 / 01 / 93 \\
12 / 01 / 93 \\
12 / 01 / 93 \\
12 / 01 / 93 \\
12 / 01 / 93 \\
12 / 01 / 93 \\
12 / 01 / 93 \\
12 / 01 / 93 \\
12 / 01 / 93 \\
12 / 01 / 93 \\
12 / 01 / 93 \\
12 / 01 / 93 \\
12 / 01 / 93 \\
12 / 01 / 93 \\
12 / 01 / 93 \\
12 / 01 / 93 \\
12 / 01 / 93 \\
12 / 01 / 93 \\
12 / 01 / 93\end{array}$ \\
\hline $\begin{array}{l}12 \\
13 \\
14 \\
15 \\
16 \\
17 \\
18 \\
19\end{array}$ & 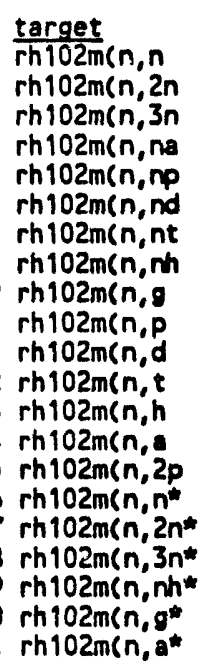 & 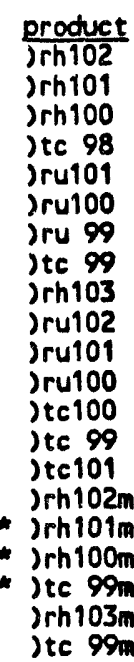 & $\begin{array}{c}m t \\
6 \\
16 \\
17 \\
22 \\
28 \\
32 \\
33 \\
34 \\
102 \\
103 \\
104 \\
105 \\
106 \\
107\end{array}$ & $\begin{array}{c}\text { Aengeries } \\
1 \\
22 \\
12 \\
63 \\
35 \\
29 \\
15 \\
7 \\
399 \\
73 \\
57 \\
51 \\
43 \\
79 \\
46 \\
50 \\
22 \\
13 \\
7 \\
399 \\
79\end{array}$ & $\begin{array}{l}\text { date } \\
12 / 01 / 93 \\
12 / 01 / 93 \\
12 / 01 / 93 \\
12 / 01 / 93 \\
12 / 01 / 93 \\
12 / 01 / 93 \\
12 / 01 / 93 \\
12 / 01 / 93 \\
12 / 01 / 93 \\
12 / 01 / 93 \\
12 / 01 / 93 \\
12 / 01 / 93 \\
12 / 01 / 93 \\
12 / 01 / 93 \\
12 / 01 / 93 \\
12 / 01 / 93 \\
12 / 01 / 93 \\
12 / 01 / 93 \\
12 / 01 / 93 \\
12 / 01 / 93 \\
12 / 01 / 93\end{array}$ \\
\hline
\end{tabular}




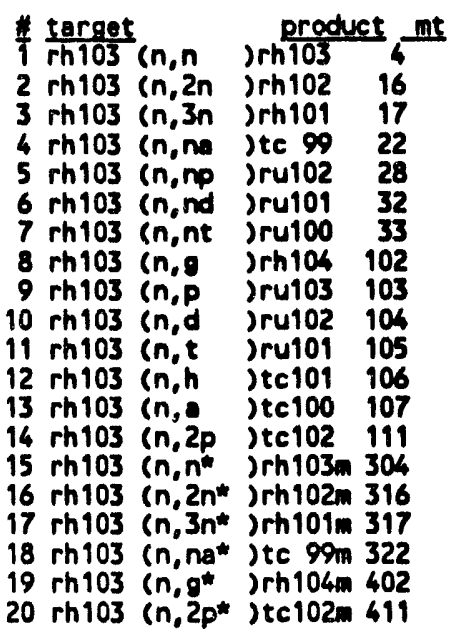

\begin{tabular}{cc} 
\# engerieg & date \\
\hline 1 & $12 / 01 / 93$ \\
29 & $12 / 01 / 93$ \\
17 & $12 / 01 / 93$ \\
61 & $12 / 01 / 93$ \\
42 & $12 / 01 / 93$ \\
17 & $12 / 01 / 93$ \\
16 & $12 / 01 / 93$ \\
1269 & $12 / 01 / 93$ \\
79 & $12 / 01 / 93$ \\
56 & $12 / 01 / 93$ \\
45 & $12 / 01 / 93$ \\
39 & $12 / 01 / 93$ \\
81 & $12 / 01 / 93$ \\
38 & $12 / 01 / 93$ \\
48 & $12 / 01 / 93$ \\
29 & $12 / 01 / 93$ \\
13 & $12 / 01 / 93$ \\
61 & $12 / 01 / 93$ \\
1269 & $12 / 01 / 93$ \\
38 & $12 / 01 / 93$
\end{tabular}

\begin{tabular}{|c|c|c|c|c|c|}
\hline 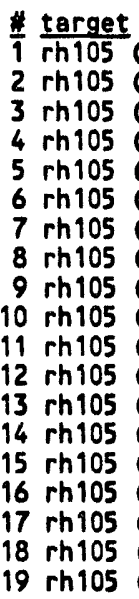 & 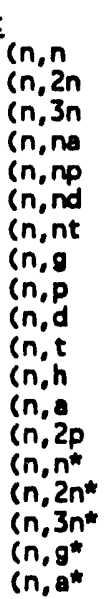 & 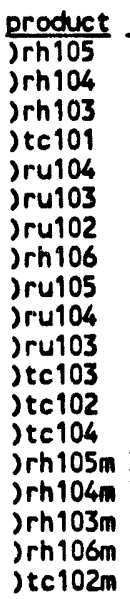 & $\begin{array}{l}\frac{m t}{4} \\
16 \\
17 \\
22 \\
28 \\
32 \\
33 \\
102 \\
103 \\
104 \\
105 \\
106 \\
107 \\
111 \\
304 \\
316 \\
317 \\
402 \\
407\end{array}$ & $\begin{array}{c}\text { Pengeries } \\
1 \\
26 \\
17 \\
60 \\
39 \\
16 \\
18 \\
447 \\
67 \\
54 \\
45 \\
33 \\
82 \\
33 \\
46 \\
26 \\
17 \\
443 \\
82\end{array}$ & $\begin{array}{l}\text { date } \\
12 / 01 / 93 \\
12 / 01 / 93 \\
12 / 01 / 93 \\
12 / 01 / 93 \\
12 / 01 / 93 \\
12 / 01 / 93 \\
12 / 01 / 93 \\
12 / 01 / 93 \\
12 / 01 / 93 \\
12 / 01 / 93 \\
12 / 01 / 93 \\
12 / 01 / 93 \\
12 / 01 / 93 \\
12 / 01 / 93 \\
12 / 01 / 93 \\
12 / 01 / 93 \\
12 / 01 / 93 \\
12 / 01 / 93 \\
12 / 01 / 93\end{array}$ \\
\hline $\begin{aligned} 1 & \text { pd100 } \\
2 & \text { pd100 } \\
3 & \text { pd100 } \\
4 & \text { pd100 } \\
5 & \text { pd100 } \\
6 & \text { pd100 } \\
7 & \text { pd100 } \\
8 & \text { pd } 100 \\
9 & \text { pd100 } \\
10 & \text { pd100 } \\
11 & \text { pd100 } \\
12 & \text { pd1100 } \\
13 & \text { pd100 } \\
14 & \text { pd100 } \\
15 & \text { pd100 } \\
16 & \text { pd100 } \\
17 & \text { pd100 } \\
18 & \text { pd100 }\end{aligned}$ & 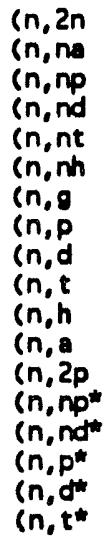 & $\begin{array}{l}\text { Jpd } 99 \\
\text { Jru } 96 \\
\text { Jrh } 99 \\
\text { jrh } 98 \\
\text { jrh } 97 \\
\text { Jru } 97 \\
\text { Jpd101 } \\
\text { Jrh100 } \\
\text { Jrh } 99 \\
\text { jrh } 98 \\
\text { Jru } 98 \\
\text { Jru } 97 \\
\text { Jru } 99 \\
\text { Jrh } 99 \mathrm{~m} \\
\text { Jrh } 98 \mathrm{~m} \\
\text { Jrh100m } \\
\text { Jrh } 99 \mathrm{~m} \\
\text { Jrh } 98 \mathrm{~m}\end{array}$ & $\begin{array}{r}16 \\
22 \\
28 \\
32 \\
33 \\
34 \\
102 \\
103 \\
104 \\
105 \\
106 \\
107 \\
111 \\
328 \\
332 \\
403 \\
404 \\
405\end{array}$ & $\begin{array}{r}23 \\
72 \\
38 \\
13 \\
8 \\
8 \\
390 \\
78 \\
55 \\
41 \\
59 \\
79 \\
55 \\
38 \\
13 \\
78 \\
55 \\
41\end{array}$ & $\begin{array}{l}12 / 01 / 93 \\
12 / 01 / 93 \\
12 / 01 / 93 \\
12 / 01 / 93 \\
12 / 01 / 93 \\
12 / 01 / 93 \\
12 / 01 / 93 \\
12 / 01 / 93 \\
12 / 01 / 93 \\
12 / 01 / 93 \\
12 / 01 / 93 \\
12 / 01 / 93 \\
12 / 01 / 93 \\
12 / 01 / 93 \\
12 / 01 / 93 \\
12 / 01 / 93 \\
12 / 01 / 93 \\
12 / 01 / 93\end{array}$ \\
\hline $\begin{array}{l}\# \frac{\text { target }}{\text { target }} \\
2 \text { pd101 } \\
2 \text { pd101 } \\
3 \text { pd101 } \\
4 \text { pd101 } \\
5 \text { pd101 } \\
6 \text { pd101 } \\
7 \text { pd101 } \\
8 \text { pd101 } \\
9 \text { pd101 } \\
10 \text { pd101 }\end{array}$ & 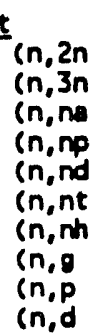 & 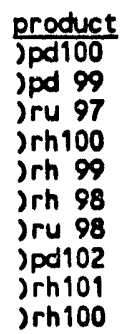 & $\begin{array}{l}\frac{m t}{16} \\
17 \\
22 \\
28 \\
32 \\
33 \\
34 \\
102 \\
103 \\
104\end{array}$ & $\begin{array}{c}\text { Engeries } \\
26 \\
8 \\
66 \\
30 \\
17 \\
9 \\
18 \\
394 \\
74 \\
51\end{array}$ & $\begin{array}{l}\text { date } \\
12 / 01 / 93 \\
12 / 01 / 93 \\
12 / 01 / 93 \\
12 / 01 / 93 \\
12 / 01 / 93 \\
12 / 01 / 93 \\
12 / 01 / 93 \\
12 / 01 / 93 \\
12 / 01 / 93 \\
12 / 01 / 93\end{array}$ \\
\hline
\end{tabular}




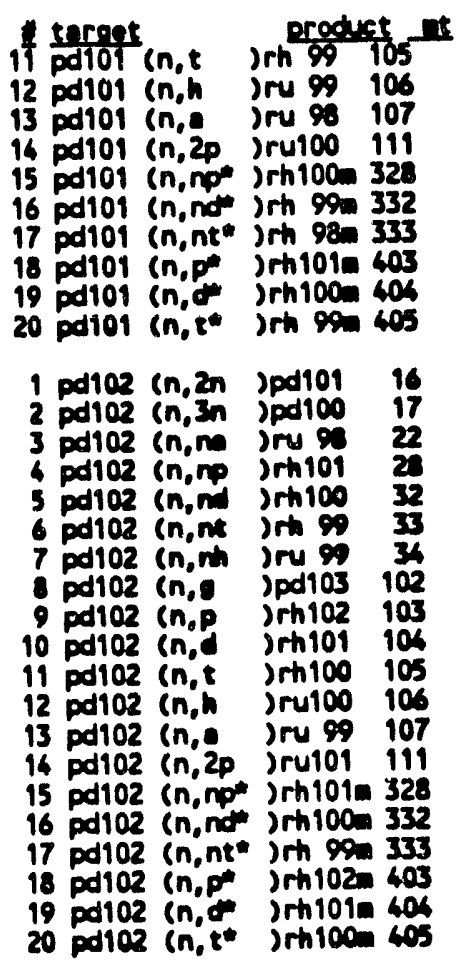

4 merie
49
55
76
61
28
17
9
74
50
69

$12 / \frac{\text { date }}{01 / 93}$

$12 / 01 / 93$

$12 / 01 / 93$

$12 / 01 / 93$

$12 / 01 / 93$

12/01/93

$12 / 01 / 93$

12/01/93

$12 / 01 / 93$

$12 / 01 / 93$

$\begin{array}{cc}22 & 12 / 01 / 93 \\ 6 & 12 / 01 / 93 \\ 69 & 12 / 01 / 93 \\ 37 & 12 / 01 / 93 \\ 12 & 12 / 01 / 93 \\ 8 & 12 / 01 / 93 \\ 6 & 12 / 01 / 93 \\ 76 & 12 / 01 / 93 \\ 80 & 12 / 01 / 93 \\ 50 & 12 / 01 / 93 \\ 61 & 12 / 01 / 93 \\ 52 & 12 / 01 / 93 \\ 82 & 12 / 01 / 93 \\ 45 & 12 / 01 / 93 \\ 35 & 12 / 01 / 93 \\ 12 & 12 / 01 / 93 \\ 8 & 12 / 01 / 93 \\ 80 & 12 / 01 / 93 \\ 50 & 12 / 01 / 93 \\ 30 & 12 / 01 / 93\end{array}$

\begin{tabular}{|c|c|c|c|c|c|}
\hline $\begin{array}{ll}4 & \text { parget } \\
4 & \text { pd103 } \\
2 & \text { pd103 } \\
3 & \text { pd103 } \\
6 & \text { pd103 } \\
5 & \text { pd103 } \\
6 & \text { pd103 } \\
7 & \text { pd103 } \\
8 & \text { pd103 } \\
9 & \text { pd103 } \\
10 & \text { pd103 } \\
11 & \text { pd103 } \\
12 & \text { pd103 } \\
13 & \text { pd103 } \\
14 & \text { pd103 } \\
15 & \text { pd103 } \\
16 & \text { pd103 } \\
17 & \text { pd103 } \\
18 & \text { pd103 } \\
19 & \text { pd103 } \\
20 & \text { pd103 }\end{array}$ & 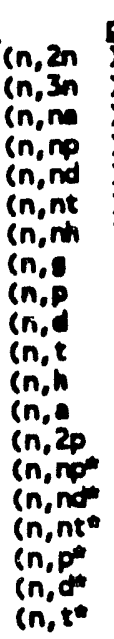 & 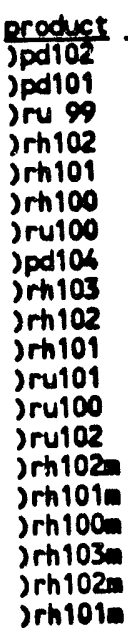 & $\begin{array}{l}9 \\
16 \\
17 \\
22 \\
28 \\
32 \\
33 \\
34 \\
102 \\
103 \\
104 \\
105 \\
106 \\
107 \\
111 \\
323 \\
332 \\
333 \\
403 \\
404 \\
405\end{array}$ & $\begin{array}{c}\text { engerien } \\
24 \\
13 \\
64 \\
31 \\
21 \\
9 \\
11 \\
389 \\
7 \\
49 \\
47 \\
40 \\
78 \\
50 \\
31 \\
21 \\
8 \\
78 \\
49 \\
47\end{array}$ & $\begin{array}{l}\text { date } \\
12 / 01 / 93 \\
12 / 01 / 93 \\
12 / 01 / 93 \\
12 / 01 / 93 \\
12 / 01 / 93 \\
12 / 01 / 93 \\
12 / 01 / 93 \\
12 / 01 / 93 \\
12 / 01 / 93 \\
12 / 01 / 93 \\
12 / 01 / 93 \\
12 / 01 / 93 \\
12 / 01 / 93 \\
12 / 01 / 93 \\
12 / 01 / 93 \\
12 / 01 / 93 \\
12 / 01 / 93 \\
12 / 01 / 93 \\
12 / 01 / 93 \\
12 / 01 / 93\end{array}$ \\
\hline 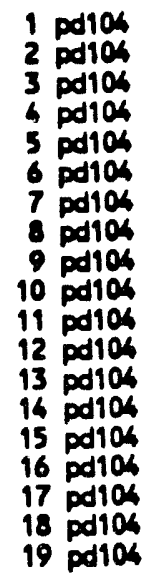 & 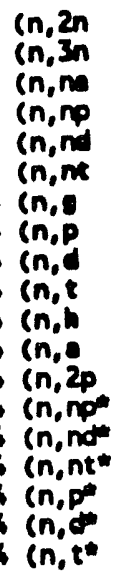 & 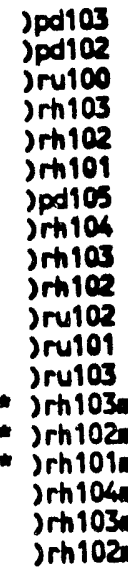 & $\begin{array}{c}16 \\
17 \\
22 \\
28 \\
32 \\
33 \\
102 \\
103 \\
104 \\
105 \\
106 \\
107 \\
111 \\
328 \\
332 \\
333 \\
403\end{array}$ & $\begin{array}{r}26 \\
15 \\
65 \\
36 \\
12 \\
9 \\
5967 \\
66 \\
67 \\
40 \\
45 \\
82 \\
39 \\
36 \\
12 \\
9 \\
66 \\
46 \\
40\end{array}$ & $\begin{array}{l}12 / 01 / 93 \\
12 / 01 / 93 \\
12 / 01 / 93 \\
12 / 01 / 93 \\
12 / 01 / 93 \\
12 / 01 / 93 \\
12 / 01 / 93 \\
12 / 01 / 93 \\
12 / 01 / 93 \\
12 / 01 / 93 \\
12 / 01 / 93 \\
12 / 01 / 93 \\
12 / 01 / 93 \\
12 / 01 / 93 \\
12 / 01 / 93 \\
12 / 01 / 93 \\
12 / 01 / 93 \\
12 / 01 / 93 \\
12 / 01 / 93\end{array}$ \\
\hline
\end{tabular}

$A-4.17$ 


\begin{tabular}{|c|c|c|c|c|c|}
\hline 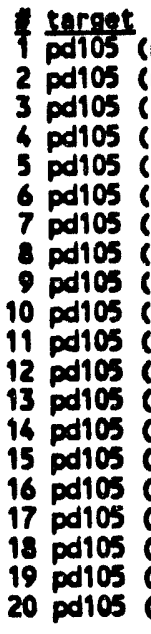 & 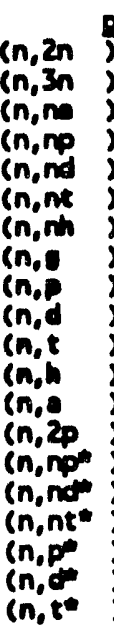 & 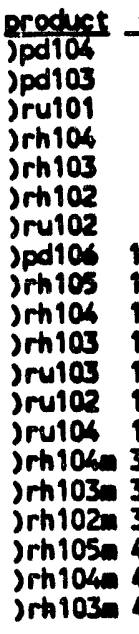 & $\begin{array}{l}10 \\
16 \\
17 \\
22 \\
28 \\
32 \\
33 \\
34 \\
102 \\
103 \\
104 \\
105 \\
106 \\
107 \\
111 \\
324 \\
332 \\
333 \\
403 \\
404 \\
405\end{array}$ & $\begin{array}{c}- \text { mogrien } \\
26 \\
17 \\
62 \\
28 \\
20 \\
10 \\
7 \\
128 \\
79 \\
47 \\
46 \\
41 \\
81 \\
43 \\
20 \\
20 \\
10 \\
80 \\
67 \\
46\end{array}$ & $\begin{array}{l}\text { date } \\
\text { 12/01/93 } \\
12 / 01 / 93 \\
12 / 01 / 93 \\
12 / 01 / 93 \\
12 / 01 / 93 \\
12 / 01 / 93 \\
12 / 01 / 93 \\
12 / 01 / 93 \\
12 / 01 / 93 \\
12 / 01 / 93 \\
12 / 01 / 93 \\
12 / 01 / 93 \\
12 / 01 / 93 \\
12 / 01 / 93 \\
12 / 01 / 93 \\
12 / 01 / 93 \\
12 / 01 / 93 \\
12 / 01 / 93 \\
12 / 01 / 93 \\
12 / 01 / 93\end{array}$ \\
\hline 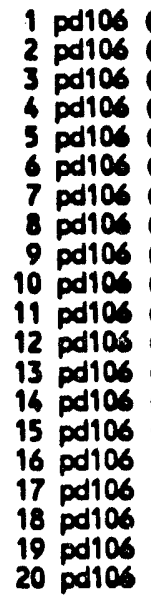 & 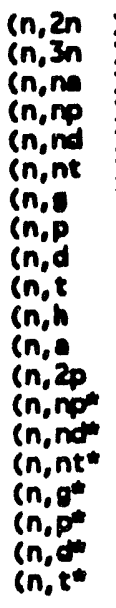 & 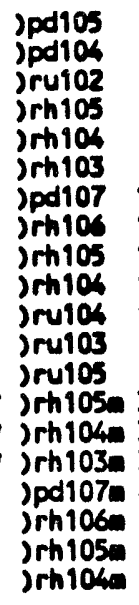 & $\begin{array}{l}16 \\
17 \\
22 \\
28 \\
32 \\
33 \\
102 \\
103 \\
104 \\
105 \\
106 \\
107 \\
111 \\
320 \\
332 \\
333 \\
402 \\
403 \\
406 \\
405\end{array}$ & $\begin{array}{r}24 \\
18 \\
64 \\
34 \\
11 \\
10 \\
6309 \\
60 \\
67 \\
30 \\
37 \\
84 \\
34 \\
32 \\
11 \\
8 \\
6313 \\
61 \\
66 \\
37\end{array}$ & $\begin{array}{l}12 / 01 / 93 \\
12 / 01 / 93 \\
12 / 01 / 93 \\
12 / 01 / 93 \\
12 / 01 / 93 \\
12 / 01 / 93 \\
12 / 01 / 93 \\
12 / 01 / 93 \\
12 / 01 / 93 \\
12 / 01 / 93 \\
12 / 01 / 93 \\
12 / 01 / 93 \\
12 / 01 / 93 \\
12 / 01 / 93 \\
12 / 01 / 93 \\
12 / 01 / 93 \\
12 / 01 / 93 \\
12 / 01 / 93 \\
12 / 01 / 93 \\
12 / 01 / 93\end{array}$ \\
\hline $\begin{array}{l}\text { target } \\
7 \text { pd107 } \\
2 \text { pd107 } \\
3 \text { pd107 } \\
4 \text { pd107 } \\
5 \text { pd107 } \\
6 \text { pd107 } \\
7 \text { pd107 } \\
8 \text { pd107 } \\
9 \text { pd107 } \\
10 \text { pd107 } \\
11 \text { pd107 } \\
12 \text { pd107 } \\
13 \text { pd107 } \\
16 \text { pd107 } \\
15 \text { pd107 } \\
16 \text { pd107 } \\
17 \text { pd107 } \\
18 \text { pd107 } \\
19 \text { pd107 } \\
20 \text { pd107 } \\
21 \text { pd107 }\end{array}$ & 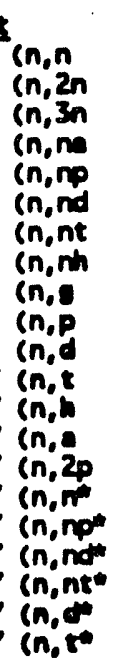 & 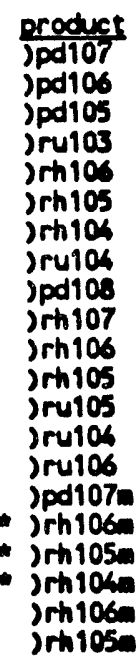 & $\begin{array}{l}16 \\
17 \\
22 \\
28 \\
32 \\
33 \\
34 \\
102 \\
103 \\
106 \\
105 \\
106 \\
107 \\
111 \\
304 \\
328 \\
332 \\
333 \\
404 \\
405\end{array}$ & $\begin{array}{c}\text { mperies } \\
1 \\
26 \\
19 \\
54 \\
26 \\
22 \\
10 \\
4 \\
7056 \\
74 \\
45 \\
46 \\
35 \\
81 \\
40 \\
52 \\
25 \\
22 \\
10 \\
45 \\
43\end{array}$ & $\begin{array}{l}\text { date } \\
\frac{12 / 01 / 93}{12 / 01 / 93} \\
12 / 01 / 93 \\
12 / 01 / 93 \\
12 / 01 / 93 \\
12 / 01 / 93 \\
12 / 01 / 93 \\
12 / 01 / 93 \\
12 / 01 / 93 \\
12 / 01 / 93 \\
12 / 01 / 93 \\
12 / 01 / 93 \\
12 / 01 / 93 \\
12 / 01 / 93 \\
12 / 01 / 93 \\
12 / 01 / 93 \\
12 / 01 / 93 \\
12 / 01 / 93 \\
12 / 01 / 93 \\
12 / 01 / 93 \\
12 / 01 / 93\end{array}$ \\
\hline $\begin{array}{l}1 \text { pdics } \\
2 \text { pd10s } \\
3 \text { pd10s } \\
4 \text { pd10s } \\
5 \text { pd10s } \\
6 \text { pd10s } \\
7 \text { pdios } \\
8 \text { pdios }\end{array}$ & $\begin{array}{l}2 n \\
\text { in } \\
\text { nn } \\
\text { non } \\
\text { nd } \\
\text { nt } \\
\text { ip } \\
\text { ip }\end{array}$ & 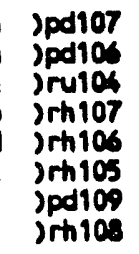 & $\begin{array}{r}16 \\
17 \\
22 \\
28 \\
32 \\
33 \\
102 \\
103\end{array}$ & $\begin{array}{r}23 \\
21 \\
59 \\
32 \\
10 \\
10 \\
5952 \\
56\end{array}$ & $\begin{array}{l}12 / 01 / 93 \\
12 / 01 / 93 \\
12 / 01 / 93 \\
12 / 01 / 93 \\
12 / 01 / 93 \\
12 / 01 / 93 \\
12 / 01 / 93 \\
12 / 01 / 93\end{array}$ \\
\hline
\end{tabular}

$$
\text { A-4. } 18
$$




\begin{tabular}{|c|c|c|c|c|c|}
\hline 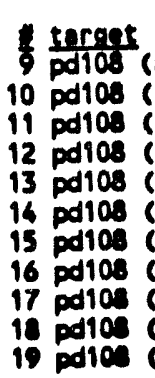 & 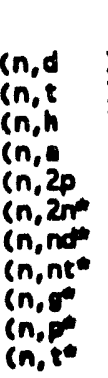 & 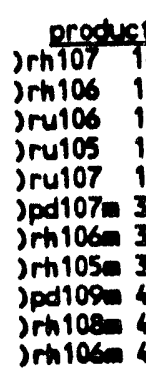 & $\begin{array}{l}10 \\
104 \\
105 \\
106 \\
107 \\
111 \\
316 \\
332 \\
333 \\
402 \\
403 \\
405\end{array}$ & $\begin{array}{l}\text { penperien } \\
65 \\
38 \\
31 \\
7 \\
30 \\
25 \\
9 \\
10 \\
5954 \\
56 \\
35\end{array}$ & $\begin{array}{l}\text { date } \\
12 / 01 / 93 \\
12 / 01 / 93 \\
12 / 01 / 93 \\
12 / 01 / 93 \\
12 / 01 / 93 \\
12 / 01 / 93 \\
12 / 01 / 93 \\
12 / 01 / 93 \\
12 / 01 / 93 \\
12 / 01 / 93 \\
12 / 01 / 93\end{array}$ \\
\hline 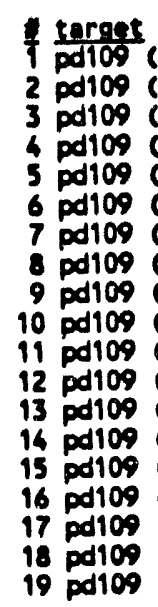 & 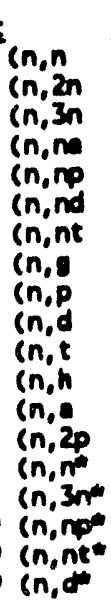 & 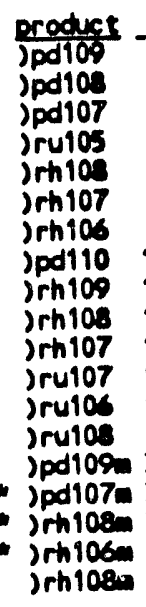 & $\begin{array}{l}16 \\
17 \\
22 \\
21 \\
32 \\
33 \\
102 \\
103 \\
104 \\
105 \\
106 \\
107 \\
111 \\
306 \\
317 \\
328 \\
333 \\
404\end{array}$ & $\begin{array}{c}\text { enperies } \\
30 \\
30 \\
18 \\
52 \\
26 \\
20 \\
11 \\
392 \\
66 \\
14 \\
45 \\
29 \\
81 \\
36 \\
56 \\
18 \\
22 \\
11 \\
46\end{array}$ & $\begin{array}{l}\text { date } \\
12 / 01 / 93 \\
12 / 01 / 93 \\
12 / 01 / 93 \\
12 / 01 / 93 \\
12 / 01 / 93 \\
12 / 01 / 93 \\
12 / 01 / 93 \\
12 / 01 / 93 \\
12 / 01 / 93 \\
12 / 01 / 93 \\
12 / 01 / 93 \\
12 / 01 / 93 \\
12 / 01 / 93 \\
12 / 01 / 93 \\
12 / 01 / 93 \\
12 / 01 / 93 \\
12 / 01 / 93 \\
12 / 01 / 93 \\
12 / 01 / 93\end{array}$ \\
\hline $\begin{array}{cc}1 & \text { pd110 } \\
2 & \text { pd110 } \\
3 & \text { pd110 } \\
4 & \text { pd110 } \\
5 & \text { pdi10 } \\
6 & \text { pd110 } \\
7 & \text { pd110 } \\
8 & \text { pd110 } \\
9 & \text { pd110 } \\
10 & \text { pd1110 } \\
11 & \text { pd110 } \\
12 & \text { pd110 } \\
13 & \text { pd110 } \\
14 & \text { pd110 } \\
15 & \text { pd110 } \\
16 & \text { pd110 } \\
17 & \text { pd110 } \\
18 & \text { pd110 } \\
19 & \text { pd110 }\end{array}$ & 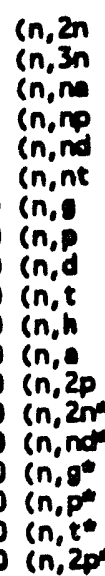 & 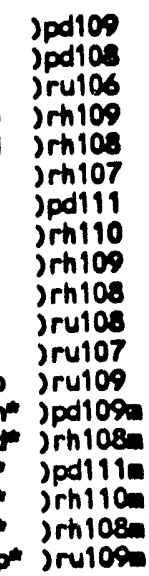 & $\begin{array}{r}16 \\
17 \\
22 \\
28 \\
32 \\
33 \\
102 \\
103 \\
106 \\
105 \\
106 \\
107 \\
111 \\
316 \\
332 \\
402 \\
403 \\
405\end{array}$ & $\begin{array}{r}32 \\
19 \\
58 \\
29 \\
10 \\
10 \\
3656 \\
52 \\
43 \\
38 \\
25 \\
71 \\
25 \\
29 \\
10 \\
3659 \\
52 \\
30 \\
25\end{array}$ & $\begin{array}{l}12 / 01 / 93 \\
12 / 01 / 93 \\
12 / 01 / 93 \\
12 / 01 / 93 \\
12 / 01 / 93 \\
12 / 01 / 93 \\
12 / 01 / 93 \\
12 / 01 / 93 \\
12 / 01 / 93 \\
12 / 01 / 93 \\
12 / 01 / 93 \\
12 / 01 / 93 \\
12 / 01 / 93 \\
12 / 01 / 93 \\
12 / 01 / 93 \\
12 / 01 / 93 \\
12 / 01 / 93 \\
12 / 01 / 93 \\
12 / 01 / 93\end{array}$ \\
\hline
\end{tabular}


WHC-EP-0727

This paes intentienally loft blenk.

$A-4.20$ 
WHC-EP-0727

Appendix A-5

Llet of Reections on flles *.es.te

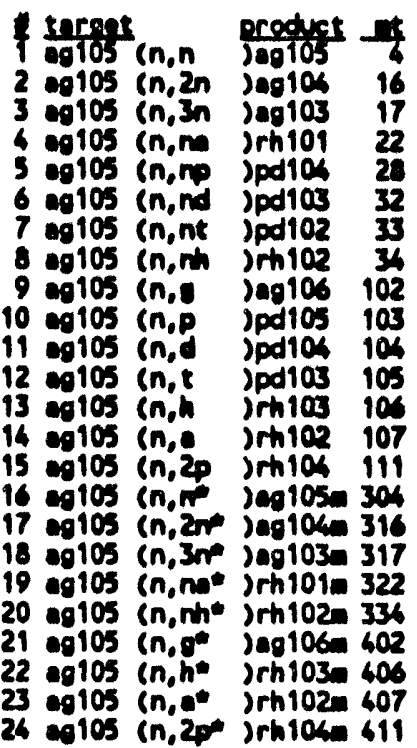

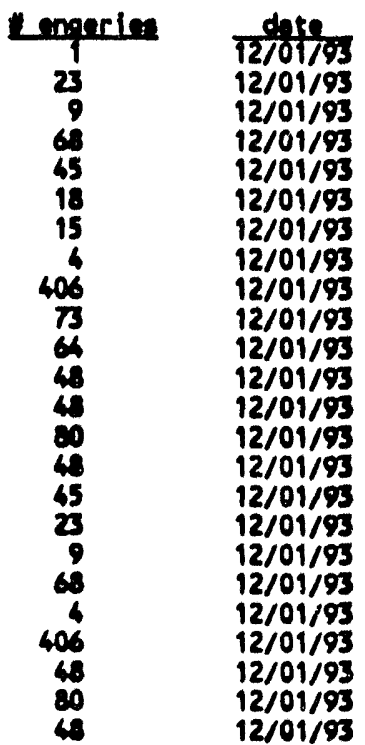

4 terant

product its

aglosem, $n$

).9106

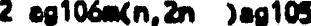

3 eolocman, in jeg10s

- 09106arn,no jrh102

5 ealo6m(n,np )pdios

6 eglobmen, nd updios

7 ogloban n, nt jpd103

8 ag106m(n,nt )rh103 3

- eglo6man, jog107 102

10 iglo6m(n,p ipdios 103

11 eglo6m(n,d ipdios 10\%

12 aglosmen,t jpdioh 105

13 oglogakn,h ith104 106

16 ag106aln,e irh103 107

15 aglo6m(n,2p )rtios 111

$1609106 \times(n, 2 m)$ ) $09105 m 316$

17 og106m(n,3n )eg104m 317

18 eq106an,n irtioz 322

19 eq106m(n,nht) rh103m 334

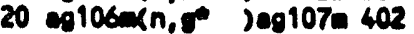

21 eglo6m(n,h* irhio4m 406

$22.9106 \times(n$, Jrh103 407

23 oglo6m(n,2p")rh105m 411

\begin{tabular}{cc} 
Lngerin & dinte \\
\hline 47 & $12 / 01 / 93$ \\
25 & $12 / 01 / 93$ \\
11 & $12 / 01 / 93$ \\
64 & $12 / 01 / 93$ \\
34 & $12 / 01 / 93$ \\
26 & $12 / 01 / 93$ \\
16 & $12 / 01 / 93$ \\
7 & $12 / 01 / 93$ \\
400 & $12 / 01 / 93$ \\
70 & $12 / 01 / 93$ \\
58 & $12 / 01 / 93$ \\
52 & $12 / 01 / 93$ \\
45 & $12 / 01 / 93$ \\
79 & $12 / 01 / 93$ \\
51 & $12 / 01 / 93$ \\
25 & $12 / 01 / 93$ \\
11 & $12 / 01 / 93$ \\
64 & $12 / 01 / 93$ \\
7 & $12 / 01 / 93$ \\
400 & $12 / 01 / 93$ \\
45 & $12 / 01 / 93$ \\
79 & $12 / 01 / 93$ \\
51 & $12 / 01 / 93$
\end{tabular}

$\begin{array}{llll}1 & 09107(n, n) & 099107 & 4 \\ 2 & 09107(n, 2 n) & \text { )ag106 } & 16\end{array}$

$209107(n, 2 n)$ ag106 16

400107 (n, na irhio3 22

5 ag107 (n,ng)pd106 28

0.0107 ( $n, n d$ )pdios 32

7 ag107 (n,nt )pdio 33

8.9107 (n.s joglos 102

- 9107 (n.p )pd107 103

10 ag107 (n,d )pdi06 104

11 eg107 (n,t ipdios 105

1200107 (n, ) rhios $10 \mathrm{~s}$

13 oglo7 in, irhios 107

$1400107 \mathrm{ln}, 2 \mathrm{p}$ ) rhics 111

$1509107(n, n)$ )eg107n 304

16 eg107 (n,2n) ag106n 316

17.9107 (n, $3 n$ jeglosm 317

$\left.18.0107(n, n)^{*}\right) r h 103 m 322$

19 ag107 (n, g*) ag108m 402

$\begin{array}{rr}1 & 12 / 01 / 93 \\ 7 & 12 / 01 / 93 \\ 2 & 12 / 01 / 93 \\ 2 & 12 / 01 / 93 \\ 66 & 12 / 01 / 93 \\ 43 & 12 / 01 / 93 \\ 17 & 12 / 01 / 93 \\ 15 & 12 / 01 / 93 \\ 4250 & 12 / 01 / 93 \\ 25 & 12 / 01 / 93 \\ 39 & 12 / 01 / 93 \\ 46 & 12 / 01 / 93 \\ 43 & 12 / 01 / 93 \\ 11 & 12 / 01 / 93 \\ 42 & 12 / 01 / 93 \\ 47 & 12 / 01 / 93 \\ 12 & 12 / 01 / 93 \\ 13 & 12 / 01 / 93 \\ 66 & 12 / 01 / 93 \\ 4249 & \end{array}$

$A-5.1$ 


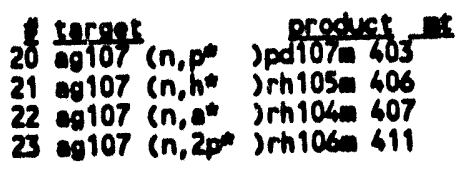

4 monria
23
43
5
42
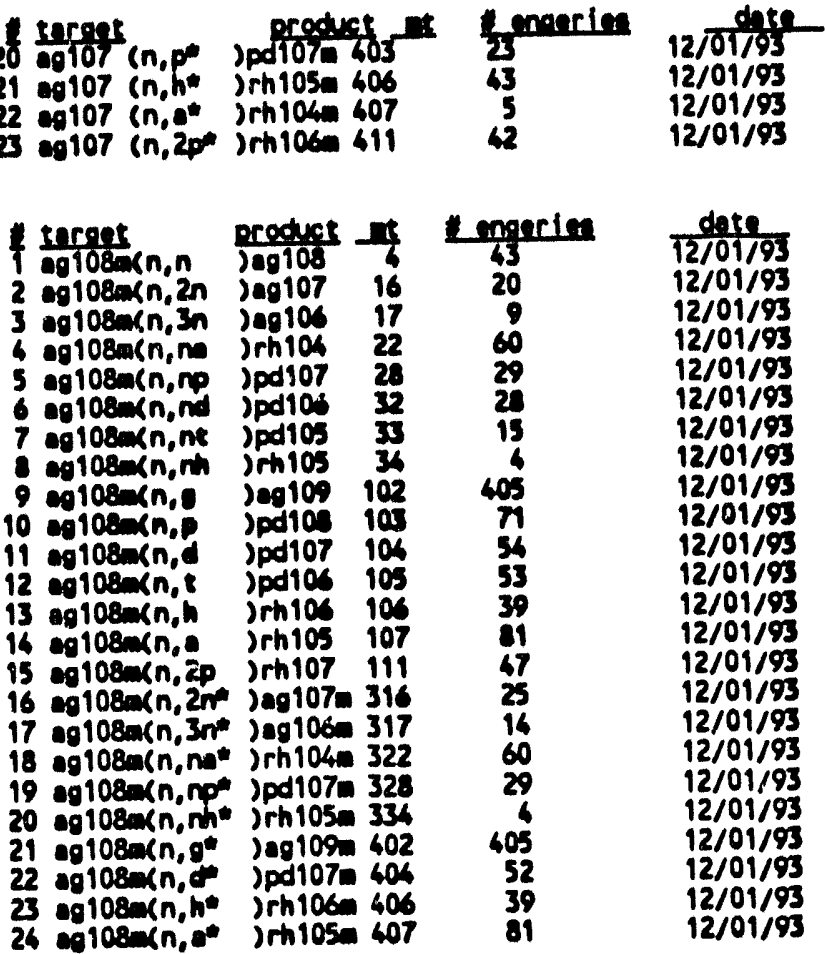

mogerie
43
20
9
60
29
28
15
4
405
74
54
53
39
81
47
25
16
60
29
6
405
52
39
81

$\frac{\text { date }}{12 / 01 / 9}$

$12 / 01 / 93$

$12 / 01 / 93$

$12 / 01 / 93$

$12 / 01 / 93$

$12 / 01 / 93$

$12 / 01 / 93$

$12 / 01 / 93$

$12 / 01 / 93$

$12 / 01 / 93$

$12 / 01 / 93$

$12 / 01 / 93$

$12 / 01 / 93$

$12 / 01 / 93$

12/01/93

$12 / 01 / 93$

$12 / 01 / 93$

$12 / 01 / 93$

$12 / 01 / 93$

$12 / 01 / 93$

$12 / 01 / 93$

$12 / 01 / 93$

$12 / 01 / 93$

$12 / 01 / 93$

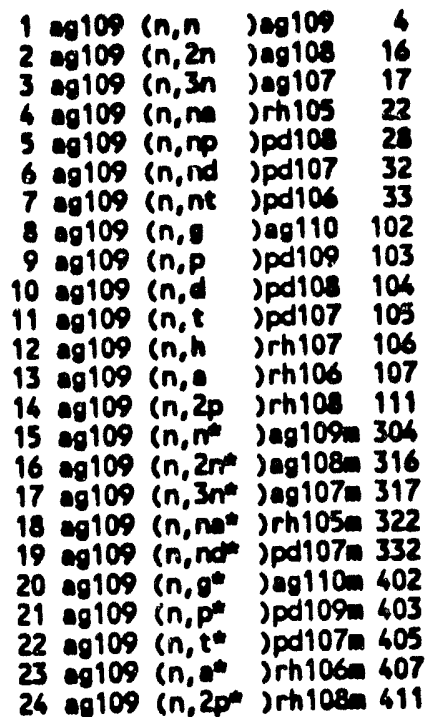

$12 / 01 / 93$

12/01/93

$12 / 01 / 93$

12/01/93

$12 / 01 / 93$

$12 / 01 / 93$

$12 / 01 / 93$

$12 / 01 / 93$

$12 / 01 / 93$

$12 / 01 / 93$

$12 / 01 / 93$

$12 / 01 / 93$

$12 / 01 / 93$

$12 / 01 / 93$

$12 / 01 / 93$

$12 / 01 / 93$

$12 / 01 / 93$

$12 / 01 / 93$

$12 / 01 / 93$

$12 / 01 / 93$

$12 / 01 / 93$

$12 / 01 / 93$

$12 / 01 / 93$

$12 / 01 / 93$

\begin{tabular}{|c|c|c|c|c|}
\hline 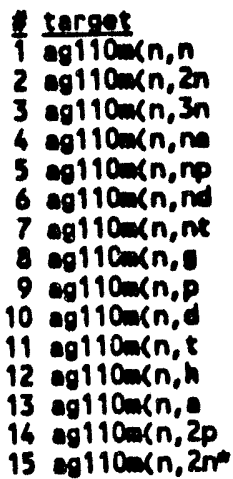 & 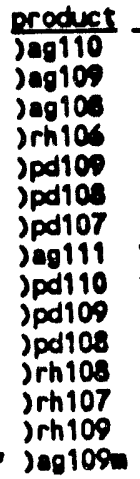 & $\begin{array}{l}17 \\
16 \\
17 \\
22 \\
22 \\
32 \\
33 \\
102 \\
103 \\
104 \\
105 \\
106 \\
107 \\
111\end{array}$ & $\begin{array}{l}209010 \\
40 \\
29 \\
17 \\
55 \\
28 \\
29 \\
15 \\
405 \\
73 \\
49 \\
55 \\
33 \\
81\end{array}$ & $\begin{array}{l}\text { date } \\
12 / 01 / 93 \\
12 / 01 / 93 \\
12 / 01 / 93 \\
12 / 01 / 93 \\
12 / 01 / 93 \\
12 / 01 / 93 \\
12 / 01 / 93 \\
12 / 01 / 93 \\
12 / 01 / 93 \\
12 / 01 / 93 \\
12 / 01 / 93 \\
12 / 01 / 93 \\
12 / 01 / 93 \\
12 / 01 / 93 \\
12 / 01 / 93\end{array}$ \\
\hline
\end{tabular}

A-5. 2 


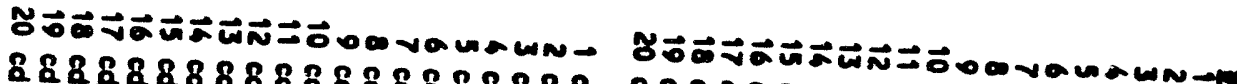

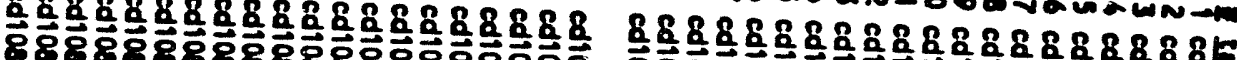

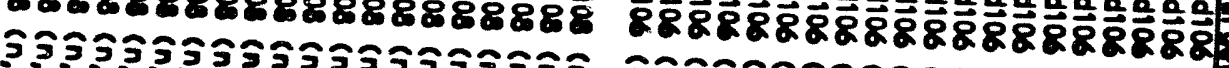

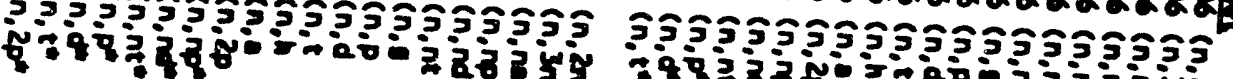

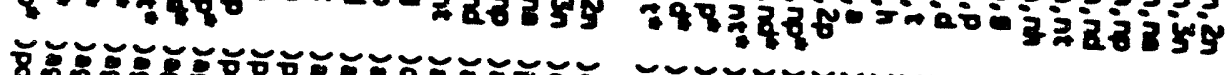

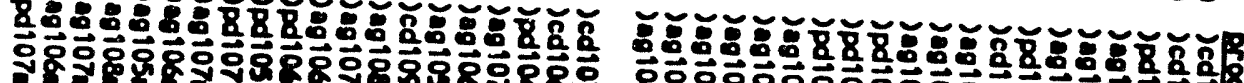

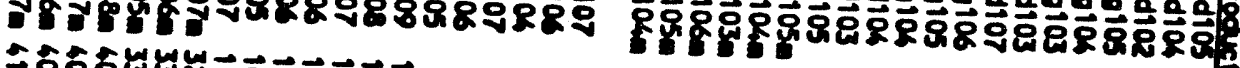

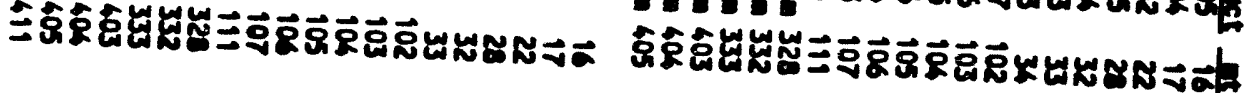

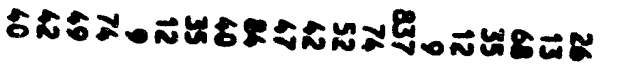

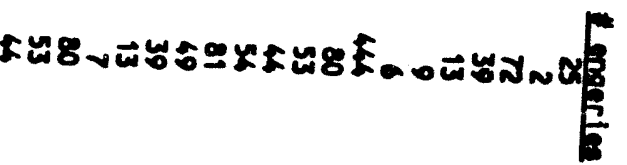

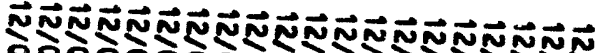

i w is

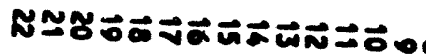

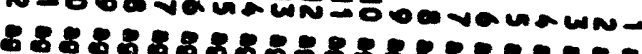

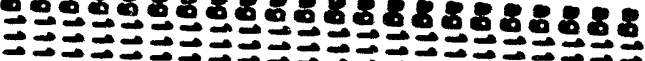

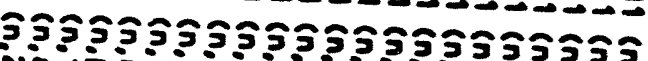

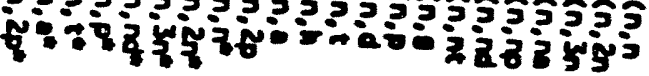

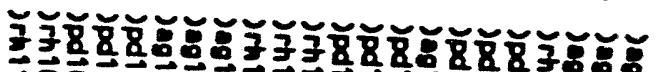

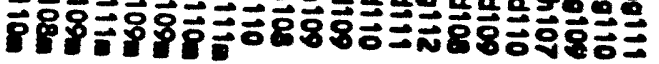

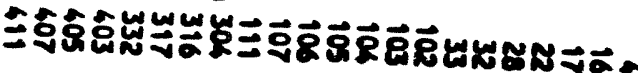

กะชลจีล 38888885 केण

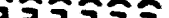
ให่

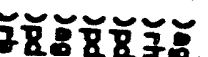

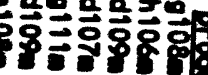

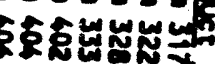

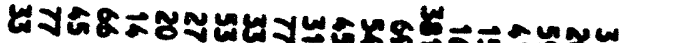

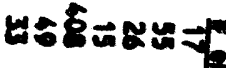

$$
\text { ह }
$$

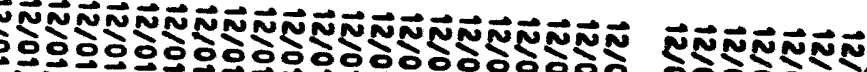

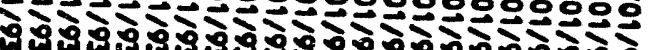

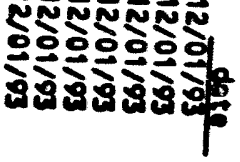




\begin{tabular}{|c|c|c|c|c|c|}
\hline 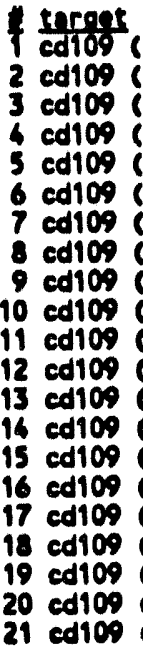 & $\left.\begin{array}{l}\text { (n,2n } \\
(n, 3 n \\
(n, n) \\
(n, n)\end{array}\right\}$ & 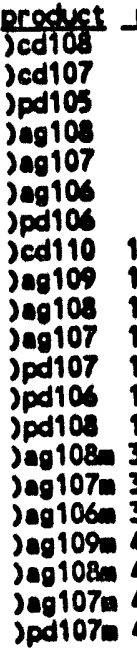 & $\begin{array}{l}7 t \\
16 \\
17 \\
22 \\
28 \\
32 \\
33 \\
34 \\
102 \\
103 \\
104 \\
105 \\
106 \\
107 \\
111 \\
328 \\
332 \\
333 \\
403 \\
406 \\
405 \\
405\end{array}$ & $\begin{array}{l}\text { menperien } \\
28 \\
15 \\
61 \\
32 \\
23 \\
10 \\
10 \\
390 \\
79 \\
40 \\
67 \\
43 \\
80 \\
45 \\
32 \\
23 \\
10 \\
79 \\
40 \\
47 \\
43\end{array}$ & $\begin{array}{l}\text { dite } \\
12 / 01 / 93 \\
12 / 01 / 93 \\
12 / 01 / 93 \\
12 / 01 / 93 \\
12 / 01 / 93 \\
12 / 01 / 93 \\
12 / 01 / 93 \\
12 / 01 / 93 \\
12 / 01 / 93 \\
12 / 01 / 93 \\
12 / 01 / 93 \\
12 / 01 / 93 \\
12 / 01 / 93 \\
12 / 01 / 93 \\
12 / 01 / 93 \\
12 / 01 / 93 \\
12 / 01 / 93 \\
12 / 01 / 93 \\
12 / 01 / 93 \\
12 / 01 / 93 \\
12 / 01 / 93\end{array}$ \\
\hline 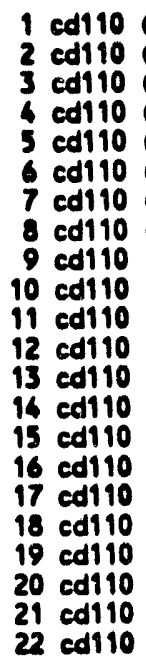 & 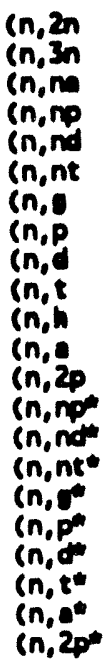 & 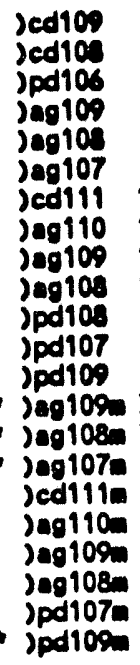 & $\begin{array}{l}16 \\
17 \\
22 \\
28 \\
32 \\
33 \\
102 \\
103 \\
106 \\
105 \\
106 \\
107 \\
111 \\
328 \\
332 \\
333 \\
402 \\
403 \\
404 \\
405 \\
407 \\
611\end{array}$ & $\begin{array}{r}30 \\
17 \\
65 \\
36 \\
11 \\
9 \\
9476 \\
64 \\
49 \\
40 \\
39 \\
85 \\
36 \\
33 \\
11 \\
9 \\
9476 \\
64 \\
49 \\
40 \\
85 \\
36\end{array}$ & $\begin{array}{l}12 / 01 / 93 \\
12 / 01 / 93 \\
12 / 01 / 93 \\
12 / 01 / 93 \\
12 / 01 / 93 \\
12 / 01 / 93 \\
12 / 01 / 93 \\
12 / 01 / 93 \\
12 / 01 / 93 \\
12 / 01 / 93 \\
12 / 01 / 93 \\
12 / 01 / 93 \\
12 / 01 / 93 \\
12 / 01 / 93 \\
12 / 01 / 93 \\
12 / 01 / 93 \\
12 / 01 / 93 \\
12 / 01 / 93 \\
12 / 01 / 93 \\
12 / 01193 \\
12 / 01 / 93 \\
12 / 01 / 93\end{array}$ \\
\hline 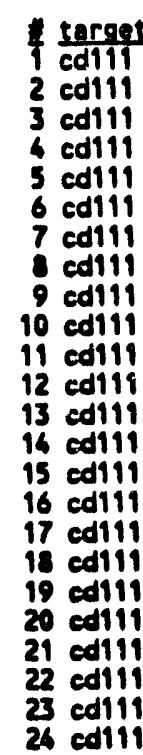 & 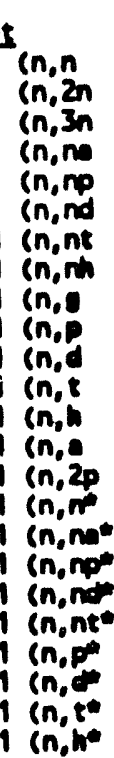 & 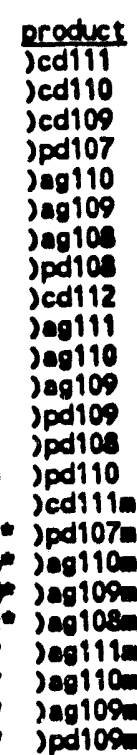 & $\begin{array}{l}07 \\
6 \\
16 \\
17 \\
22 \\
28 \\
32 \\
33 \\
36 \\
102 \\
103 \\
106 \\
105 \\
106 \\
107 \\
111 \\
304 \\
322 \\
328 \\
332 \\
333 \\
403 \\
404 \\
405\end{array}$ & $\begin{array}{c}\text { Eengerien } \\
1 \\
23 \\
13 \\
53 \\
29 \\
22 \\
10 \\
4 \\
1282 \\
80 \\
47 \\
47 \\
37 \\
81 \\
39 \\
38 \\
51 \\
29 \\
22 \\
10 \\
80 \\
45 \\
45 \\
37\end{array}$ & $\begin{array}{l}\text { date } \\
\text { 12/01/93 } \\
12 / 01 / 93 \\
12 / 01 / 93 \\
12 / 01 / 93 \\
12 / 01 / 93 \\
12 / 01 / 93 \\
12 / 01 / 93 \\
12 / 01 / 93 \\
12 / 01 / 93 \\
12 / 01 / 93 \\
12 / 01 / 93 \\
12 / 01 / 93 \\
12 / 01 / 93 \\
12 / 01 / 93 \\
12 / 01 / 93 \\
12 / 01 / 93 \\
12 / 01 / 93 \\
12 / 01 / 93 \\
12 / 01 / 93 \\
12 / 01 / 93 \\
12 / 01 / 93 \\
12 / 01 / 93 \\
12 / 01 / 93 \\
12 / 01 / 93\end{array}$ \\
\hline
\end{tabular}




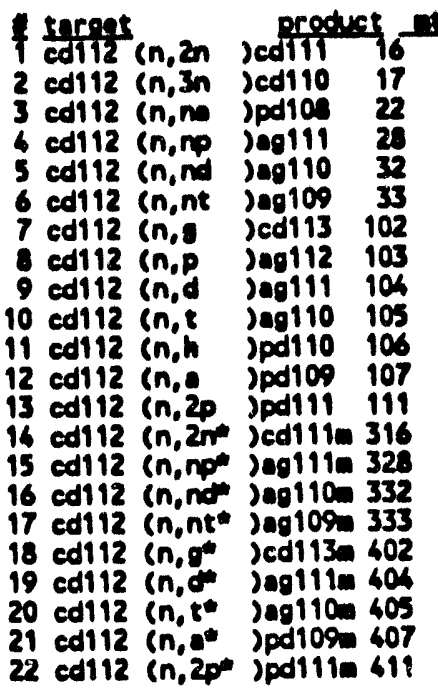

31
35
15
58
34
10
10
8301
59
67
39
33
80
32
27
34
9
8
8300
45
39
80
32

$12 / 01 / 93$

$12 / 01 / 93$

$12 / 01 / 93$

12/01/93

$12 / 01 / 93$

$12 / 01 / 93$

$12 / 01 / 93$

$12 / 01 / 93$

$12 / 01 / 93$

$12 / 01 / 93$

$12 / 01 / 93$

$12 / 01 / 93$

$12 / 01 / 93$

$12 / 01 / 93$

$12 / 01 / 93$

$12 / 01 / 93$

12/01/93

$12 / 01 / 93$

12/01/93

$12 / 01 / 93$

$12 / 01 / 93$

$12 / 01 / 93$

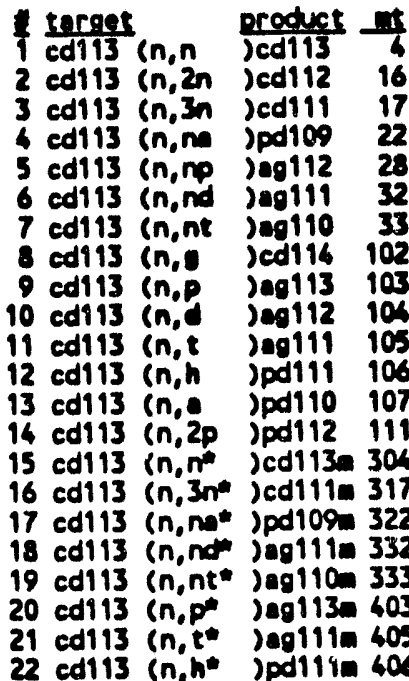

\begin{tabular}{cc} 
mengerine & $\frac{\text { date }}{12 / 01 / 93}$ \\
\hline 1 & $12 / 01 / 93$ \\
25 & $12 / 01 / 93$ \\
16 & $12 / 01 / 93$ \\
52 & $12 / 01 / 93$ \\
27 & $12 / 01 / 93$ \\
21 & $12 / 01 / 93$ \\
10 & $12 / 01 / 93$ \\
3246 & $12 / 01 / 93$ \\
69 & $12 / 01 / 93$ \\
43 & $12 / 01 / 93$ \\
46 & $12 / 01 / 93$ \\
31 & $12 / 01 / 93$ \\
81 & $12 / 01 / 93$ \\
35 & $12 / 01 / 93$ \\
35 & $12 / 01 / 93$ \\
16 & $12 / 01 / 93$ \\
30 & $12 / 01 / 93$ \\
21 & $12 / 01 / 93$ \\
10 & $12 / 01 / 93$ \\
69 & $12 / 01 / 93$
\end{tabular}

1 edi13m(n,n )cdi13

2 cd113m(n, 2n )ed112

3 cd113m(n,3n )cd111 17

4 cdil3m(n,in )pdios 22

5 cdli3m(n,no )egl12 28

6 cd113 an, ind jeg111 32

7 cdilien,n, jaglio 33

8 cdilimon,s )cdilt 102

- ed113a(n. ) 09113103

10 edilimarn,d $)$ ag112 104

11 cdilision, : jegli1 105

12 cdi13m(n.h )pdi11 106

13 cd113arn,e )pd110 107

14 cd113m(n,2p )pdi12 111

15 ed113m(n, 3n ) cd111n 317

16 ed113a(n,n* )pd109 322

17 ed113e(n,nd ) sol1 in 332

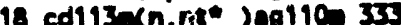

$19 \operatorname{cod} 113 \times n, 1)$ jegl13. 403

20 edi13mon, jegilim 405

21 cdi13axn, h* ipdi11m 406

$\begin{array}{rr}37 & 12 / 01 / 93 \\ 25 & 12 / 01 / 93 \\ 15 & 12 / 01 / 93 \\ 50 & 12 / 01 / 93 \\ 26 & 12 / 01 / 93 \\ 21 & 12 / 01 / 93 \\ 9 & 12 / 01 / 93 \\ 3246 & 12 / 01 / 93 \\ 64 & 12 / 01 / 93 \\ 43 & 12 / 01 / 93 \\ 43 & 12 / 01 / 93 \\ 31 & 12 / 01 / 93 \\ 81 & 12 / 01 / 93 \\ 35 & 12 / 01 / 93 \\ 13 & 12 / 01 / 93 \\ 30 & 12 / 01 / 93 \\ 21 & 12 / 01 / 93 \\ 8 & 12 / 01 / 93 \\ 64 & 12 / 01 / 93 \\ 63 & 12 / 01 / 93 \\ 31 & 12 / 01 / 93\end{array}$

$A-5.5$ 


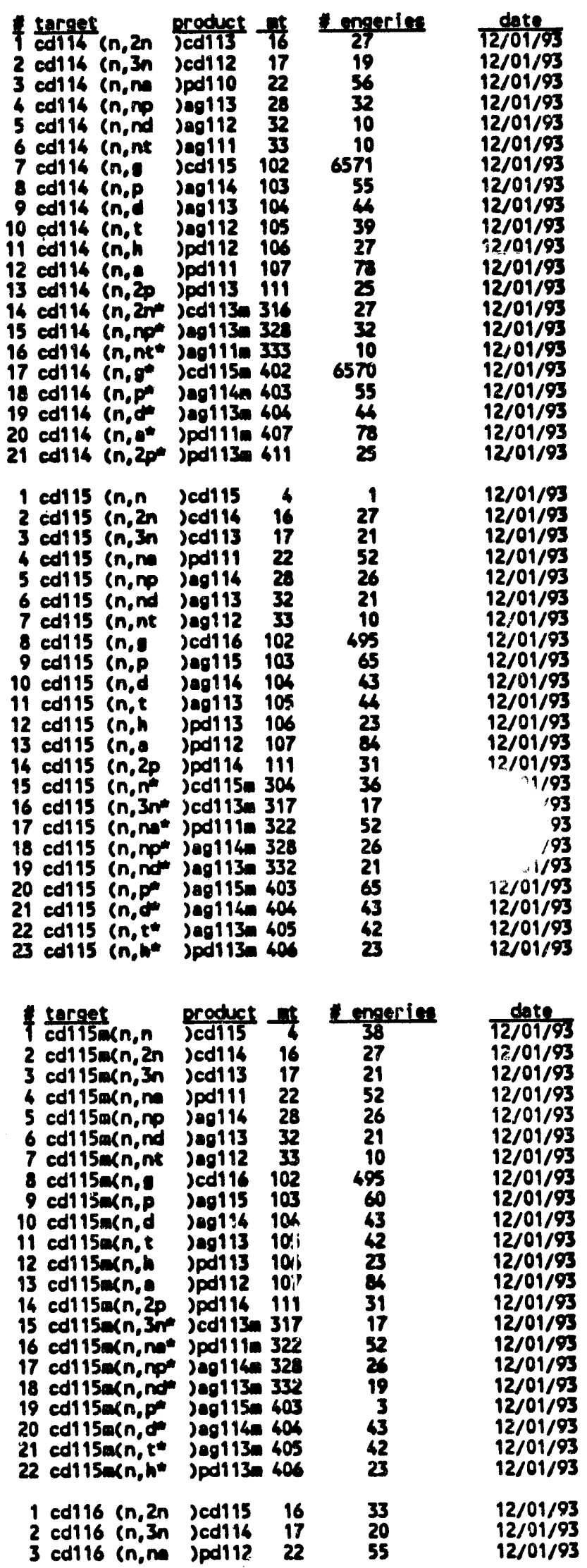

A-5. 6 


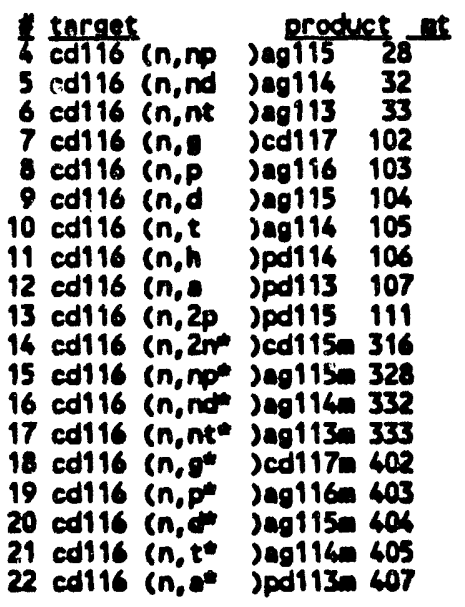

\begin{tabular}{cc} 
yenerien & dete \\
\hline 29 & $12 / 01 / 93$ \\
10 & $12 / 01 / 93$ \\
10 & $12 / 01 / 93$ \\
3335 & $12 / 01 / 93$ \\
51 & $12 / 01 / 93$ \\
43 & $12 / 01 / 93$ \\
36 & $12 / 01 / 93$ \\
23 & $12 / 01 / 93$ \\
68 & $12 / 01 / 93$ \\
19 & $12 / 01 / 93$ \\
33 & $12 / 01 / 93$ \\
29 & $12 / 01 / 93$ \\
10 & $12 / 01 / 93$ \\
10 & $12 / 01 / 93$ \\
3335 & $12 / 01 / 93$ \\
51 & $12 / 01 / 93$ \\
43 & $12 / 01 / 93$ \\
36 & $12 / 01 / 93$ \\
68 & $12 / 01 / 93$
\end{tabular}

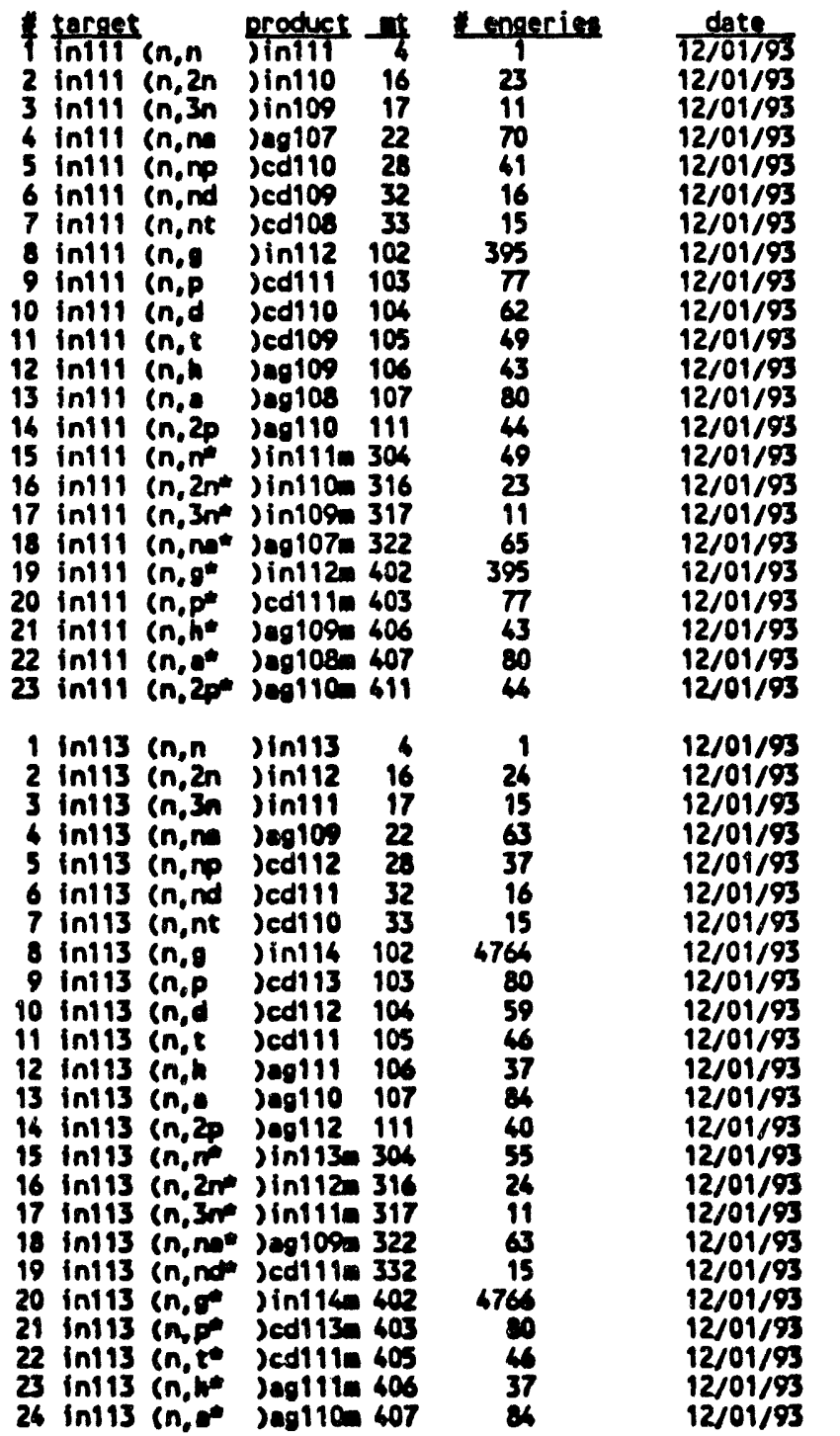




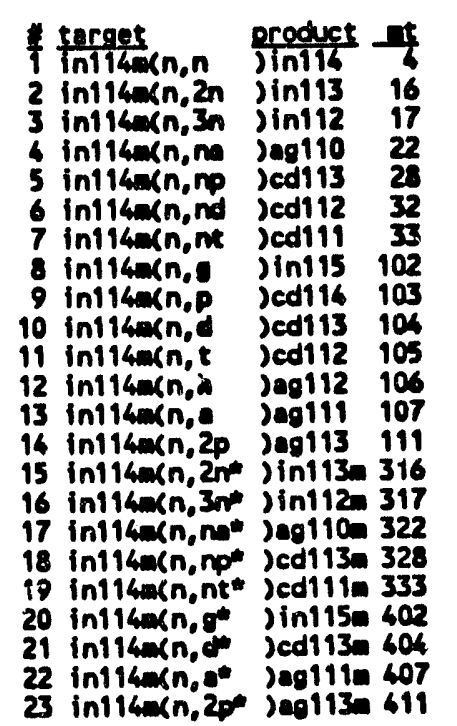

\begin{tabular}{l}
\hline engerier \\
\hline 41 \\
22 \\
13 \\
55 \\
33 \\
30 \\
13 \\
403 \\
77 \\
50 \\
51 \\
33 \\
81 \\
46 \\
22 \\
13 \\
55 \\
32 \\
13 \\
403 \\
4 \\
81 \\
46
\end{tabular}

date
$\frac{12 / 01 / 93}{12 / 01 / 93}$
$12 / 01 / 93$
$12 / 01 / 93$
$12 / 01 / 93$
$12 / 01 / 93$
$12 / 01 / 93$
$12 / 01 / 93$
$12 / 01 / 93$
$12 / 01 / 93$
$12 / 01 / 93$
$12 / 01 / 93$
$12 / 01 / 93$
$12 / 01 / 93$
$12 / 01 / 93$
$12 / 01 / 93$
$12 / 01 / 93$
$12 / 01 / 93$
$12 / 01 / 93$
$12 / 01 / 93$
$12 / 01 / 93$
$12 / 01 / 93$
$12 / 01 / 93$

$\begin{array}{ll}1 & \text { in115 (n,n )in115 } \\ 2 & \text { in115 (n,2n jini14 } \\ 3 & \text { int15 (n,3n jini13 }\end{array}$

3 int15 (n, na jegl11 22

5 ini15 (n,np) jed116 28

6 int15 ( $n$,nd )ed113 32

7 in115 (n,nt)ed112 33

- ini15 (n, jin116 102

9 ini15 (n,p )cd115 105

11 inits (n,t) cdili 105

12 in115 (n,h jag113 106

13 in115 (n,e) 09112107

16 in115 (n,2p )og114 111

15 ini15 (n, n) ini15a 304

16 in115 (n,2n*) in114m 316 17 in115 (n,3n) in113 317

18 in115 (n,no* jag111m 322

19 ini15 (n,nd )ed113m 332

20 int15 (n, $\left.g^{*}\right)$ in116m 402

21 int15 (n,p jedi15 403

22 in115 ( $n, t^{*}$ )ed113 405

23 in115 (n,h* )ag113m 406

24 int15 (n,2p*)ag114m 411

25 in115 (n, g) jin116n 702

$\begin{array}{rr}1 & 12 / 01 / 93 \\ 26 & 12 / 01 / 93 \\ 17 & 12 / 01 / 93 \\ 60 & 12 / 01 / 93 \\ 39 & 12 / 01 / 93 \\ 15 & 12 / 01 / 93 \\ 15 & 12 / 01 / 93 \\ 9374 & 12 / 01 / 93 \\ 76 & 12 / 01 / 93 \\ 54 & 12 / 01 / 93 \\ 46 & 12 / 01 / 93 \\ 31 & 12 / 01 / 93 \\ 80 & 12 / 01 / 93 \\ 35 & 12 / 01 / 93 \\ 55 & 12 / 01 / 93 \\ 26 & 12 / 01 / 93 \\ 15 & 12 / 01 / 93 \\ 60 & 12 / 01 / 93 \\ 14 & 12 / 01 / 93 \\ 9370 & 12 / 01 / 93 \\ 76 & 12 / 01 / 93 \\ 45 & 12 / 01 / 93 \\ 31 & 12 / 01 / 93 \\ 35 & 12 / 01 / 93 \\ 9375 & 12 / 01 / 93\end{array}$

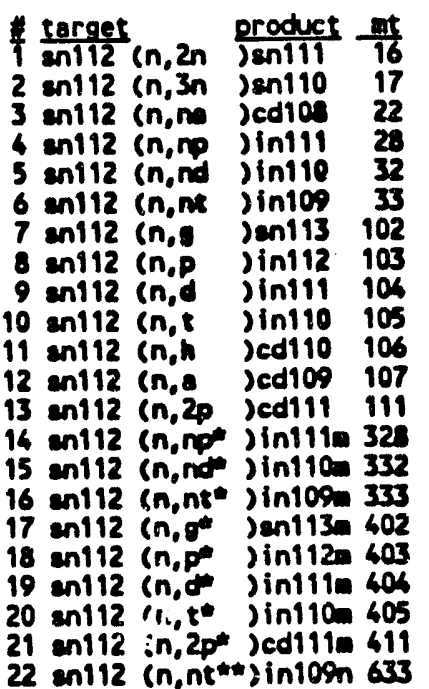

\begin{tabular}{cc} 
nengeries & date \\
\cline { 2 - 2 } 26 & $12 / 01 / 93$ \\
6 & $12 / 01 / 93$ \\
73 & $12 / 01 / 93$ \\
35 & $12 / 01 / 93$ \\
12 & $12 / 01 / 93$ \\
9 & $12 / 01 / 93$ \\
3170 & $12 / 01 / 93$ \\
80 & $12 / 01 / 93$ \\
54 & $12 / 01 / 93$ \\
43 & $12 / 01 / 93$ \\
46 & $12 / 01 / 93$ \\
81 & $12 / 01 / 93$ \\
61 & $12 / 01 / 93$ \\
35 & $12 / 01 / 93$ \\
12 & $12 / 01 / 93$ \\
9 & $12 / 01 / 93$ \\
3181 & $12 / 01 / 93$ \\
80 & $12 / 01 / 93$ \\
51 & $12 / 01 / 93$ \\
43 & $12 / 01 / 93$ \\
61 & $12 / 01 / 93$ \\
6 & $12 / 01 / 93$
\end{tabular}

$A-5.8$ 


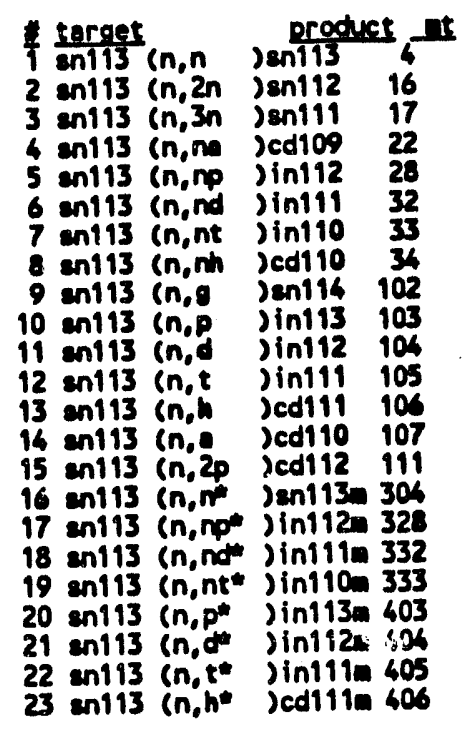

1 engerien
25
9
68
33
23
9
10
395
77
51
50
45
76
48
41
33
21
9
77
49
47
45

date
$12 / 01 / 93$
$12 / 01 / 93$
$12 / 01 / 93$
$12 / 01 / 93$
$12 / 01 / 93$
$12 / 01 / 93$
$12 / 01 / 93$
$12 / 01 / 93$
$12 / 01 / 93$
$12 / 01 / 93$
$12 / 01 / 93$
$12 / 01 / 93$
$12 / 01 / 93$
$12 / 01 / 93$
$12 / 01 / 93$
$12 / 01 / 93$
$12 / 01 / 93$
$12 / 01 / 93$
$12 / 01 / 93$
$12 / 01 / 93$
$12 / 01 / 93$
$12 / 01 / 93$
$12 / 01 / 93$

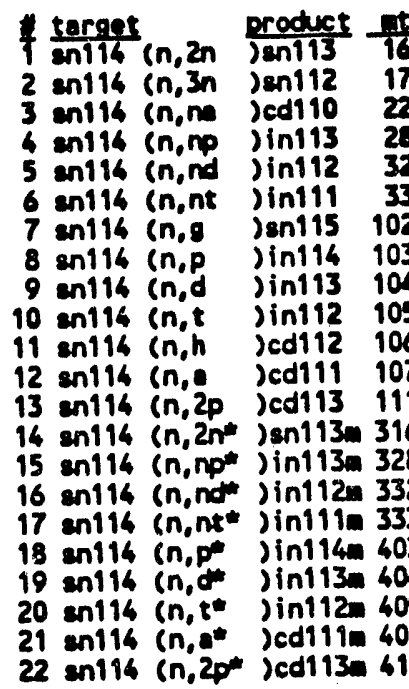

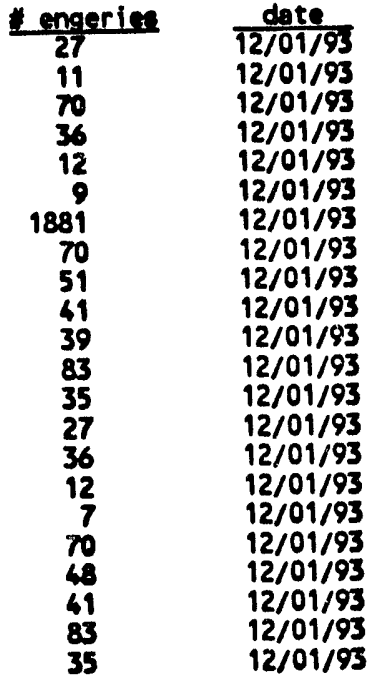

1 en115 ( $n, 2 n)$ sen114 16

2 en115 (n,3n) $\operatorname{sen} 113 \quad 17$

3 enl15 (n,ns) cdi11 22

4 en115 in, np jinill 28

5 ent15 (n,nd jint13 32

6 en115 (n,nt jin112 33

7 en115 (n,g) )en116 102

eni15 (n,p jinits 103

9 eni15 (n,d jinill 104

10 ent15 (n,t jin113 105

11 en115 (n,h )edi13 106

12 en115 (n, ) )ed112 107

13 en115 (n,2p )edi14 111

14 en115 ( $n, 3 n$ ) en113 317

15 en115 (n,n*) cd111n 322

16 en115 (n,npe in114 328

17 en115 (n, nd ) in113n 332

18 ent15 (n,nt*) int12 333

19 eni15 $(n, p)$ jin1150 403

20 en115 (n,d jini14 404

21 en115 (n, t* ) in113m 405

22 eni15 (n,h* )cdi13m 606

$\begin{array}{rr}22 & 12 / 01 / 93 \\ 11 & 12 / 01 / 93 \\ 57 & 12 / 01 / 93 \\ 28 & 12 / 01 / 93 \\ 22 & 12 / 01 / 93 \\ 9 & 12 / 01 / 93 \\ 963 & 12 / 01 / 93 \\ 81 & 12 / 01 / 93 \\ 46 & 12 / 01 / 93 \\ 47 & 12 / 01 / 93 \\ 35 & 12 / 01 / 93 \\ 80 & 12 / 01 / 93 \\ 39 & 12 / 01 / 93 \\ 11 & 12 / 01 / 93 \\ 55 & 12 / 01 / 93 \\ 26 & 12 / 01 / 93 \\ 20 & 12 / 01 / 93 \\ 9 & 12 / 01 / 93 \\ 81 & 12 / 01 / 93 \\ 48 & 12 / 01 / 93 \\ 47 & 12 / 01 / 93 \\ 35 & 12 / 01 / 93\end{array}$

A -5.9 


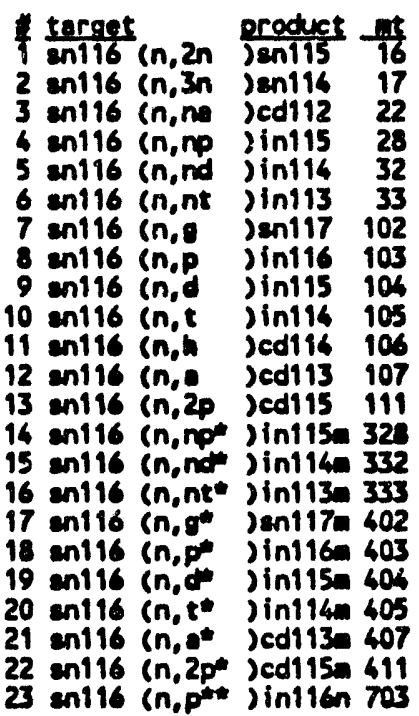

\begin{tabular}{cc} 
engerien & date \\
\cline { 2 - 2 } 26 & $12 / 01 / 93$ \\
15 & $12 / 01 / 93$ \\
65 & $12 / 01 / 93$ \\
35 & $12 / 01 / 93$ \\
11 & $12 / 01 / 93$ \\
9 & $12 / 01 / 93$ \\
2491 & $12 / 01 / 93$ \\
64 & $12 / 01 / 93$ \\
48 & $12 / 01 / 93$ \\
40 & $12 / 01 / 93$ \\
33 & $12 / 01 / 93$ \\
75 & $12 / 01 / 93$ \\
29 & $12 / 01 / 93$ \\
33 & $12 / 01 / 93$ \\
11 & $12 / 01 / 93$ \\
7 & $12 / 01 / 93$ \\
2491 & $12 / 01 / 93$ \\
64 & $12 / 01 / 93$ \\
43 & $12 / 01 / 93$ \\
38 & $12 / 01 / 93$ \\
75 & $12 / 01 / 93$ \\
29 & $12 / 01 / 93$ \\
64 & $12 / 01 / 93$
\end{tabular}

1 eni17 (n,n )en117

$\begin{array}{llll}2 & \text { en117 }(n, 2 n) \text { en } 116 \quad 16 \\ 3 & \text { en117 }(n, 3 n) \text { en115 } 17\end{array}$

$\begin{array}{lll}3 & \text { en117 ( } n, 3 n) \text { en115 } & 17 \\ 4 \text { en117 ( } n, n) & \text { cdi13 } 22\end{array}$

5 en117 (n,np) inile 28

6 en117 (n, nd ) in:15 32

7 en117 (n,nt) inils 33

- en117 (n,0 )en118 102

- sn117 (n,p) jin117 103

10 en117 (n,d jin116 104

11 eni17 $(n, t$ jini15 105

12 en117 ( $n, h$ )ed115 106

13 en117 (n, )cd116 107

14 en117 (n,2p) cd116 111

15 en117 (n, nt) jeni17a 304

16 en117 (n,n*) )cd113m 322

17 en117 (n,np) Jin116m 32

18 en117 (n,nd*) in115m 332

19 en117 (n, nt ) in114 333

20 en117 (n,p) )in117. 403

21 en117 (n, ) Jinitem 404

22 sn117 (n, t* Jini15a 405

23 en117 ( $n, h *$ ) cd115 408

26 en117 (n,now) in116n 628

25 en117 (n,du jinition 704

$\begin{array}{rr}1 & 12 / 01 / 93 \\ 26 & 12 / 01 / 93 \\ 16 & 12 / 01 / 93 \\ 54 & 12 / 01 / 93 \\ 25 & 12 / 01 / 93 \\ 21 & 12 / 01 / 93 \\ 9 & 12 / 01 / 93 \\ 3602 & 12 / 01 / 93 \\ 75 & 12 / 01 / 93 \\ 46 & 12 / 01 / 93 \\ 47 & 12 / 01 / 93 \\ 29 & 12 / 01 / 93 \\ 80 & 12 / 01 / 93 \\ 35 & 12 / 01 / 93 \\ 53 & 12 / 01 / 93 \\ 52 & 12 / 01 / 93 \\ 25 & 12 / 01 / 93 \\ 21 & 12 / 01 / 93 \\ 8 & 12 / 01 / 93 \\ 75 & 12 / 01 / 93 \\ 46 & 12 / 01 / 93 \\ 44 & 12 / 01 / 93 \\ 29 & 12 / 01 / 93 \\ 25 & 12 / 01 / 93 \\ 43 & 12 / 01 / 93\end{array}$

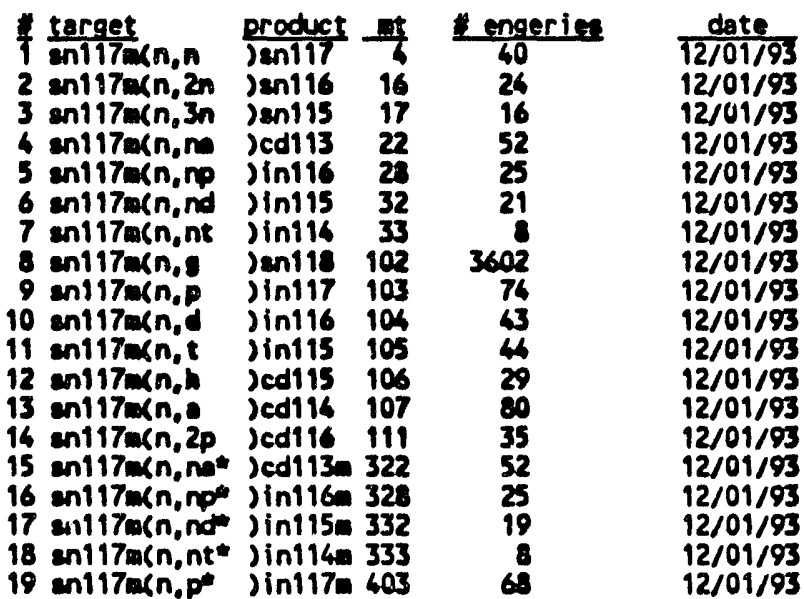

$A-5.10$ 

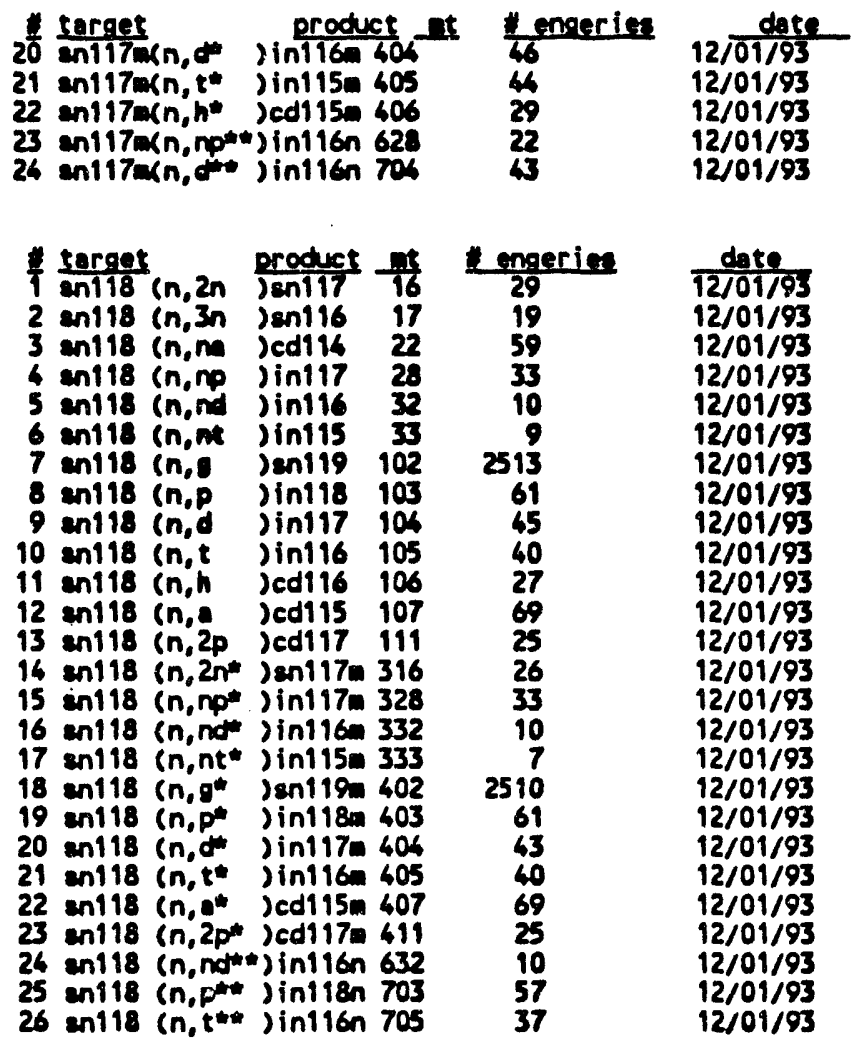

\begin{tabular}{cc} 
Lengeries & date \\
\hline 29 & $12 / 01 / 93$ \\
19 & $12 / 01 / 93$ \\
59 & $12 / 01 / 93$ \\
33 & $12 / 01 / 93$ \\
10 & $12 / 01 / 93$ \\
9 & $12 / 01 / 93$ \\
2513 & $12 / 01 / 93$ \\
61 & $12 / 01 / 93$ \\
45 & $12 / 01 / 93$ \\
40 & $12 / 01 / 93$ \\
27 & $12 / 01 / 93$ \\
69 & $12 / 01 / 93$ \\
25 & $12 / 01 / 93$ \\
26 & $12 / 01 / 93$ \\
33 & $12 / 01 / 93$ \\
10 & $12 / 01 / 93$ \\
7 & $12 / 01 / 93$ \\
2510 & $12 / 01 / 93$ \\
61 & $12 / 01 / 93$ \\
43 & $12 / 01 / 93$ \\
40 & $12 / 01 / 93$ \\
69 & $12 / 01 / 93$ \\
25 & $12 / 01 / 93$ \\
10 & $12 / 01 / 93$ \\
57 & $12 / 01 / 93$ \\
37 & $12 / 01 / 93$
\end{tabular}

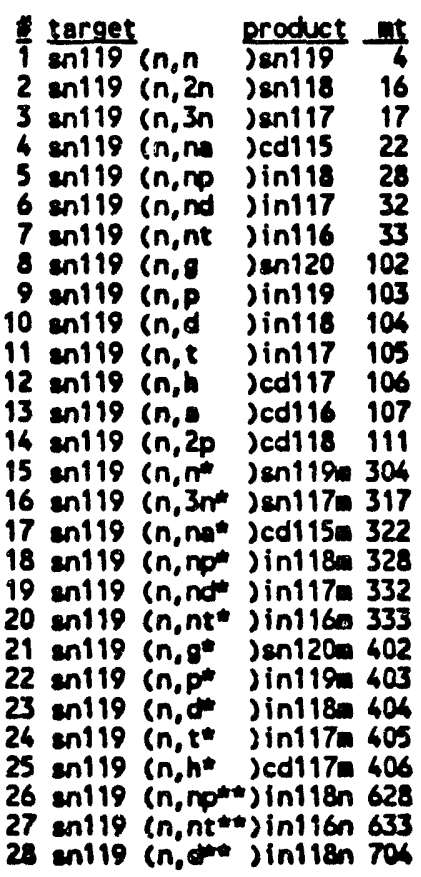

\begin{tabular}{cc} 
engeries & date \\
\hline 1 & $12 / 01 / 93$ \\
25 & $12 / 01 / 93$ \\
20 & $12 / 01 / 93$ \\
52 & $12 / 01 / 93$ \\
27 & $12 / 01 / 93$ \\
21 & $12 / 01 / 93$ \\
10 & $12 / 01 / 93$ \\
1759 & $12 / 01 / 93$ \\
68 & $12 / 01 / 93$ \\
44 & $12 / 01 / 93$ \\
45 & $12 / 01 / 93$ \\
23 & $12 / 01 / 93$ \\
83 & $12 / 01 / 93$ \\
31 & $12 / 01 / 93$ \\
55 & $12 / 01 / 93$ \\
16 & $12 / 01 / 93$ \\
50 & $12 / 01 / 93$ \\
27 & $12 / 01 / 93$ \\
19 & $12 / 01 / 93$ \\
10 & $12 / 01 / 93$ \\
1757 & $12 / 01 / 93$ \\
68 & $12 / 01 / 93$ \\
46 & $12 / 01 / 93$ \\
45 & $12 / 01 / 93$ \\
23 & $12 / 01 / 93$ \\
26 & $12 / 01 / 93$ \\
8 & $12 / 01 / 93$ \\
46 & $12 / 01 / 93$
\end{tabular}

\begin{tabular}{|c|c|c|c|c|}
\hline 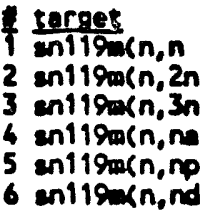 & $\begin{array}{l}\text { product } \\
\text { jen119 } \\
\text { jen118 } \\
\text { jen1117 } \\
\text { jed115 } \\
\text { jin118 } \\
\text { jin117 }\end{array}$ & $\begin{array}{l}7 \\
16 \\
17 \\
22 \\
28 \\
32\end{array}$ & $\begin{array}{c}\text { Lengeries } \\
40 \\
25 \\
16 \\
50 \\
24 \\
21\end{array}$ & $\begin{array}{l}\frac{\text { date }}{12 / 01 / 93} \\
12 / 01 / 93 \\
12 / 01 / 93 \\
12 / 01 / 93 \\
12 / 01 / 93 \\
12 / 01 / 93\end{array}$ \\
\hline
\end{tabular}

A-5. 11 


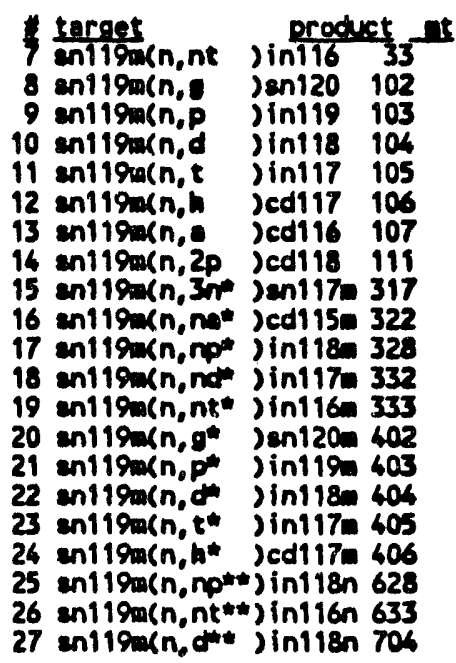

10
10
1759
68
44
45
23
83
31
16
50
27
19
10
177
6
44
45
23
26
8
61

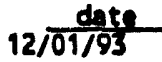

$12 / 01 / 9$

$12 / 01 / 93$

$12 / 01 / 93$

$12 / 01 / 93$

$12 / 01 / 93$

$12 / 01 / 93$

$12 / 01 / 93$

12/01/93

$12 / 01 / 93$

$12 / 01 / 93$

$12 / 01 / 93$

12/01/93

$12 / 01 / 93$

$12 / 01 / 93$

$12 / 01 / 93$

$12 / 01 / 93$

12/01/93

$12 / 01 / 93$

12/01/93

27 enl19wan, dot jinilen 70

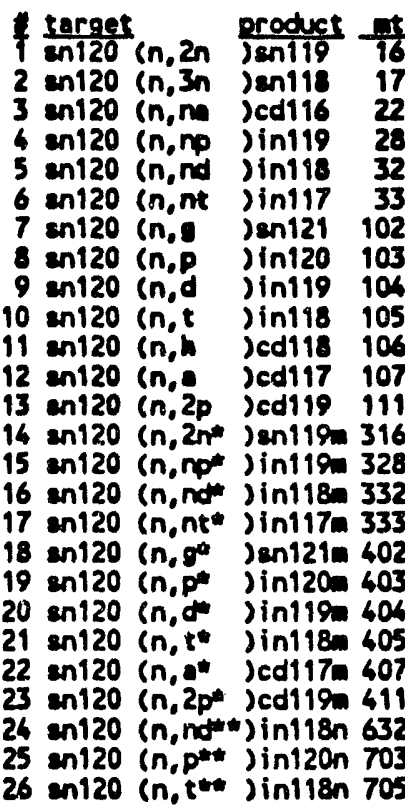

\begin{tabular}{cc} 
engerien & date \\
\hline 27 & $12 / 01 / 93$ \\
19 & $12 / 01 / 93$ \\
56 & $12 / 01 / 93$ \\
31 & $12 / 01 / 93$ \\
10 & $12 / 01 / 93$ \\
9 & $12 / 01 / 93$ \\
5780 & $12 / 01 / 93$ \\
53 & $12 / 01 / 93$ \\
43 & $12 / 01 / 93$ \\
37 & $12 / 01 / 93$ \\
19 & $12 / 01 / 93$ \\
63 & $12 / 01 / 93$ \\
19 & $12 / 01 / 93$ \\
27 & $12 / 01 / 93$ \\
29 & $12 / 01 / 93$ \\
10 & $12 / 01 / 93$ \\
7 & $12 / 01 / 93$ \\
5778 & $12 / 01 / 93$ \\
53 & $12 / 01 / 93$ \\
43 & $12 / 01 / 93$ \\
37 & $12 / 01 / 93$ \\
63 & $12 / 01 / 93$ \\
19 & $12 / 01 / 93$ \\
8 & $12 / 01 / 93$ \\
53 & $12 / 01 / 93$ \\
37 & $12 / 01 / 93$ \\
\hline
\end{tabular}

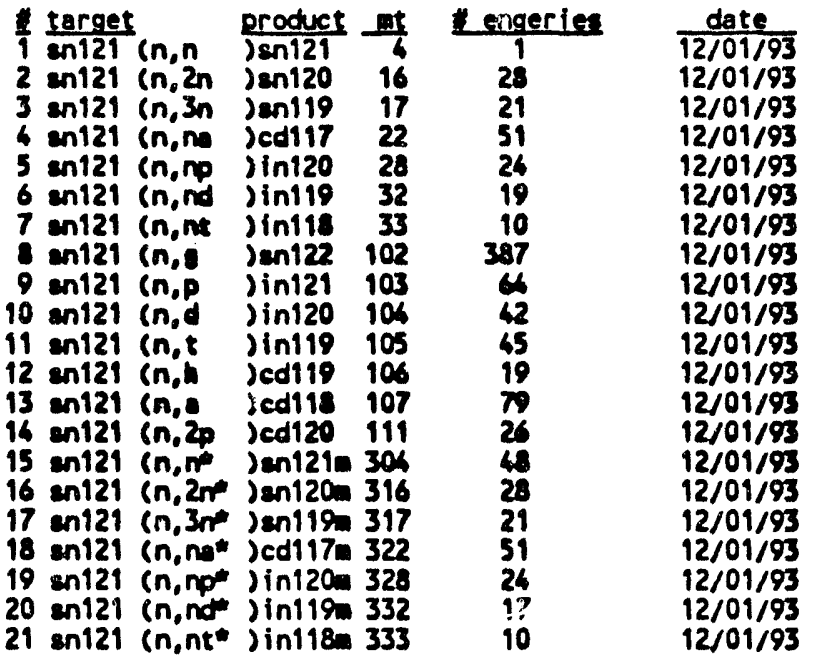

$A-5.12$ 


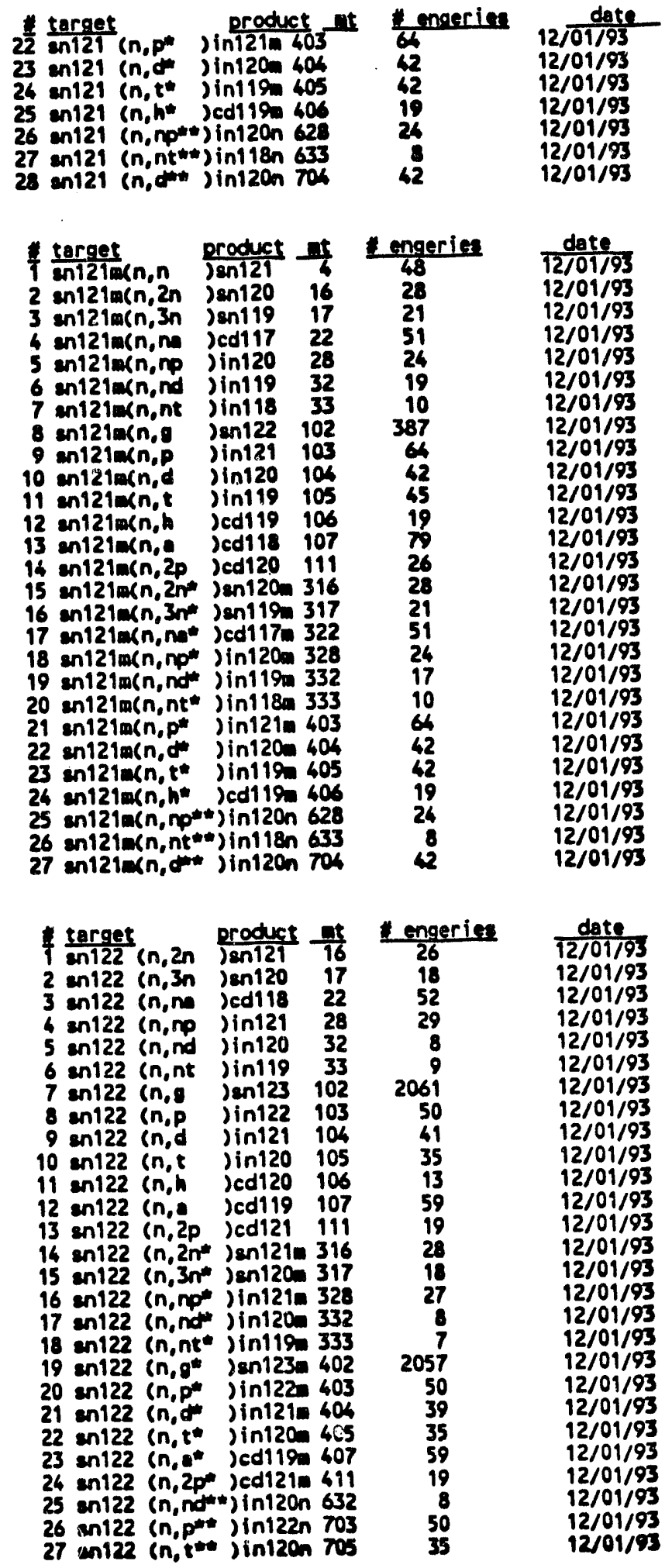

$A-5.13$ 

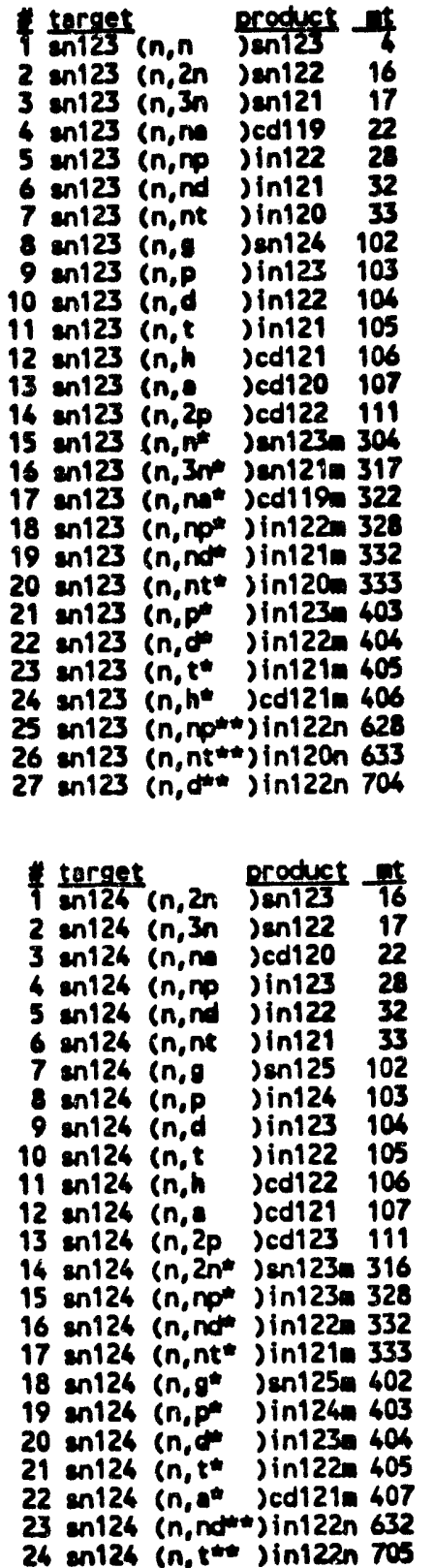

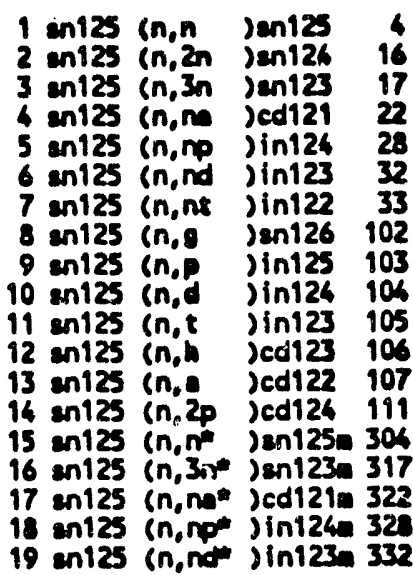

\begin{tabular}{cc} 
Lngeries & date \\
\cline { 1 - 2 } & $12 / 01 / 93$ \\
36 & $12 / 01 / 93$ \\
22 & $12 / 01 / 93$ \\
50 & $12 / 01 / 93$ \\
21 & $12 / 01 / 93$ \\
18 & $12 / 01 / 93$ \\
9 & $12 / 01 / 93$ \\
434 & $12 / 01 / 93$ \\
58 & $12 / 01 / 93$ \\
41 & $12 / 01 / 93$ \\
44 & $12 / 01 / 93$ \\
23 & $12 / 01 / 93$ \\
64 & $12 / 01 / 93$ \\
26 & $12 / 01 / 93$ \\
50 & $12 / 01 / 93$ \\
22 & $12 / 01 / 93$ \\
50 & $12 / 01 / 93$ \\
21 & $12 / 01 / 93$ \\
16 & $12 / 01 / 93$ \\
9 & $12 / 01 / 93$ \\
58 & $12 / 01 / 93$ \\
61 & $12 / 01193$ \\
41 & $12 / 01 / 93$ \\
23 & $12 / 01 / 93$ \\
21 & $12 / 01 / 93$ \\
9 & $12 / 01 / 93$ \\
38 & $12 / 01 / 93$
\end{tabular}

\begin{tabular}{cc} 
engerien & date \\
\hline 33 & $12 / 01 / 93$ \\
19 & $12 / 01 / 93$ \\
34 & $12 / 01 / 93$ \\
27 & $12 / 01 / 93$ \\
8 & $12 / 01 / 93$ \\
9 & $12 / 01 / 93$ \\
1882 & $12 / 01 / 93$ \\
48 & $12 / 01 / 93$ \\
40 & $12 / 01 / 93$ \\
33 & $12 / 01 / 93$ \\
17 & $12 / 01 / 93$ \\
64 & $12 / 01 / 93$ \\
13 & $12 / 01 / 93$ \\
33 & $12 / 01 / 93$ \\
25 & $12 / 01 / 93$ \\
8 & $12 / 01 / 93$ \\
7 & $12 / 01 / 93$ \\
1884 & $12 / 01 / 93$ \\
65 & $12 / 01 / 93$ \\
38 & $12 / 01 / 93$ \\
33 & $12 / 01 / 13$ \\
64 & $12 / 01 / 93$ \\
6 & $12 / 01 / 93$ \\
33 & $12 / 01 / 93$
\end{tabular}

12/01/93

12/01/93

$12 / 01 / 93$

$12 / 01 / 93$

12/01/93

$12 / 01 / 93$

$12 / 01 / 93$

12/01/93

$12 / 01 / 93$

12/01/93

$12 / 01 / 93$

$12 / 01 / 93$

$12 / 01 / 93$

$12 / 01 / 93$

$12 / 01 / 93$

$12 / 01 / 93$

$12 / 01 / 93$

$12 / 01 / 93$

$12 / 01 / 93$ 


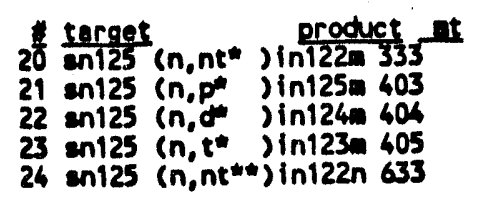

\begin{tabular}{cc} 
Henreries & date \\
\hline 9 & $12 / 01 / 93$ \\
54 & $12 / 01 / 93$ \\
37 & $12 / 01 / 93$ \\
39 & $12 / 01 / 93$ \\
7 & $12 / 01 / 93$
\end{tabular}

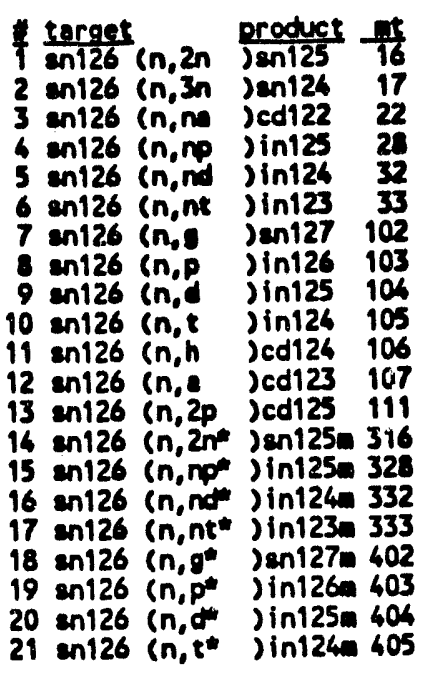

\begin{tabular}{c} 
engerin \\
\hline 36 \\
20 \\
52 \\
26 \\
7 \\
8 \\
399 \\
45 \\
37 \\
34 \\
11 \\
56 \\
4 \\
36 \\
26 \\
7 \\
8 \\
399 \\
42 \\
37 \\
32
\end{tabular}

date
$\frac{12 / 01 / 93}{12 / 01 / 93}$
$12 / 01 / 93$
$12 / 01 / 93$
$12 / 01 / 93$
$12 / 01 / 93$
$12 / 01 / 93$
$12 / 01 / 93$
$12 / 01 / 93$
$12 / 01 / 93$
$12 / 01 / 93$
$12 / 01 / 93$
$12 / 01 / 93$
$12 / 01 / 93$
$12 / 01 / 93$
$12 / 01 / 93$
$12 / 01 / 93$
$12 / 01 / 93$
$12 / 01 / 93$
$12 / 01 / 93$
$12 / 01 / 93$

1 sb119 (n,2n) 2 abl18 16

2 sb119 (n, 3n )sb117 17

3 sbilg (n,m jini1s 22

4 bile (n,np) jen118 28

5 ab119 ( $n, n d$ ) en117 32

6 sbl19 (n,nt) sn116 33

7 sb119 (n, )sb120 102

8 sb119 (n,p ) en119 103

9 sbil9 (n,d )enile 104

10 sb119 (n,t) en117 105

11 sbil9 $(n, h$ )in117 106

12 sb119 (n,e) jint16 107

13 sb119 (n,2p jin118 111

16 sb119 (n, 2n*)sb118m 316

15 sb119 (n,3n) sbi117 317

16 sb119 (n, na*) in115m 322

17 sb119 (n, nd ) sn117n 332

18 sb119 (n, ) sbi20 402

19 sb119 (n,p ) en119 403

20 sb119 (n,t*) sn117. 405

21 obl19 (n,h* Jin117m 406

22 b119 (n,** jin116m 407

23 sb119 (n,2p jin118m 411

26 ab119 (n,ate jini16n 707

25 ebl19 (n,2pen) inilea 711

$24 \quad 12 / 01 / 93$

$12 / 01 / 93$

$12 / 01 / 93$

$12 / 01 / 93$

$12 / 01 / 93$

$12 / 01 / 93$

$12 / 01 / 93$

12/01/93

$12 / 01 / 93$

$12 / 01 / 93$

12/01/93

$12 / 01 / 93$

12/01/93

$12 / 01 / 93$

$12 / 01 / 93$

$12 / 01 / 93$

$12 / 01 / 93$

$12 / 01 / 93$

$12 / 01 / 93$

$12 / 01 / 93$

$12 / 01 / 93$

$12 / 01 / 93$

$12 / 01 / 93$

$12 / 01 / 93$

$12 / 01 / 93$

\begin{tabular}{|c|c|c|c|c|}
\hline 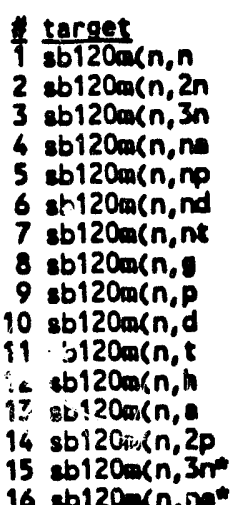 & $\begin{array}{l}\text { product } \\
\text { jeb120 } \\
\text { job119 } \\
\text { jeb1118 } \\
\text { jin1116 } \\
\text { jen1119 } \\
\text { jen118 } \\
\text { jen117 } \\
\text { jeb121 } \\
\text { jen120 } \\
\text { jen119 } \\
\text { jen118 } \\
\text { jin118 } \\
\text { jin117 } \\
\text { jin119 } \\
\text { job118m }\end{array}$ & $\begin{array}{l}7 \\
4 \\
16 \\
17 \\
22 \\
28 \\
32 \\
33 \\
102 \\
103 \\
104 \\
105 \\
106 \\
107 \\
111\end{array}$ & $\begin{array}{c}\text { Lngeries } \\
41 \\
25 \\
16 \\
66 \\
35 \\
34 \\
25 \\
396 \\
76 \\
58 \\
59 \\
35\end{array}$ & $\begin{array}{l}\text { date } \\
12 / 01 / 93 \\
12 / 01 / 93 \\
12 / 01 / 93 \\
12 / 01 / 93 \\
12 / 01 / 93 \\
12 / 01 / 93 \\
12 / 01 / 93 \\
12 / 01 / 93 \\
12 / 01 / 93 \\
12 / 01 / 93 \\
12 / 01 / 93 \\
12 / 01 / 93 \\
12 / 01 / 93 \\
12 / 01 / 93 \\
12 / 01 / 93 \\
12 / 01 / 93\end{array}$ \\
\hline
\end{tabular}




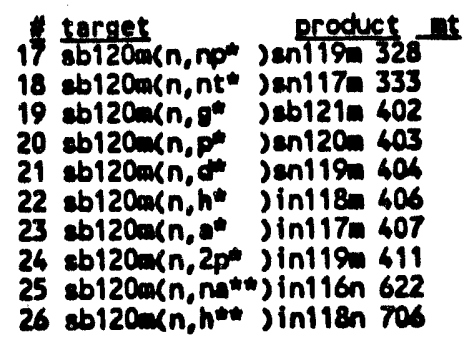

\begin{tabular}{cc} 
fongeries & date \\
\hline 35 & $12 / 01 / 93$ \\
25 & $12 / 01 / 93$ \\
396 & $12 / 01 / 93$ \\
76 & $12 / 01 / 93$ \\
56 & $12 / 01 / 93$ \\
35 & $12 / 01 / 93$ \\
79 & $12 / 01 / 93$ \\
46 & $12 / 01 / 93$ \\
66 & $12 / 01 / 93$ \\
35 & $12 / 01 / 93$
\end{tabular}

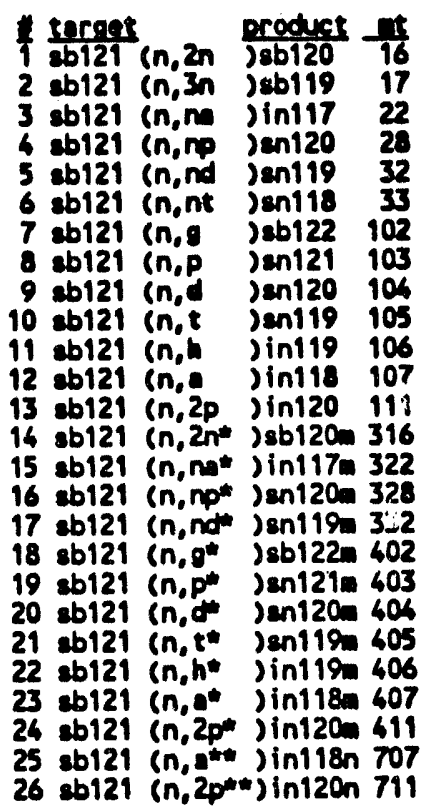

\begin{tabular}{cc} 
nengerin & date \\
\cline { 2 - 2 } 27 & $12 / 01 / 93$ \\
19 & $12 / 01 / 93$ \\
61 & $12 / 01 / 93$ \\
61 & $12 / 101 / 93$ \\
18 & $12 / 01 / 93$ \\
26 & $12 / 01 / 93$ \\
1270 & $12 / 01 / 93$ \\
84 & $12 / 01 / 93$ \\
59 & $12 / 01 / 93$ \\
51 & $12 / 01 / 93$ \\
31 & $12 / 01 / 93$ \\
80 & $12 / 01 / 93$ \\
33 & $12 / 01 / 93$ \\
27 & $12 / 01 / 93$ \\
61 & $12 / 01 / 93$ \\
41 & $12 / 01 / 93$ \\
18 & $12 / 01 / 93$ \\
1276 & $12 / 01 / 93$ \\
85 & $12 / 01 / 93$ \\
59 & $12 / 01 / 93$ \\
48 & $12 / 01 / 93$ \\
31 & $12 / 01 / 93$ \\
79 & $12 / 01 / 93$ \\
33 & $12 / 01 / 93$ \\
80 & $12 / 01 / 93$ \\
33 & $12 / 01 / 93$
\end{tabular}

\begin{tabular}{|c|c|c|c|c|c|}
\hline 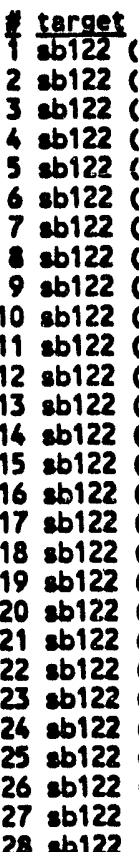 & 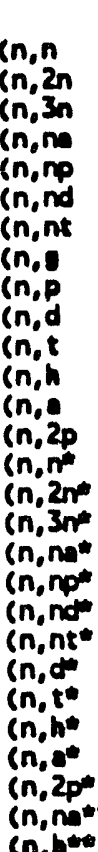 & 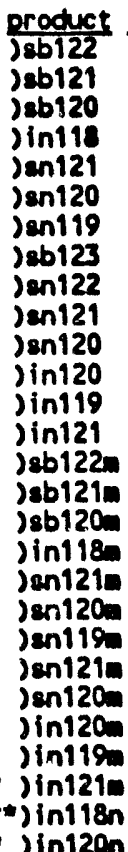 & $\begin{array}{c}7 \\
6 \\
16 \\
17 \\
22 \\
28 \\
32 \\
33 \\
102 \\
103 \\
104 \\
105 \\
106 \\
107 \\
111 \\
304 \\
316 \\
317 \\
322 \\
328 \\
332 \\
333 \\
404 \\
405 \\
406\end{array}$ & $\begin{array}{c}\text { Lengeries } \\
1 \\
31 \\
17 \\
61 \\
36 \\
32 \\
26 \\
401 \\
78 \\
56 \\
58 \\
27 \\
82 \\
37 \\
53 \\
31 \\
17 \\
61 \\
36 \\
32 \\
26 \\
56 \\
58 \\
27 \\
82 \\
37 \\
57 \\
27\end{array}$ & $\begin{array}{l}\text { date } \\
12 / 01 / 93 \\
12 / 01 / 93 \\
12 / 01 / 93 \\
12 / 01 / 93 \\
12 / 01 / 93 \\
12 / 01 / 93 \\
12 / 01 / 93 \\
12 / 01 / 93 \\
12 / 01 / 93 \\
12 / 01 / 93 \\
12 / 01 / 93 \\
12 / 01 / 93 \\
12 / 01 / 93 \\
12 / 01 / 93 \\
12 / 01 / 93 \\
12 / 01 / 93 \\
12 / 01 / 93 \\
12 / 01 / 93 \\
12 / 01 / 93 \\
12 / 01 / 93 \\
12 / 01 / 93 \\
12 / 01 / 93 \\
12 / 01 / 93 \\
12 / 01 / 93 \\
12 / 01 / 93 \\
12 / 01 / 93 \\
12 / 01 / 93 \\
12 / 01 / 93\end{array}$ \\
\hline
\end{tabular}




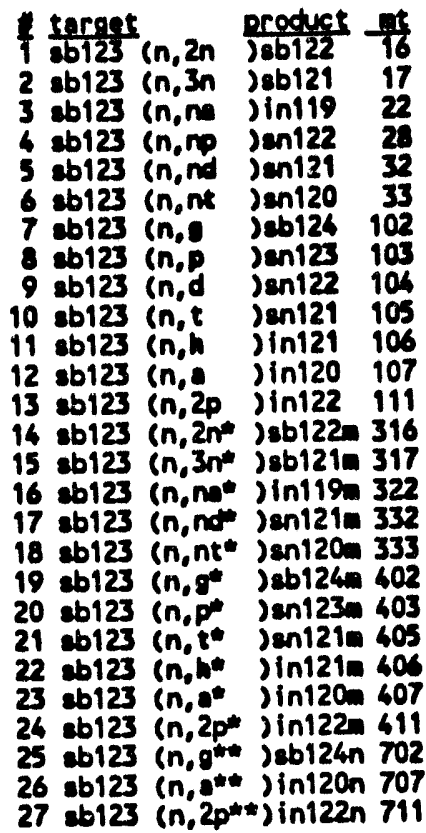

\begin{tabular}{cc} 
nengrie & date \\
\hline 26 & $12 / 01 / 93$ \\
20 & $12 / 01 / 93$ \\
59 & $12 / 01 / 93$ \\
39 & $12 / 01 / 93$ \\
16 & $12 / 01 / 93$ \\
25 & $12 / 01 / 93$ \\
8359 & $12 / 01 / 93$ \\
73 & $12 / 01 / 93$ \\
57 & $12 / 01 / 93$ \\
49 & $12 / 01 / 93$ \\
25 & $12 / 01 / 93$ \\
72 & $12 / 01 / 93$ \\
28 & $12 / 01193$ \\
26 & $12 / 01 / 93$ \\
20 & $12 / 01 / 93$ \\
57 & $12 / 01 / 93$ \\
16 & $12 / 01 / 93$ \\
25 & $12 / 01 / 93$ \\
8361 & $12 / 01 / 93$ \\
73 & $12 / 01 / 93$ \\
49 & $12 / 01 / 93$ \\
25 & $12 / 01 / 93$ \\
71 & $12 / 01 / 93$ \\
28 & $12 / 01 / 93$ \\
8358 & $12 / 01 / 93$ \\
72 & $12 / 01 / 93$ \\
28 & $12 / 01 / 93$
\end{tabular}

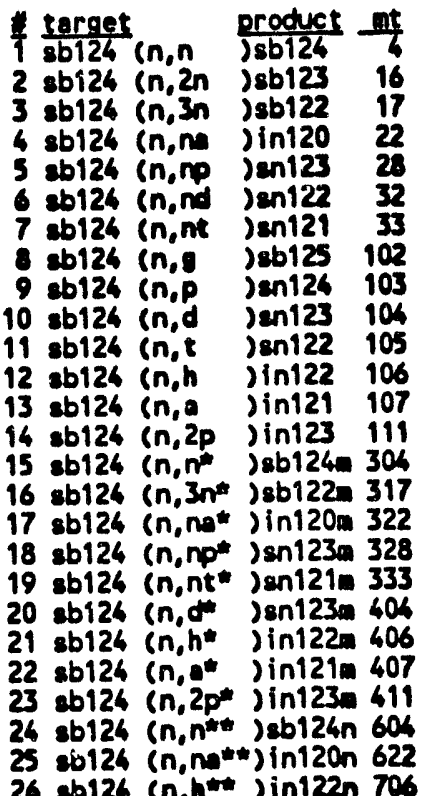

\begin{tabular}{cc} 
Eengeries & date \\
\cline { 2 - 2 } 1 & $12 / 01 / 93$ \\
27 & $12 / 01 / 93$ \\
17 & $12 / 01 / 93$ \\
57 & $12 / 01 / 93$ \\
35 & $12 / 01 / 93$ \\
31 & $12 / 01 / 93$ \\
23 & $12 / 01 / 93$ \\
396 & $12 / 01 / 93$ \\
77 & $12 / 01 / 93$ \\
54 & $12 / 01 / 93$ \\
55 & $12 / 01 / 93$ \\
21 & $12 / 01 / 93$ \\
77 & $12 / 01 / 93$ \\
33 & $12 / 01 / 93$ \\
50 & $12 / 01 / 93$ \\
17 & $12 / 01 / 93$ \\
57 & $12 / 01 / 93$ \\
35 & $12 / 01 / 93$ \\
23 & $12 / 01193$ \\
56 & $12 / 01 / 93$ \\
21 & $12 / 01 / 93$ \\
77 & $12 / 01 / 93$ \\
33 & $12 / 01 / 93$ \\
50 & $12 / 01 / 93$ \\
57 & $12 / 01 / 93$ \\
21 & $12 / 01 / 93$
\end{tabular}

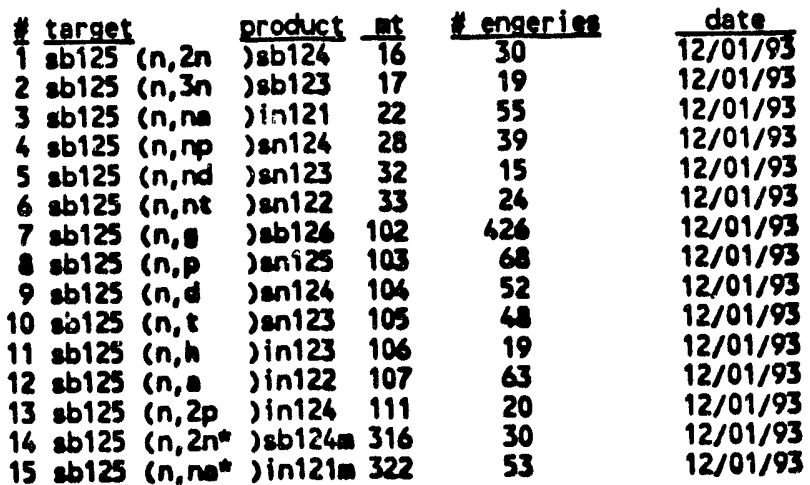




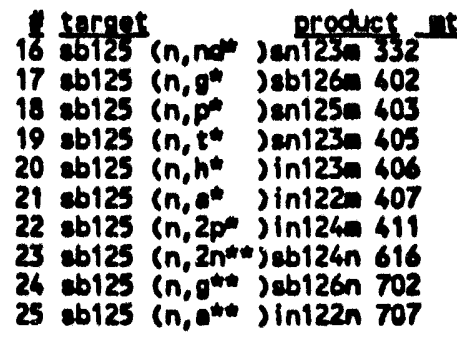

$\begin{array}{cc}\text { 4moerine } & \text { digte- } \\ 45 & 12 / 01 / 93 \\ 437 & 12 / 01 / 93 \\ 68 & 12 / 01 / 93 \\ 43 & 12 / 01 / 93 \\ 19 & 12 / 01 / 93 \\ 62 & 12 / 01 / 93 \\ 20 & 12 / 01 / 93 \\ 30 & 12 / 01 / 93 \\ 437 & 12 / 01 / 93 \\ 63 & 12 / 01 / 93\end{array}$

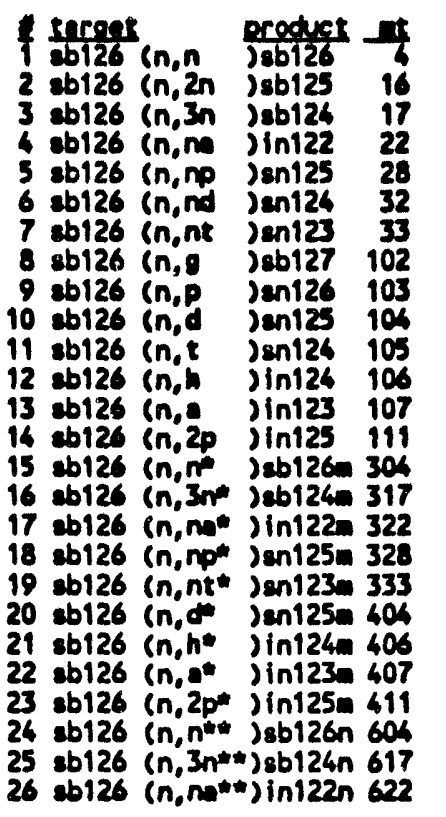

\begin{tabular}{cc} 
Lengerien & date \\
\cline { 2 - 2 } 20 & $12 / 01 / 93$ \\
29 & $12 / 01 / 93$ \\
18 & $12 / 01 / 93$ \\
52 & $12 / 01 / 93$ \\
31 & $12 / 01 / 93$ \\
30 & $12 / 01 / 93$ \\
22 & $12 / 01 / 93$ \\
450 & $12 / 01 / 93$ \\
81 & $12 / 01 / 93$ \\
49 & $12 / 01 / 93$ \\
52 & $12 / 01 / 95$ \\
17 & $12 / 01 / 93$ \\
75 & $12 / 01 / 93$ \\
26 & $12 / 01 / 93$ \\
45 & $12 / 01 / 93$ \\
18 & $12 / 01 / 93$ \\
52 & $12 / 01 / 93$ \\
31 & $12 / 01 / 93$ \\
22 & $12 / 01 / 93$ \\
49 & $12 / 01 / 93$ \\
17 & $12 / 01 / 93$ \\
75 & $12 / 01 / 93$ \\
26 & $12 / 01 / 93$ \\
49 & $12 / 01 / 93$ \\
18 & $12 / 01 / 93$ \\
52 & $12 / 01 / 93$
\end{tabular}

\begin{tabular}{|c|c|c|c|c|c|}
\hline 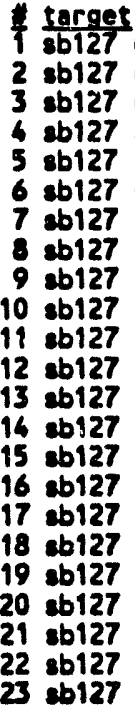 & 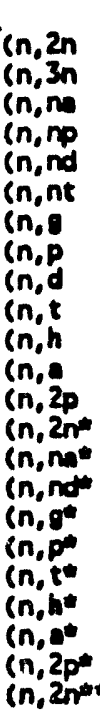 & 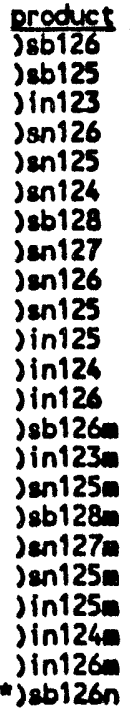 & $\begin{array}{c}17 \\
16 \\
17 \\
22 \\
28 \\
32 \\
33 \\
102 \\
103 \\
104 \\
105 \\
106 \\
107 \\
111 \\
316 \\
322 \\
332 \\
402 \\
403 \\
405 \\
406 \\
407 \\
414\end{array}$ & $\begin{array}{c}\text { Angerie } \\
38 \\
22 \\
56 \\
36 \\
15 \\
23 \\
406 \\
64 \\
51 \\
46 \\
13 \\
57 \\
9 \\
30 \\
52 \\
15 \\
406 \\
64 \\
46 \\
13 \\
57 \\
9 \\
38\end{array}$ & $\begin{array}{l}\text { date } \\
12 / 01 / 93 \\
12 / 01 / 93 \\
12 / 01 / 93 \\
12 / 01 / 93 \\
12 / 01 / 93 \\
12 / 01 / 93 \\
12 / 01 / 93 \\
12 / 01 / 93 \\
12 / 01 / 93 \\
12 / 01 / 93 \\
12 / 01 / 93 \\
12 / 01 / 93 \\
12 / 01 / 93 \\
12 / 01 / 93 \\
12 / 01 / 93 \\
12 / 01 / 93 \\
12 / 01 / 93 \\
12 / 01 / 93 \\
12 / 01 / 93 \\
12 / 01 / 93 \\
12 / 01 / 93 \\
12 / 01 / 93 \\
12 / 01 / 93\end{array}$ \\
\hline $\begin{array}{ll}1 & t e 118 \\
2 & t e 118 \\
3 & t e 118 \\
4 & t e 118 \\
5 & t e 118 \\
6 & t e 118 \\
7 & t e 118 \\
8 & t e 118\end{array}$ & $\begin{array}{l}3 n \\
n \\
n p \\
n p d \\
n d \\
n t \\
n n \\
n\end{array}$ & $\begin{array}{l}\text { Jtel17 } \\
\text { jte116 } \\
\text { jen116 } \\
\text { job117 } \\
\text { jeb116 } \\
\text { jeb115 } \\
\text { jen115 } \\
\text { Jte119 }\end{array}$ & $\begin{array}{l}16 \\
17 \\
22 \\
28 \\
32 \\
33\end{array}$ & $\begin{array}{r}22 \\
9 \\
88 \\
40 \\
16 \\
12 \\
8 \\
384\end{array}$ & $\begin{array}{l}12 / 01 / 93 \\
12 / 01 / 93 \\
12 / 01 / 93 \\
12 / 01 / 93 \\
12 / 01 / 93 \\
12 / 01 / 93 \\
12 / 01 / 93 \\
12 / 01 / 93\end{array}$ \\
\hline
\end{tabular}




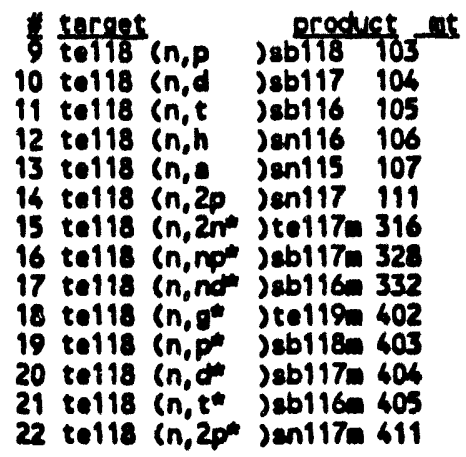

\begin{tabular}{cc} 
tengeries & date \\
\hline 81 & $12 / 01 / 93$ \\
58 & $12 / 01 / 93$ \\
47 & $12 / 01 / 93$ \\
53 & $12 / 01 / 93$ \\
79 & $12 / 01 / 93$ \\
67 & $12 / 01 / 93$ \\
22 & $12 / 01 / 93$ \\
40 & $12 / 01 / 93$ \\
16 & $12 / 01 / 93$ \\
386 & $12 / 01 / 93$ \\
80 & $12 / 01 / 93$ \\
58 & $12 / 01 / 93$ \\
44 & $12 / 01 / 93$ \\
47 & $12 / 01 / 93$
\end{tabular}

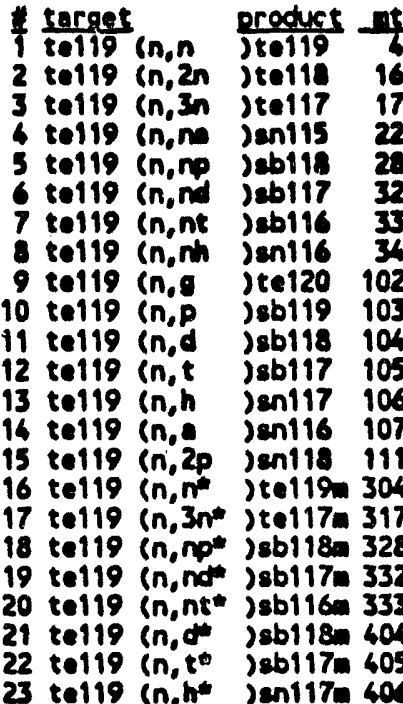

\begin{tabular}{c} 
engerie \\
\hline 1 \\
21 \\
13 \\
86 \\
35 \\
27 \\
13 \\
17 \\
398 \\
75 \\
55 \\
54 \\
50 \\
75 \\
51 \\
38 \\
9 \\
35 \\
27 \\
11 \\
53 \\
54 \\
50
\end{tabular}

$\frac{\text { date }}{12 / 01 / 93}$

$12 / 01 / 93$

$12 / 01 / 93$

$12 / 01 / 93$

$12 / 01 / 93$

$12 / 01 / 93$

$12 / 01 / 93$

$12 / 01 / 93$

$12 / 01 / 93$

$12 / 01 / 93$

$12 / 01 / 93$

$12 / 01 / 93$

$12 / 01 / 93$

$12 / 01 / 93$

$12 / 01 / 93$

$12 / 01 / 93$

$12 / 01 / 93$

$12 / 01 / 93$

12/01/93

$12 / 01 / 93$

$12 / 01 / 93$

$12 / 01 / 93$

1 tel19m(n,n )to119

2 tel19m(n,2n jte118

4 tol19m(n,n) )en115 22

5 tei19m(n,np) sbi18 28

6 tel19m(n,nd )sb117 32

7 tel19m(n,nt)sb116 33

8 tel19men,nh )an116 34

9 tell9men,g ) tel20 102

10 tell9m(n,p) seb119 103

11 tel19m(n,d )sbi18 104

12 tel19men.s sbb117 105

13 tel19m(n, )eni17 106

16 tel19man, jeni16 107

15 tel19e(n,20) an118 111

16 tel19m(n, 3n ) tel17m 317

17 tel19m(n,np* )sb118m 320

18 tel19unn, not sebi17a 332

19 tel19m(n,nt* jeb116a 333

20 tol19men, ) sbil18m 40

21 tel19-(n, tob1170 405

22 tel19ankn, ien117n 406

$12 / 01 / 93$

$\begin{array}{cc}39 & 12 / 01 / 93 \\ 21 & 12 / 01 / 93 \\ 9 & 12 / 01 / 93 \\ 84 & 12 / 01 / 93 \\ 34 & 12 / 01 / 93 \\ 25 & 12 / 01 / 93 \\ 11 & 12 / 01 / 93 \\ 17 & 12 / 01 / 93 \\ 398 & 12 / 01 / 93 \\ 75 & 12 / 01 / 93 \\ 53 & 12 / 01 / 93 \\ 51 & 12 / 01 / 93 \\ 50 & 12 / 01 / 93 \\ 75 & 12 / 01 / 93 \\ 51 & 12 / 01 / 93 \\ 9 & 12 / 1193 \\ 32 & 12 / 01193 \\ 25 & 12 / 01 / 93 \\ 10 & 12 / 01 / 93 \\ 53 & 12 / 01 / 93 \\ 51 & 12 / 01 / 93 \\ 50 & 12 / 01 / 93\end{array}$

\begin{tabular}{|c|c|c|c|}
\hline 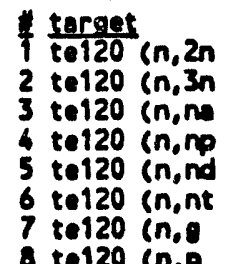 & $\begin{array}{l}\text { product } \\
\text { prol19 } \\
\text { jte118 } \\
\text { jen116 } \\
\text { job119 } \\
\text { jeb118 } \\
\text { jsb117 } \\
\text { jte121 } \\
\text { jeb120 }\end{array}$ & $\begin{array}{l}-\frac{m}{16} \\
17 \\
22 \\
28 \\
32 \\
33 \\
102 \\
103\end{array}$ & $\begin{array}{c}1 \text { engeries } \\
27 \\
11 \\
87 \\
38 \\
16 \\
12 \\
446 \\
81\end{array}$ \\
\hline
\end{tabular}

$A-5.19$ 


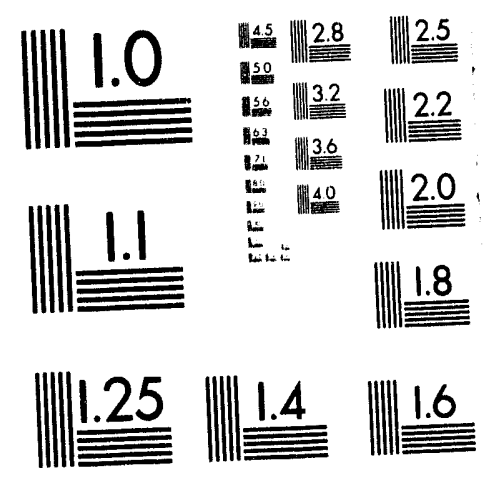




$$
\begin{aligned}
& \mathbf{N} \\
& \stackrel{o}{\omega}
\end{aligned}
$$




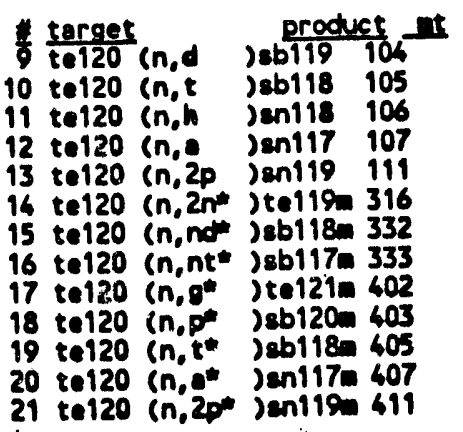

\begin{tabular}{ll} 
Hengeries & date \\
\hline 53 & $12 / 01 / 93$ \\
46 & $12 / 01 / 93$ \\
46 & $12 / 01 / 93$ \\
81 & $12 / 01 / 93$ \\
39 & $12 / 01 / 93$ \\
24 & $12 / 01 / 93$ \\
16 & $12 / 01 / 93$ \\
12 & $12 / 01 / 93$ \\
44 & $12 / 01 / 93$ \\
81 & $12 / 01 / 93$ \\
43 & $12 / 01 / 93$ \\
82 & $12 / 01 / 93$ \\
39 & $12 / 01 / 93$
\end{tabular}

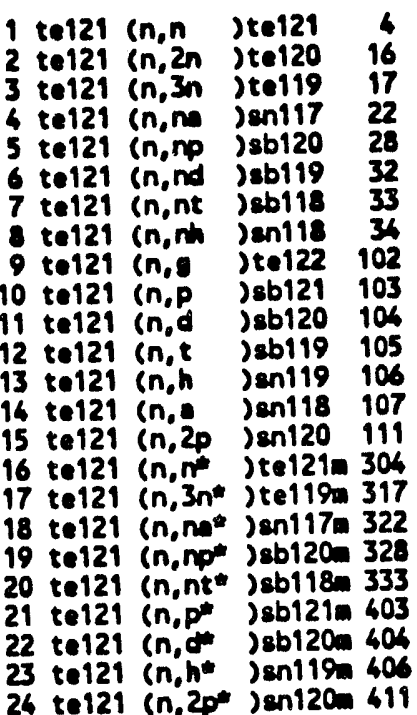

12/01/93

12/01/93

$12 / 01 / 93$

$12 / 01 / 93$

$12 / 01 / 93$

$12 / 01 / 93$

$12 / 01 / 93$

$12 / 01 / 93$

$12 / 01 / 93$

$12 / 01 / 93$

$12 / 01 / 93$

$12 / 01 / 93$

$12 / 01 / 93$

$12 / 01 / 93$

$12 / 01 / 93$

$12 / 01 / 93$

$12 / 01 / 93$

$12 / 01 / 93$

12/01/93

12/01/93

$12 / 01 / 93$

$12 / 01 / 93$

$12 / 01 / 93$

$12 / 01 / 93$

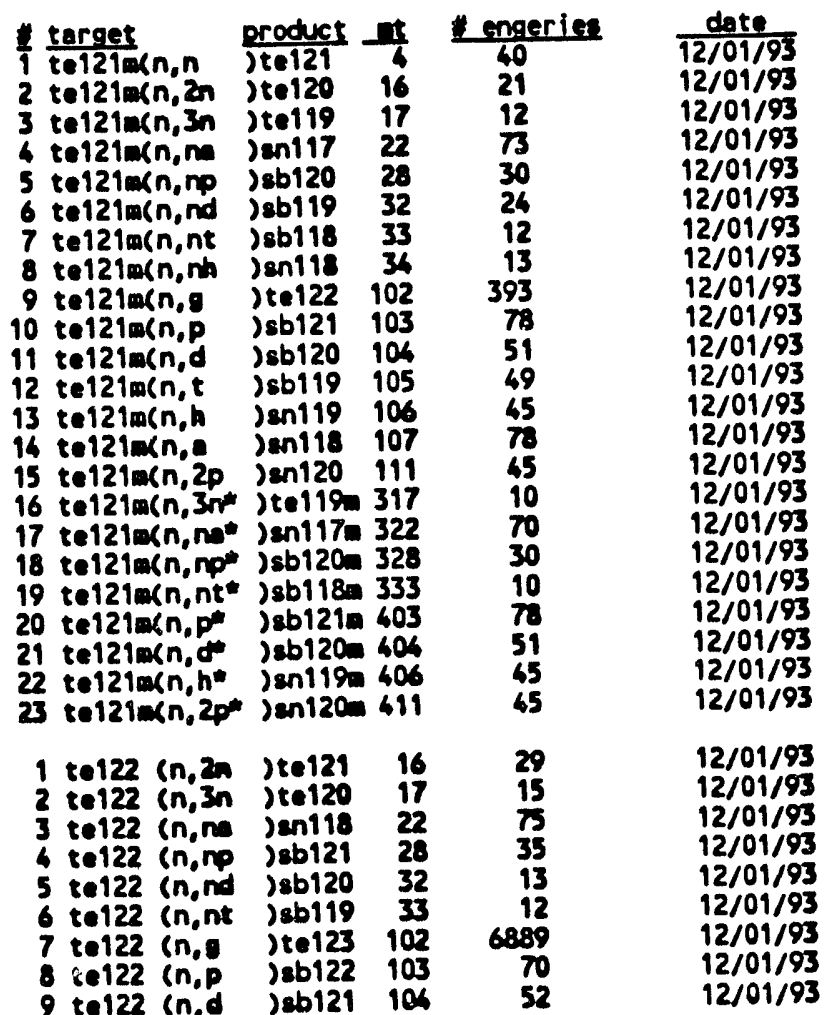

$A-5.20$ 


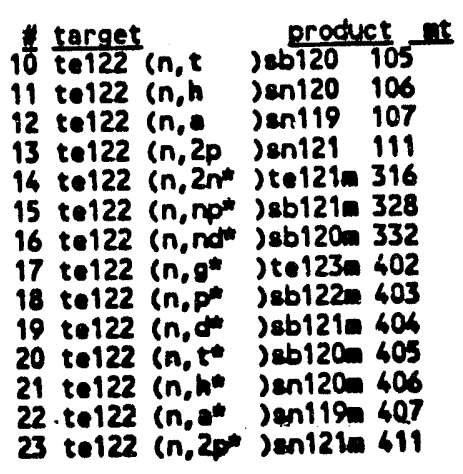

\begin{tabular}{cc} 
nengeries & date \\
\hline 46 & $12 / 1193$ \\
41 & $12 / 01 / 93$ \\
82 & $12 / 01 / 93$ \\
35 & $12 / 0193$ \\
25 & $12 / 11993$ \\
35 & $12 / 01 / 93$ \\
13 & $12 / 01 / 93$ \\
6890 & $12 / 01 / 93$ \\
70 & $12 / 01 / 93$ \\
52 & $12 / 01 / 93$ \\
44 & $12 / 01 / 93$ \\
41 & $12 / 01 / 93$ \\
82 & $12 / 01 / 93$ \\
35 & $12 / 01 / 93$
\end{tabular}

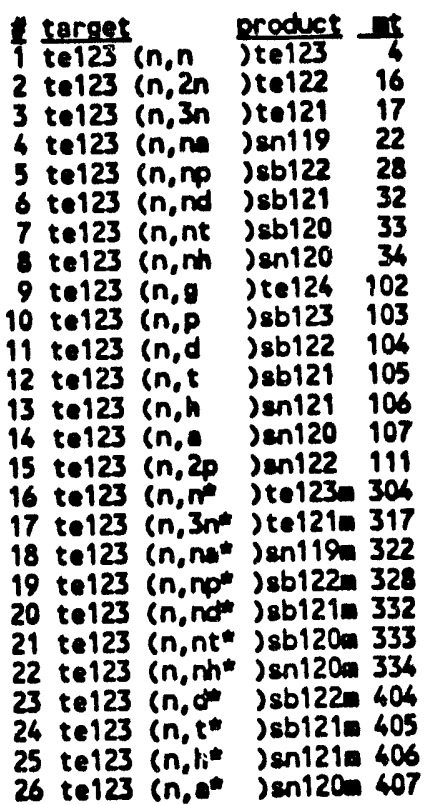

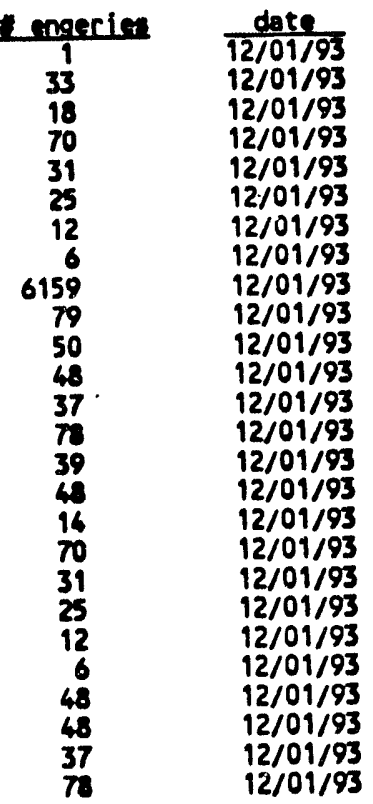

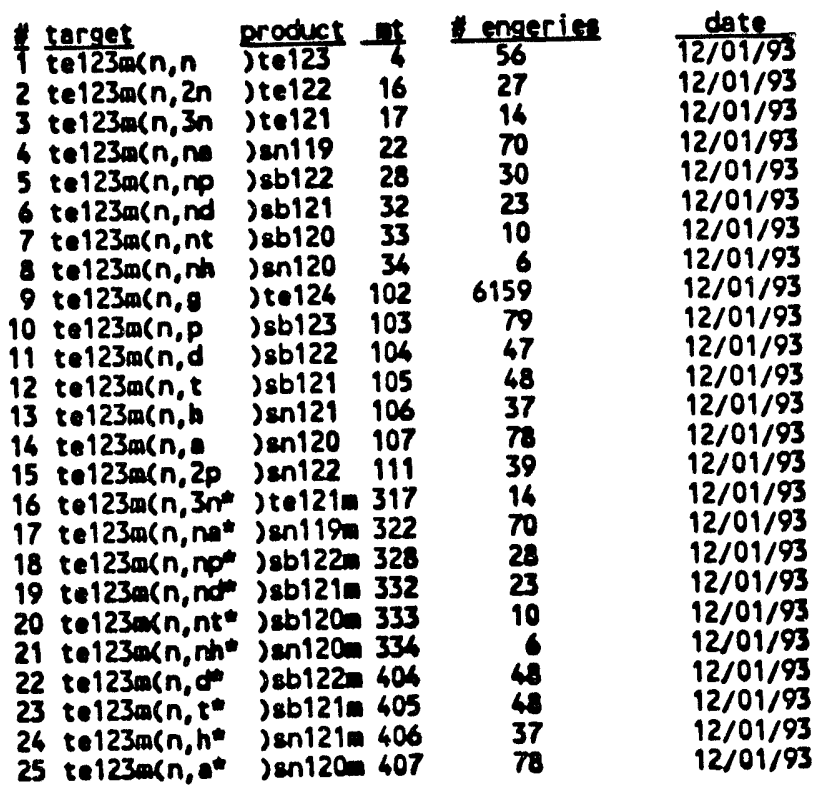




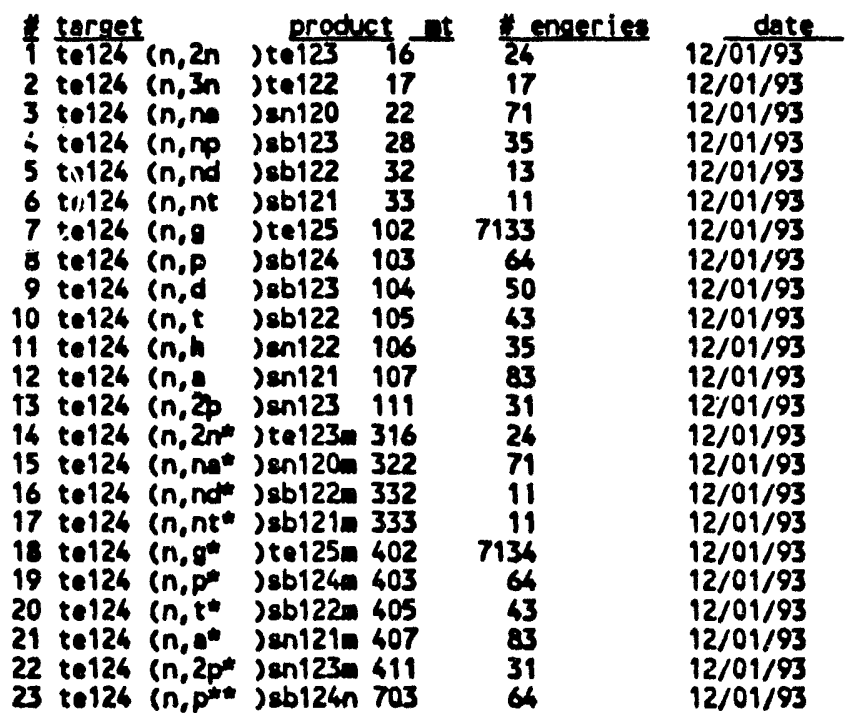

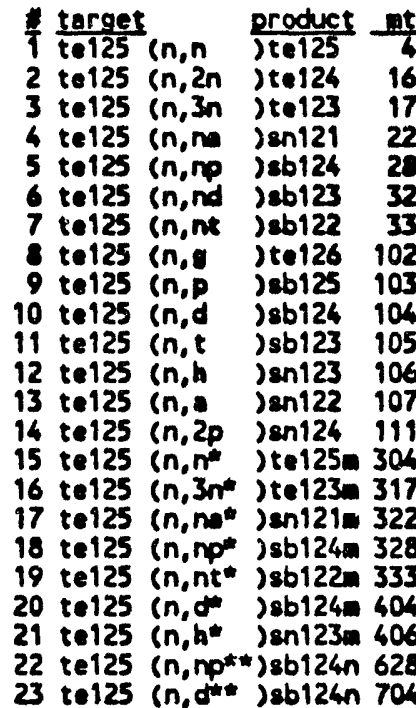

\begin{tabular}{cc} 
Engeries & $\frac{\text { date }}{12 / 01 / 93}$ \\
\hline 1 & $12 / 01 / 93$ \\
26 & $12 / 01 / 93$ \\
17 & $12 / 01 / 93$ \\
67 & $12 / 01 / 93$ \\
30 & $12 / 01 / 93$ \\
25 & $12 / 01 / 93$ \\
12 & $12 / 01 / 93$ \\
6990 & $12 / 01 / 93$ \\
81 & $12 / 01 / 93$ \\
48 & $12 / 01 / 93$ \\
50 & $12 / 01 / 93$ \\
33 & $12 / 01 / 93$ \\
70 & $12 / 01 / 93$ \\
36 & $12 / 01 / 93$ \\
48 & $12 / 01 / 93$ \\
17 & $12 / 01 / 93$ \\
67 & $12 / 01 / 93$ \\
30 & $12 / 01 / 93$ \\
12 & $12 / 01 / 93$ \\
48 & $12 / 01 / 93$ \\
33 & $12 / 01 / 93$ \\
30 & $12 / 01 / 93$
\end{tabular}

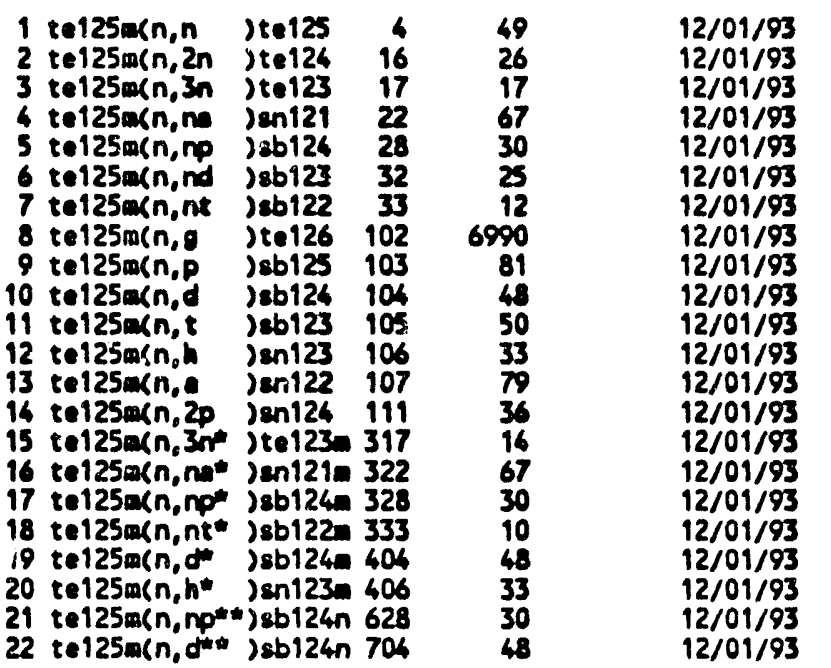


WHC-EP-0727

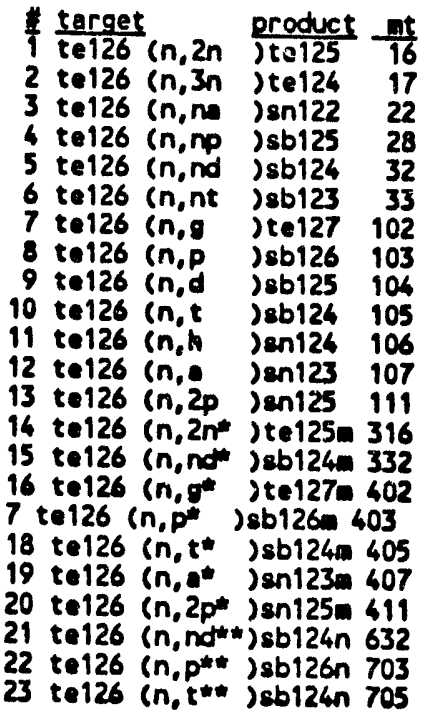

\begin{tabular}{l}
\hline engeries \\
\hline 26 \\
20 \\
68 \\
34 \\
12 \\
12 \\
4350 \\
62 \\
49 \\
43 \\
29 \\
77 \\
26 \\
26 \\
12 \\
434 \\
62 \\
43 \\
77 \\
26 \\
12 \\
62 \\
43
\end{tabular}

date

$12 / 01 / 93$

$12 / 01 / 93$

$12 / 01 / 93$

$12 / 01 / 93$

$12 / 01 / 93$

$12 / 01 / 93$

$12 / 01 / 93$

$12 / 01 / 93$

$12 / 01 / 93$

$12 / 01 / 93$

$12 / 01 / 93$

$12 / 01 / 93$

$12 / 01 / 93$

$12 / 01 / 93$

12/01/93

$12 / 01 / 93$

$12 / 01 / 93$

$12 / 01 / 93$

$12 / 01 / 93$

$12 / 01 / 93$

$12 / 01 / 93$

$12 / 01 / 93$

12/01/93

1 te127 (n,n )te127

2 tel27 (n,2n) tel26 16

3 te127 (n, 3n ) te $125 \quad 17$

4 te127 (n,n) sn123 22

5 te127 (n,np)sb126 28

$\begin{array}{lll}6 & \text { te127 (n,nd )sb125 } 32 \\ 7 & \text { te127 (n,nt ) sb124 } & 33\end{array}$

8 tel27 (n,g) tel28 102

9 tel27 (n,p )sb127 103

10 te127 (n,d )sb126 104

11 ts127 (n,t)sb125 105

12 tel27 (n,h jen125 106

13 te127 (n, ) sn124 107

14 te127 ( $n, 2 p) \operatorname{sen} 126111$

15 te127 (n, n*) te127m 304

16 te127 (n,3n*)te125m 317

17 te127 (n, not) $\operatorname{sen} 123.322$

18 te127 (n,no*)sb126m 328

19 te127 (n,nt*)sb124m 333

20 te127 (n, st) sb126 404

21 te127 (n, h*) )en125 406

22 te127 (n,np**) sb126n 628

23 te127 (n, nt*m)sb124n 633

26 te127 ( $n, d^{*}$ ) sbli26n 704

$\begin{array}{ll}1 & 12 / 01 / 93 \\ 31 & 12 / 01 / 93 \\ 17 & 12 / 101 / 93 \\ 63 & 12 / 01 / 93 \\ 29 & 12 / 01 / 93 \\ 24 & 12 / 01 / 93 \\ 12 & 12 / 01 / 93 \\ 452 & 12 / 01 / 93 \\ 76 & 12 / 01 / 93 \\ 48 & 12 / 01 / 93 \\ 50 & 12 / 01 / 93 \\ 27 & 12 / 01 / 93 \\ 82 & 12 / 01 / 93 \\ 32 & 12 / 01 / 93 \\ 48 & 12 / 01 / 93 \\ 17 & 12 / 01 / 93 \\ 63 & 12 / 01 / 93 \\ 29 & 12 / 01 / 93 \\ 12 & 12 / 01 / 93 \\ 48 & 12 / 01 / 93 \\ 27 & 12 / 01 / 93 \\ 29 & 12 / 01 / 93 \\ 12 & 12 / 01 / 93 \\ 45 & 12 / 01 / 93\end{array}$

$\frac{1}{1} \frac{\text { target }}{\text { te127m } n, n \quad \frac{\text { product }}{2 \operatorname{te} 127}-\frac{\text { nt }}{6}}$

$\begin{array}{llll}1 & t e 127 m(n, n) \\ 2 & t e 127 m(n, 2 n) t e 127 & 6\end{array}$

3 te $127 m(n, 3 n)$ te $125 \quad 17$

4 te127m(n,n) sni23 22

5 tei27m(n,np)sb126 28

6 te127m(n,nd)sb125 32

7 te127m(n,nt) sb124 33

8 tel27m(n,g)te128 102

9 te127m(n,p)sbi27 103

10 tel27m(n,d)sb126 104

11 te127m(n,t) sb125 105

12 te127m(n,h) $\operatorname{sen} 125106$

13 te127m(n,: jen126 107

14 tel27m(n,2p jen126 111

15 te127m(n, $\left.3 n^{*}\right)$ te125 317

16 te127m(n,nm) $\operatorname{sn1230} 322$

17 te127m(n,np) sb126m 328

18 te127m(n,nt )sb124m 333

19 te $127 \mathrm{~m}(\mathrm{n}, \mathrm{dm}) \mathrm{sb} 126 \mathrm{~m} 404$

20 te $127 m(n, h *)$ sn125m 406

21 te $127 m\left(n, n p^{* *}\right)$ sb126n 628

22 te $127 m\left(n, n^{* *}\right) \operatorname{sb} 124 n 633$

23 te127a(n, dtu jab126n 704

\begin{tabular}{cc} 
Engeries & date \\
\hline 50 & $12 / 01 / 93$ \\
31 & $12 / 01 / 93$ \\
17 & $12 / 01 / 93$ \\
60 & $12 / 01 / 93$ \\
29 & $12 / 01 / 93$ \\
26 & $12 / 01 / 93$ \\
12 & $12 / 01 / 93$ \\
452 & $12 / 01 / 93$ \\
76 & $12 / 01 / 93$ \\
45 & $12 / 01 / 93$ \\
67 & $12 / 01 / 93$ \\
27 & $12 / 01 / 93$ \\
82 & $12 / 01 / 93$ \\
32 & $12 / 01 / 93$ \\
17 & $12 / 01 / 93$ \\
58 & $12 / 01 / 93$ \\
29 & $12 / 01 / 93$ \\
12 & $12 / 01 / 93$ \\
48 & $12 / 01 / 93$ \\
27 & $12 / 01 / 93$ \\
29 & $12 / 01 / 93$ \\
12 & $12 / 01 / 93$ \\
45 & $12 / 01 / 93$
\end{tabular}

$A-5.23$ 


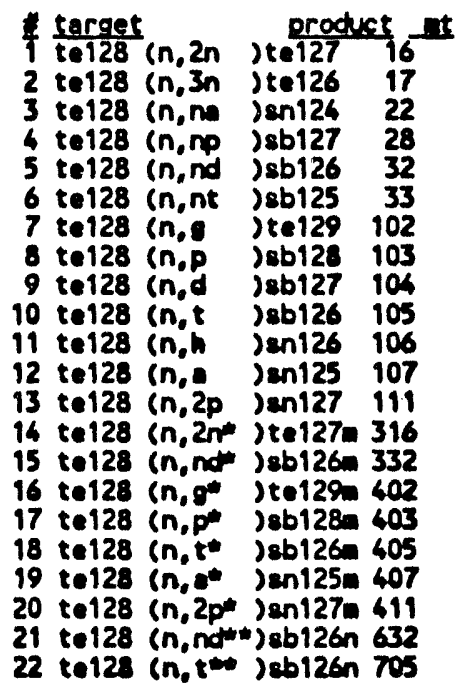

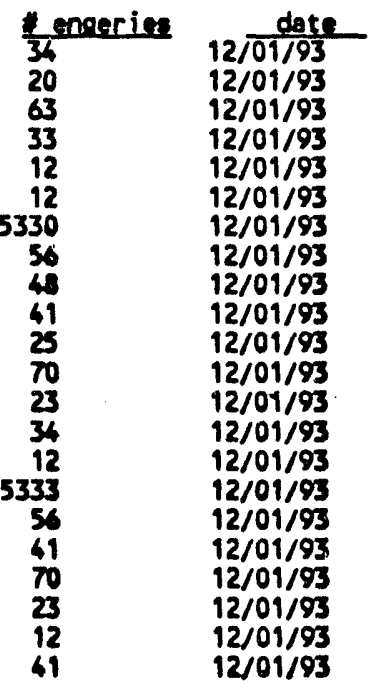

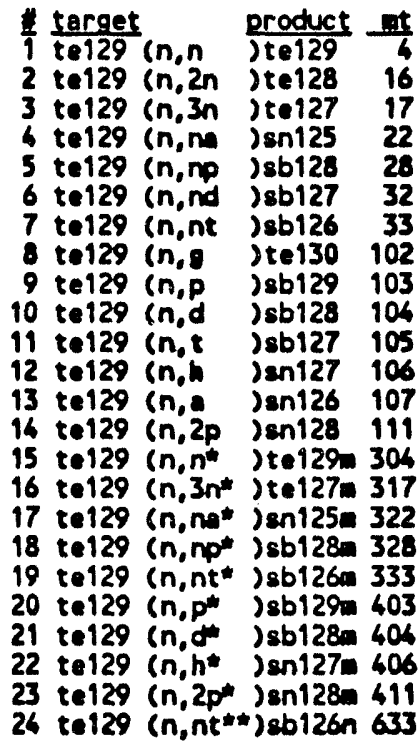

\begin{tabular}{cc} 
Hengeries & date \\
\cline { 2 - 2 } 29 & $12 / 01 / 93$ \\
29 & $12 / 01 / 93$ \\
20 & $12 / 01 / 93$ \\
55 & $12 / 01 / 93$ \\
28 & $12 / 01 / 93$ \\
26 & $12 / 01 / 93$ \\
12 & $12 / 01 / 93$ \\
432 & $12 / 01 / 93$ \\
67 & $12 / 01 / 93$ \\
46 & $12 / 01 / 93$ \\
48 & $12 / 01 / 93$ \\
23 & $12 / 01 / 93$ \\
81 & $12 / 01 / 93$ \\
27 & $12 / 01 / 93$ \\
48 & $12 / 01 / 93$ \\
18 & $12 / 01 / 93$ \\
55 & $12 / 01 / 93$ \\
28 & $12 / 01 / 93$ \\
12 & $12 / 01 / 93$ \\
67 & $12 / 01 / 93$ \\
46 & $12 / 01 / 93$ \\
23 & $12 / 01 / 93$ \\
27 & $12 / 01 / 93$ \\
12 & $12 / 01 / 93$
\end{tabular}

\begin{tabular}{|c|c|c|c|c|}
\hline 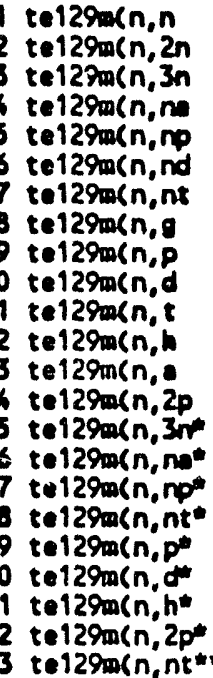 & $\begin{array}{l}\text { jte129 } \\
\text { jte128 } \\
\text { jte127 } \\
\text { jsn125 } \\
\text { jsb128 } \\
\text { jsb127 } \\
\text { jsb126 } \\
\text { jte130 } \\
\text { jsb129 } \\
\text { jsb128 } \\
\text { jsb127 } \\
\text { jen127 } \\
\text { jen126 } \\
\text { jen128 } \\
\text { jte127a } \\
\text { jen125a } \\
\text { jsb128 } \\
\text { job126a } \\
\text { jsb129m } \\
\text { jsb126 } \\
\text { jen127a } \\
\text { jen128m } \\
\text { jsb126n }\end{array}$ & $\begin{array}{r}16 \\
16 \\
17 \\
22 \\
28 \\
32 \\
33 \\
102 \\
103 \\
104 \\
105 \\
106 \\
107 \\
111 \\
317 \\
322 \\
328 \\
333 \\
403 \\
404 \\
406 \\
611 \\
633\end{array}$ & $\begin{array}{l}49 \\
29 \\
17 \\
55 \\
28 \\
26 \\
12 \\
432 \\
67 \\
43 \\
48 \\
23 \\
81 \\
27 \\
18 \\
55 \\
28 \\
12 \\
67 \\
43 \\
23 \\
27 \\
12\end{array}$ & $\begin{array}{l}12 / 01 / 93 \\
12 / 01 / 93 \\
12 / 01 / 93 \\
12 / 01 / 93 \\
12 / 01 / 93 \\
12 / 01 / 93 \\
12 / 01 / 93 \\
12 / 01 / 93 \\
12 / 01 / 93 \\
12 / 01 / 93 \\
12 / 01 / 93 \\
12 / 01 / 93 \\
12 / 01 / 93 \\
12 / 01 / 93 \\
12 / 01 / 93 \\
12 / 01 / 93 \\
12 / 01 / 93 \\
12 / 01 / 93 \\
12 / 01 / 93 \\
12 / 01 / 93 \\
12 / 01 / 93 \\
12 / 01 / 93 \\
12 / 01 / 93\end{array}$ \\
\hline
\end{tabular}




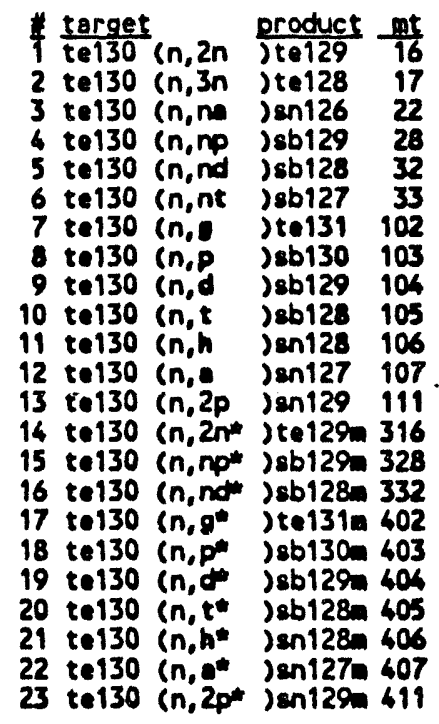

\begin{tabular}{cc} 
Engeries & date \\
\hline 40 & $12 / 01 / 93$ \\
20 & $12 / 01 / 93$ \\
58 & $12 / 01 / 93$ \\
32 & $12 / 01 / 93$ \\
12 & $12 / 01 / 93$ \\
12 & $12 / 01 / 93$ \\
4064 & $12 / 01 / 93$ \\
56 & $12 / 01 / 93$ \\
46 & $12 / 01 / 93$ \\
61 & $12 / 01 / 93$ \\
21 & $12 / 01 / 93$ \\
62 & $12 / 101 / 93$ \\
17 & $12 / 01 / 93$ \\
35 & $12 / 01 / 93$ \\
32 & $12 / 01 / 93$ \\
12 & $12 / 01 / 93$ \\
4066 & $12 / 01 / 93$ \\
54 & $12 / 01 / 93$ \\
46 & $12 / 01 / 93$ \\
61 & $12 / 01 / 93$ \\
21 & $12 / 01 / 93$ \\
63 & $12 / 01 / 93$ \\
17 & $12 / 01 / 93$
\end{tabular}

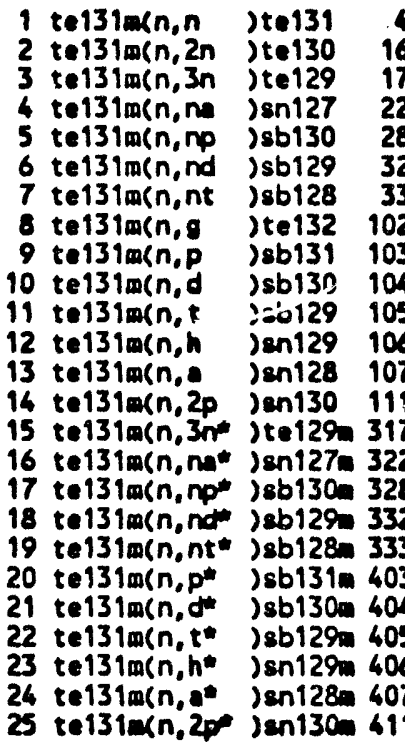

$\begin{array}{rr}53 & 12 / 01 / 93 \\ 33 & 12 / 01 / 93 \\ 19 & 12 / 01 / 93 \\ 56 & 12 / 01 / 93 \\ 28 & 12 / 01 / 93 \\ 23 & 12 / 01 / 93 \\ 12 & 12 / 01 / 93 \\ 381 & 12 / 01 / 93 \\ 60 & 12 / 01 / 93 \\ 41 & 12 / 01 / 93 \\ 45 & 12 / 01 / 93 \\ 17 & 12 / 01 / 93 \\ 77 & 12 / 01 / 93 \\ 26 & 12 / 01 / 93 \\ 19 & 12 / 01 / 93 \\ 56 & 12 / 01 / 93 \\ 28 & 12 / 01 / 93 \\ 23 & 12 / 01 / 93 \\ 12 & 12 / 01 / 93 \\ 60 & 12 / 01 / 93 \\ 41 & 12 / 01 / 93 \\ 45 & 12 / 01 / 93 \\ 17 & 12 / 01 / 93 \\ 77 & 12 / 01 / 93 \\ 26 & 12 / 01 / 93\end{array}$

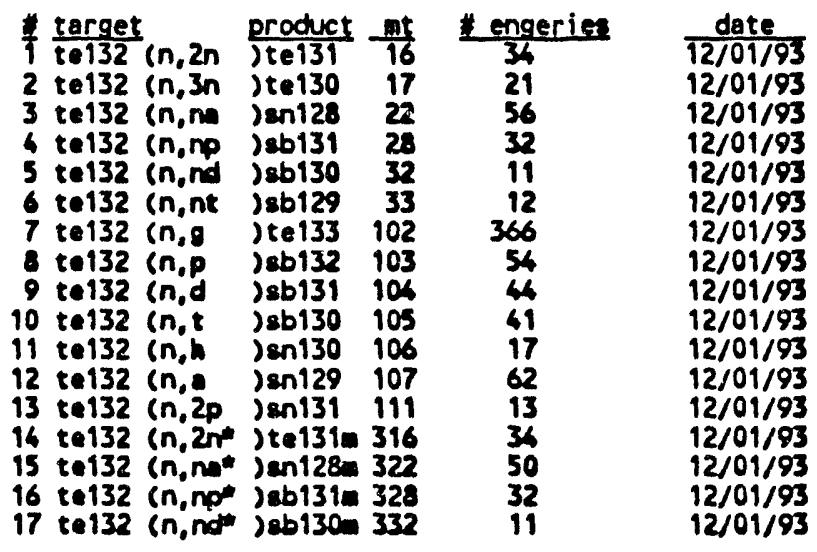


WHC-EP-0727

\begin{tabular}{|c|c|c|c|}
\hline 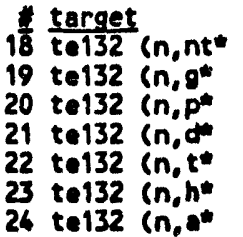 & $\begin{array}{l}\text { product } \\
\text { job129m } 333 \\
\text { jte133m } 402 \\
\text { job132m } 403 \\
\text { job131m } 404 \\
\text { job130m } 405 \\
\text { jen130m } 406 \\
\text { jen129m } 407\end{array}$ & $\begin{array}{l}1 \text { enoeries } \\
12 \\
366 \\
56 \\
46 \\
41 \\
17 \\
62\end{array}$ & $\begin{array}{l}\frac{\text { date }}{12 / 01 / 93} \\
12 / 01 / 93 \\
12 / 01 / 93 \\
12 / 01 / 93 \\
12 / 01 / 93 \\
12 / 01 / 93 \\
12 / 01 / 93\end{array}$ \\
\hline
\end{tabular}

A-5.26 
Appendix $A-6$

Liet of Reection on files *.i.en

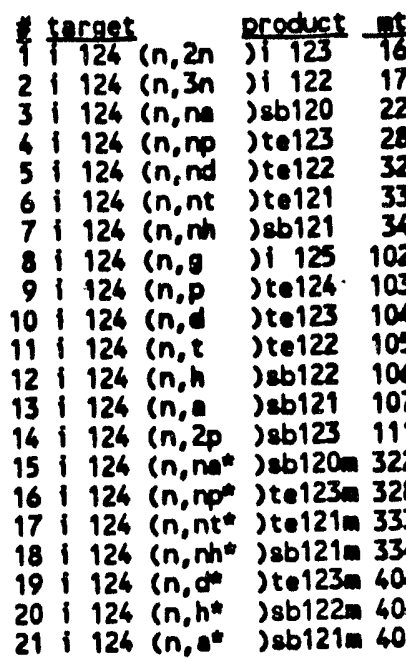

1 i $125(n, 2 n) 126 \quad 16$

2 i $125(n, 3 n)$ i $123 \quad 17$

3 i 125 (n,n) sbi21 22

4 i 125 (n,np) tel26 28

5 i 125 (n,nd)tel23 32

6 i 125 (n,nt )te122 33

$7125 \mathrm{ln}, \mathrm{s}$ )i 126102

- 125 (n,p )te125 103

9 i $125(n, d)$ te124 104

10 i $125(n, t)$ tel $123 \quad 105$

11 i $125 \mathrm{cn}, \mathrm{h}$ ) 86123106

12 in 125, sb122 107

13 i 125 (n,2p )sbi24 111

14 i 125 (n,na )sb121m 322

15 i 125 (n, nd ) te123 332

16 i 125 (n, p* )te125m 403

17 i 125 (n,t jte123m 405

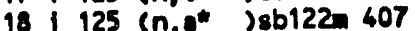

19 i $125\left(n, 2 p^{*}\right)$ sb124m 411

20 i $125\left(n, 2 p^{* m}\right)$ eb12 $12 n 711$

\begin{tabular}{l} 
engerie \\
\hline 22 \\
15 \\
73 \\
37 \\
30 \\
18 \\
6 \\
401 \\
73 \\
59 \\
61 \\
41 \\
77 \\
45 \\
73 \\
37 \\
17 \\
6 \\
57 \\
41 \\
77
\end{tabular}

date
$\frac{12 / 01 / 93}{12 / 01 / 93}$
$12 / 01 / 93$
$12 / 01 / 93$
$12 / 01 / 93$
$12 / 01 / 93$
$12 / 01 / 93$
$12 / 01 / 93$
$12 / 01 / 93$
$12 / 01 / 93$
$12 / 01 / 93$
$12 / 01 / 93$
$12 / 01 / 93$
$12 / 01 / 93$
$12 / 01 / 93$
$12 / 011933$
$12 / 01 / 93$
$12 / 01 / 93$
$12 / 01 / 93$
$12 / 01 / 93$
$12 / 01 / 93$

$26 \quad 12 / 01 / 93$

$12 / 01 / 93$

$12 / 01 / 93$

$12 / 01 / 93$

$12 / 01 / 93$

$12 / 01 / 93$

$12 / 01 / 93$

$12 / 01 / 93$

$12 / 01 / 93$

$12 / 01 / 93$

$12 / 01 / 93$

$12 / 01 / 93$

$12 / 01 / 93$

$12 / 01 / 93$

$12 / 01 / 93$

$12 / 01 / 93$

$12 / 01 / 93$

$12 / 01 / 93$

$12 / 01 / 93$

$12 / 01 / 93$

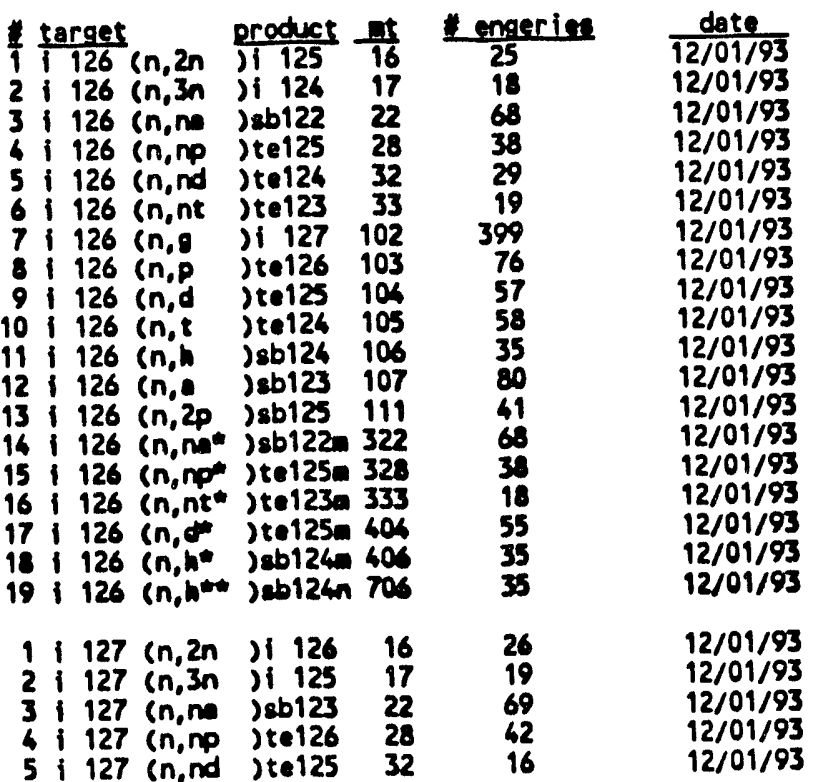

$A-6.1$ 


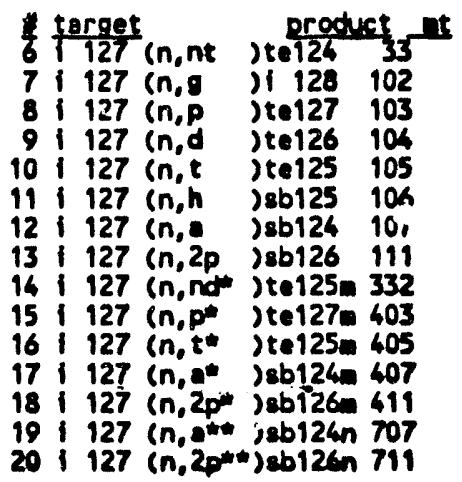

\begin{tabular}{cc} 
enoerin & date \\
\hline 23 & $12 / 01 / 93$ \\
1282 & $12 / 01 / 93$ \\
81 & $12 / 01 / 93$ \\
57 & $12 / 01 / 93$ \\
49 & $12 / 01 / 93$ \\
33 & $12 / 01 / 93$ \\
76 & $12 / 01 / 93$ \\
32 & $12 / 01 / 93$ \\
16 & $12 / 01 / 93$ \\
81 & $12 / 01 / 93$ \\
46 & $12 / 01 / 93$ \\
73 & $12 / 01 / 93$ \\
32 & $12 / 01 / 93$ \\
76 & $12 / 01 / 93$ \\
32 & $12 / 01 / 93$
\end{tabular}

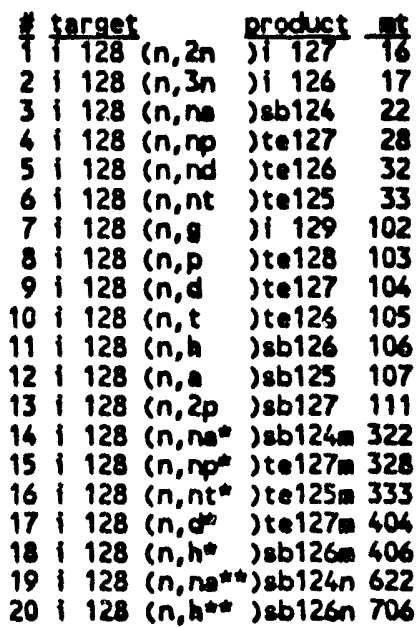

\begin{tabular}{l} 
engerie: \\
\hline 31 \\
17 \\
64 \\
35 \\
31 \\
20 \\
401 \\
78 \\
53 \\
56 \\
31 \\
81 \\
38 \\
64 \\
35 \\
20 \\
53 \\
31 \\
64 \\
31
\end{tabular}

$\frac{\text { date }}{\sqrt{2 / 01 / 93}}$

$12 / 01 / 93$

$12 / 01 / 93$

$12 / 01 / 93$

$12 / 01 / 93$

$12 / 01 / 93$

$12 / 01 / 93$

$12 / 01 / 93$

$12 / 01 / 93$

$12 / 01 / 93$

$12 / 01 / 93$

12/01/93

$12 / 01 / 93$

$12 / 01 / 93$

$12 / 01 / 93$

$12 / 01 / 93$

$12 / 01 / 93$

$12 / 01 / 93$

$12 / 01 / 93$

$12 / 01 / 93$

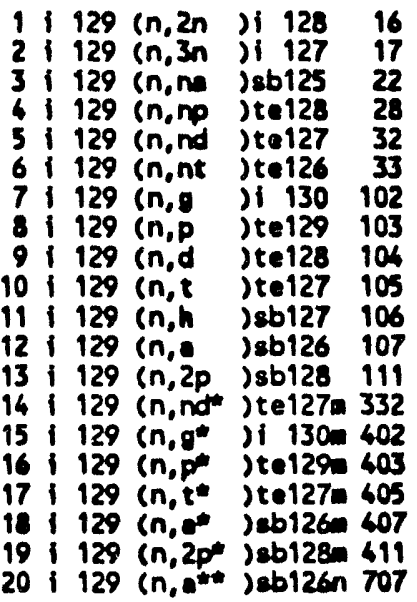

$\begin{array}{ll}29 & 12 / 01 / 93 \\ 19 & 12 / 01 / 93\end{array}$

$12 / 01 / 93$

12/01/93

$12 / 01 / 93$

$12 / 01 / 93$

$12 / 01 / 93$

12/01/93

$12 / 01 / 93$

$12 / 01 / 93$

12/01/93

$12 / 01 / 93$

$12 / 01 / 93$

$12 / 01 / 93$

12/01/93

$12 / 01 / 93$

12/01/93

$12 / 01 / 93$

$12 / 01 / 93$

1 target product $n$

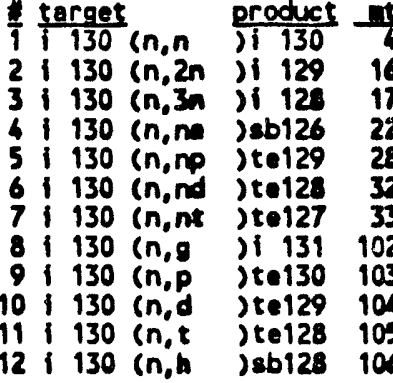

\begin{tabular}{|c|c|}
\hline $\begin{array}{c}\text { engeries } \\
1 \\
27 \\
21 \\
66 \\
34 \\
30 \\
22 \\
4 \frac{75}{79} \\
52 \\
56 \\
27\end{array}$ & $\begin{array}{l}\frac{\text { date }}{12 / 01 / 93} \\
12 / 01 / 93 \\
12 / 01 / 93 \\
12 / 01 / 93 \\
12 / 01 / 93 \\
12 / 01 / 93 \\
12 / 01 / 93 \\
12 / 01 / 93 \\
12 / 01 / 93 \\
12 / 01 / 93 \\
12 / 01 / 93 \\
12 / 01 / 93\end{array}$ \\
\hline
\end{tabular}




\begin{tabular}{|c|c|c|c|c|c|c|}
\hline $\begin{array}{l}16 \\
15 \\
16 \\
17 \\
18 \\
19 \\
20 \\
21 \\
\end{array}$ & $\begin{array}{l}\text { target } \\
130 \\
130 \\
130 \\
130 \\
130 \\
130 \\
130 \\
130 \\
130 \\
130 \\
130 \\
130 \\
130\end{array}$ & 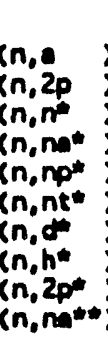 & $\begin{array}{l}\text { produc } \\
\text { jebi27 } \\
\text { jeb129 } \\
\text { ji } 130 \mathrm{~m} \\
\text { jeb126m } \\
\text { jte129m } \\
\text { jte127m } \\
\text { jte129m } \\
\text { jeb128m } \\
\text { jeb129m } \\
\text { jeb126n }\end{array}$ & $\begin{array}{l}\frac{c 4}{107} \\
111 \\
304 \\
322 \\
328 \\
333 \\
404 \\
406 \\
611 \\
622\end{array}$ & $\begin{array}{l}\frac{\text { enqerien }}{84} \\
34 \\
50 \\
66 \\
34 \\
22 \\
50 \\
27 \\
34 \\
66\end{array}$ & $\begin{array}{l}\frac{\text { date }}{12 / 01 / 93} \\
12 / 01 / 93 \\
12 / 01 / 93 \\
12 / 01 / 93 \\
12 / 01 / 93 \\
12 / 01 / 93 \\
12 / 01 / 93 \\
12 / 01 / 93 \\
12 / 01 / 93 \\
12 / 01 / 93\end{array}$ \\
\hline $\begin{array}{l}1 \\
2 \\
3 \\
4 \\
5 \\
6 \\
7 \\
8 \\
9 \\
10 \\
11 \\
12 \\
13 \\
14 \\
15 \\
16 \\
17 \\
18 \\
19 \\
20 \\
21\end{array}$ & $\begin{array}{l}131 \\
131 \\
131 \\
131 \\
131 \\
131 \\
131 \\
131 \\
131 \\
131 \\
131 \\
131 \\
131 \\
131 \\
131 \\
131 \\
131 \\
131 \\
131 \\
131 \\
131 \\
131 \\
131 \\
131 \\
131 \\
131 \\
131 \\
131\end{array}$ & 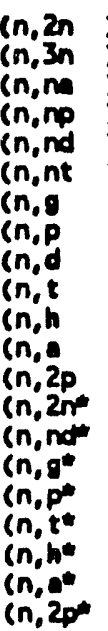 & 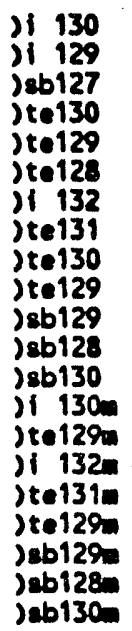 & $\begin{array}{l}16 \\
17 \\
22 \\
28 \\
32 \\
33 \\
102 \\
103 \\
104 \\
105 \\
106 \\
107 \\
111 \\
316 \\
332 \\
402 \\
403 \\
405 \\
406 \\
407 \\
411\end{array}$ & $\begin{array}{l}29 \\
20 \\
64 \\
39 \\
16 \\
22 \\
449 \\
70 \\
53 \\
49 \\
25 \\
70 \\
24 \\
29 \\
16 \\
49 \\
67 \\
49 \\
25 \\
70 \\
24\end{array}$ & $\begin{array}{l}12 / 01 / 93 \\
12 / 01 / 93 \\
12 / 01 / 93 \\
12 / 01 / 93 \\
12 / 01 / 93 \\
12 / 01 / 93 \\
12 / 01 / 93 \\
12 / 01 / 93 \\
12 / 01 / 93 \\
12 / 01 / 93 \\
12 / 01 / 93 \\
12 / 01 / 93 \\
12 / 01 / 93 \\
12 / 01 / 93 \\
12 / 01 / 93 \\
12 / 01 / 93 \\
12 / 01 / 93 \\
12 / 01 / 93 \\
12 / 01 / 93 \\
12 / 01 / 93 \\
12 / 01 / 93\end{array}$ \\
\hline $\begin{array}{c}2 \\
3 \\
4 \\
5 \\
6 \\
7 \\
8 \\
9 \\
9 \\
10 \\
11 \\
12 \\
13 \\
16 \\
15 \\
16\end{array}$ & $\begin{array}{l}\text { target } \\
x \in 124 \\
x \in 124 \\
x \in 124 \\
x=124 \\
x \in 124 \\
x=124 \\
x=126 \\
x=124 \\
x=126 \\
x=126 \\
x=124 \\
x=124 \\
x=124 \\
x=124 \\
x=124 \\
x=124\end{array}$ & 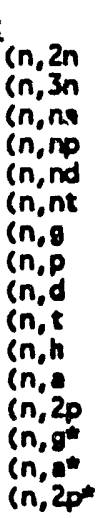 & 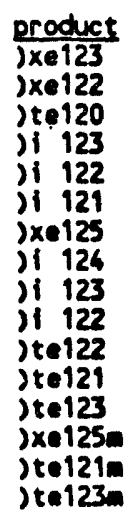 & $\begin{array}{l}\frac{1 t}{16} \\
17 \\
22 \\
28 \\
32 \\
33 \\
102 \\
103 \\
104 \\
105 \\
106 \\
107 \\
111 \\
402 \\
407 \\
411\end{array}$ & $\begin{array}{c}\text { engeries } \\
24 \\
9 \\
79 \\
39 \\
16 \\
11 \\
1443 \\
81 \\
56 \\
46 \\
48 \\
82 \\
40 \\
1433 \\
82 \\
40\end{array}$ & $\begin{array}{c}\text { date } \\
12 / 01 / 93 \\
12 / 01 / 93 \\
12 / 01 / 93 \\
12 / 01 / 93 \\
12 / 01 / 93 \\
12 / 01 / 93 \\
12 / 01 / 93 \\
12 / 01 / 93 \\
12 / 01 / 93 \\
12 / 01 / 93 \\
12 / 01 / 93 \\
12 / 01 / 93 \\
12 / 01 / 93 \\
12 / 01 / 93 \\
12 / 01 / 93 \\
12 / 01 / 93\end{array}$ \\
\hline $\begin{array}{l}8 \\
9 \\
10 \\
11 \\
12 \\
13 \\
14 \\
15 \\
16 \\
17 \\
18\end{array}$ & $\begin{array}{l}x=125 \\
x=125 \\
x=125 \\
x e 125 \\
x=125 \\
x=125 \\
x=125 \\
x=125 \\
x=125 \\
x=125 \\
x=125 \\
x=125 \\
x=125 \\
x=125 \\
x=125 \\
x=125 \\
x=125 \\
x=125\end{array}$ & 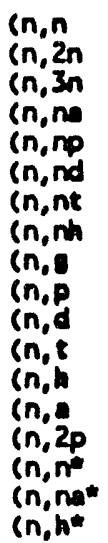 & 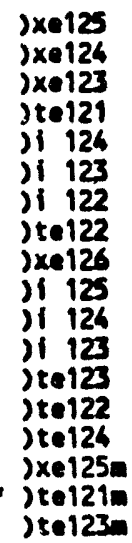 & $\begin{array}{l}6 \\
16 \\
17 \\
22 \\
28 \\
32 \\
33 \\
34 \\
102 \\
103 \\
104 \\
105 \\
106 \\
107 \\
111 \\
304 \\
322 \\
406\end{array}$ & $\begin{array}{l}1 \\
26 \\
11 \\
71 \\
34 \\
26 \\
11 \\
9 \\
394 \\
77 \\
55 \\
52 \\
45 \\
78 \\
48 \\
47 \\
71 \\
45\end{array}$ & $\begin{array}{l}12 / 01 / 93 \\
12 / 01 / 93 \\
12 / 01 / 93 \\
12 / 01 / 93 \\
12 / 01 / 93 \\
12 / 01 / 93 \\
12 / 01 / 93 \\
12 / 01 / 93 \\
12 / 01 / 93 \\
12 / 01 / 93 \\
12 / 01 / 93 \\
12 / 01 / 93 \\
12 / 01 / 93 \\
12 / 01 / 93 \\
12 / 01 / 93 \\
12 / 01 / 93 \\
12 / 01 / 93 \\
12 / 01 / 93\end{array}$ \\
\hline
\end{tabular}




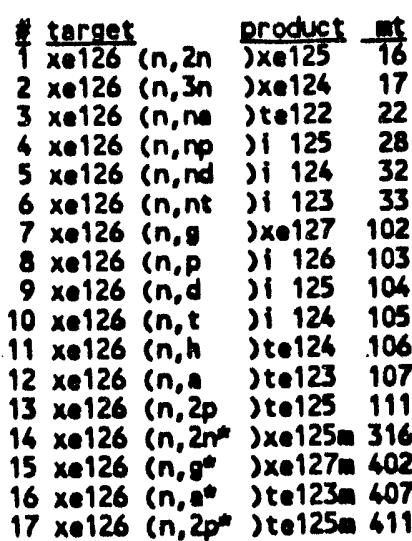

\begin{tabular}{c} 
engerie \\
\hline 23 \\
13 \\
76 \\
37 \\
13 \\
11 \\
1466 \\
82 \\
54 \\
45 \\
41 \\
83 \\
37 \\
23 \\
1459 \\
82 \\
37
\end{tabular}

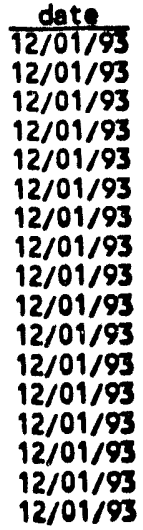

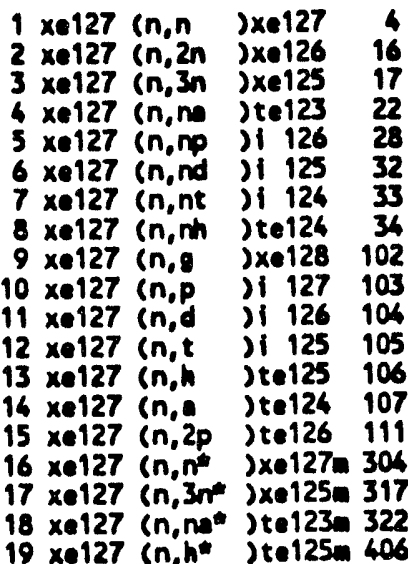

$1 \quad 12 / 01 / 93$

$12 / 01 / 93$

$12 / 01 / 93$

$12 / 01 / 93$

$12 / 01 / 93$

$12 / 01 / 93$

$12 / 01 / 93$

$12 / 01 / 93$

$12 / 01 / 93$

$12 / 01 / 93$

$12 / 01 / 93$

$12 / 01 / 93$

$12 / 01 / 93$

$12 / 01 / 93$

$12 / 01 / 93$

12/01/93

$12 / 01 / 93$

$12 / 01 / 93$

$12 / 01 / 93$

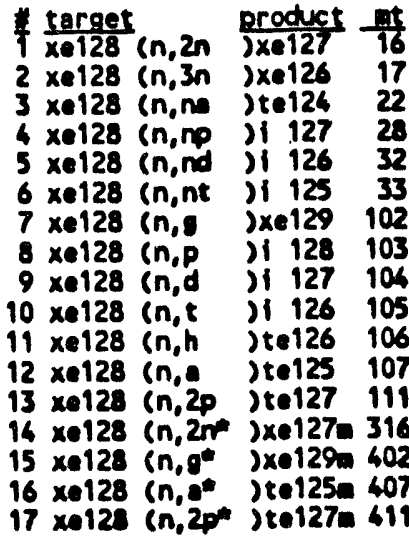

\begin{tabular}{cc} 
engeries & date \\
\hline 25 & $12 / 01 / 93$ \\
16 & $12 / 01 / 93$ \\
71 & $12 / 01 / 93$ \\
36 & $12 / 01 / 93$ \\
13 & $12 / 01 / 93$ \\
11 & $12 / 01 / 93$ \\
3653 & $12 / 01 / 93$ \\
70 & $12 / 01 / 93$ \\
53 & $12 / 01 / 93$ \\
45 & $12 / 01 / 93$ \\
37 & $12 / 01 / 93$ \\
83 & $12 / 01 / 93$ \\
32 & $12 / 01 / 93$ \\
25 & $12 / 31 / 93$ \\
3641 & $12 / 01 / 93$ \\
83 & $12 / 01 / 93$ \\
32 & $12 / 01 / 93$
\end{tabular}

\begin{tabular}{|c|c|c|}
\hline $\begin{array}{l}x \in 129 \\
x \in 129 \\
x \in 129 \\
x \in 129 \\
x \in 129 \\
x \in 129 \\
x=129 \\
x \in 129 \\
x \in 129 \\
x \in 129\end{array}$ & 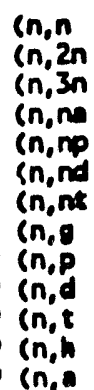 & 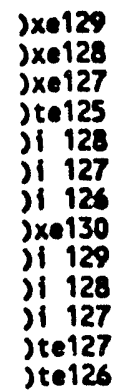 \\
\hline
\end{tabular}

$\begin{array}{rr}1 & 12 / 01 / 93 \\ 32 & 12 / 01 / 93 \\ 16 & 12 / 01 / 93 \\ 68 & 12 / 01 / 93 \\ 32 & 12 / 01 / 93 \\ 26 & 12 / 01 / 93 \\ 12 & 12 / 01 / 93 \\ 1282 & 12 / 01 / 93 \\ 81 & 12 / 01 / 93 \\ 49 & 12 / 01 / 93 \\ 49 & 12 / 01 / 93 \\ 33 & 12 / 01 / 93 \\ 80 & 12 / 01 / 93\end{array}$

A-6.4 


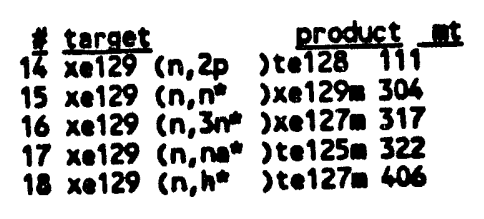

\begin{tabular}{ll} 
enaeries & $\frac{\text { date }}{38}$ \\
\hline 37 & $12 / 01 / 93$ \\
47 & $12 / 01 / 93$ \\
16 & $12 / 01 / 93$ \\
68 & $12 / 01 / 93$ \\
33 &
\end{tabular}

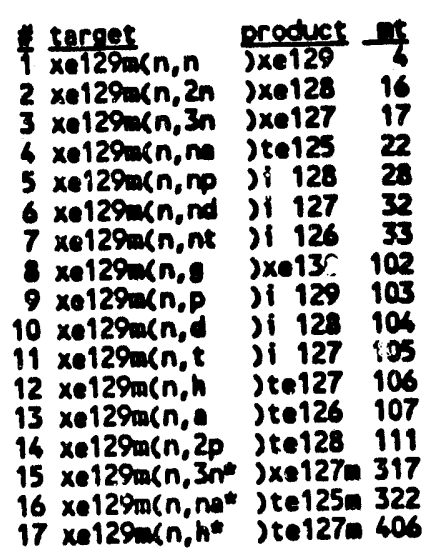

emprim
50
27
15
65
29
26
10
1281
81
69
49
33
80
38
13
63
33

$\frac{\text { date }}{12101 / 93}$

$12 / 01 / 93$

$12 / 01 / 93$

$12 / 01 / 93$

$12 / 01 / 93$

$12 / 01 / 93$

$12 / 01 / 93$

$12 / 01 / 93$

$12 / 01 / 93$

$12 / 01 / 93$

$12 / 01 / 93$

$12 / 01 / 93$

$12 / 01 / 93$

$12 / 01 / 93$

$12 / 01 / 93$

$12 / 01 / 93$

$12 / 01 / 93$

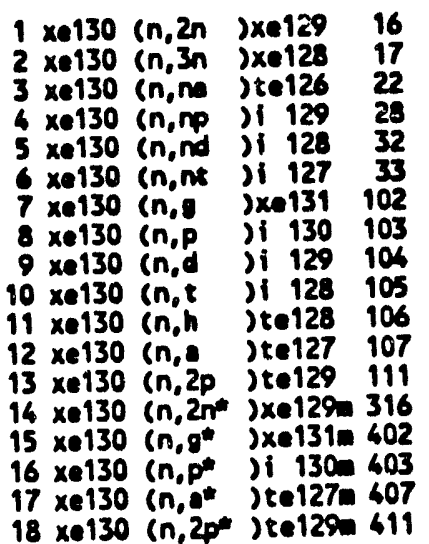

$\begin{array}{rr}25 & 12 / 01 / 93 \\ 18 & 12 / 01 / 93 \\ 71 & 12 / 01 / 93 \\ 36 & 12 / 01 / 93 \\ 13 & 12 / 01 / 93 \\ 12 & 12 / 01 / 93 \\ 3923 & 12 / 01 / 93 \\ 64 & 12 / 01 / 93 \\ 51 & 12 / 01 / 93 \\ 63 & 12 / 01 / 93 \\ 31 & 12 / 01 / 93 \\ 79 & 12 / 01 / 93 \\ 29 & 12 / 01 / 93 \\ 23 & 12 / 01 / 93 \\ 3903 & 12 / 01 / 93 \\ 64 & 12 / 01 / 93 \\ 79 & 12 / 01 / 93 \\ 29 & 12 / 01 / 93\end{array}$

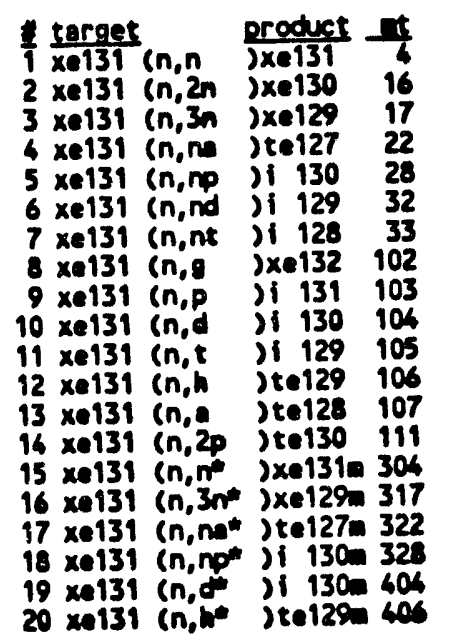

\begin{tabular}{|c|c|}
\hline $\begin{array}{c}\text { engeriet } \\
1 \\
26 \\
18 \\
65 \\
30 \\
25 \\
12 \\
8341 \\
83 \\
48 \\
50 \\
29 \\
81 \\
33 \\
4 \\
16 \\
65 \\
30 \\
48 \\
20\end{array}$ & $\begin{array}{c}\text { date } \\
12 / 01 / 93 \\
12 / 01 / 93 \\
12 / 01 / 93 \\
12 / 01 / 93 \\
12 / 01 / 93 \\
12 / 01 / 93 \\
12 / 01 / 93 \\
12 / 01 / 93 \\
12 / 01 / 93 \\
12 / 01 / 93 \\
12 / 01 / 93 \\
12 / 01 / 93 \\
12 / 01 / 93 \\
12 / 01 / 93 \\
12 / 01 / 93 \\
12 / 011 / 93 \\
12 / 01 / 93 \\
12 / 101 / 93 \\
12 / 01 / 93 \\
12 / 01 / 93\end{array}$ \\
\hline
\end{tabular}

\begin{tabular}{|c|c|}
\hline $\begin{array}{l}1 \text { xe131men,n } \\
2 \text { xe131m(n,2n } \\
3 \text { xe131m(n,3n } \\
6 x=131 \mathrm{~m}(n, n) \\
5 \text { xe131m(n,np } \\
6 x \in 131 \mathrm{~m}(n, n d\end{array}$ & $\begin{array}{l}j x e 131 \\
j x=130 \\
j x=129 \\
j \text { te } 127 \\
j i 130 \\
j i 129\end{array}$ \\
\hline
\end{tabular}

$12 / 01 / 93$
$12 / 01 / 93$
$12 / 01 / 93$
$12 / 01 / 93$
$12 / 01 / 93$
$12 / 01 / 93$

$$
\text { A-6.5 }
$$




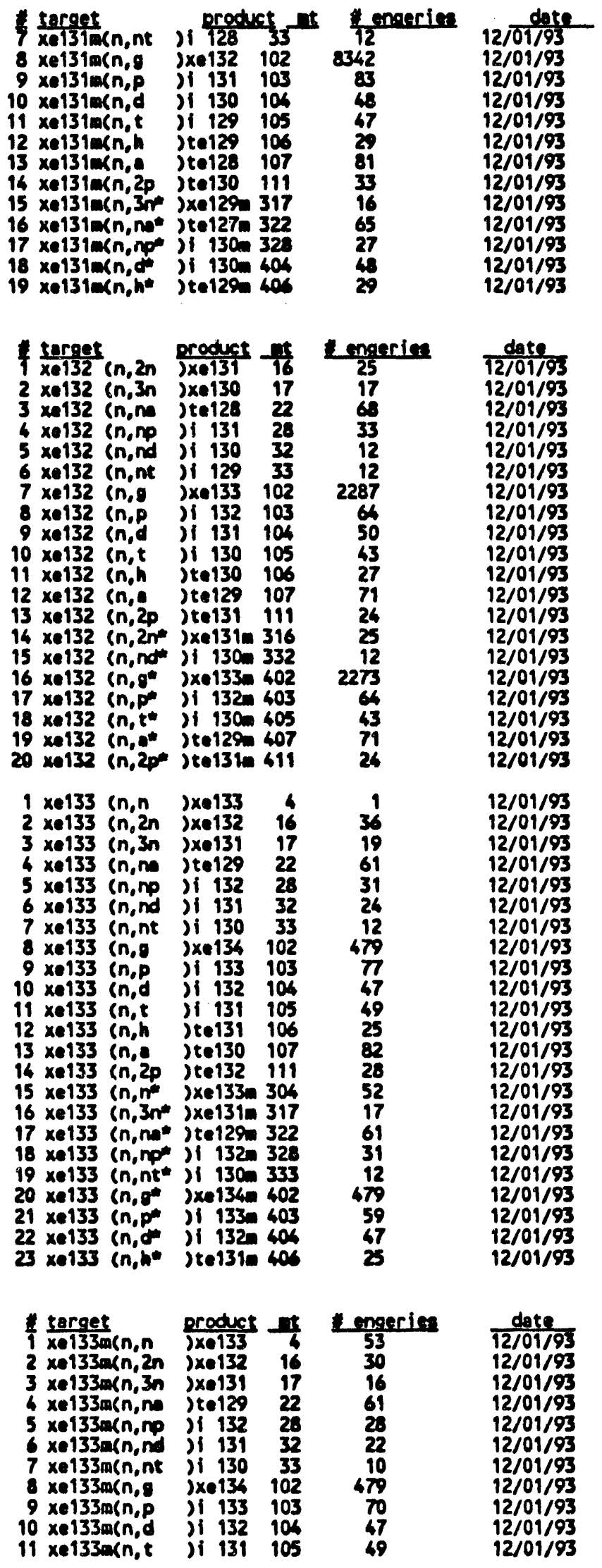

A-6. 6 


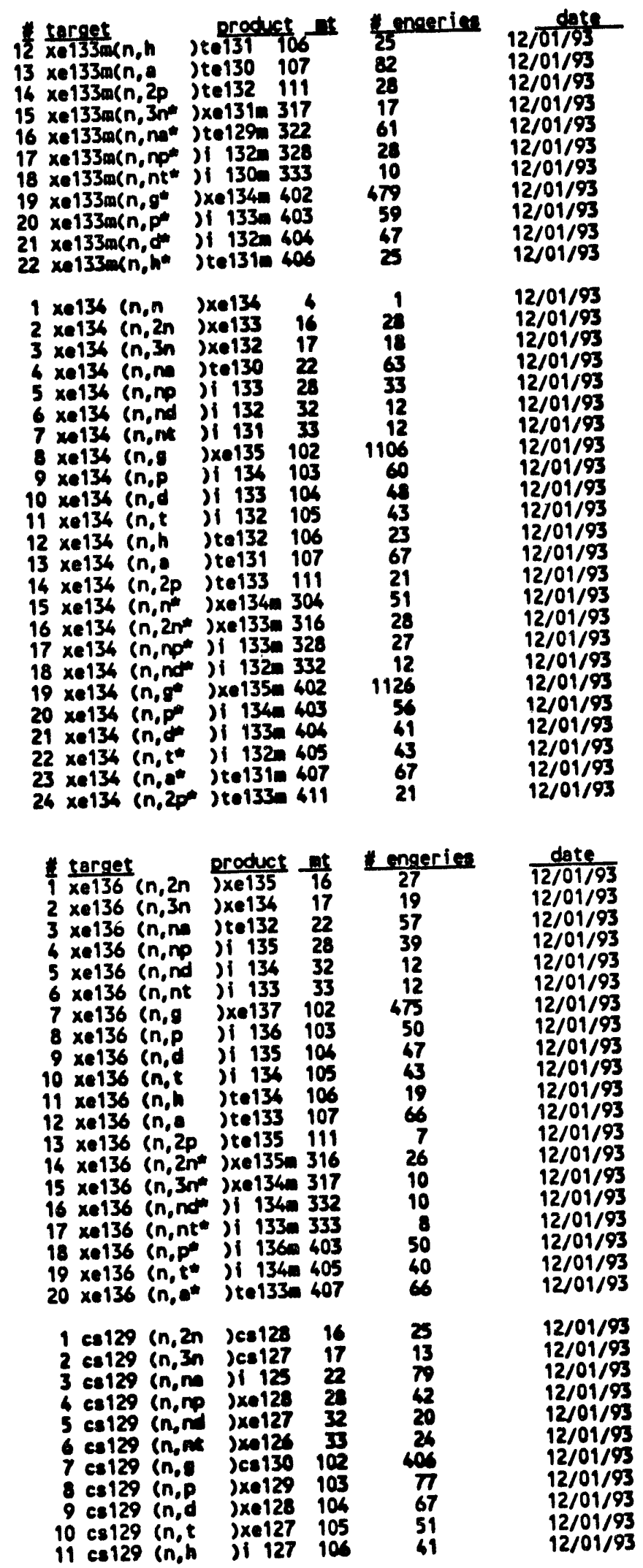




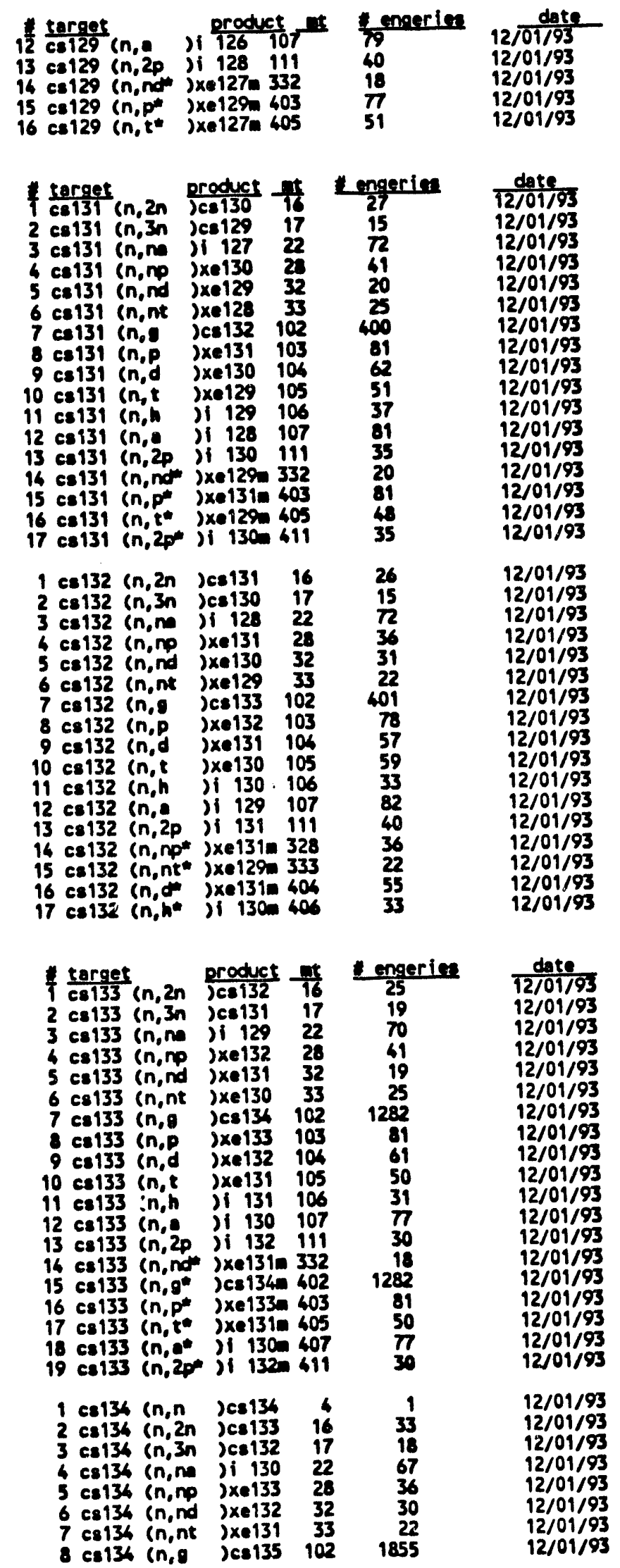

$A-6.8$ 


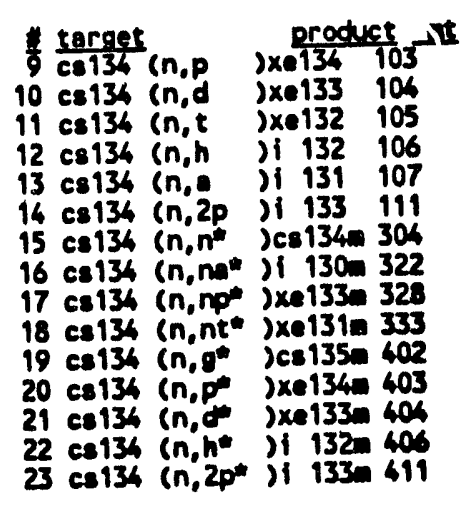

\begin{tabular}{cc} 
*enperies & $\frac{\text { date }}{12 / 01 / 93}$ \\
\hline 9 & $12 / 01 / 93$ \\
55 & $12 / 01 / 93$ \\
55 & $12 / 01 / 93$ \\
29 & $12 / 01 / 93$ \\
84 & $12 / 01 / 93$ \\
35 & $12 / 01 / 93$ \\
55 & $12 / 01 / 93$ \\
64 & $12 / 01 / 93$ \\
36 & $12 / 01 / 93$ \\
22 & $12 / 01 / 93$ \\
1854 & $12 / 01 / 93$ \\
79 & $12 / 01 / 93$ \\
53 & $12 / 01 / 93$ \\
29 & $12 / 01 / 93$ \\
35 &
\end{tabular}

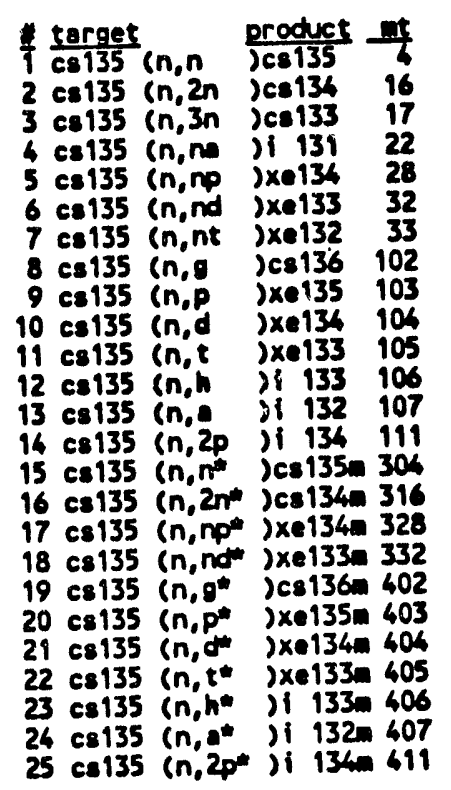

\begin{tabular}{cc} 
Engeries & dete \\
\cline { 2 - 2 } 1 & $12 / 01 / 93$ \\
31 & $12 / 101 / 93$ \\
20 & $12 / 01 / 93$ \\
68 & $12 / 01 / 93$ \\
40 & $12 / 01 / 93$ \\
17 & $12 / 01 / 93$ \\
26 & $12 / 01 / 93$ \\
2433 & $12 / 01 / 93$ \\
79 & $12 / 01 / 93$ \\
55 & $12 / 01 / 93$ \\
51 & $12 / 01 / 93$ \\
27 & $12 / 01 / 93$ \\
71 & $12 / 01 / 93$ \\
28 & $12 / 01 / 93$ \\
51 & $12 / 01 / 93$ \\
27 & $12 / 01 / 93$ \\
31 & $12 / 01 / 93$ \\
17 & $12 / 01 / 93$ \\
2433 & $12 / 01 / 93$ \\
73 & $12 / 01 / 93$ \\
46 & $12 / 01 / 93$ \\
46 & $12 / 01 / 93$ \\
27 & $12 / 01 / 93$ \\
71 & $12 / 01 / 93$ \\
28 & $12 / 01 / 93$
\end{tabular}

\begin{tabular}{|c|c|c|c|c|c|}
\hline 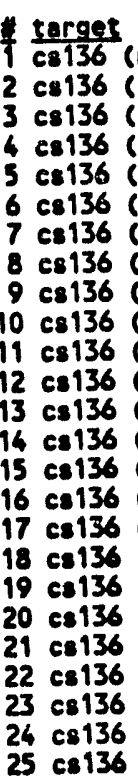 & 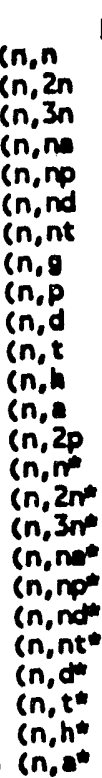 & 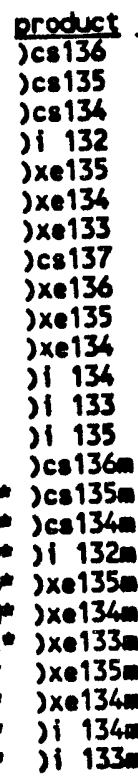 & $\begin{array}{l}7 \\
16 \\
16 \\
17 \\
22 \\
28 \\
32 \\
33 \\
102 \\
103 \\
104 \\
105 \\
106 \\
107 \\
111 \\
304 \\
316 \\
317 \\
322 \\
328 \\
332 \\
333 \\
404 \\
405 \\
406 \\
407\end{array}$ & $\begin{array}{c}\text { engeries } \\
1 \\
29 \\
20 \\
59 \\
37 \\
30 \\
20 \\
2037 \\
79 \\
52 \\
53 \\
23 \\
75 \\
30 \\
61 \\
20 \\
20 \\
59 \\
35 \\
24 \\
20 \\
50 \\
44 \\
23 \\
75\end{array}$ & $\begin{array}{l}\text { dote } \\
12 / 01 / 93 \\
12 / 01 / 93 \\
12 / 01 / 93 \\
12 / 01 / 93 \\
12 / 01 / 93 \\
12 / 01 / 93 \\
12 / 01 / 93 \\
12 / 01 / 93 \\
12 / 01 / 93 \\
12 / 01 / 93 \\
12 / 01 / 93 \\
12 / 101 / 93 \\
12 / 01 / 93 \\
12 / 01 / 93 \\
12 / 101 / 93 \\
12 / 01 / 93 \\
12 / 01 / 93 \\
12 / 1 / 903 \\
12 / 01 / 93 \\
12 / 01 / 93 \\
12 / 1193 \\
12 / 01 / 93 \\
12 / 01 / 93 \\
12 / 0193 \\
12 / 01 / 93\end{array}$ \\
\hline
\end{tabular}




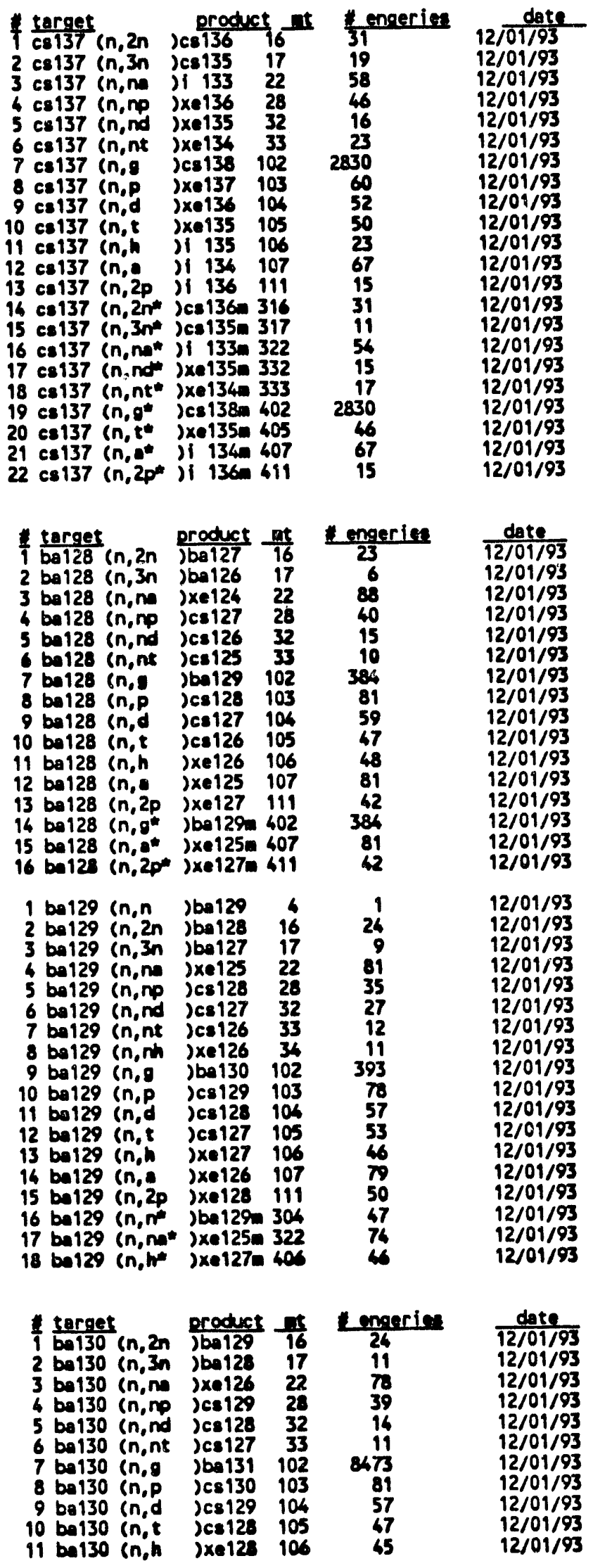

A-6. 10 


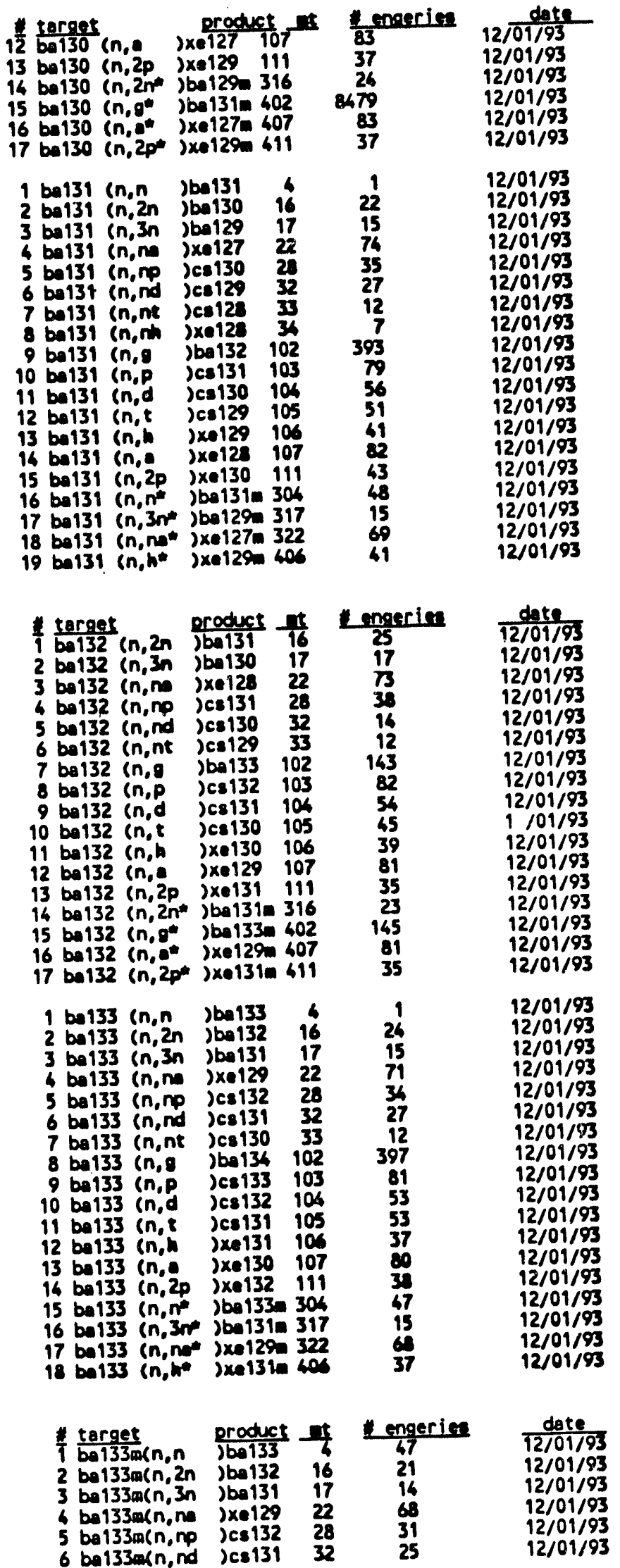

$$
\text { A-6.11 }
$$




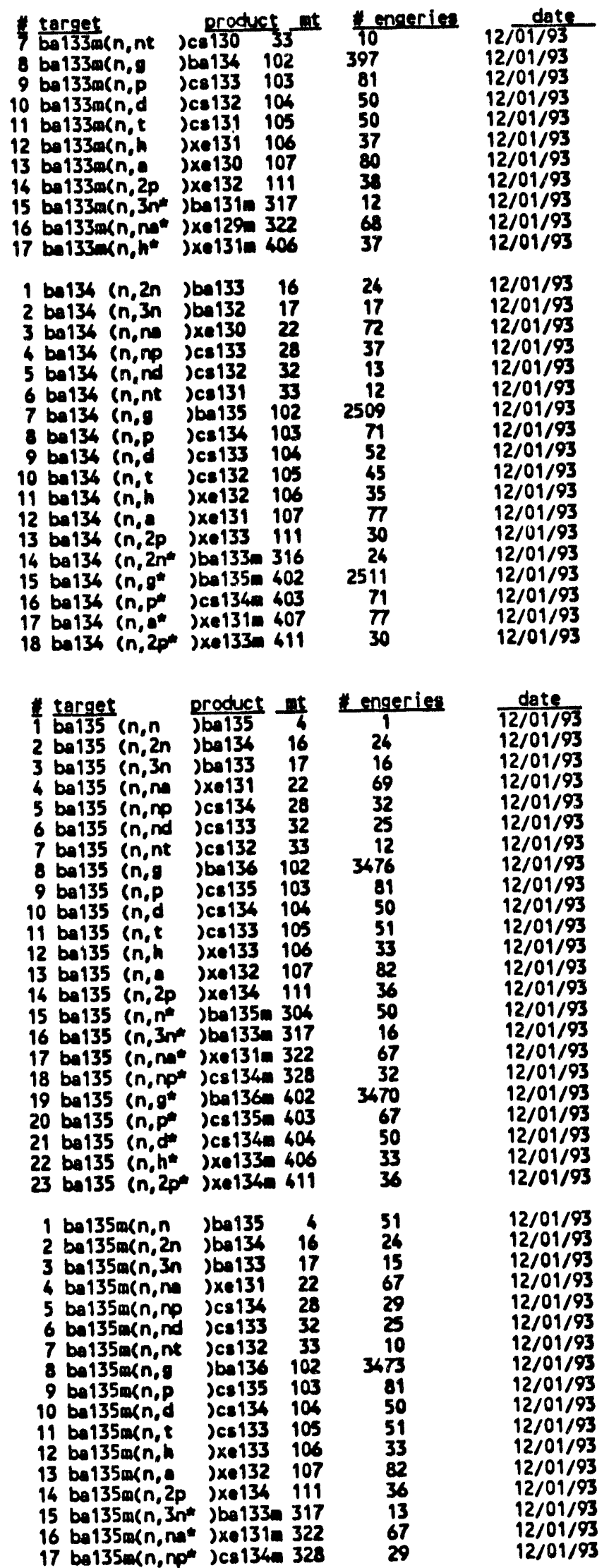




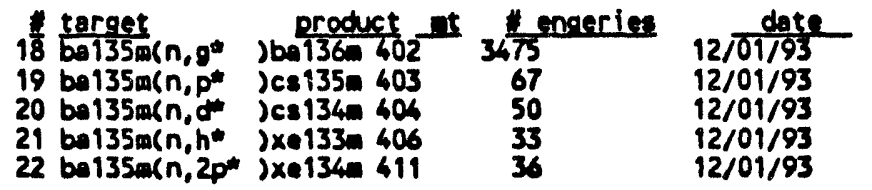

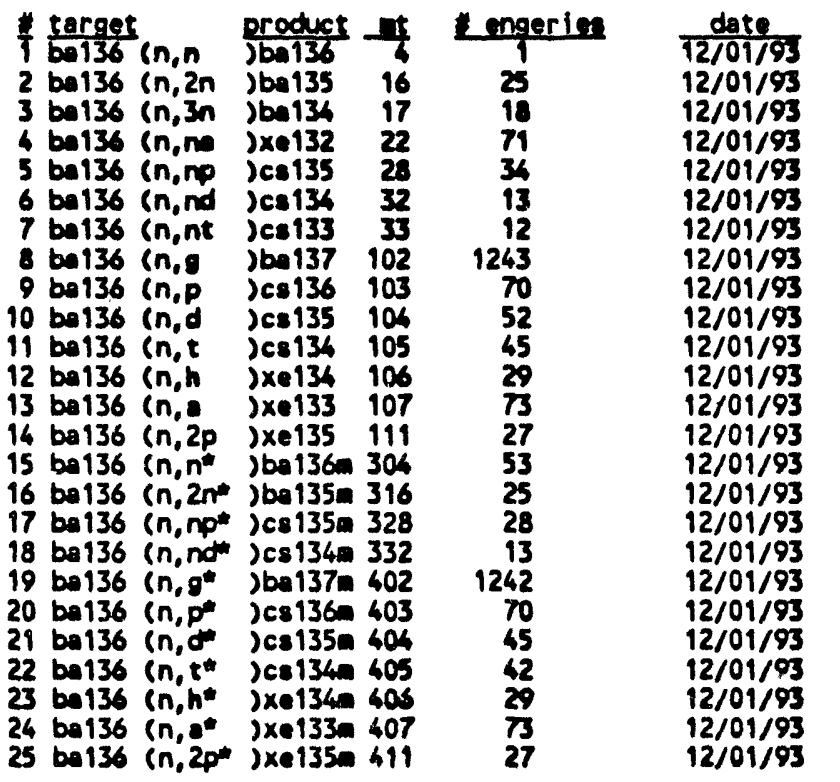

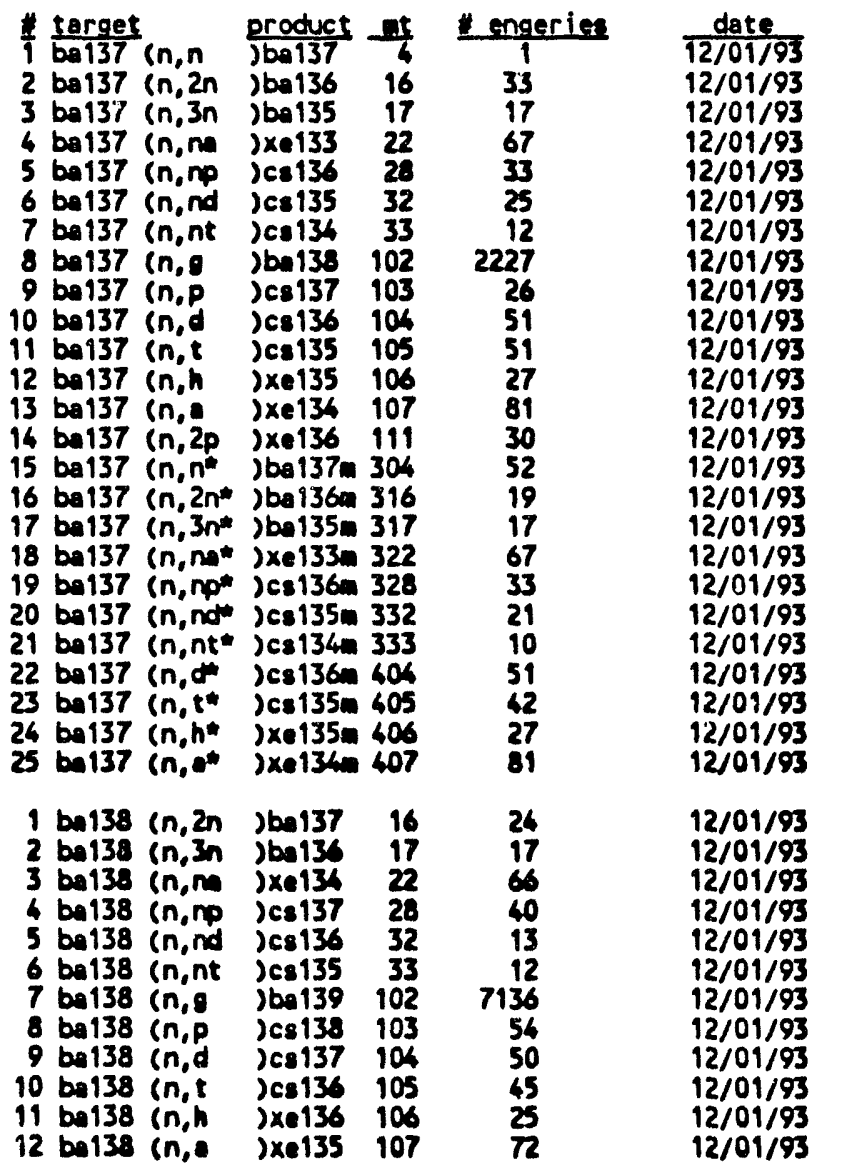

A-6. 13 

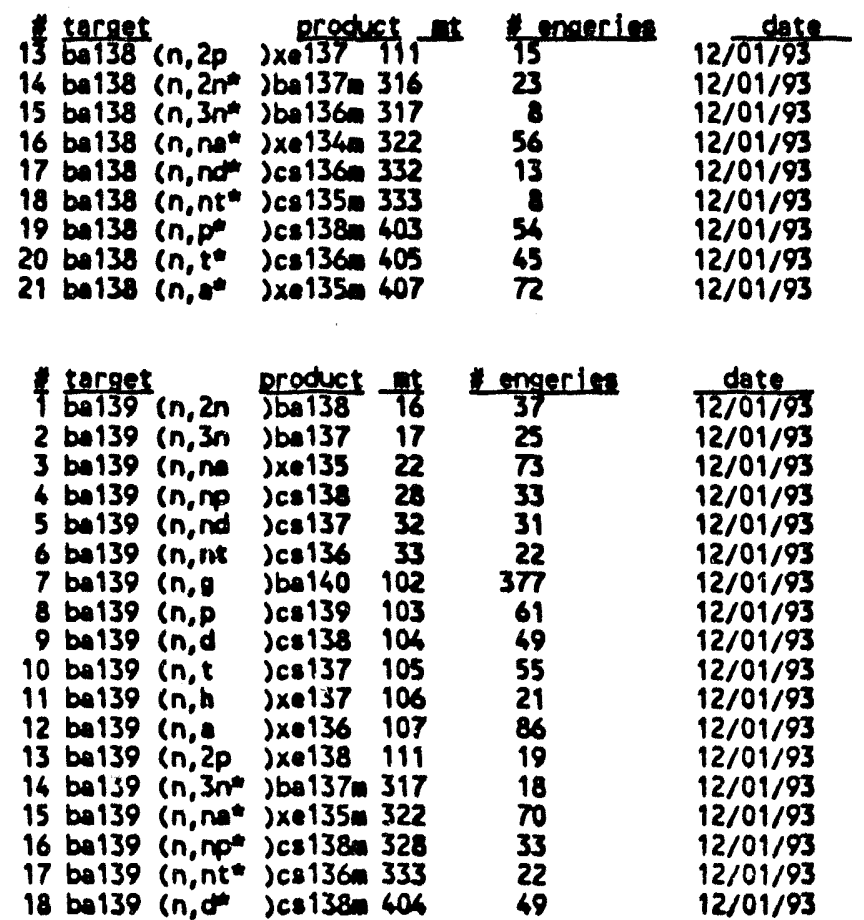

\begin{tabular}{cc} 
engerin & date \\
\hline 37 & $12 / 01 / 93$ \\
25 & $12 / 01 / 93$ \\
73 & $12 / 01 / 93$ \\
33 & $12 / 01 / 93$ \\
31 & $12 / 01 / 93$ \\
22 & $12 / 01 / 93$ \\
377 & $12 / 01 / 93$ \\
61 & $12 / 01 / 93$ \\
49 & $12 / 01 / 93$ \\
55 & $12 / 01 / 93$ \\
21 & $12 / 01 / 93$ \\
86 & $12 / 01 / 93$ \\
19 & $12 / 01 / 93$ \\
18 & $12 / 01 / 93$ \\
70 & $12 / 01 / 93$ \\
33 & $12 / 01 / 93$ \\
22 & $12 / 01 / 93$ \\
49 & $12 / 01 / 93$ \\
& $12 / 01 / 93$ \\
57 & $12 / 01 / 93$ \\
27 & $12 / 01 / 93$ \\
66 & $12 / 01 / 93$ \\
36 & $12 / 01 / 93$ \\
22 & $12 / 01 / 93$ \\
32 & $12 / 01 / 93$ \\
3353 & $12 / 01 / 93$ \\
54 & $12 / 01 / 93$ \\
40 & $12 / 01 / 93$ \\
49 & $12 / 01 / 93$ \\
19 & $12 / 01 / 93$ \\
76 & $12 / 01 / 93$ \\
11 & $12 / 01 / 93$ \\
22 & $12 / 01 / 93$ \\
46 &
\end{tabular}

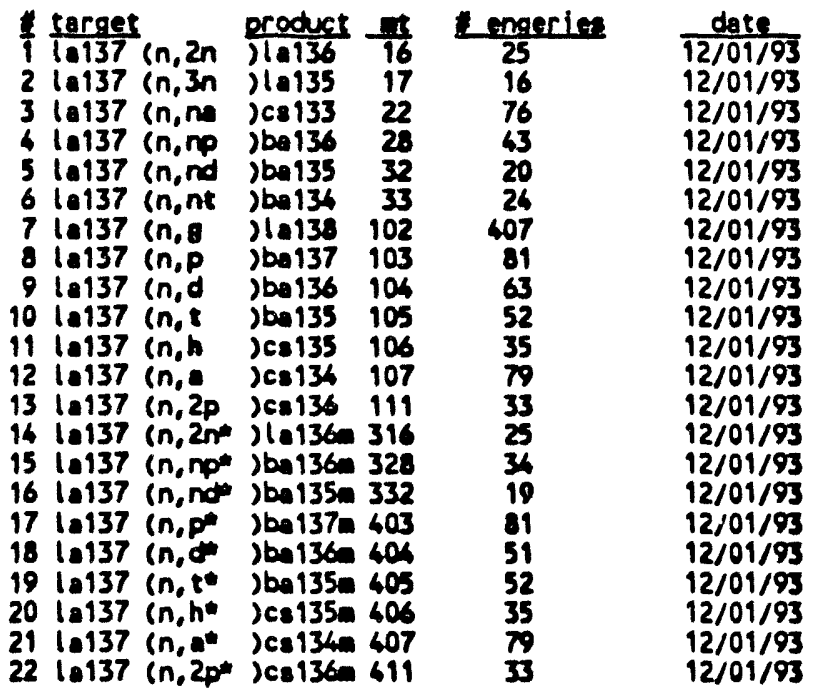




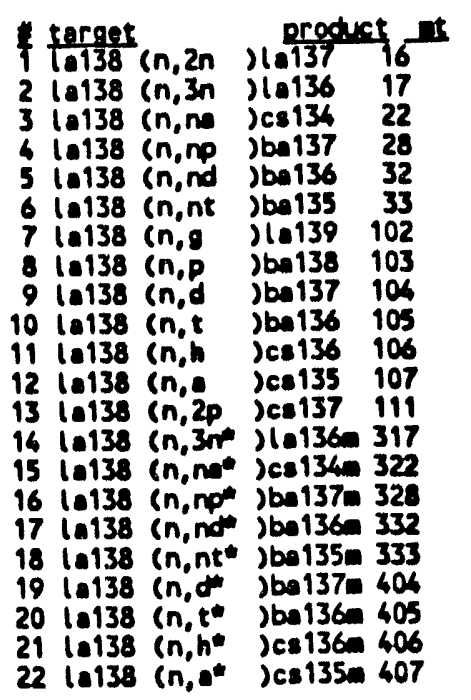

\begin{tabular}{cc} 
meneries & dete \\
\hline 26 & $12 / 01 / 93$ \\
17 & $12 / 01 / 93$ \\
70 & $12 / 01 / 93$ \\
37 & $12 / 01 / 93$ \\
31 & $12 / 01 / 93$ \\
20 & $12 / 01 / 93$ \\
1560 & $12 / 01 / 93$ \\
78 & $12 / 01 / 93$ \\
58 & $12 / 01 / 93$ \\
36 & $12 / 01 / 93$ \\
31 & $12 / 01 / 93$ \\
81 & $12 / 01 / 93$ \\
36 & $12 / 01 / 93$ \\
17 & $12 / 01 / 93$ \\
70 & $12 / 01 / 93$ \\
35 & $12 / 01 / 93$ \\
26 & $12 / 01 / 93$ \\
20 & $12 / 01 / 93$ \\
52 & $12 / 01 / 93$ \\
46 & $12 / 01 / 93$ \\
31 & $12 / 01 / 93$ \\
81 & $12 / 01 / 93$
\end{tabular}

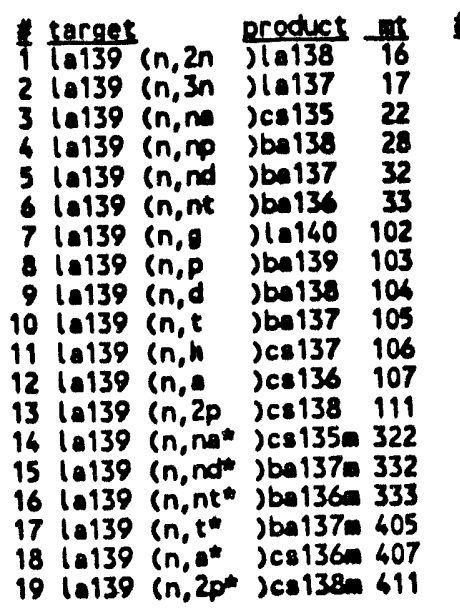

\begin{tabular}{cc} 
Lengerien & date \\
\cline { 2 - 2 } 30 & $12 / 01 / 93$ \\
19 & $12 / 01 / 93$ \\
70 & $12 / 01 / 93$ \\
47 & $12 / 01 / 93$ \\
18 & $12 / 01 / 93$ \\
26 & $12 / 01 / 93$ \\
1282 & $12 / 01 / 93$ \\
71 & $12 / 01 / 93$ \\
58 & $12 / 01 / 93$ \\
53 & $12 / 01 / 93$ \\
29 & $12 / 01 / 93$ \\
78 & $12 / 01 / 93$ \\
23 & $12 / 01 / 93$ \\
62 & $12 / 01 / 93$ \\
17 & $12 / 01 / 93$ \\
18 & $12 / 01 / 93$ \\
48 & $12 / 01 / 93$ \\
78 & $12 / 01 / 93$ \\
23 & $12 / 01 / 93$
\end{tabular}

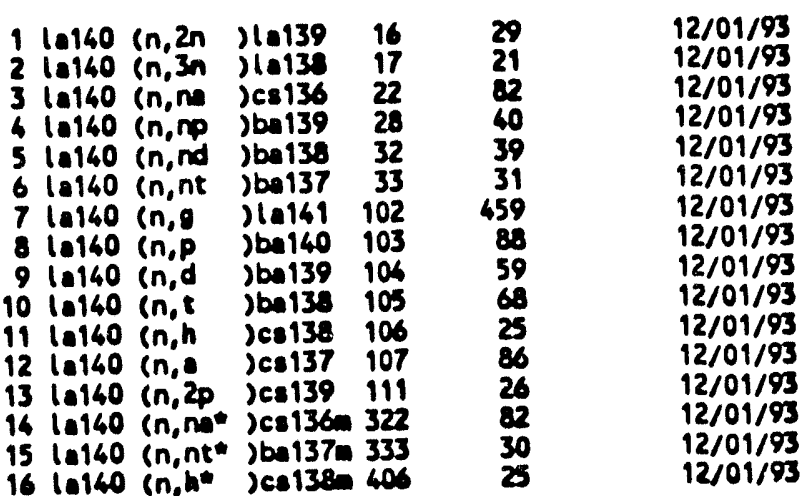

\begin{tabular}{|c|c|c|c|c|}
\hline 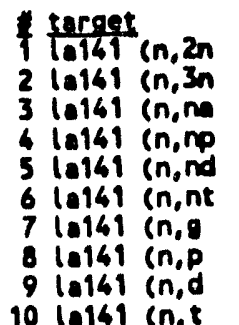 & 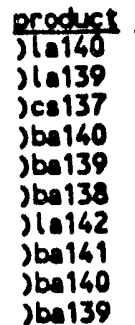 & $\begin{array}{r}-1 \\
16 \\
17 \\
22 \\
28 \\
32 \\
33 \\
102 \\
103\end{array}$ & $\begin{array}{c}\text { Lengerin } \\
67 \\
22 \\
72 \\
42 \\
29 \\
42 \\
418 \\
70 \\
57 \\
58\end{array}$ & $\begin{array}{l}\text { dote } \\
\text { 12/01/93 } \\
12 / 01 / 93 \\
12 / 01 / 93 \\
12 / 01 / 93 \\
12 / 01 / 93 \\
12 / 01 / 93 \\
12 / 01 / 93 \\
12 / 01 / 93 \\
12 / 01 / 93 \\
12 / 01 / 93\end{array}$ \\
\hline
\end{tabular}




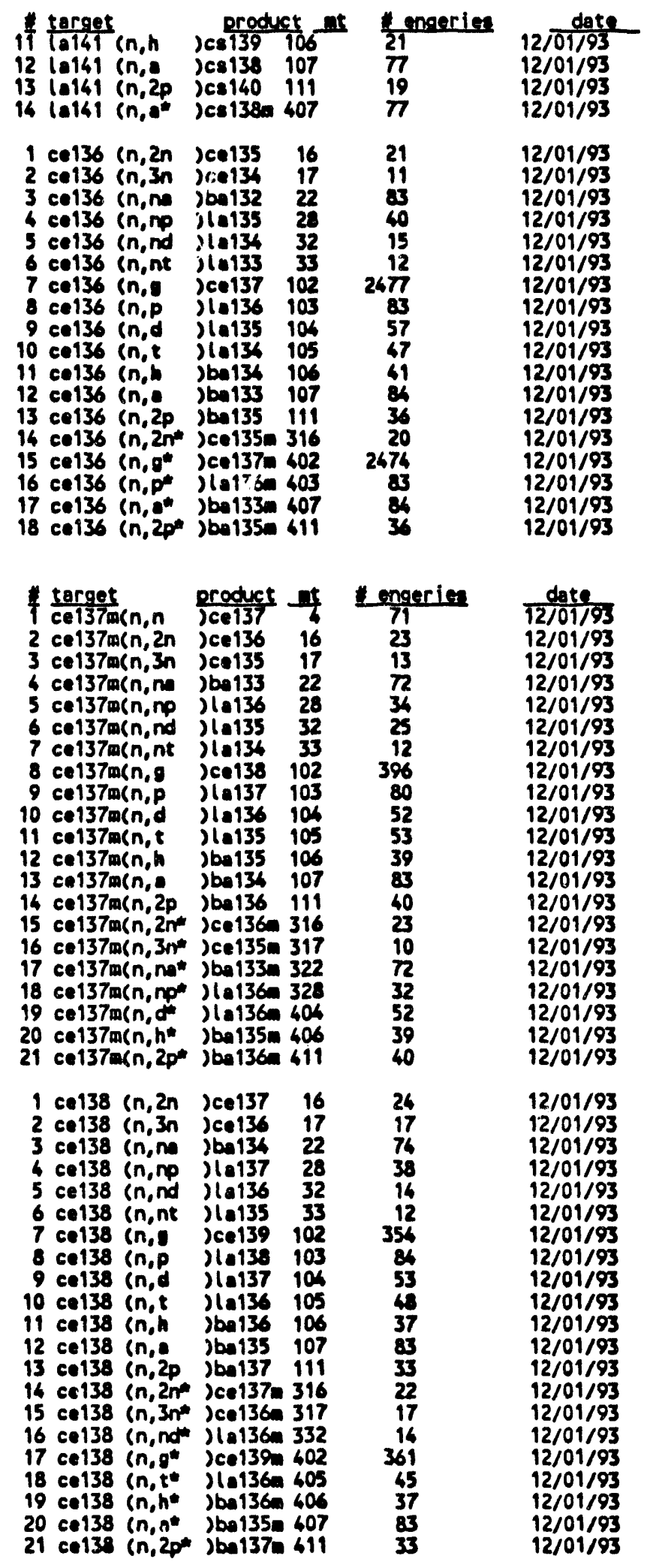




\begin{tabular}{|c|c|c|c|c|c|c|}
\hline $\begin{array}{l}7 \\
7 \\
3 \\
3 \\
5 \\
5 \\
7 \\
8 \\
8 \\
0\end{array}$ & 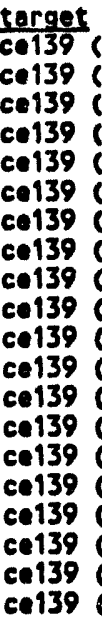 & 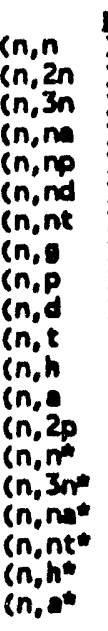 & 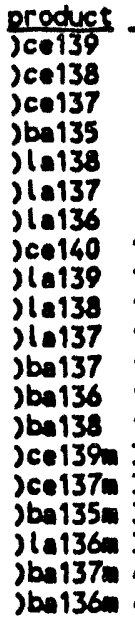 & $\begin{array}{c}94 \\
16 \\
17 \\
22 \\
28 \\
32 \\
33 \\
102 \\
103 \\
104 \\
105 \\
106 \\
107 \\
111 \\
304 \\
317 \\
322 \\
333 \\
406 \\
407\end{array}$ & $\begin{array}{c}\text { engerien } \\
26 \\
26 \\
17 \\
72 \\
34 \\
26 \\
11 \\
377 \\
81 \\
51 \\
52 \\
35 \\
81 \\
36 \\
53 \\
13 \\
72 \\
10 \\
35 \\
81\end{array}$ & $\begin{array}{l}\text { dare } \\
12 / 01 / 93 \\
12 / 01 / 93 \\
12 / 01 / 93 \\
12 / 01 / 93 \\
12 / 01 / 93 \\
12 / 01 / 93 \\
12 / 01 / 93 \\
12 / 01 / 93 \\
12 / 01 / 93 \\
12 / 01 / 93 \\
12 / 01 / 93 \\
12 / 01 / 93 \\
12 / 01 / 93 \\
12 / 01 / 93 \\
12 / 01 / 93 \\
12 / 01 / 93 \\
12 / 01 / 93 \\
12 / 01 / 93 \\
12 / 01 / 93 \\
12 / 01 / 93\end{array}$ \\
\hline $\begin{array}{l}1 \\
2 \\
3 \\
6 \\
5 \\
6 \\
7 \\
8 \\
9 \\
10 \\
11 \\
12 \\
13 \\
14\end{array}$ & 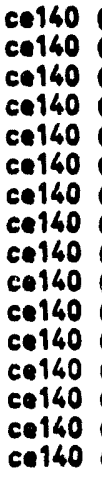 & 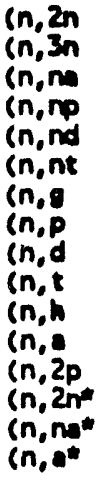 & 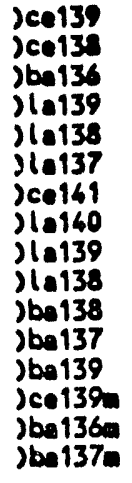 & $\begin{array}{l}16 \\
17 \\
22 \\
28 \\
32 \\
33 \\
102 \\
103 \\
104 \\
105 \\
106 \\
107 \\
111 \\
316 \\
322 \\
407\end{array}$ & $\begin{array}{r}26 \\
18 \\
73 \\
61 \\
16 \\
11 \\
1831 \\
66 \\
54 \\
67 \\
31 \\
79 \\
25 \\
21 \\
62 \\
79\end{array}$ & $\begin{array}{l}12 / 01 / 93 \\
12 / 01 / 93 \\
12 / 01 / 93 \\
12 / 01 / 93 \\
12 / 01 / 93 \\
12 / 01 / 93 \\
12 / 01 / 93 \\
12 / 01 / 93 \\
12 / 01 / 93 \\
12 / 01 / 93 \\
12 / 01 / 93 \\
12 / 01 / 93 \\
12 / 01 / 93 \\
12 / 01 / 93 \\
12 / 01 / 93 \\
12 / 01 / 93\end{array}$ \\
\hline $\begin{array}{l}10 \\
11 \\
12 \\
13\end{array}$ & 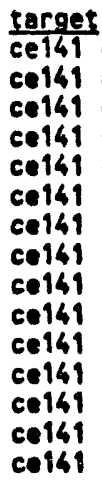 & 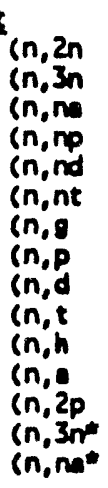 & 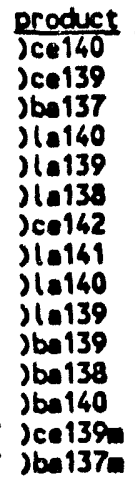 & $\begin{array}{c}\frac{. t}{16} \\
17 \\
22 \\
28 \\
32 \\
33 \\
102 \\
103 \\
104 \\
105 \\
106 \\
107 \\
111 \\
317\end{array}$ & $\begin{array}{c}\text { engeries } \\
38 \\
22 \\
78 \\
36 \\
33 \\
19 \\
6313 \\
76 \\
51 \\
55 \\
29 \\
83 \\
28 \\
18 \\
76\end{array}$ & $\begin{array}{l}\frac{\text { date }}{12 / 01 / 93} \\
12 / 01 / 93 \\
12 / 01 / 93 \\
12 / 01 / 93 \\
12 / 01 / 93 \\
12 / 01 / 93 \\
12 / 01 / 93 \\
12 / 01 / 93 \\
12 / 01 / 93 \\
12 / 01 / 93 \\
12 / 01 / 93 \\
12 / 01 / 93 \\
12 / 01 / 93 \\
12 / 01 / 93 \\
12 / 01 / 93\end{array}$ \\
\hline $\begin{array}{l}1 \\
1 \\
1\end{array}$ & $\begin{array}{l}c e 142 \\
c e 142 \\
c e 142 \\
c e 142 \\
c e 142 \\
c e 142 \\
c e 142 \\
c e 142 \\
c e 142 \\
c e 142 \\
c e 142 \\
c e 142 \\
c e 142\end{array}$ & 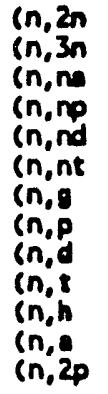 & 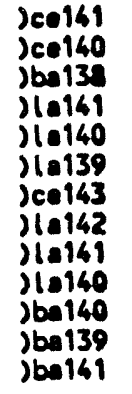 & $\begin{array}{l}16 \\
17 \\
22 \\
28 \\
32 \\
33 \\
102 \\
103 \\
104 \\
105 \\
106 \\
107 \\
111\end{array}$ & $\begin{array}{r}42 \\
26 \\
72 \\
37 \\
23 \\
30 \\
1317 \\
62 \\
51 \\
50 \\
25 \\
83 \\
19\end{array}$ & $\begin{array}{l}12 / 01 / 93 \\
12 / 01 / 93 \\
12 / 01 / 93 \\
12 / 01 / 93 \\
12 / 01 / 93 \\
12 / 01 / 93 \\
12 / 01 / 93 \\
12 / 01 / 93 \\
12 / 01 / 93 \\
12 / 01 / 93 \\
12 / 01 / 93 \\
12 / 01 / 93 \\
12 / 01 / 93\end{array}$ \\
\hline
\end{tabular}




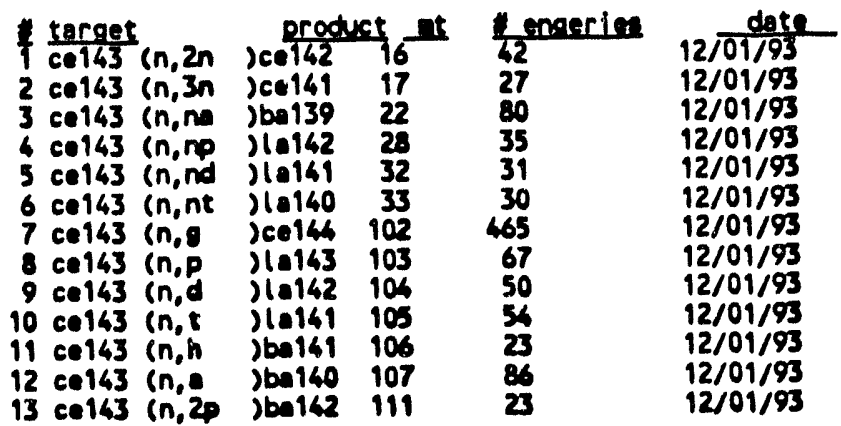

\begin{tabular}{|c|c|c|c|c|c|}
\hline 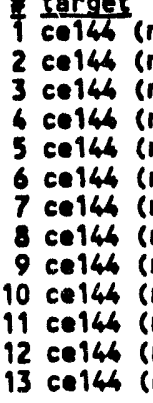 & $\begin{array}{l}(n, 2 n) \\
(n, 3 n) \\
(n, n) \\
(n, n p) \\
\text { (n,nd }) \\
(n, n t) \\
(n, 9) \\
(n, p) \\
(n, d) \\
(n, t) \\
(n, n) \\
(n, 2) \\
(n, 2 p)\end{array}$ & 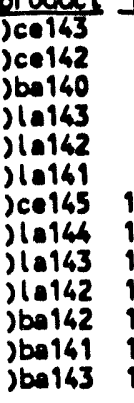 & $\begin{array}{l}17 \\
17 \\
22 \\
22 \\
32 \\
33 \\
102 \\
103 \\
104 \\
105 \\
106 \\
107 \\
111\end{array}$ & $\begin{array}{l}50 \\
23 \\
70 \\
39 \\
26 \\
29 \\
3857 \\
56 \\
49 \\
50 \\
19 \\
73 \\
13\end{array}$ & $\begin{array}{l}12 / 01 / 93 \\
12 / 01 / 93 \\
12 / 01 / 93 \\
12 / 01 / 93 \\
12 / 01 / 93 \\
12 / 01 / 93 \\
12 / 01 / 93 \\
12 / 01 / 93 \\
12 / 01 / 93 \\
12 / 01 / 93 \\
12 / 01 / 93 \\
12 / 01 / 93 \\
12 / 01 / 93\end{array}$ \\
\hline 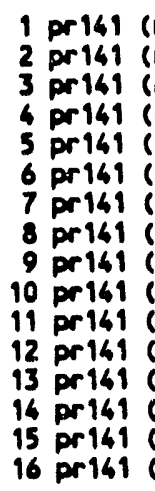 & 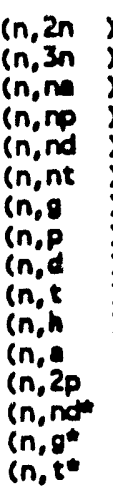 & 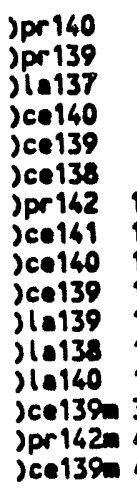 & $\begin{array}{l}16 \\
17 \\
22 \\
28 \\
32 \\
33 \\
102 \\
103 \\
104 \\
105 \\
106 \\
107 \\
111 \\
332 \\
402 \\
405\end{array}$ & $\begin{array}{r}31 \\
17 \\
76 \\
49 \\
20 \\
21 \\
1282 \\
84 \\
64 \\
55 \\
35 \\
84 \\
31 \\
16 \\
1282 \\
50\end{array}$ & $\begin{array}{l}12 / 01 / 93 \\
12 / 01 / 93 \\
12 / 01 / 93 \\
12 / 01 / 93 \\
12 / 01 / 93 \\
12 / 01 / 93 \\
12 / 01 / 93 \\
12 / 01 / 93 \\
12 / 01 / 93 \\
12 / 01 / 93 \\
12 / 01 / 93 \\
12 / 01 / 93 \\
12 / 01 / 93 \\
12 / 01 / 93 \\
12 / 01 / 93 \\
12 / 01 / 93\end{array}$ \\
\hline $\begin{array}{l}1 \text { pr } 142 \\
2 \text { pr } 142 \\
3 \text { pr } 142 \\
4 \text { pr } 142 \\
5 \text { pr } 142 \\
6 \text { pr } 142 \\
7 \text { pr } 142 \\
8 \text { pr } 142 \\
9 \text { pr } 142 \\
10 \text { pr } 142 \\
11 \text { pr } 142 \\
12 \text { pr } 142 \\
13 \text { pr } 142 \\
14 \text { pr } 142 \\
15 \text { pr } 142 \\
16 \text { pr } 142 \\
17 \text { pr } 142\end{array}$ & 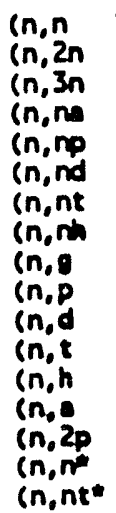 & 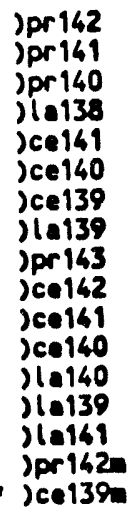 & $\begin{array}{l}4 \\
16 \\
17 \\
22 \\
28 \\
32 \\
33 \\
34 \\
102 \\
103 \\
104 \\
105 \\
106 \\
107 \\
111 \\
304 \\
333\end{array}$ & $\begin{array}{l}1 \\
32 \\
20 \\
83 \\
42 \\
39 \\
32 \\
6 \\
465 \\
86 \\
64 \\
68 \\
33 \\
84 \\
34 \\
55 \\
28\end{array}$ & $\begin{array}{l}12 / 01 / 93 \\
12 / 01 / 93 \\
12 / 01 / 93 \\
12 / 01 / 93 \\
12 / 01 / 93 \\
12 / 21 / 93 \\
12 / 01 / 93 \\
12 / 01 / 93 \\
12 / 01 / 93 \\
12 / 01 / 93 \\
12 / 01 / 93 \\
12 / 01 / 93 \\
12 / 01 / 93 \\
12 / 01 / 93 \\
12 / 01 / 93 \\
12 / 01 / 93 \\
12 / 01 / 93\end{array}$ \\
\hline $\begin{array}{l}\frac{1}{1} \frac{\text { target }}{1} \text { pr } 143 \\
2 \text { pr } 143 \\
3 \text { pr } 143 \\
6 \text { pr } 143 \\
5 \text { pr } 143 \\
6 \text { pr } 143 \\
7 \text { pr } 143\end{array}$ & $\begin{array}{l}\text { (n, 2n } \\
(n, 3 n \\
(n, n) \\
(n, n) \\
(n, n d \\
(n, n) \\
(n, \theta)\end{array}$ & $\begin{array}{l}\text { Jpr } 142 \\
\text { Jpr } 161 \\
\text { Jle139 } \\
\text { Jce162 } \\
\text { Jce161 } \\
\text { Jce140 } \\
\text { Jpr } 144\end{array}$ & $\begin{array}{r}17 \\
22 \\
28 \\
32 \\
33 \\
102\end{array}$ & $\begin{array}{l}\text { engerie } \\
46 \\
25 \\
80 \\
65 \\
30 \\
42 \\
469\end{array}$ & $\begin{array}{l}\text { date } \\
12 / 01 / 93 \\
12 / 01 / 93 \\
12 / 01 / 93 \\
12 / 01 / 93 \\
12 / 01 / 93 \\
12 / 01 / 93 \\
12 / 01 / 93\end{array}$ \\
\hline
\end{tabular}

$A-6.18$ 


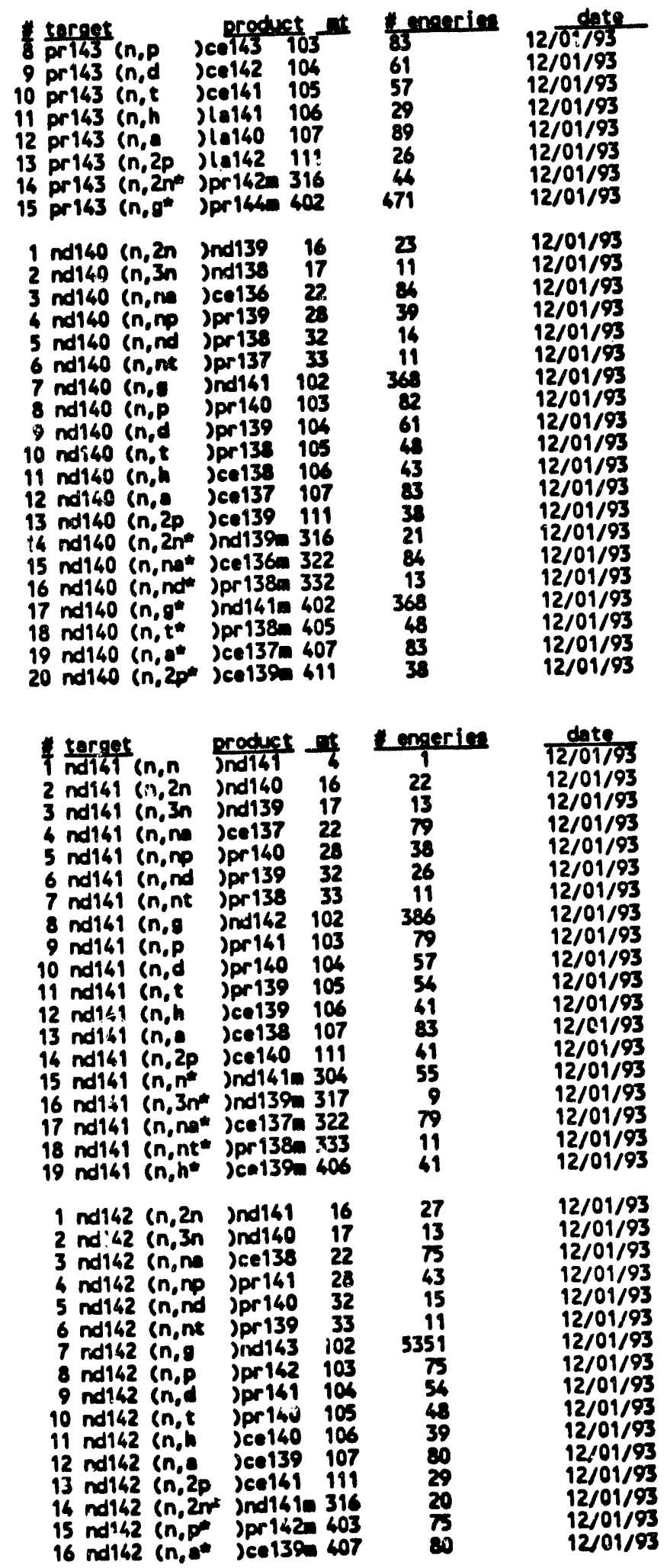




\begin{tabular}{|c|c|c|c|c|c|}
\hline $\begin{array}{l}85 \\
15 n \\
2 n \\
3 n \\
4 n \\
5 n \\
6 n \\
7 n \\
8 n \\
9 n \\
10 n \\
11 n \\
12 n \\
13 n \\
14 n \\
15 n \\
16 n \\
17 n \\
18 n\end{array}$ & 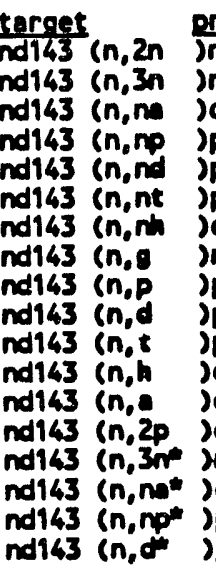 & 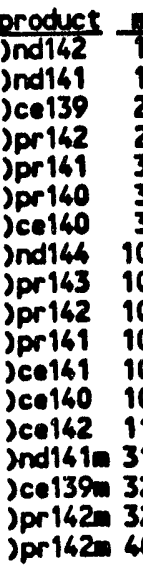 & $\begin{array}{l}16 \\
16 \\
17 \\
22 \\
28 \\
32 \\
33 \\
34 \\
102 \\
103 \\
104 \\
105 \\
106 \\
107 \\
111 \\
317 \\
322 \\
328 \\
404\end{array}$ & $\begin{array}{c}\text { engerien } \\
27 \\
16 \\
84 \\
36 \\
32 \\
15 \\
7 \\
1282 \\
88 \\
56 \\
59 \\
35 \\
82 \\
34 \\
16 \\
84 \\
36 \\
56\end{array}$ & $\begin{array}{l}\text { date } \\
12 / 01 / 93 \\
12 / 01 / 93 \\
12 / 01 / 93 \\
12 / 01 / 93 \\
12 / 011993 \\
12 / 01 / 93 \\
12 / 01 / 93 \\
12 / 01 / 93 \\
12 / 01 / 93 \\
12 / 01 / 93 \\
12 / 01 / 93 \\
12 / 01 / 93 \\
12 / 01 / 93 \\
12 / 01 / 93 \\
12 / 01 / 93 \\
12 / 01 / 93 \\
12 / 01 / 93 \\
12 / 01 / 93\end{array}$ \\
\hline $\begin{array}{c}8 \\
9 \\
10 \\
11 \\
12 \\
13 \\
16 \\
15 \\
16\end{array}$ & 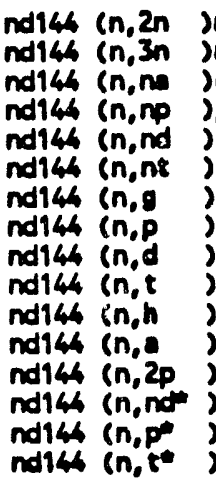 & 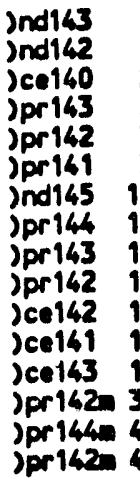 & $\begin{array}{l}16 \\
17 \\
22 \\
28 \\
32 \\
33 \\
102 \\
103 \\
104 \\
105 \\
106 \\
107 \\
111 \\
332 \\
403 \\
405\end{array}$ & $\begin{array}{r}39 \\
21 \\
81 \\
38 \\
24 \\
27 \\
7664 \\
70 \\
54 \\
52 \\
33 \\
09 \\
27 \\
24 \\
70 \\
52\end{array}$ & $\begin{array}{l}12 / 01 / 93 \\
12 / 01 / 93 \\
12 / 01 / 93 \\
12 / 01 / 93 \\
12 / 01 / 93 \\
12 / 01 / 93 \\
12 / 01 / 93 \\
12 / 01 / 93 \\
12 / 01 / 93 \\
12 / 01 / 93 \\
12 / 01 / 93 \\
12 / 01 / 93 \\
12 / 01 / 93 \\
12 / 01 / 93 \\
12 / 01 / 93 \\
12 / 01 / 93\end{array}$ \\
\hline $\begin{array}{l}10 \\
10\end{array}$ & $\begin{array}{l}\text { terget } \\
\text { nd145 (n, 2n } \\
\text { nd145 (n,3n } \\
\text { nd145 (n,ne } \\
\text { nd145 (n,np } \\
\text { nd145 (n,nd } \\
\text { nd145 (n,nt } \\
\text { nd145 in,e } \\
\text { nd145 in,p } \\
\text { nd145 in,d } \\
\text { nd145 (n,t } \\
\text { nd145 (n,n } \\
\text { nd145 (n,e } \\
\text { nd145 (n,2p } \\
\text { nd145 (n,np } \\
\text { nd145 in,nt* } \\
\text { nd145 in,d }\end{array}$ & $\begin{array}{l}\text { product } \\
\text { ond144 } \\
\text { jnd143 } \\
\text { jce141 } \\
\text { jpr144 } \\
\text { jpr143 } \\
\text { jpr142 } \\
\text { jnd146 } \\
\text { jpr145 } \\
\text { jpr144 } \\
\text { jpr143 } \\
\text { jee143 } \\
\text { jce142 } \\
\text { jce144 } \\
\text { jpr1444 } \\
\text { jpr142. } \\
\text { Jpr144 }\end{array}$ & $\begin{array}{l}-9 t \\
16 \\
17 \\
22 \\
28 \\
32 \\
33 \\
102 \\
103 \\
104 \\
105 \\
106 \\
107 \\
111 \\
328 \\
333 \\
404\end{array}$ & $\begin{array}{c}\text { engeries } \\
40 \\
24 \\
86 \\
36 \\
31 \\
28 \\
1282 \\
75 \\
54 \\
55 \\
29 \\
85 \\
29 \\
36 \\
28 \\
54\end{array}$ & $\begin{array}{l}\text { date } \\
12 / 01 / 93 \\
12 / 01 / 93 \\
12 / 01 / 93 \\
12 / 01 / 93 \\
12 / 01 / 93 \\
12 / 01 / 93 \\
12 / 01 / 93 \\
12 / 01 / 93 \\
12 / 01 / 93 \\
12 / 01 / 93 \\
12 / 01 / 93 \\
12 / 01 / 93 \\
12 / 01 / 93 \\
12 / 01 / 93 \\
12 / 01 / 93 \\
12 / 01 / 93\end{array}$ \\
\hline $\begin{array}{l}9 \\
10 \\
11 \\
12 \\
13 \\
14 \\
15\end{array}$ & 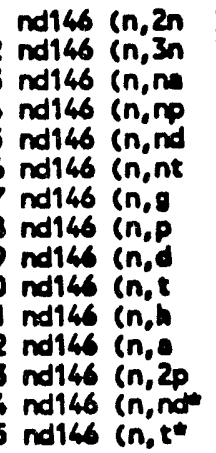 & 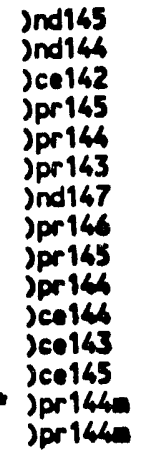 & $\begin{array}{l}16 \\
17 \\
22 \\
28 \\
32 \\
33 \\
102 \\
103 \\
104 \\
105 \\
106 \\
107 \\
111 \\
332 \\
405\end{array}$ & $\begin{array}{r}39 \\
26 \\
77 \\
38 \\
25 \\
27 \\
1282 \\
65 \\
53 \\
50 \\
27 \\
81 \\
21 \\
25 \\
50\end{array}$ & $\begin{array}{l}12 / 01 / 93 \\
12 / 01 / 93 \\
12 / 01 / 93 \\
12 / 01 / 93 \\
12 / 01 / 93 \\
12 / 01 / 93 \\
12 / 01 / 93 \\
12 / 01 / 93 \\
12 / 01 / 93 \\
12 / 01 / 93 \\
12 / 01 / 93 \\
12 / 01 / 93 \\
12 / 01 / 93 \\
12 / 01 / 93 \\
12 / 01 / 93\end{array}$ \\
\hline
\end{tabular}




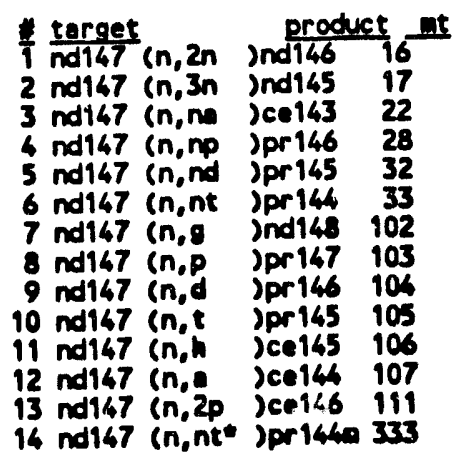

\begin{tabular}{l} 
tengeries \\
\hline 45 \\
26 \\
86 \\
36 \\
32 \\
30 \\
1804 \\
76 \\
52 \\
55 \\
25 \\
83 \\
25 \\
30
\end{tabular}

date
$12 / 01 / 93$
$12 / 01 / 93$
$12 / 01 / 93$
$12 / 01 / 93$
$12 / 01 / 93$
$12 / 01 / 93$
$12 / 01 / 93$
$12 / 01 / 93$
$12 / 01 / 93$
$12 / 01 / 93$
$12 / 01 / 93$
$12 / 01 / 93$
$12 / 01 / 93$
$12 / 01 / 93$

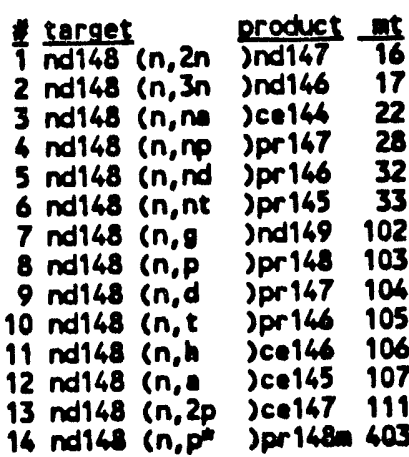

\begin{tabular}{c} 
engeries \\
\hline 46 \\
26 \\
70
\end{tabular}

$\frac{\text { date }}{12 / 01 / 93}$

$12 / 01 / 93$

$12 / 01 / 93$

$12 / 01 / 93$

$12 / 01 / 93$

$12 / 01 / 93$

$12 / 01 / 93$

$12 / 01 / 93$

$12 / 01 / 93$

$12 / 01 / 93$

$12 / 01 / 93$

$12 / 01 / 93$

$12 / 01 / 93$

16 ndits (n,p )pritim 403

1 nd149 (n,2n )nd148 16

2 nd149 (n,3n )nd147 17

3 nd149 (n,na ) cel45 22

4 nd149 (n,np )pr148 28

5 nd149 (n,nd)pr147 32

6 nd149 (n,nt )pr146 33

7 nd149 (n,g) ind150 102

8 nd149 (n,p )pr149 103

9 nd149 (n,d jpr148 104

10 nd149 (n,t jpr147 105

11 nd149 (n,h ) ce147 106

12 nd149 (n, )ce146 107

13 nd149 (n,2p )ce148 111

14 nd149 (n,np* )pr148m 328

15 nd149 (n, ) )pr148m 404

1 nd150 (n,2n )nd149 16

2 nd150 (n,3n )nd14 17

3 nd150 (n,n) $)$ ce146 22

4 nd150 (n,np)pr149 28

5 nd150 (n,nd )pr148 32

6 nd150 (n,nt ipr167 33

7 nd150 $\mathrm{ln}, \mathrm{g}$ ind151 102

8 nd150 (n.p )pr150 103

9 nd150 (n,d jpr149 104

10 nd150 (n,t )pr148 105

11 nd150 (n,h )ce148 106

12 nd150 (n, ) ce147 107

13 nd150 (n,2p )ce149 111

14 nd150 (n, ndt )pr148n 332

15 nd150 (n,t* Jpr148 405

\begin{tabular}{|c|c|c|c|c|}
\hline 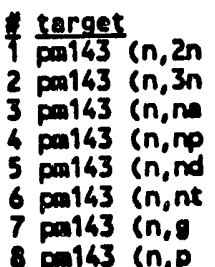 & 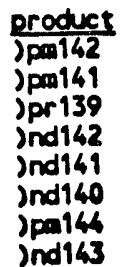 & $\begin{array}{l}\frac{n t}{16} \\
17 \\
22 \\
28 \\
32 \\
33 \\
102 \\
103\end{array}$ & $\begin{array}{c}\text { engeries } \\
22 \\
9 \\
79 \\
53 \\
21 \\
17 \\
395 \\
79\end{array}$ & $\begin{array}{l}\text { date } \\
12 / 01 / 93 \\
12 / 01 / 93 \\
12 / 01 / 93 \\
12 / 01 / 93 \\
12 / 01 / 93 \\
12 / 01 / 93 \\
12 / 01 / 93 \\
12 / 01 / 93\end{array}$ \\
\hline
\end{tabular}

$A-6.21$ 


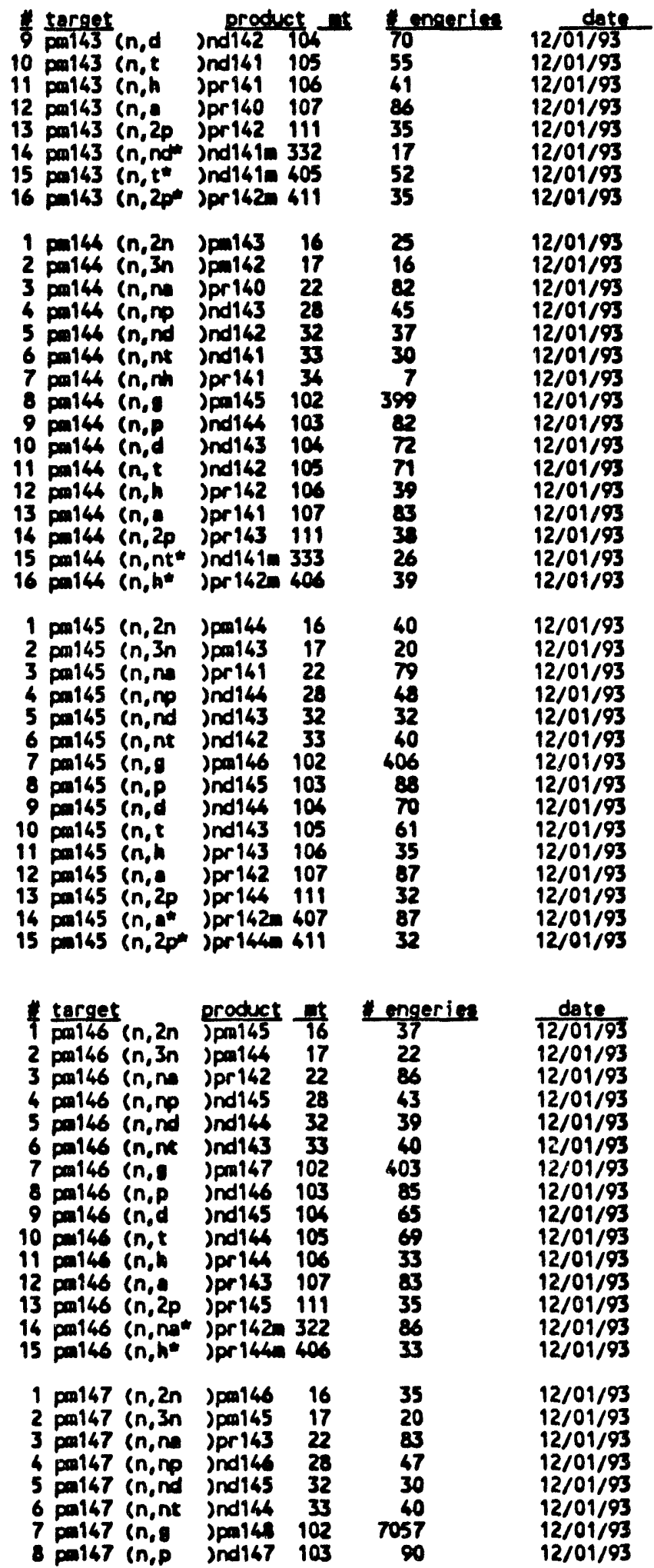




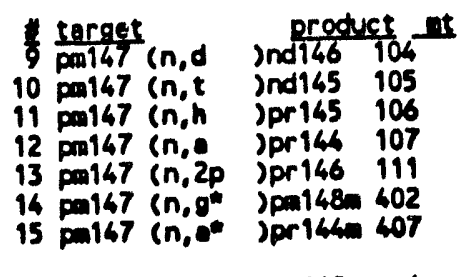

1 engerias
65
57
31
81
28
7058
81

date
$12 / 01 / 93$
$12 / 01 / 93$
$12 / 01 / 93$
$12 / 01 / 93$
$12 / 01 / 93$
$12 / 01 / 93$
$12 / 01 / 93$

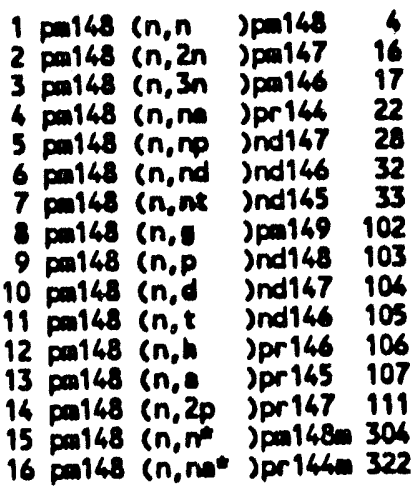

$12 / 01 / 93$

$12 / 01 / 93$

$12 / 01 / 93$

$12 / 01 / 93$

$12 / 01 / 93$

$12 / 01 / 93$

$12 / 01 / 93$

$12 / 01 / 93$

$12 / 01 / 93$

$12 / 01 / 93$

$12 / 01 / 93$

$12 / 01 / 93$

$12 / 01 / 93$

$12 / 01 / 93$

$12 / 01 / 93$

$12 / 01 / 93$

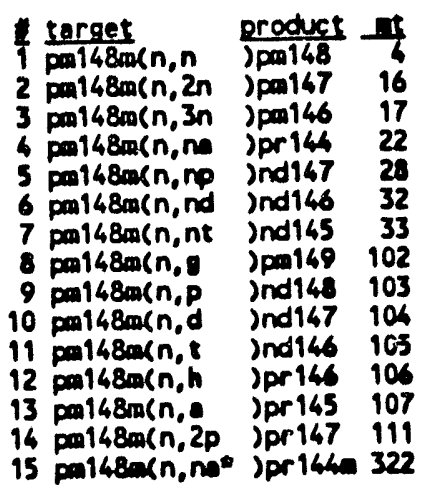

\begin{tabular}{cc} 
Eengerion & date \\
\cline { 2 - 2 } 75 & $12 / 01 / 93$ \\
38 & $12 / 01 / 93$ \\
26 & $12 / 01 / 93$ \\
87 & $12 / 01 / 93$ \\
62 & $12 / 01 / 93$ \\
38 & $12 / 01 / 93$ \\
37 & $12 / 01 / 93$ \\
582 & $12 / 01 / 93$ \\
86 & $12 / 01 / 93$ \\
61 & $12 / 01 / 93$ \\
69 & $12 / 01 / 93$ \\
27 & $12 / 01 / 93$ \\
83 & $12 / 01 / 93$ \\
31 & $12 / 01 / 93$ \\
87 & $12 / 01 / 93$
\end{tabular}

1 pol49 (n,2n)pmi48 16

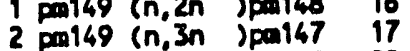

3 pol49 ( $n, n$ )pr145 22

40149 (n,no indi48 28

5 pmi49 ( $n$,nd ind147 32

6 plis (n, int ind14s 33

7 pm149 (n,g )po150 102

8 por149 (n,p )nd149 103

9 pol149 (n,d )nd148 104

10 pmils (n,t ind147 105

11 pmise (n,h )pr147 106

12 pm149 (n, ) pr146 107

13 pm149 (n,2p )pr148 111

14 pal49 (n,2n") pal48m 316

15 pallo (n,2p)pr148 611

$\begin{array}{cc}46 & 12 / 01 / 93 \\ 25 & 12 / 01 / 93 \\ 73 & 12 / 01 / 93 \\ 46 & 12 / 01 / 93 \\ 30 & 12 / 01 / 93 \\ 61 & 12 / 01 / 93 \\ 466 & 12 / 01 / 93 \\ 85 & 12 / 01 / 93 \\ 63 & 12 / 01 / 93 \\ 59 & 12 / 01 / 93 \\ 25 & 12 / 01 / 93 \\ 76 & 12 / 01 / 93 \\ 23 & 12 / 01 / 93 \\ 44 & 12 / 01 / 93 \\ 23 & 12 / 01 / 93\end{array}$

1 pa150 (n, 2n )pmi49 16

2 polso (n,3n )pol40 17

3 150 (n,m jprl46 22

4 polso en, no ind149 28

5 pmiso (n,nd ind148 32

6 colso en,nt ind167 33

7 polso (n, )po151 102

8 pmiso (n,p ind150 103

9 pmiso (n,d ind149 10

10 pmiso (n, indits 105

11 pmiso (n,h )pri48 106

12 polso (n,: jprit4 107

13 pm150 (n,2p )pr149 111

16 pol150 (n,3n*)pm148m 317

15 palso (n, $h$ )pritem 406

$\begin{array}{ll}41 & 12 / 01 / 93 \\ 27 & 12 / 01 / 93 \\ 79 & 12 / 01 / 93 \\ 39 & 12 / 01 / 93 \\ 39 & 12 / 01 / 93 \\ 40 & 12 / 01 / 93 \\ 403 & 12 / 01 / 93 \\ 86 & 12 / 01 / 93 \\ 61 & 12 / 01 / 93 \\ 65 & 12 / 01 / 93 \\ 23 & 12 / 01 / 93 \\ 70 & 12 / 01 / 93 \\ 28 & 12 / 01 / 93 \\ 22 & 12 / 01 / 93 \\ 23 & 12 / 01 / 93\end{array}$

$A-6.23$ 


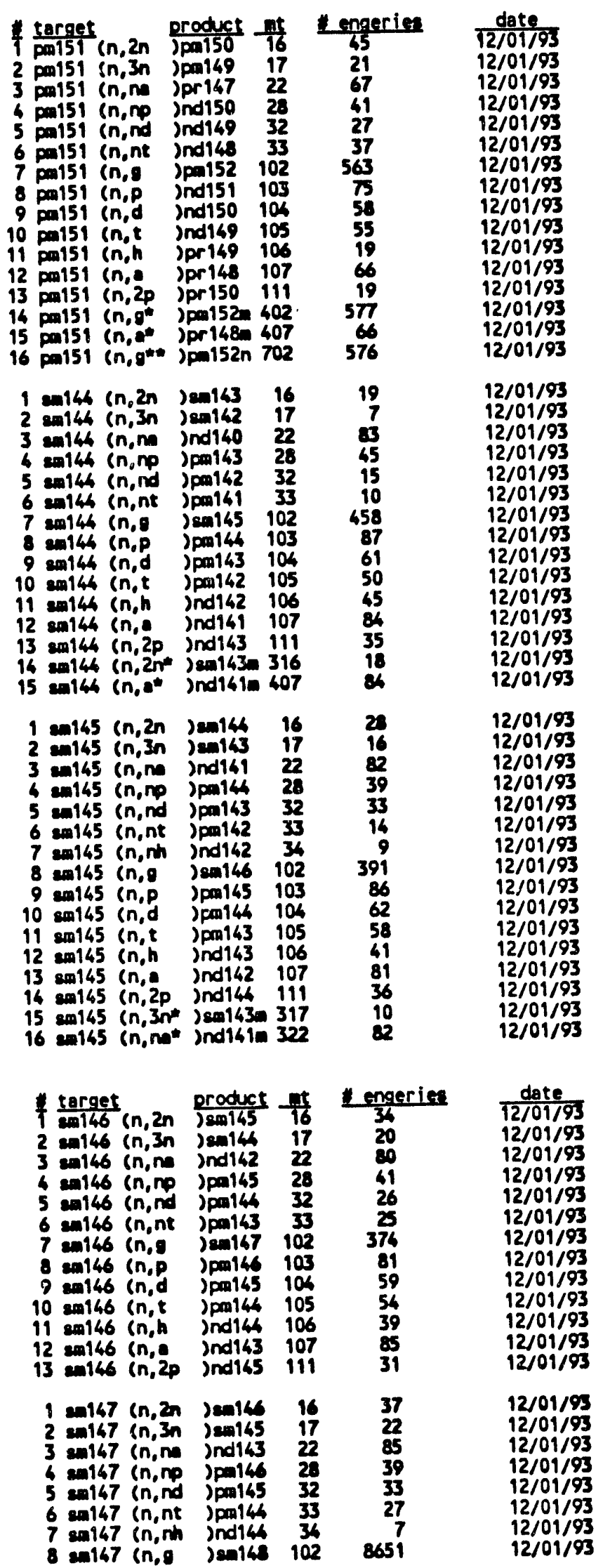




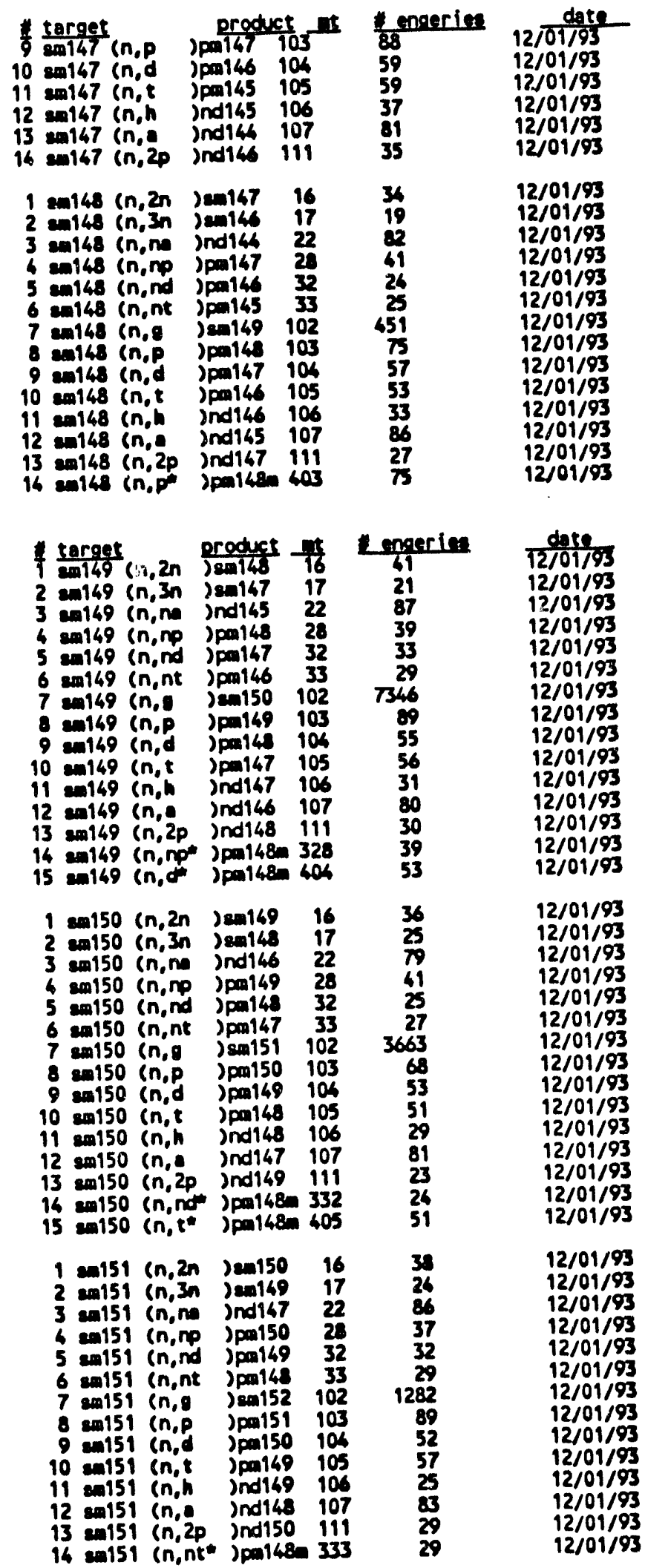




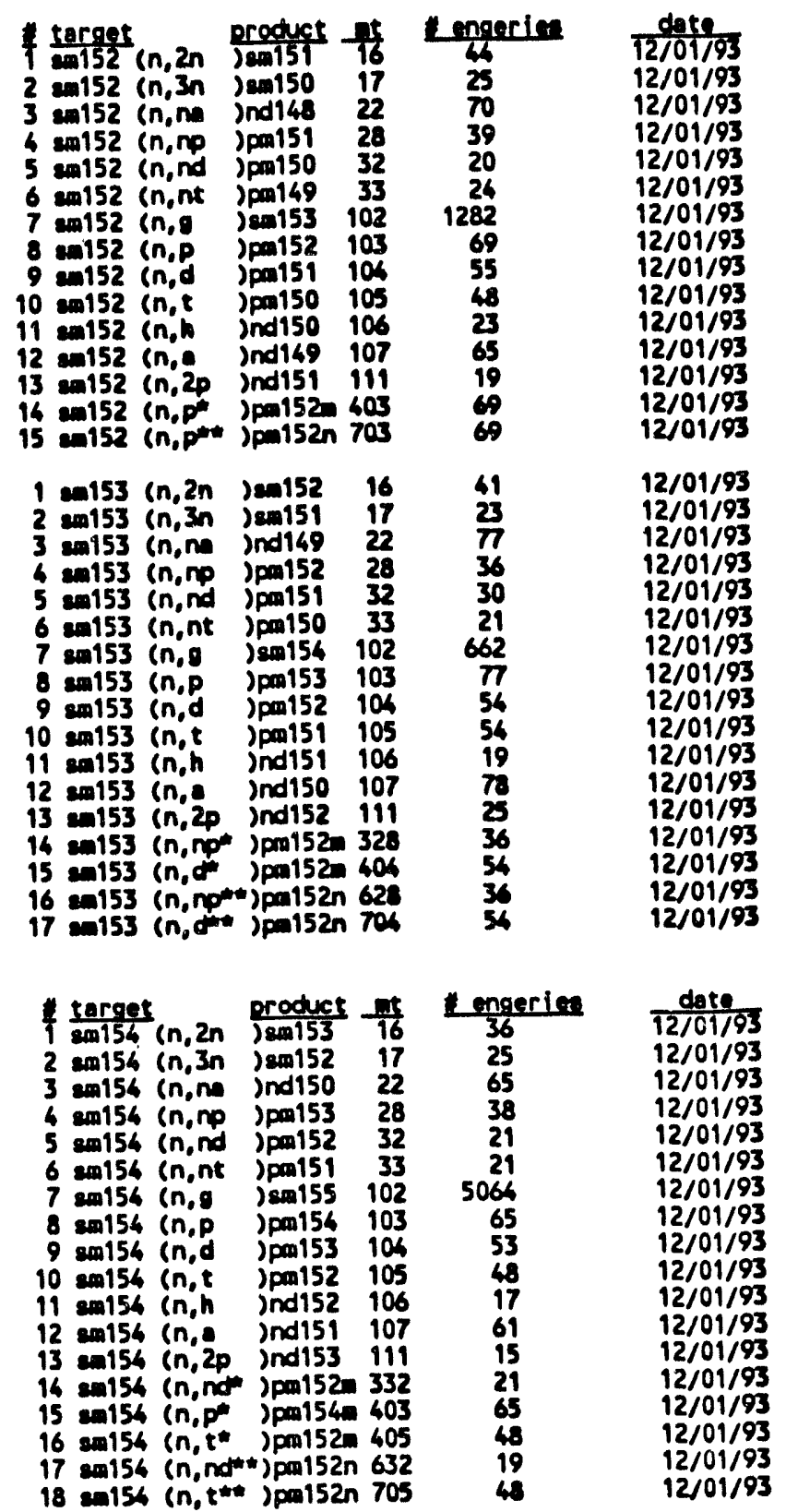


Appendix A-7

List of Reaction on flles ".eu.lu

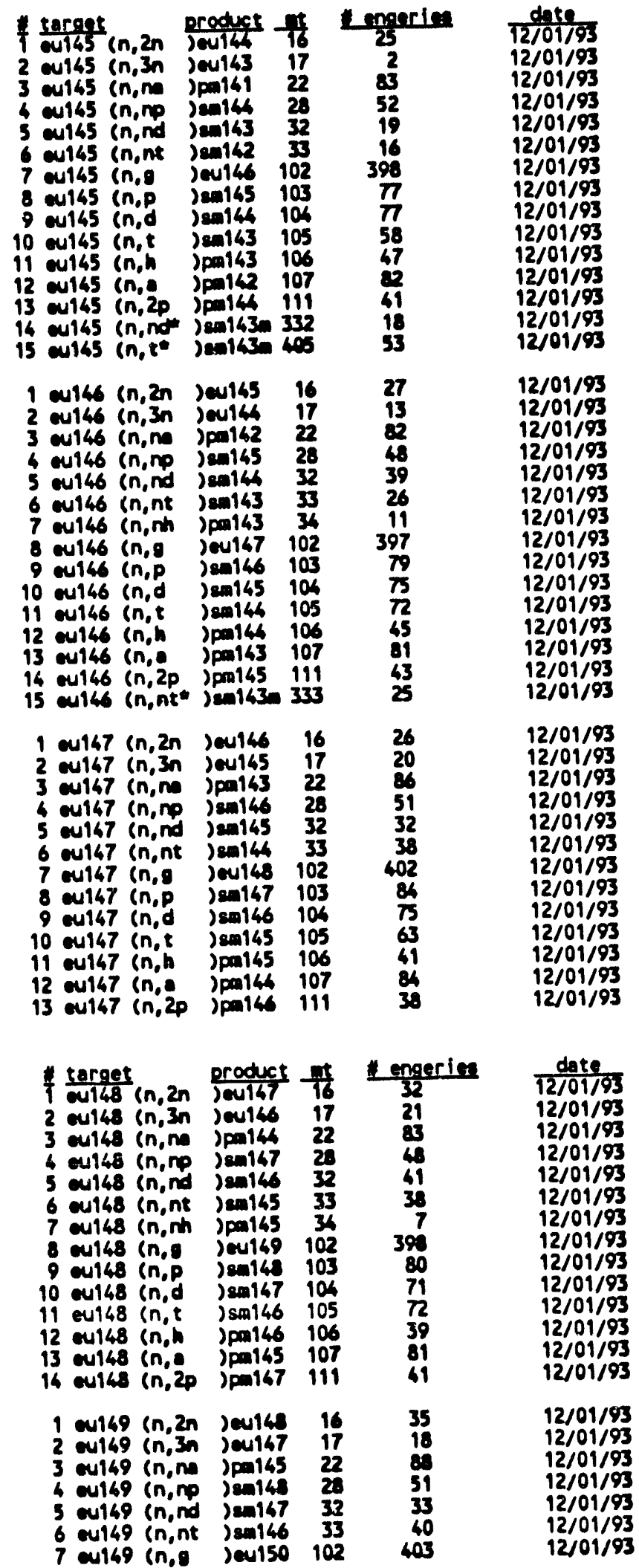

$A-7.1$ 


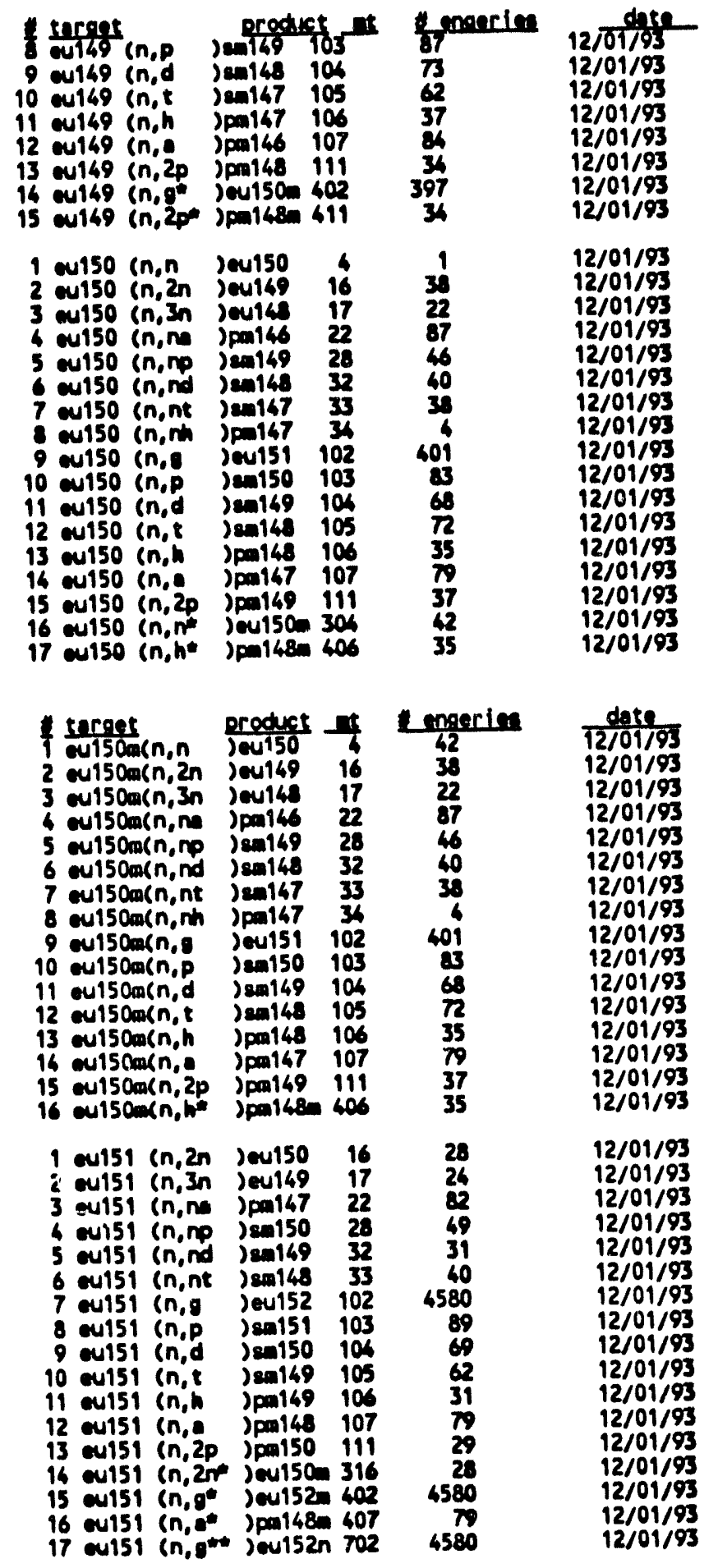

A-7.2 


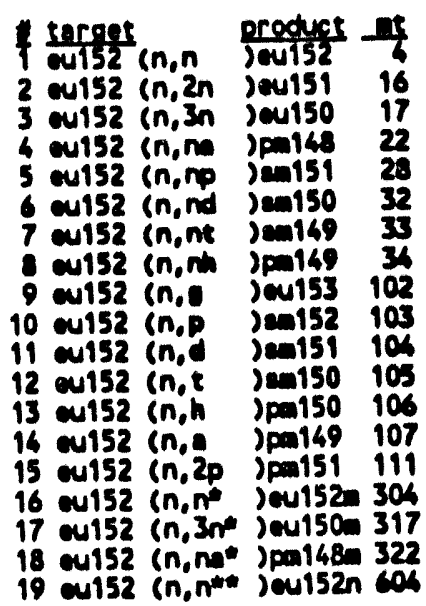

\begin{tabular}{cc} 
Lengerien & date \\
\hline 1 & $12 / 01 / 93$ \\
40 & $12 / 01 / 93$ \\
23 & $12 / 01 / 93$ \\
87 & $12 / 01 / 93$ \\
62 & $12 / 01 / 93$ \\
39 & $12 / 01 / 93$ \\
37 & $12 / 01 / 93$ \\
2 & $12 / 01 / 93$ \\
5926 & $12 / 01 / 93$ \\
83 & $12 / 01 / 93$ \\
65 & $12 / 01 / 93$ \\
70 & $12 / 01 / 93$ \\
29 & $12 / 011993$ \\
83 & $12 / 01 / 93$ \\
34 & $12 / 01 / 93$ \\
40 & $12 / 01 / 93$ \\
23 & $12 / 01 / 93$ \\
87 & $12 / 01 / 93$ \\
40 & $12 / 01 / 93$
\end{tabular}

1 cu152-an,n 204152

2 ou152a(n,2n jeu1si 16

3 cu152akn,3n jeuiso if

5 culs2akn,m )palw 22

6u152a(n,nd )en150 32

7 eu152m(n,nt ) emi69 33

- eu152m(n,8 )evis3 102

- culs2arn.p )en152 103

10 eu152m(n,d)emisi 106

11 euls2man,t jew150 105

12 eu152a(n,h jpe150 106

13 eu152m(n, )pm149 107

14 eu152m(n,2p )pm151 111

15 eu152m(n,3n* )ev150m 317

16 e152m(n,nt)pa148m 322

17 cu152m(n,ntw ) ievis2n cos

$\begin{array}{rr}1 & 12 / 01 / 93 \\ 40 & 12 / 01 / 93 \\ 23 & 12 / 01 / 93 \\ 87 & 12 / 01 / 93 \\ 42 & 12 / 01 / 93 \\ 39 & 12 / 01 / 93 \\ 37 & 12 / 01 / 93 \\ 5926 & 12 / 01 / 93 \\ 83 & 12 / 01 / 93 \\ 65 & 12 / 01 / 93 \\ 70 & 12 / 01 / 93 \\ 20 & 12 / 011 / 93 \\ 83 & 12 / 01 / 93 \\ 34 & 12 / 01 / 93 \\ 23 & 12 / 01 / 93 \\ 87 & 12 / 01 / 93 \\ 40 & 12 / 01 / 93\end{array}$

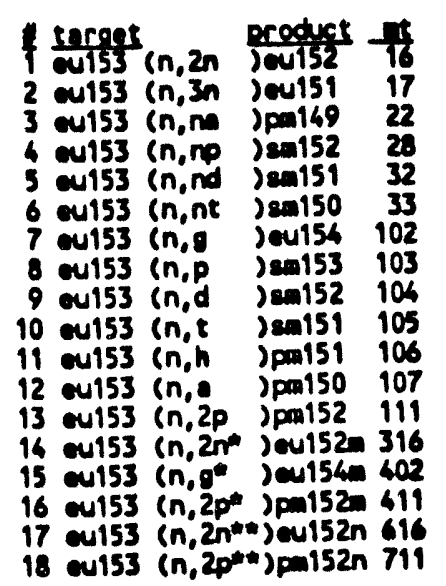

\begin{tabular}{cc} 
Lmerias & dute \\
\hline 33 & $12 / 01 / 93$ \\
23 & $12 / 01 / 93$ \\
73 & $12 / 01 / 93$ \\
46 & $12 / 01 / 93$ \\
27 & $12 / 01 / 93$ \\
36 & $12 / 01193$ \\
6584 & $12 / 101 / 93$ \\
90 & $12 / 01 / 93$ \\
63 & $12 / 01 / 93$ \\
56 & $12 / 01 / 93$ \\
25 & $12 / 01 / 93$ \\
69 & $12 / 01 / 93$ \\
26 & $12 / 01 / 93$ \\
33 & $12 / 01 / 93$ \\
6583 & $12 / 01 / 93$ \\
26 & $12 / 01 / 93$ \\
33 & $12 / 01 / 93$ \\
26 & $12 / 01 / 93$
\end{tabular}

\begin{tabular}{|c|c|c|c|c|c|}
\hline 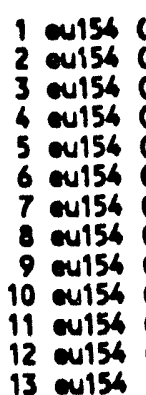 & $\begin{array}{l}(n, n) \\
(n, 2 n \\
(n, 3 n \\
(n, n) \\
(n, n) \\
(n, n d \\
(n, n) \\
(n, s) \\
(n, p) \\
(n, d) \\
(n, i) \\
(n, n) \\
(n, i)\end{array}$ & 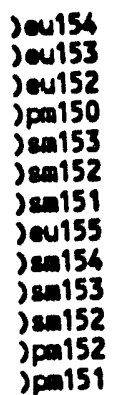 & $\begin{array}{l}16 \\
17 \\
22 \\
28 \\
32 \\
33 \\
102 \\
103 \\
104 \\
105 \\
106 \\
107\end{array}$ & $\begin{array}{r}1 \\
39 \\
18 \\
77 \\
42 \\
37 \\
32 \\
5385 \\
86 \\
61 \\
64\end{array}$ & $\begin{array}{l}12 / 01 / 93 \\
12 / 01 / 93 \\
12 / 01 / 93 \\
12 / 01 / 93 \\
12 / 01 / 93 \\
12 / 01 / 93 \\
12 / 01 / 93 \\
12 / 01 / 93 \\
12 / 01 / 93 \\
12 / 01 / 93 \\
12 / 01 / 93 \\
12 / 01 / 93 \\
12 / 01 / 93\end{array}$ \\
\hline
\end{tabular}

$A-7.3$ 


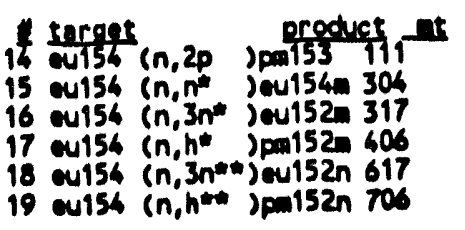

$\begin{array}{ll}\text { yenoncien } & 12 / 01 / 93 \\ 29 & 12 / 01 / 93 \\ 41 & 12 / 01 / 93 \\ 18 & 12 / 01 / 93 \\ 23 & 12 / 01 / 93 \\ 18 & 12 / 01 / 93 \\ 23 & \end{array}$

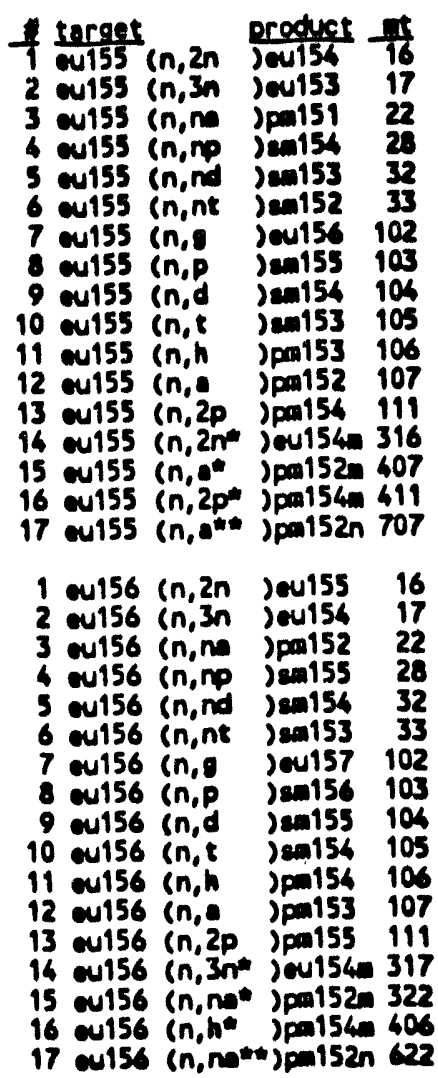

\begin{tabular}{cc} 
Lngerim & dats \\
\cline { 2 - 2 } 35 & $12 / 01 / 93$ \\
21 & $12 / 01 / 93$ \\
67 & $12 / 01 / 93$ \\
63 & $12 / 01 / 93$ \\
27 & $12 / 01 / 93$ \\
32 & $12 / 01 / 93$ \\
946 & $12 / 01 / 93$ \\
83 & $12 / 01 / 93$ \\
61 & $12 / 01 / 93$ \\
56 & $12 / 01 / 93$ \\
21 & $12 / 01 / 93$ \\
64 & $12 / 01 / 93$ \\
21 & $12 / 01 / 93$ \\
35 & $12 / 01 / 93$ \\
63 & $12 / 01 / 93$ \\
21 & $12 / 01 / 93$ \\
64 & $12 / 01 / 93$ \\
39 & $12 / 01 / 93$ \\
39 & $12 / 01 / 93$ \\
19 & $12 / 01 / 93$ \\
75 & $12 / 01 / 93$ \\
41 & $12 / 01 / 93$ \\
34 & $12 / 01 / 93$ \\
29 & $12 / 01 / 93$ \\
515 & $12 / 01 / 93$ \\
88 & $12 / 01 / 93$ \\
58 & $12 / 01 / 93$ \\
59 & $12 / 01 / 93$ \\
19 & $12 / 01 / 93$ \\
69 & $12 / 01 / 93$ \\
23 & $12 / 01 / 93$ \\
19 & $12 / 01 / 93$ \\
73 & $12 / 01 / 93$ \\
19 & $12 / 01 / 93$ \\
73 &
\end{tabular}

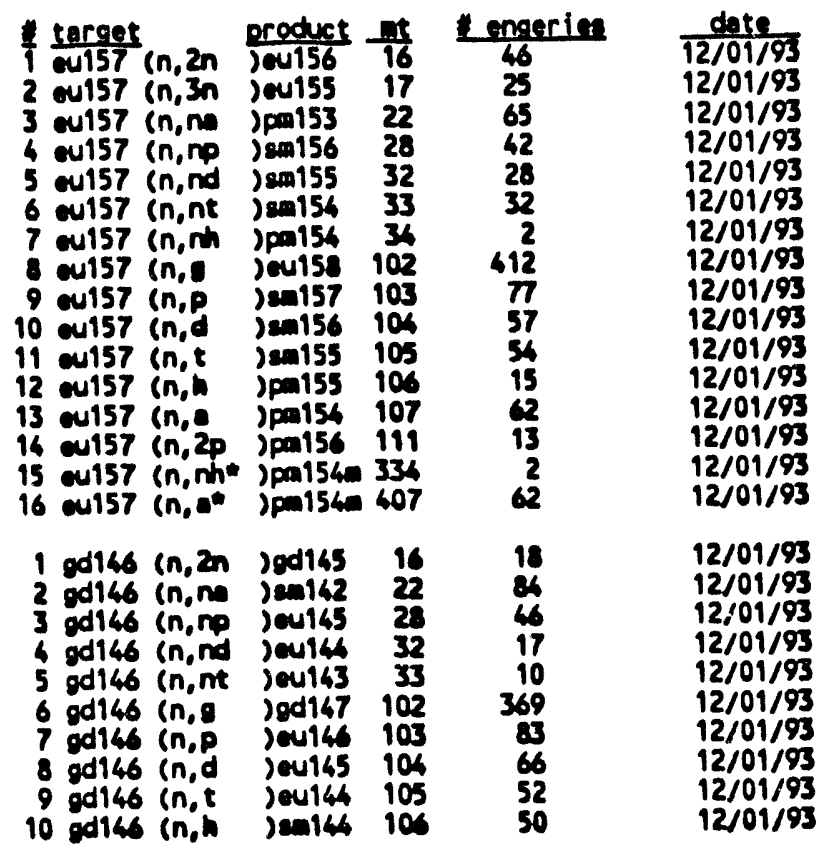

$$
\text { A-7.4 }
$$




\begin{tabular}{|c|c|c|c|c|}
\hline 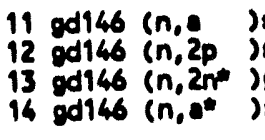 & 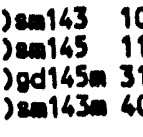 & $\begin{array}{l}107 \\
111 \\
316 \\
07\end{array}$ & $\begin{array}{l}82 \\
61 \\
17 \\
82\end{array}$ & $\begin{array}{l}12 / 01 / 93 \\
12 / 01 / 93 \\
12 / 01 / 93 \\
12 / 01 / 93\end{array}$ \\
\hline 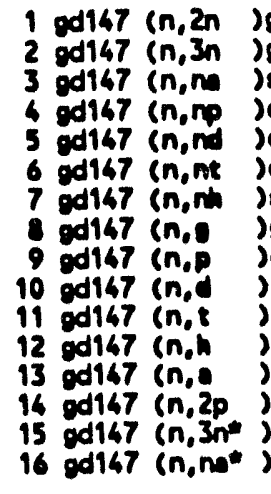 & 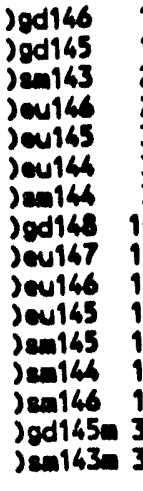 & $\begin{array}{l}16 \\
17 \\
22 \\
28 \\
32 \\
33 \\
34 \\
102 \\
103 \\
104 \\
105 \\
100 \\
107 \\
111 \\
317 \\
322\end{array}$ & $\begin{array}{l}28 \\
8 \\
78 \\
43 \\
35 \\
16 \\
15 \\
393 \\
84 \\
67 \\
62 \\
48 \\
77 \\
43 \\
6 \\
76\end{array}$ & $\begin{array}{l}12 / 01 / 93 \\
12 / 01 / 93 \\
12 / 01 / 93 \\
12 / 01 / 93 \\
12 / 01 / 93 \\
12 / 01 / 93 \\
12 / 01 / 93 \\
12 / 01 / 93 \\
12 / 01 / 93 \\
12 / 01 / 93 \\
12 / 01 / 93 \\
12 / 01 / 93 \\
12 / 01 / 93 \\
12 / 01 / 93 \\
12 / 01 / 93 \\
12 / 01 / 93\end{array}$ \\
\hline 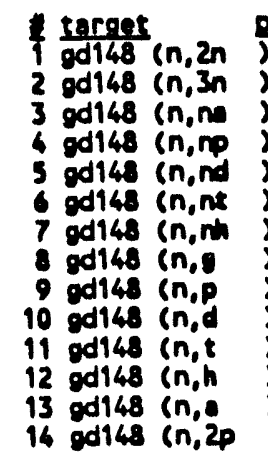 & 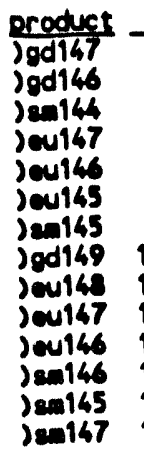 & $\begin{array}{l}\frac{x}{16} \\
17 \\
22 \\
28 \\
32 \\
33 \\
34 \\
102 \\
103 \\
104 \\
105 \\
106 \\
107 \\
111\end{array}$ & $\begin{array}{c}\text { engeries } \\
25 \\
15 \\
85 \\
43 \\
27 \\
26 \\
4 \\
390 \\
83 \\
65 \\
55 \\
45 \\
82 \\
36\end{array}$ & $\begin{array}{l}\text { date } \\
12 / 01 / 93 \\
12 / 01 / 93 \\
12 / 01 / 93 \\
12 / 01 / 93 \\
12 / 01 / 93 \\
12 / 01 / 93 \\
12 / 01 / 93 \\
12 / 01 / 93 \\
12 / 01 / 93 \\
12 / 01 / 93 \\
12 / 01 / 93 \\
12 / 01 / 93 \\
12 / 01 / 93 \\
12 / 01 / 93\end{array}$ \\
\hline 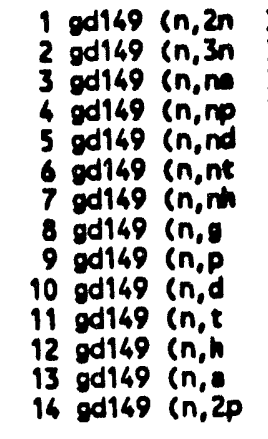 & 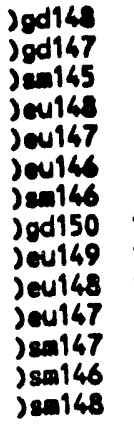 & $\begin{array}{l}16 \\
17 \\
22 \\
23 \\
32 \\
33 \\
34 \\
102 \\
103 \\
104 \\
105 \\
106 \\
107 \\
111\end{array}$ & $\begin{array}{r}34 \\
16 \\
82 \\
41 \\
34 \\
27 \\
11 \\
387 \\
87 \\
64 \\
60 \\
43 \\
78 \\
37\end{array}$ & $\begin{array}{l}12 / 01 / 93 \\
12 / 01 / 93 \\
12 / 01 / 93 \\
12 / 01 / 93 \\
12 / 01 / 93 \\
12 / 01 / 93 \\
12 / 01 / 93 \\
12 / 01 / 93 \\
12 / 01 / 93 \\
12 / 01 / 93 \\
12 / 01 / 93 \\
12 / 01 / 93 \\
12 / 01 / 93 \\
12 / 01 / 93\end{array}$ \\
\hline 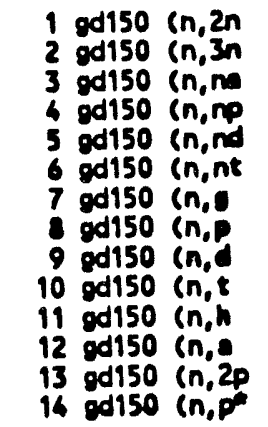 & 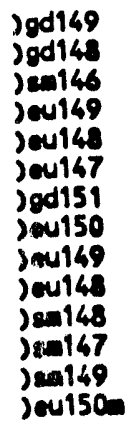 & $\begin{array}{l}16 \\
17 \\
22 \\
28 \\
32 \\
33 \\
102 \\
103 \\
104 \\
105 \\
106 \\
107 \\
111 \\
403\end{array}$ & $\begin{array}{l}12 \\
20 \\
87 \\
46 \\
26 \\
25 \\
391 \\
91 \\
62 \\
55 \\
41 \\
82 \\
32 \\
91\end{array}$ & $\begin{array}{l}12 / 01 / 93 \\
12 / 01 / 93 \\
12 / 01 / 93 \\
12 / 01 / 93 \\
12 / 01 / 93 \\
12 / 01 / 93 \\
12 / 01 / 93 \\
12 / 01 / 93 \\
12 / 01 / 93 \\
12 / 01 / 93 \\
12 / 01193 \\
12 / 01 / 93 \\
12 / 01 / 93 \\
12 / 01 / 93\end{array}$ \\
\hline
\end{tabular}




\begin{tabular}{|c|c|c|c|c|}
\hline 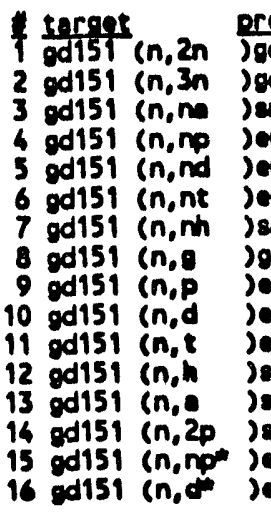 & 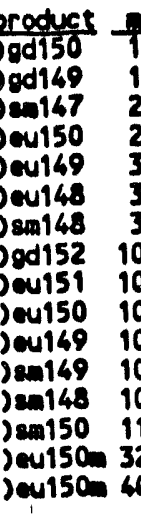 & $\begin{array}{l}7 \\
16 \\
17 \\
22 \\
28 \\
32 \\
33 \\
34 \\
02 \\
02 \\
143 \\
104 \\
105 \\
106 \\
107 \\
112 \\
328 \\
604\end{array}$ & $\begin{array}{l}\text { mangin } \\
16 \\
20 \\
86 \\
41 \\
35 \\
28 \\
7 \\
388 \\
88 \\
59 \\
60 \\
37 \\
70 \\
36 \\
61 \\
59\end{array}$ & $\begin{array}{l}\frac{\text { date }}{12 / 01 / 93} \\
12 / 01 / 93 \\
12 / 01 / 93 \\
12 / 01 / 93 \\
12 / 01 / 93 \\
12 / 01 / 93 \\
12 / 01 / 93 \\
12 / 01 / 93 \\
12 / 01 / 93 \\
12 / 01 / 93 \\
12 / 01 / 93 \\
12 / 01 / 93 \\
12 / 01 / 93 \\
12 / 01 / 93 \\
12 / 01 / 93 \\
12 / 01 / 93\end{array}$ \\
\hline 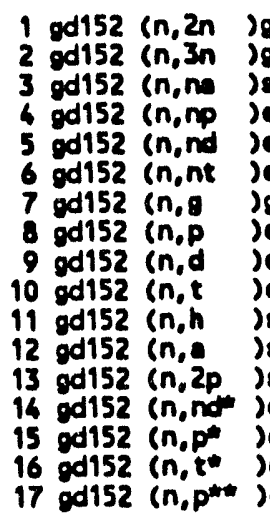 & 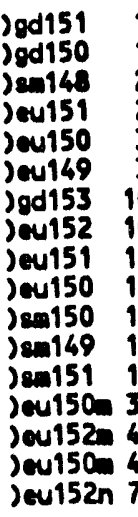 & $\begin{array}{l}16 \\
17 \\
22 \\
28 \\
32 \\
33 \\
102 \\
103 \\
104 \\
105 \\
106 \\
107 \\
111 \\
332 \\
403 \\
405 \\
703\end{array}$ & $\begin{array}{r}32 \\
18 \\
82 \\
44 \\
25 \\
26 \\
1282 \\
77 \\
57 \\
53 \\
35 \\
78 \\
28 \\
25 \\
77 \\
53 \\
77\end{array}$ & $\begin{array}{l}12 / 01 / 93 \\
12 / 01 / 93 \\
12 / 01 / 93 \\
12 / 01 / 93 \\
12 / 01 / 93 \\
12 / 01 / 93 \\
12 / 01 / 93 \\
12 / 01 / 93 \\
12 / 01 / 93 \\
12 / 01 / 93 \\
12 / 01 / 93 \\
12 / 01 / 93 \\
12 / 01 / 93 \\
12 / 01 / 93 \\
12 / 01 / 93 \\
12 / 01 / 93 \\
12 / 01 / 93\end{array}$ \\
\hline 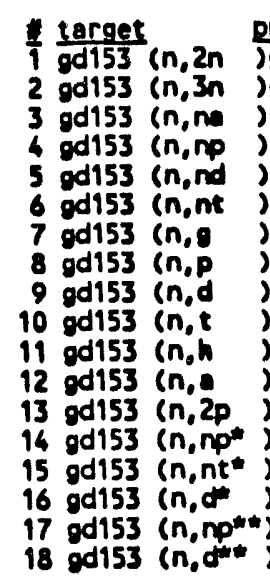 & $\begin{array}{l}\text { product } \\
\text { jgd152 } \\
\text { jgd151 } \\
\text { jem149 } \\
\text { jeu152 } \\
\text { jeu151 } \\
\text { jeu150 } \\
\text { jod154 } \\
\text { jeu153 } \\
\text { jou152 } \\
\text { jeu15i } \\
\text { jem151 } \\
\text { jem150 } \\
\text { jem152 } \\
\text { jeu152a } \\
\text { jeu150a } \\
\text { jeu152n } \\
\text { jeu152n } \\
\text { jeu152n }\end{array}$ & $\begin{array}{l}\frac{8}{16} \\
17 \\
22 \\
28 \\
32 \\
33 \\
102 \\
103 \\
104 \\
105 \\
106 \\
107 \\
114 \\
328 \\
333 \\
404 \\
628 \\
704\end{array}$ & $\begin{array}{c}\text { Lengeries } \\
31 \\
20 \\
86 \\
37 \\
32 \\
26 \\
399 \\
88 \\
56 \\
58 \\
31 \\
83 \\
33 \\
37 \\
26 \\
56 \\
37 \\
56\end{array}$ & $\begin{array}{l}\frac{\text { date }}{12 / 01 / 93} \\
12 / 01 / 93 \\
12 / 01 / 93 \\
12 / 01 / 93 \\
12 / 01 / 93 \\
12 / 01 / 93 \\
12 / 01 / 93 \\
12 / 01 / 93 \\
12 / 01 / 93 \\
12 / 01 / 93 \\
12 / 01 / 93 \\
12 / 01 / 93 \\
12 / 01 / 93 \\
12 / 01 / 93 \\
12 / 01 / 93 \\
12 / 01 / 93 \\
12 / 01 / 93 \\
12 / 01 / 93\end{array}$ \\
\hline 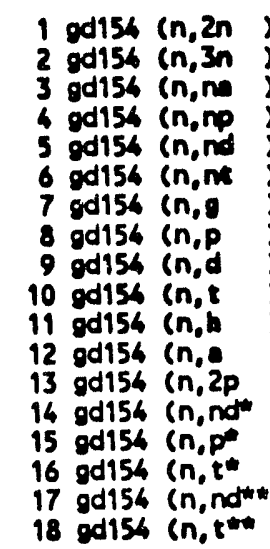 & $\begin{array}{l}\text { jod153 } \\
\text { jod152 } \\
\text { jea150 } \\
\text { jeu153 } \\
\text { jeu152 } \\
\text { jeu151 } \\
\text { jod155 } \\
\text { jeu154 } \\
\text { jeu153 } \\
\text { jeu152 } \\
\text { jen152 } \\
\text { jen151 } \\
\text { jem153 } \\
\text { jeu152a } \\
\text { jeu154m } \\
\text { jeu152n } \\
\text { jeu152n } \\
\text { jeu152n }\end{array}$ & $\begin{array}{r}16 \\
17 \\
22 \\
28 \\
32 \\
33 \\
102 \\
103 \\
104 \\
105 \\
106 \\
107 \\
111 \\
332 \\
403 \\
405 \\
632 \\
705\end{array}$ & $\begin{array}{r}32 \\
20 \\
72 \\
42 \\
21 \\
21 \\
4301 \\
76 \\
57 \\
50 \\
29 \\
72 \\
27 \\
21 \\
76 \\
50 \\
21 \\
50\end{array}$ & $\begin{array}{l}12 / 01 / 93 \\
12 / 01 / 93 \\
12 / 01 / 93 \\
12 / 01 / 93 \\
12 / 01 / 93 \\
12 / 01 / 93 \\
12 / 01 / 93 \\
12 / 01 / 93 \\
12 / 01 / 93 \\
12 / 01 / 93 \\
12 / 01 / 93 \\
12 / 01 / 93 \\
12 / 01 / 93 \\
12 / 01 / 93 \\
12 / 01 / 93 \\
12 / 01 / 93 \\
12 / 01 / 93 \\
12 / 01 / 93\end{array}$ \\
\hline
\end{tabular}

A-7. 6 


\begin{tabular}{|c|c|c|c|c|}
\hline 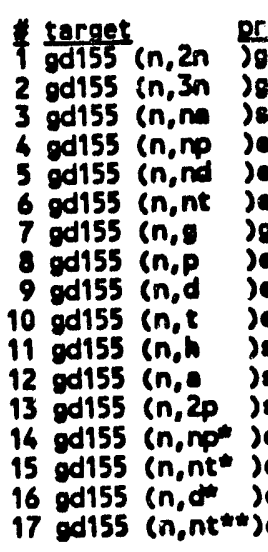 & 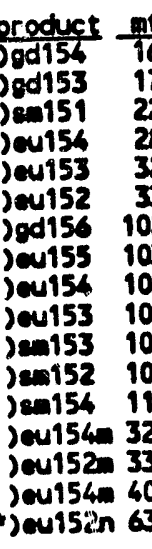 & $\begin{array}{l}n t \\
16 \\
17 \\
22 \\
28 \\
32 \\
33 \\
02 \\
03 \\
04 \\
05 \\
06 \\
07 \\
111 \\
28 \\
333 \\
604 \\
333\end{array}$ & $\begin{array}{c}\text { engeries } \\
39 \\
20 \\
80 \\
39 \\
32 \\
20 \\
6687 \\
89 \\
57 \\
56 \\
27 \\
81 \\
31 \\
39 \\
20 \\
54 \\
20\end{array}$ & $\begin{array}{l}\text { date } \\
12 / 01 / 93 \\
12 / 01 / 93 \\
12 / 01 / 93 \\
12 / 01 / 93 \\
12 / 01 / 93 \\
12 / 01 / 93 \\
12 / 01 / 93 \\
12 / 01 / 93 \\
12 / 01 / 93 \\
12 / 01 / 93 \\
12 / 01 / 93 \\
12 / 01 / 93 \\
12 / 01 / 93 \\
12 / 01 / 93 \\
12 / 01 / 93 \\
12 / 01 / 93 \\
12 / 01 / 93\end{array}$ \\
\hline 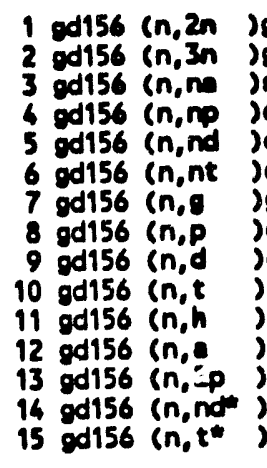 & $\begin{array}{l}\text { jod155 } \\
\text { jod156 } \\
\text { jemi52 } \\
\text { jeu155 } \\
\text { jeu154 } \\
\text { jeu153 } \\
\text { jod157 } \\
\text { jeu156 } \\
\text { jeu155 } \\
\text { jeu154 } \\
\text { jen154 } \\
\text { jem153 } \\
\text { jen155 } \\
\text { jeu1540 } \\
\text { jev154m }\end{array}$ & $\begin{array}{l}16 \\
17 \\
22 \\
23 \\
32 \\
33 \\
102 \\
103 \\
104 \\
105 \\
106 \\
107 \\
111 \\
332 \\
405\end{array}$ & $\begin{array}{r}33 \\
17 \\
70 \\
41 \\
21 \\
20 \\
8741 \\
75 \\
56 \\
50 \\
25 \\
67 \\
23 \\
21 \\
50\end{array}$ & $\begin{array}{l}12 / 01 / 93 \\
12 / 01 / 93 \\
12 / 01 / 93 \\
12 / 01 / 93 \\
12 / 01 / 93 \\
12 / 01 / 93 \\
12 / 01 / 93 \\
12 / 01 / 93 \\
12 / 01 / 93 \\
12 / 01 / 93 \\
12 / 01 / 93 \\
12 / 01 / 93 \\
12 / 01 / 93 \\
12 / 01 / 93 \\
12 / 01 / 93\end{array}$ \\
\hline 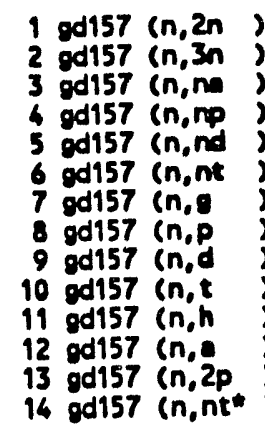 & $\begin{array}{l}\text { jod156 } \\
\text { jod155 } \\
\text { jemis3 } \\
\text { jeu156 } \\
\text { jeu155 } \\
\text { jeu154 } \\
\text { jod158 } \\
\text { jeu157 } \\
\text { jeu156 } \\
\text { jeu155 } \\
\text { jem155 } \\
\text { jem156 } \\
\text { jem156 } \\
\text { jeu154a }\end{array}$ & $\begin{array}{l}16 \\
17 \\
22 \\
28 \\
32 \\
33 \\
102 \\
103 \\
104 \\
105 \\
106 \\
107 \\
111 \\
333\end{array}$ & $\begin{array}{r}37 \\
20 \\
78 \\
38 \\
30 \\
20 \\
6401 \\
83 \\
55 \\
56 \\
23 \\
73 \\
26 \\
20\end{array}$ & $\begin{array}{l}12 / 01 / 93 \\
12 / 01 / 93 \\
12 / 01 / 93 \\
12 / 01 / 93 \\
12 / 01 / 93 \\
12 / 01 / 93 \\
12 / 01 / 93 \\
12 / 01 / 93 \\
12 / 01 / 93 \\
12 / 01 / 93 \\
12 / 01 / 93 \\
12 / 01 / 93 \\
12 / 01 / 93 \\
12 / 01 / 93\end{array}$ \\
\hline 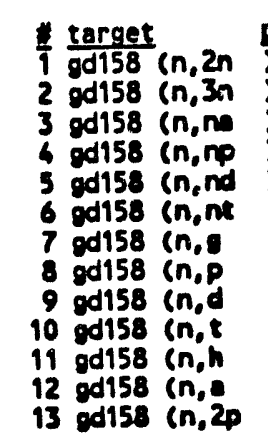 & $\begin{array}{l}\text { product } \\
\text { jod157 } \\
\text { jod156 } \\
\text { jen156 } \\
\text { jeu157 } \\
\text { jeu156 } \\
\text { jeu155 } \\
\text { jod159 } \\
\text { jeu158 } \\
\text { jeu157 } \\
\text { jeu156 } \\
\text { jen156 } \\
\text { jea155 } \\
\text { jen157 }\end{array}$ & $\begin{array}{l}\frac{9 t}{16} \\
17 \\
22 \\
28 \\
32 \\
33 \\
102 \\
103 \\
104 \\
105 \\
106 \\
107 \\
111\end{array}$ & $\begin{array}{c}\text { engeries } \\
43 \\
26 \\
68 \\
40 \\
24 \\
22 \\
7627 \\
70 \\
56 \\
50 \\
19 \\
63 \\
17\end{array}$ & $\begin{array}{l}\text { dete } \\
12 / 01 / 93 \\
12 / 01 / 93 \\
12 / 01 / 93 \\
12 / 01 / 93 \\
12 / 01 / 93 \\
12 / 01 / 93 \\
12 / 01 / 93 \\
12 / 01 / 93 \\
12 / 01 / 93 \\
12 / 01 / 93 \\
12 / 01 / 93 \\
12 / 01 / 93 \\
12 / 01 / 93\end{array}$ \\
\hline $\begin{array}{l}1 \text { gd159 (n, 2n } \\
2 \text { gd159 (n, 3n } \\
3 \text { gd159 (n,nn } \\
4 \text { gd159 (n,np } \\
5 \text { gd159 (n,nd } \\
6 \text { gd159 (n,nt } \\
7 \text { gd159 (n, } \\
8 \text { gd159 (n,p } \\
9 \text { gd159 (n,d }\end{array}$ & $\begin{array}{l}\text { jod158 } \\
\text { jod157 } \\
\text { jem155 } \\
\text { jeu158 } \\
\text { jer } 07 \\
\text { jeu156 } \\
\text { jod160 } \\
\text { jeu150 } \\
\text { jeu15: }\end{array}$ & $\begin{array}{l}16 \\
17 \\
22 \\
28 \\
32 \\
33 \\
102 \\
103 \\
104\end{array}$ & $\begin{array}{r}63 \\
22 \\
72 \\
38 \\
31 \\
23 \\
397 \\
76 \\
55\end{array}$ & $\begin{array}{l}12 / 01 / 93 \\
12 / 01 / 93 \\
12 / 01 / 93 \\
12 / 01 / 93 \\
12 / 01 / 93 \\
12 / 01 / 93 \\
12 / 01 / 93 \\
12 / 01 / 93 \\
12 / 01 / 93\end{array}$ \\
\hline
\end{tabular}

A-7.7 


\begin{tabular}{|c|c|c|c|c|}
\hline 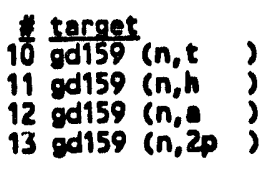 & 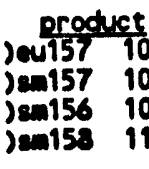 & $\begin{array}{l}1 \\
05 \\
06 \\
07 \\
11\end{array}$ & $\begin{array}{l}\frac{18}{54} \\
17 \\
70 \\
19\end{array}$ & $\begin{array}{l}\text { date } \\
12 / 01 / 93 \\
12 / 01 / 93 \\
12 / 01 / 93 \\
12 / 01 / 93\end{array}$ \\
\hline 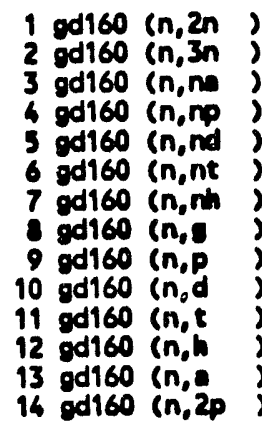 & $\begin{array}{l}\text { jod159 } \\
\text { jod158 } \\
\text { jem156 } \\
\text { jev159 } \\
\text { jeu158 } \\
\text { jeu157 } \\
\text { jen157 } \\
\text { jed161 } \\
\text { jeu160 } \\
\text { jeu159 } \\
\text { jeu158 } \\
\text { jen158 } \\
\text { jem157 } \\
\text { jea159 }\end{array}$ & $\begin{array}{l}16 \\
17 \\
22 \\
28 \\
32 \\
33 \\
31 \\
102 \\
103 \\
104 \\
105 \\
106 \\
107 \\
111\end{array}$ & $\begin{array}{l}31 \\
23 \\
63 \\
60 \\
25 \\
23 \\
2 \\
5085 \\
64 \\
52 \\
50 \\
11 \\
62 \\
11\end{array}$ & $\begin{array}{l}12 / 01 / 93 \\
12 / 01 / 93 \\
12 / 01 / 93 \\
12 / 01 / 93 \\
12 / 01 / 93 \\
12 / 01 / 93 \\
12 / 01 / 93 \\
12 / 01 / 93 \\
12 / 01 / 93 \\
12 / 01 / 93 \\
12 / 01 / 93 \\
12 / 01 / 93 \\
12 / 01 / 93 \\
12 / 01 / 93\end{array}$ \\
\hline 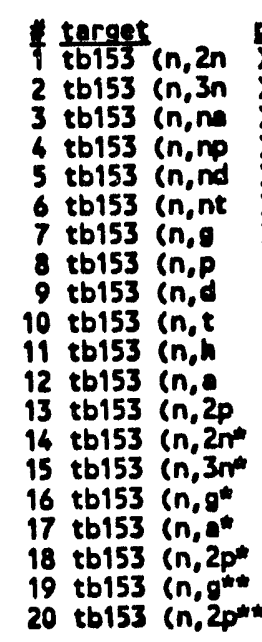 & $\begin{array}{l}\text { product } \\
\text { jtb152 } \\
\text { jtb151 } \\
\text { jeu149 } \\
\text { jod152 } \\
\text { jod151 } \\
\text { jod150 } \\
\text { jtb154 } \\
\text { jod153 } \\
\text { jod152 } \\
\text { jod151 } \\
\text { jeu151 } \\
\text { jeu150 } \\
\text { jeu152 } \\
\text { jtb152n } \\
\text { jtb151n } \\
\text { jtb154m } \\
\text { jeu150m } \\
\text { jeu152m } \\
\text { jtb154n } \\
\text { ieu152n }\end{array}$ & $\begin{array}{l}-1 \\
16 \\
17 \\
22 \\
28 \\
32 \\
33 \\
102 \\
103 \\
104 \\
105 \\
106 \\
107 \\
1111 \\
316 \\
317 \\
402 \\
407 \\
411 \\
702 \\
711\end{array}$ & $\begin{array}{c}\text { Engerien } \\
27 \\
17 \\
88 \\
52 \\
34 \\
39 \\
380 \\
86 \\
75 \\
61 \\
37 \\
84 \\
35 \\
25 \\
17 \\
399 \\
84 \\
35 \\
399 \\
35\end{array}$ & $\begin{array}{l}\text { date } \\
12 / 01 / 93 \\
12 / 01 / 93 \\
12 / 01 / 93 \\
12 / 01 / 93 \\
12 / 01 / 93 \\
12 / 01 / 93 \\
12 / 01 / 93 \\
12 / 01 / 93 \\
12 / 01 / 93 \\
12 / 01 / 93 \\
12 / 01 / 93 \\
12 / 01 / 93 \\
12 / 01 / 93 \\
12 / 01 / 93 \\
12 / 01 / 93 \\
12 / 01 / 93 \\
12 / 01 / 93 \\
12 / 01 / 93 \\
12 / 01 / 93 \\
12 / 01 / 93\end{array}$ \\
\hline 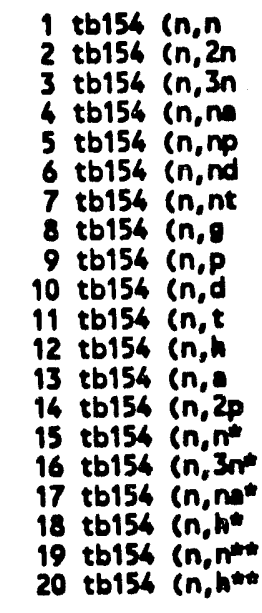 & $\begin{array}{l}\text { jtb154 } \\
\text { jtb153 } \\
\text { jtb152 } \\
\text { jeu150 } \\
\text { jod153 } \\
\text { jgd152 } \\
\text { jod151 } \\
\text { jtb155 } \\
\text { jod154 } \\
\text { jgd153 } \\
\text { jod152 } \\
\text { jev152 } \\
\text { jeu151 } \\
\text { jeu153 } \\
\text { jtb154m } \\
\text { jtb152 } \\
\text { jeu150 } \\
\text { jeu152n } \\
\text { jtb154n } \\
\text { jeu152n }\end{array}$ & $\begin{array}{r}6 \\
16 \\
17 \\
22 \\
28 \\
32 \\
33 \\
102 \\
103 \\
106 \\
105 \\
106 \\
107 \\
111 \\
304 \\
317 \\
322 \\
406 \\
604 \\
706\end{array}$ & $\begin{array}{l}1 \\
28 \\
17 \\
86 \\
48 \\
61 \\
37 \\
404 \\
80 \\
71 \\
73 \\
35 \\
80 \\
39 \\
35 \\
16 \\
86 \\
35 \\
38 \\
35\end{array}$ & $\begin{array}{l}12 / 01 / 93 \\
12 / 01 / 93 \\
12 / 01 / 93 \\
12 / 01 / 93 \\
12 / 01 / 93 \\
12 / 01 / 93 \\
12 / 01 / 93 \\
12 / 01 / 93 \\
12 / 01 / 93 \\
12 / 01 / 93 \\
12 / 01 / 93 \\
12 / 01 / 93 \\
12 / 01 / 93 \\
12 / 01 / 93 \\
12 / 01 / 93 \\
12 / 01 / 93 \\
12 / 01 / 93 \\
12 / 01193 \\
12 / 01 / 93 \\
12 / 01 / 93\end{array}$ \\
\hline $\begin{array}{l}\text { target } \\
1 \text { tb155 (n,2n } \\
2 \text { tb155 (n,3n } \\
3 \text { tb155 (n,n } \\
4 \text { tb155 (n,np } \\
5 \text { tb155 (n,nd } \\
6 \text { tb155 (n,nt } \\
7 \text { tb155 (n, } \\
8 \text { tb155 (n,p }\end{array}$ & $\begin{array}{l}\text { product } \\
\text { jtb156 } \\
\text { jtb153 } \\
\text { jeu1si } \\
\text { jod154 } \\
\text { jod153 } \\
\text { jgd152 } \\
\text { jtb156 } \\
\text { jod155 }\end{array}$ & $\begin{array}{l}\frac{71}{16} \\
17 \\
22 \\
28 \\
32 \\
33 \\
102 \\
103\end{array}$ & $\begin{array}{c}\text { engerie: } \\
28 \\
16 \\
79 \\
51 \\
30 \\
34 \\
384 \\
87\end{array}$ & $\begin{array}{c}\text { date } \\
\frac{\text { dato1/93 }}{12 / 01 / 93} \\
12 / 01 / 93 \\
12 / 01 / 93 \\
12 / 01 / 93 \\
12 / 01 / 93 \\
12 / 01 / 93 \\
12 / 01 / 93 \\
12 / 01 / 93\end{array}$ \\
\hline
\end{tabular}

$A-7.8$ 


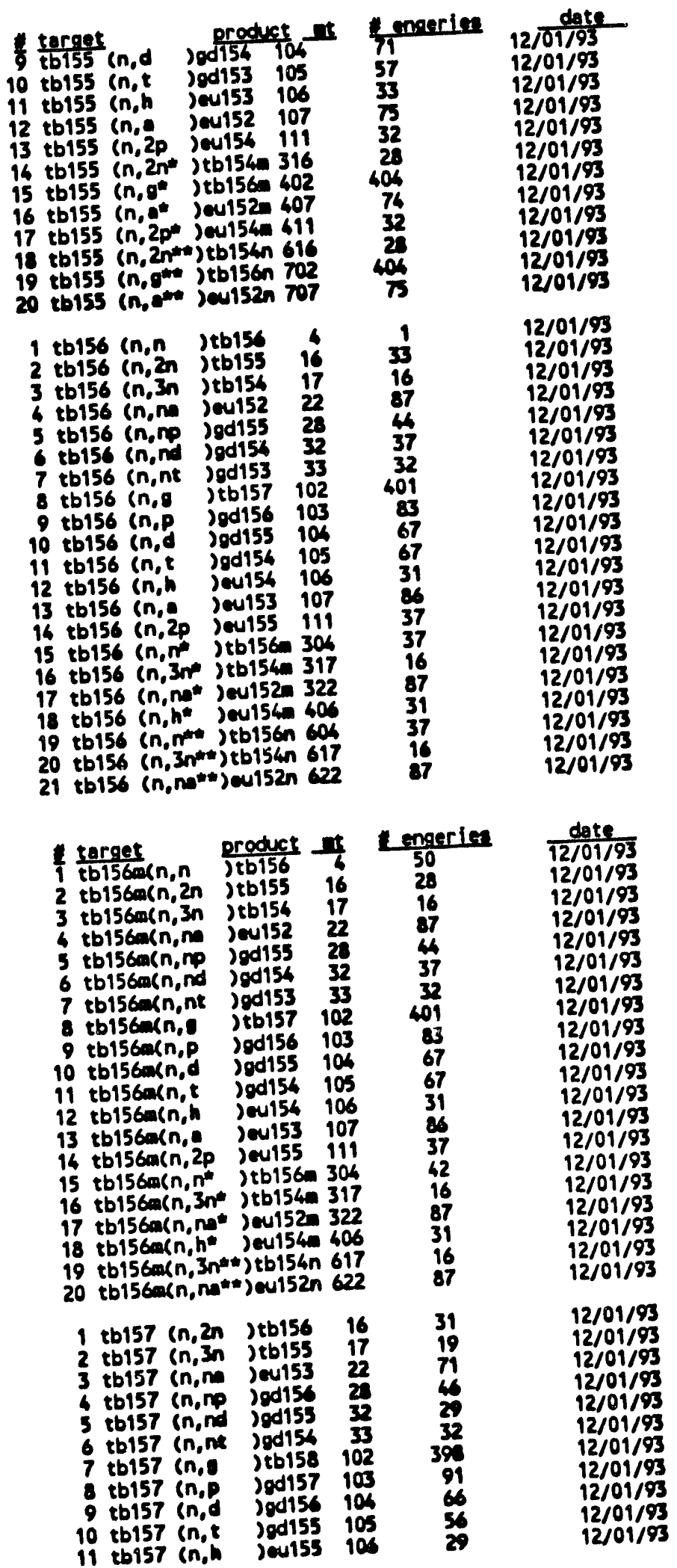

$A-7.9$ 


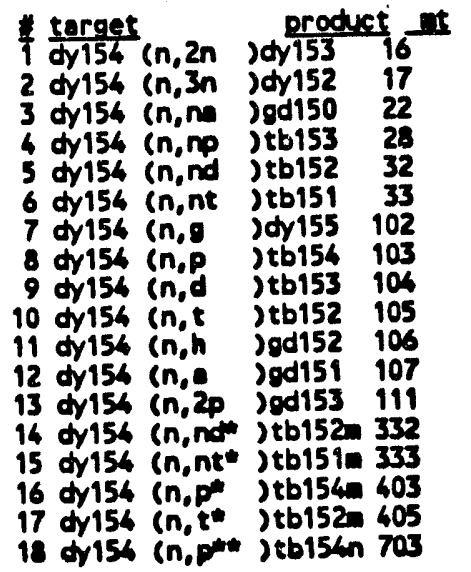

\begin{tabular}{cc} 
1enoeries & date \\
\hline 30 & $12 / 01 / 93$ \\
16 & $12 / 01 / 93$ \\
89 & $12 / 01 / 93$ \\
45 & $12 / 01 / 93$ \\
27 & $12 / 01 / 93$ \\
26 & $12 / 01 / 93$ \\
397 & $12 / 01 / 93$ \\
91 & $12 / 01 / 93$ \\
62 & $12 / 01 / 93$ \\
55 & $12 / 01 / 93$ \\
41 & $12 / 01 / 93$ \\
84 & $12 / 01 / 93$ \\
35 & $12 / 01 / 93$ \\
25 & $12 / 01 / 93$ \\
26 & $12 / 01 / 93$ \\
91 & $12 / 01 / 93$ \\
52 & $12 / 01 / 93$ \\
91 & $12 / 01 / 93$
\end{tabular}

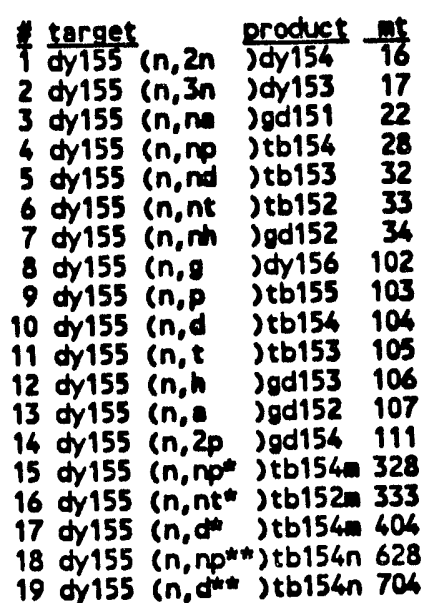

2 eng

$\begin{array}{cc}\text { engerine } & \text { date } \\ & 12 / 01 / 93 \\ 19 & 12 / 01 / 93 \\ 84 & 12 / 01 / 93 \\ 41 & 12 / 01 / 93 \\ 33 & 12 / 01 / 93 \\ 26 & 12 / 01 / 93 \\ 7 & 12 / 01 / 93 \\ 392 & 12 / 01 / 93 \\ 86 & 12 / 01 / 93 \\ 60 & 12 / 01 / 93 \\ 60 & 12 / 01 / 93 \\ 39 & 12 / 01 / 93 \\ 76 & 12 / 01 / 93 \\ 40 & 12 / 01 / 93 \\ 41 & 12 / 01 / 93 \\ 26 & 12 / 01 / 93 \\ 60 & 12 / 01 / 93 \\ 41 & 12 / 01 / 93 \\ 60 & 12 / 01 / 93\end{array}$

1 dy156 (n,2n )dy155 16 2 dy156 (n,3n) dy156 17 3 dy156 (n,m) odis2 22 4 dy 156 (n,np) tb155 28 5 dy156 (n,nd )tb154 32 6 dy156 (n,nt) jtb153 33 7 dy156 (n,g) jy 157102 8 dy156 (n,p )tb156 103 9 dy156 (n,d )tb155 104 10 dy156 (n,t )tb154 105 11 dy156 (n,h jod154 106 12 dy156 (n, od153 107 13 dy156 (n,2p) gd155 111 14 dy156 (n,nd ) tb154m 332 15 dr156 (n,p jtb156m 403 16 dy156 (n,t )tb154m 405 17 dy156 (n,ndm)tb154n 632 18 dis6 (n, pote jtb156n 703 19 dy156 (n, tw ) tb154n 705

$\begin{array}{rr}32 & 12 / 01 / 93 \\ 19 & 12 / 01 / 93 \\ 84 & 12 / 01 / 93 \\ 43 & 12 / 01 / 93 \\ 23 & 12 / 01 / 93 \\ 20 & 12 / 01 / 93 \\ 3335 & 12 / 01 / 93 \\ 89 & 12 / 01 / 93 \\ 63 & 12 / 01 / 93 \\ 52 & 12 / 01 / 93 \\ 37 & 12 / 01 / 93 \\ 79 & 12 / 01 / 93 \\ 32 & 12 / 01 / 93 \\ 23 & 12 / 01 / 93 \\ 89 & 12 / 01 / 93 \\ 52 & 12 / 01 / 93 \\ 23 & 12 / 01 / 93 \\ 89 & 12 / 01 / 93 \\ 52 & 12 / 01 / 93\end{array}$

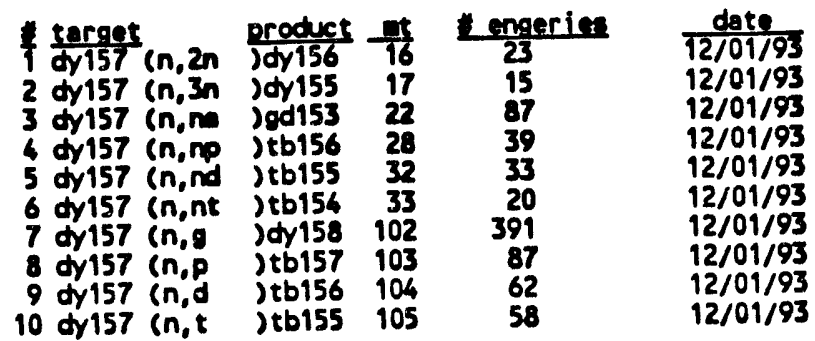

$A-7.11$ 


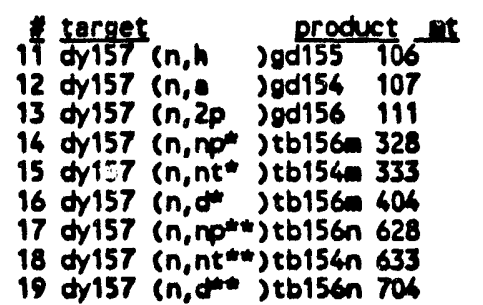

$\frac{1}{33}$ enories
82
36
39
20
62
39
20
58

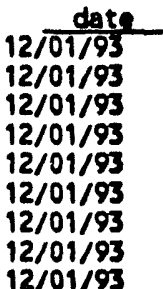

1 dy 158 (n, 2n )dy157 16
2 dy $158(n, 3 n$ dy $156 \quad 17$

3 dris8 (n,m) od15h 22

6 dy158 (n,np) tb157

5 dy158 (n,nd )tb150

6 dy158 (n,m) jtb155

7 or 158 (n,s )dyiso 102

8 dy158 (n,p )tb158 103

9 dy158 (n,d )tb157 106

10 dy158 (n,t )tb156 105

11 dy158 (n,h )gd156 106

12 dy158 (n,e )gd155 107

13 dy158 (n,2p) od157 111

14 dy158 (n, nd ) tb156m 332

15 dy158 (n,p) jtb158m 403

16 dy158 (n,t*)tb156m 405

17 dy158 (n,ndt*) tb156n 632

18 dy 158 (n, t*t) tb156n 705

$\begin{array}{rr}27 & 12 / 01 / 93 \\ 16 & 12 / 01 / 93 \\ 77 & 12 / 01 / 93 \\ 45 & 12 / 01 / 93 \\ 23 & 12 / 01 / 93 \\ 21 & 12 / 01 / 93 \\ 1220 & 12 / 01 / 93 \\ 92 & 12 / 01 / 93 \\ 60 & 12 / 01 / 93 \\ 52 & 12 / 01 / 93 \\ 31 & 12 / 01 / 93 \\ 76 & 12 / 011 / 93 \\ 30 & 12 / 01 / 93 \\ 23 & 12 / 01 / 93 \\ 92 & 12 / 01 / 93 \\ 52 & 12 / 01 / 93 \\ 23 & 12 / 01 / 93 \\ 52 & 12 / 01 / 93\end{array}$

terget

$\frac{\text { product }}{20158} \frac{\text { gt }}{16}$

dy159 (n, 2n ) dy158 $\frac{}{16}$

3 dy159 (n, na jod155 22

4 dy159 (n,np )tb158 28

5 dy159 ( $n$,nd ) tb157 32

6 dy159 (n,nt) tb156 33

7 dy159 (n,g idy160 102

8 dy159 (n,p tb159 103

9 dy159 (n,d )tb158 104

10 dy159 (n,t )tb157 105

11 dy159 (n,h )gd157 106

12 dy159 (n,. jod156 107

13 dy159 (n,2p) gd158 111

16 dy 159 ( $n, n p^{\circ}$ ) tb158m 328

15 dy159 (n,nt*) tb156a 333

16 dy159 (n, d ) tb158m 40\%

17 oy159 (n,nt**) tb156n 633

\begin{tabular}{cc} 
nengerien & date \\
\cline { 2 - 2 } 31 & $12 / 01 / 93$ \\
18 & $12 / 01 / 93$ \\
88 & $12 / 01 / 93$ \\
39 & $12 / 01 / 93$ \\
31 & $12 / 01 / 93$ \\
20 & $12 / 01 / 93$ \\
392 & $12 / 01 / 93$ \\
88 & $12 / 01 / 93$ \\
59 & $12 / 01 / 93$ \\
58 & $12 / 01 / 93$ \\
29 & $12 / 01 / 93$ \\
78 & $12 / 01 / 93$ \\
32 & $12 / 01 / 93$ \\
39 & $12 / 01 / 93$ \\
20 & $12 / 01 / 93$ \\
59 & $12 / 01 / 93$ \\
20 & $12 / 01 / 93$
\end{tabular}

1 dy160 ( $n, 2 n)$ dy159 16

2 dy 160 (n, 3n )dy158 17

3 oy $160(n, n)$ gdis6 22

4 dy160 (n,np) tb159 28

5 dy160 ( $n$,nd ) tb158 32

6 dy160 (n,nt) tb157 33

7 dy 160 (n,g) dy161 102

8 dy160 (n,p )tb160 103

9 dy 160 (n,d )tb159 104

10 dy 160 (n,t )tb158 105

11 dy $160(n, h)$ jod158 106

12 dy160 (n, jod157 107

13 dy160 (n,2p) gd159 111

16 dy 160 ( $n$, nd ) tb158m 332

15 dy 160 (n, t* )tb158m 405

1 dy161 (n,2n )dy160

2 dy161 (n,3n )dy159

3 dy161 ( $n, n$ ) gd157

4 dy161 (n, np) tb160

5 dy161 (n,nd) tb159 32

6 dy161 (n,nt) tb158 33

7 dy161 (n,g)dy162 102

8 dy161 (n,p)tb161 103

9 dy161 (n,d) tb160 ios

$\begin{array}{rr}30 & 12 / 01 / 93 \\ 16 & 12 / 01 / 93 \\ 72 & 12 / 01 / 93 \\ 46 & 12 / 01 / 93 \\ 25 & 12 / 01 / 93 \\ 22 & 12 / 01 / 93 \\ 1247 & 12 / 01 / 93 \\ 80 & 12 / 01 / 93 \\ 58 & 12 / 01 / 93 \\ 52 & 12 / 01 / 93 \\ 27 & 12 / 01 / 93 \\ 63 & 12 / 01 / 93 \\ 25 & 12 / 01 / 93 \\ 23 & 12 / 01 / 93 \\ 52 & 12 / 01 / 93\end{array}$

$12 / 01 / 93$

$12 / 01 / 93$

$12 / 01 / 93$

$12 / 01 / 93$

$12 / 01 / 93$

$12 / 01 / 93$

$\begin{array}{rr}4015 & 12 / 01 / 93 \\ 89 & 12 / 01 / 93 \\ 58 & 12 / 01 / 93\end{array}$

$\begin{array}{rr}4015 & 12 / 01 / 93 \\ 89 & 12 / 01 / 93 \\ 58 & 12 / 01 / 93\end{array}$

$A-7.12$ 


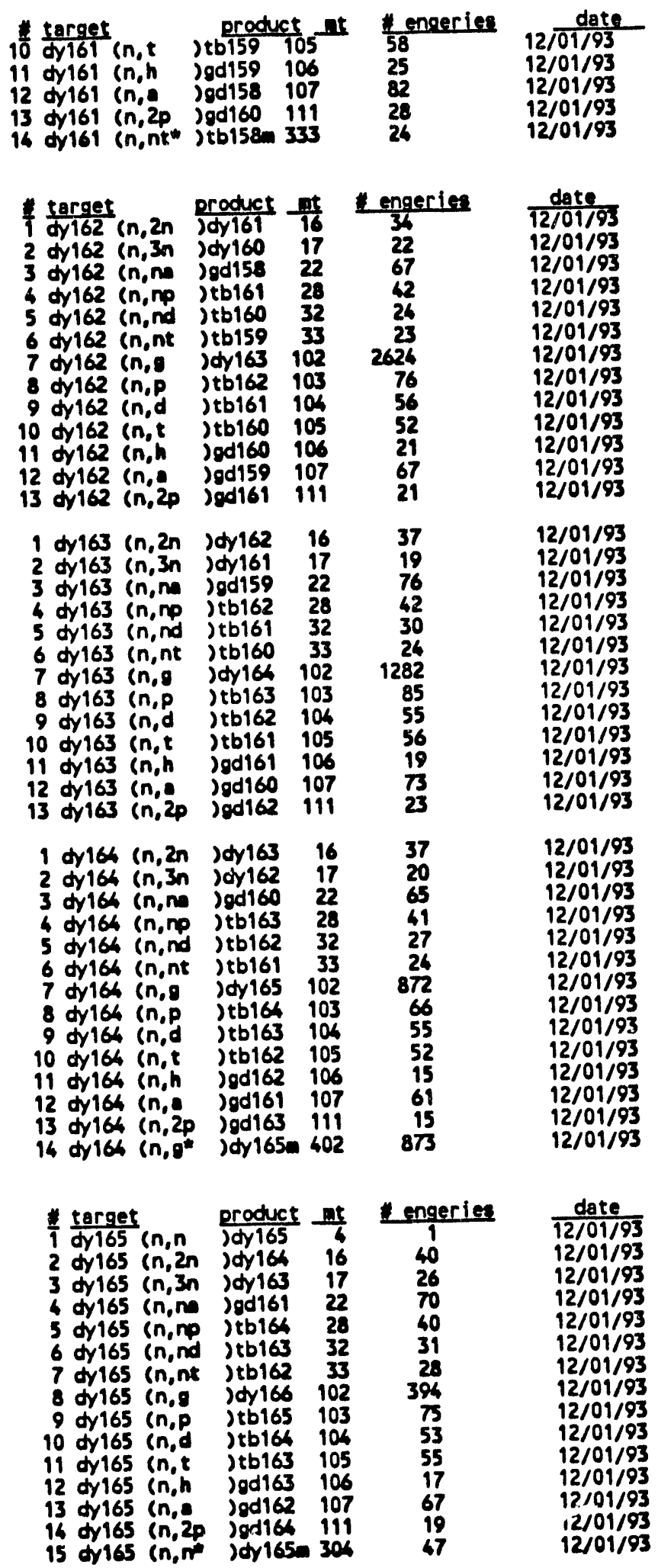




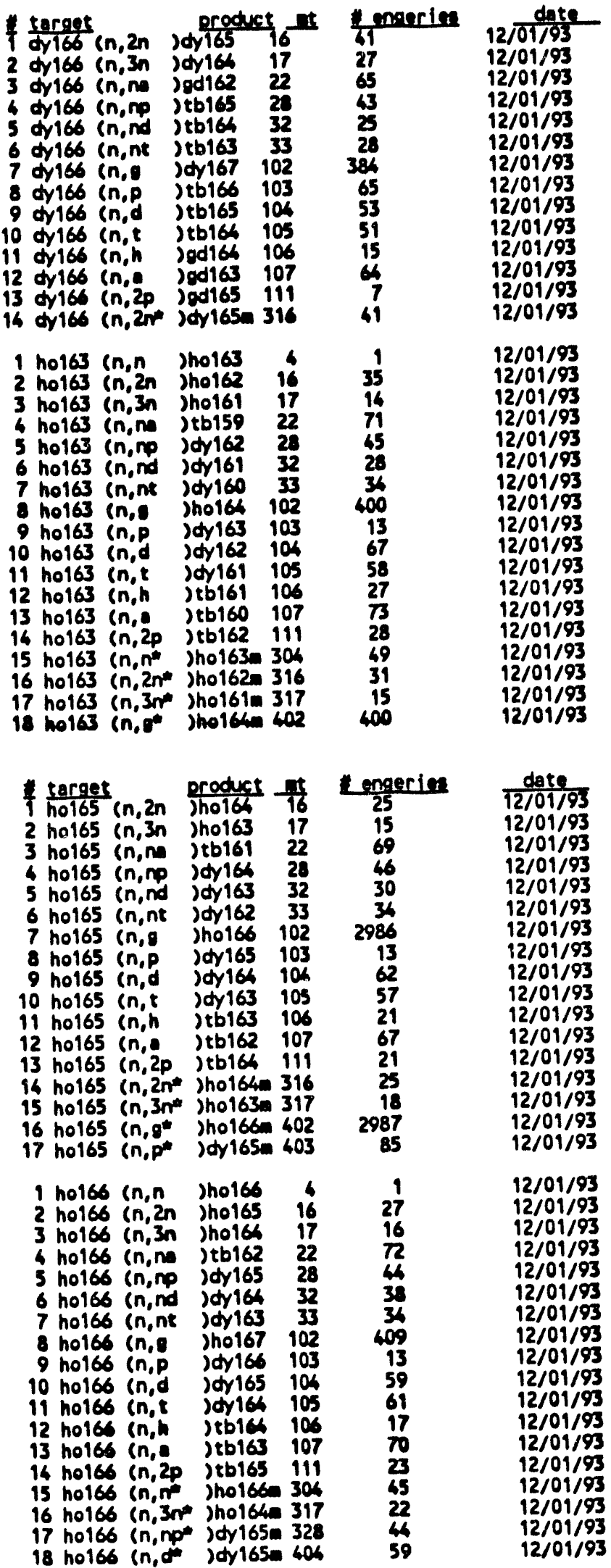




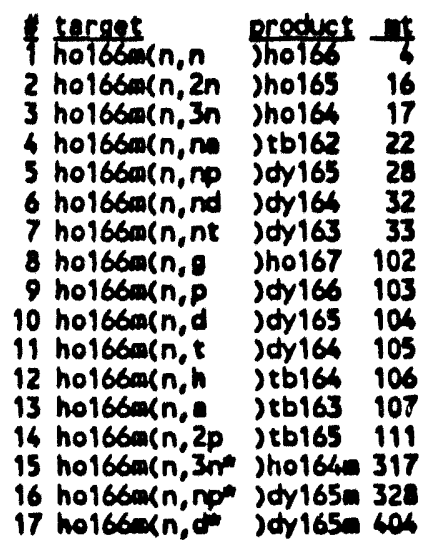

snoris
50
27
16
72
46
38
34
409
13
59
61
17
70
23
18
46
59

$\frac{\text { date }}{\text { तints }}$

$12101 / 93$

$12 / 01 / 93$

$12 / 01 / 93$

$12 / 01 / 93$

$12 / 01 / 93$

$12 / 01 / 93$

12/01/93

$12 / 01 / 93$

$12 / 01 / 93$

$12 / 01 / 93$

$12 / 01 / 93$

$12 / 01 / 93$

$12 / 01 / 93$

$12 / 01 / 93$

$12 / 01 / 93$

$12 / 01 / 93$

1 er162 (n, 2n )er161 16

2 er162 ( $n, 3 n$ )er160 17

3 or162 (n,m )dy15s 22

6 or 162 (n,np ino161 28

5 er162 (n,nd hol60 32

$6 \operatorname{er162}(n, n t)$ hols9 33

$7 \operatorname{or} 162(n, g)$ )er163 102

o er162 $\mathrm{kn,d}$ hol61 104

10 er162 (n,t iho160 105

11 er162 (n, h joy160 106

12 er162 (n, )dy159 107

13 er162 (n,2p )dy161 111

16 or162 (n,npt ho16ia 320

15 er 162 in, num hho160m 332

16 er162 (n,nt* )ho159m 333

17 er162 (n,p) holsan 403

18 or162 $(n, d$ Jho161m 40 h

19 er162 (n,t") ho160 405

20 er162 (n,ndtuthol6on 632

21 or 162 ( $n, t^{+*}$ )hol6on 705

$\begin{array}{cc}26 & 12 / 01 / 93 \\ 15 & 12 / 01 / 93 \\ 84 & 12 / 01 / 93 \\ 46 & 12 / 01 / 93 \\ 25 & 12 / 01 / 93 \\ 20 & 12 / 01 / 93 \\ 3833 & 12 / 01 / 93 \\ 92 & 12 / 01 / 93 \\ 63 & 12 / 01 / 93 \\ 53 & 12 / 01 / 93 \\ 35 & 12 / 01 / 93 \\ 80 & 12 / 01 / 93 \\ 31 & 12 / 01 / 93 \\ 43 & 12 / 01 / 93 \\ 25 & 12 / 01 / 93 \\ 20 & 12 / 01 / 93 \\ 92 & 12 / 01 / 93 \\ 63 & 12 / 01 / 93 \\ 53 & 12 / 01 / 93 \\ 25 & 12 / 01 / 93 \\ 51 & 12 / 01 / 93 \\ & 12103\end{array}$

\begin{tabular}{|c|c|c|c|c|c|}
\hline 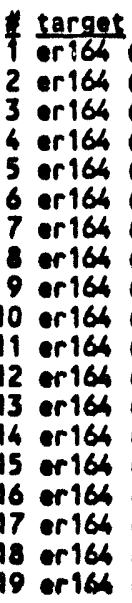 & 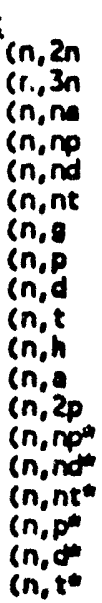 & 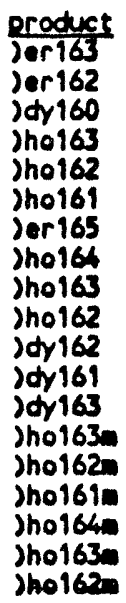 & $\begin{array}{l}7 \\
76 \\
17 \\
22 \\
28 \\
32 \\
33 \\
102 \\
103 \\
104 \\
105 \\
106 \\
107 \\
111 \\
328 \\
332 \\
333 \\
403 \\
404 \\
405\end{array}$ & $\begin{array}{c}\text { engeries } \\
31 \\
19 \\
77 \\
45 \\
25 \\
22 \\
6739 \\
92 \\
60 \\
53 \\
29 \\
72 \\
23 \\
42 \\
25 \\
22 \\
92 \\
60 \\
53\end{array}$ & $\begin{array}{l}\text { date } \\
12 / 01 / 93 \\
12 / 01 / 93 \\
12 / 01 / 93 \\
12 / 01 / 93 \\
12 / 01 / 93 \\
12 / 01 / 93 \\
12 / 01 / 93 \\
12 / 01 / 93 \\
12 / 01 / 93 \\
12 / 01 / 93 \\
12 / 01 / 93 \\
12 / 01 / 93 \\
12 / 01 / 93 \\
12 / 01 / 93 \\
12 / 01 / 93 \\
12 / 01 / 93 \\
12 / 01 / 93 \\
12 / 01 / 93 \\
12 / 01 / 93\end{array}$ \\
\hline $\begin{array}{l}\operatorname{er} 165 \\
\operatorname{er} 165 \\
\operatorname{er} 165 \\
\operatorname{er} 165 \\
\operatorname{er} 165 \\
\operatorname{er} 165 \\
\operatorname{er} 165 \\
\operatorname{er} 165 \\
\operatorname{or} 165 \\
\operatorname{er} 165 \\
\operatorname{er} 165 \\
\operatorname{er} 165\end{array}$ & $\begin{array}{l}(n, 2 n \\
(n, 3 n \\
(n, n) \\
(n, n) \\
(n, n) \\
(n, n) \\
(n, 8) \\
(n, p) \\
(n, d \\
l n, t \\
(n, n) \\
(n, 0)\end{array}$ & $\begin{array}{l}\text { Jer164 } \\
\text { Jer163 } \\
\text { Jdy161 } \\
\text { Jho164 } \\
\text { Jho163 } \\
\text { Jho162 } \\
\text { Jer166 } \\
\text { Jho165 } \\
\text { Jho164 } \\
\text { Jho163 } \\
\text { Jdy163 } \\
\text { Joy162 }\end{array}$ & $\begin{array}{c}16 \\
17 \\
22 \\
28 \\
32 \\
33 \\
102 \\
103 \\
104 \\
105 \\
106 \\
107\end{array}$ & $\begin{array}{c}28 \\
17 \\
81 \\
40 \\
34 \\
25 \\
390 \\
91 \\
61\end{array}$ & $\begin{array}{l}12 / 01 / 93 \\
12 / 01 / 93 \\
12 / 01 / 93 \\
12 / 01 / 93 \\
12 / 01 / 93 \\
12 / 01 / 93 \\
12 / 01 / 93 \\
12 / 01 / 93 \\
12 / 01 / 93 \\
12 / 01 / 93 \\
12 / 01 / 93 \\
12 / 01 / 93\end{array}$ \\
\hline
\end{tabular}

$A-7.15$ 


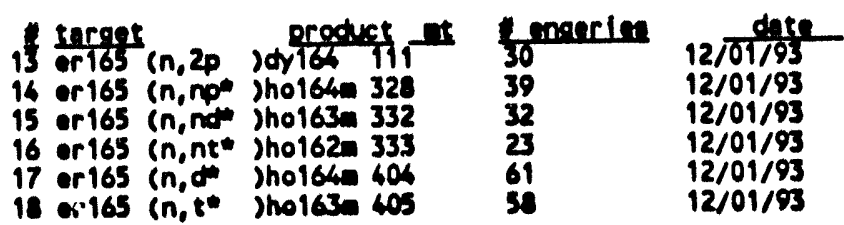

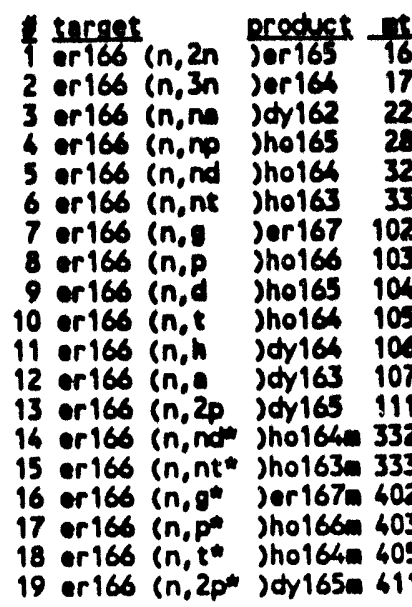

$\begin{array}{cc}\text { monerin } & \text { diate } \\ & 12 / 01 / 93 \\ 20 & 12 / 01 / 93 \\ 73 & 12 / 01 / 93 \\ 45 & 12 / 01 / 93 \\ 26 & 12 / 01 / 93 \\ 26 & 12 / 01 / 93 \\ 8798 & 12 / 01 / 93 \\ 80 & 12 / 01 / 93 \\ 58 & 12 / 01 / 93 \\ 53 & 12 / 01 / 93 \\ 25 & 12 / 01 / 93 \\ 70 & 12 / 01 / 93 \\ 23 & 12 / 01 / 93 \\ 26 & 12 / 01 / 93 \\ 26 & 12 / 01 / 93 \\ 8801 & 12 / 01 / 93 \\ 80 & 12 / 01 / 93 \\ 51 & 12 / 01 / 93 \\ 23 & 12 / 01 / 93 \\ & 12 / 01 / 93 \\ 1 & 12 / 01 / 93 \\ 40 & 12 / 01 / 93 \\ 18 & 12 / 01 / 93 \\ 76 & 12 / 01 / 93 \\ 41 & 12 / 01 / 93 \\ 32 & 12 / 01 / 93 \\ 25 & 12 / 01 / 93 \\ 5093 & 12 / 01 / 93 \\ 91 & 12 / 01 / 93 \\ 56 & 12 / 01 / 93 \\ 57 & 12 / 01 / 93 \\ 21 & 12 / 01 / 93 \\ 77 & 12 / 01 / 93 \\ 25 & 12 / 01 / 93 \\ 45 & 12 / 01 / 93 \\ 61 & 12 / 01 / 93 \\ 26 & 12 / 01 / 93 \\ 56 & 12 / 01 / 93 \\ 21 & \end{array}$

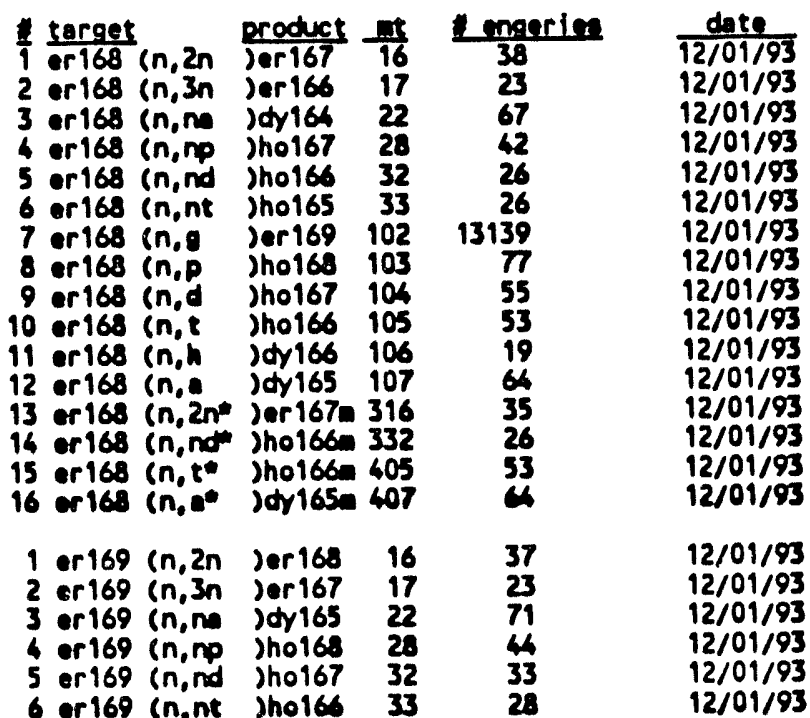

A-7. 16 


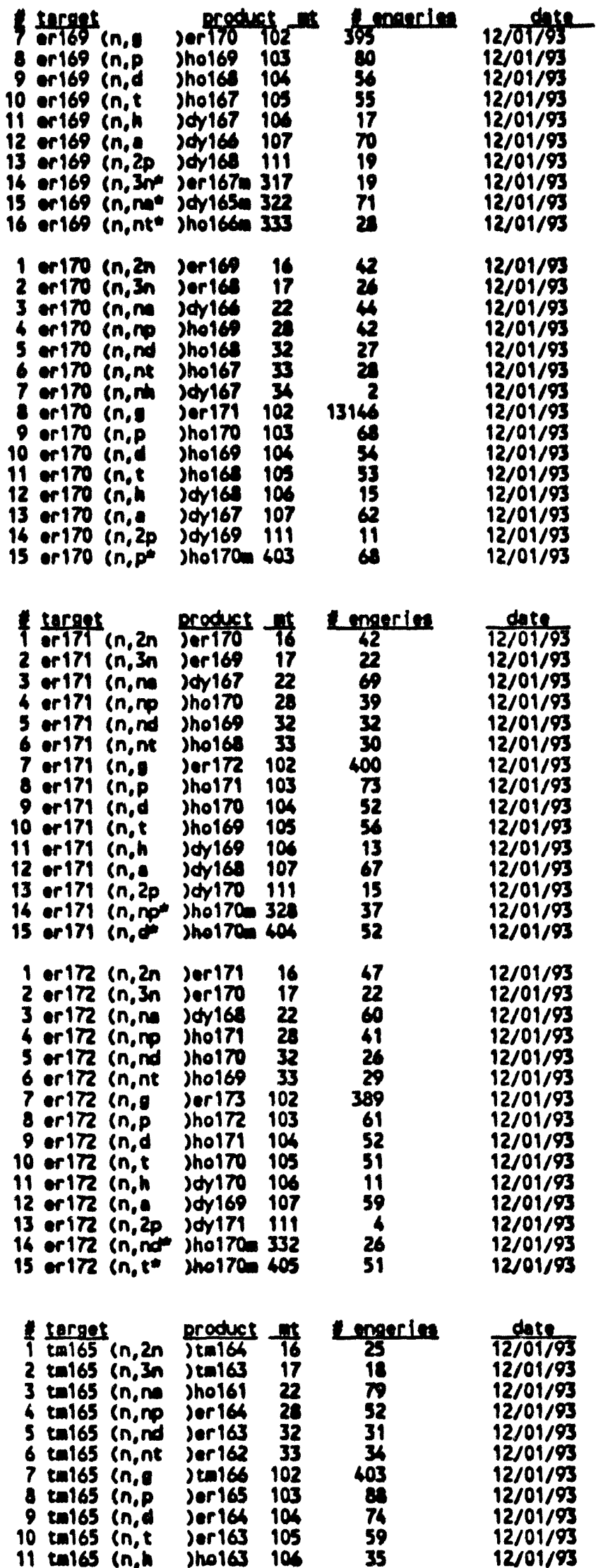

$A-7.17$ 


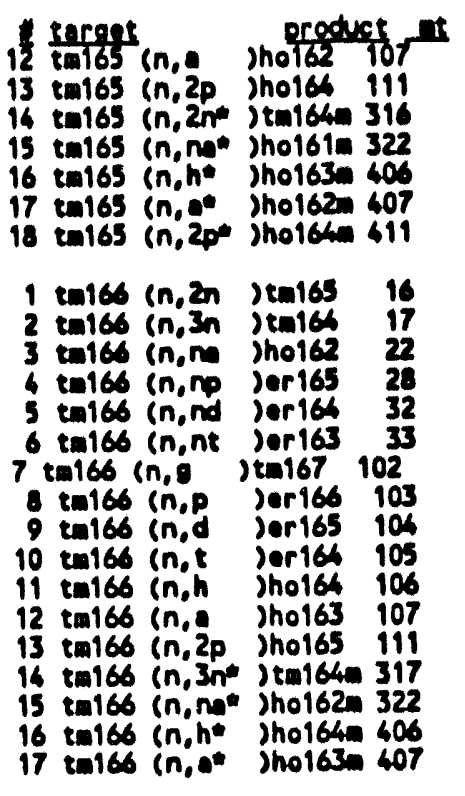

\begin{tabular}{cc} 
monerin & date \\
\hline 80 & $12 / 01 / 93$ \\
34 & $12 / 01 / 93$ \\
23 & $12 / 01 / 93$ \\
79 & $12 / 01 / 93$ \\
35 & $12 / 01 / 93$ \\
80 & $12 / 01 / 93$ \\
36 & $12 / 01 / 93$ \\
23 & $12 / 01 / 93$ \\
23 & $12 / 01 / 93$ \\
16 & $12 / 01 / 93$ \\
89 & $12 / 01 / 93$ \\
67 & $12 / 01 / 93$ \\
40 & $12 / 01 / 93$ \\
36 & $12 / 01 / 93$ \\
400 & $12 / 01 / 93$ \\
85 & $12 / 01 / 93$ \\
70 & $12 / 01 / 93$ \\
68 & $12 / 01 / 93$ \\
33 & $12 / 01 / 93$ \\
84 & $12 / 01 / 93$ \\
36 & $12 / 01 / 93$ \\
16 & $12 / 01 / 93$ \\
89 & $12 / 01 / 93$ \\
33 & $12 / 01 / 93$ \\
84 &
\end{tabular}

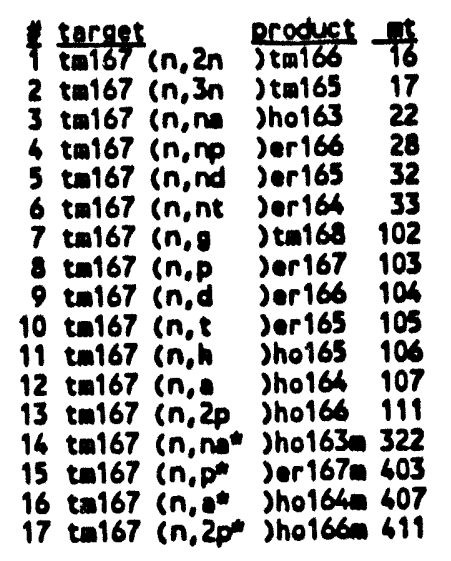

\begin{tabular}{cc} 
moneries & dote \\
\hline 30 & $12 / 01 / 93$ \\
20 & $12 / 01 / 93$ \\
76 & $12 / 01 / 93$ \\
52 & $12 / 01 / 93$ \\
30 & $12 / 01 / 93$ \\
36 & $12 / 01 / 93$ \\
406 & $12 / 01 / 93$ \\
89 & $12 / 01 / 93$ \\
69 & $12 / 01 / 93$ \\
59 & $12 / 01 / 93$ \\
20 & $12 / 01 / 93$ \\
77 & $12 / 01 / 93$ \\
29 & $12 / 01 / 93$ \\
76 & $12 / 01 / 93$ \\
89 & $12 / 01 / 93$ \\
77 & $12 / 01 / 93$ \\
29 & $12 / 01 / 93$ \\
33 & $12 / 01 / 93$ \\
18 & $12 / 01 / 93$ \\
82 & $12 / 01 / 93$ \\
46 & $12 / 01 / 93$ \\
39 & $12 / 01 / 93$ \\
33 & $12 / 01 / 93$ \\
400 & $12 / 01 / 93$ \\
90 & $12 / 01 / 93$ \\
66 & $12 / 01 / 93$ \\
65 & $12 / 01 / 93$ \\
27 & $12 / 01 / 93$ \\
81 & $12 / 01 / 93$ \\
31 & $12 / 01 / 93$ \\
82 & $12 / 01 / 93$ \\
43 & $12 / 01 / 93$ \\
66 & $12 / 01 / 93$ \\
27 & $12 / 01 / 93$
\end{tabular}

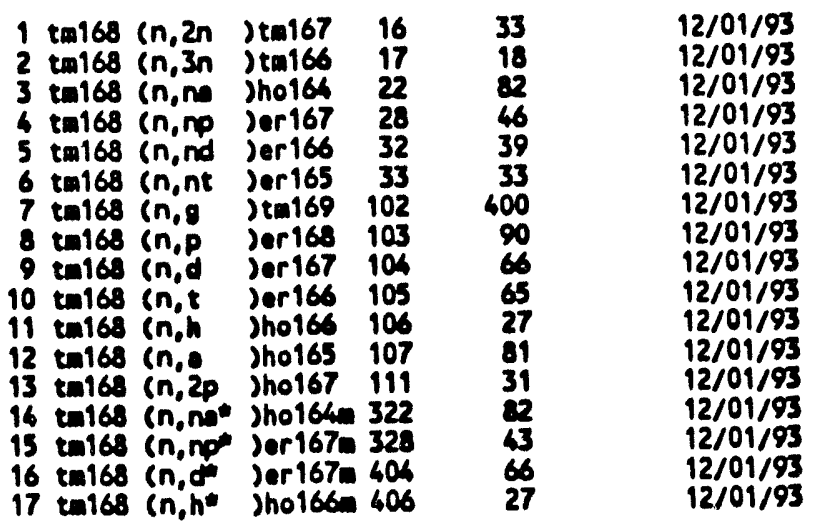

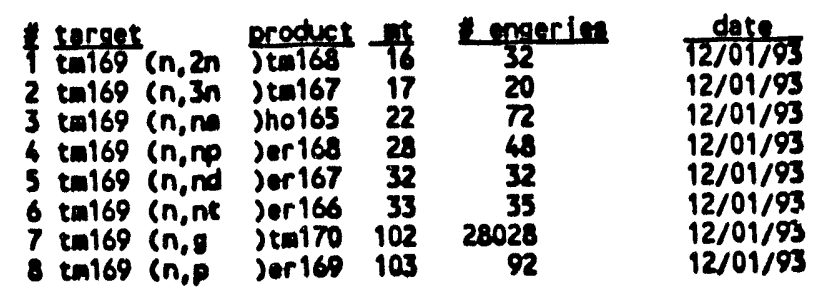




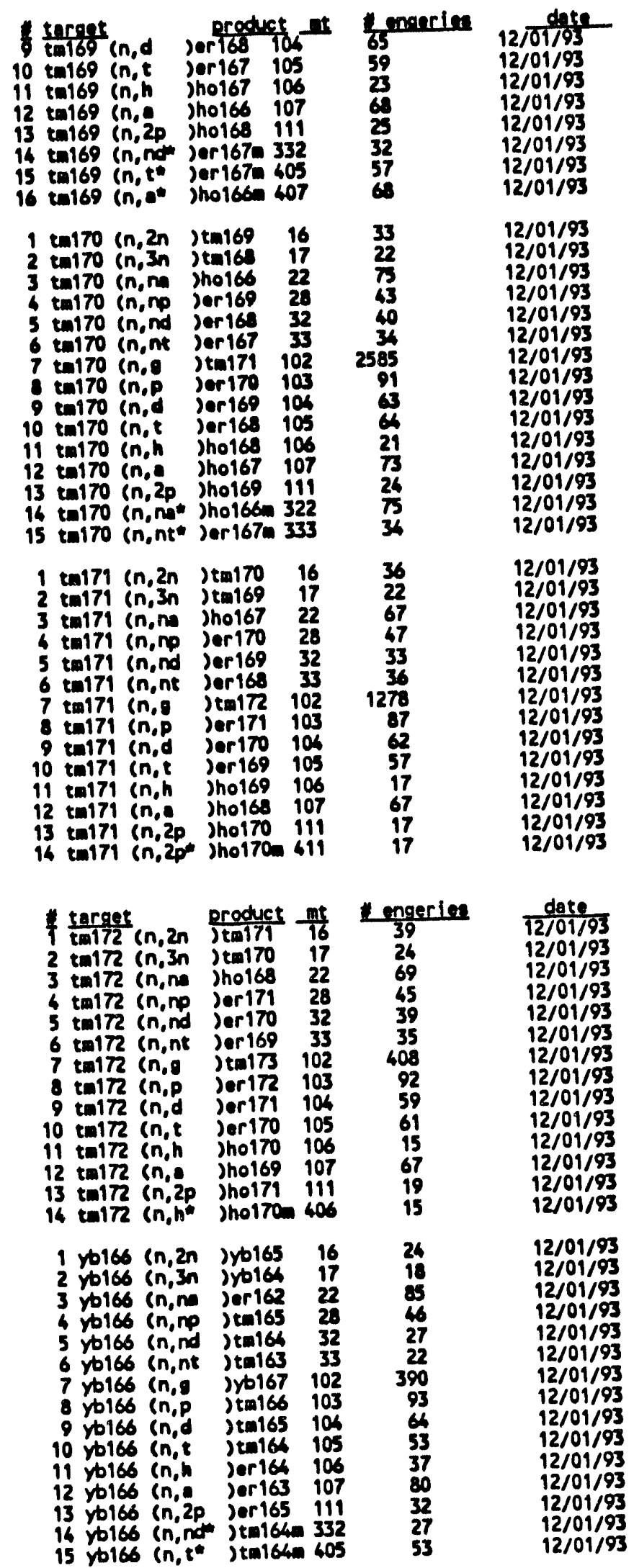

A-7. 19 


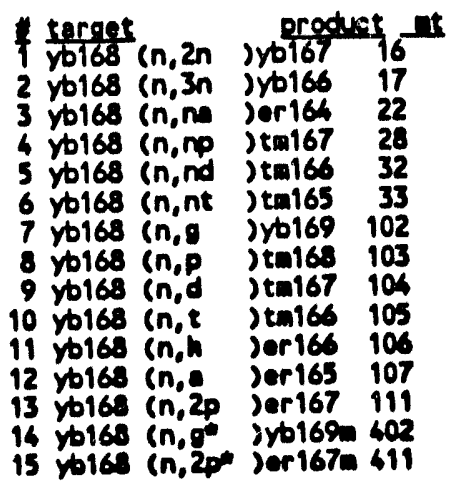

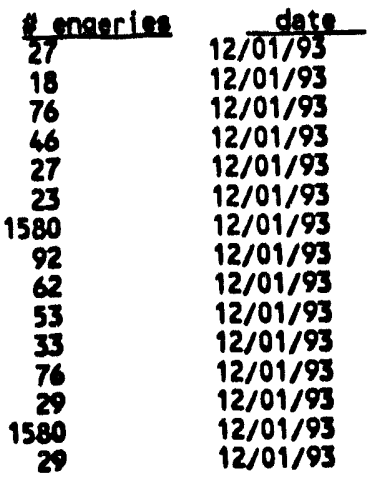

\begin{tabular}{|c|c|c|c|c|}
\hline 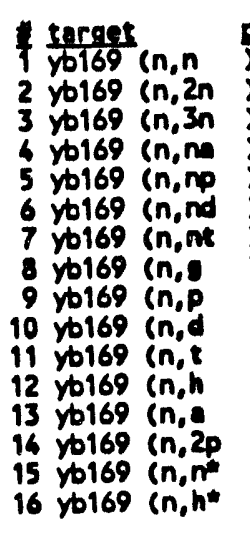 & 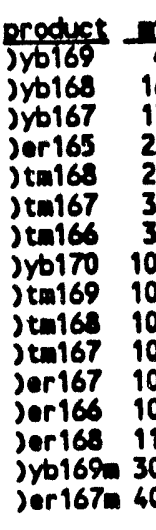 & $\begin{array}{c}22 \\
28 \\
32 \\
33 \\
13 \\
102 \\
103 \\
106 \\
105 \\
106 \\
107 \\
119 \\
304\end{array}$ & $\begin{array}{c}\text { mperine } \\
1 \\
31 \\
16 \\
89 \\
45 \\
34 \\
23 \\
3086 \\
92 \\
61 \\
59 \\
31 \\
85 \\
32 \\
52 \\
31\end{array}$ & $\begin{array}{l}\frac{\text { date }}{12 / 01 / 93} \\
12 / 01 / 93 \\
12 / 1 / 93 \\
12 / 01 / 93 \\
12 / 01 / 93 \\
12 / 01 / 93 \\
12 / 01 / 93 \\
12 / 01 / 93 \\
12 / 01 / 93 \\
12 / 01 / 93 \\
12 / 01 / 93 \\
12 / 01 / 93 \\
12 / 01 / 93 \\
12 / 01 / 93 \\
12 / 01 / 93 \\
12 / 01 / 93\end{array}$ \\
\hline 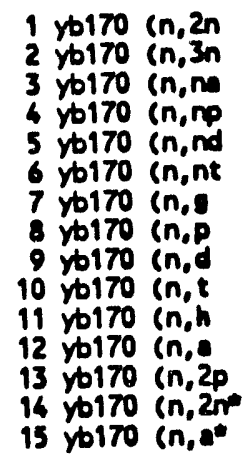 & 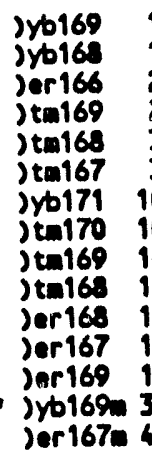 & $\begin{array}{l}16 \\
17 \\
22 \\
28 \\
32 \\
33 \\
102 \\
103 \\
106 \\
105 \\
106 \\
107 \\
111 \\
316 \\
407\end{array}$ & $\begin{array}{r}29 \\
21 \\
77 \\
46 \\
28 \\
25 \\
5475 \\
91 \\
59 \\
55 \\
27 \\
73 \\
25 \\
29 \\
73\end{array}$ & $\begin{array}{l}12 / 01 / 93 \\
12 / 01 / 93 \\
12 / 01 / 93 \\
12 / 01 / 93 \\
12 / 1193 \\
12 / 01 / 93 \\
12 / 01 / 93 \\
12 / 01 / 93 \\
12 / 0193 \\
12 / 01 / 93 \\
12 / 01 / 93 \\
12 / 01 / 93 \\
12 / 01 / 93 \\
12 / 01 / 93 \\
12 / 01 / 93\end{array}$ \\
\hline 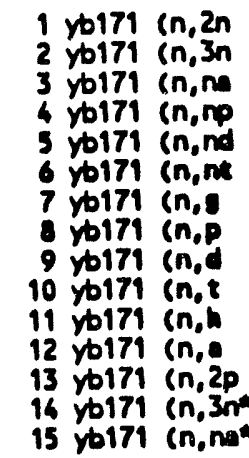 & 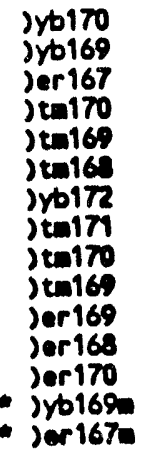 & $\begin{array}{l}16 \\
17 \\
22 \\
28 \\
32 \\
33 \\
102 \\
103 \\
104 \\
105 \\
106 \\
107 \\
111 \\
317 \\
322\end{array}$ & $\begin{array}{r}30 \\
19 \\
82 \\
44 \\
36 \\
27 \\
29802 \\
91 \\
59 \\
59 \\
25 \\
80\end{array}$ & $\begin{array}{l}12 / 01 / 93 \\
12 / 01 / 93 \\
12 / 01 / 93 \\
12 / 01 / 93 \\
12 / 01 / 93 \\
12 / 01 / 93 \\
12 / 01 / 93 \\
12 / 01 / 93 \\
12 / 01 / 93 \\
12 / 01 / 93 \\
12 / 01 / 93 \\
12 / 01 / 93 \\
12 / 01 / 93 \\
12 / 01 / 93 \\
12 / 01 / 93\end{array}$ \\
\hline
\end{tabular}




\begin{tabular}{|c|c|c|c|c|}
\hline 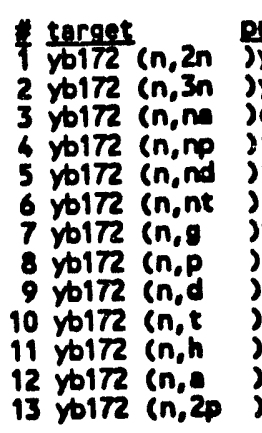 & 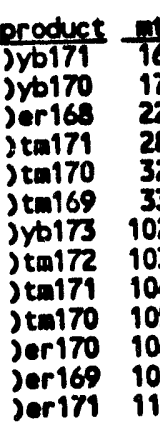 & $\begin{array}{l}11 \\
16 \\
17 \\
22 \\
28 \\
32 \\
33 \\
102 \\
103 \\
104 \\
105 \\
106 \\
107 \\
111\end{array}$ & $\begin{array}{c}\text { enger } \\
33 \\
22 \\
69 \\
45 \\
28 \\
27 \\
12717 \\
80 \\
57 \\
55 \\
23 \\
70 \\
21\end{array}$ & $\begin{array}{l}\text { date } \\
\frac{\text { dat }}{12 / 01 / 93} \\
12 / 01 / 93 \\
12 / 01 / 93 \\
12 / 01 / 93 \\
12 / 01 / 93 \\
12 / 01 / 93 \\
12 / 01 / 93 \\
12 / 01 / 93 \\
12 / 01 / 93 \\
12 / 01 / 93 \\
12 / 01 / 93 \\
12 / 01 / 93 \\
12 / 01 / 93\end{array}$ \\
\hline 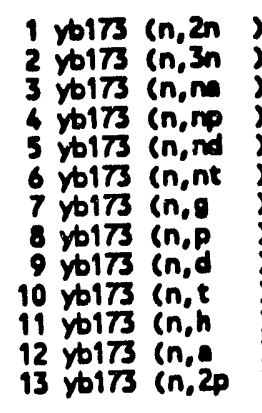 & 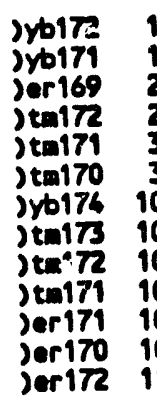 & $\begin{array}{l}16 \\
17 \\
22 \\
28 \\
32 \\
33 \\
102 \\
103 \\
104 \\
105 \\
106 \\
107 \\
111\end{array}$ & $\begin{array}{r}39 \\
21 \\
76 \\
45 \\
33 \\
28 \\
27311 \\
87 \\
57 \\
58 \\
19 \\
76 \\
21\end{array}$ & $\begin{array}{l}12 / 01 / 93 \\
12 / 01 / 93 \\
12 / 01 / 93 \\
12 / 01 / 93 \\
12 / 01 / 93 \\
12 / 01 / 93 \\
12 / 01 / 93 \\
12 / 01 / 93 \\
12 / 01 / 93 \\
12 / 01 / 93 \\
12 / 01 / 93 \\
12 / 01 / 93 \\
12 / 01 / 93\end{array}$ \\
\hline 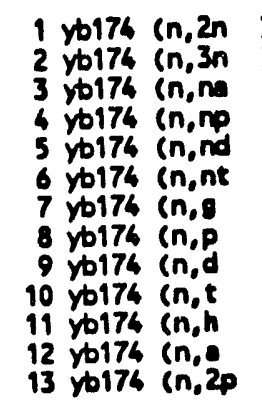 & 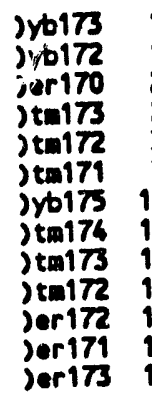 & $\begin{array}{l}16 \\
17 \\
22 \\
28 \\
32 \\
33 \\
102 \\
103 \\
104 \\
105 \\
106 \\
107 \\
111\end{array}$ & $\begin{array}{r}37 \\
25 \\
65 \\
43 \\
29 \\
28 \\
6446 \\
76 \\
56 \\
55 \\
17 \\
65 \\
13\end{array}$ & $\begin{array}{l}12 / 01 / 93 \\
12 / 01 / 93 \\
12 / 01 / 93 \\
12 / 01 / 93 \\
12 / 01 / 93 \\
12 / 01 / 93 \\
12 / 01 / 93 \\
12 / 01 / 93 \\
12 / 01 / 93 \\
12 / 01 / 93 \\
12 / 01 / 93 \\
12 / 01 / 93 \\
12 / 01 / 93\end{array}$ \\
\hline 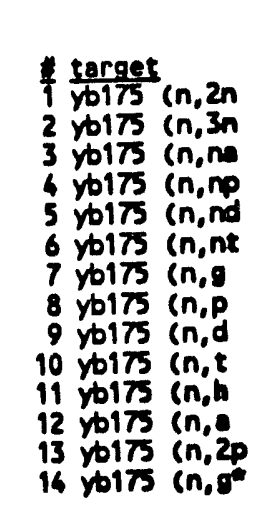 & 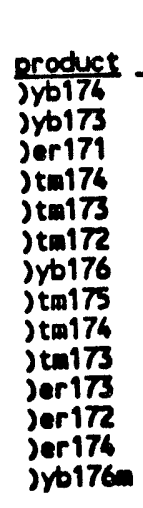 & $\begin{array}{l}\frac{m t}{16} \\
17 \\
22 \\
28 \\
32 \\
33 \\
102 \\
103 \\
104 \\
105 \\
105 \\
107 \\
111 \\
402\end{array}$ & $\begin{array}{c}\text { Lengerien } \\
45 \\
26 \\
70 \\
43 \\
34 \\
30 \\
400 \\
76 \\
56 \\
58 \\
15 \\
71 \\
17 \\
400\end{array}$ & $\begin{array}{c}\text { date } \\
12 / 01 / 93 \\
12 / 01 / 93 \\
12 / 01 / 93 \\
12 / 01 / 93 \\
12 / 01 / 93 \\
12 / 01 / 93 \\
12 / 01 / 93 \\
12 / 01 / 93 \\
12 / 01 / 93 \\
12 / 01 / 93 \\
12 / 01 / 93 \\
12 / 01 / 93 \\
12 / 01 / 93 \\
12 / 01 / 93\end{array}$ \\
\hline 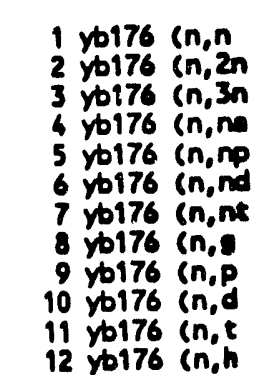 & 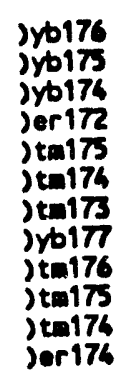 & $\begin{array}{r}16 \\
17 \\
22 \\
28 \\
32 \\
33 \\
102 \\
103 \\
104 \\
105 \\
106\end{array}$ & $\begin{array}{r}1 \\
69 \\
26 \\
63 \\
43 \\
30 \\
30 \\
6975 \\
66 \\
54 \\
53 \\
13\end{array}$ & $\begin{array}{l}12 / 01 / 93 \\
12 / 01 / 93 \\
12 / 01 / 93 \\
12 / 01 / 93 \\
12 / 01 / 93 \\
12 / 01 / 93 \\
12 / 01 / 93 \\
12 / 01 / 93 \\
12 / 01 / 93 \\
12 / 01 / 93 \\
12 / 01 / 93 \\
12 / 01 / 93\end{array}$ \\
\hline
\end{tabular}

A-7.21 


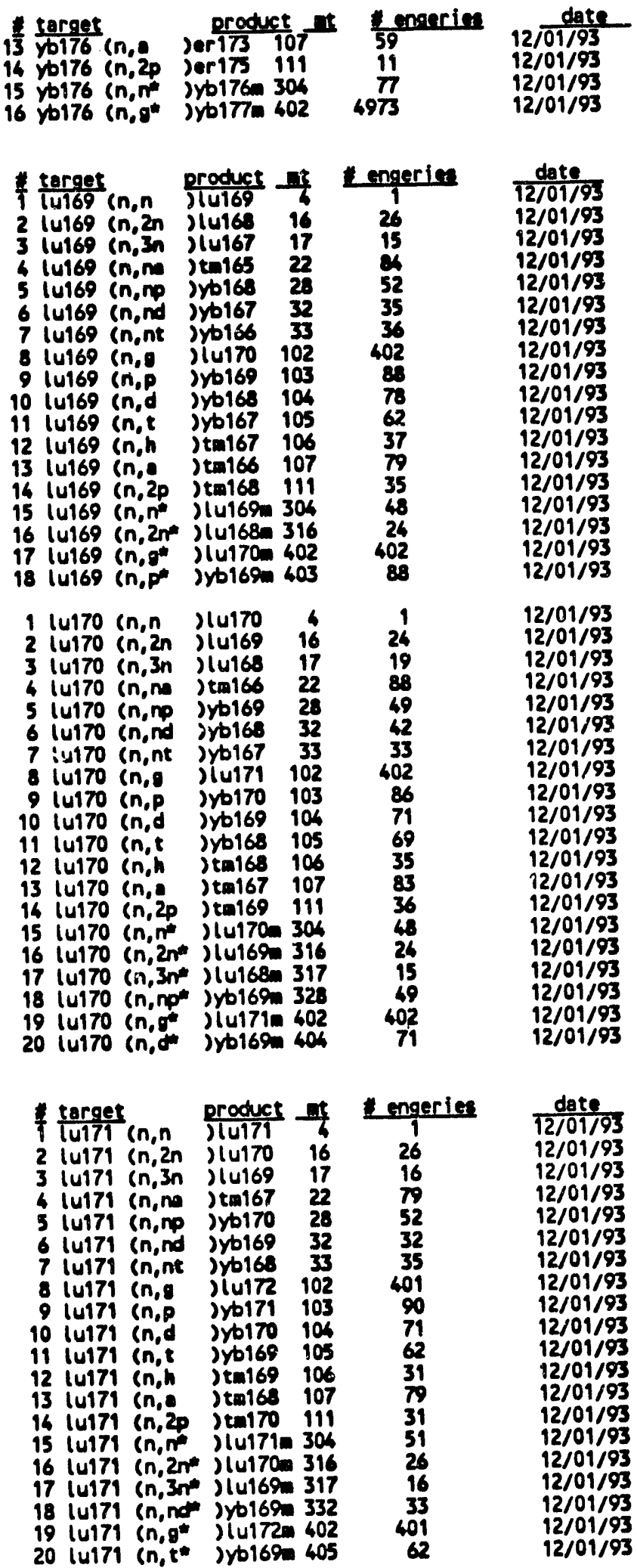




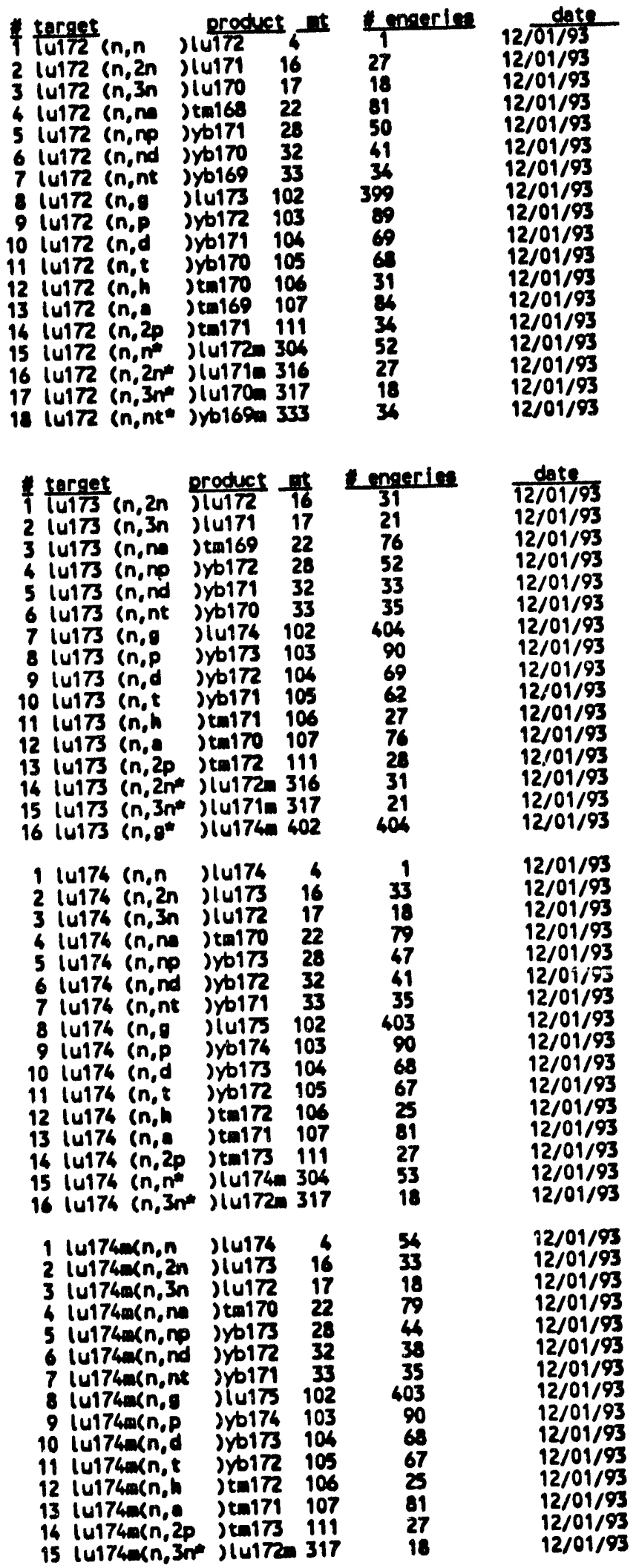




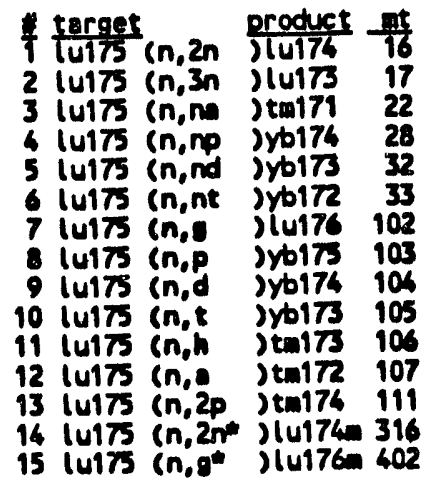

\begin{tabular}{c}
\hline engeries \\
\hline 27 \\
7 \\
69 \\
48 \\
34 \\
37 \\
3268 \\
92 \\
67 \\
59 \\
21 \\
71 \\
21 \\
26 \\
3267
\end{tabular}

\begin{tabular}{l} 
date \\
\hline $12 / 01 / 93$ \\
$12 / 01 / 93$ \\
$12 / 01 / 93$ \\
$12 / 01 / 93$ \\
$12 / 01 / 93$ \\
$12 / 01 / 93$ \\
$12 / 01 / 93$ \\
$12 / 01 / 93$ \\
$12 / 01 / 93$ \\
$12 / 01 / 93$ \\
$12 / 01 / 93$ \\
$12 / 01 / 93$ \\
$12 / 01 / 93$ \\
$12 / 011 / 93$ \\
$12 / 01 / 93$
\end{tabular}

1 lu176 (n,n) )lu176

2 lui76 $(n, 2 n$ jluits

3 lu176 (n,3n) )lu174

4 lu176 (n,n) $\operatorname{ttmi72}$

5 lu176 (n,np jybits

6 lu176 (n,nd)yb174

7 lu176 (n,nt jybits 33

8 lu176 $\mathrm{n,g}$ jlu177 102

- lu176 (n,p jyb176 103

10 lu176 (n,d Jybits 104

11 lui76 (n,t jybi74 105

12 lu176 (n,h jtm174 106

13 lu176 (n, ) tmit3 107

14 lu176 (n,2p) tmits 111

15 lu176 (n, n jlu176m 304

16 lu176 $(n, 3 n$ ) lu174m 317

17 lu176 $\left(n, g^{*}\right.$ jlu177m 402

18 lu176 (n,p") Jybi76m 403

$\begin{array}{rr}1 & 12 / 01 / 93 \\ 28 & 12 / 01 / 93 \\ 18 & 12 / 01 / 93 \\ 76 & 12 / 01 / 93 \\ 46 & 12 / 101 / 93 \\ 40 & 12 / 01 / 93 \\ 37 & 12 / 01 / 93 \\ 3707 & 12 / 01 / 93 \\ 91 & 12 / 01 / 93 \\ 63 & 12 / 01 / 93 \\ 67 & 12 / 01 / 93 \\ 19 & 12 / 01 / 93 \\ 73 & 12 / 01 / 93 \\ 23 & 12 / 01 / 93 \\ 46 & 12 / 01 / 93 \\ 17 & 12 / 01 / 93 \\ 3703 & 12 / 01 / 93 \\ 91 & 12 / 01 / 93\end{array}$

target product $\frac{\text { At }}{10}$

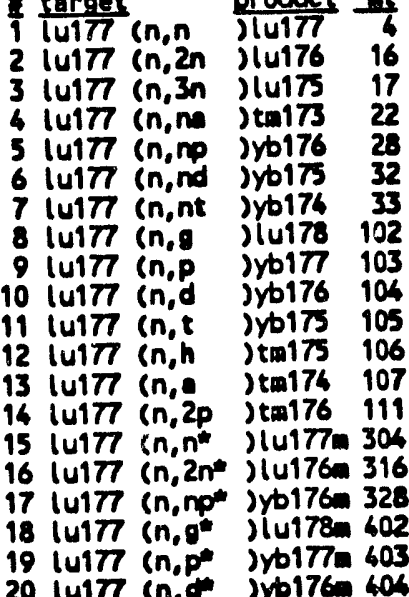

Lengerien

35

date

$\frac{d}{12 / 01 / 93}$

$12 / 01 / 93$

$12 / 01 / 93$

$12 / 01 / 93$

$12 / 01 / 93$

$12 / 01 / 93$

$12 / 01 / 93$

$12 / 01 / 93$

$12 / 01 / 93$

$12 / 01 / 93$

$12 / 01 / 93$

12/01/93

$12 / 01 / 93$

12/01/93

$12 / 01 / 93$

12/01/93

12/01/93

12/01/93

$12 / 01 / 93$

12/01/93

9 luit7men,n Juit7

2 luit7men, 2n ) lui76 16

3 luit7a(n, 3n jiuits 17

4 luit7m(n,m) timits 22

5 lu1T7m(n,no) yb176 28

6 luimorn,nd jybirs 32

7 luimmen,nt jybi74 33

luit7men,g jlui7e 102

- luimakn,p jybit7 103

10 luit7men,d ixbi76 100

11 luit7men,t jybits 105

12 luit7men, ) tmits 106

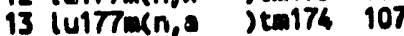

14 luir7mo(11,2p )tmi76 111

15 lu177m(n,2n ) lu176m 316

16 luit7men, no" Jybit6a 328

$12 / 01 / 93$

$12 / 01 / 93$

$12 / 01 / 93$

$12 / 01 / 93$

$12 / 01 / 93$

$12 / 01 / 93$

$12 / 01 / 93$

12/01/93

$12 / 01 / 93$

$12 / 01 / 93$

12/01/93

12/01/93

$12 / 01 / 93$

$12 / 01 / 93$

$12 / 01 / 93$

12/01/93

A-7. 24 
WHC-EP-0727

This page intentionally loft blenk.

$A-7.26$ 
WHC-EP-0727

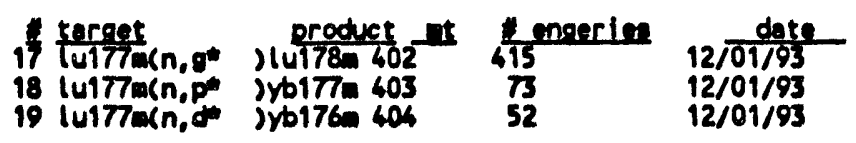

$A-7.25$ 
WHC-EP-0727

Appendix A-8

List of Reection on files".hf.po

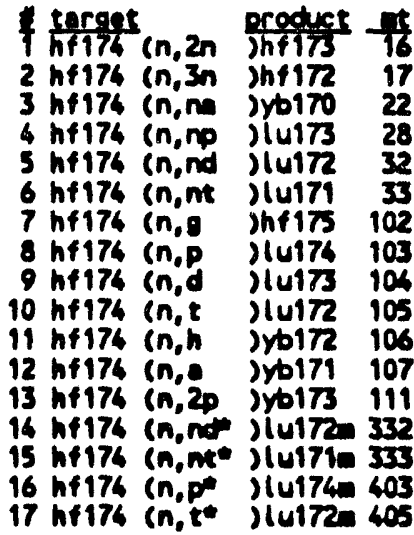

\begin{tabular}{|c|c|}
\hline $\begin{array}{c}1 \text { engeriat } \\
30 \\
19 \\
76 \\
46 \\
30 \\
26 \\
4456 \\
93 \\
62 \\
55 \\
31 \\
76 \\
28 \\
30 \\
26 \\
93 \\
55\end{array}$ & $\begin{array}{l}\frac{\text { dete }}{12 / 01 / 93} \\
12 / 01 / 93 \\
12 / 01 / 93 \\
12 / 01 / 93 \\
12 / 01 / 93 \\
12 / 01 / 93 \\
12 / 01 / 93 \\
12 / 01 / 93 \\
12 / 01 / 93 \\
12 / 01 / 93 \\
12 / 01 / 93 \\
12201 / 93 \\
12 / 01 / 93 \\
12 / 01 / 93 \\
12201 / 93 \\
12 / 01 / 93 \\
12 / 01 / 93\end{array}$ \\
\hline $\begin{array}{l}32 \\
17 \\
82 \\
46 \\
36 \\
26 \\
392 \\
92 \\
62 \\
60 \\
29 \\
87 \\
31 \\
46 \\
26 \\
62\end{array}$ & $\begin{array}{l}12 / 01 / 93 \\
12 / 01 / 93 \\
12 / 01 / 93 \\
12 / 01 / 93 \\
12 / 01 / 93 \\
12201 / 93 \\
12 / 01 / 93 \\
12 / 01 / 93 \\
12 / 01 / 93 \\
12 / 01 / 93 \\
12101 / 93 \\
12 / 01 / 93 \\
12 / 101 / 93 \\
1201 / 193 \\
12 / 01 / 93 \\
12 / 01 / 93\end{array}$ \\
\hline
\end{tabular}

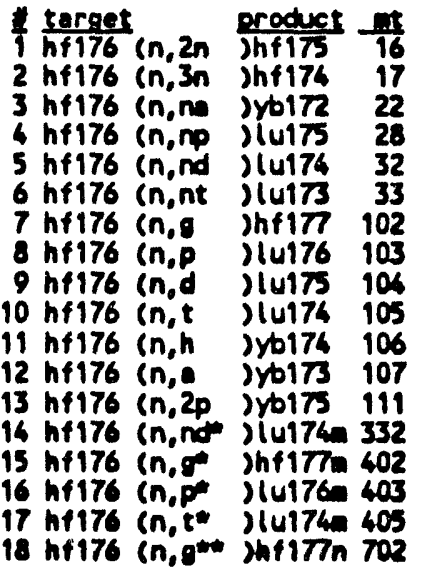

$\frac{1 \text { maeria: }}{33}$

$\frac{\text { dete }}{12 / 01 / 93}$

$12 / 01 / 93$

12/01/93

12/01/93

12/01/93

12/01/93

$12 / 01 / 93$

$12 / 01 / 93$

$12 / 01 / 93$

12/01/93

$12 / 01 / 93$

$12 / 01 / 93$

$12 / 01 / 93$

$12 / 01 / 93$

$12 / 01 / 93$

$12 / 01 / 93$

$12 / 01 / 93$

$12 / 01 / 93$

\begin{tabular}{|c|c|c|c|c|c|}
\hline $\begin{array}{ll}1 & h+177 \\
2 & h+177 \\
3 & h+17 \\
4 & h+177 \\
5 & h+17 \\
6 & h+177 \\
7 & h+177 \\
8 & h+177 \\
9 & h+17 \\
10 & h+177 \\
11 & h+17 \\
12 & h+17 \\
13 & h+177 \\
14 & h+177\end{array}$ & 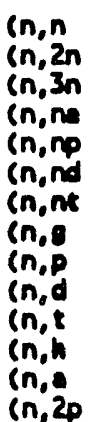 & 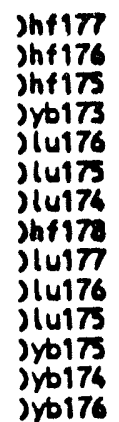 & $\begin{array}{r}16 \\
16 \\
17 \\
22 \\
28 \\
32 \\
33 \\
102 \\
103 \\
104 \\
105 \\
106 \\
107\end{array}$ & $\begin{array}{r}1 \\
40 \\
18 \\
79 \\
47 \\
37 \\
29 \\
1282 \\
93 \\
59 \\
59 \\
25\end{array}$ & $\begin{array}{l}12 / 01 / 93 \\
12101 / 93 \\
12 / 01 / 93 \\
12 / 101 / 93 \\
12 / 01 / 93 \\
12 / 01 / 93 \\
12101 / 93 \\
12 / 01 / 93 \\
12 / 01 / 93 \\
1201 / 193 \\
12 / 01 / 93 \\
12101 / 93 \\
12 / 01 / 93 \\
12 / 01 / 93\end{array}$ \\
\hline
\end{tabular}

A-8. 1 


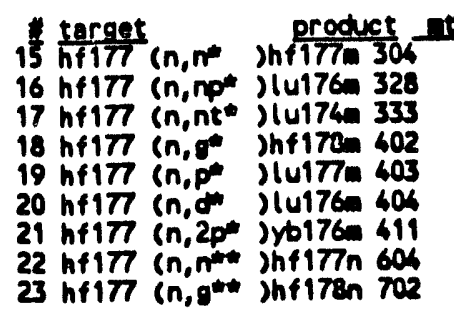

\begin{tabular}{cc} 
yenceries & $\frac{\text { date }}{42}$ \\
\hline 42 & $12 / 1193$ \\
47 & $12 / 0193$ \\
29 & $12 / 1193$ \\
1281 & $12 / 01 / 93$ \\
84 & $12 / 01 / 93$ \\
59 & $12 / 0193$ \\
55 & $12 / 01 / 93$ \\
61 & $12 / 01 / 93$ \\
1282 & $12 / 01 / 93$
\end{tabular}

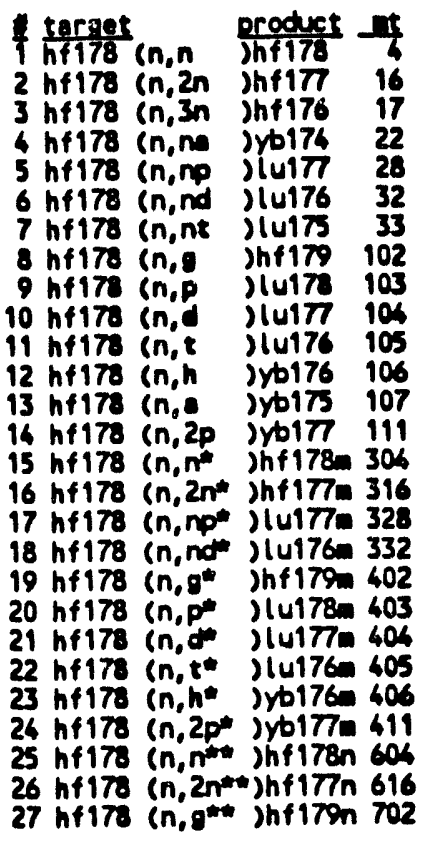

\begin{tabular}{cc} 
Lengerins & date \\
\cline { 2 - 2 } 1 & $12 / 01 / 93$ \\
37 & $12 / 01 / 93$ \\
20 & $12 / 01193$ \\
70 & $12 / 01 / 93$ \\
46 & $12 / 01 / 93$ \\
31 & $12 / 01 / 93$ \\
29 & $12 / 01 / 93$ \\
1282 & $12 / 01 / 93$ \\
79 & $12 / 01 / 93$ \\
58 & $12 / 01 / 93$ \\
55 & $12 / 01 / 93$ \\
21 & $12 / 01 / 93$ \\
70 & $12 / 01 / 93$ \\
19 & $12 / 01 / 93$ \\
32 & $12 / 01 / 93$ \\
37 & $12 / 01 / 93$ \\
61 & $12 / 01 / 93$ \\
31 & $12 / 01 / 93$ \\
1282 & $12 / 01 / 93$ \\
77 & $12 / 01 / 93$ \\
56 & $12 / 01 / 93$ \\
55 & $12 / 01 / 93$ \\
21 & $12 / 01 / 93$ \\
19 & $12 / 01 / 93$ \\
30 & $12 / 01 / 93$ \\
28 & $12 / 01 / 93$ \\
1282 & $12 / 01 / 93$
\end{tabular}

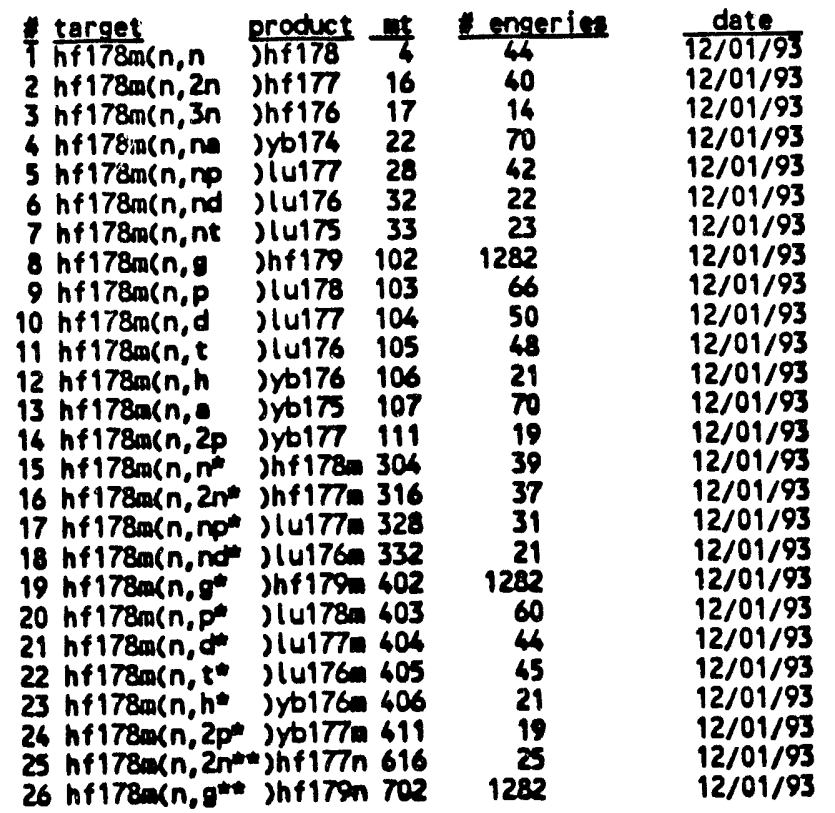




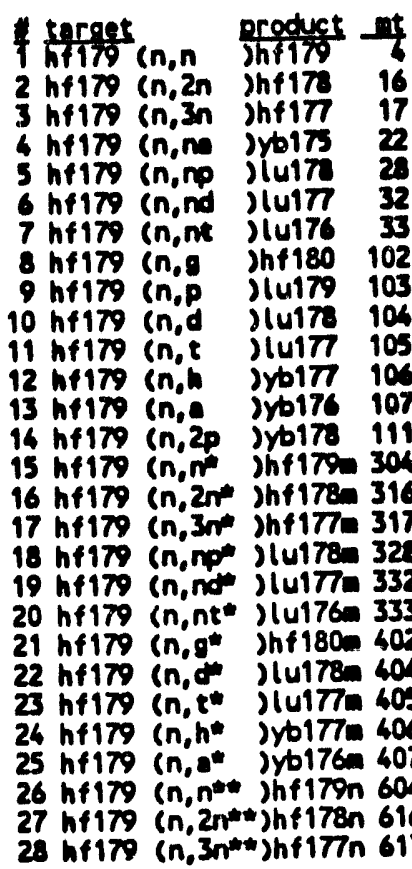

\begin{tabular}{cc} 
mengerine & date \\
\cline { 2 - 2 } & $12 / 01 / 93$ \\
38 & $12 / 01 / 93$ \\
21 & $12 / 01 / 93$ \\
73 & $12 / 01 / 93$ \\
46 & $12 / 01 / 93$ \\
35 & $12 / 01 / 93$ \\
29 & $12 / 01 / 93$ \\
1281 & $12 / 01 / 93$ \\
23 & $12 / 01 / 93$ \\
56 & $12 / 01 / 93$ \\
58 & $12 / 01 / 93$ \\
19 & $12 / 01 / 93$ \\
70 & $12 / 01 / 93$ \\
21 & $12 / 01 / 93$ \\
17 & $12 / 01 / 93$ \\
39 & $12 / 01 / 93$ \\
21 & $12 / 01 / 93$ \\
63 & $12 / 01 / 93$ \\
31 & $12 / 01 / 93$ \\
29 & $12 / 01 / 93$ \\
1282 & $12 / 01 / 93$ \\
56 & $12 / 01 / 93$ \\
56 & $12 / 01 / 93$ \\
19 & $12 / 01 / 93$ \\
71 & $12 / 01 / 93$ \\
79 & $12 / 01 / 93$ \\
37 & $12 / 01 / 93$ \\
12 & $12 / 01 / 93$
\end{tabular}

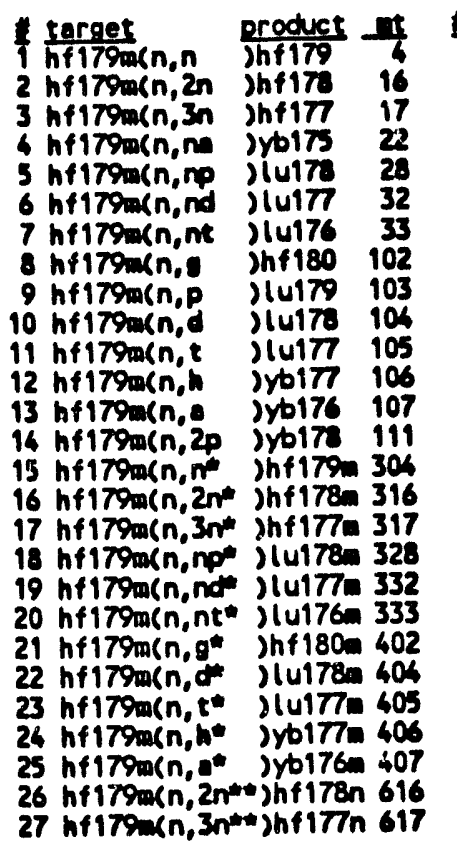

\begin{tabular}{cc} 
nengeries & date \\
\cline { 2 - 2 } 59 & $12 / 01 / 93$ \\
35 & $12 / 01 / 93$ \\
17 & $12 / 01 / 93$ \\
75 & $12 / 01 / 93$ \\
46 & $12 / 01 / 93$ \\
31 & $12 / 01 / 93$ \\
29 & $12 / 01 / 93$ \\
1281 & $12 / 01 / 93$ \\
23 & $12 / 01 / 93$ \\
54 & $12 / 01 / 93$ \\
56 & $12 / 01 / 93$ \\
19 & $12 / 01 / 93$ \\
70 & $12 / 01 / 93$ \\
21 & $12 / 01 / 93$ \\
17 & $12 / 01 / 93$ \\
39 & $12 / 01 / 93$ \\
21 & $12 / 01 / 93$ \\
39 & $12 / 01 / 93$ \\
27 & $12 / 01 / 93$ \\
29 & $12 / 01 / 93$ \\
1282 & $12 / 01 / 93$ \\
52 & $12 / 01 / 93$ \\
50 & $12 / 01 / 93$ \\
19 & $12 / 01 / 93$ \\
71 & $12 / 01 / 93$ \\
34 & $12 / 01 / 93$ \\
8 & $12 / 01 / 93$
\end{tabular}

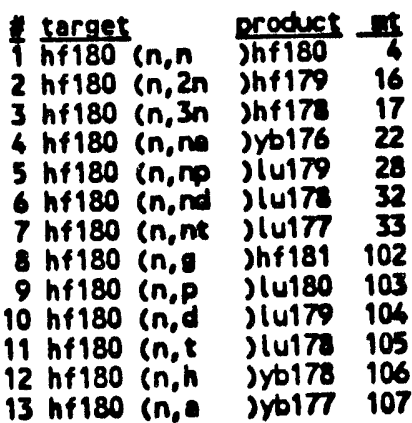

\begin{tabular}{cc} 
Lengeries & date \\
\cline { 2 - 2 } 1 & $12 / 01 / 93$ \\
18 & $12 / 01 / 93$ \\
21 & $12 / 01 / 93$ \\
65 & $12 / 01 / 93$ \\
43 & $12 / 01 / 93$ \\
30 & $12 / 01 / 93$ \\
29 & $12 / 01 / 93$ \\
1282 & $12 / 01 / 93$ \\
23 & $12 / 01 / 93$ \\
55 & $12 / 01 / 93$ \\
53 & $12 / 01 / 93$ \\
15 & $12 / 01 / 93$ \\
61 & $12 / 01 / 93$
\end{tabular}

A-8. 3 


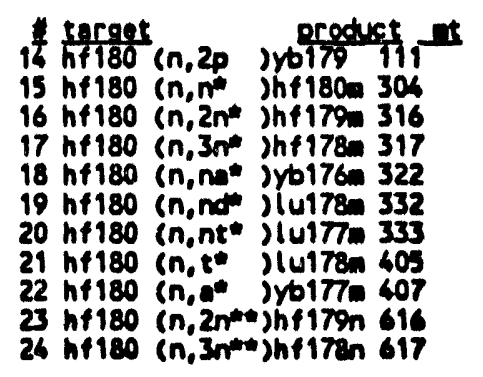

$\begin{array}{ll}\text { yemencin } & \text { date } \\ 11 & 12 / 01 / 93 \\ 51 & 12 / 01 / 93 \\ 18 & 12 / 01 / 93 \\ 21 & 12 / 01 / 93 \\ 65 & 12 / 01 / 93 \\ 30 & 12 / 01 / 93 \\ 29 & 12 / 01 / 93 \\ 53 & 12 / 01 / 93 \\ 61 & 12 / 01 / 93 \\ 16 & 12 / 01 / 93 \\ 10 & 12 / 01 / 93\end{array}$

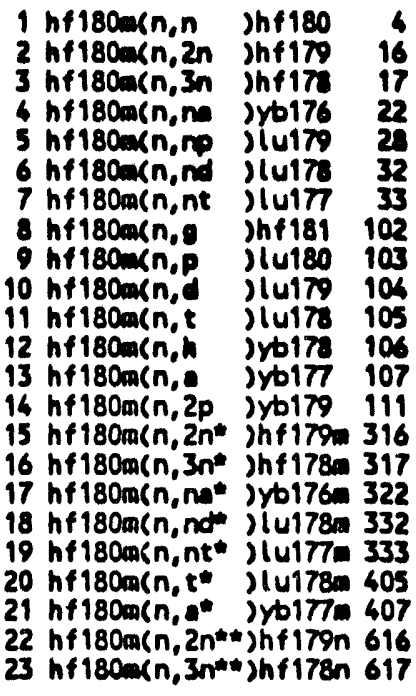

$\begin{array}{rr}18 & 12 / 01 / 93 \\ 16 & 12 / 01 / 93 \\ 17 & 12 / 01 / 93 \\ 65 & 12 / 01 / 93 \\ 38 & 12 / 01 / 93 \\ 25 & 12 / 01 / 93 \\ 26 & 12 / 01 / 93 \\ 1282 & 12 / 01 / 93 \\ 23 & 12 / 01 / 93 \\ 53 & 12 / 01 / 93 \\ 49 & 12 / 01 / 93 \\ 15 & 12 / 01 / 93 \\ 61 & 12 / 01 / 93 \\ 11 & 12 / 01 / 93 \\ 18 & 12 / 01 / 93 \\ 21 & 12 / 01 / 93 \\ 65 & 12 / 01 / 93 \\ 26 & 12 / 01 / 93 \\ 23 & 12 / 01 / 93 \\ 49 & 12 / 01 / 93 \\ 61 & 12 / 01 / 93 \\ 13 & 12 / 01 / 93 \\ 8 & 12 / 01 / 93\end{array}$

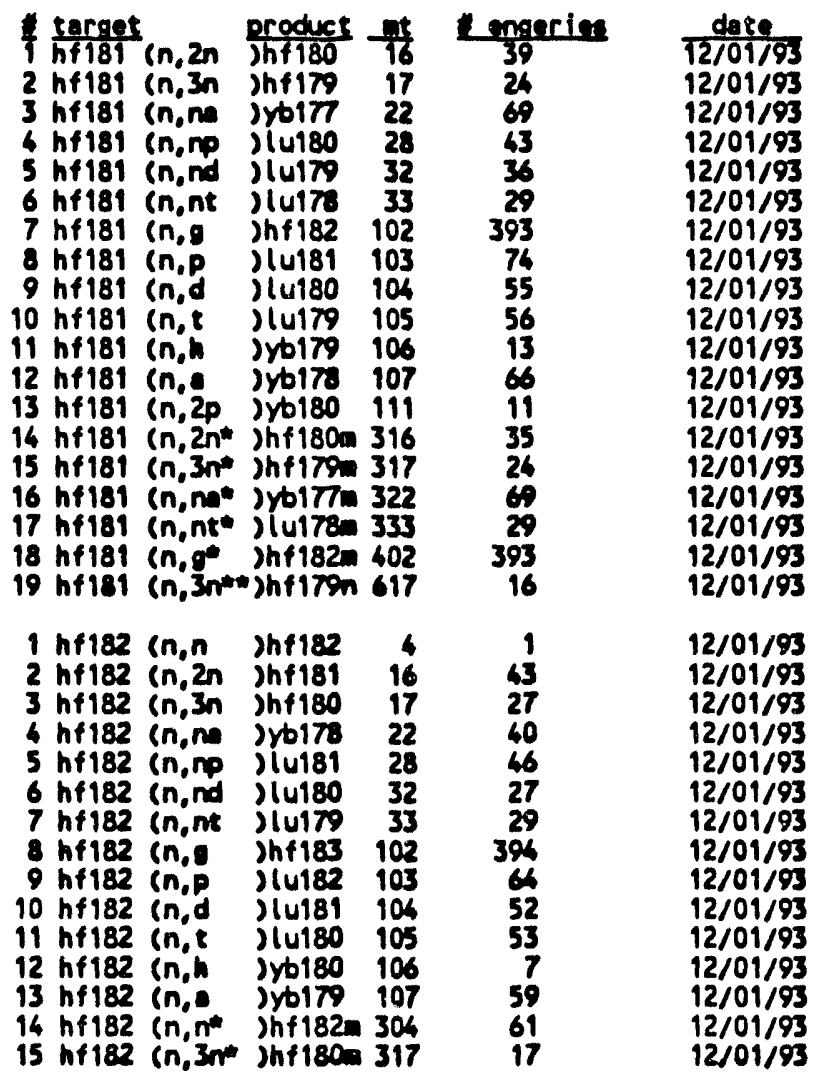




\begin{tabular}{|c|c|c|c|c|c|}
\hline 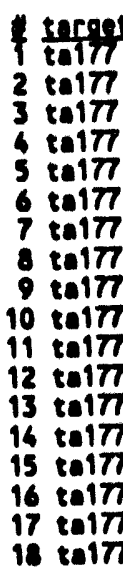 & $\left.\begin{array}{l}(n, 2 n \\
(n, 3 n \\
(n, n) \\
(n, n) \\
\text { (n, nd } \\
\text { (n,nt }\end{array}\right\}$ & 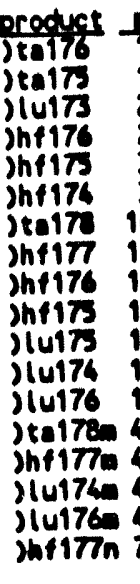 & $\begin{array}{l}18 \\
16 \\
17 \\
22 \\
28 \\
32 \\
33 \\
102 \\
103 \\
104 \\
105 \\
108 \\
107 \\
111 \\
602 \\
603 \\
607 \\
611 \\
703\end{array}$ & $\begin{array}{l}\text { mperim } \\
33 \\
17 \\
80 \\
33 \\
37 \\
35 \\
404 \\
93 \\
69 \\
62 \\
29 \\
76 \\
29 \\
404 \\
93 \\
76 \\
29 \\
4\end{array}$ & $\begin{array}{l}\text { digte } \\
12 / 01 / 93 \\
12 / 01 / 93 \\
12 / 01 / 93 \\
12 / 01 / 93 \\
12 / 01 / 93 \\
12 / 01 / 93 \\
12 / 01 / 93 \\
12 / 01 / 93 \\
12 / 01 / 93 \\
12 / 01 / 93 \\
12 / 01 / 93 \\
12 / 01 / 93 \\
12 / 01 / 93 \\
12 / 01 / 93 \\
12 / 01 / 93 \\
12 / 01 / 93 \\
12 / 01 / 93 \\
12 / 01 / 93\end{array}$ \\
\hline $\begin{array}{lll}1 & t a 17 \\
2 & t a 17 \\
3 & t a 17 \\
6 & t a 17 \\
5 & t a 17 \\
6 & t a 17 \\
7 & t a 17 \\
8 & t a 17 \\
9 & t a 17 \\
10 & t a 17 \\
11 & t a 17 \\
12 & t a 17 \\
13 & t a 17 \\
16 & t a 17 \\
15 & t a 17 \\
16 & t a 17 \\
17 & t a 17 \\
18 & t a 17 \\
19 & t a 17 \\
20 & t a 17 \\
21 & t a 17 \\
22 & t a 17 \\
23 & t a 1 \\
26 & t a 1 \\
25 & t a 1 \\
26 & t a 1 \\
27 & t a 1 \\
28 & t a 1\end{array}$ & 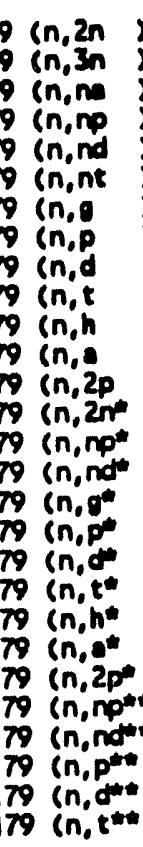 & 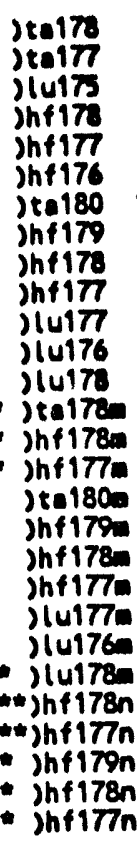 & $\begin{array}{l}16 \\
17 \\
22 \\
28 \\
32 \\
33 \\
102 \\
103 \\
104 \\
105 \\
106 \\
107 \\
111 \\
316 \\
328 \\
332 \\
402 \\
403 \\
404 \\
405 \\
406 \\
407 \\
611 \\
628 \\
632 \\
703 \\
704 \\
705\end{array}$ & $\begin{array}{l}39 \\
22 \\
73 \\
50 \\
35 \\
35 \\
406 \\
92 \\
66 \\
39 \\
25 \\
72 \\
25 \\
39 \\
50 \\
35 \\
406 \\
92 \\
66 \\
59 \\
25 \\
72 \\
25 \\
39 \\
25 \\
92 \\
53 \\
49\end{array}$ & $\begin{array}{l}12 / 01 / 93 \\
12 / 01 / 93 \\
12 / 01 / 93 \\
12 / 01 / 93 \\
12 / 01 / 93 \\
12 / 01 / 93 \\
12 / 01 / 93 \\
12 / 01 / 93 \\
12 / 01 / 93 \\
12 / 01 / 93 \\
12 / 01 / 93 \\
12 / 01 / 93 \\
12 / 01 / 93 \\
12 / 01 / 93 \\
12 / 01 / 93 \\
12 / 01 / 93 \\
12 / 01 / 93 \\
12 / 01 / 93 \\
12 / 01 / 93 \\
12 / 01 / 93 \\
12 / 01 / 93 \\
12 / 01 / 93 \\
12 / 01 / 93 \\
12 / 01 / 93 \\
12 / 01 / 93 \\
12 / 01 / 93 \\
12 / 01 / 93 \\
12 / 01 / 93\end{array}$ \\
\hline 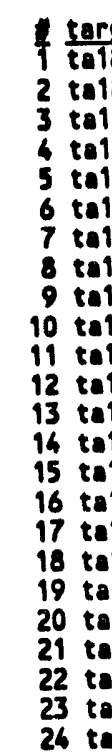 & 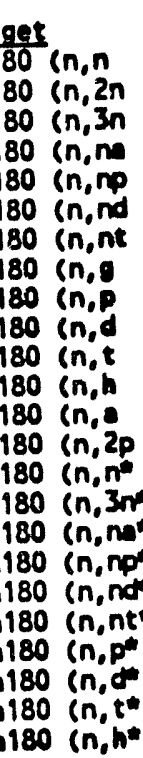 & 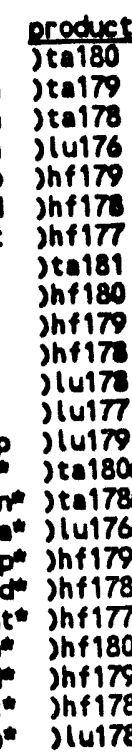 & 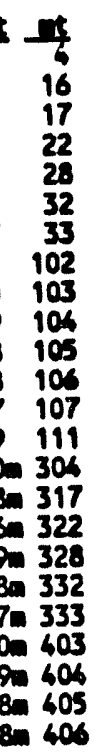 & $\begin{array}{c}\text { mengerie } \\
1 \\
33 \\
18 \\
76 \\
48 \\
40 \\
33 \\
396 \\
91 \\
64 \\
46 \\
21 \\
71 \\
26 \\
43 \\
18 \\
76 \\
48 \\
40 \\
33 \\
91 \\
64 \\
64 \\
21\end{array}$ & $\begin{array}{c}\text { date } \\
12 / 01 / 93 \\
12 / 01 / 93 \\
12 / 01 / 93 \\
12 / 01 / 93 \\
12 / 01 / 93 \\
12 / 01 / 93 \\
12 / 01 / 93 \\
12 / 01 / 93 \\
12 / 01 / 93 \\
12 / 01 / 93 \\
12 / 01 / 93 \\
12 / 01 / 93 \\
12 / 01 / 93 \\
12 / 01 / 93 \\
12 / 01 / 93 \\
12 / 01 / 93 \\
12 / 01 / 93 \\
12 / 01 / 93 \\
12 / 01 / 93 \\
12 / 01 / 93 \\
12 / 01 / 93 \\
12 / 01 / 93 \\
12 / 01 / 93 \\
12 / 01 / 93\end{array}$ \\
\hline
\end{tabular}

A-8. 5 


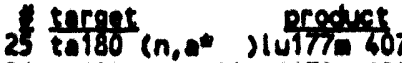
26 te180 (n,nomintitin 62 27 ta180 (n,nde)hf17an 632 28 tal80 (n,ntw)ht177n 633 20 talso $(n, d$ ore infiron 704 30 ta180 (n, twit inf17en 705

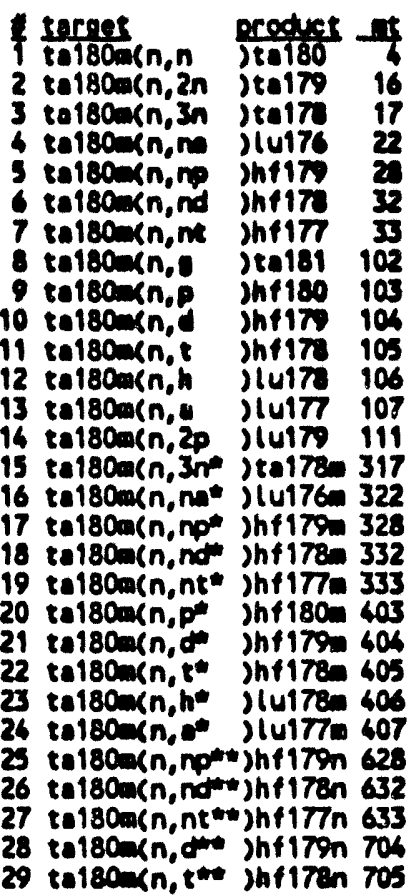

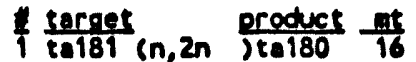

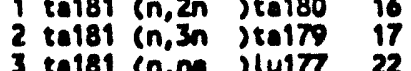
3 tolsi $n, n$ lluitr 22

4 ta181 (n,no inf180 28

5 tal81 (n,nd infi79 32

6 ta18i in,nt inf17s 33

7 ta181 (n.) ta182 102

8 tolsi (n,p inf181 103

9 to181 (n,d inf180 104

10 ta181 (n,t shfi79 105

11 talsi (n,h Jlui79 106

12 talsi $(n, a)$ jluirs 107

13 tal81 (n,2p) )lu180 111

16 ta18i (n,2n) te180 316

is talsi (n,m* jlu177m 322

16 tal81 (n,np jht 380 m 328

17 tal81 ( $n, n d$ ) hf179 332

18 talsi (n,nt* )hf178m 333

19 ta181 $\left(n, g^{*}\right)$ tals2m 402 20 ta181 (n, Jhf180 uon

21 ta181 (n,t* )hf179 405

22 ta18i $\left(n, e^{*}\right.$ Jlu178 407 23 ta181 (n, ndwshfiron 632 26 to181 (n,ntw)hf178n 633 25 to181 (n, g* )ta182n 702 26 talsi $(n, t)$ infirion 705

$\begin{array}{ll}\text { ymancin } & 12 / 01 / 93 \\ 47 & 12 / 01 / 93 \\ 63 & 12 / 01 / 93 \\ 29 & 12 / 01 / 93 \\ 27 & 12 / 01 / 93 \\ 54 & 12 / 01 / 93 \\ 34 & \end{array}$

\begin{tabular}{cc} 
monerien & date \\
\hline 43 & $12 / 01 / 93$ \\
33 & $12 / 01 / 93$ \\
18 & $12 / 01 / 93$ \\
76 & $12 / 01 / 93$ \\
46 & $12 / 01 / 93$ \\
40 & $12 / 01 / 93$ \\
33 & $12 / 01 / 93$ \\
39 & $12 / 01 / 93$ \\
91 & $12 / 01 / 93$ \\
44 & $12 / 01 / 93$ \\
64 & $12 / 01 / 93$ \\
21 & $12 / 01 / 93$ \\
71 & $12 / 01 / 93$ \\
26 & $12 / 01 / 93$ \\
18 & $12 / 01 / 93$ \\
76 & $12 / 01 / 93$ \\
40 & $12 / 01 / 93$ \\
40 & $12 / 01 / 93$ \\
33 & $12 / 01 / 93$ \\
91 & $12 / 01 / 93$ \\
64 & $12 / 01 / 93$ \\
64 & $12 / 01 / 93$ \\
21 & $12 / 01 / 93$ \\
71 & $12 / 01 / 93$ \\
43 & $12 / 01 / 93$ \\
29 & $12 / 01 / 93$ \\
27 & $12 / 01 / 93$ \\
58 & $12 / 01 / 93$ \\
54 & $12 / 01 / 93$
\end{tabular}

\begin{tabular}{cc} 
Lengerien & dote \\
\cline { 2 - 2 } 36 & $92 / 101 / 93$ \\
7 & $12 / 01 / 93$ \\
15 & $12 / 01 / 93$ \\
50 & $12 / 01 / 93$ \\
34 & $12 / 01 / 93$ \\
31 & $12 / 01 / 93$ \\
1282 & $12 / 01 / 93$ \\
6 & $12 / 01 / 93$ \\
59 & $12 / 01 / 93$ \\
56 & $12 / 01 / 93$ \\
17 & $12 / 01 / 93$ \\
61 & $12 / 01 / 93$ \\
17 & $12 / 01 / 93$ \\
37 & $12 / 01 / 93$ \\
15 & $12 / 01 / 93$ \\
44 & $12 / 01 / 93$ \\
34 & $12 / 01 / 93$ \\
31 & $12 / 01 / 93$ \\
1282 & $12 / 01 / 93$ \\
59 & $12 / 01 / 93$ \\
56 & $12 / 01 / 93$ \\
61 & $12 / 01 / 93$ \\
30 & $12 / 01 / 93$ \\
27 & $12 / 01 / 93$ \\
1282 & $12 / 01 / 93$ \\
53 & $12 / 01 / 93$
\end{tabular}


WHC-EP-0727

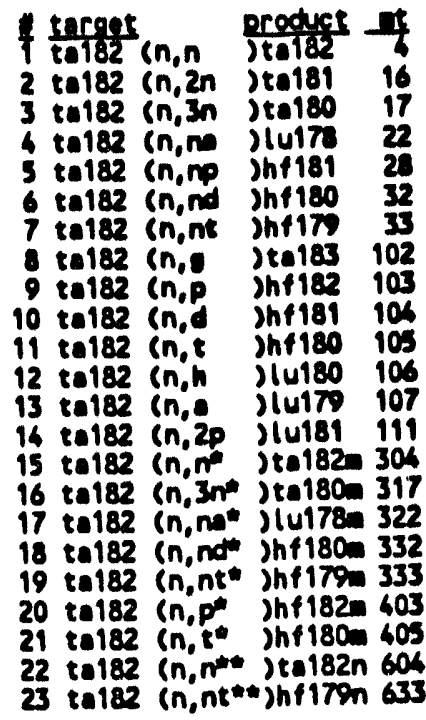

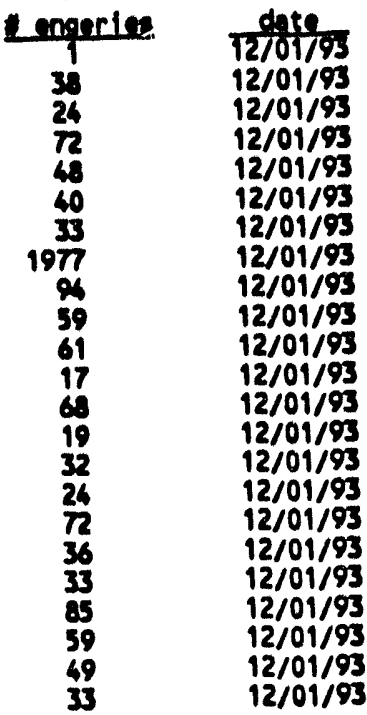

1 tale3 (n,2n )ta182 16 2 ta183 $(n, 3 n$ )ta181 17 3 tels3 (n,na juits 22 4 tal83 (n,np infle2 28 5 ta183 (n,nd )hf181 32 6 to183 $(n, n t$ inf180 33 7 tais3 (n, ) tolsh 102 8 tais3 $\mathrm{kn}, \mathrm{p}$ hit183 103 9 tais3 $\mathrm{in,d} \mathrm{jhf182} 10$ 10 te183 (n,t Jhfisi 105 $11 \mathrm{ta183} \mathrm{cm}, \mathrm{h}$ jlu181 106

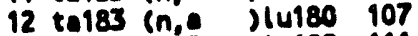
13 ta183 (n,2p Jlu182 111 16 ta183 (n,2n*)ta182n 316 is tal83 (n,np jhf182m 328 16 tal83 (n,nt* inf180 333 17 ta183 $\left(n, \alpha^{*}\right.$ inf182m 404 18 tal83 (n, 2ntm)ta182n 616

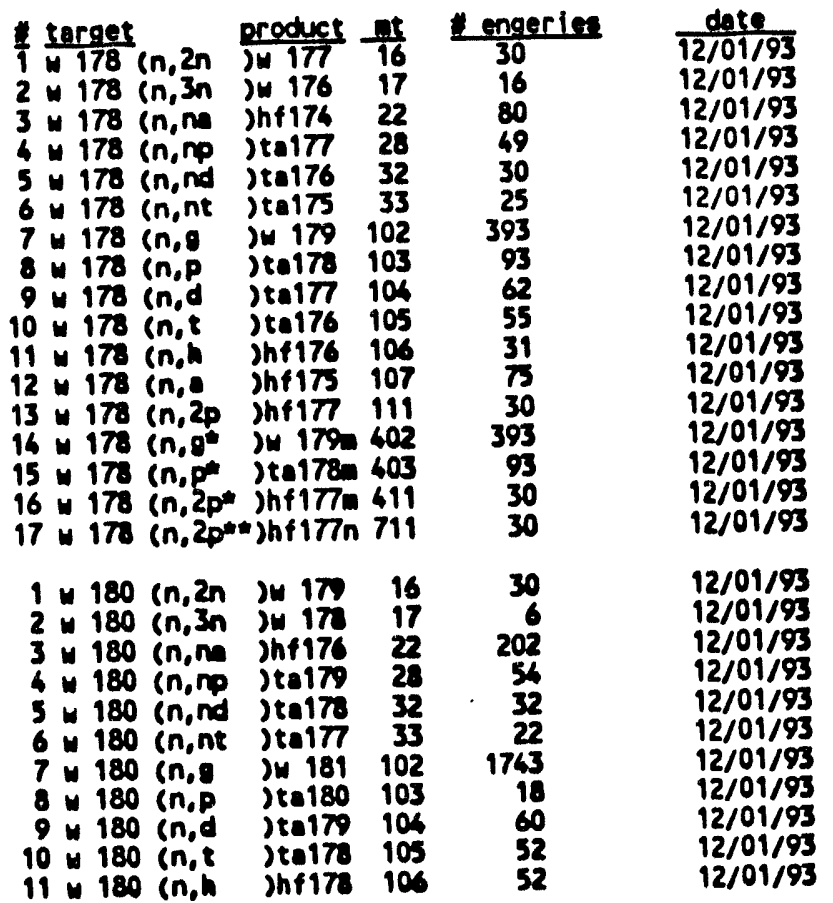

$A-8.7$ 


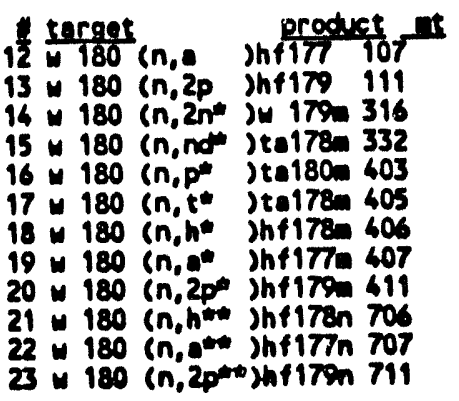

$\begin{array}{cc}\text { yonerien } & \text { date } \\ 201 & 12 / 01 / 93 \\ 46 & 12 / 01 / 93 \\ 30 & 12 / 01 / 93 \\ 32 & 12 / 01 / 93 \\ 18 & 12 / 01 / 93 \\ 52 & 12 / 01 / 93 \\ 122 & 12 / 01 / 93 \\ 17 & 12 / 01 / 93 \\ 120 & 12 / 01 / 93 \\ 67 & 12 / 01 / 93 \\ 17 & 12 / 01 / 93 \\ 45 & 12 / 01 / 93\end{array}$

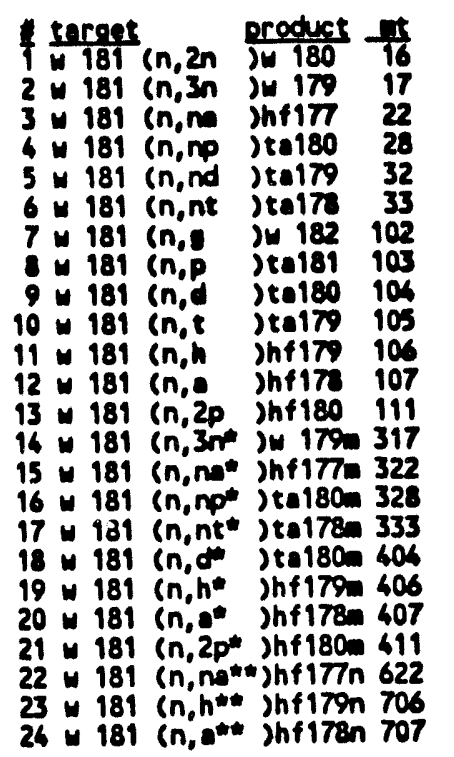

\begin{tabular}{l} 
mogrin \\
\hline 32 \\
18 \\
80 \\
45 \\
35 \\
25 \\
391 \\
92 \\
59 \\
58 \\
23 \\
76 \\
26 \\
18 \\
80 \\
45 \\
25 \\
59 \\
23 \\
73 \\
26 \\
80 \\
23 \\
74
\end{tabular}

date
12/01/93
$12 / 01 / 93$
$12 / 01 / 93$
$12 / 01 / 93$
$12 / 01 / 93$
$12 / 01 / 93$
$12 / 01 / 93$
$12 / 01 / 93$
$12 / 01 / 93$
$12 / 01 / 93$
$12 / 01 / 93$
$12 / 01 / 93$
$12 / 01 / 93$
$12 / 01 / 93$
$12 / 01 / 93$
$12 / 01 / 93$
$12 / 01 / 93$
$12 / 01 / 93$
$12 / 01 / 93$
$12 / 01 / 93$
$12 / 01 / 93$
$12 / 01 / 93$
$12 / 01 / 93$
$12 / 01 / 93$

1 w $182(n, 2 n)$ ) $181 \quad 16$

2 * $182(n, 3 n)$ in 18017

3 - 182 in,n jhf170 22

4 182 (n,mo )ta181 28

5 y 182 in,nd jtaiso 32

6 * 182 (n,nt jtal79 33

7 * 182 (n, is 183102

8 * 182 (n,p jta182 103

9 * 182 (n,d jtalsi 104

$10 \div 182$ (n,t jta180 105

$11182 \mathrm{kn}, \mathrm{h}$ inf180 106

12 - 182 (n,s inf179 107

$13=182$ (n,2p inf181 111

16 * $182(n, n$ * )hf178m 322

15 w 182 ( $n, n$, )te180 332

16 w $182\left(n, 0^{*}\right.$ is $183 m 402$

17 * 182 (n, it )to182m 403

18 * 182 ( $n, t$ ) ta180 405

19 * 182 (n, h* inf180 606

20182 $n$, inf17\% 407

21 y 182 (n,nutusfiren 622

22 * $182\left(n, p^{\text {tw }}\right)$ tal82n 703

23 w 182 (n,a* ) hifirin 707

$\begin{array}{rr}41 & 12 / 01 / 93 \\ 6 & 12 / 01 / 93 \\ 199 & 12 / 01 / 93 \\ 49 & 12 / 01 / 93 \\ 32 & 12 / 01 / 93 \\ 22 & 12 / 01 / 93 \\ 1282 & 12 / 01 / 93 \\ 42 & 12 / 01 / 93 \\ 66 & 12 / 01 / 93 \\ 52 & 12 / 01 / 93 \\ 45 & 12 / 01 / 93 \\ 29 & 12 / 01 / 93 \\ 16 & 12 / 01 / 93 \\ 166 & 12 / 01 / 93 \\ 32 & 12 / 01 / 93 \\ 1282 & 12 / 01 / 93 \\ 42 & 12 / 01 / 93 \\ 52 & 12 / 01 / 93 \\ 41 & 12 / 01 / 93 \\ 12 & 12 / 01 / 93 \\ 97 & 12 / 01 / 93 \\ 42 & 12 / 01 / 93 \\ 12 & 12 / 01 / 93\end{array}$

\begin{tabular}{|c|c|c|c|c|}
\hline 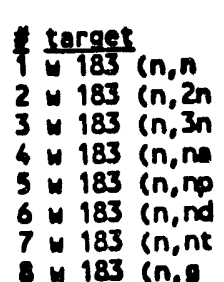 & $\begin{array}{l}\text { product } \\
\text { ow } 183 \\
\text { in } 182 \\
\text { jw } 181 \\
\text { jhf } 179 \\
\text { jta182 } \\
\text { jto181 } \\
\text { jte180 } \\
\text { ju } 184\end{array}$ & $\begin{array}{l}16 \\
17 \\
22 \\
28 \\
32 \\
33 \\
102\end{array}$ & $\begin{array}{c}\text { Lemeries } \\
17 \\
17 \\
23 \\
205 \\
36 \\
38 \\
24 \\
1282\end{array}$ & $\begin{array}{l}\text { dete } \\
12 / 01 / 93 \\
12 / 01 / 93 \\
12 / 01 / 93 \\
12 / 01 / 93 \\
12 / 01 / 93 \\
12 / 01 / 93 \\
12 / 01 / 93 \\
12 / 01 / 93\end{array}$ \\
\hline
\end{tabular}

$A-8.8$ 


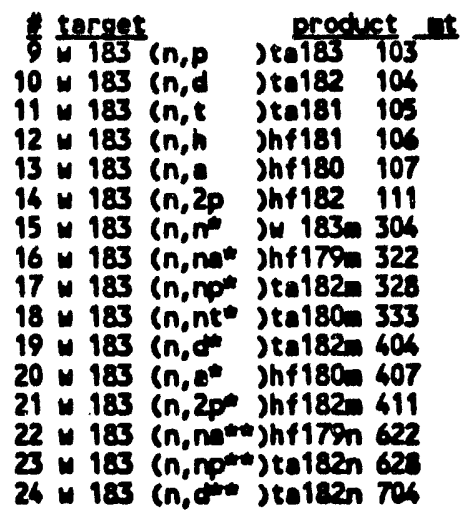

\begin{tabular}{ll} 
yenerien & date \\
\hline 38 & $12 / 01 / 93$ \\
56 & $12 / 01 / 93$ \\
56 & $12 / 01 / 93$ \\
16 & $12 / 01 / 93$ \\
26 & $12 / 01 / 93$ \\
18 & $12 / 01 / 93$ \\
69 & $12 / 01 / 93$ \\
13 & $12 / 01 / 93$ \\
36 & $12 / 01 / 93$ \\
26 & $12 / 01 / 93$ \\
56 & $12 / 01 / 93$ \\
26 & $12 / 01 / 93$ \\
18 & $12 / 01 / 93$ \\
13 & $12 / 01 / 93$ \\
36 & $12 / 01 / 93$ \\
56 & $12 / 01 / 93$
\end{tabular}

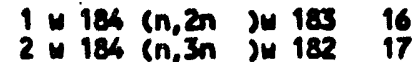

3 is6 (n,m) jhf180 22

4 w 184 (n,np )ta183 28

5 y 184 (n, nd ) ta182 32

6 w 184 (n,nt jta181 33

7 w 184 (n,s in 185102

8 w 184 (n.p jta184 103

9 - 184 (n,d jte183 104

10 w 184 (n,t jtel82 105

11 - $184 \mathrm{Cn,h}$ inf182 106

12 * 184 (n, $)$ jhf18) 107

13 w 184 (n,2p Jhf183 111

14 y 184 (n,2n* in $183 n 316$

15 * 184 (n,na* )hf180- 322

16 * 184 ( $n$, nd ) ta182n 332

17 w $184\left(n, \theta^{\circ}\right.$ iw $185 m 402$

18 * 184 (n,t* )ta182 405

19 - $184 \mathrm{Cn}, \mathrm{h}$ inf182 406

20 * $184(n, n d-i)$ tal $182 n 632$

21 - 184 ( $n, t$ w ) tals2n 705

$17 \quad 12 / 01 / 93$

$12 / 01 / 93$

$12 / 01 / 93$

$12 / 01 / 93$

$12 / 01 / 93$

$12 / 01 / 93$

$12 / 01 / 93$

$12 / 01 / 93$

$12 / 01 / 93$

$12 / 01 / 93$

$12 / 01 / 93$

$12 / 01 / 93$

$12 / 01 / 93$

12/01/93

12/01/93

$12 / 01 / 93$

$12 / 01 / 93$

12/01/93

$12 / 01 / 93$

$12 / 01 / 93$

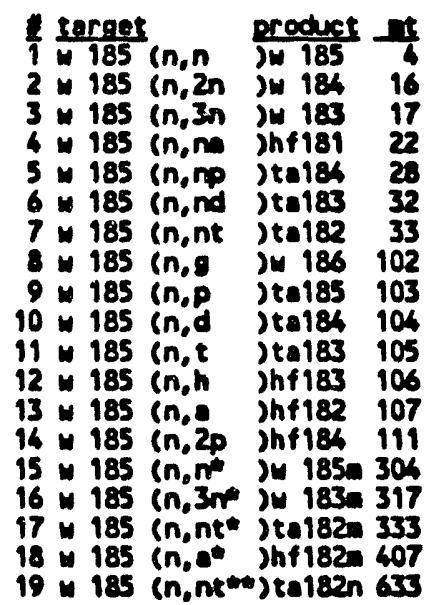

1
62
26
70
45
36
27
600
79
55
56
15
67
15
66
21
27
67
27

$12 / 01 / 93$

\begin{tabular}{|c|c|c|c|c|c|}
\hline 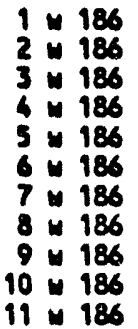 & $\begin{array}{l}(n, 2 n \\
(n, 3 n \\
(n, n) \\
(n, n) \\
(n, n d \\
(n, n t \\
(n, g) \\
(n, p) \\
(n, d \\
(n, t) \\
(n, n\end{array}$ & 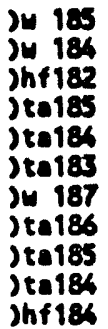 & $\begin{array}{r}16 \\
17 \\
22 \\
28 \\
32 \\
33 \\
102 \\
103 \\
104 \\
105 \\
106\end{array}$ & $\begin{array}{r}38 \\
8 \\
62 \\
63 \\
30 \\
26 \\
4232 \\
77 \\
52 \\
50 \\
8\end{array}$ & $\begin{array}{l}12 / 01 / 93 \\
12 / 01 / 93 \\
12 / 01 / 93 \\
12 / 01 / 93 \\
12 / 01 / 93 \\
12 / 01 / 93 \\
12 / 01 / 93 \\
12 / 01 / 93 \\
12 / 01 / 93 \\
12 / 01 / 93 \\
12 / 01 / 93\end{array}$ \\
\hline
\end{tabular}

A-8.9 


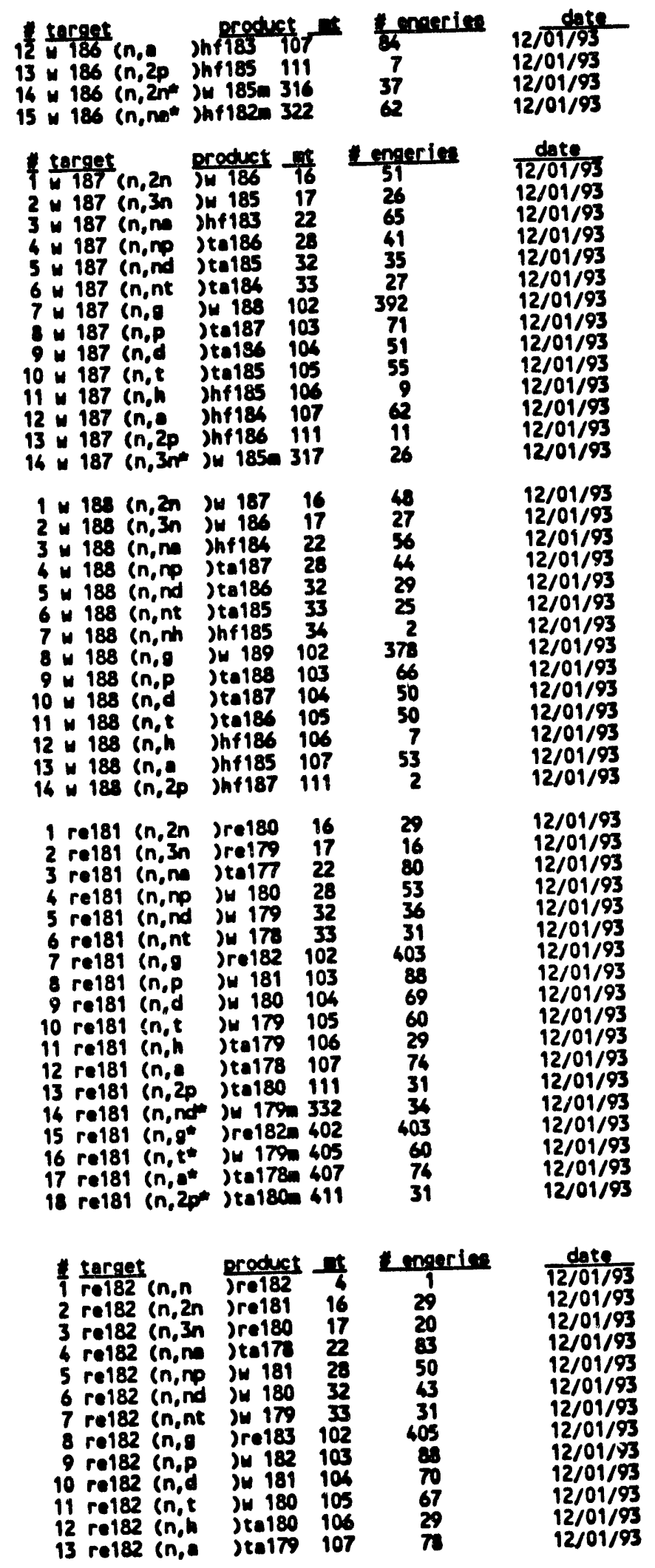

$A-8.10$ 


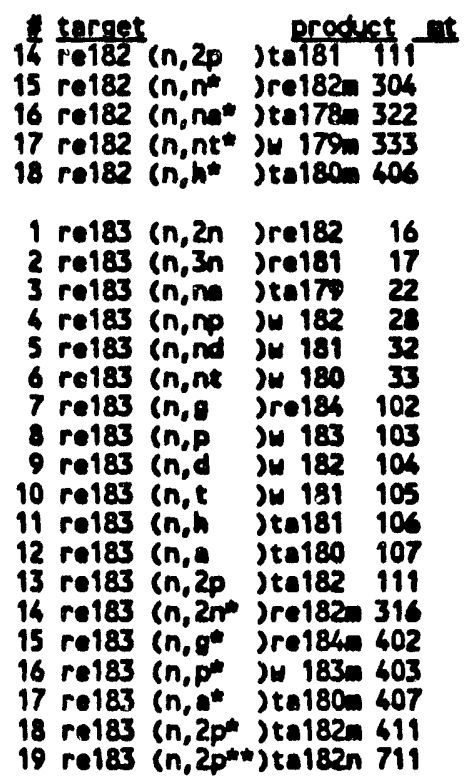

\begin{tabular}{cc} 
moneries & date \\
\hline 32 & $12 / 01 / 93$ \\
45 & $12 / 01 / 93$ \\
83 & $12 / 01 / 93$ \\
31 & $12 / 01 / 93$ \\
29 & $12 / 01 / 93$ \\
36 & $12 / 01 / 93$ \\
16 & $12 / 01 / 93$ \\
73 & $12 / 01 / 93$ \\
53 & $12 / 01 / 93$ \\
35 & $12 / 01 / 93$ \\
31 & $12 / 01 / 93$ \\
406 & $12 / 01 / 93$ \\
92 & $12 / 01 / 93$ \\
67 & $12 / 01 / 93$ \\
58 & $12 / 01 / 93$ \\
25 & $12 / 01 / 93$ \\
60 & $12 / 01 / 93$ \\
25 & $12 / 01 / 93$ \\
32 & $12 / 01 / 93$ \\
406 & $12 / 01 / 93$ \\
92 & $12 / 01 / 93$ \\
68 & $12 / 01 / 93$ \\
25 & $12 / 01 / 93$ \\
25 & $12 / 01 / 93$
\end{tabular}

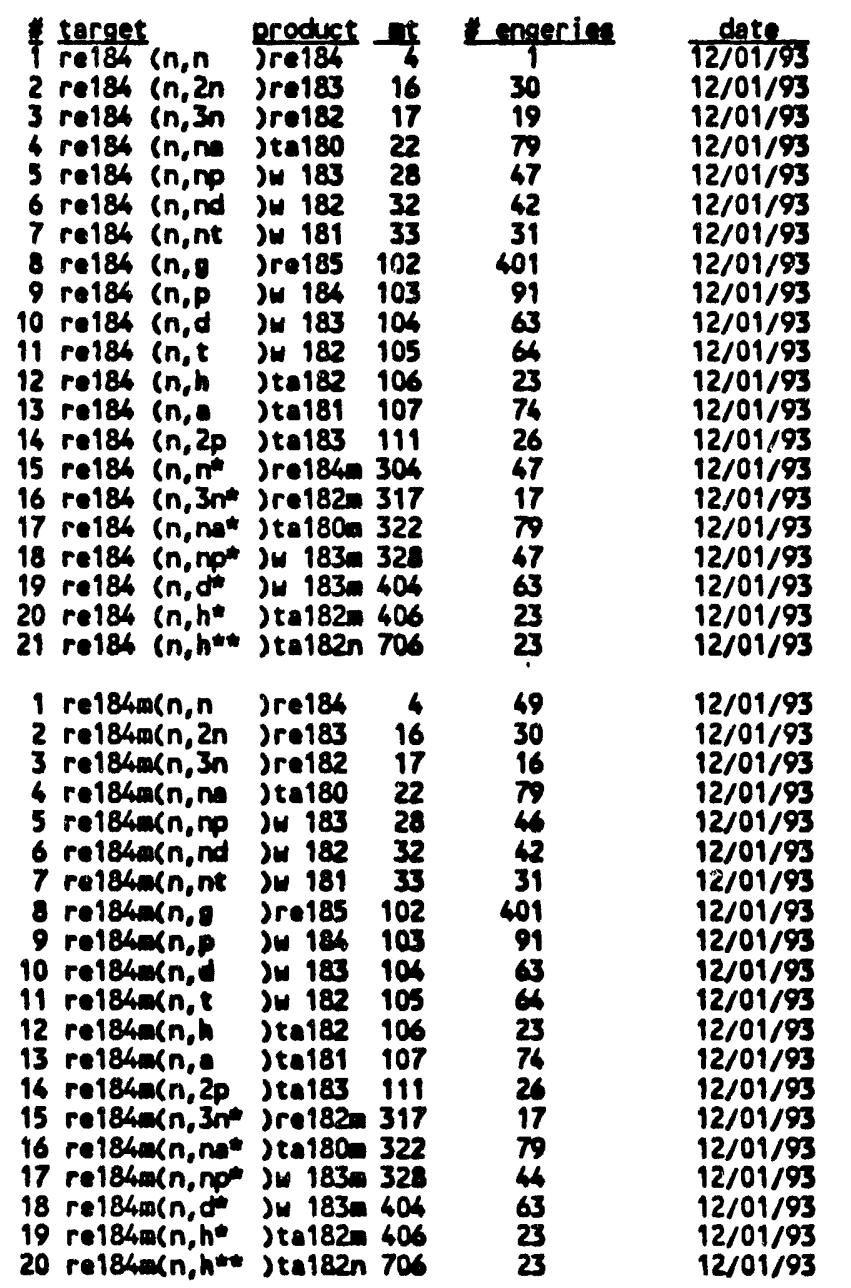




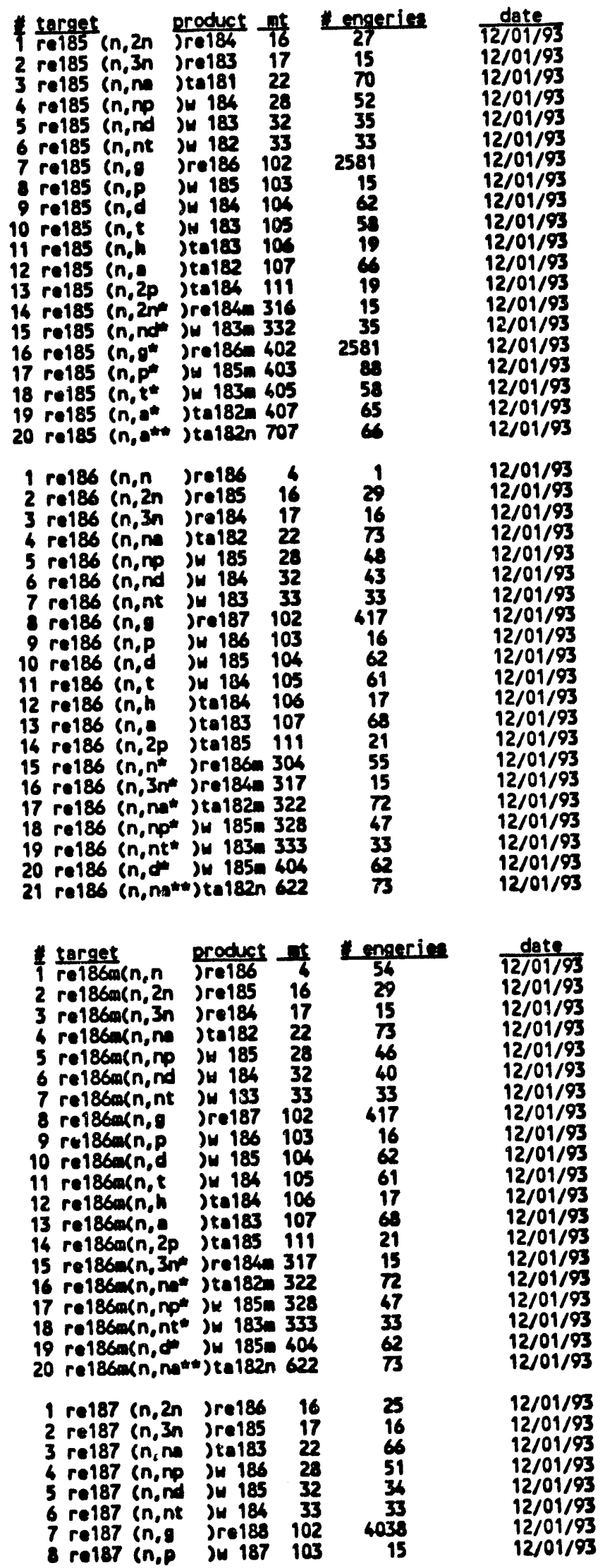

A-8.12 


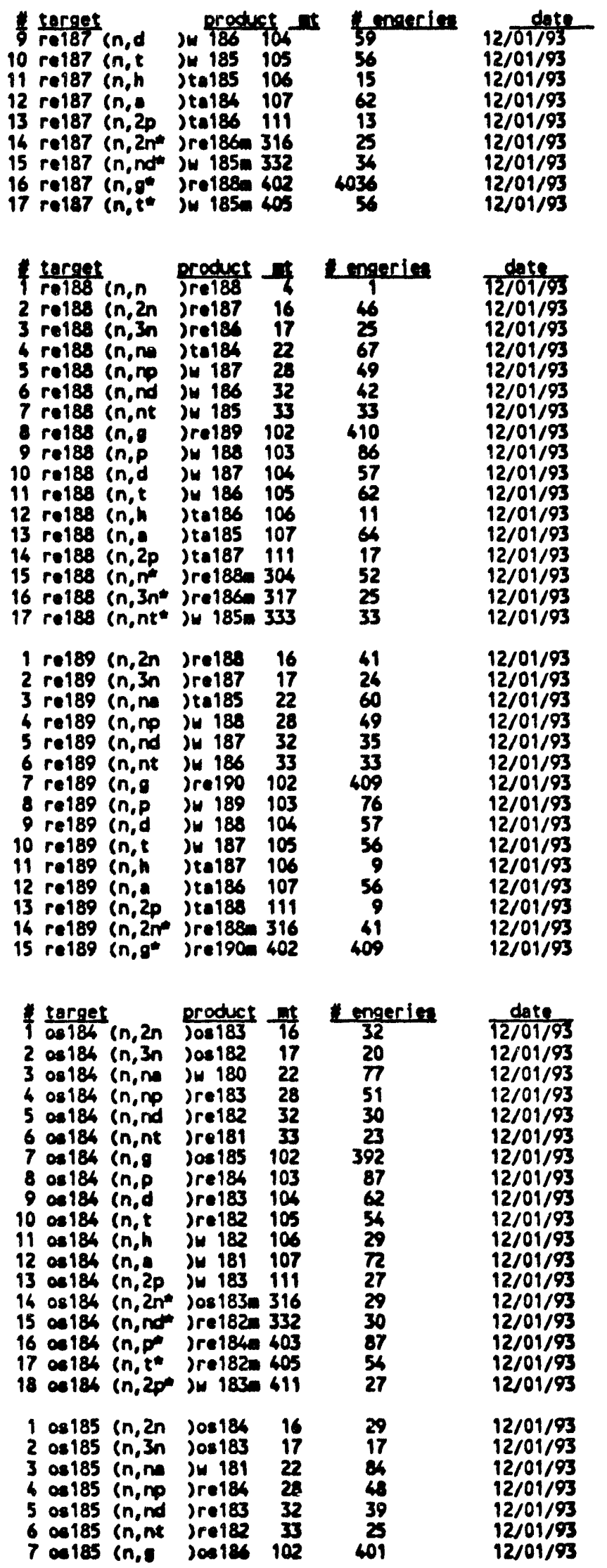

$A-8.13$ 


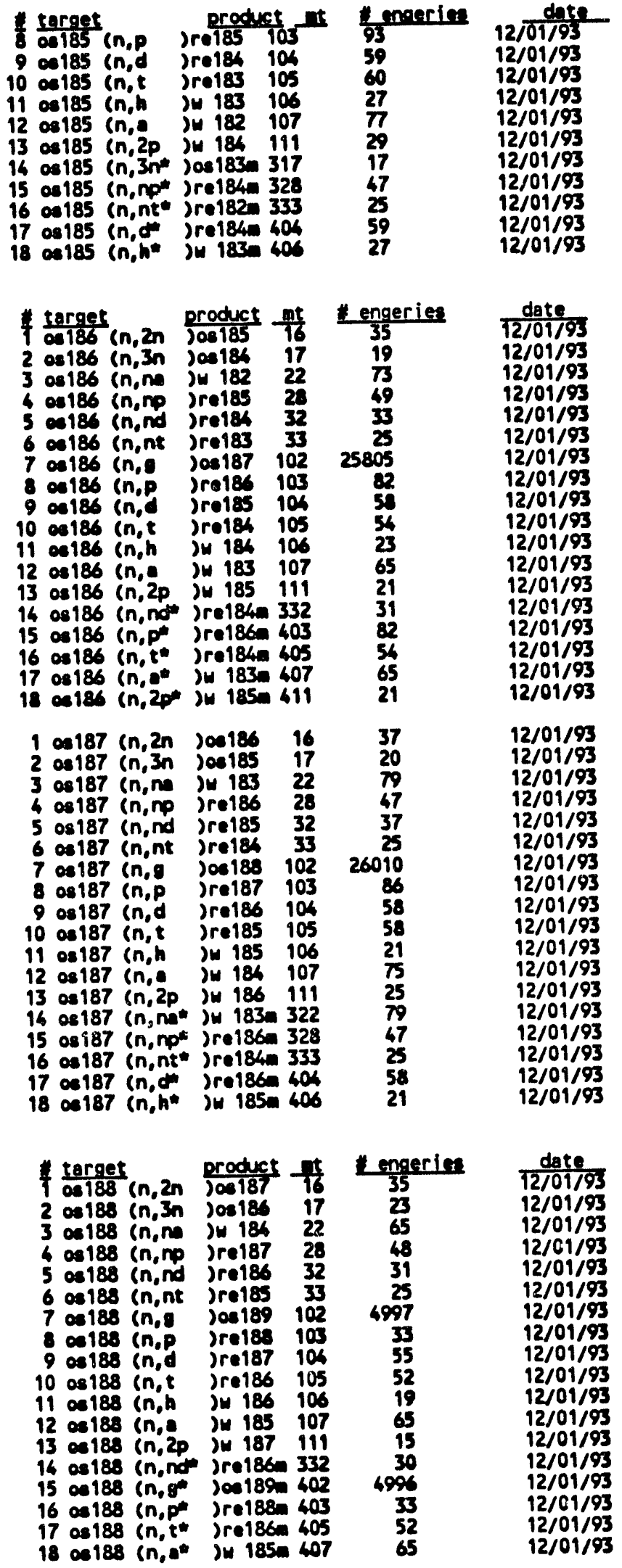




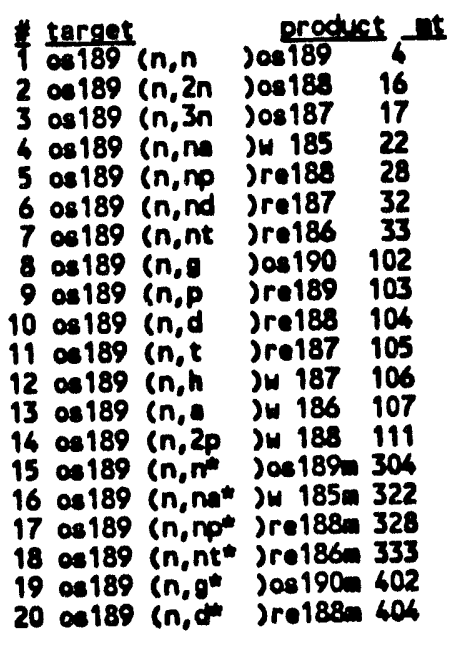

1
1
40
22
72
45
38
25
1243
82
54
56
17
68
19
53
72
45
25
1243
54

$12 / \frac{\text { date }}{01 / 93}$

$12 / 01 / 93$

$12 / 01 / 93$

$12 / 01 / 93$

$12 / 01 / 93$

$12 / 01 / 93$

$12 / 01 / 93$

$12 / 01 / 93$

$12 / 01 / 93$

$12 / 01 / 93$

$12 / 01 / 93$

$12 / 01 / 93$

$12 / 01 / 93$

$12 / 01 / 93$

$12 / 01 / 93$

$12 / 01 / 93$

$12 / 01 / 93$

$12 / 01 / 93$

$12 / 01 / 93$

$12 / 01 / 93$

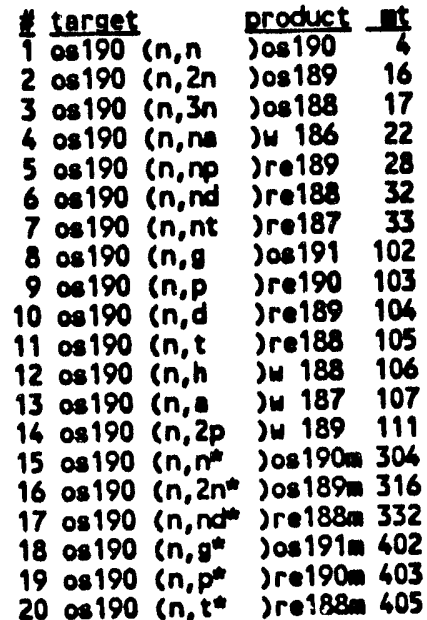

\begin{tabular}{cc} 
Engerien & date \\
\cline { 2 - 2 } 1 & $12 / 01 / 93$ \\
61 & $12 / 01 / 93$ \\
26 & $12 / 01 / 93$ \\
60 & $12 / 01 / 93$ \\
45 & $12 / 01 / 93$ \\
30 & $12 / 01 / 93$ \\
23 & $12 / 01 / 93$ \\
2294 & $12 / 01 / 93$ \\
69 & $12 / 01 / 93$ \\
53 & $12 / 01 / 93$ \\
50 & $12 / 01 / 93$ \\
13 & $12 / 01 / 93$ \\
15 & $12 / 01 / 93$ \\
9 & $12 / 01 / 93$ \\
56 & $12 / 01 / 93$ \\
41 & $12 / 01 / 93$ \\
29 & $12 / 01 / 93$ \\
2293 & $12 / 01 / 93$ \\
69 & $12 / 01 / 93$ \\
50 & $12 / 01 / 93$
\end{tabular}

$100191(n, n) 2001996$

$208191(n, 2 n) 0819016$

3 os199 $(n, 3 n$ jos189 17

408191 (n,na in $187 \quad 22$

508191 (n,np ) re190 28

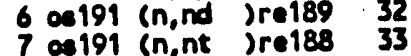

7 os191 (n,nt )re188 33

08191 (n,p jre191 103

$\begin{array}{rrrr}9 & 08191 & (n, p) & \text { rel91 } \\ 10 & 03191 & (n, d) & \text { )re190 } 104\end{array}$

11 os191 (n,t )re189 105

$1208191 \mathrm{cn}, \mathrm{h}$ iw $189 \quad 106$

1308191 (n, is is 188 107

1408199 (n, 2p iw 190111

150191 (n, n jos1910 30

16 os191 (n,2n*) 20s190m 316

17 cal91 (n,3n ) 00189 m 317

18 os191 (n,np )re190- 328

1908191 (n,nt*) re188m 333

$200191\left(n, g^{+}\right) 00192 \pi, 402$

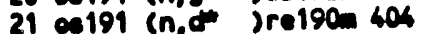

$\begin{array}{rr}1 & 12 / 01 / 93 \\ 39 & 12 / 01 / 93 \\ 21 & 12 / 01 / 93 \\ 68 & 12 / 01 / 93 \\ 43 & 12 / 01 / 93 \\ 35 & 12 / 01 / 93 \\ 23 & 12 / 01 / 93 \\ 396 & 12 / 01 / 93 \\ 76 & 12 / 01 / 93 \\ 51 & 12 / 01 / 93 \\ 53 & 12 / 01 / 93 \\ 9 & 12 / 01 / 93 \\ 62 & 12 / 01 / 93 \\ 13 & 12 / 01 / 93 \\ 50 & 12 / 01 / 93 \\ 30 & 12 / 011993 \\ 21 & 12 / 01 / 93 \\ 43 & 12 / 01 / 93 \\ 23 & 12 / 01 / 93 \\ 394 & 12 / 01 / 93 \\ 51 & 12 / 01 / 93\end{array}$

$A-8.15$ 


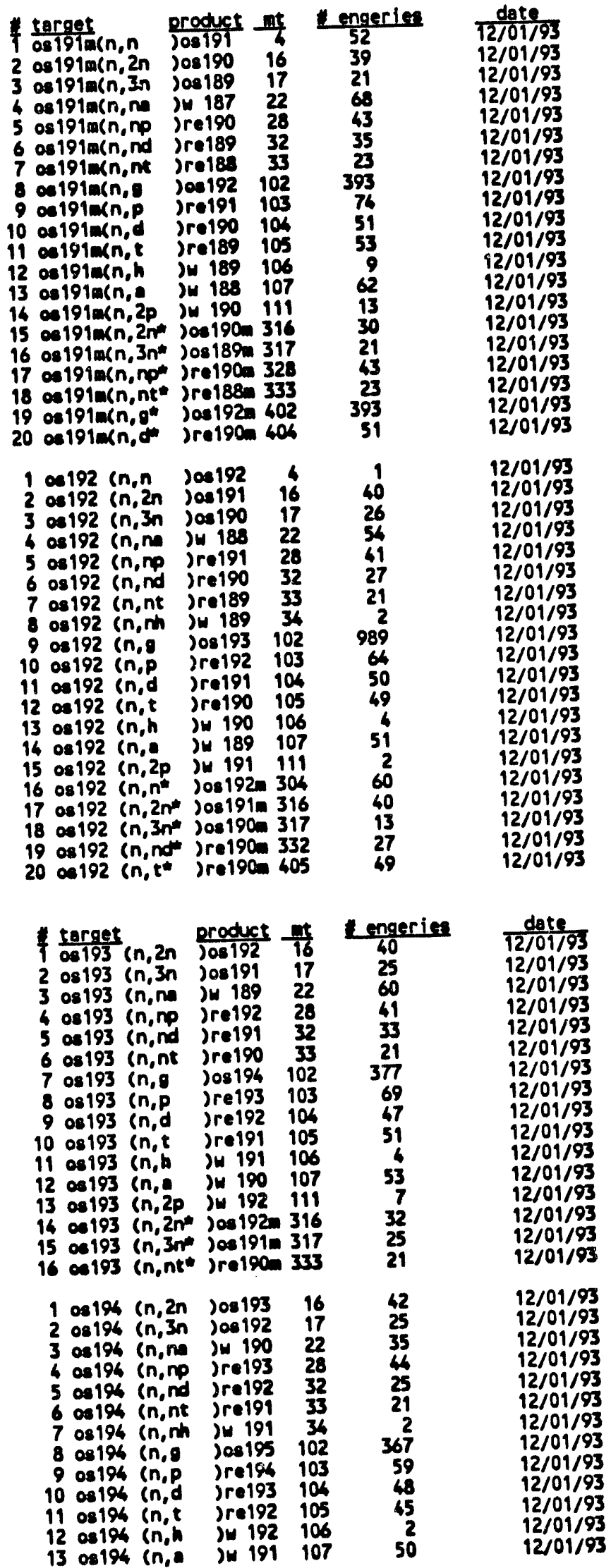

A-8.16 


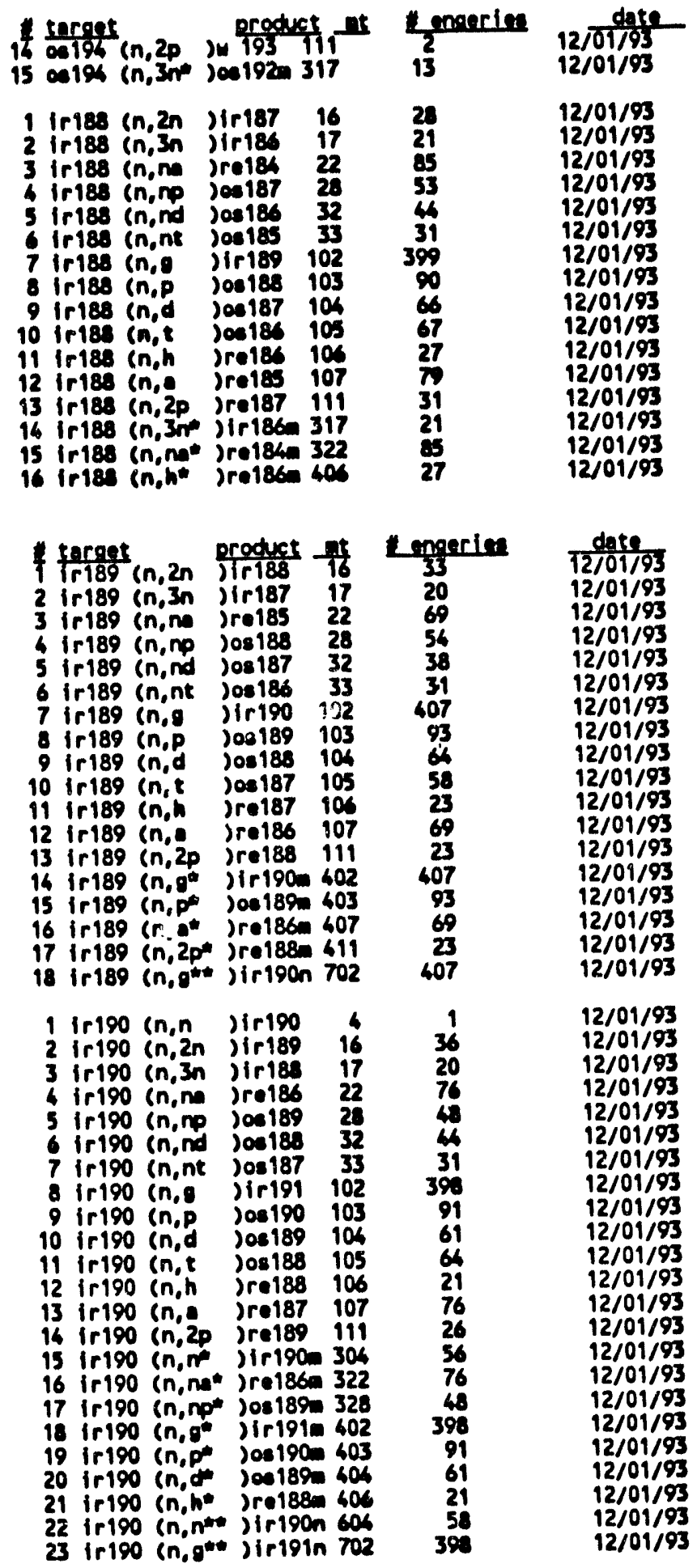




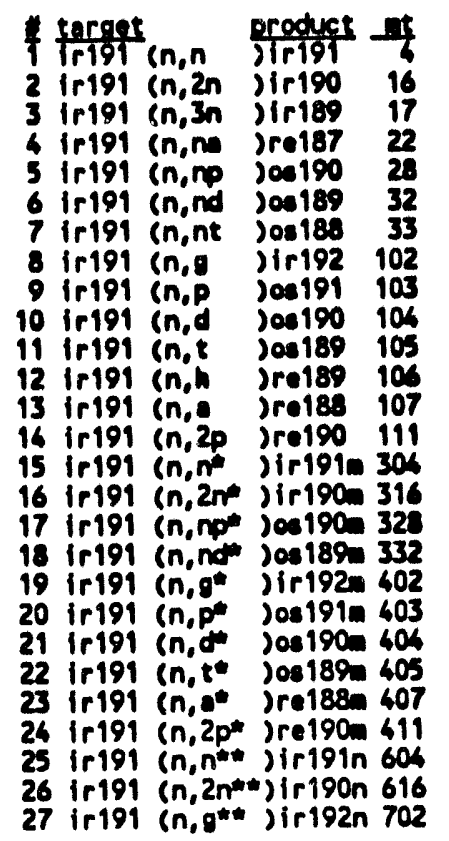

\begin{tabular}{cc} 
congerien & dote \\
\cline { 2 - 2 } 31 & $12 / 01 / 93$ \\
31 & $12 / 01 / 93$ \\
23 & $12 / 01 / 93$ \\
64 & $12 / 01 / 93$ \\
54 & $12 / 01 / 93$ \\
36 & $12 / 01 / 93$ \\
31 & $12 / 01 / 93$ \\
7426 & $12 / 01 / 93$ \\
88 & $12 / 01 / 93$ \\
62 & $12 / 01 / 93$ \\
57 & $12 / 01 / 93$ \\
17 & $12 / 01 / 93$ \\
61 & $12 / 01 / 93$ \\
17 & $12 / 01 / 93$ \\
66 & $12 / 01 / 93$ \\
31 & $12 / 01 / 93$ \\
45 & $12 / 01 / 93$ \\
33 & $12 / 01 / 93$ \\
7431 & $12 / 01 / 93$ \\
86 & $12 / 01 / 93$ \\
60 & $12 / 01 / 93$ \\
57 & $12 / 01 / 93$ \\
61 & $12 / 01 / 93$ \\
17 & $12 / 01 / 93$ \\
57 & $12 / 01 / 93$ \\
31 & $12 / 01 / 93$ \\
7421 & $12 / 01 / 93$
\end{tabular}

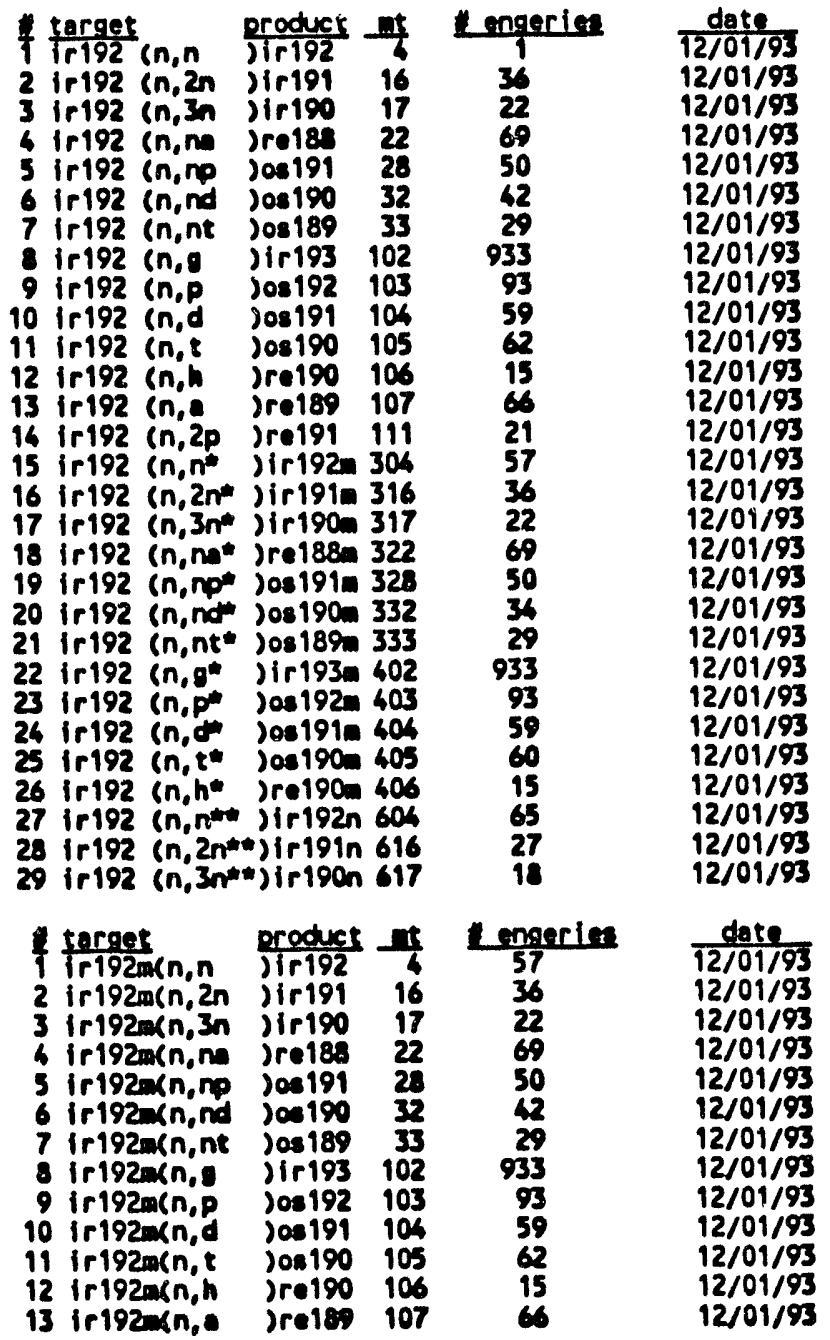




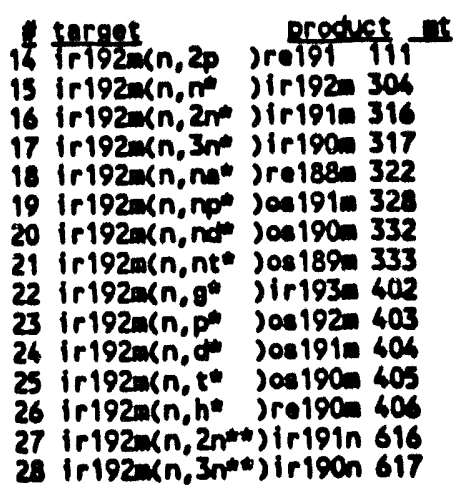

\begin{tabular}{cc} 
moneries & date \\
\hline 21 & $12 /$ c1/93 \\
57 & $12 / 01 / 93$ \\
36 & $12 / 101 / 93$ \\
22 & $12 / 101 / 93$ \\
69 & $12 / 01 / 93$ \\
50 & $12 / 01 / 93$ \\
34 & $12 / 01 / 93$ \\
29 & $12 / 01 / 93$ \\
933 & $12 / 01 / 93$ \\
93 & $12 / 01 / 93$ \\
59 & $12 / 01 / 93$ \\
60 & $12 / 01 / 93$ \\
15 & $12 / 01 / 93$ \\
27 & $12 / 01 / 93$ \\
18 & $12 / 01 / 93$
\end{tabular}

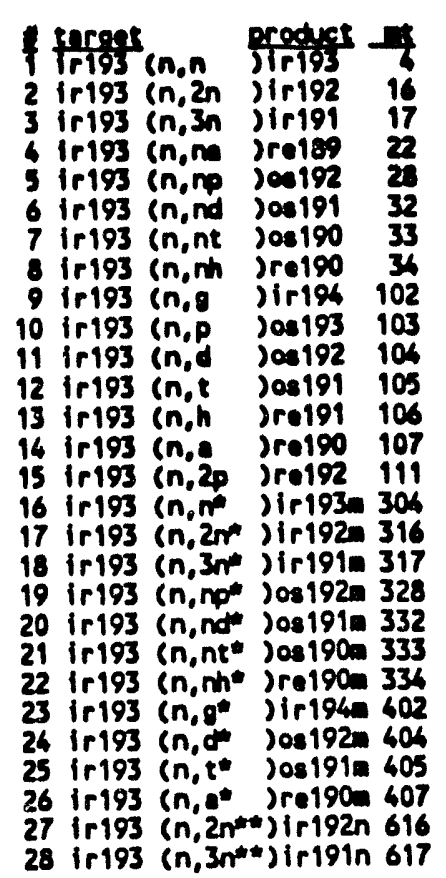

\begin{tabular}{cc} 
Lmpele & ditotes \\
\hline $12 / 01 / 93$ \\
33 & $12 / 01 / 93$ \\
20 & $12 / 01 / 93$ \\
61 & $12 / 01 / 93$ \\
52 & $12 / 01 / 93$ \\
34 & $12 / 01 / 93$ \\
29 & $12 / 01 / 93$ \\
2 & $12 / 01 / 93$ \\
7677 & $12 / 01 / 93$ \\
84 & $12 / 01193$ \\
58 & $12 / 01193$ \\
55 & $12 / 01 / 93$ \\
11 & $12 / 01 / 93$ \\
55 & $12 / 01 / 93$ \\
11 & $12 / 01 / 93$ \\
57 & $12 / 01 / 93$ \\
33 & $12 / 01 / 93$ \\
19 & $12 / 01 / 93$ \\
43 & $12 / 01 / 93$ \\
34 & $12 / 01 / 93$ \\
29 & $12 / 01 / 93$ \\
2 & $12 / 01 / 93$ \\
7701 & $12 / 01 / 93$ \\
56 & $12 / 01 / 93$ \\
55 & $12 / 01 / 93$ \\
55 & $12 / 01 / 93$ \\
33 & $12 / 01 / 93$ \\
10 & $12 / 01 / 93$
\end{tabular}

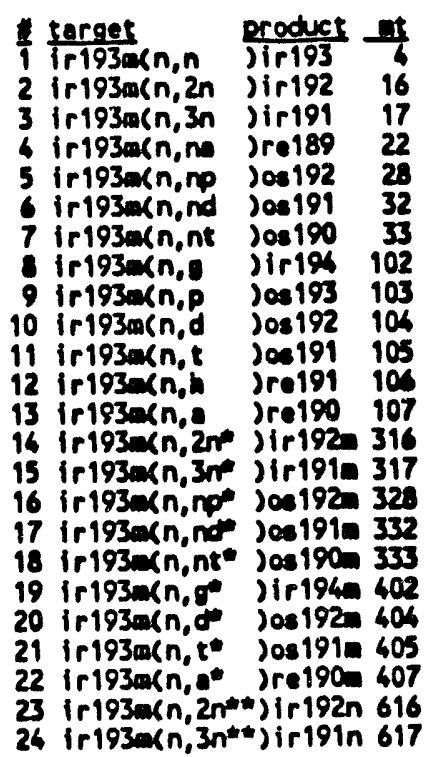

\begin{tabular}{cc} 
moneries & date \\
\cline { 2 - 2 } 60 & $12 / 01 / 93$ \\
33 & $12 / 01 / 93$ \\
20 & $12 / 01 / 93$ \\
61 & $12 / 01 / 93$ \\
49 & $12 / 01 / 93$ \\
36 & $12 / 01 / 93$ \\
29 & $12 / 01199$ \\
7677 & $12 / 01 / 93$ \\
84 & $12 / 01 / 93$ \\
58 & $12 / 01 / 93$ \\
55 & $12 / 01 / 93$ \\
11 & $12 / 01 / 93$ \\
55 & $12 / 01 / 93$ \\
33 & $12 / 01 / 93$ \\
19 & $12 / 01 / 93$ \\
61 & $12 / 01 / 93$ \\
36 & $12 / 01 / 93$ \\
29 & $12 / 01 / 93$ \\
701 & $12 / 01 / 93$ \\
56 & $12 / 01 / 93$ \\
55 & $12 / 01 / 93$ \\
55 & $12 / 01 / 93$ \\
29 & $12 / 01 / 93$ \\
10 & $12 / 01 / 93$ \\
&
\end{tabular}

A-8. 19 


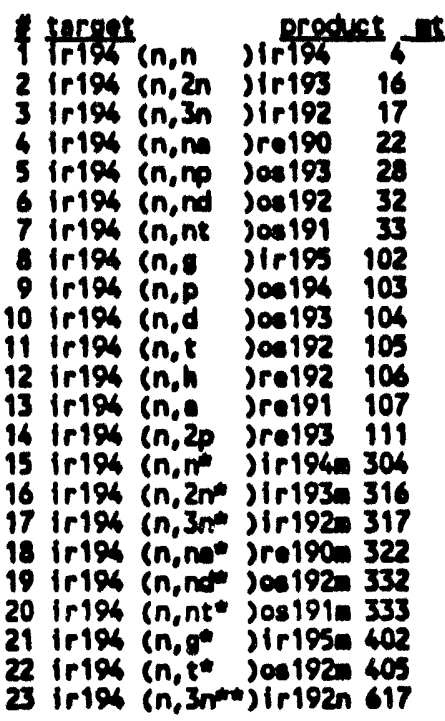

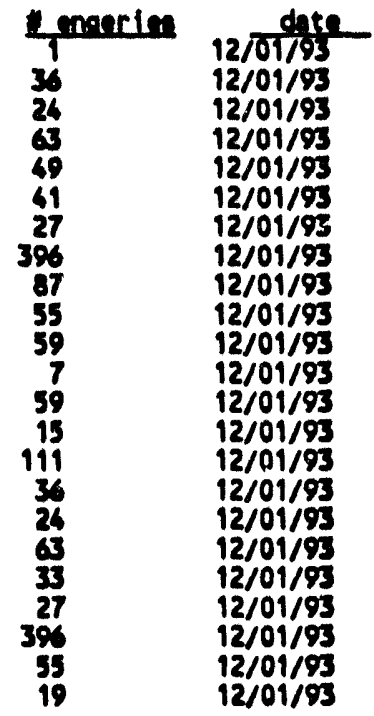

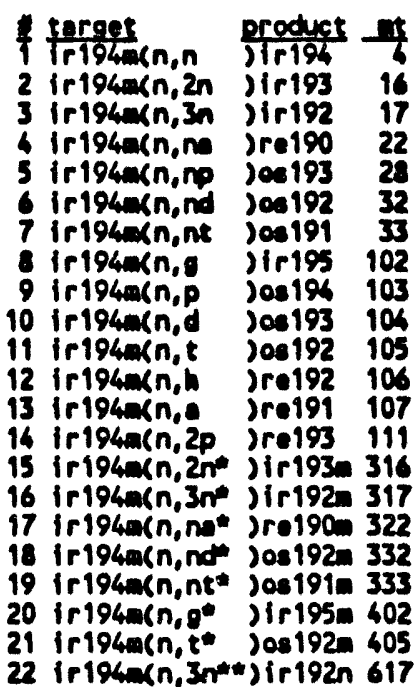

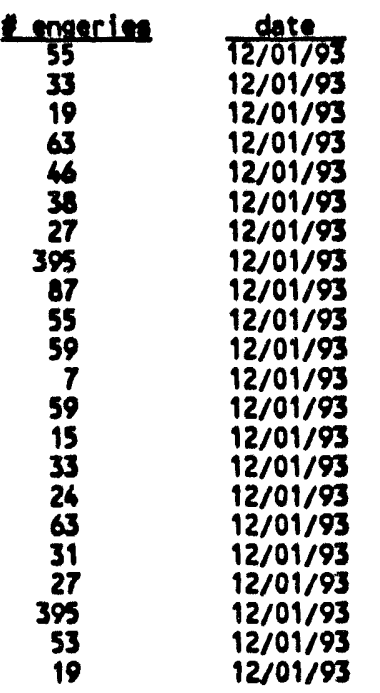

\begin{tabular}{|c|c|c|c|c|}
\hline 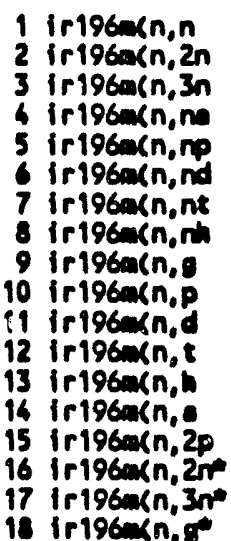 & 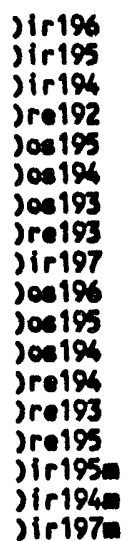 & $\begin{array}{l}6 \\
16 \\
17 \\
22 \\
22 \\
32 \\
33 \\
34 \\
102 \\
103 \\
104 \\
105 \\
106 \\
107 \\
111 \\
316 \\
317 \\
402\end{array}$ & $\begin{array}{r}57 \\
41 \\
20 \\
60 \\
46 \\
38 \\
29 \\
2 \\
368 \\
79 \\
56 \\
58 \\
2 \\
54 \\
7 \\
41 \\
20 \\
365\end{array}$ & $\begin{array}{l}12 / 01 / 93 \\
12 / 01 / 93 \\
12 / 01 / 93 \\
12 / 01 / 93 \\
12 / 01 / 93 \\
12 / 01 / 93 \\
12 / 01 / 93 \\
12 / 01 / 93 \\
12 / 01 / 93 \\
12 / 01 / 93 \\
12 / 01 / 93 \\
12 / 01 / 93 \\
12 / 01 / 93 \\
12 / 01 / 93 \\
12 / 01 / 93 \\
12 / 01 / 93 \\
12 / 01 / 93 \\
12 / 01 / 93\end{array}$ \\
\hline
\end{tabular}




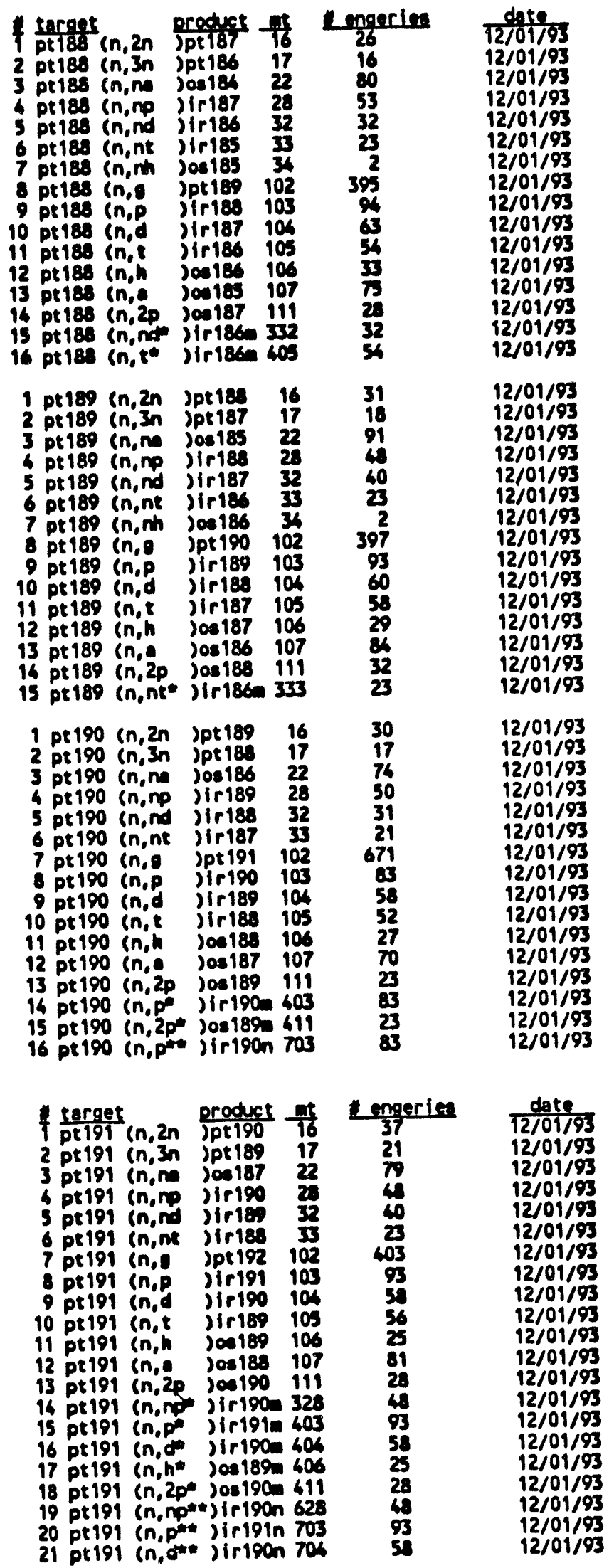

A-8. 21 


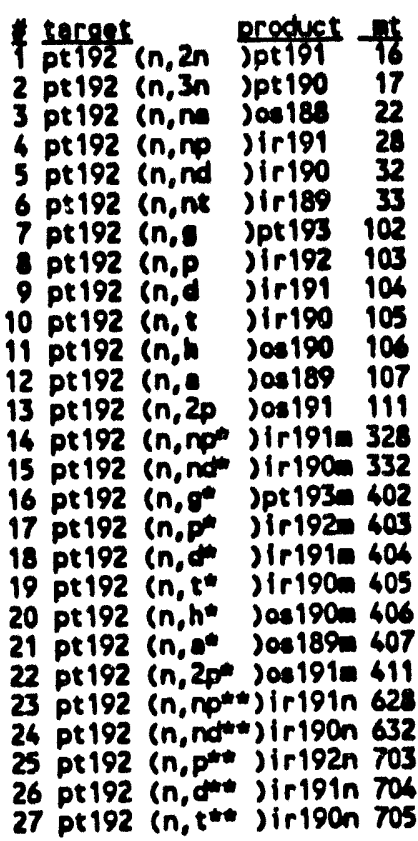

\begin{tabular}{cc} 
Lngecle & date \\
\hline 30 & $12 / 01 / 93$ \\
20 & $12 / 01 / 93$ \\
65 & $12 / 01 / 93$ \\
49 & $12 / 01 / 93$ \\
29 & $12 / 01 / 93$ \\
21 & $12 / 01 / 93$ \\
871 & $12 / 01 / 93$ \\
80 & $12 / 01 / 93$ \\
55 & $12 / 01 / 93$ \\
50 & $12 / 01 / 93$ \\
21 & $12 / 01 / 93$ \\
64 & $12 / 01 / 93$ \\
17 & $12 / 01 / 93$ \\
49 & $12 / 01 / 93$ \\
29 & $12 / 01 / 93$ \\
871 & $12 / 01 / 93$ \\
80 & $12 / 01 / 93$ \\
55 & $12 / 01 / 93$ \\
50 & $12 / 01 / 93$ \\
21 & $12 / 01 / 93$ \\
66 & $12 / 01 / 93$ \\
17 & $12 / 01 / 93$ \\
60 & $12 / 01 / 93$ \\
29 & $12 / 01 / 93$ \\
80 & $12 / 01 / 93$ \\
53 & $12 / 01 / 93$ \\
50 & $12 / 01 / 93$
\end{tabular}

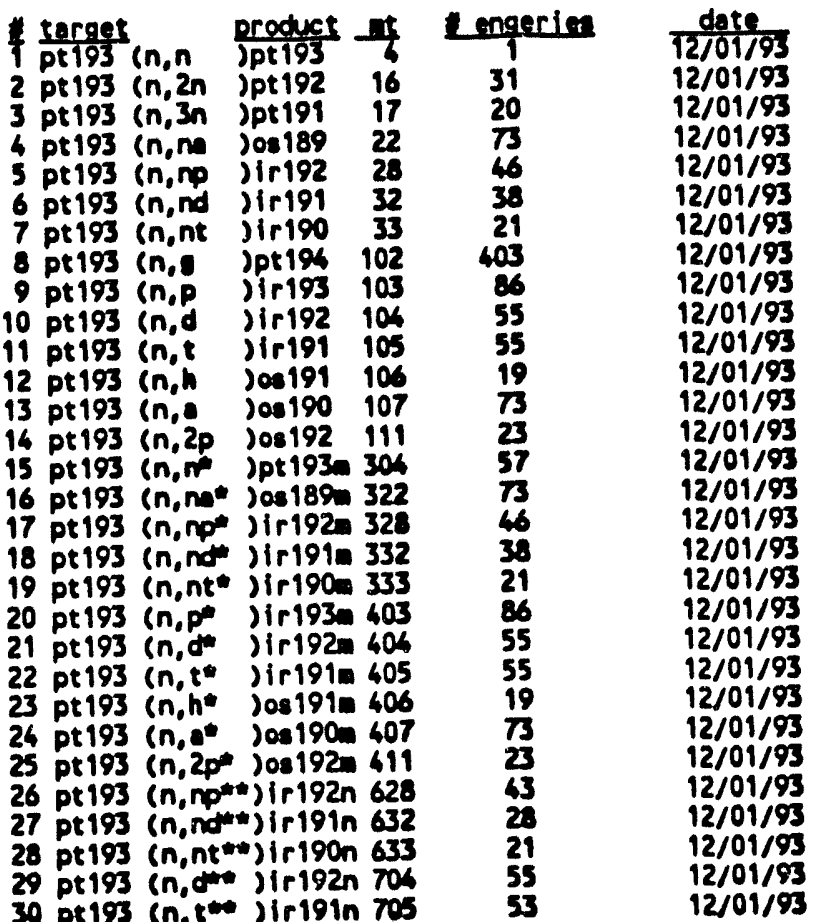

\begin{tabular}{|c|c|c|c|c|}
\hline 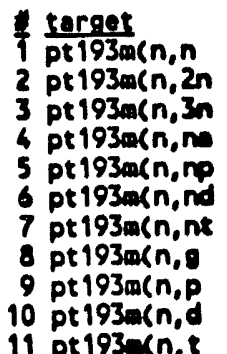 & $\begin{array}{l}\text { product } \\
\text { Ppt193 } \\
\text { jpt192 } \\
\text { jpt191 } \\
\text { jos189 } \\
\text { jir192 } \\
\text { Jir191 } \\
\text { Jir190 } \\
\text { jpt194 } \\
\text { jir193 } \\
\text { Jir192 } \\
\text { Jir191 }\end{array}$ & $\begin{array}{c}. t \\
6 \\
16 \\
17 \\
22 \\
28 \\
32 \\
33 \\
102 \\
103 \\
104 \\
105\end{array}$ & $\begin{array}{c}\text { engerien } \\
58 \\
31 \\
17 \\
73 \\
43 \\
36 \\
21 \\
403 \\
86 \\
55 \\
55\end{array}$ & $\begin{array}{l}\frac{\text { date }}{12 / 01 / 93} \\
12 / 01 / 93 \\
12 / 01 / 93 \\
12 / 01 / 93 \\
12 / 01 / 93 \\
12 / 01 / 93 \\
12 / 01 / 93 \\
12 / 01 / 93 \\
12 / 01 / 93 \\
12 / 01 / 93 \\
12 / 01 / 93\end{array}$ \\
\hline
\end{tabular}

A-8.22 


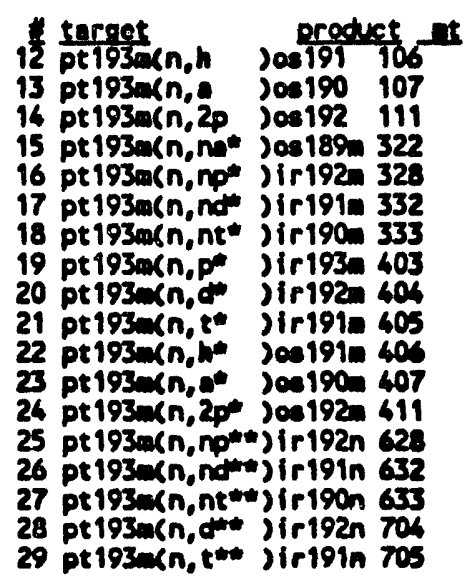

\begin{tabular}{ll} 
marien & date \\
\hline 19 & $12 / 01 / 93$ \\
73 & $12 / 01 / 93$ \\
23 & $12 / 01 / 93$ \\
73 & $12 / 01 / 93$ \\
46 & $12 / 01 / 93$ \\
38 & $12 / 01 / 93$ \\
21 & $12 / 01 / 93$ \\
86 & $12 / 01 / 93$ \\
55 & $12 / 01 / 93$ \\
55 & $12 / 01 / 93$ \\
19 & $12 / 01 / 93$ \\
73 & $12 / 01 / 93$ \\
23 & $12 / 01 / 93$ \\
63 & $12 / 01 / 93$ \\
23 & $12 / 01 / 93$ \\
21 & $12 / 01 / 93$ \\
55 & $12 / 01 / 93$ \\
53 & $12 / 01 / 93$
\end{tabular}

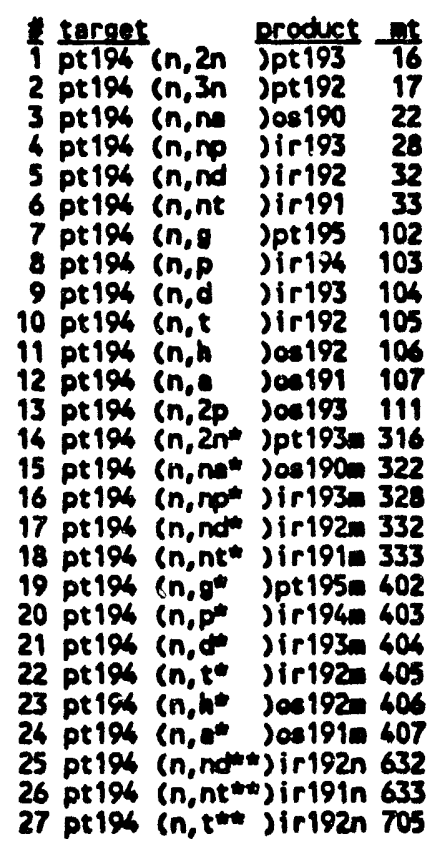

\begin{tabular}{cc} 
engerien & date \\
\hline 30 & $12 / 01 / 93$ \\
22 & $12 / 01 / 93$ \\
61 & $12 / 01 / 93$ \\
47 & $12 / 01 / 93$ \\
28 & $12 / 01 / 93$ \\
19 & $12 / 01 / 93$ \\
1858 & $12 / 01 / 93$ \\
72 & $12 / 01 / 93$ \\
53 & $12 / 01 / 93$ \\
49 & $12 / 01 / 93$ \\
15 & $12 / 01 / 93$ \\
56 & $12 / 01 / 93$ \\
13 & $12 / 01 / 93$ \\
30 & $12 / 01 / 93$ \\
61 & $12 / 01 / 93$ \\
47 & $12 / 01 / 93$ \\
28 & $12 / 01 / 93$ \\
19 & $17 / 04 / 93$ \\
1860 & \\
72 & \\
53 & \\
49 & \\
15 & $12 / 41 / 13$ \\
56 & $12 / 01 / 93$ \\
28 & $12 / 01 / 93$ \\
19 & $12 / 01 / 93$ \\
49 & $12 / 01 / 93$
\end{tabular}

\begin{tabular}{|c|c|c|c|c|c|}
\hline 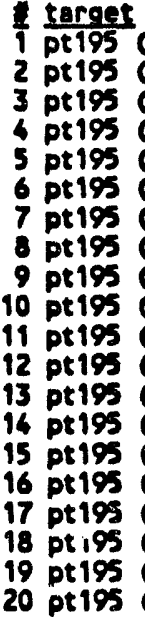 & 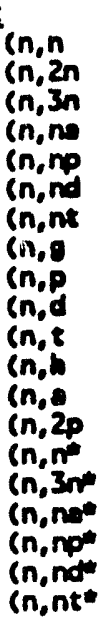 & $\begin{array}{l}\text { product } \\
\text { jpt195 } \\
\text { jpt194 } \\
\text { jpt193 } \\
\text { jos191 } \\
\text { jir194 } \\
\text { jir193 } \\
\text { jir192 } \\
\text { jpt19\% } \\
\text { jir19\% } \\
\text { jir194 } \\
\text { jir193 } \\
\text { jos193 } \\
\text { jos192 } \\
\text { jos194 } \\
\text { jpt1950 } \\
\text { jpt193 } \\
\text { jos1910 } \\
\text { jir1944 } \\
\text { jirr1934 } \\
\text { jir1920 }\end{array}$ & $\begin{array}{c}0 t \\
4 \\
16 \\
17 \\
22 \\
28 \\
32 \\
33 \\
102 \\
103 \\
104 \\
105 \\
105 \\
107 \\
111 \\
304 \\
317 \\
322 \\
328 \\
332 \\
333\end{array}$ & $\begin{array}{c}\text { Lngeries } \\
1 \\
32 \\
19 \\
67 \\
44 \\
36 \\
19 \\
697 \\
76 \\
53 \\
53 \\
13 \\
64 \\
17 \\
56 \\
19 \\
67\end{array}$ & $\begin{array}{l}\text { date } \\
12 / 01 / 93 \\
12 / 01 / 93 \\
12 / 01 / 93 \\
12 / 01 / 93 \\
12 / 01 / 93 \\
12 / 01 / 93 \\
12 / 01 / 93 \\
12 / 01 / 93 \\
12 / 01 / 93 \\
12 / 01 / 93 \\
12 / 011993 \\
12 / 01 / 93 \\
12 / 01 / 93 \\
12 / 01 / 93 \\
12 / 01 / 93 \\
12 / 01 / 93 \\
12 / 01 / 93 \\
12 / 01 / 93 \\
12 / 01 / 93 \\
12 / 01 / 93\end{array}$ \\
\hline
\end{tabular}




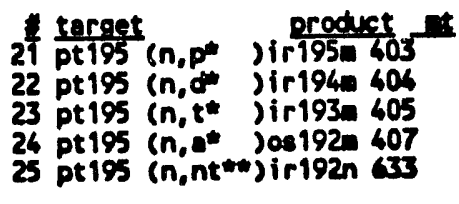

$\begin{array}{cc}\text { eneerini } & \frac{\text { date }}{76} \\ 53 & 12 / 01 / 93 \\ 53 & 12 / 1 / 93 \\ 53 & 12 / 1 / 93 \\ 64 & 12 / 01 / 93 \\ 19 & 12 / 01 / 93\end{array}$

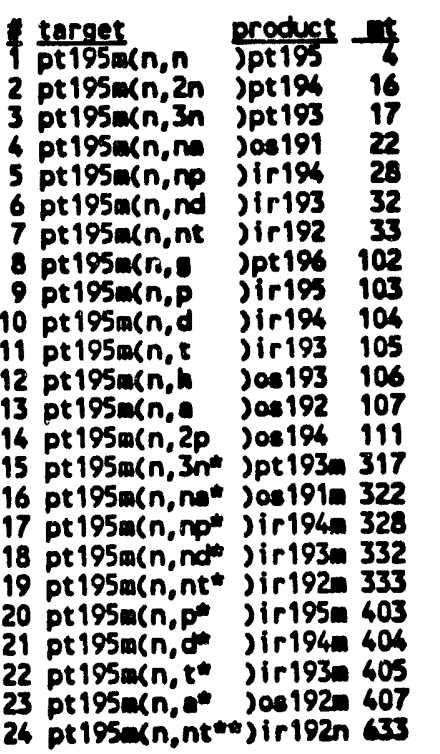

\begin{tabular}{cc} 
mangerien & date \\
\hline 57 & $12 / 11 / 93$ \\
32 & $1201 / 93$ \\
19 & $12 / 01 / 93$ \\
67 & $12 / 11 / 93$ \\
46 & $12 / 11 / 93$ \\
35 & $12 / 01 / 93$ \\
19 & $12 / 1 / 93$ \\
8977 & $12 / 01 / 93$ \\
76 & $12 / 11 / 93$ \\
53 & $12 / 1193$ \\
53 & $12 / 01 / 93$ \\
13 & $12 / 01 / 93$ \\
64 & $12 / 1193$ \\
17 & $12 / 01 / 93$ \\
19 & $12 / 01 / 93$ \\
67 & $12 / 0193$ \\
61 & $12 / 01 / 93$ \\
35 & $12 / 01 / 93$ \\
19 & $12 / 0193$ \\
76 & $12 / 01 / 93$ \\
53 & $12 / 01 / 93$ \\
53 & $12 / 0193$ \\
64 & $12 / 01 / 93$ \\
19 & $12 / 01 / 93$
\end{tabular}

1 pt196 (n, 2n )pt195 16

2 pt196 (n, 3n pptiq 17

3 pt196 in, on jos192 22

5 pt196 (n, nd jirigh 32

5 pt196 (n,nd jirigh 33

7 pt196 (n,g jpt197 102

8 pt196 in,p irige 103

9 pt196 in,d jir195 106

10 pt196 $\mathrm{cn,t}$ jir1\% 105

11 pt 196 (n,h jos $194 \quad 106$

12 pt196 (n, ) 00193107

13 pt196 (n, 2p 20:195 111

14 pt196 (n,2n*)pt195n 316

15 pt 196 (n,ne* jos 192a 322

16 pt196 (n,npe jir195m 328

17 pt196 (n, now )irr194m 332

18 pt196 (n,nt* jir193m 333

19 pt196 (n, $g^{*}$ )pt 197a 402

20 pt196 $(n, p$ jir196m 403

21 pt196 $(n, \infty)$ ir ig5m 404

22 pt196 $(n, t$, Jir194m 405

$\begin{array}{rr}40 & 12 / 01 / 93 \\ 20 & 12 / 101 / 93 \\ 59 & 12 / 01 / 93 \\ 46 & 12 / 01 / 93 \\ 29 & 12 / 01 / 93 \\ 19 & 12 / 01 / 93 \\ 1595 & 12 / 01 / 93 \\ 67 & 12 / 01 / 93 \\ 50 & 12 / 01 / 93 \\ 47 & 12 / 01 / 93 \\ 9 & 12 / 01 / 93 \\ 53 & 12 / 01 / 93 \\ 7 & 12 / 01 / 93 \\ 35 & 12 / 01 / 93 \\ 59 & 12 / 01 / 93 \\ 46 & 12 / 01 / 93 \\ 27 & 12 / 01 / 93 \\ 19 & 12 / 01 / 93 \\ 1611 & 12 / 01 / 93 \\ 67 & 12 / 01 / 93 \\ 50 & 12 / 01 / 93 \\ 67 & 12 / 01 / 93\end{array}$

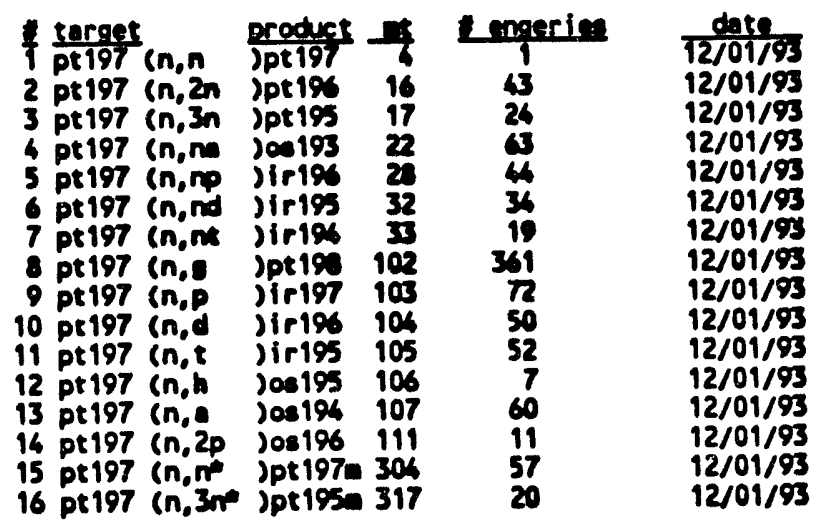




\begin{tabular}{|c|c|c|c|c|c|c|}
\hline & $\begin{array}{l}\text { target: } \\
\text { pt197 } \\
\text { pt197 } \\
\text { pt197 } \\
\text { pt197 } \\
\text { pt197 } \\
\text { pt197 }\end{array}$ & 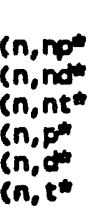 & 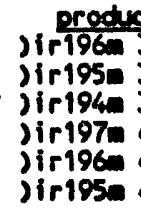 & $\begin{array}{l}\frac{c t}{328} \\
332 \\
333 \\
403 \\
406 \\
405\end{array}$ & $\begin{array}{l}\frac{1}{40} \text { merien } \\
41 \\
33 \\
19 \\
72 \\
50 \\
52\end{array}$ & $\begin{array}{l}\text { date } \\
12 / 01 / 93 \\
12 / 01 / 93 \\
12 / 01 / 93 \\
12 / 01 / 93 \\
12 / 01 / 93 \\
12 / 01 / 93\end{array}$ \\
\hline $\begin{array}{r}1 \\
2 \\
3 \\
4 \\
5 \\
6 \\
7 \\
8 \\
9 \\
10 \\
11 \\
12 \\
13 \\
14 \\
15 \\
16 \\
17 \\
18 \\
19 \\
20 \\
21\end{array}$ & $\begin{array}{l}\text { pt198 } \\
\text { pt198 } \\
\text { pt198 } \\
\text { pt198 } \\
\text { pt198 } \\
\text { pt198 } \\
\text { pt198 } \\
\text { pt198 } \\
\text { pt198 } \\
\text { pt } 198 \\
\text { pt198 } \\
\text { pt198 } \\
\text { pt198 } \\
\text { pt } 198 \\
\text { pt198 } \\
\text { pt } 198 \\
\text { pt198 } \\
\text { pt198 } \\
\text { pt } 198 \\
\text { pt } 198 \\
\text { pt } 198\end{array}$ & 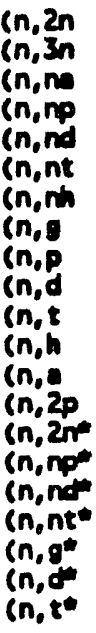 & 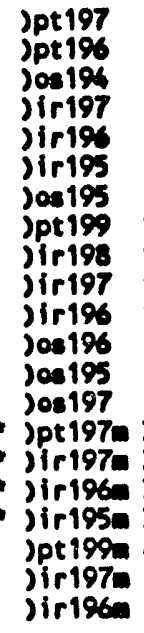 & $\begin{array}{l}16 \\
17 \\
22 \\
28 \\
32 \\
33 \\
34 \\
102 \\
103 \\
104 \\
105 \\
106 \\
107 \\
111 \\
316 \\
328 \\
332 \\
333 \\
402 \\
404 \\
405\end{array}$ & $\begin{array}{r}38 \\
23 \\
55 \\
45 \\
27 \\
19 \\
2 \\
1677 \\
62 \\
49 \\
45 \\
2 \\
50 \\
2 \\
38 \\
45 \\
25 \\
19 \\
1482 \\
49 \\
45\end{array}$ & $\begin{array}{l}12 / 01 / 93 \\
12 / 01 / 93 \\
12 / 01 / 93 \\
12 / 01 / 93 \\
12 / 01 / 93 \\
12 / 01 / 93 \\
12 / 01 / 93 \\
12 / 01 / 93 \\
12 / 01 / 93 \\
12 / 01 / 93 \\
12 / 01 / 93 \\
12 / 01 / 93 \\
12 / 01 / 93 \\
12 / 01 / 93 \\
12 / 01 / 93 \\
12 / 01 / 93 \\
12 / 01 / 93 \\
12 / 01 / 93 \\
12 / 01 / 93 \\
12 / 01 / 93 \\
12 / 01 / 93\end{array}$ \\
\hline $\begin{array}{l}7 \\
1 \\
2 \\
3 \\
4 \\
5 \\
6 \\
7 \\
8 \\
9 \\
10 \\
11 \\
12 \\
13 \\
16 \\
15 \\
16 \\
17 \\
18 \\
19 \\
20 \\
21 \\
22 \\
23 \\
26 \\
25 \\
26 \\
27 \\
28\end{array}$ & 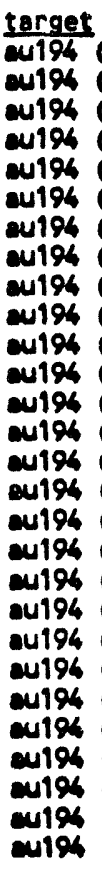 & 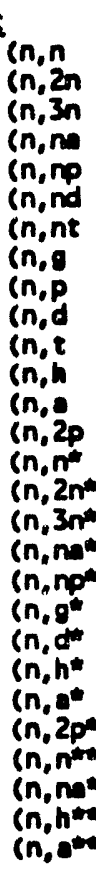 & 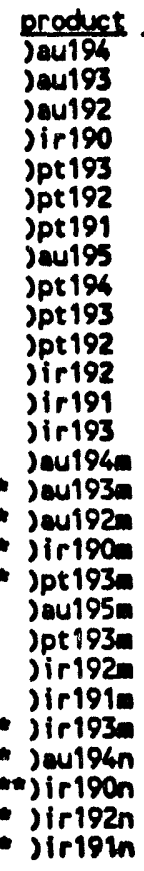 & $\begin{array}{c}7 \\
4 \\
16 \\
17 \\
22 \\
28 \\
32 \\
33 \\
102 \\
103 \\
104 \\
105 \\
106 \\
107 \\
111 \\
304 \\
316 \\
317 \\
322 \\
328 \\
402 \\
404 \\
406 \\
407 \\
411 \\
604 \\
622 \\
706\end{array}$ & $\begin{array}{c}\text { Lengeries } \\
1 \\
27 \\
20 \\
71 \\
49 \\
43 \\
25 \\
406 \\
91 \\
62 \\
59 \\
21 \\
73 \\
28 \\
63 \\
26 \\
16 \\
70 \\
49 \\
406 \\
62 \\
21 \\
72 \\
28 \\
63 \\
71 \\
21 \\
73\end{array}$ & $\begin{array}{c}\text { date } \\
12 / 01 / 93 \\
12 / 01 / 93 \\
12 / 01 / 93 \\
12 / 01 / 93 \\
12 / 01 / 93 \\
12 / 01 / 93 \\
12 / 01 / 93 \\
12 / 01 / 93 \\
12 / 01 / 93 \\
12 / 01 / 93 \\
12 / 01 / 93 \\
12 / 01 / 93 \\
12 / 01 / 93 \\
12 / 01 / 93 \\
12 / 01 / 93 \\
12 / 01 / 93 \\
12 / 01 / 93 \\
12 / 011993 \\
12 / 01 / 93 \\
12 / 01 / 93 \\
12 / 01 / 93 \\
12 / 01 / 93 \\
12 / 01 / 93 \\
12 / 01 / 93 \\
12 / 01 / 93 \\
12 / 01 / 93 \\
12 / 01 / 93 \\
12 / 01 / 93\end{array}$ \\
\hline $\begin{array}{r}2 \\
3 \\
6 \\
5 \\
6 \\
7 \\
8 \\
9 \\
10 \\
11 \\
12\end{array}$ & 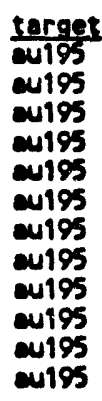 & $\begin{array}{l}\text { (n, 3n } \\
\text { (n, n) } \\
(n, n) \\
\text { (n, nd } \\
\text { (n, nt } \\
(n, 8) \\
(n, p) \\
(n, d) \\
(n, k) \\
(n, n)\end{array}$ & $\begin{array}{l}\text { product } \\
\text { Jou195 } \\
\text { jau194 } \\
\text { jeu193 } \\
\text { jir191 } \\
\text { jpt194 } \\
\text { jpt193 } \\
\text { jpt192 } \\
\text { jau196 } \\
\text { jpt195 } \\
\text { jpt194 } \\
\text { jpt193 } \\
\text { jir193 }\end{array}$ & $\begin{array}{r}16 \\
17 \\
22 \\
25 \\
32 \\
33 \\
102 \\
103 \\
104 \\
105 \\
106\end{array}$ & $\begin{array}{c}\text { menerin } \\
1 \\
32 \\
21 \\
62 \\
53 \\
34 \\
25 \\
403 \\
87 \\
60 \\
53 \\
17\end{array}$ & $\begin{array}{l}\text { date } \\
\frac{12 / 01 / 93}{12 / 01 / 93} \\
12 / 01 / 93 \\
12 / 01 / 93 \\
12 / 01 / 93 \\
12 / 01 / 93 \\
12 / 01 / 93 \\
12 / 01 / 93 \\
12 / 01 / 93 \\
12 / 01 / 93 \\
12 / 01 / 93 \\
12 / 01 / 93\end{array}$ \\
\hline
\end{tabular}




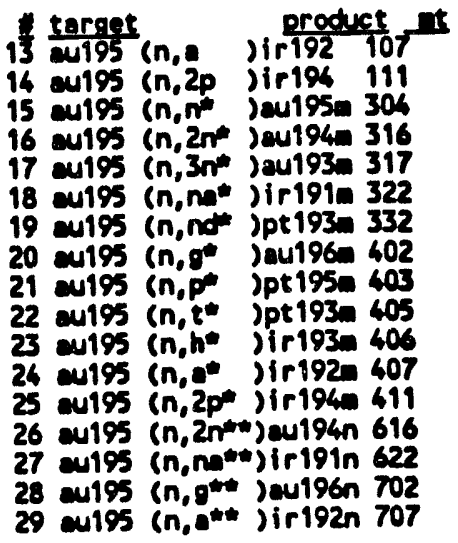

\begin{tabular}{cc} 
1eneries & date \\
\hline 60 & $12 / 01 / 93$ \\
19 & $12 / 01 / 93$ \\
67 & $12 / 01 / 93$ \\
32 & $12 / 01 / 93$ \\
17 & $12 / 01 / 93$ \\
62 & $12 / 01 / 93$ \\
36 & $12 / 01 / 93$ \\
403 & $12 / 01 / 93$ \\
87 & $12 / 01 / 93$ \\
53 & $12 / 01 / 93$ \\
17 & $12 / 01 / 93$ \\
59 & $12 / 01 / 93$ \\
19 & $12 / 01 / 93$ \\
28 & $12 / 01 / 93$ \\
62 & $12 / 01 / 93$ \\
403 & $12 / 01 / 93$ \\
60 & $12 / 01 / 93$
\end{tabular}

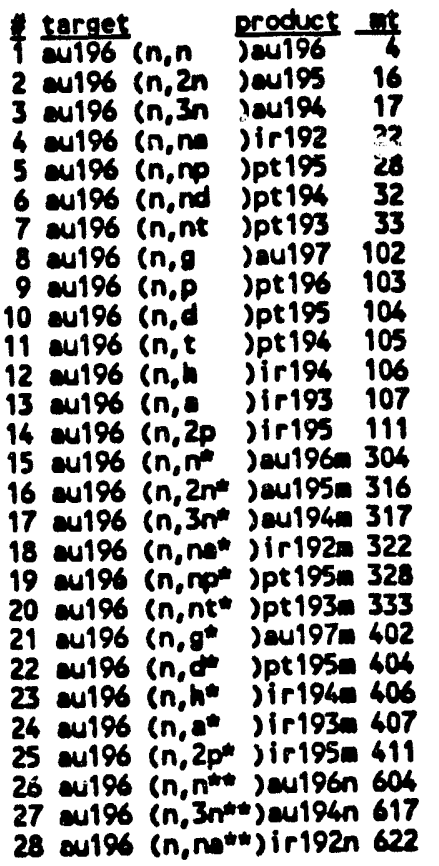

\begin{tabular}{cc} 
nengerien & date \\
\cline { 2 - 2 } 1 & $12 / 01 / 93$ \\
27 & $12 / 01 / 93$ \\
18 & $12 / 01 / 93$ \\
63 & $12 / 01 / 93$ \\
50 & $12 / 01 / 93$ \\
42 & $12 / 01 / 93$ \\
23 & $12 / 01 / 93$ \\
403 & $12 / 01 / 93$ \\
93 & $12 / 01 / 93$ \\
57 & $12 / 01 / 93$ \\
60 & $12 / 01 / 93$ \\
15 & $12 / 01 / 93$ \\
64 & $12 / 01 / 93$ \\
23 & $12 / 01 / 93$ \\
39 & $12 / 01 / 93$ \\
25 & $12 / 01 / 93$ \\
18 & $12 / 01 / 93$ \\
67 & $12 / 01 / 93$ \\
50 & $12 / 01 / 93$ \\
23 & $12 / 01 / 93$ \\
403 & $12 / 01 / 93$ \\
57 & $12 / 01 / 93$ \\
15 & $12 / 01 / 93$ \\
64 & $12 / 01 / 93$ \\
23 & $12 / 01 / 93$ \\
63 & $12 / 01 / 93$ \\
15 & $12 / 01 / 93$ \\
68 & $12 / 01 / 93$
\end{tabular}

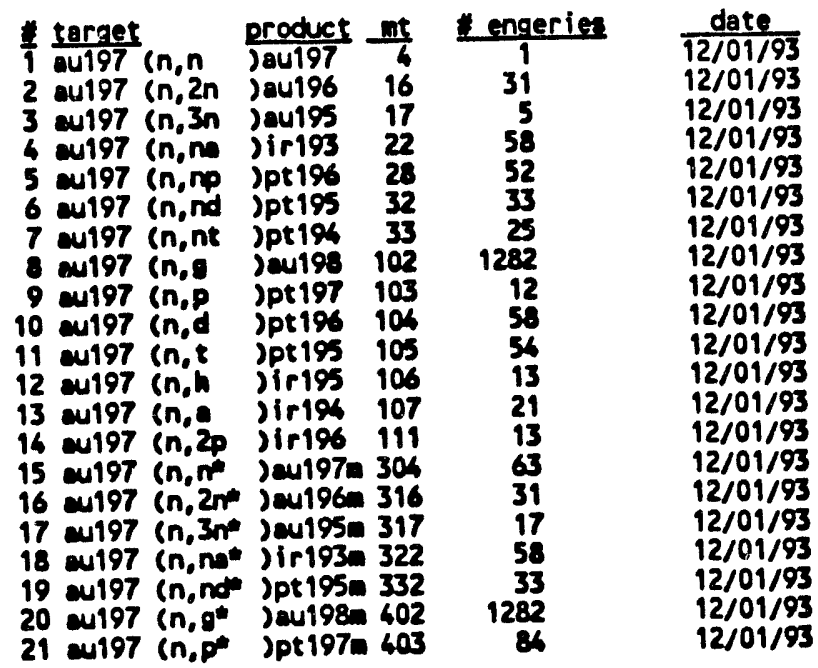




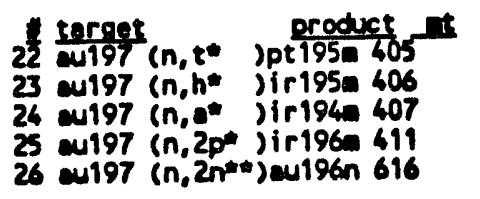

$\begin{array}{ll}\text { Henoerien } & \frac{\text { date }}{34} \\ 13 & 12 / 01 / 93 \\ 21 & 12 / 01 / 93 \\ 13 & 12 / 01 / 93 \\ 31 & 12 / 01 / 93 \\ 31 & 12 / 01 / 93\end{array}$

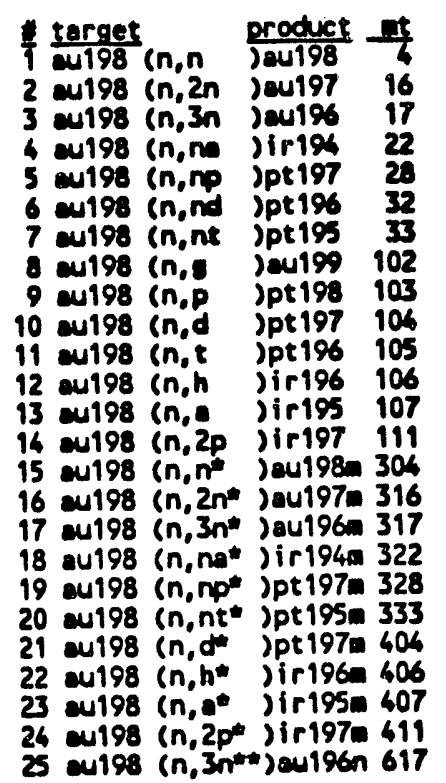

\begin{tabular}{l} 
engerie \\
\hline 1 \\
32 \\
19 \\
62 \\
40 \\
41 \\
23 \\
416 \\
83 \\
56 \\
56 \\
9 \\
59 \\
15 \\
55 \\
32 \\
19 \\
62 \\
46 \\
23 \\
56 \\
9 \\
59 \\
15 \\
16
\end{tabular}

date

12/01/93

$12 / 01 / 95$

$12 / 01 / 93$

$12 / 01 / 93$

$12 / 01 / 93$

$12 / 01 / 93$

$12 / 01 / 93$

$12 / 01 / 93$

12/01/93

$12 / 01 / 93$

$12 / 01 / 93$

$12 / 01 / 93$

$12 / 01 / 93$

$12 / 01 / 93$

$12 / 01 / 93$

$12 / 01 / 93$

$12 / 01 / 93$

$12 / 01 / 93$

$12 / 01 / 93$

$12 / 01 / 93$

$12 / 01 / 93$

$12 / 01 / 93$

$12 / 01 / 93$

$12 / 01 / 93$

$12 / 01 / 93$

$4 \frac{\text { target }}{\text { w198m } n, n} \frac{\text { product }}{\text { ) tou198 }}$

2 w198m(n,2n jou197 16

4 eu198m(n,ne jir19h 22

5 eul98m(n,np) jpt197 28

6 eu198m(n,nd )pt196 32

7 eu198m(n,nt jpt195 33

8 eu198min,s jau199 102

- w198m(n,p jpt198 103

10 eu198m(n,d )pt197 104

11 eul98arn,t jpt196 105

12 eu198m(n,h jir196 106

13 wu198m(n,. jir195 107

16 su198m(n,2p )ir197 111

15 au198m(n, $2 n$ *) au197m 316

16 ou198m(n,3n*) au196m 317

17 su198m(n,n* )ir194m 322

18 w 328

19 cu198m(n,nt* )pt195m 333

20 eu198m(n,\&) pt197m 406

21 culg8men, jir196 406

22 cu198m(n, jir195n 407

23 cu198m(n,2p Jir197m 611

24 w198m(n, 3ntw) jev196n 617

\begin{tabular}{cc} 
mogerien & date \\
\cline { 2 - 2 } 39 & $12 / 01 / 93$ \\
30 & $12 / 01 / 93$ \\
16 & $12 / 01 / 93$ \\
62 & $12 / 01 / 93$ \\
44 & $12 / 01 / 93$ \\
38 & $12 / 01 / 93$ \\
23 & $12 / 01 / 93$ \\
416 & $12 / 01 / 93$ \\
88 & $12 / 01 / 93$ \\
56 & $12 / 01 / 93$ \\
56 & $12 / 01 / 93$ \\
9 & $12 / 01 / 93$ \\
59 & $12 / 01 / 93$ \\
15 & $12 / 01 / 93$ \\
27 & $12 / 01 / 93$ \\
19 & $12 / 01 / 93$ \\
62 & $12 / 01 / 93$ \\
42 & $12 / 01 / 93$ \\
23 & $12 / 01 / 93$ \\
56 & $12 / 01 / 93$ \\
9 & $12 / 01 / 93$ \\
59 & $12 / 01 / 93$ \\
15 & $12 / 01 / 93$ \\
11 & $12 / 01 / 93$
\end{tabular}

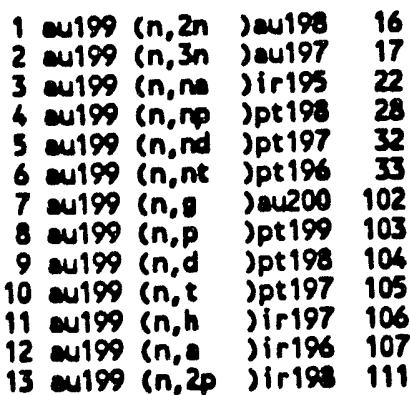

$\begin{array}{cc}35 & 12 / 01 / 93 \\ 22 & 12 / 01 / 93 \\ 55 & 12 / 01 / 93 \\ 49 & 12 / 01 / 93 \\ 32 & 12 / 01 / 93 \\ 25 & 12 / 01 / 93 \\ 384 & 12 / 01 / 93 \\ 76 & 12 / 101 / 93 \\ 55 & 12 / 01 / 93 \\ 52 & 12 / 01 / 93 \\ 7 & 12 / 01 / 93 \\ 52 & 12 / 01 / 93 \\ 7 & 12 / 01 / 93\end{array}$

A-8.27 


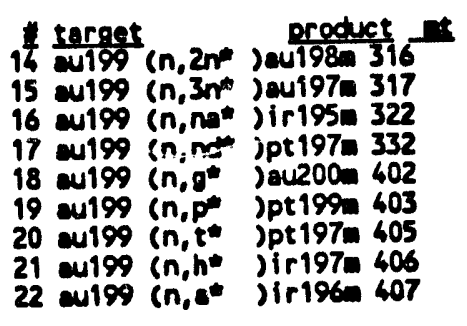

$\begin{array}{cc}\text { Lenoerias } & \text { date } \\ 32 & 12 / 01 / 93 \\ 19 & 12 / 01 / 93 \\ 55 & 12 / 01 / 93 \\ 31 & 12 / 01 / 93 \\ 38 & 12 / 01 / 93 \\ 76 & 12 / 01 / 93 \\ 52 & 12 / 01 / 93 \\ 7 & 12 / 01 / 93 \\ 52 & 12 / 01 / 93\end{array}$

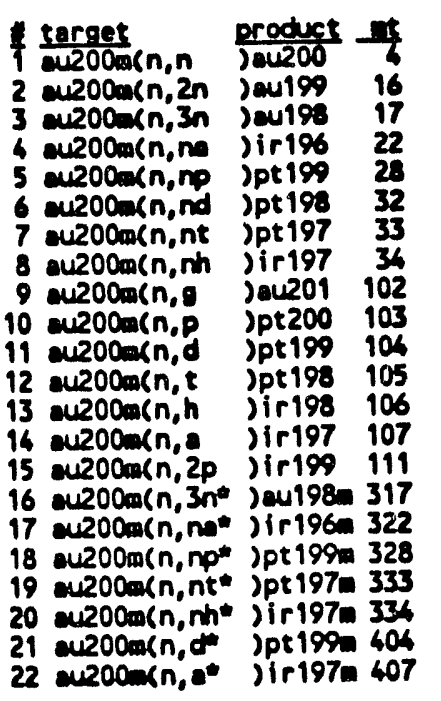

12

\begin{tabular}{cc} 
sngerien & date \\
\cline { 2 - 2 } 61 & $12 / 01 / 93$ \\
31 & $12 / 01 / 93$ \\
17 & $12 / 101 / 93$ \\
56 & $12 / 01 / 93$ \\
62 & $12 / 01 / 93$ \\
36 & $12 / 01 / 93$ \\
23 & $12 / 01 / 93$ \\
2 & $12 / 01 / 93$ \\
371 & $12 / 01 / 93$ \\
83 & $12 / 01 / 93$ \\
53 & $12 / 01 / 93$ \\
54 & $12 / 01 / 93$ \\
2 & $12 / 01 / 93$ \\
54 & $12 / 01 / 93$ \\
9 & $12 / 01 / 93$ \\
12 & $12 / 01 / 93$ \\
56 & $12 / 01 / 93$ \\
40 & $12 / 01 / 93$ \\
23 & $12 / 01 / 93$ \\
2 & $12 / 01 / 93$ \\
53 & $12 / 01 / 93$ \\
56 & $12 / 01 / 93$
\end{tabular}

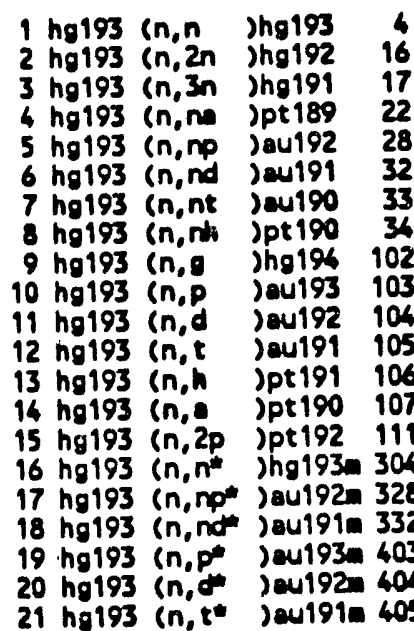

$\begin{array}{rr}1 & 12 / 01 / 93 \\ 25 & 12 / 01 / 93 \\ 18 & 12 / 01 / 93 \\ 83 & 12 / 01 / 93 \\ 48 & 12 / 01 / 93 \\ 38 & 12 / 01 / 93 \\ 16 & 12 / 01 / 93 \\ 2 & 12 / 01 / 93 \\ 400 & 12 / 01 / 93 \\ 91 & 12 / 01 / 93 \\ 61 & 12 / 01 / 93 \\ 57 & 12 / 01 / 93 \\ 29 & 12 / 01 / 93 \\ 81 & 12 / 01193 \\ 32 & 12 / 01 / 93 \\ 112 & 12 / 01 / 93 \\ 45 & 12 / 01 / 93 \\ 37 & 12 / 01 / 93 \\ 91 & 12 / 01 / 93 \\ 61 & 12 / 01 / 93 \\ 57 & 12 / 01 / 93\end{array}$

\begin{tabular}{|c|c|c|c|c|}
\hline 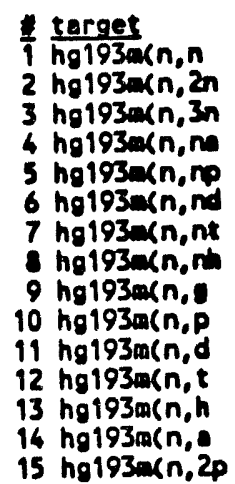 & $\begin{array}{l}\text { Product } \\
\text { Phg193 } \\
\text { jhg192 } \\
\text { jhg191 } \\
\text { jpt189 } \\
\text { jeu192 } \\
\text { jev191 } \\
\text { jou190 } \\
\text { jpt190 } \\
\text { jhg194 } \\
\text { jou193 } \\
\text { jau192 } \\
\text { jou191 } \\
\text { jpt191 } \\
\text { jpt190 } \\
\text { jpt192 }\end{array}$ & $\begin{array}{c}\mathrm{nt} \\
6 \\
16 \\
17 \\
22 \\
28 \\
32 \\
33 \\
31 \\
102 \\
103 \\
104 \\
105\end{array}$ & $\begin{array}{c}\text { engeries } \\
70 \\
25 \\
18 \\
83 \\
48 \\
30 \\
16 \\
2 \\
400 \\
91 \\
61 \\
57 \\
29 \\
81 \\
32\end{array}$ & $\begin{array}{l}\text { dete } \\
12 / 01 / 93 \\
12 / 01 / 93 \\
12 / 01 / 93 \\
12 / 01 / 93 \\
12 / 01 / 93 \\
12 / 01 / 93 \\
12 / 01 / 93 \\
12 / 01 / 93 \\
12 / 01 / 93 \\
12 / 01 / 93 \\
12 / 01 / 93 \\
12 / 01 / 93 \\
12 / 01 / 93 \\
12 / 01 / 93 \\
12 / 01 / 93\end{array}$ \\
\hline
\end{tabular}




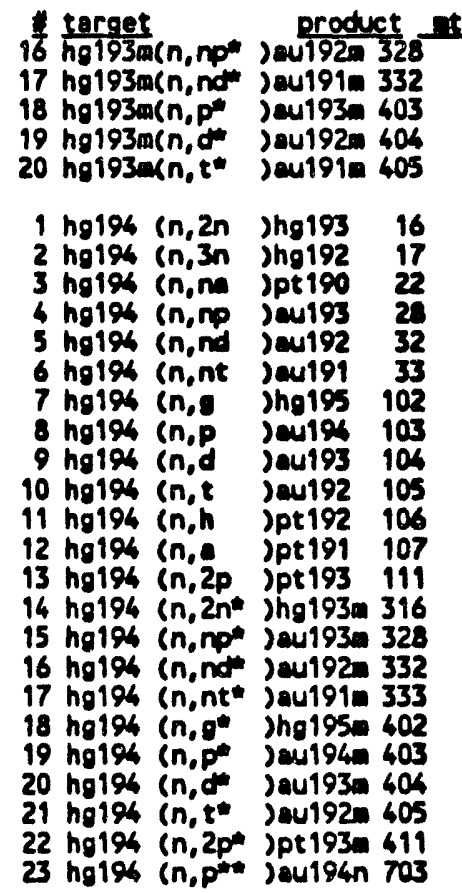

\begin{tabular}{ll} 
Lenoeriee & $\frac{\text { date }}{45}$ \\
\hline 37 & $12 / 01 / 93 / 93$ \\
91 & $12 / 01 / 93$ \\
61 & $12 / 11 / 93$ \\
57 & $12 / 01 / 93$
\end{tabular}

$\begin{array}{cc}27 & 12 / 01 / 93 \\ 17 & 12 / 01 / 93 \\ 64 & 12 / 01 / 93 \\ 50 & 12 / 01 / 93 \\ 20 & 12 / 01 / 93 \\ 16 & 12 / 01 / 93 \\ 383 & 12 / 01 / 93 \\ 38 & 12 / 01 / 93 \\ 57 & 12 / 01 / 93 \\ 57 & 12 / 01 / 93 \\ 69 & 12 / 01 / 93 \\ 25 & 12 / 01 / 93 \\ 68 & 12 / 01 / 93 \\ 23 & 12 / 01 / 93 \\ 27 & 12 / 01 / 93 \\ 50 & 12 / 01 / 93 \\ 27 & 12 / 01 / 93 \\ 16 & 12 / 01 / 93 \\ 388 & 12201 / 93 \\ 86 & 12 / 01 / 93 \\ 57 & 12 / 01 / 93 \\ 49 & 12201 / 93 \\ 23 & 12 / 01 / 93 \\ 87 & \end{array}$

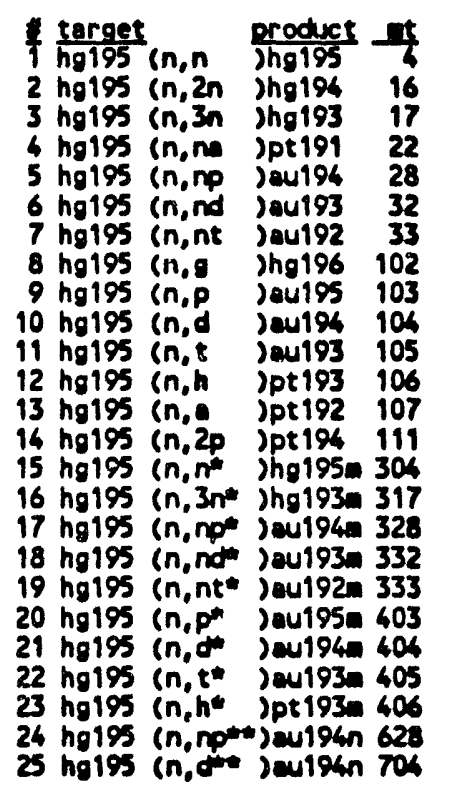

\begin{tabular}{cc} 
Lengeries & dete \\
\hline 12 & $12 / 01 / 93$ \\
33 & $12 / 01 / 93$ \\
19 & $12 / 01 / 93$ \\
74 & $12 / 01 / 93$ \\
47 & $12 / 01 / 93$ \\
36 & $12 / 01 / 93$ \\
16 & $12 / 01 / 93$ \\
394 & $12 / 01 / 93$ \\
92 & $12 / 01 / 93$ \\
57 & $12 / 01 / 93$ \\
55 & $12 / 01 / 93$ \\
23 & $12 / 01 / 93$ \\
75 & $12 / 01 / 93$ \\
28 & $12 / 01 / 93$ \\
66 & $12 / 01 / 93$ \\
19 & $12 / 01 / 93$ \\
47 & $12 / 01 / 93$ \\
35 & $12 / 01 / 93$ \\
16 & $12 / 01 / 93$ \\
92 & $12 / 01 / 93$ \\
57 & $12 / 01 / 93$ \\
55 & $12 / 01 / 93$ \\
23 & $12 / 01 / 93$ \\
46 & $12 / 01 / 93$ \\
57 & $12 / 01 / 93$
\end{tabular}

\begin{tabular}{|c|c|c|c|c|}
\hline 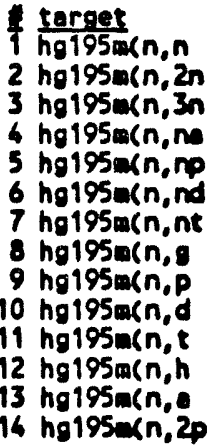 & 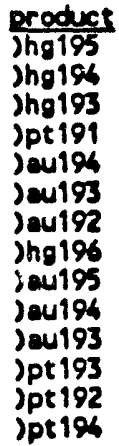 & $\begin{array}{c}17 \\
22 \\
28 \\
32 \\
33 \\
102 \\
103 \\
106 \\
105 \\
106 \\
107 \\
111\end{array}$ & $\begin{array}{l}\text { engerie } \\
67 \\
28 \\
19 \\
74 \\
47 \\
35 \\
16 \\
396 \\
92 \\
57 \\
55 \\
23 \\
78 \\
28\end{array}$ & $\begin{array}{l}\text { date } \\
12 / 01 / 93 \\
12 / 01 / 93 \\
12 / 01 / 93 \\
12 / 01 / 93 \\
12 / 01 / 93 \\
12 / 01 / 93 \\
12 / 01 / 93 \\
12 / 01 / 93 \\
12 / 01 / 93 \\
12 / 01 / 93 \\
12 / 01 / 93 \\
12 / 01 / 93 \\
12 / 01 / 93 \\
12 / 01 / 93\end{array}$ \\
\hline
\end{tabular}




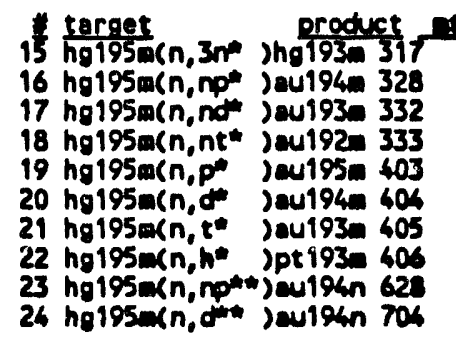

\begin{tabular}{ll} 
enqerien & date \\
\hline 15 & $12 / 01 / 93$ \\
47 & $12 / 01 / 93$ \\
35 & $12 / 01 / 93$ \\
16 & $12 / 01 / 93$ \\
92 & $12 / 01 / 93$ \\
57 & $12 / 01 / 93$ \\
55 & $12 / 01 / 93$ \\
23 & $12 / 01 / 93$ \\
44 & $12 / 01 / 93$ \\
57 & $12 / 01 / 93$
\end{tabular}

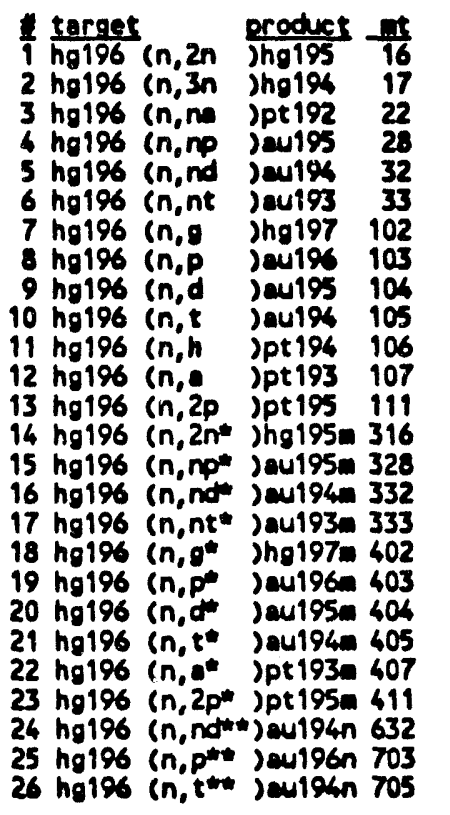

\begin{tabular}{cc} 
engeries & date \\
\cline { 2 - 2 } 31 & $12 / 01 / 93$ \\
20 & $12 / 01 / 93$ \\
62 & $12 / 01 / 93$ \\
49 & $12 / 01 / 93$ \\
29 & $12 / 01 / 93$ \\
14 & $12 / 01 / 93$ \\
75 & $12 / 01 / 93$ \\
73 & $12 / 01 / 93$ \\
83 & $12 / 01 / 93$ \\
55 & $12 / 01 / 93$ \\
69 & $12 / 01 / 93$ \\
21 & $12 / 01 / 93$ \\
63 & $12 / 01 / 93$ \\
19 & $12 / 01 / 93$ \\
27 & $12 / 01 / 93$ \\
69 & $12 / 01 / 93$ \\
29 & $12 / 01 / 93$ \\
16 & $12 / 01 / 93$ \\
797 & $12 / 01 / 93$ \\
83 & $12 / 01 / 93$ \\
55 & $12 / 01 / 93$ \\
49 & $12 / 01 / 93$ \\
63 & $12 / 01 / 93$ \\
19 & $12 / 01 / 93$ \\
27 & $12 / 01 / 93$ \\
83 & $12 / 01 / 93$ \\
69 &
\end{tabular}

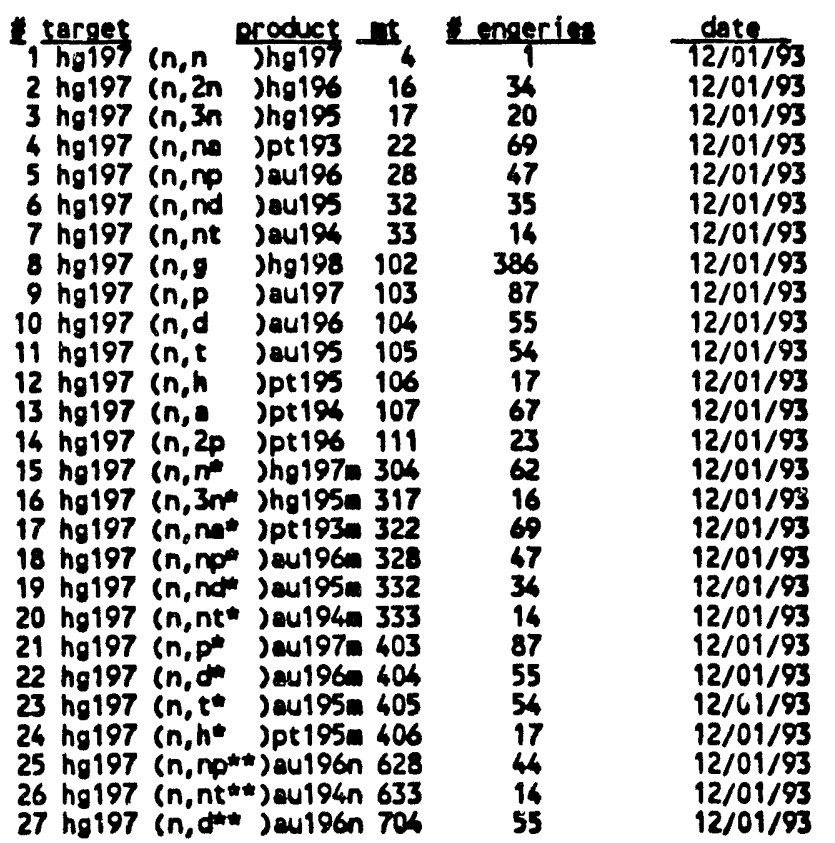




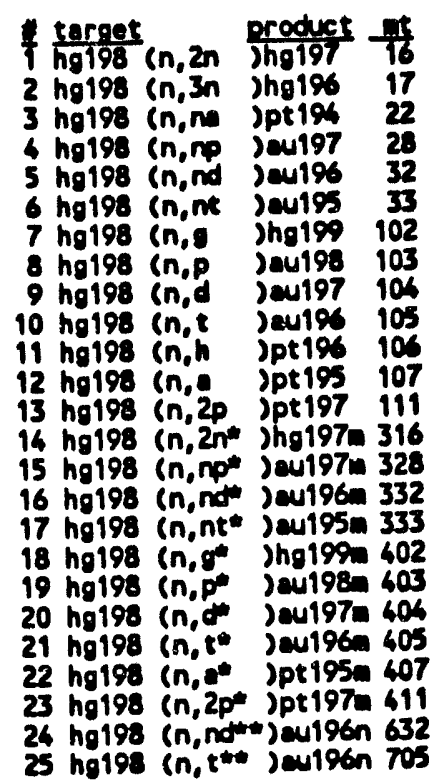

\begin{tabular}{cc} 
congerie & date \\
\hline 31 & $12 / 01 / 93$ \\
21 & $12 / 01 / 93$ \\
61 & $12 / 01 / 93$ \\
46 & $12 / 01 / 93$ \\
28 & $12 / 01 / 93$ \\
16 & $12 / 01 / 93$ \\
1782 & $12 / 01 / 93$ \\
76 & $12 / 01 / 93$ \\
53 & $12 / 01 / 93$ \\
67 & $12 / 01 / 93$ \\
15 & $12 / 01 / 93$ \\
58 & $12 / 01 / 93$ \\
15 & $12 / 01 / 93$ \\
28 & $12 / 01 / 93$ \\
46 & $12 / 01 / 93$ \\
28 & $12 / 01 / 93$ \\
16 & $12 / 01 / 93$ \\
1819 & $12 / 01 / 93$ \\
76 & $12 / 01 / 93$ \\
53 & $12 / 01 / 93$ \\
47 & $12 / 01 / 93$ \\
58 & $12 / 01 / 93$ \\
15 & $12 / 01 / 93$ \\
26 & $12 / 01 / 93$ \\
47 & $12 / 01 / 93$
\end{tabular}

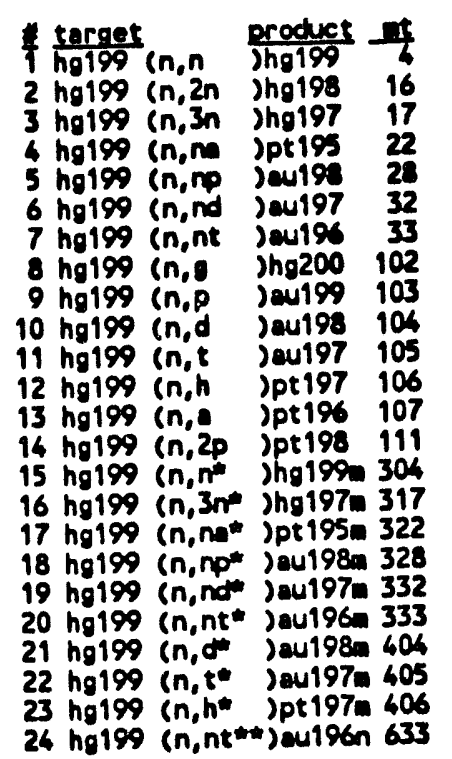

\begin{tabular}{cc} 
Lengerin & date \\
\cline { 2 - 2 } 30 & $12 / 01 / 93$ \\
30 & $12 / 01 / 93$ \\
18 & $12 / 01 / 93$ \\
65 & $12 / 01 / 93$ \\
47 & $12 / 01 / 93$ \\
34 & $12 / 01 / 93$ \\
16 & $12 / 01 / 93$ \\
2373 & $12 / 01 / 93$ \\
82 & $12 / 01 / 93$ \\
54 & $12 / 01 / 93$ \\
52 & $12 / 01 / 93$ \\
13 & $12 / 01 / 93$ \\
61 & $12 / 01 / 93$ \\
17 & $12 / 01 / 93$ \\
66 & $12 / 01 / 93$ \\
18 & $12 / 01 / 93$ \\
65 & $12 / 01 / 93$ \\
42 & $12 / 01 / 93$ \\
33 & $12 / 01 / 93$ \\
16 & $12 / 01 / 93$ \\
54 & $12 / 01 / 93$ \\
52 & $12 / 01 / 93$ \\
13 & $12 / 01 / 93$ \\
16 & $12 / 01 / 93$
\end{tabular}

\begin{tabular}{|c|c|c|c|c|c|}
\hline 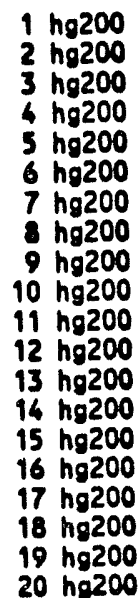 & 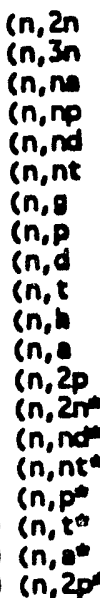 & 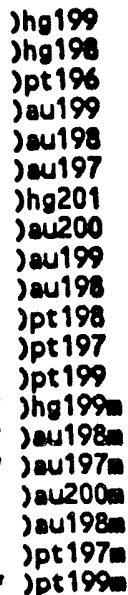 & $\begin{array}{l}16 \\
17 \\
22 \\
28 \\
32 \\
33 \\
102 \\
103 \\
104 \\
105 \\
106 \\
107 \\
111 \\
316 \\
332 \\
333 \\
403 \\
405 \\
407 \\
411\end{array}$ & $\begin{array}{r}30 \\
21 \\
56 \\
67 \\
28 \\
16 \\
1615 \\
70 \\
52 \\
47 \\
11 \\
56 \\
9 \\
29 \\
26 \\
16\end{array}$ & $\begin{array}{l}12 / 01 / 93 \\
12 / 01 / 93 \\
12 / 01 / 93 \\
12 / 01 / 93 \\
12 / 01 / 93 \\
12 / 01 / 93 \\
12 / 01 / 93 \\
12 / 01 / 93 \\
12 / 01 / 93 \\
12 / 01 / 93 \\
12 / 01 / 93 \\
12 / 01 / 93 \\
12 / 01 / 93 \\
12 / 01 / 93 \\
12 / 01 / 93 \\
12 / 01 / 93 \\
12 / 01 / 93 \\
12 / 01 / 93 \\
12 / 01 / 93 \\
12 / 01 / 93\end{array}$ \\
\hline
\end{tabular}




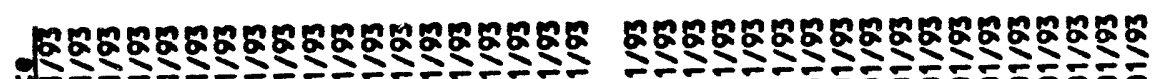

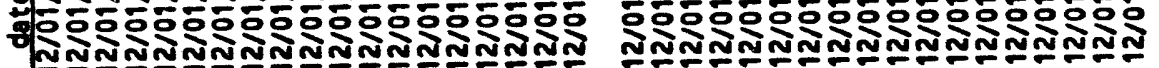

화

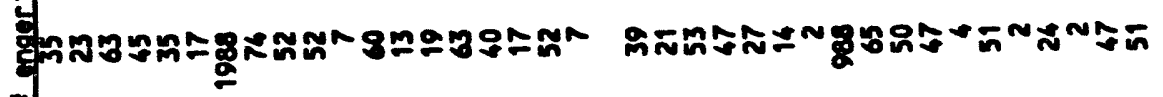

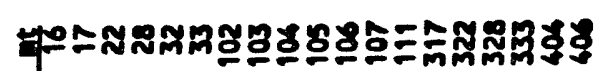

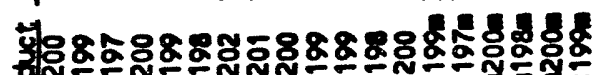

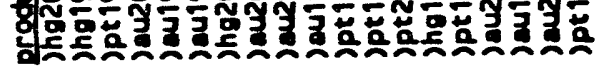

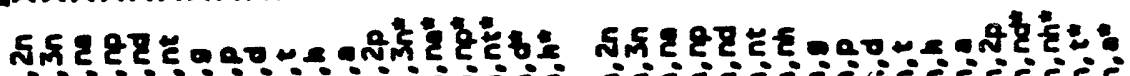

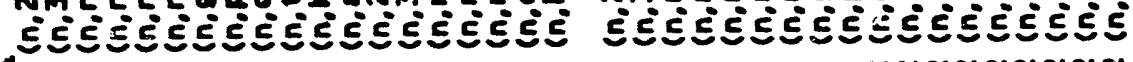

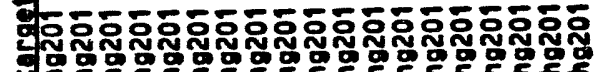

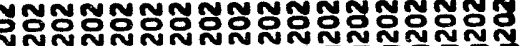

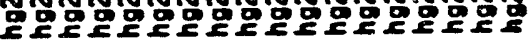

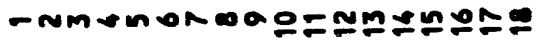

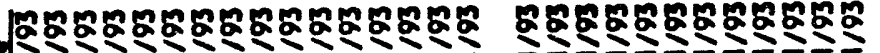

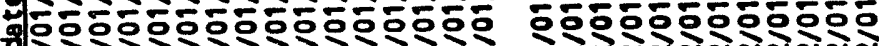

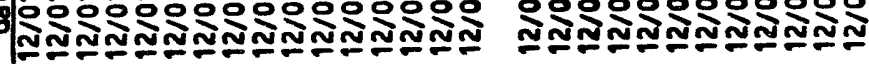

$\stackrel{\sim}{m}$

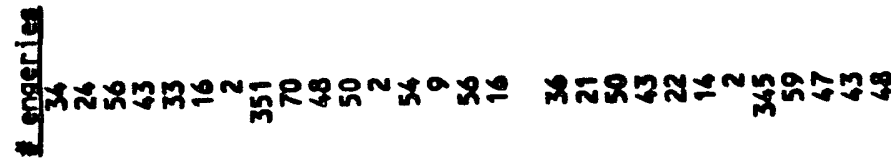

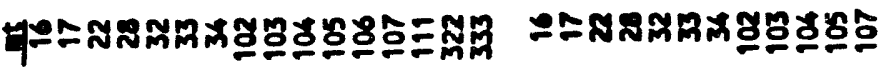

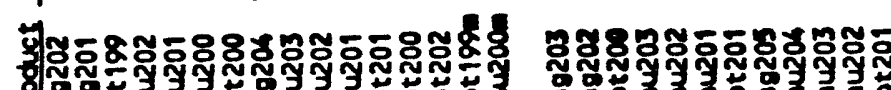

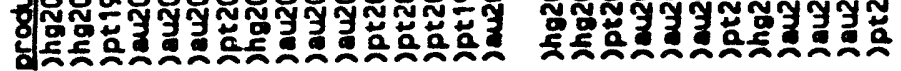

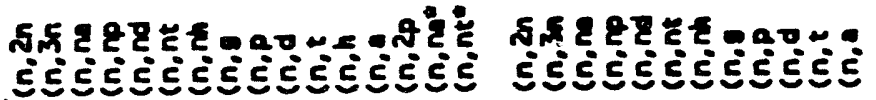

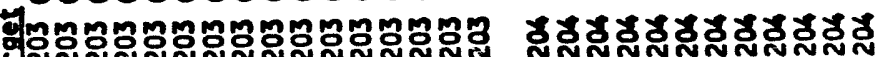

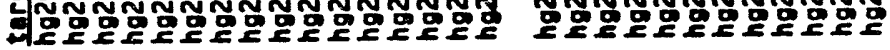

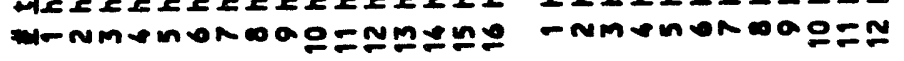




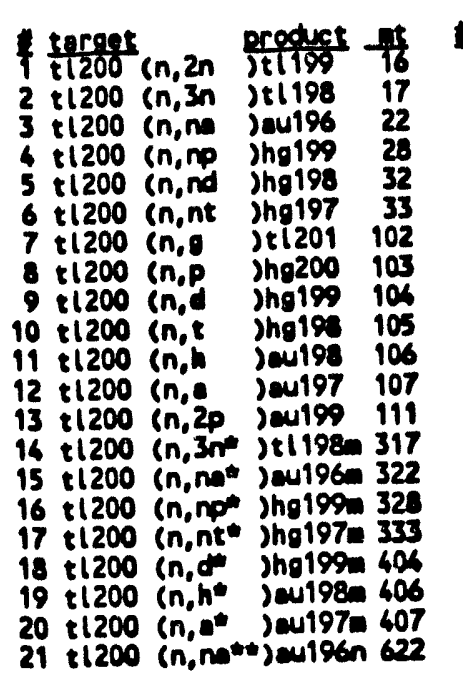

engeries
26
20
67
51
43
23
396
92
59
58
19
68
26
16
66
49
23
59
19
69
67

$\frac{\text { date }}{12 / 01 / 93}$

$12 / 01 / 93$

$12 / 01 / 93$

$12 / 01 / 93$

$12 / 01 / 93$

$12 / 01 / 93$

$12 / 01 / 93$

$12 / 01 / 93$

$12 / 01 / 93$

$12 / 01 / 93$

$12 / 01 / 93$

$12 / 01 / 93$

$12 / 01 / 93$

$12 / 01 / 93$

$12 / 01 / 93$

$12 / 01 / 93$

$12 / 01 / 93$

$12 / 01 / 93$

$12 / 01 / 93$

$12 / 01 / 93$

21 t1200 (n,nat) aulo6n 622

$12 / 01 / 93$

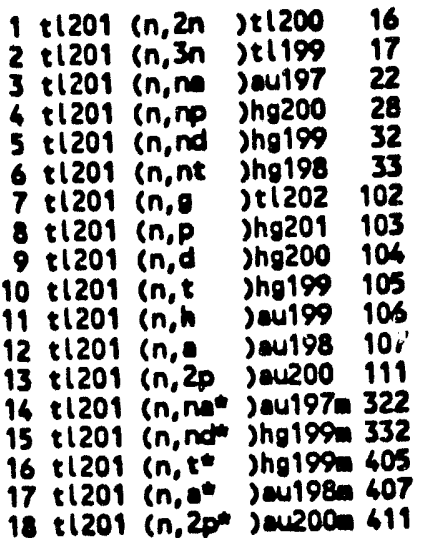

$28 \quad 12 / 01 / 93$

$12 / 01 / 93$

$12 / 01 / 93$

$12 / 01 / 93$

$12 / 01 / 93$

$12 / 01 / 93$

$12 / 01 / 93$

$12 / 01 / 93$

$12 / 01 / 93$

$12 / 01 / 93$

$12 / 01 / 93$

$12 / 01 / 93$

12/01/93

$12 / 01 / 93$

$12 / 01 / 93$

$12 / 01 / 93$

$12 / 01 / 93$

$12 / 01 / 93$
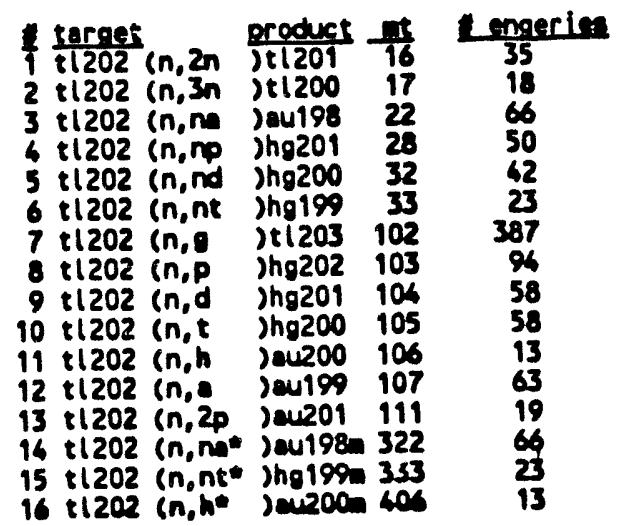

35

18

date

(1/93

$12 / 01 / 93$

$12 / 01 / 93$

$12 / 01 / 93$

$12 / 01 / 93$

$12 / 01 / 93$

$12 / 01 / 93$

$12 / 01 / 93$

$12 / 01 / 93$

$12 / 01 / 93$

$12 / 01 / 93$

$12 / 01 / 93$

$12 / 01 / 93$

$12 / 01 / 93$

$12 / 01 / 93$

$12 / 01 / 93$

1 t1203 (n, 2n ) t1202 16

2 t $1203(n, 3 n) t 1201 \quad 17$

3 t 1203 (n, $n$ jeu199 22

t 1203 (n, no )ha202 28

5 t1203 (n, nd )hg201 32

6 t203 in.nt )hg200 33

7 t1203 (n, )ti204 102

8 tl203 (n,p )hg203 103

9 t1203 (n,d jhg202 104

10 t1203 (n,t jhg201 105

11 t1203 (n, ) e 201106

12 t1203 (n, jaw200 107

13 t1203 (n, 2p ) ou202 111

$16 t 1203\left(n, a^{+}\right)$) 200007407

$12 / 01 / 93$

$12 / 01 / 93$

$12 / 01 / 93$

$12 / 01 / 93$

$12 / 01 / 93$

$12 / 01 / 93$

$12 / 01 / 93$

$12 / 01 / 93$

$12 / 01 / 93$

$12 / 01 / 93$

$12 / 01 / 93$

$12 / 01 / 93$

$12 / 01 / 93$

$12 / 01 / 93$

$A-8.33$ 


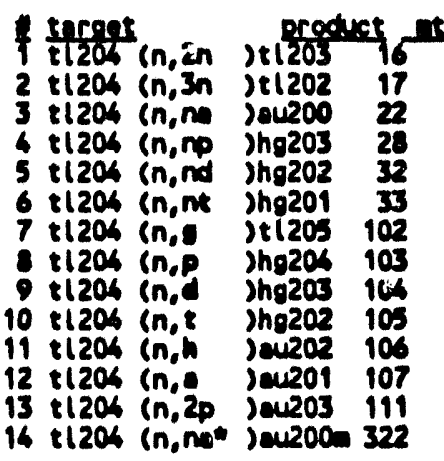

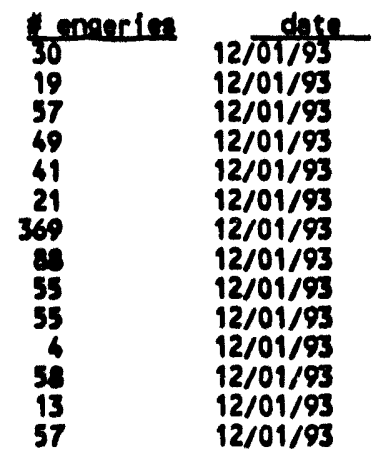

4 target $\frac{\text { product }}{\mathrm{t}(205}$ (n, 2n

2 t1205 (n, 3n $) t 1203 \quad 17$

3 t 1205 (n,m jeupot 22

4 t205 (n,np inges

5 tl20s (n,nd ing203

6 t1205 (n,nt )hg202

7 t1205 (n, nh) aupo2

- tl20s (n.9) tel206 102

9 t1205 in.p jho20s 103

10 : 1205 in.d sha204 104

11 t 1205 (n,t jhges 105

12 ti205 (n,h jeu203 105

13 t 1205 (n, jeu202 107

$14+1205$ (n.2p) sau20 111

15 t 1205 in, je jt1206m 402

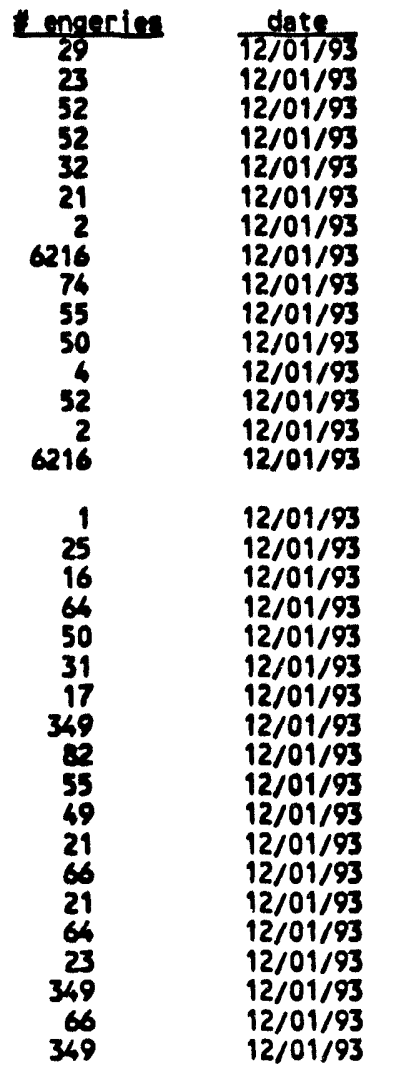

1 pb202 (n,n Jpb202

2 pb202 (n,2n jpb201 16

3 pb20: (n,3n )pb200 17

4 pb202 (n,na )ho198 22

5 pb202 (n,np) )tl201 28

6 pb202 (n,nd jt 120032

7 pb202 (n,nt) t1199 33

- pb202 (n, )ob203 102

9 pb202 (n,p )ti202 103

10 pb202 (n,d )ti201 104

11 b202 (n, )t $1200 \quad 105$

12 pb202 (n,h jhg200 106

13 pb202 (n, )ho199 107

14 pb202 (n,2p )ha201 111

15 pb202 (n, nt)pb202 304

16 pb202 (n,2n )pb201m 316

17 pb202 (n, jpb203m 402

18 pb202 (n, )ho199w 407

19 pb202 (n, g** jpb203n 702

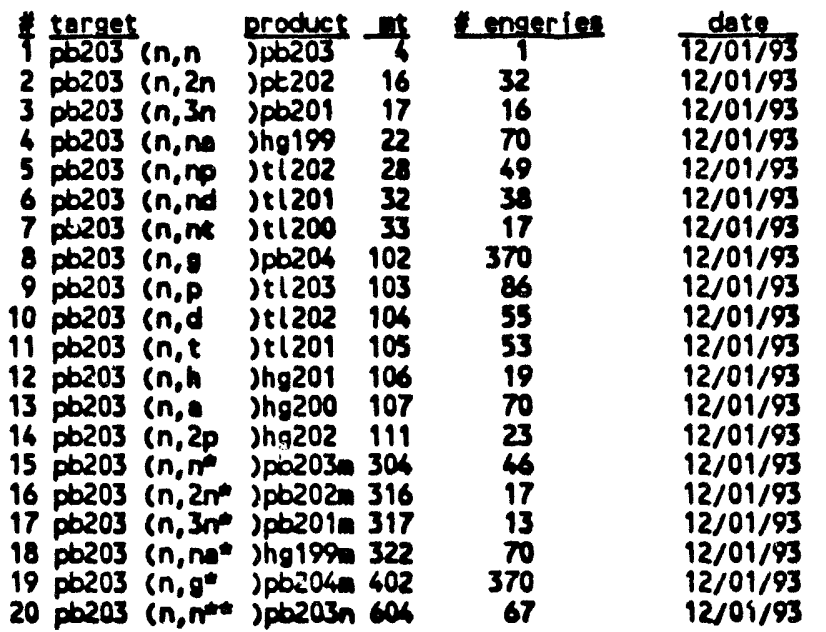

$A-8 \cdot 34$ 


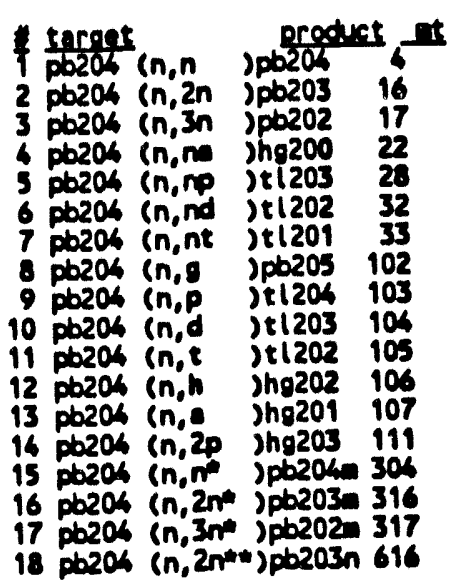

\begin{tabular}{cc} 
temeries & date \\
\hline 1 & $12 / 01 / 93$ \\
22 & $12 / 01 / 93$ \\
6 & $12 / 01 / 93$ \\
60 & $12 / 01 / 93$ \\
49 & $12 / 01 / 93$ \\
32 & $12 / 01 / 93$ \\
16 & $12 / 01 / 93$ \\
4596 & $12 / 01 / 93$ \\
18 & $12 / 01 / 93$ \\
34 & $12 / 01 / 93$ \\
49 & $12 / 01 / 93$ \\
17 & $12 / 01 / 93$ \\
61 & $12 / 01 / 93$ \\
15 & $12 / 01 / 93$ \\
59 & $12 / 01 / 93$ \\
22 & $12 / 01 / 93$ \\
7 & $12 / 01 / 93$ \\
17 & $12 / 01 / 93$
\end{tabular}

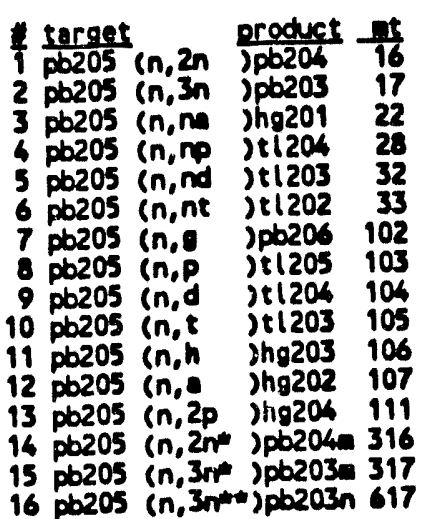

$\begin{array}{cc}\text { Lengerin } & \text { date } \\ 16 & 12 / 01 / 93 \\ 6 & 12 / 01 / 93 \\ 65 & 12 / 01 / 93 \\ 48 & 12 / 01 / 93 \\ 36 & 12 / 01 / 93 \\ 16 & 12 / 01 / 93 \\ 358 & 12 / 01 / 93 \\ 82 & 12 / 01 / 93 \\ 56 & 12 / 01 / 93 \\ 52 & 12 / 01 / 95 \\ 13 & 12 / 01 / 93 \\ 65 & 12 / 01 / 93 \\ 17 & 12 / 01 / 93 \\ 20 & 12 / 01 / 93 \\ 21 & 12 / 01 / 93 \\ 6 & 12 / 01 / 93\end{array}$

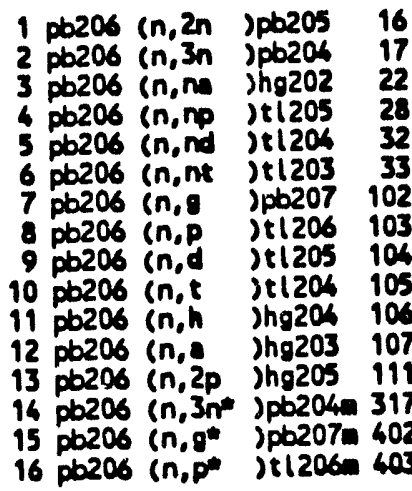

$\begin{array}{rr}22 & 12 / 01 / 93 \\ 6 & 12 / 01 / 93 \\ 56 & 12 / 01 / 93 \\ 48 & 12 / 01 / 93 \\ 29 & 12 / 01 / 93 \\ 16 & 12 / 01 / 93 \\ 7375 & 12 / 01 / 93 \\ 76 & 12 / 01 / 93 \\ 52 & 12 / 01 / 93 \\ 67 & 12 / 01 / 93 \\ 11 & 12 / 01 / 93 \\ 28 & 12 / 01 / 93 \\ 9 & 12 / 01 / 93 \\ 8 & 12 / 01 / 93 \\ 737 & 12 / 01 / 93 \\ 68 & 12 / 01 / 93\end{array}$

\begin{tabular}{|c|c|c|c|c|c|}
\hline $\begin{array}{l}\text { terget } \\
\text { pb207 } \\
\text { pb207 } \\
\text { pb207 } \\
\text { pb207 } \\
\text { pb207 } \\
\text { pb207 } \\
\text { pb207 } \\
\text { pb207 } \\
9 \text { pb207 } \\
0 \text { pb207 } \\
1 \text { pb207 } \\
2 \text { pb207 } \\
3 \text { pb207 } \\
4 \text { pb207 } \\
5 \text { pb207 } \\
6 \text { pb207 } \\
7 \text { pb207 }\end{array}$ & 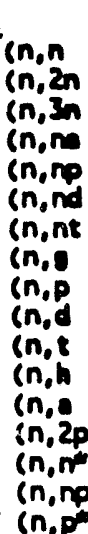 & $\begin{array}{l}\text { product } \\
\text { jpb207 } \\
\text { jpb206 } \\
\text { jpb205 } \\
\text { jhg203 } \\
\text { jt1206 } \\
\text { jt1205 } \\
\text { jt1204 } \\
\text { jpb206 } \\
\text { jt1207 } \\
\text { jti206 } \\
\text { jt1205 } \\
\text { jhg205 } \\
\text { jhg206 } \\
\text { Jhg206 }\end{array}$ & $\begin{array}{c}16 \\
17 \\
22 \\
26 \\
32 \\
33 \\
102 \\
103 \\
106 \\
105 \\
106\end{array}$ & $\begin{array}{r}2091 \\
14 \\
6 \\
57 \\
45 \\
32 \\
11 \\
3768 \\
71 \\
50 \\
50 \\
7 \\
59 \\
9 \\
63 \\
34 \\
71\end{array}$ & $\begin{array}{l}\text { date } \\
2 / 01 / 93 \\
12 / 01 / 93 \\
12 / 01 / 93 \\
12 / 01 / 93 \\
12 / 01 / 93 \\
12 / 01 / 93 \\
12 / 01 / 93 \\
12 / 01 / 93 \\
12 / 01 / 93 \\
12 / 01 / 93 \\
12 / 01 / 93 \\
12 / 01 / 93 \\
12 / 01 / 93 \\
12 / 01 / 93 \\
12 / 01 / 93 \\
12 / 01 / 93 \\
12 / 01 / 93\end{array}$ \\
\hline
\end{tabular}

$A-8.35$ 


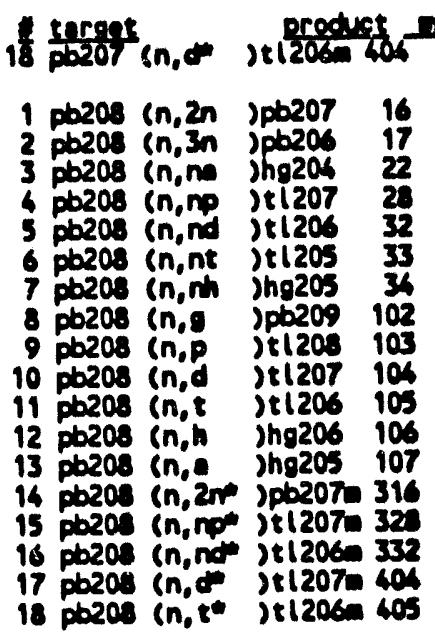

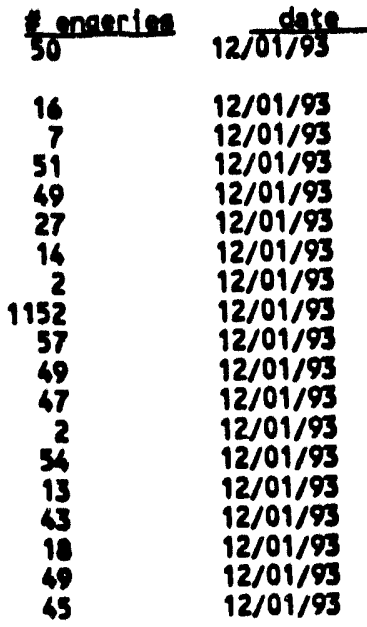

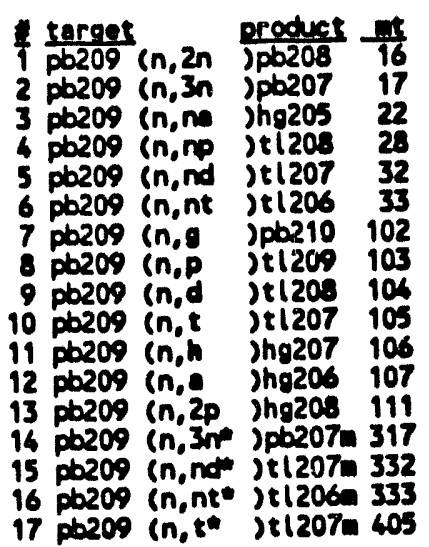

\begin{tabular}{cc} 
Engerien & date \\
\hline 45 & $12 / 01 / 93$ \\
29 & $12 / 01 / 93$ \\
67 & $12 / 01 / 93$ \\
46 & $12 / 01 / 93$ \\
43 & $12 / 01 / 93$ \\
26 & $12 / 1193$ \\
353 & $12 / 91 / 93$ \\
63 & $12 / 01 / 93$ \\
51 & $12 / 01 / 93$ \\
56 & $12 / 01 / 93$ \\
19 & $12 / 01 / 93$ \\
59 & $12 / 01 / 93$ \\
9 & $12 / 01 / 93$ \\
19 & $12 / 01 / 93$ \\
36 & $12 / 01 / 93$ \\
24 & $12 / 01 / 93$ \\
56 & $12 / 01 / 93$ \\
55 & $12 / 01 / 93$ \\
55 & $12 / 01 / 93$ \\
30 & $12 / 01 / 93$ \\
30 & $12 / 01 / 93$ \\
43 & $12 / 01 / 93$ \\
36 & $12 / 01 / 93$ \\
32 & $12 / 01 / 93$ \\
2 & $12 / 01 / 93$ \\
34 & $12 / 01 / 93$ \\
58 & $12 / 01 / 93$ \\
49 & $12 / 01 / 93$ \\
52 & $12 / 01 / 93$ \\
21 & $12 / 01 / 93$ \\
71 & $12 / 01 / 93$ \\
2 & $12 / 01 / 93$ \\
32 &
\end{tabular}

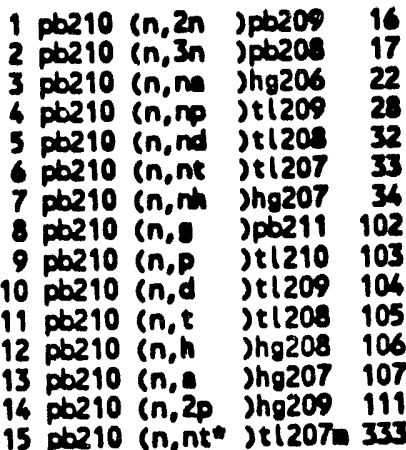

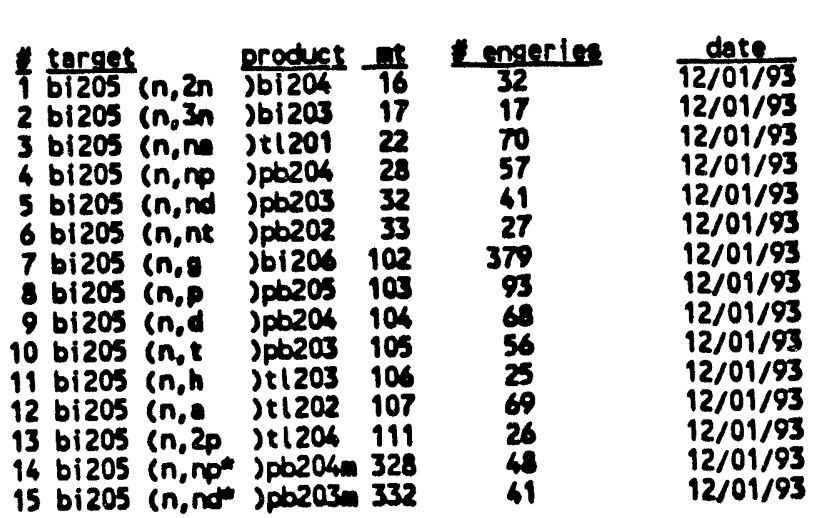

A-8.36 


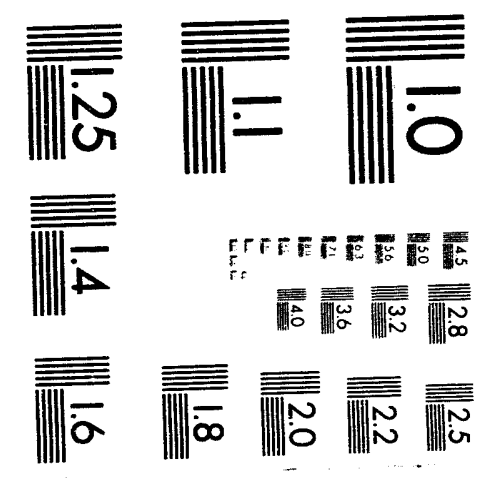



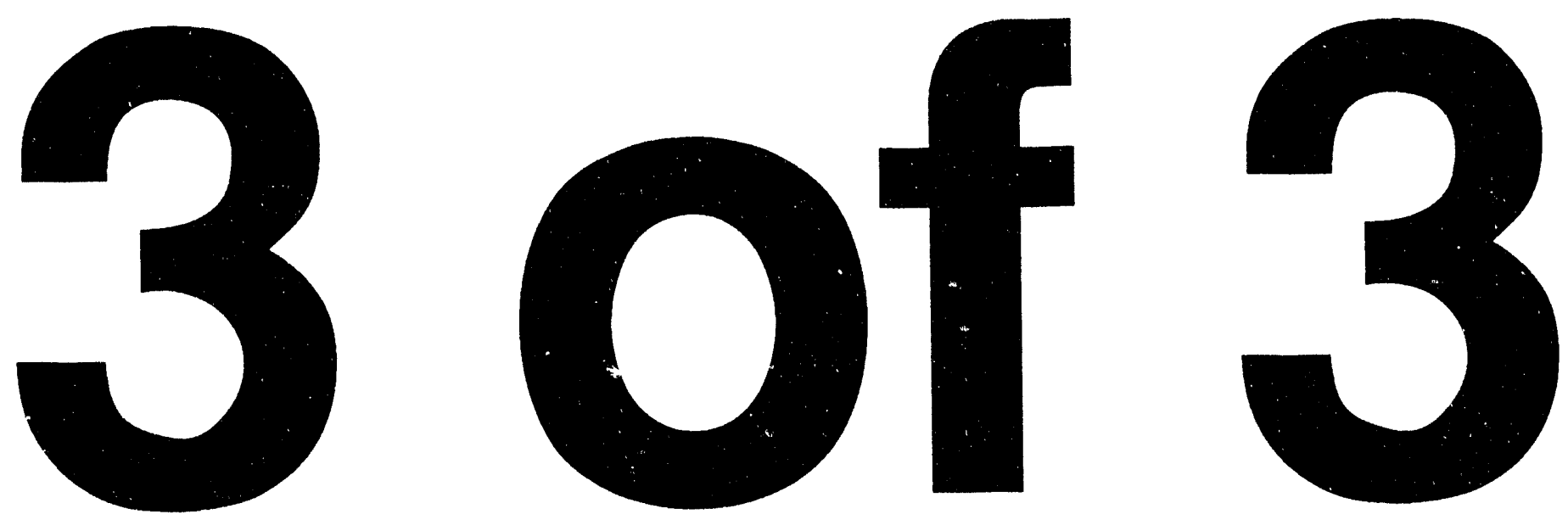


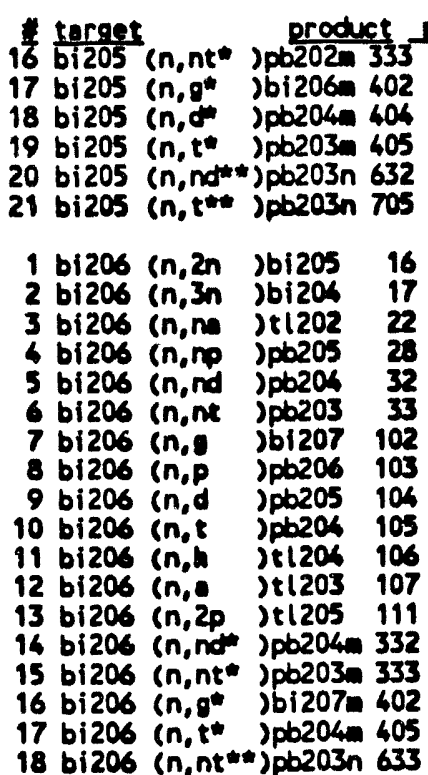

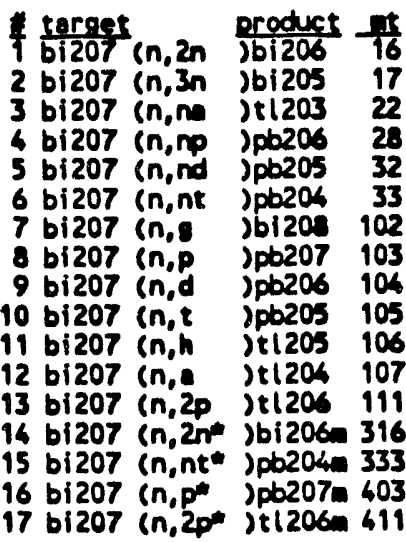

1 bi20s (n, 2n )bizo7 16

2 bi208 (n,3n) bi206 17

3 bi20s (n,m )tl204 22

4 bi20s (n,no jpb207 28

5 bi208 (n,nd jpb206 32

6 bi208 (n,nt )pb2Js 33

7 bi208 (n, )bizos 102

8 bi208 (n,p )pb208 103

9 bi20s in,d jpb207 104

10 bi20s (n,t jpb20s 105

11 bi20s (n, ) ti20s 106

12 bi20s (,.$)$ it 1205107

13 bi20s (n,2p) ti207 11

16 bi20s (n,2r) bi207- 316

15 bi20s (n,3n) bi206m 317

16 bi20s (n,npm)pb207a 328

17 bi20s en,d jpb207 404

18 bi208 (n,h )t 1206 m 406

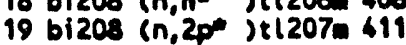

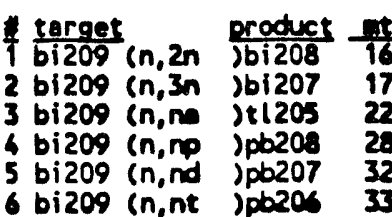

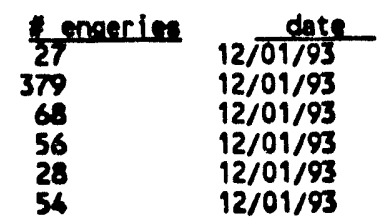

$12 / 01 / 93$

$12 / 01 / 93$

$12 / 01 / 93$

$12 / 01 / 93$

$12 / 01 / 93$

$12 / 01 / 93$

$12 / 01 / 93$

$12 / 01 / 93$

$12 / 01 / 93$

$12 / 01 / 93$

$12 / 01 / 93$

$12 / 01 / 93$

$12 / 01 / 93$

$12 / 01 / 93$

$12 / 01 / 93$

$12 / 01 / 93$

$12 / 01 / 93$

$12 / 01 / 93$

\begin{tabular}{cc} 
2ngerien & date \\
\hline 28 & $12 / 01 / 93$ \\
21 & $12 / 01 / 93$ \\
67 & $12 / 01 / 93$ \\
55 & $12 / 01 / 93$ \\
61 & $12 / 01 / 93$ \\
27 & $12 / 01 / 93$ \\
366 & $12 / 01 / 93$ \\
92 & $12 / 01 / 93$ \\
65 & $12 / 01 / 93$ \\
56 & $12 / 01 / 93$ \\
21 & $12 / 01 / 93$ \\
68 & $12 / 01 / 93$ \\
23 & $12 / 01 / 93$ \\
28 & $12 / 01 / 93$ \\
27 & $12 / 01 / 93$ \\
92 & $12 / 01 / 93$ \\
23 & $12 / 01 / 93$ \\
36 & $12 / 01 / 93$ \\
18 & $12 / 01 / 93$ \\
69 & $12 / 01 / 93$ \\
56 & $12 / 01 / 93$ \\
45 & $12 / 01 / 93$ \\
26 & $12 / 01 / 93$ \\
363 & $12 / 01 / 93$ \\
90 & $12 / 01 / 93$ \\
64 & $12 / 01 / 93$ \\
59 & $12 / 01 / 93$ \\
19 & $12 / 01 / 93$ \\
70 & $12 / 01 / 93$ \\
22 & $12 / 01 / 93$ \\
21 & $12 / 01 / 93$ \\
18 & $12 / 01 / 93$ \\
47 & $12 / 01 / 93$ \\
64 & $12 / 01 / 93$ \\
19 & $12 / 01 / 93$ \\
22 & $12 / 01 / 93$
\end{tabular}

date

12/01/93

$12 / 01 / 93$

$12 / 01 / 95$

$12 / 01 / 93$

$12 / 01 / 93$

$12 / 01 / 93$

$A-8.37$ 


\begin{tabular}{|c|c|c|c|c|c|}
\hline $\begin{array}{l}\text { target } \\
7 \text { bi209 } \\
8 \text { bi209 } \\
9 \text { bi209 } \\
10 \text { bi209 } \\
11 \text { bi } 209 \\
12 \text { bi209 } \\
13 \text { bi209 } \\
14 \text { bi } 209 \\
15 \text { bi209 } \\
16 \text { bi209 } \\
17 \text { bi } 209 \\
18 \text { bi209 } \\
19 \text { bi } 209\end{array}$ & 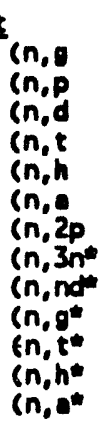 & 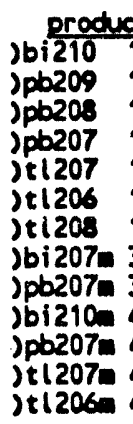 & $\begin{array}{l}\frac{c t}{102}= \\
103 \\
104 \\
105 \\
106 \\
107 \\
111 \\
317 \\
332 \\
402 \\
405 \\
406 \\
407\end{array}$ & $\begin{array}{c}4 \text { enqeries } \\
1168 \\
76 \\
62 \\
58 \\
17 \\
65 \\
9 \\
10 \\
34 \\
1168 \\
58 \\
17 \\
65\end{array}$ & $\begin{array}{l}\frac{\text { date }}{12 / 01 / 93} \\
12 / 01 / 93 \\
12 / 01 / 93 \\
12 / 01 / 93 \\
12 / 01 / 93 \\
12 / 01 / 93 \\
12 / 01 / 93 \\
12 / 01 / 93 \\
12 / 01 / 93 \\
12 / 01 / 93 \\
12 / 01 / 93 \\
12 / 01 / 93 \\
12 / 01 / 93\end{array}$ \\
\hline $\begin{array}{l}1 \text { bi210 } \\
2 \text { bi210 } \\
3 \text { bi210 } \\
4 \text { bi210 } \\
5 \text { bi210 } \\
6 \text { bi210 } \\
7 \text { bi210 } \\
8 \text { bi210 } \\
9 \text { bi210 } \\
10 \text { bi210 } \\
11 \text { bi210 } \\
12 \text { bi210 } \\
13 \text { bi210 } \\
14 \text { bi210 } \\
15 \text { bi210 } \\
16 \text { bi210 } \\
17 \text { bi210 } \\
18 \text { bi210 }\end{array}$ & 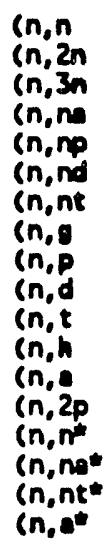 & $\begin{array}{l}\text { Jbi210 } \\
\text { jbi209 } \\
\text { Jbi20s } \\
\text { jt1206 } \\
\text { jpb200 } \\
\text { jpb208 } \\
\text { jpb207 } \\
\text { jbi211 } \\
\text { jpb210 } \\
\text { jpb209 } \\
\text { jpb208 } \\
\text { jt1208 } \\
\text { jt1207 } \\
\text { jt } t 209 \\
\text { jbi210 } \\
\text { jt1206m } \\
\text { jpb207m } \\
\text { jt1207 }\end{array}$ & $\begin{array}{l}4 \\
16 \\
17 \\
22 \\
28 \\
32 \\
33 \\
102 \\
103 \\
104 \\
105 \\
106 \\
107 \\
111 \\
304 \\
322 \\
333 \\
407\end{array}$ & $\begin{array}{r}1 \\
36 \\
27 \\
81 \\
58 \\
56 \\
36 \\
370 \\
83 \\
64 \\
68 \\
13 \\
76 \\
11 \\
116 \\
81 \\
36 \\
76\end{array}$ & $\begin{array}{l}12 / 01 / 93 \\
12 / 01 / 93 \\
12 / 01 / 93 \\
12 / 01 / 93 \\
12 / 01 / 93 \\
12 / 01 / 93 \\
12 / 01 / 93 \\
12 / 01 / 93 \\
12 / 01 / 93 \\
12 / 01 / 93 \\
12 / 01 / 93 \\
12 / 01 / 93 \\
12 / 01 / 93 \\
12 / 01 / 93 \\
12 / 01 / 93 \\
12 / 01 / 93 \\
12 / 01 / 93 \\
12 / 01 / 93\end{array}$ \\
\hline 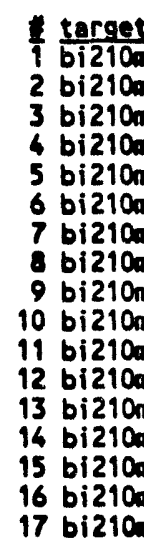 & & 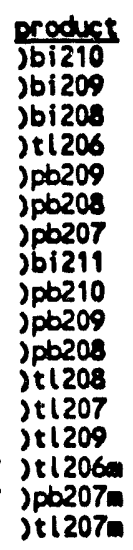 & $\begin{array}{l}n \\
6 \\
16 \\
17 \\
22 \\
28 \\
32 \\
33 \\
102 \\
103 \\
104 \\
105 \\
106 \\
107 \\
111 \\
322 \\
333 \\
407\end{array}$ & $\begin{array}{c}\text { moeries } \\
60 \\
36 \\
27 \\
81 \\
58 \\
54 \\
36 \\
370 \\
83 \\
64 \\
68 \\
13 \\
76 \\
11 \\
81 \\
36 \\
76\end{array}$ & $\begin{array}{c}\text { date } \\
12 / 01 / 93 \\
12 / 01 / 93 \\
12 / 01 / 93 \\
12 / 01 / 93 \\
12 / 01 / 93 \\
12 / 01 / 93 \\
12 / 01 / 93 \\
12 / 01 / 93 \\
12 / 01 / 93 \\
12 / 01 / 93 \\
12 / 01 / 93 \\
12 / 01 / 93 \\
12 / 01 / 93 \\
12 / 01 / 93 \\
12 / 01 / 93 \\
12 / 01 / 93 \\
12 / 01 / 93\end{array}$ \\
\hline 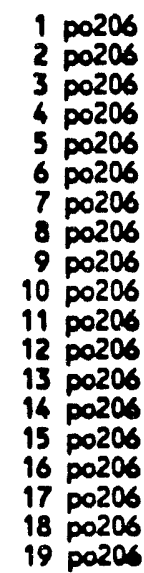 & 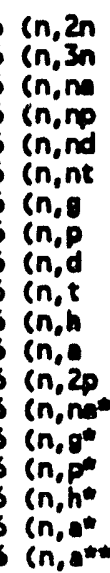 & $\begin{array}{l}\text { jpo205 } \\
\text { jpo204 } \\
\text { jpb202 } \\
\text { jbi205 } \\
\text { jbi204 } \\
\text { jbi203 } \\
\text { jpo207 } \\
\text { jbi206 } \\
\text { jbi205 } \\
\text { jbi204 } \\
\text { jpb204 } \\
\text { jpb203 } \\
\text { jpb205 } \\
\text { jpb2022 } \\
\text { jpo2077 } \\
\text { jbi2066 } \\
\text { jpb2044 } \\
\text { jpb2033 } \\
\text { jpb203n }\end{array}$ & $\begin{array}{l}16 \\
17 \\
22 \\
28 \\
32 \\
33 \\
102 \\
103 \\
104 \\
105 \\
106 \\
107 \\
111 \\
322 \\
402 \\
403 \\
406 \\
407 \\
707\end{array}$ & $\begin{array}{r}27 \\
15 \\
79 \\
57 \\
36 \\
19 \\
342 \\
94 \\
59 \\
54 \\
33 \\
81 \\
29 \\
79 \\
342 \\
44 \\
33 \\
80 \\
81\end{array}$ & $\begin{array}{l}12 / 01 / 93 \\
12 / 01 / 93 \\
12 / 01 / 93 \\
12 / 01 / 93 \\
12 / 01 / 93 \\
12 / 01 / 93 \\
12 / 01 / 93 \\
12 / 01 / 93 \\
12 / 01 / 93 \\
12 / 01 / 93 \\
12 / 01 / 93 \\
12 / 01 / 93 \\
12 / 01 / 93 \\
12 / 01 / 93 \\
12 / 011993 \\
12 / 011 / 93 \\
12 / 011 / 93 \\
121011 / 93 \\
12 / 01 / 93\end{array}$ \\
\hline
\end{tabular}




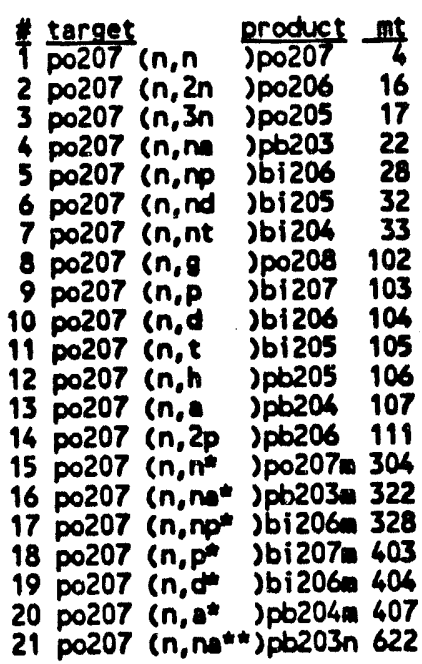

\begin{tabular}{c}
\hline engeries \\
\hline 1 \\
27 \\
20 \\
88 \\
55 \\
63 \\
21 \\
361 \\
92 \\
60 \\
56 \\
31 \\
85 \\
31 \\
56 \\
88 \\
55 \\
92 \\
60 \\
85 \\
88
\end{tabular}

date

$2 / 01 / 93$

$12 / 01 / 93$

$12 / 01 / 93$

$12 / 01 / 93$

$12 / 01 / 93$

$12 / 01 / 93$

$12 / 01 / 93$

$12 / 01 / 93$

$12 / 01 / 93$

$12 / 01 / 93$

$12 / 01 / 93$

$12 / 01 / 93$

$12 / 01 / 93$

$12 / 01 / 93$

$12 / 01 / 93$

$12 / 01 / 93$

$12 / 01 / 93$

$12 / 01 / 93$

$12 / 01 / 93$

$12 / 01 / 93$

1 po208 (n, 2n )po207 16

2 p0208 (n,3n )po206 17

3 p0208 (n,n) )pb204 22

4 po208 (n,np )bi207 28

5 p0208 ( $n$, nd ) bi206 32

6 po208 (n,nt )biz05 33

7 po208 (n,g)po209 102

8 po208 (n,p jbi208 103

9 po208 (n,d jbi207 106

10 po208 (n,t jbi206 105

11 po208 (n,h jpb206 106

12 po208 (n, ) pb205 107

13 po208 (n,2p)pb207 111

14 po208 in, 2n* )po207m 316

15 po208 (n,na*)pb204m 322

16 po208 (n,np )bi207m 328

17 po208 (n, nd ) bi206m 332

18 po208 (n,d) jbi207m 404

19 po208 (n,t) Jbi206m 405

20 po208 (n,2p")pb207m 411

$32 \quad 12 / 01 / 93$

$12 / 01 / 93$

$12 / 01 / 93$

$12 / 01 / 93$

$12 / 01 / 93$

$12 / 01 / 93$

$12 / 01 / 93$

$12 / 01 / 93$

$12 / 01 / 93$

$12 / 01 / 93$

$12 / 01 / 93$

$12 / 01 / 93$

$12 / 01 / 93$

$12 / 01 / 93$

$12 / 01 / 93$

$12 / 0 i / 93$

$12 / 01 / 93$

$12 / 01 / 93$

$12 / 01 / 93$

$12 / 01 / 93$

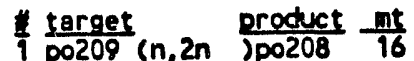

2 po209 (n,3n)po207 17

3 po209 (n,n) pb205 22

4 po209 (n,np jbi208 28

5 po209 (n,nd jbi207 32

6 po209 (n,nt) bi206 33

7 po209 (n,s)po210 102

8 po209 (n,p jbi209 103

9 po209 (n,d )bi208 104

10 po209 in,t jbi207 105

11 po209 (n,h jpb207 106

12 po209 (n, )pb206 107

13 po209 (n,20 )pb208 111

14 p0209 $(n, 3 n *)$ po207m 317

15 p209 (n,nd )bi207- 332

16 po209 (n,nt* ibi206m 333

17 po209 (n,t * jbi207m 405

18 po209 (n,h* )pb207m 406

\begin{tabular}{cc} 
Eengeries & date \\
\cline { 2 - 2 } 28 & $12 / 01 / 93$ \\
22 & $12 / 01 / 93$ \\
82 & $12 / 01 / 93$ \\
56 & $12 / 01 / 93$ \\
41 & $12 / 01 / 93$ \\
18 & $12 / 01 / 93$ \\
335 & $12 / 01 / 93$ \\
93 & $12 / 01 / 93$ \\
59 & $12 / 01 / 93$ \\
57 & $12 / 01 / 93$ \\
29 & $12 / 01 / 93$ \\
80 & $12 / 01 / 93$ \\
27 & $12 / 01 / 93$ \\
12 & $12 / 01 / 93$ \\
31 & $12 / 01 / 93$ \\
18 & $12 / 01 / 93$ \\
57 & $12 / 01 / 93$ \\
29 & $12 / 01 / 93$
\end{tabular}

1 po210 (n,2n )po209 16

2p0210 (n,3n)po208 17

3 po210 (n,na )pb206 22

4 po210 (n,np jbi209 28

5 po210 (n,nd)bizos 32

6 po210 (n,nt )bi207 33

7 po210 (n,g jpo211 102

8 po210 (n,p bi210 103

9 po210 (n,d jbizos 104

10 p0210 (n,t Jbiz0s 105

$\begin{array}{rr}31 & 12 / 01 / 93 \\ 23 & 12 / 01 / 93 \\ 75 & 12 / 01 / 93 \\ 57 & 12 / 01 / 93 \\ 38 & 12 / 01 / 93 \\ 20 & 12 / 01 / 93 \\ 350 & 12 / 01 / 93 \\ 76 & 12 / 01 / 93 \\ 58 & 12 / 01 / 93 \\ 53 & 12 / 01 / 93\end{array}$

$A-8.39$ 
WHC-EP-0727

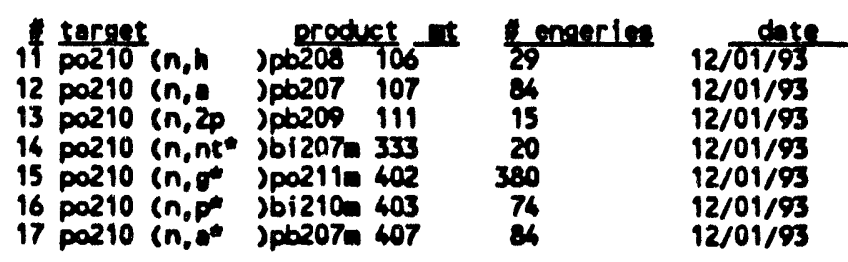


WHC-EP-0727

\section{DISTRIBUTION}

\section{Number of copies}

\section{ONSITE}

U.S. Department of Enerqy-

Richland Operations office

5 D. D. Green (5) K8-50

Westinghouse Hanford Company

35 F. M. Mann (32) HO-36

Information Release
Administration (3) L8-07

Distr-1 


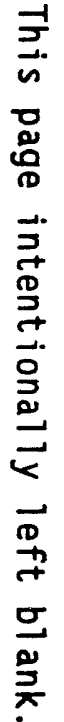

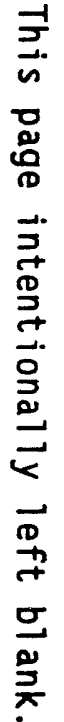

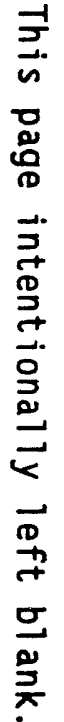

1 

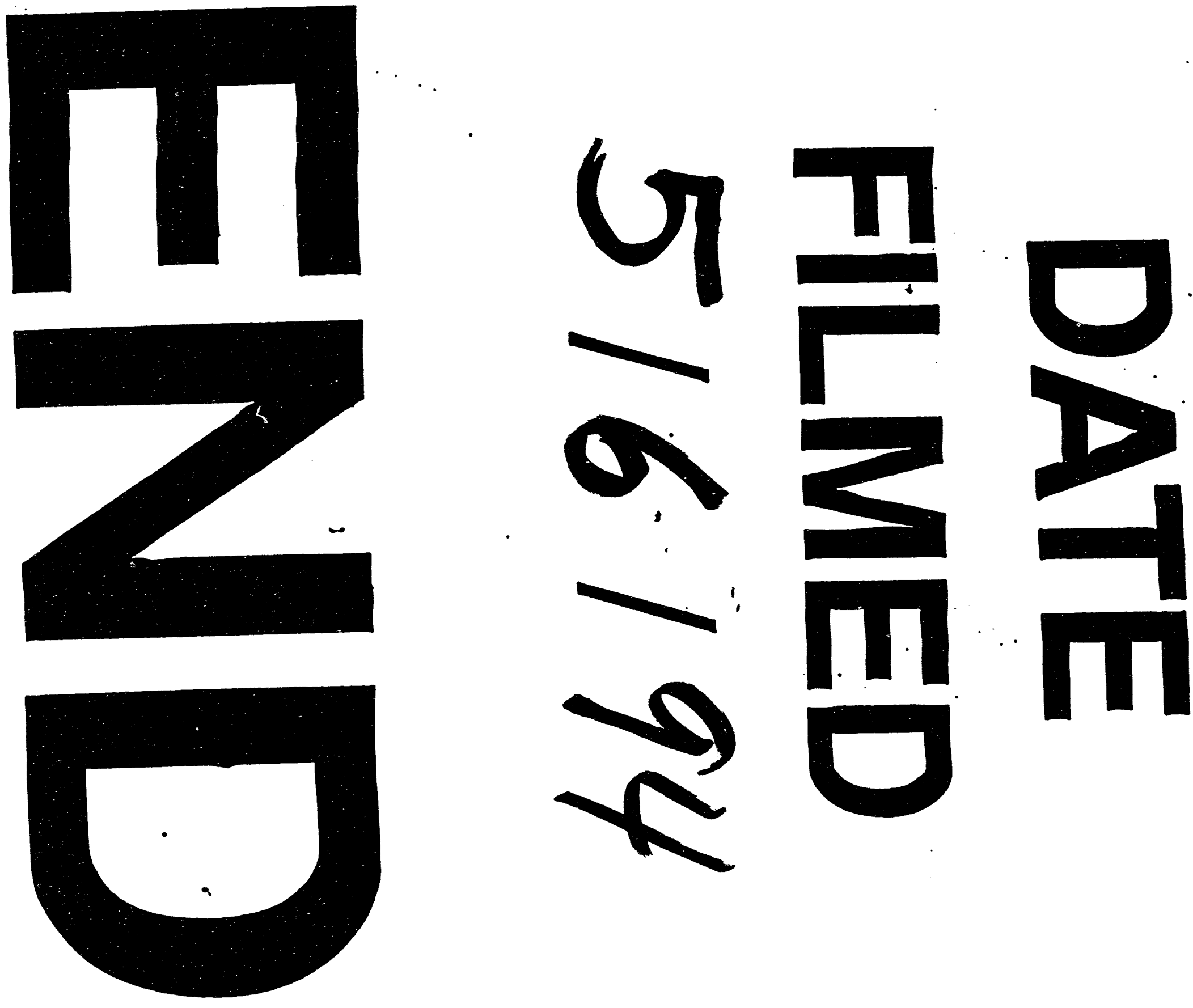

II

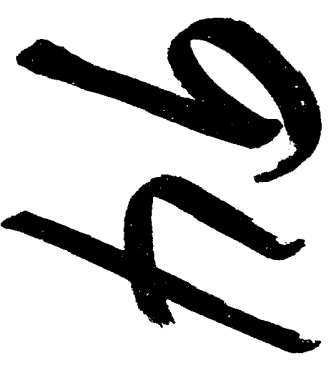




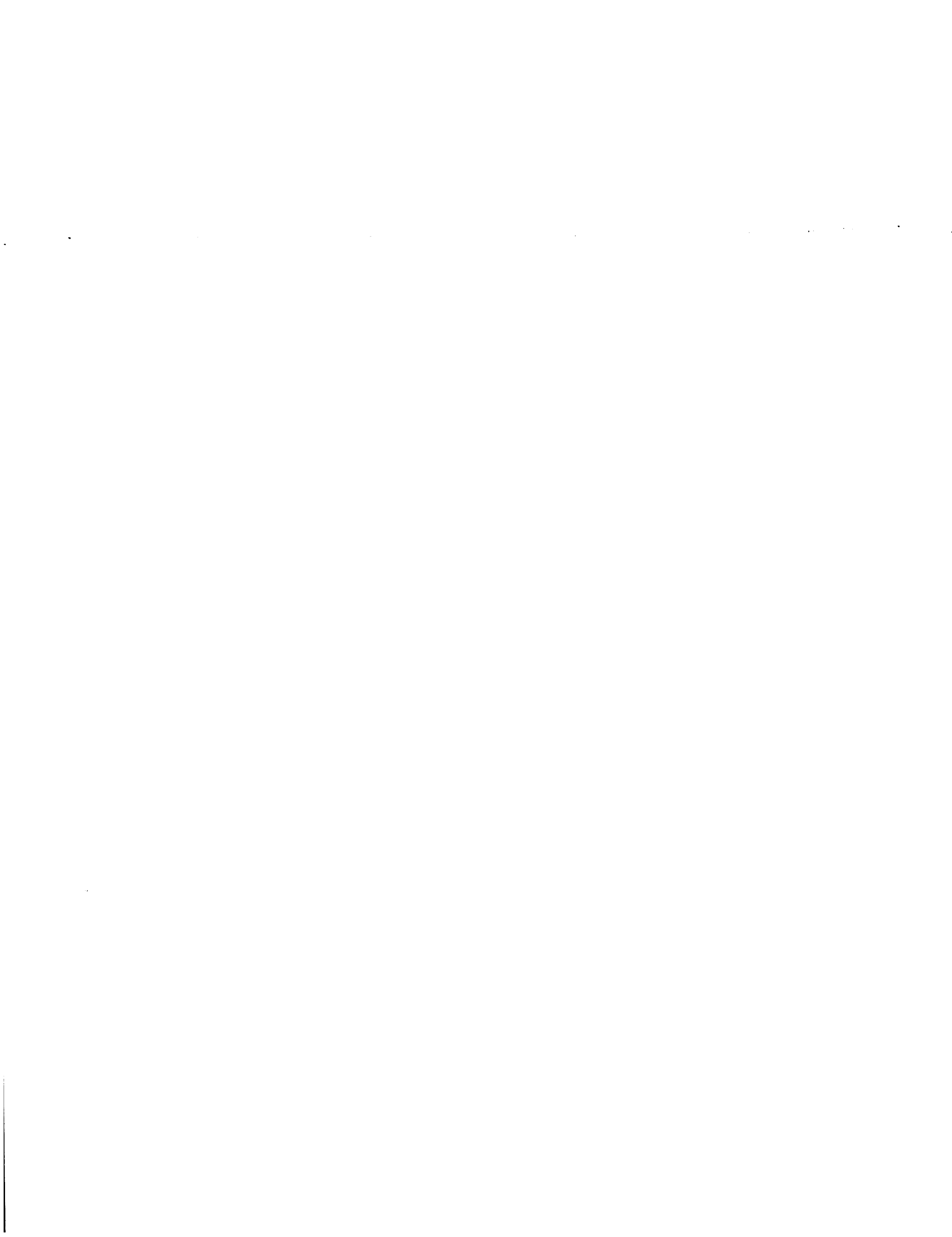

